\title{
Assessing the Air Pollution Carrying Capacity of the Northern Puget Sound Region: An Application of TAPAS
}

September 1980

Prepared for the

U.S. Department of Energy Assistant Secretary for Environment Office of Environmental Assessments under Contract DE-AC06-76RLO 1830 


\section{NOTICE}

This repon was prepared as an account of work sponsored by the United States Government. Neither the United States nor the Department of Energy, nor any of their employees, nor any of their contractors, subcontractors. or their employees, makes any warranty, express or implied, or assumes any legal liability or responsibility for the accuracy. completeness or usefulness of any information, apparatus, product or process disclosed, or represents that its use would not infringe privately owned rights.

The views, opinions and conclusions contained in this report are those of the contractor and do not necessarily represent those of the United States Government or the United States Department of Energy.
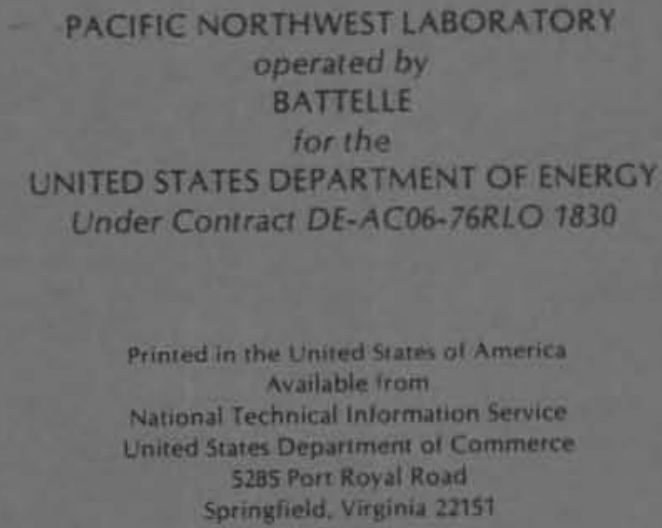

Price: Printed Copy 5 $\therefore$ Microfiche $\$ 3.00$

* Pages Seliing Price

$\begin{array}{ll}001-025 & \$ 4.00 \\ 026-050 & \$ 4.50 \\ 051-075 & \$ 5.25 \\ 076-100 & \$ 6.00 \\ 101-125 & \$ 6.30 \\ 126-150 & \$ 7.25 \\ 157-175 & \$ 6.00 \\ 176-200 & 59.00 \\ 207-225 & \$ 9.25 \\ 226-250 & 59.50 \\ 251-275 & 510.75 \\ 276-300 & \$ 11.00\end{array}$


ASSESSING THE AIR POLLUTION CARRYING CAPACITY

OF THE NORTHERN PUGET SOUND REGION:

AN APPLICATION OF TAPAS

September 1980

Prepared by Martha C. Wood Joseph E. Childs William E. Marlatt

of Marlatt \& Associates

3611 Richmond Drive

Fort Collins, Colorado 80526

Prepared for the

U.S. Department of Energy Assistant Secretary for Environment Office of Environmental Assessments under Contract DE-AC06-76RLO 1830

Pacific Northwest Laboratory

Richland, Washington 99352 


\section{ACKNOWLEDGEMENTS}

The work reported here was conducted under the sponsorship of and with the funding from the U.S. Department of Energy, Office of Environmental Assessments, Regional Impacts Division through Pacific Northwest Laboratory. The Pacific Northwest Laboratory Project Manager was Dave S. Renne. The authors wish to acknowledge the support of the Division, managed by Dr. Roger D. Shu11, under Contract DE-ACO6-76RL0-1830. 

LIST OF TABLES ..................... v v LIST OF FIGURES ..................... . . . . . . . . EXECUTIVE SUMMARY .................... X xi

I. INTRODUCTION . . . . . . . . . . . . . . . . . 1

II. DATA COLLECTION ................. 11 III. MODEL DESCRIPTIONS . . . . . . . . . . 25 WINDS .................. . . 25

Input . . . . . . . . . . . . . 25

Output . . . . . . . . . . . . . . . 34

TOPOGRAPHIC AIR POLLUTION ANALYSIS SYSTEM (TAPAS) • • . 34

Input . . . . . . . . . . . . . . . 35

Output ........................... 36

IV. RESULTS . . . . . . . . . . . . . . 39

WIND FLOW SIMULATION ANALYSES . . . . . . . . . 40

January 3,1975 . . . . . . . . . . . . 57

January 24,1975 . . . . . . . . . . . . 58

January 30,1975 . . . . . . . . . . . 60

February 12,1975 . . . . . . . . . . . 61

AIR QUALITY EMISSION LIMITATION ANALYSES . . . . . . 62

Daily Cases ................. 65

Annual Case . . . . . . . . . . . . . 73

DISCUSSION OF RESULTS . . . . . . . . . . . . 82

Wind Flow Simulation . . . . . . . . . . . 82

TAPAS Results . . . . . . . . . . . . . 85 
V. SUMMARY AND CONCLUSIONS . . . . . . . . . . . 89

VI. REFERENCES ..................... . . 95

APPENDIX A: Estimating Airflow Patterns Over Complex Terrain

APPENDIX B: Topographic Analysis System (TOPAS)

APPENDIX C: Wind Flow Patterns in Northeastern Washington

APPENDIX D: A Topographic Air Pollution Analysis System

APPENDIX E: Data and Results for Daily and Annual Cases 
LIST OF TABLES

Table

$\underline{\text { Page }}$

1. Air Quality Standards for $\mathrm{SO}_{2}$. . . . . . . .

2

2. Total Suspended Particulate Levels Measured at Mount Vernon and Lake Terrell in 1975 and 1976 . . . . . .

3. Stations Exceeding 3-Hour Average Limit of 50 Parts Per Hundred Million $\mathrm{SO}_{2}$ or 24-Hour Average Limit of 10 Parts Per Hundred Million $\mathrm{SO}_{2}$ in 1975 and 1976 . . . . .

4. Major Sources of $\mathrm{SO}_{2}$ in the Northern Puget Sound Area in 1976

5. Locations From Which Meteorological Data Were Obtained for Model Validation . . . . . . . . . . . . .

6. Seasonal Cycle of Winds Along Washington Coast for Five Year Period (1961-1965) . . . . . . . . . . .

7. Vegetation Types, Associated Map Codes and Roughness Lengths Used for WINDS Model Input . . . . . . . .

8. Final Input Data Used in Wind Flow Simulation . . . .

9. Comparison of Winds Results With Observed Data -January 3, 1975 . . . . . . . . . . . . . .

10. Fraction of the Predicted Values Within a Given Number of Compass Points or Meters per Second of Observed Values for January 3, 1975 Simulation . . . . . .

11. Analysis of Simulation Accuracy for Each Station for All January 3, 1975 Cases ..............

12. Comparison of Ferndale Stations and $850 \mathrm{mb}$. Wind Flow From Quillayute Soundings with Background Flow Used as Final Input to WINDS Model (Jan. 3, 1975 Simulation) .

13. Comparison of Winds Results With Observed Data -January 24,1975 . . . . . . . . . . . . .

14. Fraction of the Predicted Values Within a Given Number of Compass Points or Meters per Second of Observed Values for January 24, 1975 Simulation . . . . . . .

15. Analysis of Simulation Accuracy for Each Station for All January 24, 1975 Cases . . . . . . . . . . . 
(Continued)

16. Comparison of Ferndale Stations and $850 \mathrm{mb}$. Wind Flow From Quillayute Soundings with Background Flow Used as Final Input to WINDS Model (Jan. 24, 1975 Simulation) .

17. Comparison of Winds Results With Observed Data -January 30, 1975 . . . . . . . . . . . . .

18. Fraction of the Predicted Values Within a Given Number of Compass Points or Meters per Second of Observed Values for January 30, 1975 Simulation . . . . . .

19. Analysis of Simulation Accuracy for Each Station for All January 30, 1975 Cases ............

20. Comparison of Ferndale Stations with $850 \mathrm{mb}$. Wind Flow From Quillayute Soundings with Background Flow Used as Final Input to WINDS Model (Jan. 30, 1975 Simulation) •

21. Comparison of Winds Results With Observed Data -February 12, 1975 . . . . . . . . . . . . .

22. Fraction of the Predicted Values Within a Given Number of Compass Points or Meters per Second of Observed Values for February 12, 1975 Simulation . . . . . .

23. Analysis of Simulation Accuracy for Each Station for All February 12, 1975 Cases . . . . . . . . .

24. Comparison of Ferndale Stations and $850 \mathrm{mb}$. Wind Flow From Quillayute Soundings with Background Flow Used as Final Input to WINDS Model (Feb. 12, 1975 Simulation) .

25. Major $\mathrm{SO}_{2}$ Source Grid Locations for Northern Puget Sound Study Area . . . . . . . . . . . . . . .

26. Emission Strength That Would Result in Ambient $\mathrm{SO}_{2}$ Concentrations Equal to Short and Long Term Standard Limitations (.40 ppm and $.10 \mathrm{ppm}$, respectively) at Locations Indicated -- January 3, 1975 Simulation . . .

27. Emission Strength That Would Result in Ambient $\mathrm{SO}_{2}$ Concentrations Equal to Short and Long Term Standard Limitations (.40 ppm and $.10 \mathrm{ppm}$, respectively) at Locations Indicated -- January 24, 1975 Simulation . . . 


\section{LIST OF TABLES}

(Continued)

Table

$\underline{\text { Page }}$

28. Emission Strength That Would Result in Ambient $\mathrm{SO}_{2}$ Concentrations Equal to Short and Long Term Standard Limitations (.40 ppm and $.10 \mathrm{ppm}$, respectively) at Locations Indicated -- January 30, 1975 Simulation . . .

29. Emission Strength That Would Result in Ambient $\mathrm{SO}_{2}$ Concentrations Equal to Short and Long Term Standard Limitations (.40 ppm and $.10 \mathrm{ppm}$, respectively) at Locations Indicated -- February 12, 1975 Simulation . .

30. Emission Rate or Total Emission Into Core Area Mixing Volume That Would Result in Ambient Concentrations Equal to Standard Limitations (.40 ppm SO2, 1-Hour standard; $.10 \mathrm{ppm} \mathrm{SO} 2,24$-Hour standard) for January 3, 1975 Simulation ...................

31. Emission Rate or Total Emission Into Core Area Mixing Volume That Would Result in Ambient Concentrations Equal to Standard Limitations (.40 ppm SO2, 1-Hour standard; $.10 \mathrm{ppm} \mathrm{SO} 2,24$-Hour standard) for January 24, 1975 Simulation ..................

32. Emission Rate or Total Emission Into Core Area Mixing Volume That Would Result in Ambient Concentrations Equal to Standard Limitations (.40 ppm SO 2,1 -Hour standard; $.10 \mathrm{ppm} \mathrm{SO} 2,24$-Hour standard) for January 30, 1975 Simulation . . . . . . . . . . . . . . . .

33. Emission Rate or Total Emission Into Core Area Mixing Volume That Would Result in Ambient Concentrations Equal to Standard Limitations (.40 ppm $\mathrm{SO}_{2}$, 1-Hour standard; $.10 \mathrm{ppm} \mathrm{SO} 2,24$-Hour standard) for February 12, 1975 Simulation ..................

34. Annual Total Emissions of $\mathrm{SO}_{2}$ That Would Result in Ambient Concentrations of $80,60,40$ or $20 \mu \mathrm{g} / \mathrm{m}^{3}$ at Present or Projected Source Location Grid Cells . . . . . . . .

35. Total Allowable Emissions Within Core Area on an Annual Basis to Achieve Ambient Concentrations of $80,60,40$ and $20 \mathrm{\mu g} / \mathrm{m}^{3} \mathrm{SO}_{2}$. . . . . . . . . . . . . . . 


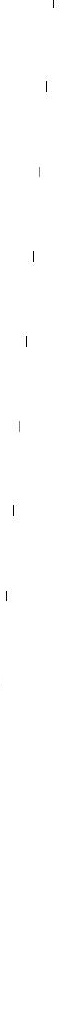


1. Monitoring Locations for $\mathrm{SO}_{2}$ and TSP Data ......

2. Northern Puget Sound Study Area and "Core" Area of Interest .. . . . . . . . . . . . . . . .

3. Northern Puget Sound Region Showing Potential Petroleum Port and Refinery Development . . . . . . . . .

4. Locations From Which Meteorological Data Were Obtained for Model Validation .. . . . . . . . . . 13

5. Surface Wind Roses for Washington Stations - January . • 17

6. Surface Wind Roses for Washington Stations - July . . 18

7. Surface Wind Roses for Washington Stations - Annual . . 19

8. Grid Point Elevations for the Northern Puget Sound Study Area . . . . . . . . . . . . . . . .

9. Grid Point Vegetation Codes for Northern Puget Sound Study Area . . . . . . . . . . . . . . .

10. Northern Puget Sound Region Showing Three Low Level Interconnecting Channels: Hoquiam-Puget Sound, Strait of Juan de Fuca, Strait of Georgia . . . . . . . .

11. Location of "Observed" Data Points in the Northern Section of Puget Sound and Strait of Juan de Fuca . . . 30

12. Location of Major $\mathrm{SO}_{2}$ Sources for Northern Puget Sound ....................

13. Combined PI( $\pi$ )-Matrix for the Annual Case; $\mathrm{SO}_{2}$ Standards are $20 \mu \mathrm{g} / \mathrm{m}^{3}$..................

14. Combined PI( $\pi)$-Matrix for the Annual Case; $\mathrm{SO}_{2}$ Standards are $40 \mathrm{\mu g} / \mathrm{m}^{3}$..................

15. Combined PI( $\pi)$-Matrix for the Annual Case; $\mathrm{SO}_{2}$ Standards are $60 \mathrm{\mu g} / \mathrm{m}^{3}$. . . . . . . . . . . . . . .

16. Combined PI( $\pi)$-Matrix for the Annual Case; $\mathrm{SO}_{2}$ Standards are $80 \mathrm{\mu g} / \mathrm{m}^{3}$.................... 

EXECUTIVE SUMMARY

\section{INTRODUCTION}

The utility of traditional air quality diffusion models is limited in areas of complex topography. At the same time, the air quality constraints on facilities' siting, "carrying capacity"* requirements and land use planning alternatives may be particularly important in complex terrain. Such areas present a real challenge for the air quality modeler -- local topographic influences dominate the surface flow and dispersion potential patterns much of the time and these patterns can be expected to change drastically with a shift in the background flow conditions. The technique discussed in this report (the TAPAS system) was developed to provide valid information on the dispersion capability of the lower atmosphere in areas characterized by complex terrain features.

The northern Puget Sound region of Washington (U.S.A.) and British Columbia (Canada) was chosen as an ideal location in which to test the utility of the TAPAS system. The coastal areas of Washington and British Columbia are characterized by complex terrain. Data from the limited number of wind monitoring sites in the study area indicate the controlling influence that topographic features (including mountains, large and small islands and ocean passages) have on surface wind patterns. Under these conditions, ordinary diffusion modeling techniques using non-varying

*The term carrying capacity for air pollution is used here in the same manner as the range manager uses it to determine the number of animals to be permitted to graze on a certain pasture. Just as knowledge of the interaction of soils, plants and precipitation are the primary input to grazing capacity calculations, information on terrain, wind patterns and mixing depth are the inputs to determining air pollution carrying capacity. 
wind speed and direction assumptions could not be expected to provide valid information on the air pollution "carrying capacity" of the region.

The northern Puget Sound is in a Class II air quality region. Air quality measurements indicate that the air in this region is fairly clean with respect to total suspended particulates. Short-term violations of federal and state ambient air quality standards for $\mathrm{SO}_{2}$, however, were recorded at several locations during 1975 and 1976, although the present $\mathrm{SO}_{2}$ monitoring network is not adequate to provide detailed information about the spatial distribution of this pollutant (NWAPA, 1977). A study was undertaken to assess the air quality $\left(\mathrm{SO}_{2}\right)$ impacts associated with energy development in the region and to determine the constraints on energy development due to regulated limitations on the amount of air quality degradation allowable in the area. The northern Puget Sound region already contains a number of refinery and wharf locations which represent substantial sources of $\mathrm{SO}_{2}$ emission. Projected expansion of these facilities, along with possible new energy development sites, provide a potential for significant air quality deterioration within the region.

\section{MODEL DESCRIPTION}

The Topographic Air Pollution Analysis System (TAPAS) consists of two major sections. The first part is a wind flow model (WINDS) which is capable of computing spatial and temporal variations in the low level wind field due to changes in topography, surface roughness and surface heating. Information about the study area is reduced to a system of grid points. Input for the flow model includes the prevailing 
background flow (speed and direction) over the study area plus elevation, surface roughness and surface temperature information for each grid point. Output from the WINDS model consists of a series of maps of wind direction and speed (Figure 1) at each grid point, for each day and time. A mixing volume index, based primarily on wind speed and convergence or divergence of air parcels, is also calculated. This index provides information on the relative potential for pollutant accumulation at each grid cell location.

The second section of the TAPAS system combines the wind field output from WINDS, the mixing volume index array and the Pollutant Standards Index (PSI) (Thom and Ott, 1976) to determine the total emission from each grid cell that would result in selected ambient air quality levels over the entire study area. The resultant index, called the PI( $\pi)$-Matrix, contains no specific source information but provides an atmospheric constraint matrix (i.e. an array of contaminant "carrying capacity" values) for land use planning. An example of the PI-Matrix output is shown in Figure 2 .

The modeling techniques used were developed by the Department of Earth Resources, Colorado State University; the Mountain Meteorology program of the Rocky Mountain Forest and Range Experiment Station (USFS); and Marlatt and Associates, Fort Collins, Colorado.

\section{DATA ANALYSIS}

Four days were selected for testing the model in the northern Puget Sound region. These days were dates on which high concentrations of sulfur dioxide or particulate matter were recorded in the northern Puget Sound region and represent meteorological conditions with background flow 


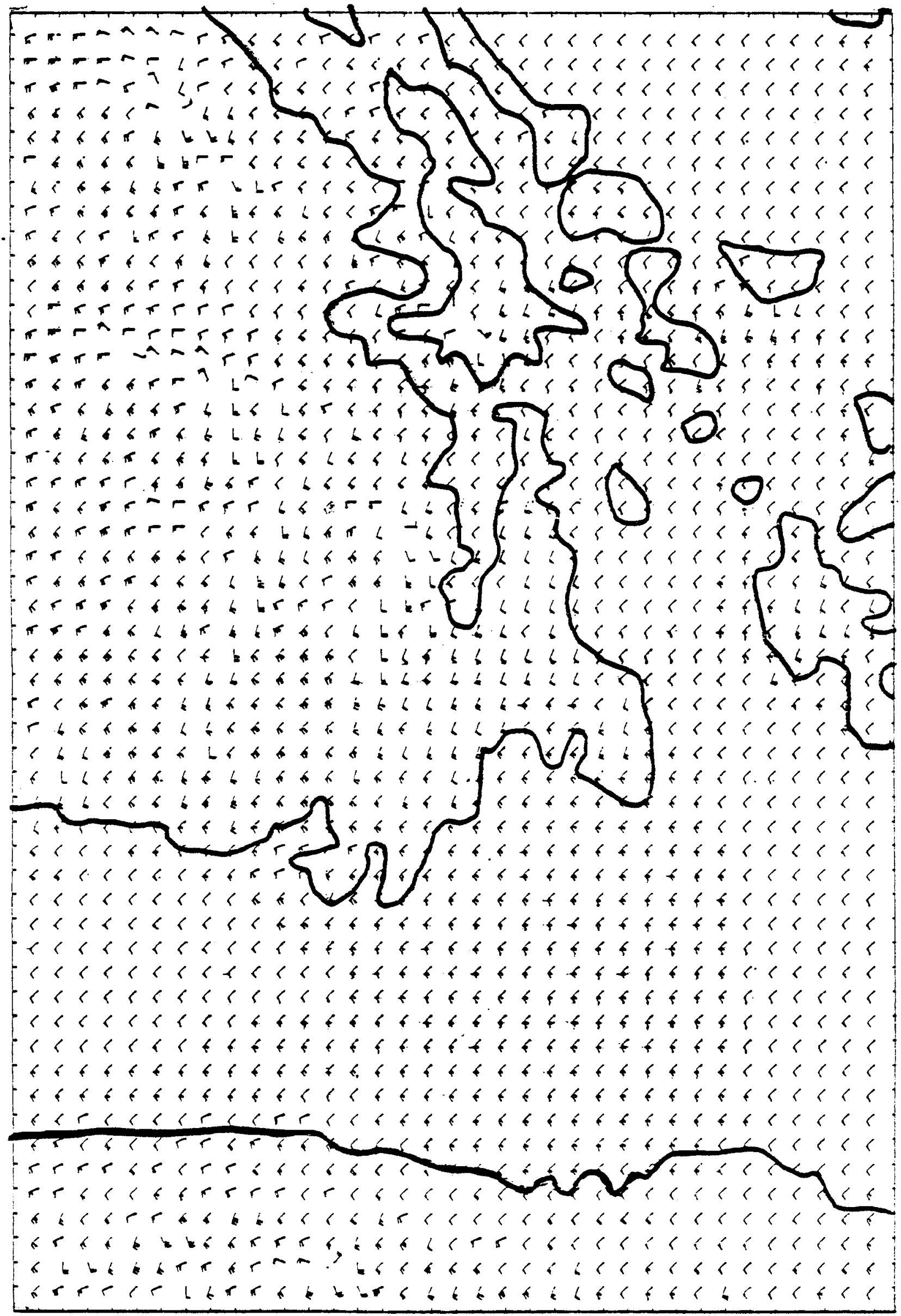




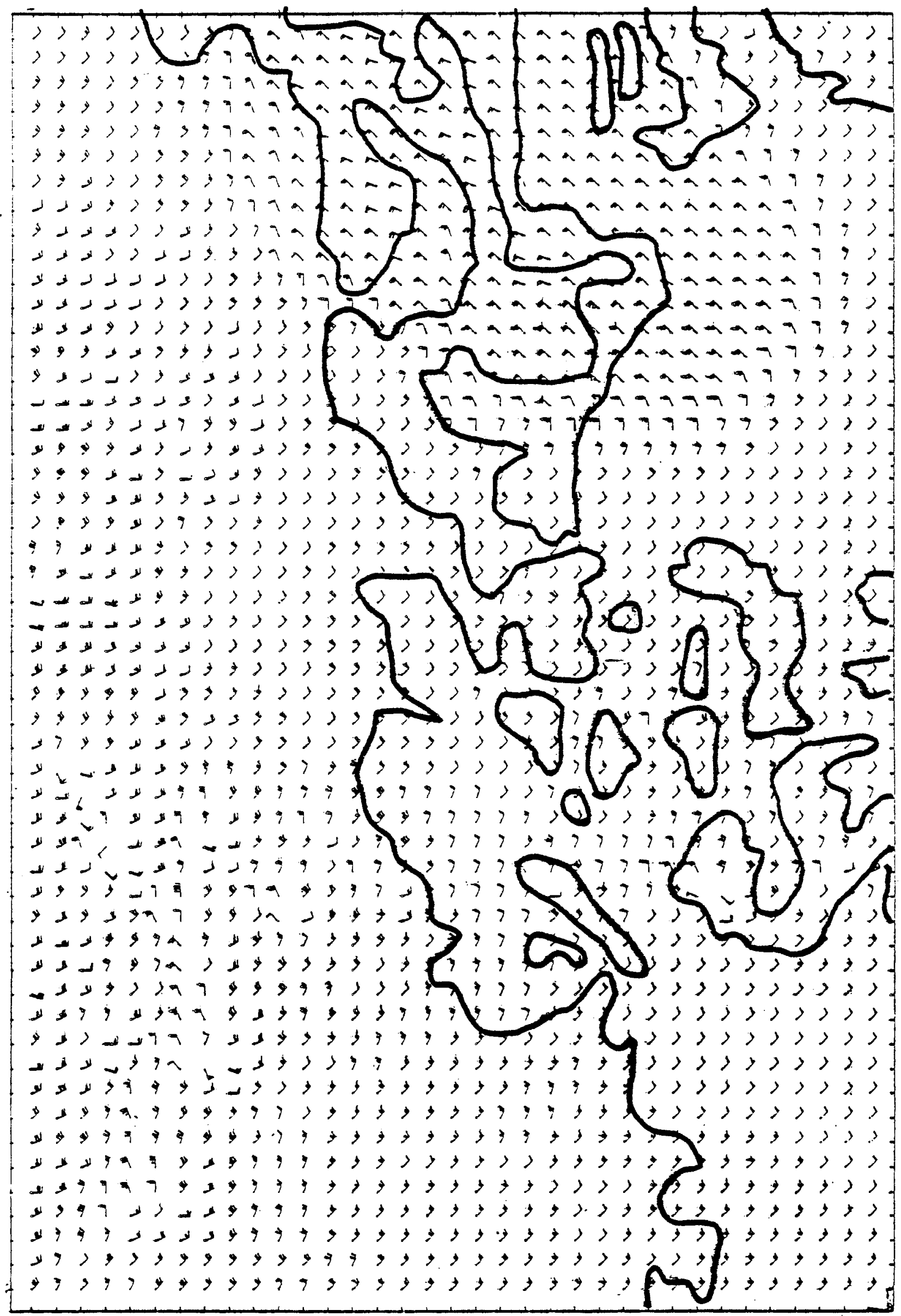


Figure 2. PI( $\pi)$-Matrix For January 3, 1975 at 0300.

This figure illustrates the $\mathrm{SO}_{2}$ emissions in each $2 \mathrm{~km}^{2}$ grid cell which would result in ambient $\mathrm{SO}_{2}$ concentrations of $.4 \mathrm{ppm}$ (Washington one hour ambient standard limitation) over the entire study area.

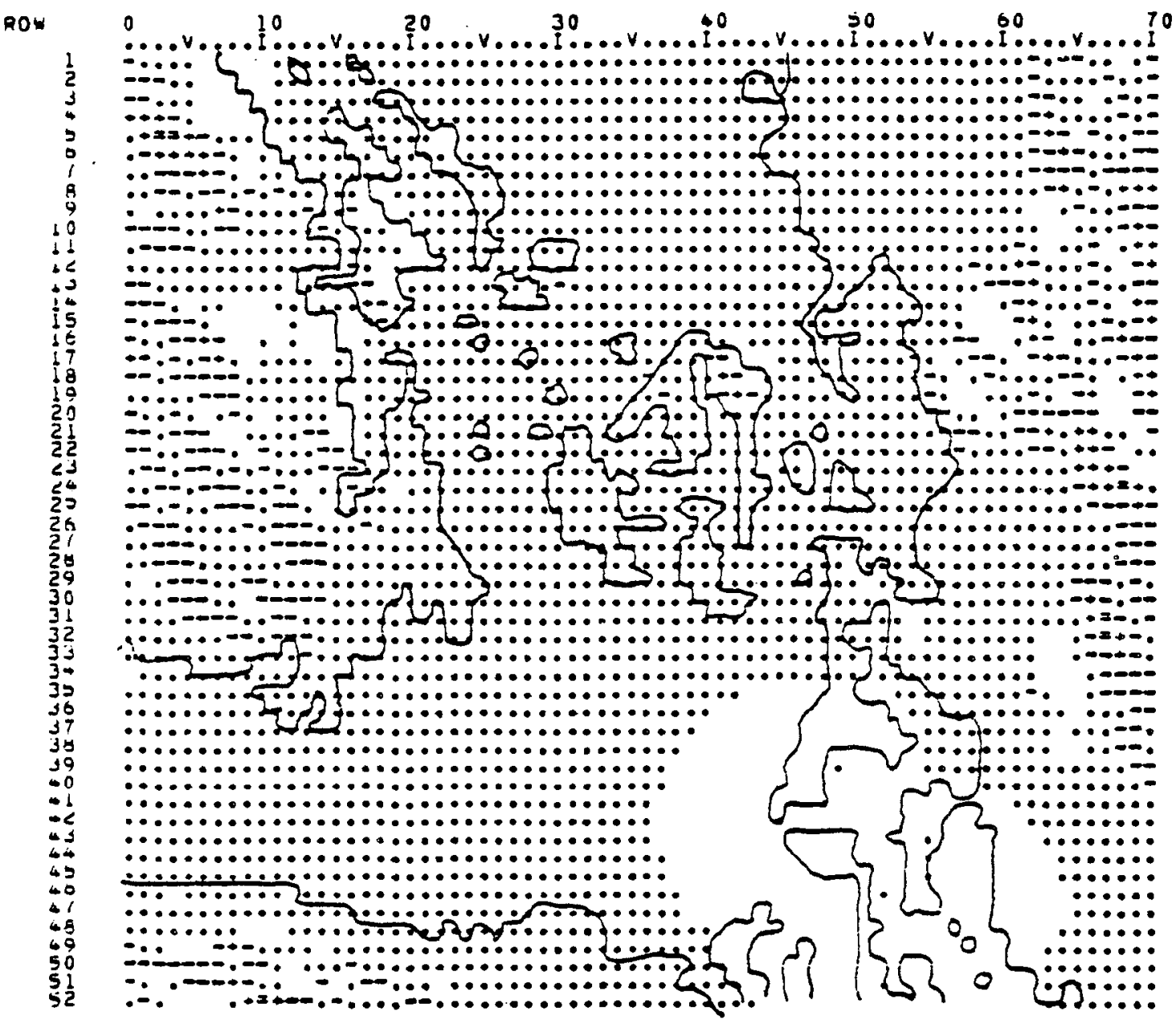

zassion RzSTRLFTON CODES:

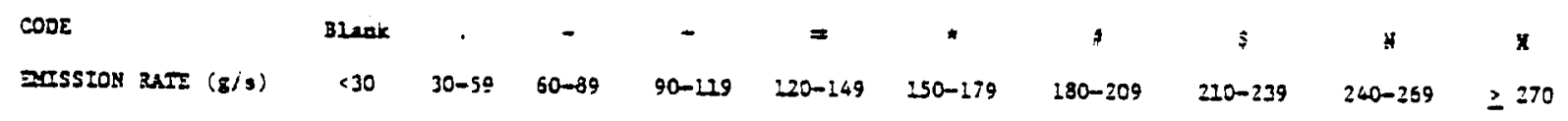


directions varying from $360^{\circ}$ clockwise to $247^{\circ}$ and background wind speeds ranging from 0.5 miles per hour to over 13 miles per hour in the study area.

Short-term PI-Matrices, representing instantaneous emission rate limitations to achieve ambient concentrations of $0.4 \mathrm{ppm} \mathrm{SO}_{2}$ (Washington one hour ambient standards (PSAPCA, 1977)), were calculated at four hour intervals for each of the dates analyzed. In addition, a 24-hour PIMatrix representing total emissions to achieve an ambient concentration of $0.1 \mathrm{ppm} \mathrm{SO}_{2}$ (Washington 24-hour ambient standard (PSAPCA, 1977)), was calculated for each day simulated. An annual PI-Matrix was also calculated from the annual mixing volume index, based on annual wind rose data from Bellingham, Washington.

\section{RESULTS}

Table 1 shows a comparison of WINDS results to observed meteorology at 11 locations in the northern Puget Sound region for one of the four days analyzed. Due to rounding and grid point location discrepancies between the observed and simulated situations, the mean error figures, rather than errors at individual grid locations, were relied on most heavily in analyzing WINDS model performance. The table shows that the mean errors for the entire study area, and for a "core" area of approximately $3118 \mathrm{~km}^{2}$ centered around the refinery and petrochemical developments near Ferndale and Anacortes, were generally within one meter per second or one compass point for this case. The standard deviation of error, used as a measure of spread in the simulation comparison, were also generally less than or equal to two compass points or two meters per second for this simulation. Similar comparisons were made for the other three days modeled. 
Tab1e 1. Comparison of WINDS Results With Observed Data -- January 3, 1975. The numbers represent amounts by which simulation differs from observed flow conditions. Units are compass points for direction (16-point compass; + for clockwise deviation, - for counterclockwise error) and meters per second for speed ( + for overprediction, - for underprediction).

\begin{tabular}{|c|c|c|c|c|c|c|c|c|c|c|c|c|}
\hline \multirow{3}{*}{$\begin{array}{l}\text { Station code } \\
\text { FARC }\end{array}$} & \multicolumn{12}{|c|}{ Tline } \\
\hline & \multicolumn{2}{|c|}{$\begin{array}{c}0300 \\
\mathrm{DIr} / \mathrm{spd}\end{array}$} & \multicolumn{2}{|c|}{$\begin{array}{c}0700 \\
\mathrm{n} / \mathrm{r} / \mathrm{spd}\end{array}$} & \multicolumn{2}{|c|}{$\begin{array}{c}1100 \\
n ! r / S p d\end{array}$} & \multicolumn{2}{|c|}{$\begin{array}{c}1500 \\
\mathrm{Dl} / \mathrm{spd}\end{array}$} & \multicolumn{2}{|c|}{$\begin{array}{c}1900 \\
\mathrm{DIr} / \mathrm{spd}\end{array}$} & \multicolumn{2}{|c|}{$1 \mathrm{DI} / \mathrm{spd}$} \\
\hline & $\mathbf{0}$ & -3 & 0 & -1 & -1 & 0 & 0 & -2 & 0 & -3 & +1 & 1.2 \\
\hline FHRI. & o & 0 & -1 & -1 & +1 & $\mathbf{0}$ & -1 & $\mathbf{0}$ & -1 & +1 & -1 & -2 \\
\hline BFILA & +1 & -1 & 0 & -3 & 0 & 0 & 0 & 0 & +2 & -3 & +1 & 0 \\
\hline BH,C: & o & 0 & 1.2 & 0 & +2 & $\mathbf{0}$ & +2 & +1 & 0 & +1 & +1 & 0 \\
\hline BLILT & o & 0 & +1 & o & +1 & +1 & +2 & +1 & 13 & -1 & o & 0 \\
\hline AAVI:* & +1 & 0 & -1 & -1 & -1 & -2 & -1 & -2 & 11 & -2 & -4 & 0 \\
\hline BHII. & -1 & +4 & -7 & $\mathbf{0}$ & +2 & $\mathbf{0}$ & 11 & -1 & +1 & +1 & $\mathbf{0}$ & -1 \\
\hline WIIII & 0 & -1 & +2 & +1 & -1 & -1 & +1 & +1 & 0 & +1 & $\mathbf{0}$ & +1 \\
\hline SHTII & +1 & -2 & 0 & -1 & - & - & +1 & -4 & +1 & -2 & - & - \\
\hline VICA & -1 & +1 & -2 & +1 & -1. & o & -1 & -3 & 0 & -5 & o & -3 \\
\hline VICII & 0 & -2 & -1 & 0 . & +1 & -2 & -1 & -2 & 0 & -6 & -1 & -4 \\
\hline \multicolumn{13}{|l|}{ Mean Error } \\
\hline A11 Stat Lone & .09 & -.36 & -.64 & -.45 & .30 & -.40 & .27 & -1.00 & $.6 / 4$ & -1.64 & -.30 & -.90 \\
\hline $\begin{array}{l}\text { Stmidard Devlat } \\
\text { of Frror } \\
\text { All stat Lons }\end{array}$ & 70 & & & 11 & 25 & & & & & 95 & & 1.97 \\
\hline $\begin{array}{l}\text { Mean Firor } \\
\text { Core Area }\end{array}$ & .33 & -.67 & .17 & -1.00 & .33 & -.17 & .31 & -.31 & .83 & -1.17 & -.33 & 0.00 \\
\hline $\begin{array}{l}\text { Stambleral bevlat i } \\
\text { of lirror } \\
\text { core Area }\end{array}$ & .52 & 1.21 & 1.17 & 1.10 & 1.21 & .98 & 1.37 & 1.37 & 1.47 & 1.81 & 1.97 & 1.26 \\
\hline
\end{tabular}


Comparisons of observed meteorology and simulation predictions for the four days analyzed showed various degress of accuracy in model performance under the differing metecrological situations encountered. This allowed the assessment of meteorological and grid size limitations to model use. The result is a set of guidelines detailing data requirements, area size limitations and methods of adjusting the WINDS mode1 to fit individual situations.

The emission limitation (PI-Matrix) calculations were dealt with in two ways. Emission rates or amounts from each grid cell that would result in ambient $\mathrm{SO}_{2}$ concentrations equal to Washington one hour or 24-hour standard limitations were calculated for each of the simulation periods. In addition, a core area total emission amount was calculated by assuming complete mixing and, therefore, uniform concentrations over the entire core area mixing volume. The individual grid cell rates represent conservative emission restrictions while the core area totals represent an upper limit to the $\mathrm{SO}_{2}$ emissions that could be tolerated while maintaining acceptable levels of air quality.

An annual case was also calculated which shows emission limitations necessary under the most prevalent flow conditions to meet federal and state annual average $\mathrm{SO}_{2}$ standards and Class II and Class III PSD increments. Table 2 shows annual emission constraints at grid cells representing the locations of present or proposed $\mathrm{SO}_{2}$ point and area sources, along with 1976 NEDS (EPA-NEDS, 1977) estimates of actual $\mathrm{SO}_{2}$ emissions at some of these locations. Table 3 shows core area total emission limitations, along with the NEDS 1976 total estimated point source emissions for the area. 
Table 2. Annual Total Emissions of $\mathrm{SO}_{2}$ That Would Result in Ambient Concentrations of $80,60,40$ or $20 \mu \mathrm{g} / \mathrm{m}^{3}$ at Present or Projected Source Iocation Grid Ce1ls. Emission estimates for current sources for 1976 are also 1isted (EPA, NEDS, 1976). Units are $10^{3}$ kilograms $\mathrm{SO}_{2}$.

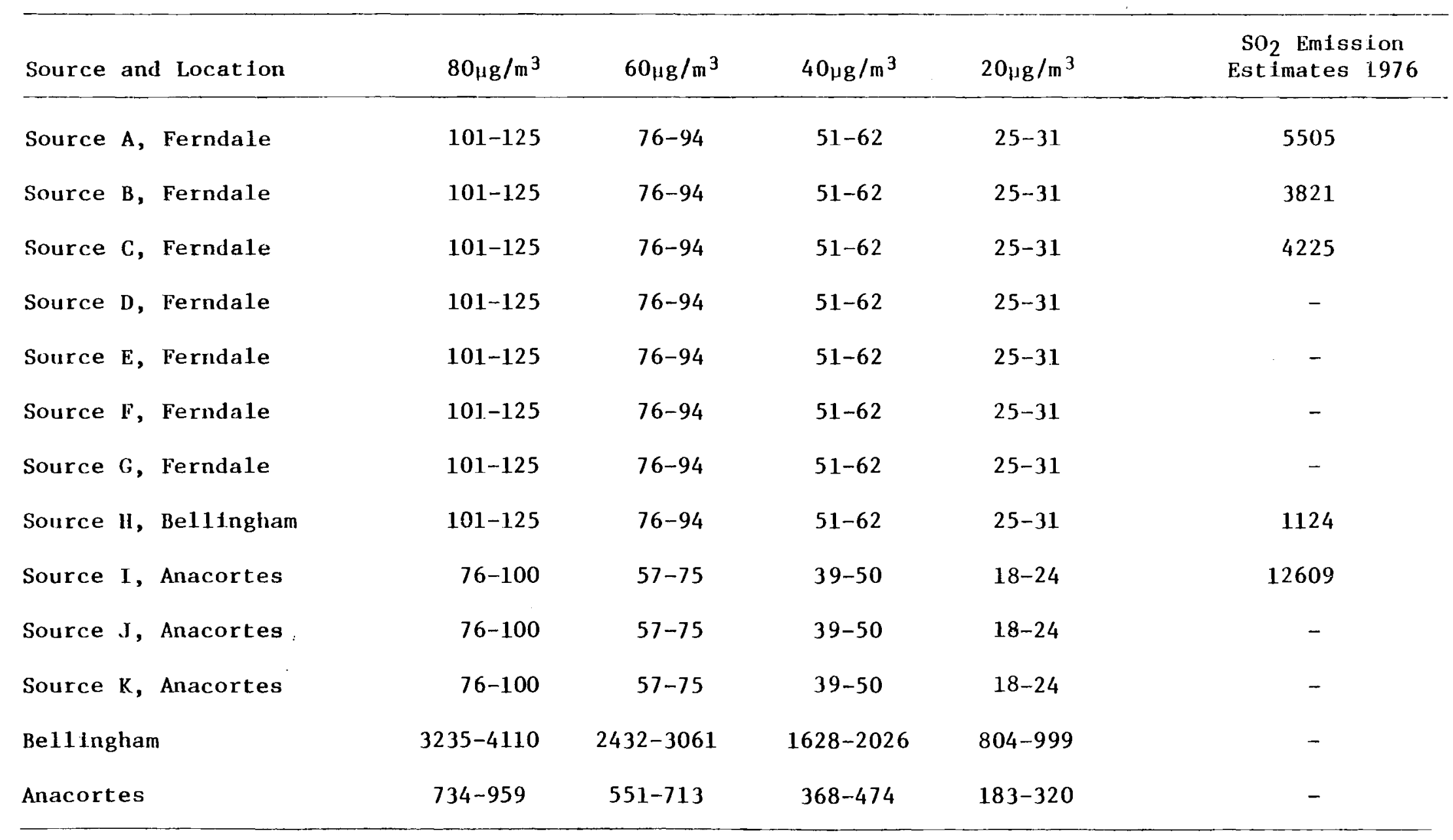


Table 3. Total Allowable Emissions Within Core Area on an Annual Basis to Achieve Ambient Concentrations of $80,60,40$ and $20 \mu \mathrm{g} / \mathrm{m}^{3} \mathrm{SO}_{2}$. Units are $10^{6} \mathrm{kilograms} \mathrm{SO}_{2}$.

\begin{tabular}{cc}
\hline $\mathrm{SO}_{2}$ Concentration & Emission Total \\
\hline $80 \mu \mathrm{g} / \mathrm{m}^{3}$ & 95.58 \\
$60 \mathrm{\mu g} / \mathrm{m}^{3}$ & 71.68 \\
$40 \mathrm{\mu g} / \mathrm{m}^{3}$ & 47.79 \\
$20 \mu \mathrm{g} / \mathrm{m}^{3}$ & 23.89 \\
1976 Point Source Estimated Emissions $=27.29$ \\
(total core area)
\end{tabular}

\section{CONCLUSION}

The emission constraint analysis for the northern Puget Sound region indicated that total $\mathrm{SO}_{2}$ emissions in the core area (on an annual basis) are less than half of the amount that could be tolerated in the entire core region while maintaining ambient concentrations within state and federal annual average limitations. However, comparison of source characteristics with source location and grid cell emission restrictions indicated that localized areas adjacent to the major point sources are already far in excess of the $\mathrm{SO}_{2}$ "carrying capacity". This conclusion is supported by air quality monitoring data which indicate state standard violations at several receptor locations within the study area -- in some cases at points many grid cells "downwind" from the source of contaminant emission (NWAPA, 1977).

The annual maps of wind patterns and PI-Matrix values show areas of both good and poor dispersion characteristics under the most prevalent 
flow conditions. Localized sites of excellent dispersion occur within the higher portions of Vancouver Island, the Olympic Peninsula and the foothills of the Cascades -- these generally coincide with areas with high wind speed predictions and probably represent exposed sites such as ridge top locations. Areas of reduced dispersion occur from Port Angeles west along the northern edge of the Olympic Peninsula, along the eastern edge of Vancouver Island and from the Bellingham Bay area south to Mt. Vernon and Camano Island and north along the edge of the higher terrain to the east.

The wind flow and emission constraint analyses performed for this study provide valuable information regarding the model's utility for subsequent analyses of a similar nature. Mixing volume index maps may be used to indicate areas of high and low "pollution potential" -- in effect, where to site or not site future emission sources. The PI-Matrix calculations could then be used to set allowable emission rates or amounts for each grid cell or, for clusters of grid cells, with reference to any standard of air quality desired. The TAPAS system may also prove useful in preparing attainment plans for areas where contaminant concentrations are currently in excess of standard limitations by defining levels to which emissions must be reduced to insure acceptable air quality at all locations. 
Figure 1. Windfield Analysis of Northern Puget Sound Study Area for January 3, 1975 at 0300 PST. Background flow input: 225.0 degrees at 5.0 meters per second.

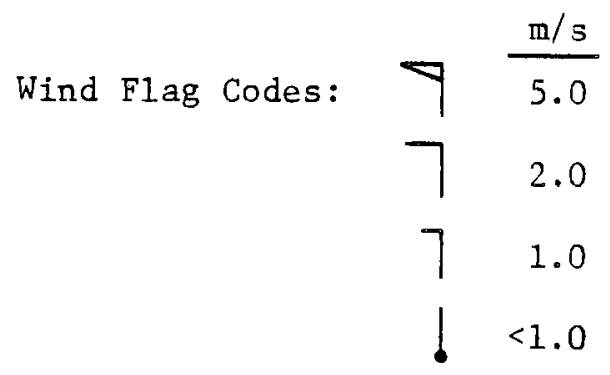



CHAPTER I

INTRODUCTION

The Topographic Air Pollution Analysis System (TAPAS) has been developed for use as a land use planning and facilities siting tool. It can provide the information needed to determine regional and local land use constraints that may be necessary due to regulated limitations on air quality degradation. In this study, the TAPAS system has been used to determine the carrying capacity of a region with respect to sources of $\mathrm{SO}_{2}$; to establish, in effect, the level of $\mathrm{SO}_{2}$ emissions that can be tolerated within the study area while maintaining acceptable levels of air quality. "Acceptable" levels of $\mathrm{SO}_{2}$ are those defined by the National Ambient Air Quality Standards and, for the region under study, standards of the State of Washington. These standards are shown in Table 1.

The purpose of this project was to test the performance of the TAPAS system in an area of complex terrain. The northern Puget Sound region of Washingt on was chosen for this analysis. The TAPAS system has been used to determine emission limitations that would be necessary to attain various regional air quality levels under annual average and "worst case" episodic conditions. Overall model performance and the utility of this technique for future analyses of a similar nature have been evaluated.

The magnitude of contaminant emissions that can be tolerated in any area is primarily determined by the character and frequency of relatively short-term episodes (hours to days) when prevailing meteorological conditions inhibit pollutant dispersal or otherwise interact with emissions to cause high groundlevel concentrations. Consequently, an 
Table 1. Air Quallty Standards for $\mathrm{SO}_{2}$. Units are micrograms per cubic meter $\left(\mu \mathrm{g} / \mathrm{m}^{3}\right)$ and parts per million (ppm) (PSAPCA, 1977; PL 95-95, 1977).

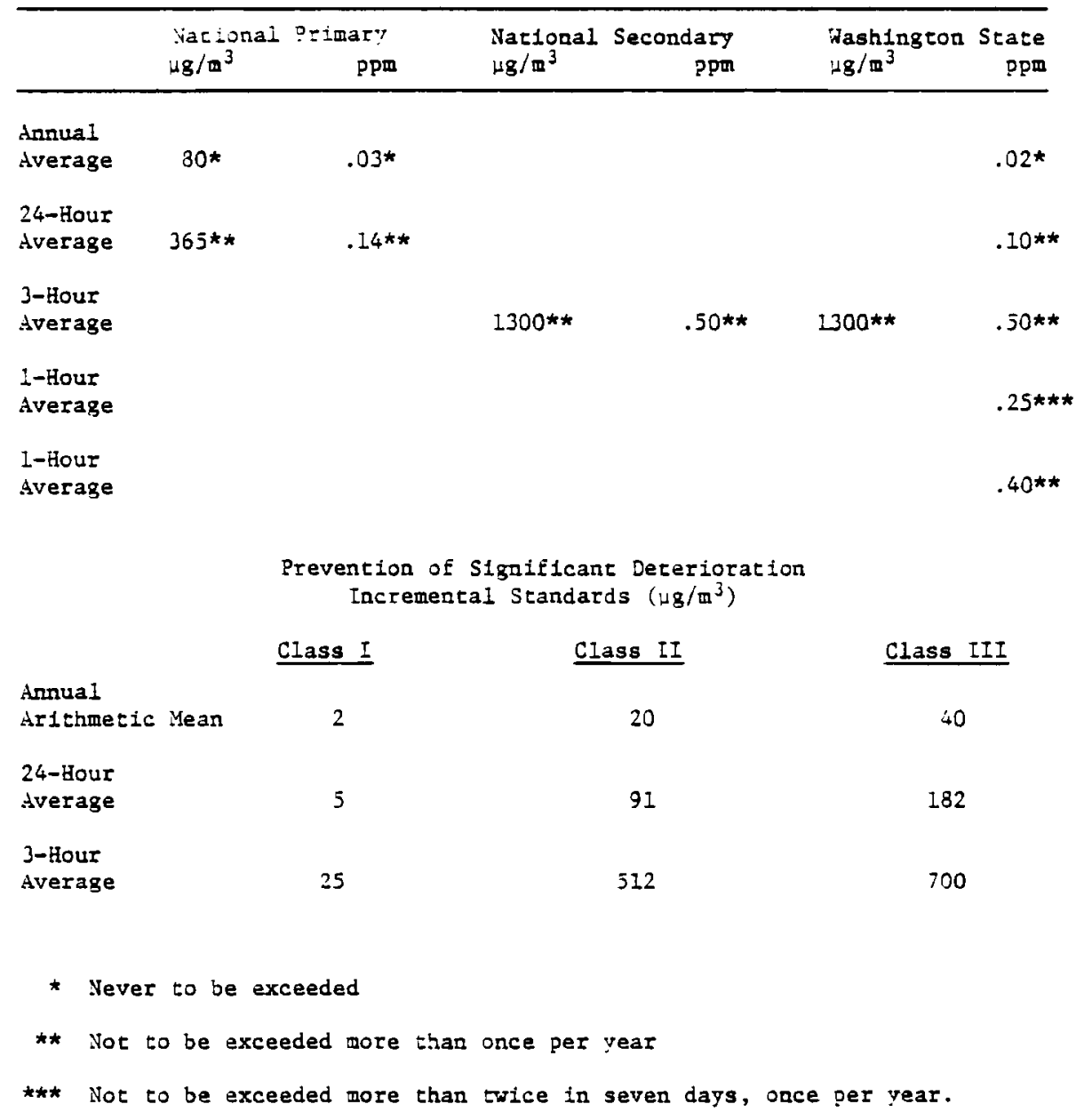


attempt was made to determine several synoptic conditions that were associated with high pollutant levels in the northern Puget Sound region and then to run the wind flow and air quality models for three to four specific days representative of these conditions. This information was then used to estimate the allowable $\mathrm{SO}_{2}$ emissions for the area under similar meteorological conditions. Air quality and meteorological data from the northern Puget Sound area were collected to define needed input variables and to compare with the model results for validation.

Several area agencies (Northwest Air Pollution Association (NWAPA), Puget Sound Air Pollution Control Agency (PSAPCA) and representatives from Western Washington State College) were contacted in an effort to collect available air quality and meteorological data. While information specifically concerning the meteorological conditions most likely to be associated with episodes of poor air quality was not available, data concerning total suspended particulate (TSP) and $\mathrm{SO}_{2}$ measurements taken in the region through 1975 and 1976 were obtained and are summarized in Tables 2 and 3. Particulates were monitored at the Lake Terre11 Game Refuge and at the NWAPA office building in Mt. Vernon. The NWAPA $\mathrm{SO}_{2}$ station network shows several monitoring locations within the study region and violations were recorded at four of the stations over the two year period. Monitoring locations for both TSP and $\mathrm{SO}_{2}$ from which data were obtained are shown in Figure 1.

The air quality data indicate that the northern Puget Sound region is fairly clean with respect to particulates, but that $\mathrm{SO}_{2}$ levels have occasionally exceeded standards at several locations. The highest levels of $\mathrm{SO}_{2}$ and $\mathrm{TSP}$ recorded during 1975 and 1976 were assumed to be indicative of meteorological conditions that might cause periods of high pollutant 
Table 2. Total Suspended Particulate Levels Measured at Mount Vernon and Lake Terrell in 1975 and 1976. Four highest days recorded for each year are shown, along with annual means for 1975 (units are micrograms per cubic meter) (NWAPA, 1977).*

\begin{tabular}{|c|c|c|c|c|}
\hline \multirow[b]{2}{*}{ Year } & \multicolumn{2}{|c|}{ Mount Vernon } & \multicolumn{2}{|c|}{ Lake Terrell } \\
\hline & Date & $\begin{array}{c}\text { Concentration } \\
\left(\mu \mathrm{g} / \mathrm{m}^{3}\right)\end{array}$ & Date & $\begin{array}{c}\text { Concentration } \\
\left(\mathrm{\mu g} / \mathrm{m}^{3}\right)\end{array}$ \\
\hline \multirow[t]{4}{*}{1975} & January 24 & 128 & February 5 & 78 \\
\hline & January 30 & 119 & July 23 & 56 \\
\hline & February 5 & 115 & June 11 & 45 \\
\hline & March 7 & 105 & June 17 & 42 \\
\hline
\end{tabular}

Annual

Arithmetric Mean 44.5 Geometric Mean 38.2

1976

$\begin{array}{lr}\text { January 20 } & 117 \\ \text { February 6 } & 116 \\ \text { May 1 } & 94 \\ \text { October 22 } & 88\end{array}$

Annual

Arithmetric Mean 20.5

Geometric Mean 17.0

May 1

April $28 \quad 55$

February $6 \quad 48$

July 24

*Applicable standards are $75 \mu \mathrm{g} / \mathrm{m}^{3}$, federal primary standard for annual geometric mean; $260 \mathrm{\mu g} / \mathrm{m}^{3}$, federal primary standard for 24-hour average; $60 \mathrm{\mu g} / \mathrm{m}^{3}$, federal secondary and Washington State standard for annual geometric mean; and $150 \mu \mathrm{g} / \mathrm{m}^{3}$, federal secondary and Washington State standard for 24-hour average concentration. 
Table 3. Stations exceeding 3-hour averaga liate of 50 parts per hundred million $\mathrm{SO}_{2}$ or 24-hour average limite of 10 pares per hundred million $\mathrm{SO}_{2}$ in 1975 and 1975. Only the highest 3- or 24-hour average concentration is shown for each day. See Figure 1 for location of numbered stations. (NWAPA, 1977)

\begin{tabular}{|c|c|c|c|c|c|}
\hline Year & Date & Location & Time & Pertod & $\begin{array}{c}\text { Concentration } \\
\text { (pphm) }\end{array}$ \\
\hline \multirow[t]{17}{*}{1975} & January 3 & 30 (Bellingham) & & Hour & 69 \\
\hline & January 8 & 30 (Bellingham) & & Hour & 77 \\
\hline & December 4 & 30 (Bellingham) & & Hour & 67 \\
\hline & January 6 & 25 (B1rch Bay) & 24 & Hour & 11 \\
\hline & January 3 & 30 (Bell1ngham) & 24 & Hour & 21 \\
\hline & January $j$ & 30 (Bel1Ingiam) & 24 & Hour & 11 \\
\hline & January 6 & 30 (Bellingham) & 24 & Hour & 19 \\
\hline & January 7 & 30 (Beilingham) & 24 & Jour & 11 \\
\hline & January 3 & 30 (Bellinginam) & 24 & Hour & 21 \\
\hline & January 9 & 30 (Bell1ngham) & 24 & Bour & 21 \\
\hline & February 10 & 30 (Bell1nghan) & 24 & Hour & 12 \\
\hline & Eebruary 11 & 30 (Belilngham) & 24 & Hour & 14 \\
\hline & February 12 & 30 (Bell1ngham) & 24 & Hour & 40 \\
\hline & February 13 & 30 (Bellingham) & 26 & Bour & 12 \\
\hline & December 1 & 30 (Bellingham) & 24 & Hour & 18 \\
\hline & December 2 & 30 (Bellingham) & 24 & Hour & 18 \\
\hline & December 3 & 30 (BellLngham) & 24 & Bour & 18 \\
\hline \multirow[t]{13}{*}{1976} & dprt1 22 & 32 (March Polne) & 3 & Hour & 74 \\
\hline & June 20 & 25 (B1rch Bay) & 24 & Hour & 14 \\
\hline & June 2i & 25 (B1rch Bay) & 24 & Rour & 11 \\
\hline & Eebruary 3 & 23 (Narch Po1nt) & 24 & Hour & 13 \\
\hline & February 4 & 28(March Polne) & 24 & Hour & 18 \\
\hline & January 9 & 30 (Ballingham) & 24 & Hour & 11 \\
\hline & February 16 & 30 (Ballingham) & 24 & Hour & 16 \\
\hline & Eebruary 17 & 30 (Bell1ngham) & 24 & Hour & 11 \\
\hline & Eebruary 18 & 30 (5all Lngham) & 24 & Hour & 15 \\
\hline & Eebruary 19 & 30 (Bellingham) & 24 & Bour & 11 \\
\hline & March 26 & 30 (Bellingham) & 24 & Hour & 12 \\
\hline & April 22 & 32 (Mareh Point) & 24 & Hour & 18 \\
\hline & Apr11 23 & 32 (March 2oine) & 24 & Hour & 18 \\
\hline
\end{tabular}




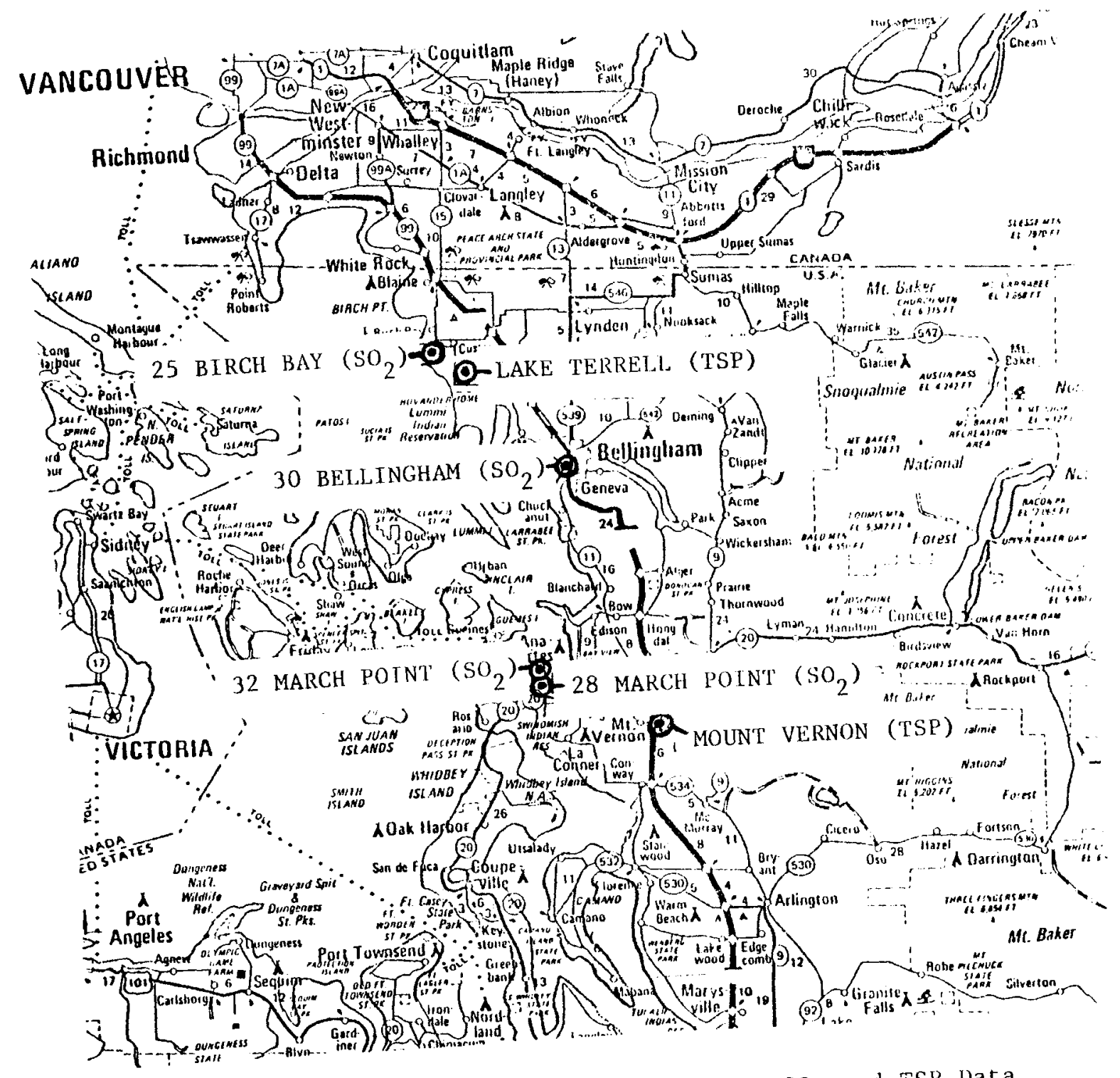

Pigure 1. Monitoring Jocations for $\mathrm{SO}_{2}$ and $\mathrm{TSP}$ Data
(NWAPA, 1977). 
concentrations at other times as well. Four days were chosen for the wind flow and air quality analyses based on the air quality data presented and the availability of meteorological data for the study area on the appropriate dates. These dates are January 24 and January 30, 1975 (showing the highest TSP concentrations for Mt. Vernon in the two years monitored) and January 3 and February 12, 1975 (when violations of $\mathrm{SO}_{2}$ standards occurred at Bellingham). These four days also represent differing synoptic conditions (and associated background wind speed and direction) over the northern Puget Sound region.

To include the pertinent terrain features (including islands and ocean passages) that influence wind flow in the northern Puget Sound study area as originally described, the modeling area was enlarged to include the northern portions of the Olympic Peninsula, Washington; and the southern portion of Vancouver Island, British Columbia, Canada. The entire area is shown in Figure 2, along with the "core area" of interest, centered around Bellingham and the refinery and wharf developments near Ferndale and Anacortes. An enlarged view of this core region is shown in Figure 3. 


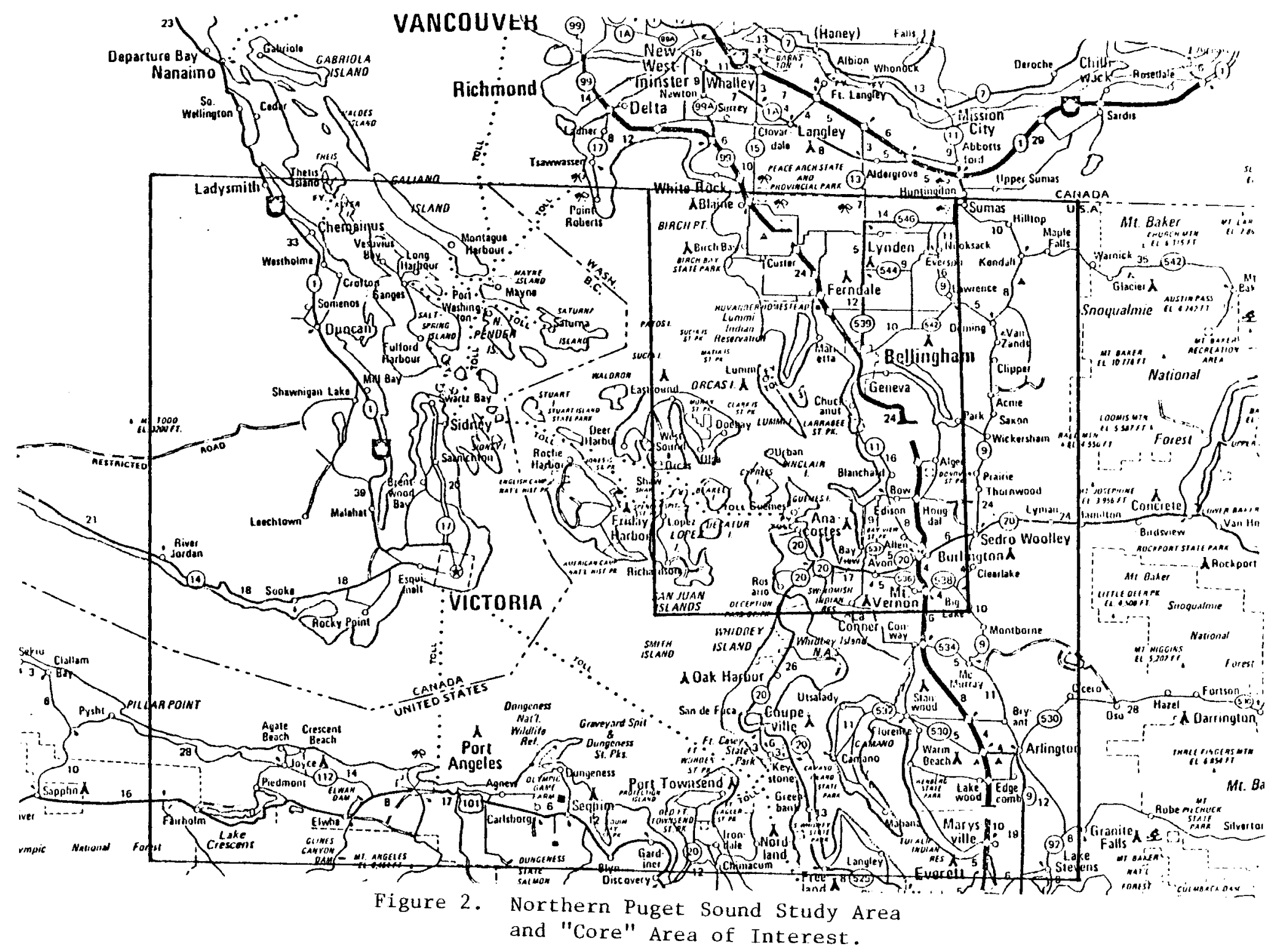




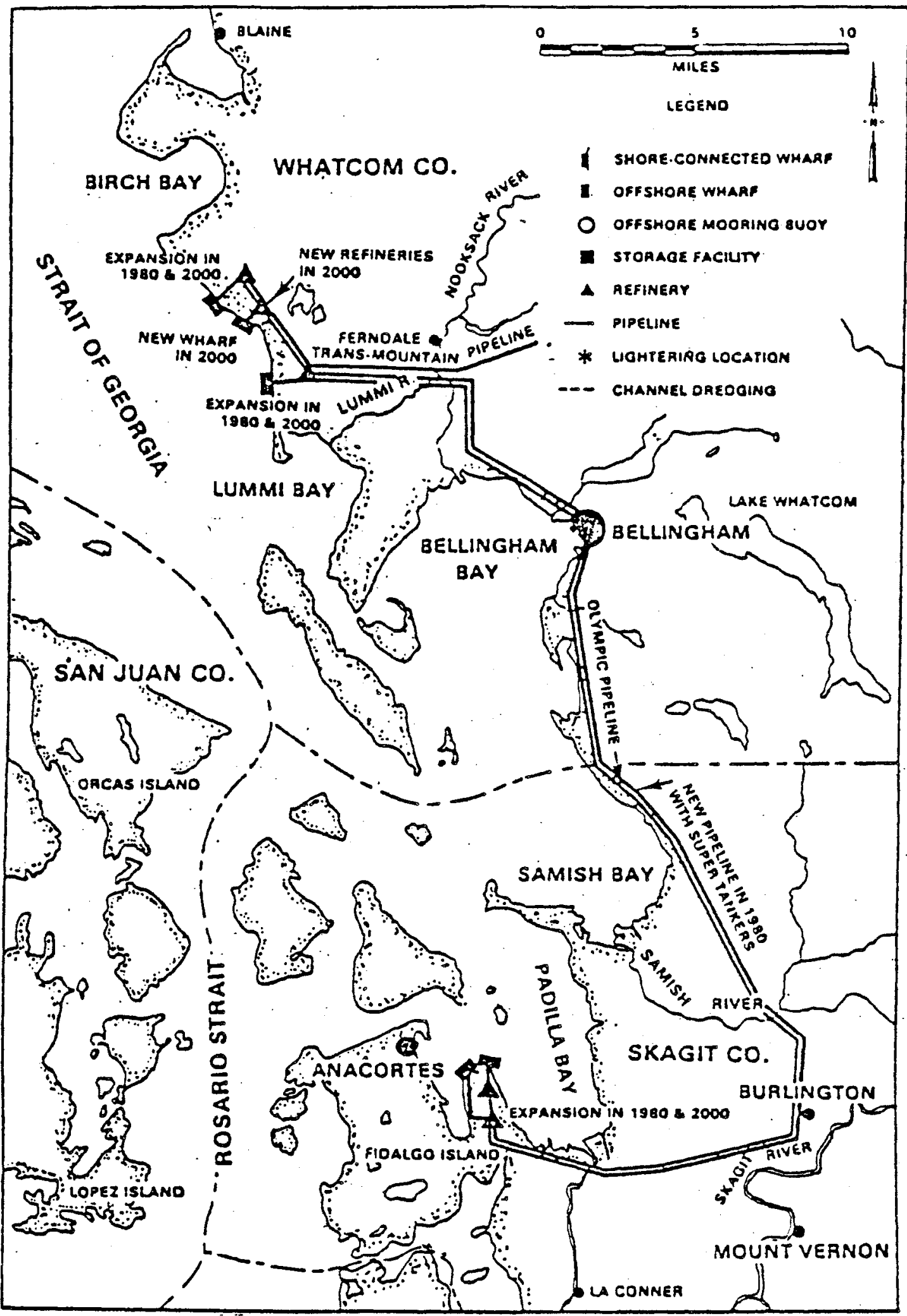

Figure 3. Northern Puget Sound Region Showing Potential Petroleum Port and Refinery Development (Battelle Pacific Northwest Laboratories, 1977). 

An inventory of major sources of $\mathrm{SO}_{2}$ in the northern Puget Sound region is available from the National Emissions Data System files (NEDS). A list of these source locations and total $\mathrm{SO}_{2}$ emissions for 1976 is shown in Table 4, along with the probable area sources of emission in the region.

Table 4. Major Sources of $\mathrm{SO}_{2}$ in the Northern Puget Sound Area in 1976 (EPA-NEDS, 1977).

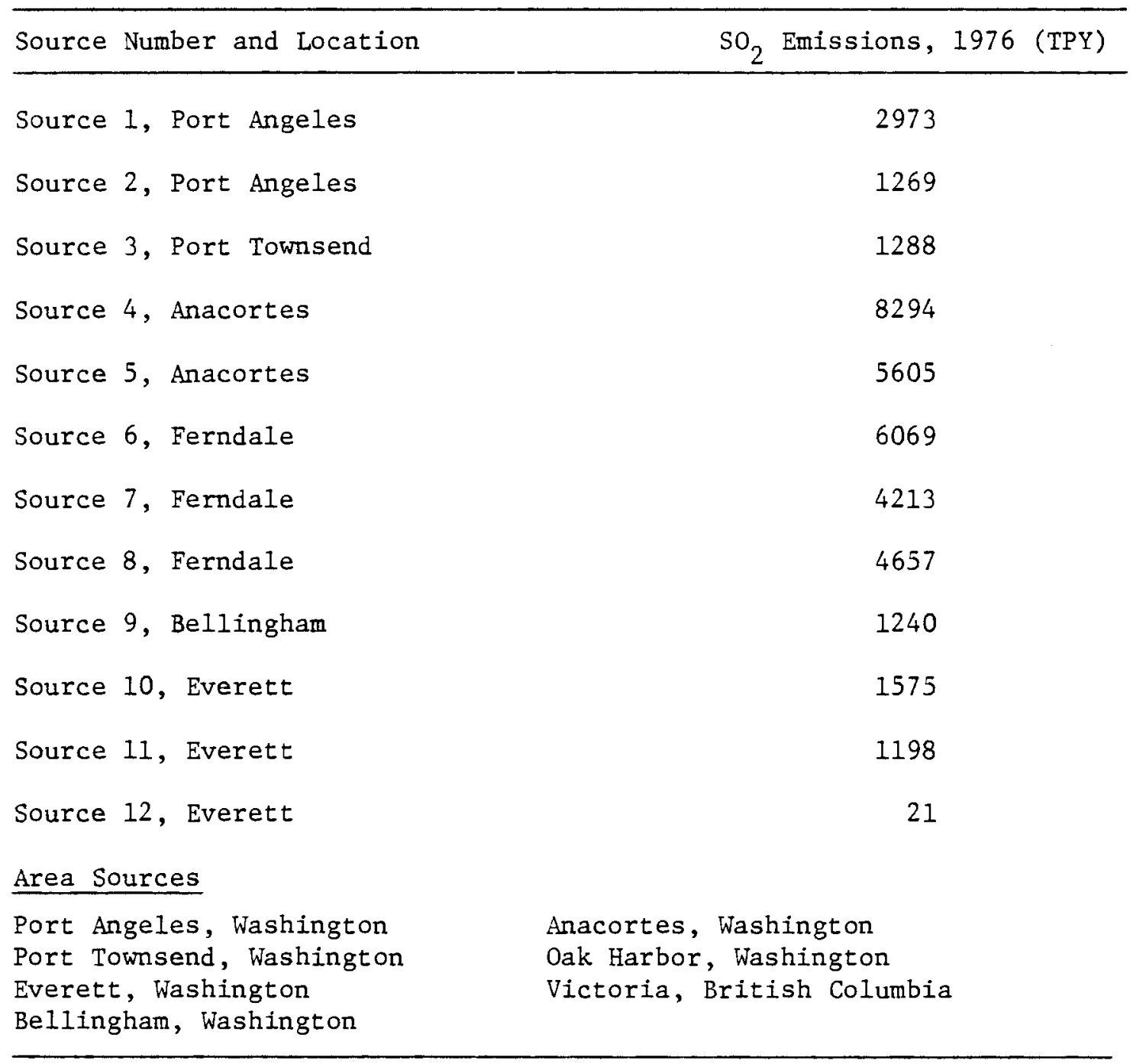


Meteorological data was collected from stations in the study area. These included locations with data on record with the National Climatic Center in Asheville, North Carolina and also a number of private data collection points operated by industries in the region. Table 5 is a list of all locations in the study area for which data were obtained. The station at Everett, which is just outside the modeled area, was used to check input for the wind flow model. A station at Quillayute, on the western side of the Olympic Peninsula, is also included. Upper air soundings were available for this location and were used to generate input for the wind flow model. Data for each of the four days used in the modeling effort are presented under each separate case later in this paper. The locations of the validation points are shown in Figure 4. The four days chosen for analysis represent differing synoptic situations -- the one characteristic all have in common is that high pollutant concentrations occurred within the northern Puget Sound region on each of the four days. January 3, 1975 and February 12, 1975 were marked by violations of short-term $\mathrm{SO}_{2}$ standards at Bellingham while January 24 and 30, 1975 showed the highest particulate levels (although within standard limitations) that were recorded over the two year period (1975-1976) at Mt. Vernon. Some relationships between synoptic meteorology and air quality are apparent from these four cases and their relationship to average meteorological conditions is also of interest.

The Washington coast is located approximately in the center of a zone of prevailing westerlies with local winds varying from northwest to southwest throughout most of the year. The seasonal cycle of winds on the Pacific Northwest coast is largely determined by the circulation about the northern Pacific high pressure area and the Aleutian low 
Table 5. Locations From Which Meteorological Data were Obtained for Model Validation.

\begin{tabular}{lc}
\hline \multicolumn{1}{c}{ Name and Location } & Code \\
\hline ARCO Refinery, Ferndale, Washington & FARC \\
Mobile Refinery, Ferndale, Washington & FMBL \\
Airport, Bellingham, Washington & BELA \\
Columbia Cement, Bellingham, Washington & BELC \\
PAMS Station, Bellingham, Washington & BELT \\
Shell Refinery, Anacortes, Washington & ASHL \\
Allied Chemical, Anacortes, Washington & AALC \\
Northwest Petrochemical, Anacortes, Washington & ANWP \\
Texaco Refinery, Anacortes, Washington & ATEX \\
Puget Sound Power and Light, Bacus Hill, Washington & BHIL \\
Naval Air Station, Whidbey Island, Washington & WHID \\
Coast Guard Light Station, Smith Island, Washington & SMTH \\
Medical-Dental Building, Everett, Washington & EVRT \\
Victoria Int'l. Airport, Vancouver Island, British Columbia & VICA \\
Gonzales Heights, Vancouver Island, Victoria, British Columbiaa & VICH \\
Pearson Aircraft, Port Angeles, Washington & PAPA \\
Coast Guard, Ediz Hook, Port Angeles, Washington & PACG \\
Quillayute Airport, Quillayute, Washington & \\
\hline & \\
\hline
\end{tabular}




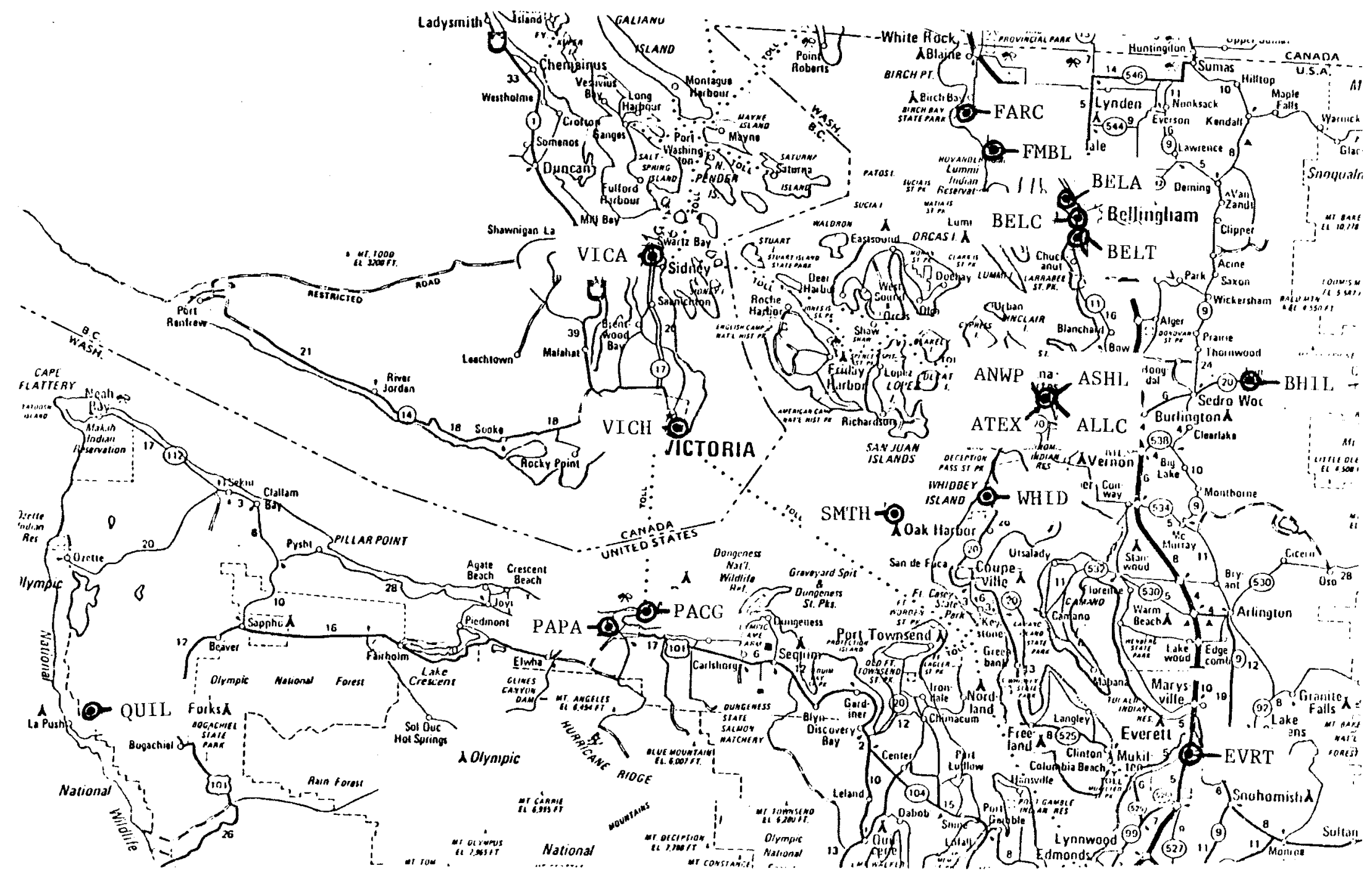

Figure 4. Locations From Which Meteorological Data Were Obtained for Model Validation. 
pressure area. During summer the north Pacific high reaches its greatest development (approximately $1025 \mathrm{mb}$ ) and is centered about $30-40^{\circ} \mathrm{N}$ and $150^{\circ} \mathrm{W}$; the Aleutian low is weak during this period. The interaction of these pressure zones favors the development of summer winds generally from the northwest quadrant over the nearshore and coastal areas of Washington.

During winter the north Pacific high weakens and its center shifts about $10^{\circ}$ southward while the Aleutian low intensifies. The resulting winds, frequently at gale force, approach the Washington coast from the southwest quadrant.

Extra-tropical cyclones occur most frequently in winter and the strongest winds are associated with the more intense winter storms moving east across the ocean. Depending upon the location of the center as it impinges on the coast, winds may be from northwest to southeasterly. In western Washington, circulation of air around the low centers approaching land results in a high frequency of strong southeasterly and southerly winds along the coast and strong easterly winds in the Strait of Juan de Fuca.

The barrier presented by the mountains of the coast range influences the general wind pattern, deflecting the winds so that they tend to align with the trend of the coast. In regions where the mountains are low, the deflecting effect is minimal and normal oceanic wind conditions prevail. The low level passages through the mountains at Hoquiam, Washington; the Strait of Juan de Fuca; and the Strait of Georgia show this pattern and influence the overall wind flow through the study area. 
Table 6 shows the seasonal cycle of winds approaching the Washington coast, measured at the Umatilla Lightship located off Cape Alava of the Olympic Peninsula at $48^{\circ} 10.0^{\prime} \mathrm{N}, 124^{\circ} 50.0^{\prime} \mathrm{W}$. The table shows resultant wind speed and direction (vector sum of all observations taken each month) and mean scalar speed over a five year period. Figures 5, 6 and 7 show January, July and annual surface wind roses at various locations in western Washington. The channeling effect of the Straits of Georgia and Juan de Fuca and the Hoquiam-Puget Sound passage are evident at several stations. The air quality data collected for the years 1975 and 1976 show that short-term $\mathrm{SO}_{2}$ violations in the study area occur most often in several day "episodes" during the winter period (i.e. January 3-9, 1975; February 10-13, 1975; December 1-4, 1975; February 16-19, 1976). The highest TSP levels recorded, on the other hand, show little discernable pattern, although the occurrence of several day episodes such as are seen in the $\mathrm{SO}_{2}$ data would not be apparent here because measurements of particulate concentrations are only taken every fourth day.

Meteorological data were collected for the January $3-9$ and the February 10-13, 1975 periods. Some similarity in synoptic conditions may be ascertained: data for both time periods show low pressure centers at the surface in the immediate vicinity of the study area or moving across the northern Puget Sound region, with associated fronts passing through the area under study. Low pressure systems were apparent at $850 \mathrm{mb}$. in the northwest quadrant relative to the study area with flow from the south, southwest or west. Flow at 700 and $500 \mathrm{mb}$. was from the northwest, west or southwest around the Aleutian low with an additional low pressure center at $700 \mathrm{mb}$. to the west of the study area on some occasions. 
Table 6. Seasonal Cycle of Winds Along Washington Coast for Five Year Period (1961-1965) (EPA, 1972).

\begin{tabular}{|c|c|c|c|c|c|}
\hline Month & $\begin{array}{r}\text { Resultant } \\
\text { (degr }\end{array}$ & $\begin{array}{l}\text { Direction } \\
\text { rees) }\end{array}$ & $\begin{array}{c}\text { Resultant Speed } \\
(\text { knots })\end{array}$ & $\begin{array}{l}\text { Scalar Speed } \\
\text { (knots) }\end{array}$ & $\begin{array}{l}\text { Number of } \\
\text { Observations }\end{array}$ \\
\hline January & 182 & $\mathrm{~S}$ & 7 & 18 & 557 \\
\hline February & 167 & SSE & 4 & 16 & 675 \\
\hline March & 210 & SW & 3 & 14 & 693 \\
\hline April & 208 & SW & 5 & 15 & 713 \\
\hline May & 265 & W & 5 & 13 & 745 \\
\hline June & 266 & W & 5 & 12 & 719 \\
\hline Ju1y & 238 & WSW & 4 & 10 & 860 \\
\hline August & 206 & SW & 2 & 7 & 925 \\
\hline September & 179 & $\mathrm{~S}$ & 2 & 8 & 900 \\
\hline October & 167 & SSE & 8 & 13 & 923 \\
\hline November & 161 & SSE & 9 & 16 & 898 \\
\hline December & 168 & SSE & 9 & 17 & 930 \\
\hline
\end{tabular}



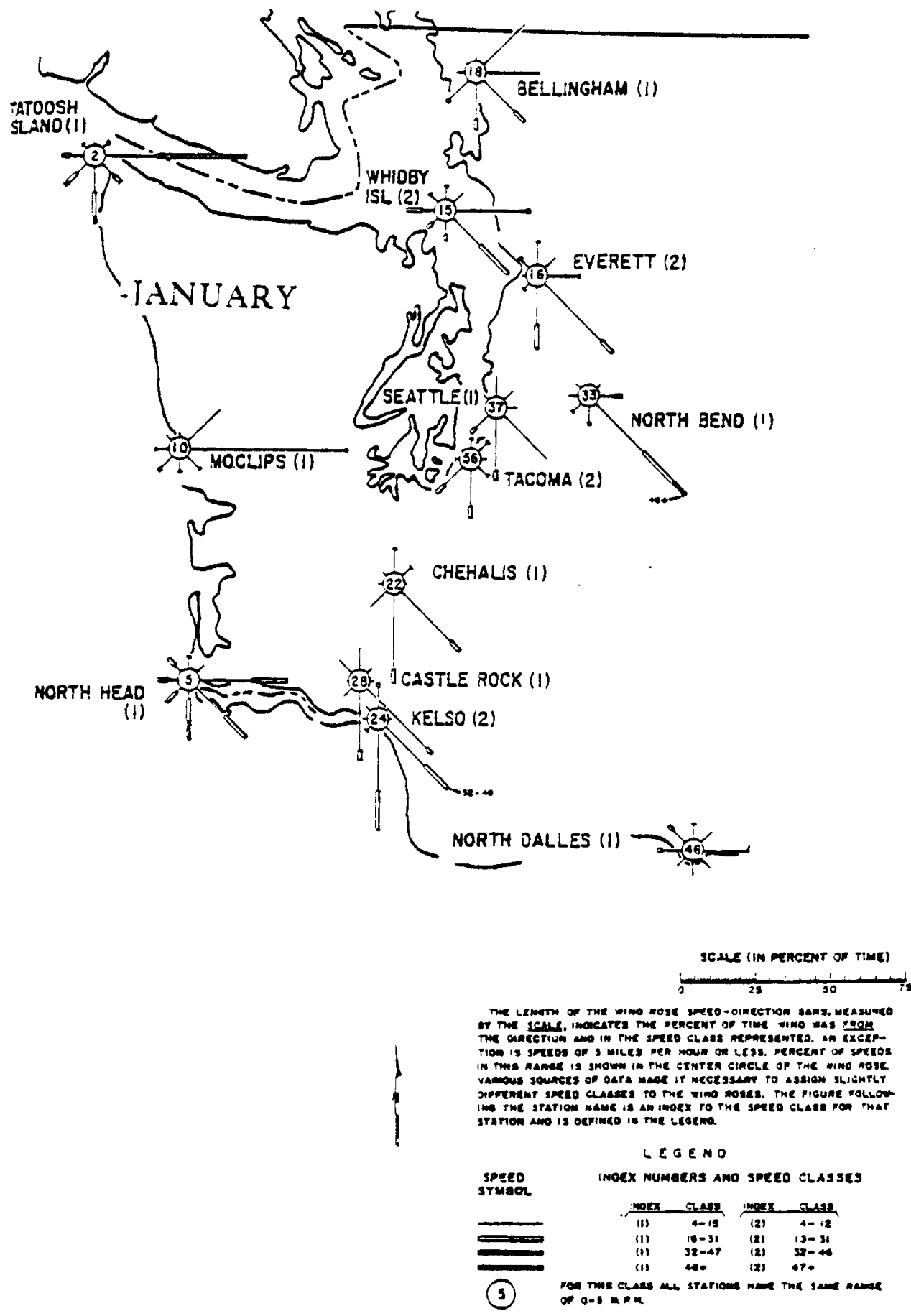

Figure 5. Surface Wind Roses for Washington Stations - January. (Washingt on State University, 1972) 

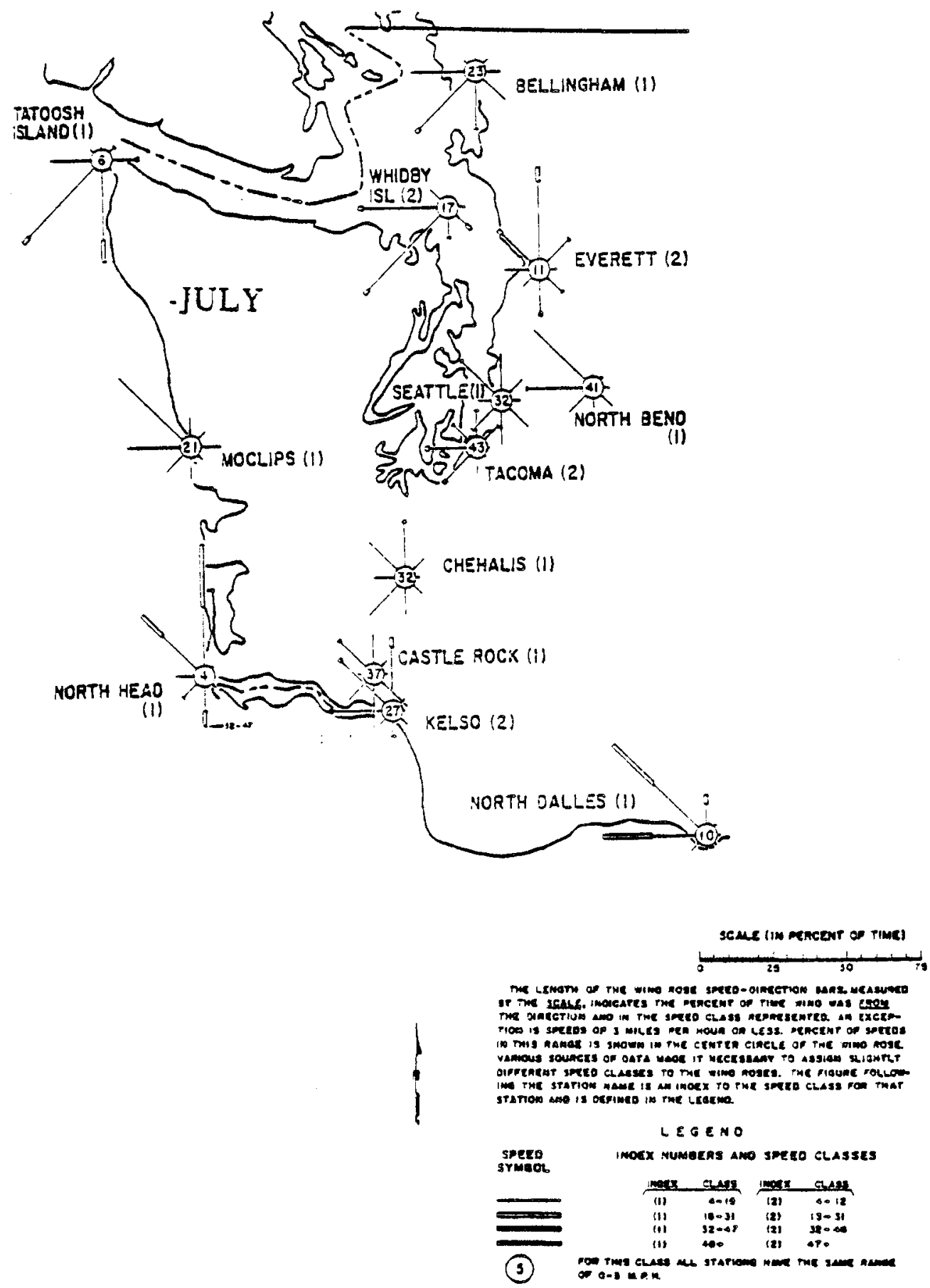

Figure 6. Surface Wind Roses for Washington Stations - July. (Washington State University, 1972) 

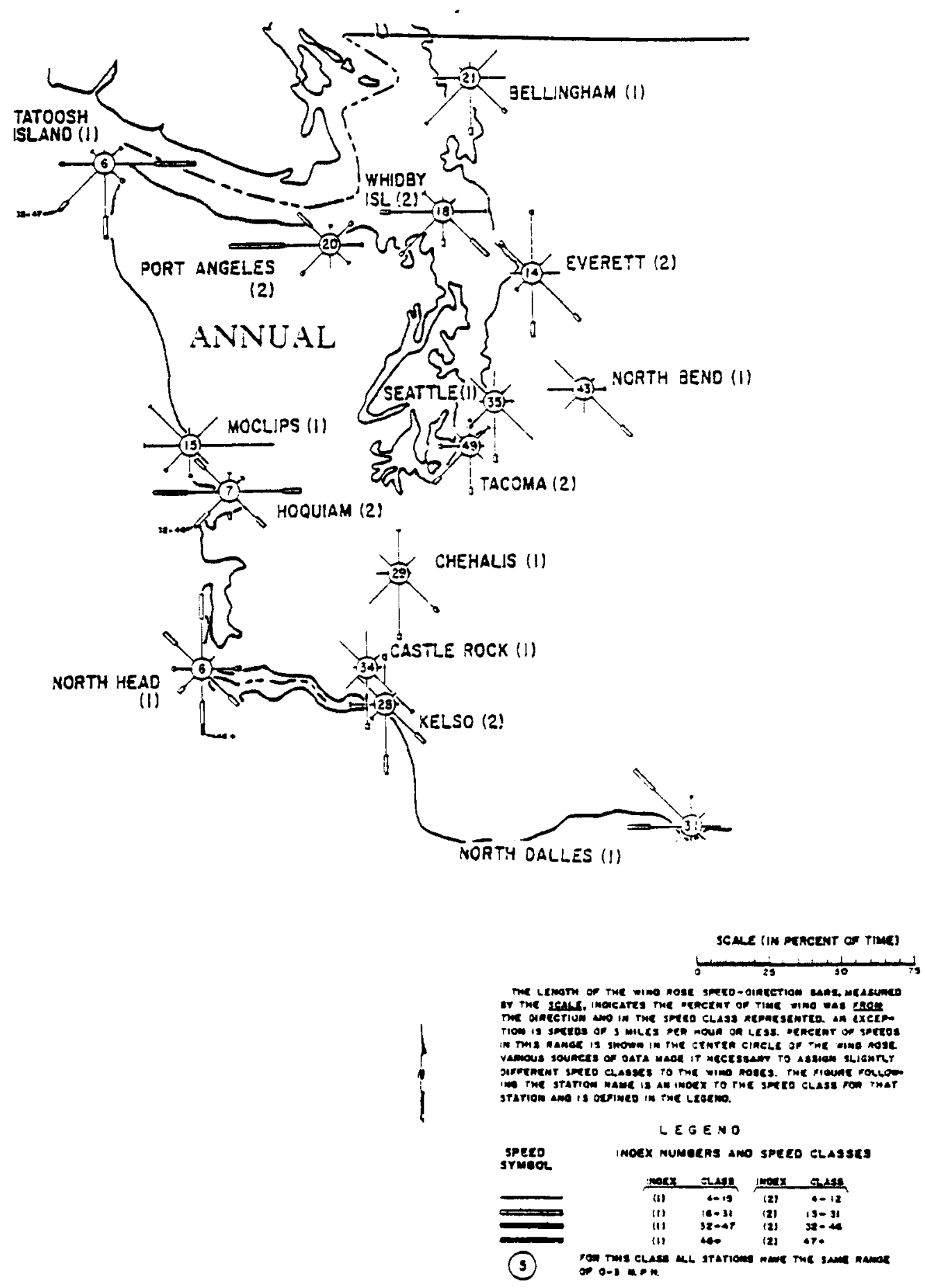

Figure 7. Surface Wind Roses for Washington Stations - Annual (Washington State University, 1972) 
It would appear that a synoptic situation which is associated with high $\mathrm{SO}_{2}$ concentrations in the study area is the passage of extra-tropical cyclones to the north or through the area and, in particular, a rapid succession of several cyclones passing through the region over the period of a few days. This situation is likely to be most common during the winter months.

The occurrence of $\mathrm{SO}_{2}$ short-term standard violations during such periods may reflect the configuration of major $\mathrm{SO}_{2}$ sources and $\mathrm{SO}_{2}$ sampling locations in the region. Three-quarters of the violations recorded over the two year period were at the Bellingham locations and both episodes discussed (January 3-9 and February 10-13, 1975) showed violations at this location on each day. The presence of low pressure systems to the northwest of the study area at $850 \mathrm{mb}$. and to the north, northeast or northwest at the surface would bring prevailing flow over the northern Puget Sound region (at plume height) from the south to southwestern sector. Under such circumstances, the Bellingham location would be "downwind" from the refinery complexes at March Point (which represent major sources of $\mathrm{SO}_{2}$ in the area) much of the time.

January 3, 1975 provides a good example of a typical wintertime situation. A low pressure center developed to the west of the study area at $850 \mathrm{mb}$. and moved eastward across the region through the period of analysis. Surface conditions show the development of a depression directly east of the study area during the simulation. Presumably, 24 hour $\mathrm{SO}_{2}$ standard violations at Bellingham were primarily caused by flow conditions from the southern quadrant during the first part of the day. (More detailed discussions of meteorological conditions are given under each separate case in the appendicies included with this paper.) 
February 12 represents a similar situation with a strong low pressure system at the surface, $850 \mathrm{mb}, 700 \mathrm{mb}$. and $500 \mathrm{mb}$. to the west of the study area. Flow was generally from the southwest at all levels, although the position of an occluded front through the study area complicated the surface flow situation. This pattern is also likely to occur during the winter months as extra-tropical cyclones develop in the Gulf of Alaska and move south and eastward.

The two days chosen for simulation on the basis of TSP measurements are quite different synoptically. January 24, 1975 was characterized by light winds with fairly high pressure conditions at the surface in northern Puget Sound. Flow at the upper levels was westerly around the Aleutian low. Stagnation conditions generally inhibit dispersion of pollutants and such uncharacteristically light wind speeds through the northern Puget Sound region would allow pollutant buildup in the study area. This situation would not be typical of the winter months and might be expected more frequently in summer.

January 30, 1975, in contrast, showed a more typical winter pattern with cyclones developing off the Pacific coast. This resulted in steady southeasterly flow at the surface over northern Puget Sound and southerly to southwesterly flow at upper levels. Winds were strong over much of the region and blowing dust may have contributed a significant portion of the TSP load measured on this occasion.

The four days chosen for analysis indicate that high pollutant concentrations may occur in the northern Puget Sound region under a variety of meteorological conditions. The rapid passage of extra-tropical cyclones that can occur during the winter months is apparently conductive to high $\mathrm{SO}_{2}$ levels in some portions of the study area. This may reflect 
source/receptor configuration to some extent. Stagnation conditions, though probably less common in the area, can occur and would tend to inhibit dispersion of any pollutants introduced into the atmosphere over northern Puget Sound. Conditions that offer a potential for pollutant concentration may also be accompanied by southeasterly flow over the region -- a fairly common occurrence during the winter period. The individual cases to be modeled show a variety of flow conditions with background directions $360.0^{\circ}$ clockwise to $247.5^{\circ}$ represented and wind speeds from $1 \mathrm{~m} / \mathrm{s}$ to $6.5 \mathrm{~m} / \mathrm{s}$. Flow from the west to northwest, which would be a common summertime situation, is not represented. Each case reflects a situation where the meteorological conditions could interact with contaminant emissions in such a way that high groundlevel concentrations of pollutants would result. 


\section{CHAPTER III}

MODEL DESCRIPTIONS

WINDS

The WINDS model is a simple one-layer model of atmospheric boundary layer flow that was developed for use in complex terrain. The model was derived through simplification of the fundamental Navier-Stokes flow equations. This simplification was gained by neglecting advection terms in the equations of motion and by assuming an impulse solution. Although the equations do not describe all flow characteristics, the resultant solution describes a diagnostic model of the vector flow field. Procedures for solving the equations involve serial approximations which superimpose a new physical effect on the previous solution. The first step in the solution is to transfer the large scale background wind into a terrain-induced modification of the throughflow. Next, thermal and frictional modification of the vorticity and divergence are introduced and superimposed on the terrain-induced flow. Finally, stream functions and velocity potentials are calculated so that the wind speed and direction can be defined at all interior points. The WINDS model is described more fully in a U. S. Forest Service technical paper (see Appendix A) (Fosberg, Marlatt and Krupnak, 1976) and a user's manual (Childs and Marlatt, 1978).

Input

An array of grid points, 54 rows by 72 columns, with a spacing of 2,032 meters between points was used to represent the study area. The major inputs required to run the WINDS model and the information sources used in this analysis to arrive at these inputs include: 
1. ELEV - an array of elevations in meters at each grid point. Elevations for this study were obtained from the TOPAS map tapes developed by the U. S. Army (Topographic Analysis System User's Guide, U.S.D.A. Forest Service, 1976), see Appendix B.

2. IVEG - an array containing types of vegetation growing at each grid point. This information was taken from U.S.G.S topographic maps $(1: 250,000$ scale). Vegetation types used and associated roughness lengths are listed in Table 7. A map of vegetation types in the study area appears as Figure 9.

3. T70, T80, H70, H80 - temperatures and heights of upper level surfaces. T70 $\left({ }^{\circ} \mathrm{K}\right)$ is the temperature of the $700 \mathrm{mb}$. surface. T80 ( $\left.{ }^{\circ} \mathrm{K}\right)$ is the temperature of the $850 \mathrm{mb}$. surface. $H 70$ (m) is the height of the $700 \mathrm{mb}$. surface. H80 (m) is the height of the $850 \mathrm{mb}$. surface.

These variables were determined from upper atmosphere soundings taken from the Quillayute station in the northwestern section of the Olympic Peninsula and from upper air maps. Soundings and map readings are available at 12 hour intervals, at approximately 0300 PST and 1500 PST in the Puget Sound region.

The variables described are used to compute SIGMA, the lapse rate of the atmosphere over the study area and THETABR, the surface potential temperature. In conjunction with the elevation arrays, these variables are used to compute potential surface temperature $\left({ }^{\circ} \mathrm{K}\right)$ at each grid point (array THETA). The computed potential temperatures were used for all grid points with an elevation greater than zero (sea level). Surface 


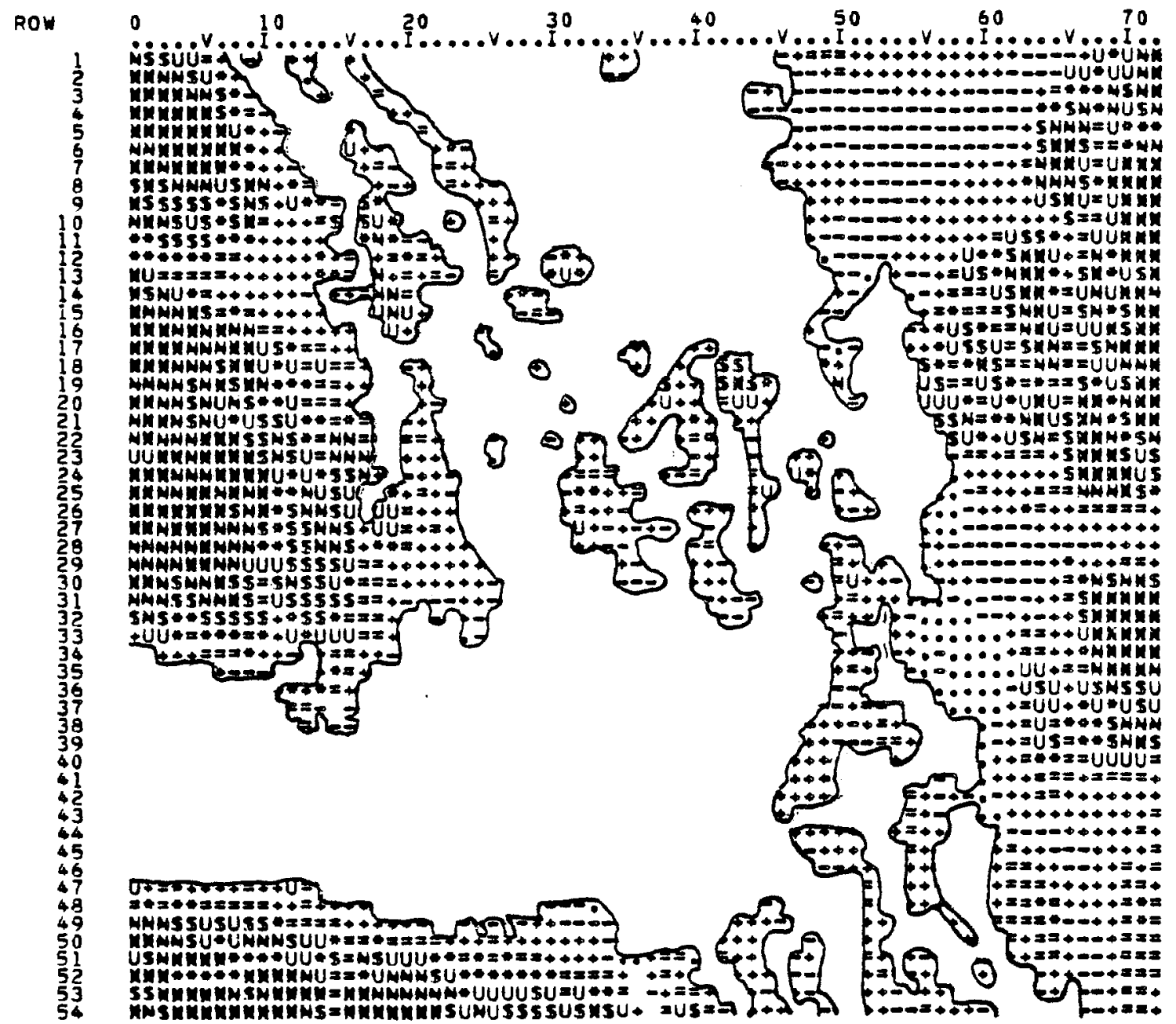

\begin{tabular}{|c|c|}
\hline Code & Elevation (ft.) \\
\hline Blank & 0.0 \\
\hline & $.1-10.0$ \\
\hline - & $10.1-100.0$ \\
\hline+ & $100.1-300.0$ \\
\hline$=$ & $300.1-500.0$ \\
\hline * & $500.1-750.0$ \\
\hline $\mathrm{U}$ & $750.1-1000.0$ \\
\hline$\$$ & $1000.1-1500.0$ \\
\hline H & $1500.1-2000.0$ \\
\hline K & $>2000.0$ \\
\hline
\end{tabular}

Figure 8. Grid Point Elevations for the Northern Puget Sound Study Area. 
Table 7. Vegetation Types, Associated Map Codes and Roughness Lengths Used for WINDS Model Input (Fosberg, Marlatt, Krupnak, 1976).

\begin{tabular}{lcc} 
Vegetation Type & Map Code & Roughness Length (m) \\
\hline Forest & F & 2.80 \\
Rough Water & $\mathrm{R}$ & 0.10 \\
Smooth or Protected Water & W & 0.01 \\
Thin Grass up to 50 cm High & $\mathrm{G}$ & 0.05 \\
Cultivated Fields & $\mathrm{S}$ & 1.00 \\
Towns & $\mathrm{T}$ & 1.65 \\
City or Urban Areas & $\mathrm{V}$ & 2.00 \\
Orchard & $\mathrm{O}$ & 1.98 \\
Industrial & $\mathrm{I}$ & 1.65 \\
\hline
\end{tabular}

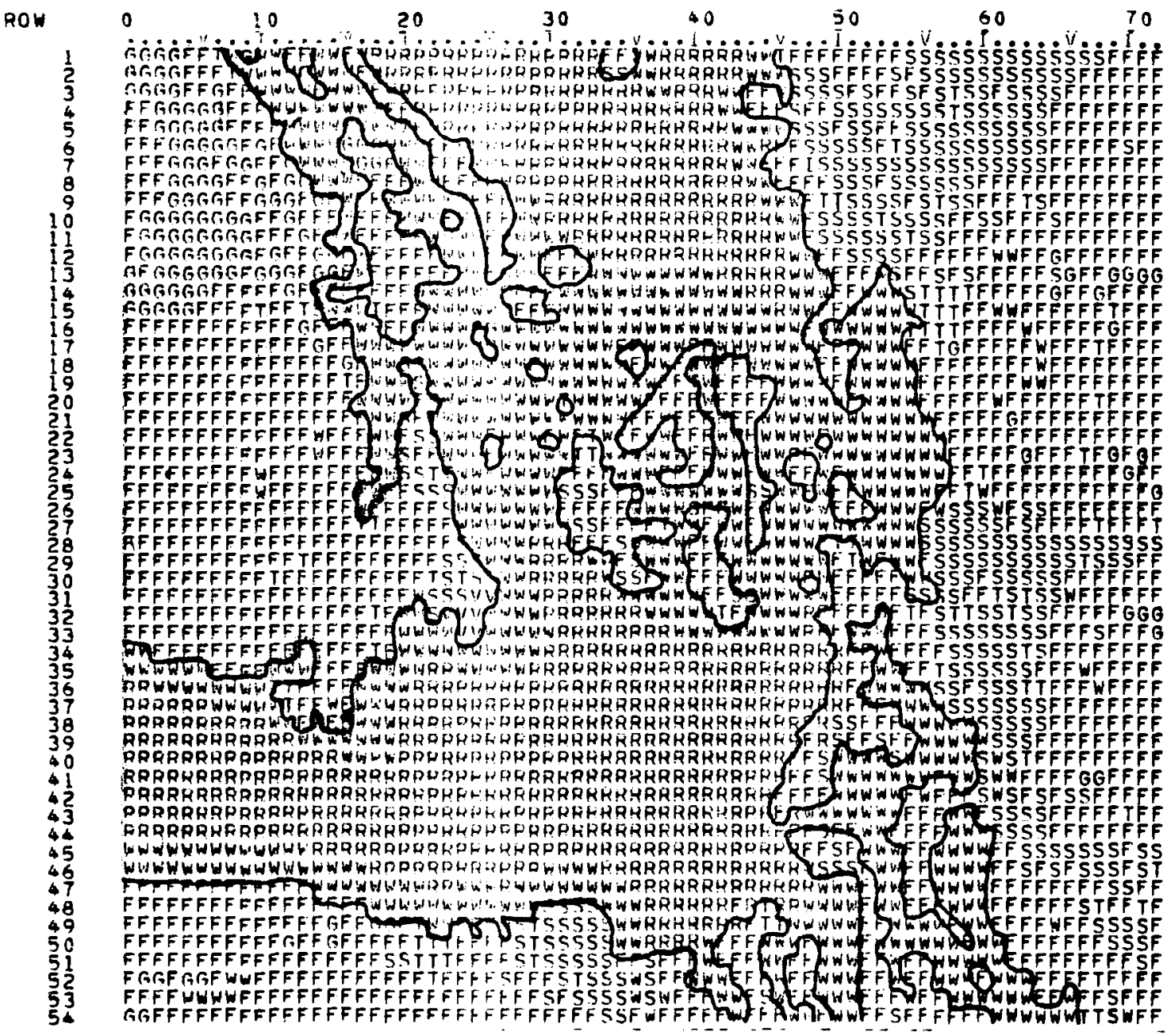

Figure 9. Grid Point Vegetation Codes for Northern Puget Sound Study Area. 
water temperatures were determined from information on average January and February temperatures for the northern Puget Sound area (Washington State University, 1972). A value of $282.16^{\circ} \mathrm{K}$ was used for all ocean surface grid points. The non-dimensional surface pressure array, PSTR, was also calculated from the above variables and the ELEV array.

4. ZHT (m) - vertical height of the air slab (6 meters used).

5. DEGRES (deg.) and SPD ( $\left.\mathrm{m} / \mathrm{sec}_{.}\right)$- the background wind direction over the entire grid, based on a 16-point compass, and the speed of the prevailing background flow (m/sec.)

The WINDS model requires a single prevailing background flow as input. For most situations, however, it is impossible to realistically assume a single prevailing flow over the entire grid area as the flow field will have been channelized and perturbed by surrounding terrain features before it reaches the study area.

The northern Puget Sound region seen in Figure 10 shows three interconnected low level channels. These are Puget Sound to the southeast, the east-west oriented Strait of Juan de Fuca in the southwestern portion of the study area and the Strait of Georgia, filling the northcentral portion of the area and oriented somewhat northwest-southeast. The interior of Vancouver Island, the Olympic Peninsula and the foothills of the Cascades on the eastern edge of the area surround the sea level passages with much higher terrain. Wind speed and direction at the study area boundary will depend on the direction from which winds approach the coast, coupled with the influence of channelization through the three low level passages. This can result in quite different prevailing background flow characteristics for various sections of the grid area. This situation is discussed further in Appendix C. 


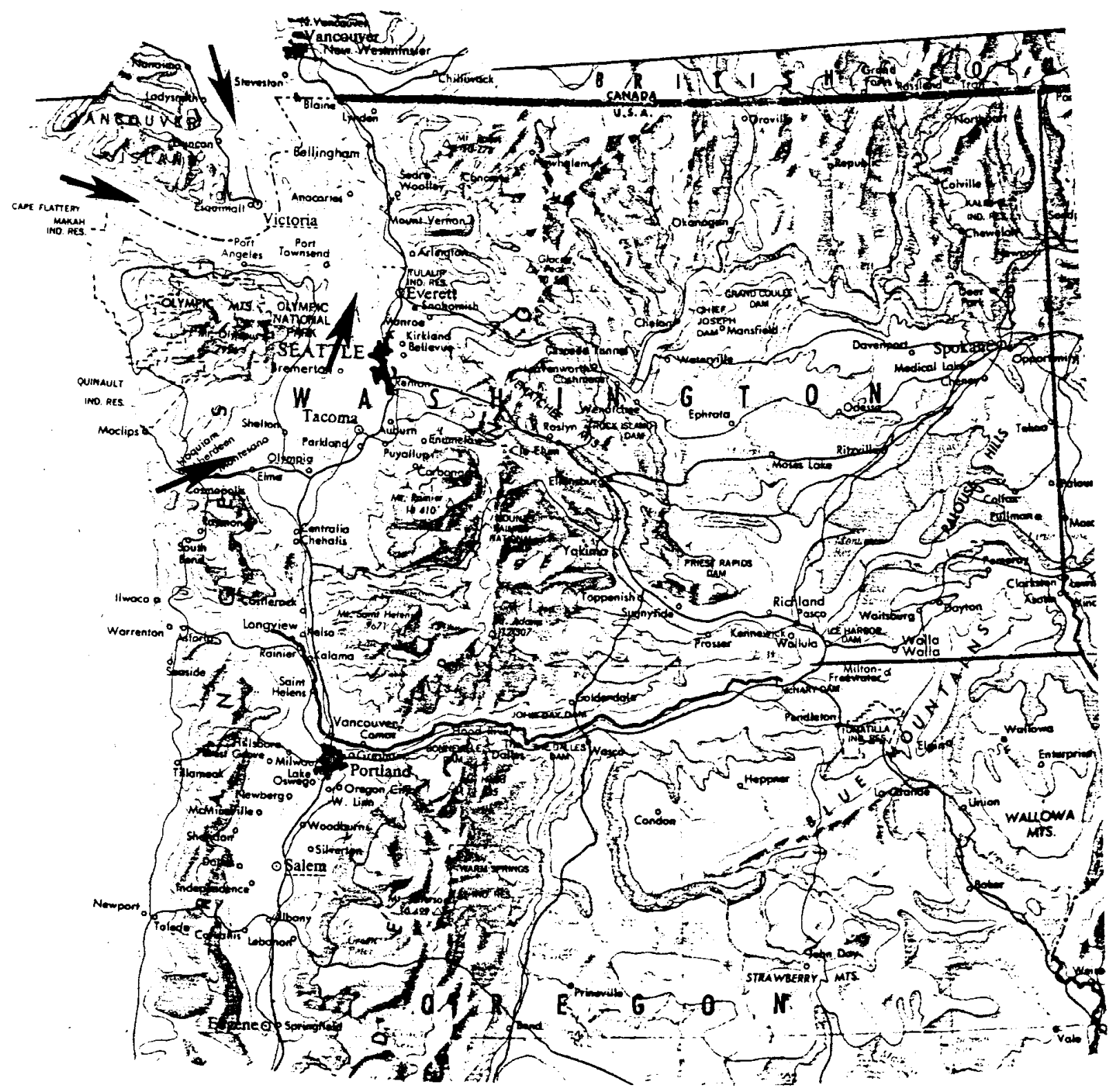

Figure 10. Northern Puget Sound Region Showing Three Low Leve1 Interconnecting Channels: Hoquiam-Puget Sound, Strait of Juan de Fuca, Strait of Georgia. 
The capability to input observed wind data at any grid point provides some flexibility in dealing with a complex flow situation such as occurs in the study area. Required inputs are the $\mathrm{x}$ and $\mathrm{y}$ coordinates of the observed data points, the wind direction in degrees and speed in meters per second. The points are set to the observed data and then interpolated into the initial background wind field. Each point influences speed and direction out through a circular area with a radius of three grid points and with the observed data at the center.

To simulate the complex flow characteristics in the study area, a scatter of points in the northern section of Puget Sound and another set of points in the Strait of Juan de Fuca were used as "observed" data points. These were located so as to allow the user to define a trend in flow in either passage that would differ from the background speed and direction over the grid as a whole, without directly affecting any of the validation stations. The location of the points used are shown in Figure 11 .

The Puget Sound points could be set either to the background flow or to a southeasterly flow direction of $135^{\circ}$, at any speed. Data points in Juan de Fuca Strait could be set to indicate an easterly flow $\left(90^{\circ}\right)$, a westerly flow $\left(270^{\circ}\right.$ ) or to the background speed and direction. These points were only used when the meteorological data collected indicated channelization in one passage or the other that differed significantly from the apparent background speed or direction over the rest of the grid. The station at Everett and, to a lesser extent, the stations at Smith and Whidbey Islands were used as indicators of flow from Puget Sound. The Victoria-Gonzales Heights station was examined with reference to wind flow characteristics in the Juan de Fuca region. 


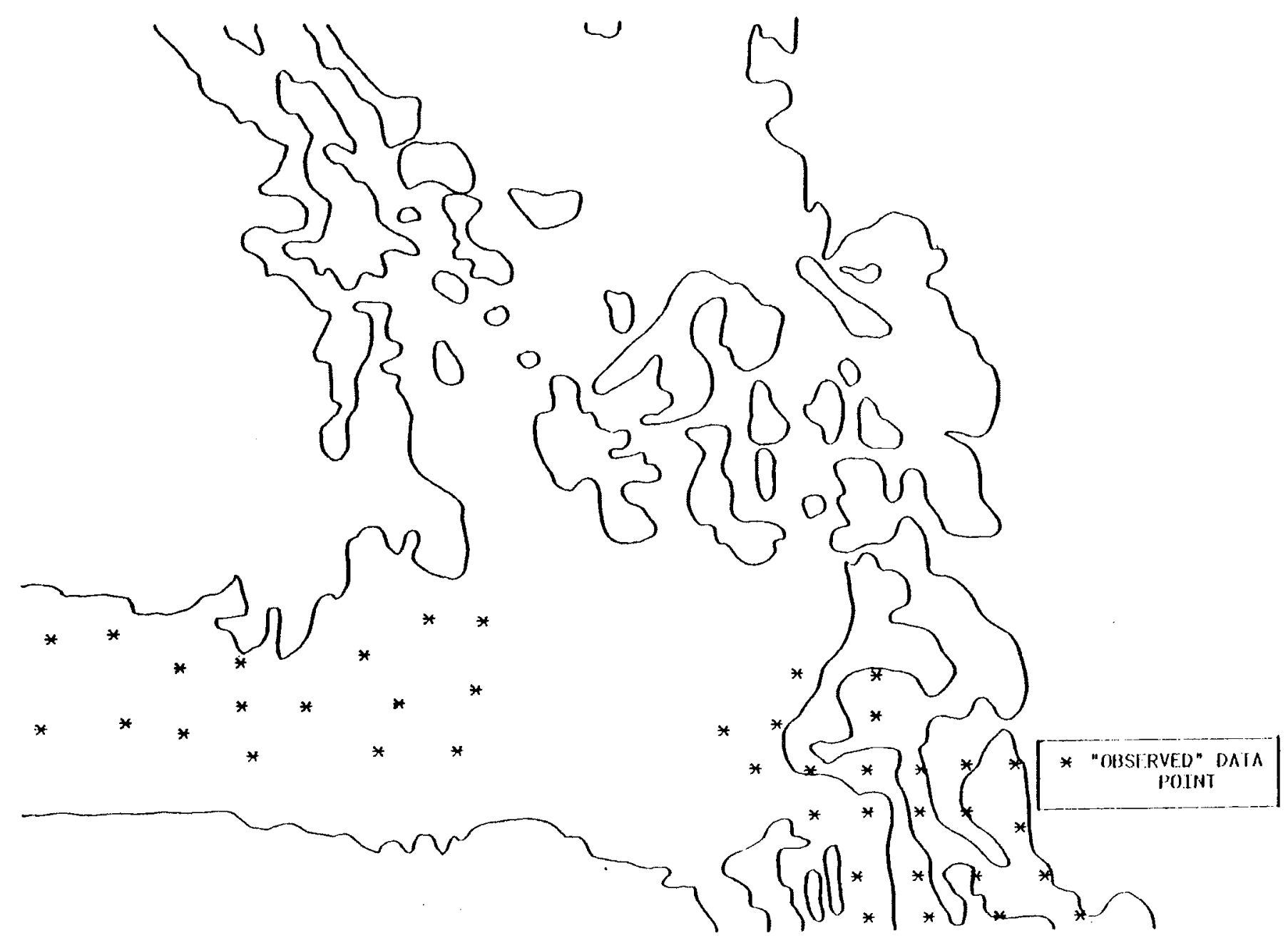

Figure 11. Location of "Observed" Data Points in the Northern Section of Puget Sound and Strait of Juan de Fuca. 
The two Port Angeles stations are also located in the Strait of Juan de Fuca area but were excluded from the analyses. Examination of the data collected at these locations showed a large percentage of data missing. Another source (Jensen and Danard, 1975) indicates an apparent sheltering effect of surrounding land forms that may render at least one of the Port Angeles stations unrepresentative.

The problem remained of choosing a prevailing background speed and direction to input as the initial wind field over the entire grid. Most of the stations (including Quillayute) are located such that a strong influence of surrounding topography on the measured speeds and directions would be expected and is indeed apparent from the data collected. The locations least affected by local terrain and so most representative of prevailing flow conditions would appear to be at the refinery locations to the north of the grid area (FARC and FMBL stations), although complications can arise even here due to their location near the edge of the study area (see discussion of January 30,1975 cases). An attempt to correlate the prevailing background flow with the $850 \mathrm{mb}$. level flow measured at Quillayute proved unsatisfactory. This station is located a considerable distance outside the study area and is on the other side of the Olympic Mountains, which block flow into the northern Puget Sound region from some directions at the $850 \mathrm{mb}$. level. Also, soundings are only available at 12 hour intervals (approximately 0315 PST and 1515 PST), while simulations were run for six periods on each of the four days chosen $(0300,0700,1100,1500,1900$ and 2300 hours PST).

The procedure used was to estimate a background flow based on a rough average of the core area stations, set the flow in Puget Sound and Juan de Fuca Strait as described above and simulate the windfield. 
The errors in direction and speed for all stations were averaged and, on the basis of these average deviations, the initial background speed or direction adjusted and the simulation rerun. If necessary, this procedure was repeated to minimize the mean error over the entire grid area. The flows actually used were then compared to the observed conditions at the data station locations to see which matched most closely; in effect, to analyze what information would have provided the best approximation of measured flow if used in the WINDS model initially. This is discussed in more detail under each case simulation.

Output

Output from the WINDS model consists of a series of maps showing wind direction (based on a 16-point compass) and wind speed (meters/ second) for each grid point for each day and time. A mixing volume index which describes the relative pollution potential of each grid cell, based primarily on wind speed and convergence or divergence of air parcels, is also calculated. The array of mixing volume indices calculated in the WINDS model is used as input to the TAPAS system (A Topographic Air Pollution Analysis System, Fosberg and Fox, 1977).

\section{Topographic Air Pollution Analysis System (TAPAS)}

The Topographic Air Pollution Analysis System (TAPAS) was developed from meteorological and air pollution dispersion models to allow the incorporation of air quality considerations into land use planning procedures. The windfield (calculated in WINDS) is combined with a dispersion model to determine a mixing volume which, in conjunction with 
the Pollutant Standards Index, provides a means of determining total emissions at specified air quality levels. The resultant index, the PI ( $\pi)$-Matrix, contains no source information but rather forms an atmospheric constraint matrix when applied to land use planning. Allowable emissions at each grid point are calculated for the PI( $\pi)$ index matrix and the ambient air quality standards. (See more detailed discussion of the TAPAS system in Fosberg and Fox (1977) in Appendix D.)

Input

The major inputs to the TAPAS system include:

1. UD, UN

Arrays of wind speeds (m/s) at each grid point (from WINDS model) for day or night cases, respectively.

2. AINDXD, AINDXN

Mixing volume indices for each grid point (from WINDS model), day or night case. This index represents the volume that would be incorporated by a plume located within each computational grid cell. Basically, this is the air available for mixing the effluent. The theoretical development of this index is based on the Gaussian Plume model. The index, I, is defined as $\frac{X}{Q} \times 10^{6}$ where $X$ is the ambient pollutant concentration, $Q$ is the pollutant emission rate and $10^{6}$ is a scaling factor based on Turner's (1969) maximum value of $\chi \frac{\mathrm{U}}{\mathrm{Q}}$ from a groundlevel source with Class $\mathrm{F}$ stability, where $\mathrm{u}$ represents wind speed. 
3. PSI

The Pollutant Standards Index ( $\Psi$ ) (Thom and Ott, 1976) which is defined: $\Psi=a+b\left(\frac{x}{\chi_{s}}\right)$ where $x_{s}$ is the ambient air quality standard for a particular pollutant; $a$ and $b$ are constant scaling factors (set to 0 and 100 , respectively, for the current analysis) and $x$ is the ambient concentration of the pollutant.

2. LCHIS, SCHIS

Long-term ( $>3$ hours) and short-term ( 3 hours or less) air quality standards for the effluent being analyzed. The standards considered under the TAPAS section of this study were federal and state annual average standards for $\mathrm{SO}_{2}(.03 \mathrm{ppm}$ $\left(80 \mu \mathrm{g} / \mathrm{m}^{3}\right)$ and $.02 \mathrm{ppm}\left(60 \mu \mathrm{g} / \mathrm{m}^{3}\right)$, respectively), the Washington 24 hour average standards for $\mathrm{So}_{2}\left(.10 \mathrm{ppm}\left(260 \mathrm{\mu g} / \mathrm{m}^{3}\right)\right)$, the state one hour average standard for $\mathrm{SO}_{2}$, not to be exceeded more than once per year $\left(.40 \mathrm{ppm}\left(1047 \mu \mathrm{g} / \mathrm{m}^{3}\right)\right)$ and federal incremental standards for Class II and Class III areas (.0076 and $.015 \mathrm{ppm}$ (20 and $\left.40 \mu \mathrm{g} / \mathrm{m}^{3}\right)$, respectively).

\section{Output}

The output from the TAPAS system is a PI-Matrix of the same dimensions as the mixing volume index. The PI-Matrix represents maximum allowable emissions in each grid cell that will insure that the standards are not exceeded. Two forms of the PI-Matrix are used, based on different averaging times:

1. For application to short-term standards, no longer than three hours, a short-term PI-Matrix is calculated. The $\pi_{s}$ values, in dimensions of mass per unit time, represent total emission rates 
which will result in air quality of a given $\Psi$ value within each grid cell. For all the PI-Matrix calculations in this analysis, values of $X$ were set equal to $x_{s}$ in the Pollution Standards Index calculations. The short-term $\pi_{s}$ value represents the instantaneous emission rate allowable.

2. For standards longer than three hours averaging time, a different PI-Index is calculated. The long-term index $\pi_{L}$ is arrived at by using Gaussian dispersion to define a mixing cross-sectional area, and then using concepts similar to the box model (Fox, 1975) to compute the volume. The Gaussian form of the mixing volume index is transformed to a ventilated box model. The $\pi_{L}$ index obtained is in units of mass. An emission rate must be multiplied by the length of time it is operating to give the allowable pollution loading for a given cell.

Two averaging periods were used to calculate long-term $\pi_{L}$ matrix values. A 24 hour average value was calculated using a daytime situation (1500 PST) and a nighttime situation (0300 PST) for each day simulated. An annual PI-Matrix was calculated from the annual mixing volume index based on annual wind rose data from Bellingham. 


\section{CHAPTER IV}

RESULTS

The results of the wind flow modeling and associated PI-Matrix values for each of the four days chosen are shown in the following section. For the wind flow analyses all input, observations, data points and resultant wind directions were rounded to the nearest $22.5^{\circ}$ sector (16-point compass). Likewise, speeds were rounded to the nearest meter per second. Stations were located as nearly as possible on a topographic map $(1: 250,000$ scale) and then assigned to the closest grid point. As some data stations only report wind direction to the nearest $45^{\circ}$ and since all input, observations and output were rounded to the nearest compass point, a tolerance of \pm one compass point $\left(22.5^{\circ}\right)$ was assumed for the simulation results. Thus, the wind flow directions were considered correctly modeled if the simulated value was within $22.5^{\circ}$ of the direction reported (after rounding to the nearest compass point). Wind speeds were assumed correctly simulated if within \pm one meter per second of the reported value.

The necessity of assigning each station a grid point location introduced some additional variance into the validation procedure. In several cases, the exact location of the site was uncertain enough that it could easily have been assigned to one of the other surrounding grid points. Since adjacent grid points often have different wind flows, the model may have, in some cases, correctly simulated the true wind flow at the station location even though the predicted value was more than one compass point or one meter per second different from the reported value. For this reason, the average deviations were relied on most heavily in evaluating WINDS model performance. 
Appendix E, included with this report, contains more detailed information concerning the four case analyses. The appendix contains a description of the synoptic meteorology influencing the northern Puget Sound region during each period of analysis, along with sounding data from Quillayute, Washington. Observed and predicted wind flow conditions at data station localities are presented for each hour of the analyses. Wind flow modeling results for the entire northern Puget Sound region are summarized in wind flag maps and the PI-Matrix results are shown in a series of shaded maps.

\section{WIND FLOW SIMULATION ANALYSES}

Comparison tables for the wind flow analyses use the following conventions: notations are an indication of magnitude and direction that predicted winds err from observed wind data. Units are meters per second for wind speed and compass points (16-point compass) for direction. Positive (+) deviations for direction indicate error in a clockwise direction and negative (-) deviations indicate error in a counter-clockwise direction from the observed winds. Likewise, positive $(+)$ deviations in wind speed indicate over-prediction and negative $(-)$ notations indicate predicted wind speeds below recorded values.

The mean error between observed and simulated values of wind speed and direction and the standard deviations of error were calculated for each case. Mean and standard deviation values were calculated for all stations shown in the WINDS results tables and also for the stations in the "core" area only (FARC, FMBL, BELA, BELC, BELA, BELT and the Anacortes-March Point stations). 
The mean error provides a point estimation of the overall accuracy with which wind flow conditions were simulated for a given time period. The standard deviation of error has been used as a measure of spread. These parameters facilitate comparison between simulation results for different time periods. As discussed previously, deviations of less than or equal to one compass point or one meter per second are within the tolerance units expected of the simulation due to time, grid point and rounding discrepancies between the collected data and the simulated situations. Unfortunately, the nature of the data precludes the estimation of a precise rejection region by standard statistical methods (i.e. nonnormality of error distributions invalidate the matched pair t-test; ties and zero difference scores violate the assumptions of non-parametric procedures such as the Wilcoxon test, etc.). It is apparent, however, that small errors ( $\leq 1-2$ compass points or meters per second) are relatively uninformative while large discrepancies may provide useful information regarding model limitations or station anomalies. Since much of the data collected was used, at least indirectly, to generate input variables, the comparison of observed and simulated flow conditions at the 11 data locations does not represent a validation procedure but simply an analysis of how closely the observed situation could be simulated by the model.

The Allied Chemical station at March Point (AALC) was eliminated from the analysis as examination of the January 3 and February 12, 1975 meteorological data showed frequent large deviations from the other three nearby stations. Since these four stations (AALC, ASHL, ATEX and ANWP) are located at the same grid point for modeling purposes, the elimination of this station seemed justifiable. The three March Point stations were treated as one data point in the analyses by averaging the deviations of 
the simulated wind flow at that grid location from the three observed values.

Table 8 is a summary of final input flows, temperature and pressure data used in the wind flow analyses. The speed and direction to which the "observed" data points were set is indicated under the Puget Sound and Juan de Fuca designations.

Tables 9, 13, 17 and 21 are comparisons of simulation results with the observed meteorological data. Deviations are recorded as compass points (16-point compass) and meters per second. "Core" area stations are FARC, FMBL, BELA, BELC, BELT and the Anacortes Stations (AAVE).

Tables $10,14,18$ and 22 represent more detailed comparisons of WINDS results to the observed data. The fractions of the predicted values within a given number of compass points or meters per second of the observed values are shown for each hour simulated.

Tables 11, 15, 19 and 23 show analyses of observed versus predicted values for each station for each day simulated. Mean error values and the standard deviation of error are given -- units are compass points (16-point compass) for direction and meters per second for speed. Tables 12, 16, 20 and 24 are comparisons of the Ferndale station (FARC and FMBL) observations and the $850 \mathrm{mb}$. sounding data from Quillayute with the background direction and speed used in the analyses. 
Table 8. Final Input Data Used in Wind Flow Simulation. Units are degrees for direction, meters per second for speed, ${ }^{\circ} \mathrm{K}$ for temperature and meters for heights.

\begin{tabular}{|c|c|c|c|c|c|c|c|c|c|c|}
\hline \multirow[b]{2}{*}{ Time } & \multicolumn{2}{|c|}{ Background } & \multicolumn{2}{|c|}{ Puget Sound } & \multicolumn{2}{|c|}{ Juan de Fuca } & \multirow[b]{2}{*}{$\mathrm{T} 70$} & \multirow[b]{2}{*}{$\mathrm{T} 80$} & \multirow[b]{2}{*}{$\mathrm{H} 70$} & \multirow[b]{2}{*}{$\mathrm{H} 8 \mathrm{C}$} \\
\hline & Dir & Speed & Dir & $\overline{\text { Speed }}$ & Dir & Speed & & & & \\
\hline \multicolumn{11}{|c|}{ January 3,1975} \\
\hline 0300 & 225.0 & 5.0 & 135.0 & 3.0 & 225.0 & 5.0 & 258.7 & 267.7 & 3003 & 1511 \\
\hline 0700 & 157.5 & 3.0 & 135.0 & 2.0 & 270.0 & 2.0 & 260.1 & 268.5 & 2972 & 1464 \\
\hline 1100 & 112.5 & 2.0 & 135.0 & 3.0 & 112.5 & 2.0 & 261.5 & 269.3 & 2940 & 1417 \\
\hline 1500 & 67.5 & 3.5 & 135.0 & 4.0 & 67.5 & 3.5 & 262.9 & 270.1 & 2909 & 1390 \\
\hline 1900 & 90.0 & 4.0 & 135.0 & 5.0 & 90.0 & 4.0 & 261.5 & 269.3 & 2878 & 1363 \\
\hline 2300 & 22.5 & 4.0 & 135.0 & 8.5 & 22.5 & 4.0 & 260.1 & 268.5 & 2846 & 1336 \\
\hline \multicolumn{11}{|c|}{ January 24,1975} \\
\hline 0300 & 180.0 & 3.0 & 180.0 & 3.0 & 180.0 & 3.0 & 267.6 & 270.2 & 3017 & 1496 \\
\hline 0700 & 135.0 & 2.0 & 135.0 & 2.0 & 270.0 & 2.0 & 267.0 & 270.5 & 3010 & 1486 \\
\hline 1100 & 157.5 & 2.0 & 135.0 & 2.0 & 270.0 & 2.0 & 266.4 & 270.8 & 3003 & 1476 \\
\hline 1500 & 180.0 & 1.0 & 135.0 & 1.0 & 270.0 & 4.0 & 265.8 & 271.2 & 2996 & 1466 \\
\hline 1900 & 225.0 & 1.0 & 225.0 & 1.0 & 270.0 & 3.5 & 264.3 & 270.5 & 2987 & 1464 \\
\hline 2300 & 0.0 & 1.0 & 360.0 & 1.0 & 270.0 & 2.0 & 262.8 & 269.9 & 2978 & 1462 \\
\hline
\end{tabular}


Table 8 . Continued

\begin{tabular}{|c|c|c|c|c|c|c|c|c|c|c|}
\hline \multirow[b]{2}{*}{ Time } & \multicolumn{2}{|c|}{ Background } & \multicolumn{2}{|c|}{ Puget Sound } & \multicolumn{2}{|c|}{ Juan de Fuca } & \multirow[b]{2}{*}{ T70 } & \multirow[b]{2}{*}{$\mathrm{T} 80$} & \multirow[b]{2}{*}{ H70 } & \multirow[b]{2}{*}{ H80 } \\
\hline & Dir & $\overline{\text { Speed }}$ & Dir & Speed & Dir & Speed & & & & \\
\hline \multicolumn{11}{|c|}{ January 30,1975} \\
\hline 0300 & 112.5 & 4.0 & 135.0 & 6.0 & 90.0 & 7.0 & 258.6 & 266.8 & 2918 & 1427 \\
\hline 0700 & 112.5 & 4.0 & 135.0 & 7.0 & 90.0 & 10.0 & 258.6 & 266.9 & 2944 & 1398 \\
\hline 1100 & 112.5 & 5.5 & 135.0 & 6.5 & 90.0 & 16.0 & 258.6 & 267.0 & 2960 & 1369 \\
\hline 1500 & 112.5 & 4.0 & 135.0 & 12.0 & 90.0 & 15.0 & 258.6 & 267.0 & 2840 & 1340 \\
\hline 1900 & 112.5 & 6.5 & 135.0 & 6.5 & 90.0 & 20.0 & 259.1 & 267.4 & 2822 & 1319 \\
\hline 2300 & 112.5 & 6.0 & 135.0 & 10.0 & 90.0 & 18.0 & 259.6 & 267.8 & 2804 & 1298 \\
\hline \multicolumn{11}{|c|}{ February 12,1975} \\
\hline 0300 & 112.5 & 5.0 & 135.0 & 12.0 & 112.5 & 5.0 & 268.8 & 276.2 & 2777 & 1229 \\
\hline 0700 & 157.5 & 6.0 & 135.0 & 11.0 & 90.0 & 2.0 & 266.4 & 274.4 & 2787 & 1249 \\
\hline 1100 & 180.0 & 6.5 & 135.0 & 8.5 & 90.0 & 3.0 & 263.9 & 272.7 & 2797 & 1269 \\
\hline 1500 & 247.5 & 5.5 & 247.5 & 5.5 & 270.0 & 13.0 & 261.4 & 271.0 & 2806 & 1290 \\
\hline 1900 & 157.5 & 2.5 & 157.5 & 2.5 & 270.0 & 8.0 & 261.7 & 270.8 & 2821 & 1320 \\
\hline 2300 & 157.5 & 1.5 & 135.0 & 1.5 & 270.0 & 4.0 & 262.0 & 270.5 & 2836 & 1320 \\
\hline
\end{tabular}


Table 9. Comparison of Winds Results WIth Olsserved Data -- Jamuary 3, 1975.

lintits are compass points (16 point compass) for dircetion and

meters per second for speed conpartsons.

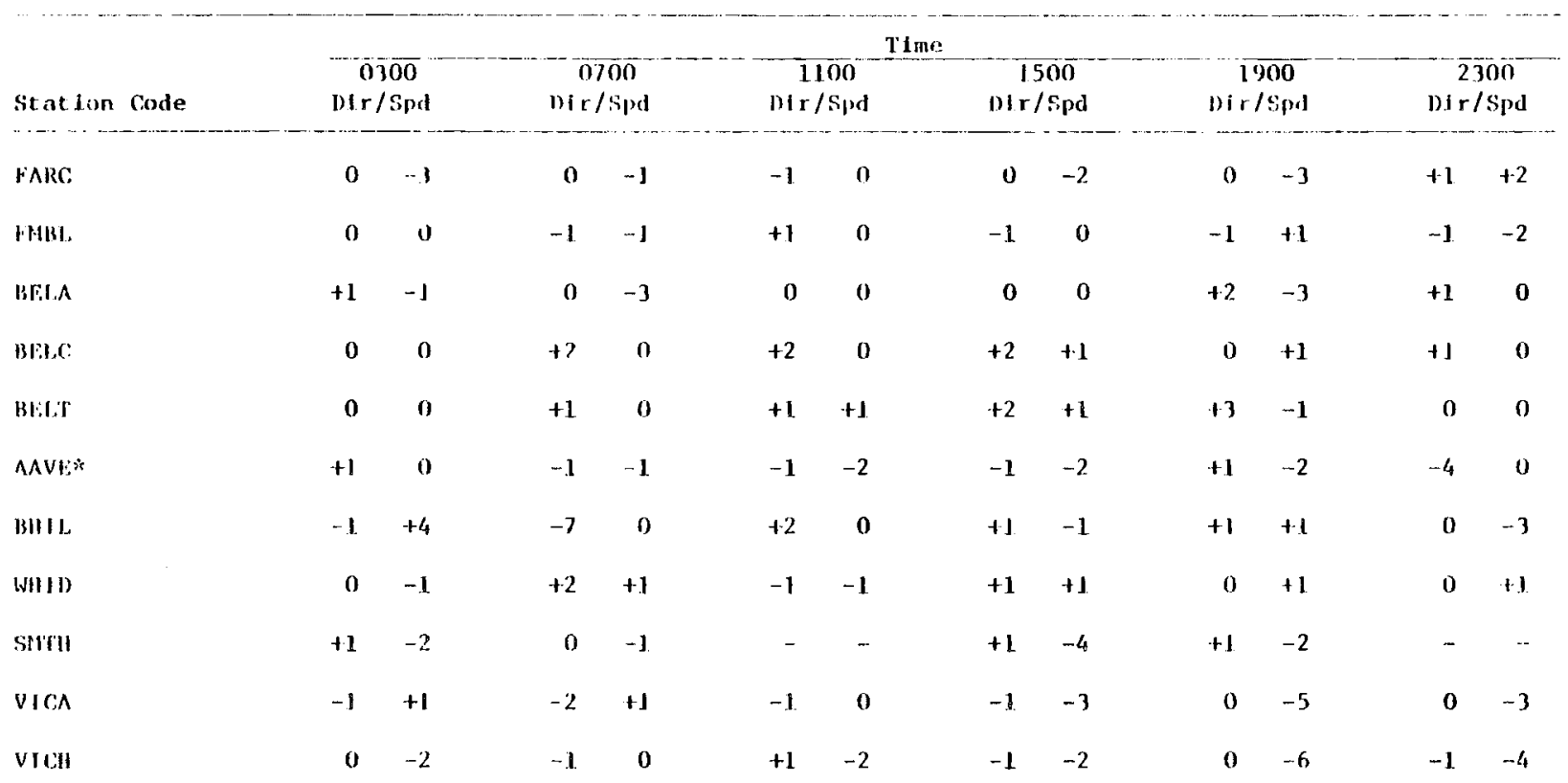

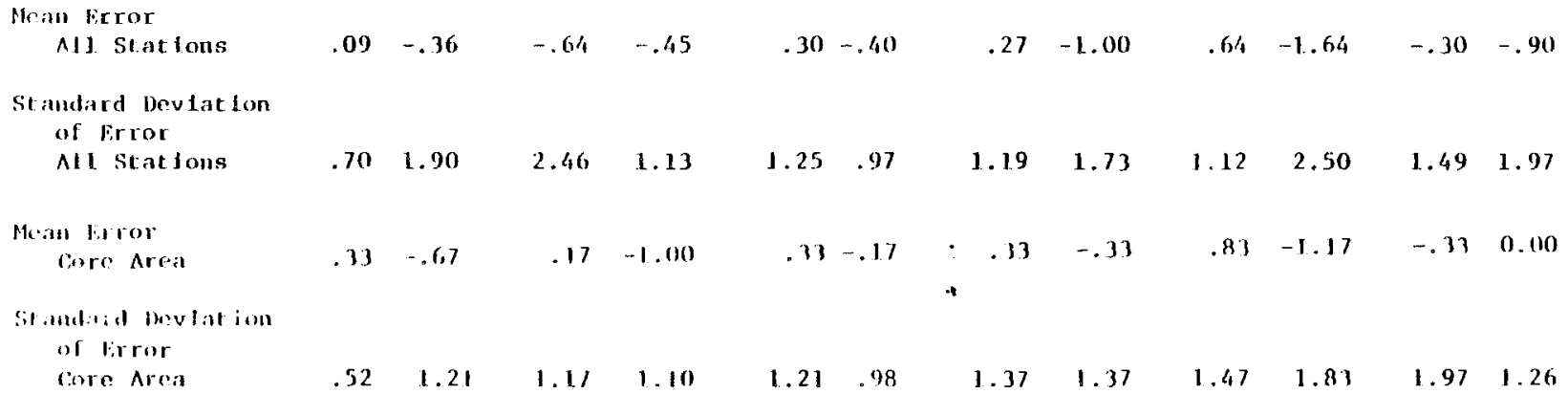


Table 10. Fraction of the Predicted Values Within a Given Number of Compass Points or Meters per Second of Observed Values for January 3, 1975 Simulation.

\begin{tabular}{lllllll}
\hline 0300 & 0700 & 1100 & 1500 & 1900 & 2300
\end{tabular}

\section{Al1 Stations}

$\begin{array}{llrrrrr} \pm 1 \text { Compass Point } & 1.00 & .64 & .80 & .82 & .82 & .90 \\ \pm 2 \text { Compass Points } & 1.00 & .91 & 1.00 & 1.00 & .91 & .90 \\ \pm 1 \mathrm{~m} / \mathrm{s} & .64 & .91 & .80 & .54 & .45 & .50 \\ \pm 2 \mathrm{~m} / \mathrm{s} & .82 & .91 & 1.00 & .82 & .64 & .70 \\ \pm 3 \mathrm{~m} / \mathrm{s} & .91 & 1.00 & 1.00 & .91 & .82 & .90\end{array}$

"Core" stations

\begin{tabular}{lrrrrrr} 
\pm 1 Compass Point & 1.00 & .83 & .83 & .67 & .67 & .83 \\
\pm 2 Compass Points & 1.00 & 1.00 & 1.00 & 1.00 & .83 & .83 \\
$\pm 1 \mathrm{~m} / \mathrm{s}$ & .83 & .83 & .83 & .67 & .50 & .67 \\
$\pm 2 \mathrm{~m} / \mathrm{s}$ & .83 & .83 & 1.00 & 1.00 & .67 & 1.00 \\
$\pm 3 \mathrm{~m} / \mathrm{s}$ & 1.00 & 1.00 & 1.00 & 1.00 & 1.00 & 1.00 \\
\hline
\end{tabular}


Table 11. Analysis of Simulation Accuracy for Each Station for All January 3, 1975 Cases. Units are compass points (16-point compass) and meters per second.

\begin{tabular}{lcccc}
\hline \multirow{2}{*}{ Station Code } & \multicolumn{2}{c}{ Mean Error } & \multicolumn{2}{c}{ Standard Deviation of Error } \\
\cline { 2 - 3 } Firection & Speed & -1.17 & .63 & Speed \\
\hline FMRC & 0.00 & -.33 & .84 & 1.94 \\
BELA & -.50 & -1.17 & .82 & 1.03 \\
BELC & .67 & .33 & .98 & 1.47 \\
BELT & 1.17 & .17 & 1.17 & .52 \\
AAVE & 1.17 & -1.17 & 1.83 & .75 \\
BHIL & -.83 & .17 & 3.27 & .98 \\
WHID & -.67 & .33 & 1.03 & 2.32 \\
SMTH & .33 & -2.25 & .50 & 1.03 \\
VICA & .75 & -1.50 & .75 & 2.51 \\
VICH & -.83 & -2.67 & .82 & 2.07 \\
& -.33 & & & \\
\hline
\end{tabular}

Table 12. Comparison of Ferndale Stations and $850 \mathrm{mb}$. Wind Flow From Quillayute Soundings with Background Flow Used as Final Input to WINDS Mode1 (Jan. 3, 1975 Simulation). Units are degrees and meters per second.

\begin{tabular}{lccccccccccccc}
\hline & $\begin{array}{c}0300 \\
\text { Dir/Spd }\end{array}$ & $\begin{array}{c}0700 \\
\text { Dir/Spd }\end{array}$ & $\begin{array}{c}1100 \\
\text { Dir/Spd }\end{array}$ & $\begin{array}{c}1500 \\
\text { Dir/Spd }\end{array}$ & $\begin{array}{c}1900 \\
\text { Dir/Spd }\end{array}$ & $\begin{array}{c}2300 \\
\text { Dir/Spd }\end{array}$ \\
\hline $\begin{array}{l}\text { Background } \\
\text { Flow WINDS }\end{array}$ & 225 & 5.0 & 158 & 3.0 & 113 & 2.0 & 68 & 2.0 & 90 & 4.0 & 23 & 4.0 \\
FARC Obs. & 225 & 8.0 & 158 & 4.0 & 135 & 2.0 & 68 & 6.0 & 90 & 7.0 & 360 & 3.0 \\
FMBL Obs. & 225 & 6.0 & 180 & 5.0 & 113 & 2.0 & 90 & 3.0 & 113 & 3.0 & 45 & 6.0 \\
$\begin{array}{l}\text { QUIL 850 mb. } \\
\text { Observation }\end{array}$ & 225 & 14.0 & - & - & - & - & 140 & 41.0 & - & - & - & - \\
\hline
\end{tabular}


Table 13. Comparison of Winds Results With Observed Data -. Minuary 24, 1975.

Inits are composs points (16 - polut compass) for dlrect lon and meters per second for speed compartsons.

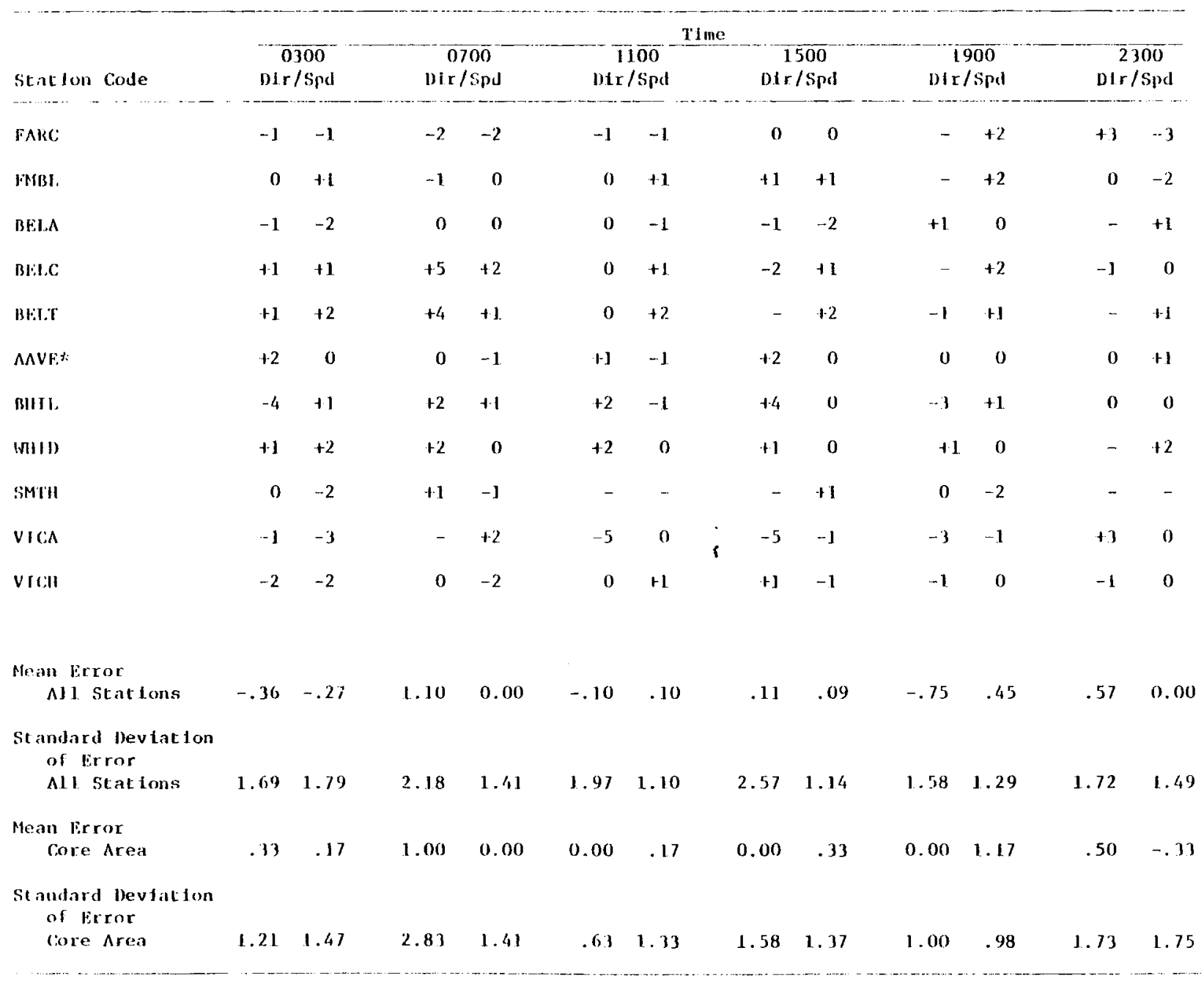

*AAVE, represents the average of ASHI, ANIP and ATEX results. 
Table 14. Fraction of the Predicted Values Within a Given Number of Compass Points or Meters per Second of Observed Values for January 24, 1975 Simulation.

\begin{tabular}{lrrrrrr}
\hline & 0300 & 0700 & 1100 & 1500 & 1900 & 2300 \\
\hline Al1 Stations & & & & & & \\
\pm 1 Compass Point & .73 & .50 & .70 & .56 & .75 & .71 \\
\pm 2 Compass Points & .91 & .80 & .90 & .78 & .75 & .71 \\
$\pm 1 \mathrm{~m} / \mathrm{s}$ & .45 & .64 & .90 & .82 & .64 & .70 \\
$\pm 2 \mathrm{~m} / \mathrm{s}$ & .91 & 1.00 & 1.00 & 1.00 & 1.00 & .90 \\
$\pm 3 \mathrm{~m} / \mathrm{s}$ & 1.00 & 1.00 & 1.00 & 1.00 & 1.00 & 1.00 \\
$\pm \mathrm{Core}$ "Stations & & & & & & \\
$\pm 1 \mathrm{Compass}$ Point & .83 & .50 & 1.00 & .60 & 1.00 & .75 \\
$\pm 2 \mathrm{Compass}$ Points & 1.00 & .67 & 1.00 & 1.00 & 1.00 & .75 \\
$\pm 1 \mathrm{~m} / \mathrm{s}$ & .67 & .67 & .83 & .67 & .50 & .67 \\
$\pm 2 \mathrm{~m} / \mathrm{s}$ & 1.00 & 1.00 & 1.00 & 1.00 & 1.00 & .83 \\
$\pm 3 \mathrm{~m} / \mathrm{s}$ & 1.00 & 1.00 & 1.00 & 1.00 & 1.00 & 1.00 \\
\hline & & & & & & \\
\hline
\end{tabular}


Table 15. Analysis of Simulation Accuracy for Each Station for All January 24, 1975 Cases.

\begin{tabular}{lcccc}
\hline \multirow{2}{*}{ Station Code } & \multicolumn{2}{c}{ Mean Error } & \multicolumn{2}{c}{ Standard Deviation of Error } \\
\cline { 2 - 3 } Firection & Speed & & Direction & Speed \\
\hline FMBL & -.20 & 0.83 & 1.92 & 1.72 \\
BELA & 0.00 & .50 & .71 & 1.38 \\
BELC & -.20 & 0.67 & .84 & 1.21 \\
BELT & .60 & 1.17 & 2.70 & .75 \\
AAVE & 1.60 & 1.50 & 1.52 & .55 \\
BHIL & .83 & -.17 & .98 & .82 \\
WHID & .17 & .33 & 3.13 & 1.03 \\
SMTH & 1.40 & .67 & .55 & 1.00 \\
VICA & 0.00 & -1.00 & 1.41 & 1.64 \\
VICH & -1.60 & -.50 & 2.97 & 1.21 \\
\hline & -.50 & -.67 & 1.05 & \\
\hline
\end{tabular}

Table 16. Comparison of Ferndale Stations and $850 \mathrm{mb}$. Wind Flow From Quillayute Soundings with Background Flow Used as Final Input to WINDS Model (Jan. 24, 1975 Simulation). Direction rounded to nearest compass point (16-point compass).

\begin{tabular}{|c|c|c|c|c|c|c|}
\hline & $\begin{array}{c}0300 \\
\mathrm{Dir} / \mathrm{Spd}\end{array}$ & $\begin{array}{c}0700 \\
\text { Dir/Spd }\end{array}$ & $\begin{array}{c}1100 \\
\operatorname{Dir} / \text { Spd }\end{array}$ & $\begin{array}{c}1500 \\
\operatorname{Dir} / \mathrm{Spd}\end{array}$ & $\begin{array}{c}1900 \\
\mathrm{Dir} / \mathrm{Spd}\end{array}$ & $\begin{array}{c}2300 \\
\text { Dir/Spd }\end{array}$ \\
\hline Flow WINDS & 1803.0 & 1352.0 & 1582.0 & 1801.0 & 2751.0 & 01.0 \\
\hline FARC Obs. & 2034.0 & 1804.0 & 1803.0 & 1802.0 & Calm & 2934.0 \\
\hline FMBL Obs. & 1803.0 & 1803.0 & 1802.0 & 1801.0 & Calm & 3153.0 \\
\hline $\begin{array}{l}\text { QUIL } 850 \mathrm{mb} \text {. } \\
\text { Observation }\end{array}$ & 2439.0 & - & - & 3159.0 & - & - \\
\hline
\end{tabular}


Table 17. Comparisom of Winds Resulls With Observed Data -- banuary 30, 1975. thits are compiss poluts (16-polut compriss) for direction and ineters per second for speed comparisoms.

\begin{tabular}{|c|c|c|c|c|c|c|c|c|c|c|c|c|}
\hline \multirow{3}{*}{$\begin{array}{l}\text { Station Code } \\
\text { FARC }\end{array}$} & \multicolumn{12}{|c|}{ Tine } \\
\hline & \multicolumn{2}{|c|}{$\begin{array}{c}0300 \\
D / r / S p d\end{array}$} & \multicolumn{2}{|c|}{$\begin{array}{c}0700 \\
\mathrm{Dir} / \mathrm{spd}\end{array}$} & \multicolumn{2}{|c|}{$\begin{array}{l}1100 \\
01 \mathrm{r} / \mathrm{s} d \mathrm{~d}\end{array}$} & \multicolumn{2}{|c|}{$\begin{array}{l}1500 \\
0.1 \mathrm{r} / \mathrm{Spd}\end{array}$} & \multicolumn{2}{|c|}{$\begin{array}{c}1.900 \\
\mathrm{Dir} / \mathrm{spd}\end{array}$} & \multicolumn{2}{|c|}{$\begin{array}{c}2300 \\
\mathrm{DLr} / \mathrm{spd}\end{array}$} \\
\hline & +1 & -1 & +1 & 0 & +1 & 0 & +1 & 0 & +1 & +1 & +1 & -2 \\
\hline FMBL. & 0 & +1 & 0 & 0 & 0 & +1 & 0 & 0 & 0 & 0 & +1 & -1 \\
\hline BFI:A & +4 & -1 & +3 & +1 & 14 & -1 & +5 & +1 & +1 & -1 & +1 & +2 \\
\hline BELC: & - & - & - & - & - & - & - & - & - & - & - & - \\
\hline BELT & $+t_{3}$ & +1 & +3 & +1 & +5 & +1 & 0 & +1 & +1 & 0 & -1 & +2 \\
\hline$\triangle \Lambda \cup E^{*}$ & -1 & 0 & -1 & -2 & -2 & -1 & -1 & -2 & 0 & +3 & +1 & +3 \\
\hline Blil.1. & +2 & 0 & +2 & 0 & +1 & 12 & +2 & +2 & +1 & +4 & +1 & +3 \\
\hline WIIID & +1 & 0 & +1 & 0 & +1 & -1 & 0 & $\mathbf{0}$ & 11 & +2 & +1 & 0 \\
\hline SNTH & 0 & -3 & 0 & -5 & - & - & 0 & -2 & +1 & -6 & - & - \\
\hline VICA & 0 & 0 & -2 & $-h_{6}$ & -1 & -6 & -1 & -7 & -1 & -2 & 0 & -3 \\
\hline Vich & -2 & -2 & -2 & -2 & -2 & -2 & -2 & -7 & 0 & -6 & -2 & -5 \\
\hline
\end{tabular}

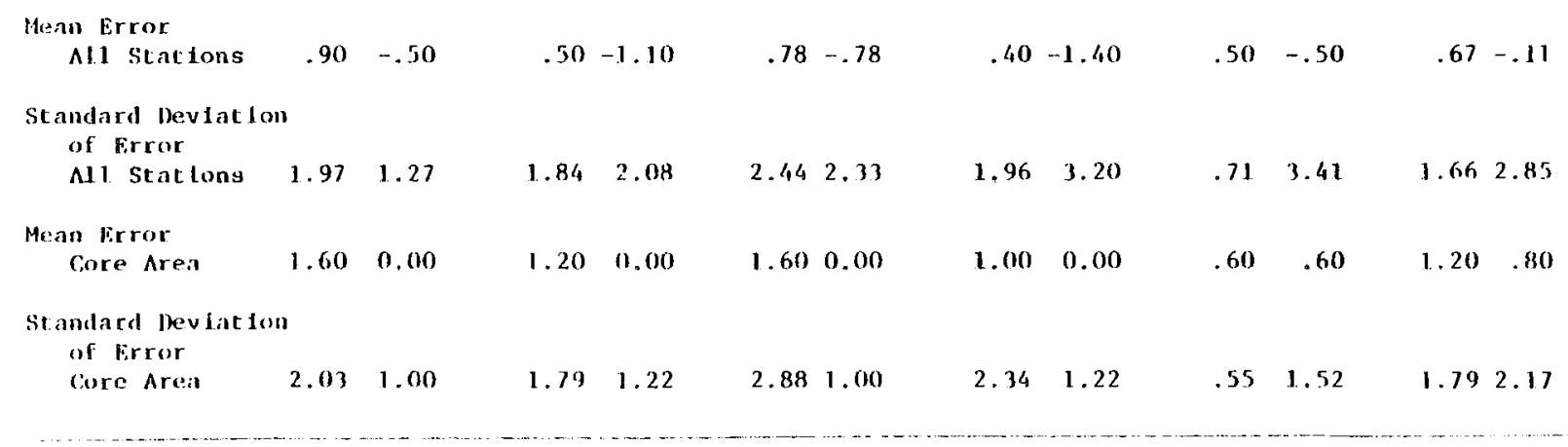

*AAVE represents the average of ASHI., ANWP and ATtix results. 
Tab1e 18. Fraction of the Predicted Values Within a Given Number of Compass Points or Meters per Second of Observed Values for January 30, 1975 Simulation.

\begin{tabular}{lrrrrrr}
\hline & 0300 & 0700 & 1100 & 1500 & 1900 & 2300 \\
\hline $\begin{array}{l}\text { A11 Stations } \\
\pm 1 \text { Compass Point }\end{array}$ & .60 & .50 & .55 & .70 & 1.00 & .78 \\
\pm 2 Compass Points & .80 & .80 & .78 & .90 & 1.00 & .89 \\
$\pm 1 \mathrm{~m} / \mathrm{s}$ & .80 & .60 & .67 & .50 & .40 & .22 \\
$\pm 2 \mathrm{~m} / \mathrm{s}$ & .90 & .80 & .89 & .80 & .60 & .56 \\
$\pm 3 \mathrm{~m} / \mathrm{s}$ & 1.00 & .80 & .89 & .80 & .70 & .89
\end{tabular}

\section{"Core" Stations}

$\begin{array}{lrrrrrr} \pm 1 \text { Compass Point } & .60 & .60 & .40 & .80 & 1.00 & .80 \\ \pm 2 \text { Compass Points } & .60 & .60 & .60 & .80 & 1.00 & .80 \\ \pm 1 \mathrm{~m} / \mathrm{s} & 1.00 & .80 & 1.00 & .80 & .80 & .20 \\ \pm 2 \mathrm{~m} / \mathrm{s} & 1.00 & 1.00 & 1.00 & 1.00 & .80 & .80 \\ \pm 3 \mathrm{~m} / \mathrm{s} & 1.00 & 1.00 & 1.00 & 1.00 & 1.00 & 1.00\end{array}$


Table 19. Analysis of Simulation Accuracy for Each Station for All January 30, 1975 Cases.

\begin{tabular}{|c|c|c|c|c|}
\hline \multirow[b]{2}{*}{ Station Code } & \multicolumn{2}{|c|}{ Mean Error } & \multirow{2}{*}{\multicolumn{2}{|c|}{$\frac{\text { Standard Deviation of Error }}{\text { Direction }}$}} \\
\hline & Direction & Speed & & \\
\hline FARC & 1.00 & -.33 & 0.00 & 1.03 \\
\hline FMBL & .17 & .17 & .41 & .75 \\
\hline BELA & 3.50 & .17 & 1.38 & 1.33 \\
\hline BELC & - & - & - & - \\
\hline BELT & 2.00 & 1.00 & 2.37 & .63 \\
\hline AAVE & -.67 & .17 & 1.03 & 2.32 \\
\hline BHIL & 1.50 & 1.83 & .55 & 1.60 \\
\hline WHID & .83 & .17 & .41 & .98 \\
\hline SMTH & .25 & -4.00 & .50 & 1.83 \\
\hline VICA & -.83 & -3.67 & .75 & 2.58 \\
\hline VICH & -1.67 & -4.00 & .82 & 2.28 \\
\hline
\end{tabular}

Table 20. Comparison of Ferndale Stations with $850 \mathrm{mb}$. Wind Flow From Quillayute Soundings with Background Flow Used as Final Input to WINDS Model (Jan. 30, 1975 Simulation). Direction rounded to nearest compass point (16-point compass).

\begin{tabular}{lccccccccccccc}
\hline & $\begin{array}{c}0300 \\
\text { Dir/Spd }\end{array}$ & $\begin{array}{c}0700 \\
\text { Dir/Spd }\end{array}$ & $\begin{array}{c}1100 \\
\text { Dir/Spd }\end{array}$ & $\begin{array}{c}1500 \\
\text { Dir/Spd }\end{array}$ & $\begin{array}{c}1900 \\
\text { Dir/Spd }\end{array}$ & $\begin{array}{c}2300 \\
\text { Dir/Spd }\end{array}$ \\
\hline $\begin{array}{l}\text { Background } \\
\text { Flow WINDS }\end{array}$ & 112 & 4.0 & 112 & 4.0 & 112 & 5.5 & 112 & 4.0 & 112 & 6.5 & 112 & 6.0 \\
FARC Obs. & 90 & 5.0 & 90 & 4.0 & 90 & 6.0 & 90 & 4.0 & 90 & 6.0 & 90 & 8.0 \\
FMBL Obs. & 112 & 3.0 & 112 & 4.0 & 112 & 4.0 & 112 & 4.0 & 112 & 6.0 & 90 & 7.0 \\
QUIL 850 mb. & 205 & 8.0 & - & - & - & - & 170 & 18.0 & - & - & - & - \\
Observation & 205 & & & & & & & & & & & &
\end{tabular}




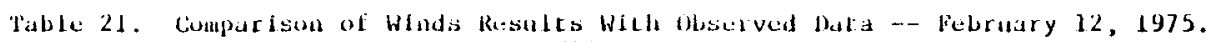
Unitis are compass points (16-point compass) for direction and meters per secund for speed comparlsons.

\begin{tabular}{|c|c|c|c|c|c|c|c|c|c|c|c|c|}
\hline \multirow{3}{*}{$\begin{array}{l}\text { Station codte } \\
\text { PARe }\end{array}$} & \multicolumn{10}{|c|}{ litu= } & \multirow{2}{*}{\multicolumn{2}{|c|}{$\begin{array}{l}2300 \\
\mathrm{Dir} / \mathrm{spd}\end{array}$}} \\
\hline & \multicolumn{2}{|c|}{$\begin{array}{c}0300 \\
\text { Dirisipui }\end{array}$} & \multicolumn{2}{|c|}{$\begin{array}{c}0700 \\
\mathrm{Dic} / \mathrm{spd}\end{array}$} & \multicolumn{2}{|c|}{$\begin{array}{c}1100 \\
\text { Dis/Spd }\end{array}$} & \multicolumn{2}{|c|}{$\begin{array}{c}1500 \\
\text { vir } / \mathrm{spd}\end{array}$} & \multicolumn{2}{|c|}{$\begin{array}{c}1900 \\
\mathrm{Dir} / \mathrm{S}_{\mathrm{p}} \mathrm{d}\end{array}$} & & \\
\hline & - & - & - & - & -4 & 0 & -6 & -1 & +6 & -3 & +7 & 0 \\
\hline t'Miss. & - & +5 & -3 & +1 & 0 & il & -1 & +3 & 0 & 0 & 0 & +1 \\
\hline$B E L A$ & +5 & 0 & 0 & -1 & 0 & 0 & 11 & $-?$ & -1 & -1 & -1 & 0 \\
\hline BSELCC & 0 & i) & +1 & -1 & +1 & -1 & +1 & +2 & 0 & +1 & +1 & 0 \\
\hline BESIT & 15 & 11 & +1 & -2 & 11 & -1 & +3 & +2 & 0 & +1 & - & 12 \\
\hline AAVL:* & -2 & -4 & 0 & -1 & 0 & -2 & -1 & -2 & 0 & 0 & +1 & -3 \\
\hline BHIL L & +1 & -8 & +1 & -5 & +1 & -6 & $1 \cdot 1$ & -2 & 14 & -1 & +4 & -5 \\
\hline Wllid & 0 & 0 & -1 & 0 & 0 & -1 & -1 & 0 & - & $t-2$ & 0 & +1 \\
\hline sertu & 0 & -2 & 0 & +1 & - & - & -1 & -2 & -5 & -1 & - & - \\
\hline ViceA & -7 & +1 & - & +6 & +1 & +2 & +1 & -7 & -2 & +1 & -6 & -2 \\
\hline vicil & -1 & -6 & +5 & +2 & -1 & -4 & +1 & -2 & -1 & -2 & -1 & -5 \\
\hline
\end{tabular}

Nean Error

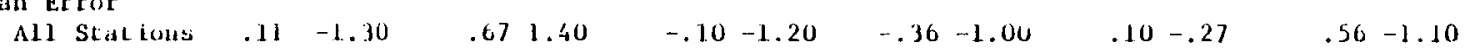

Standard bevlation

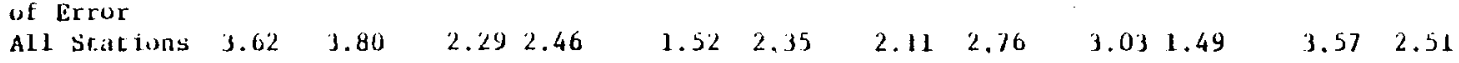

Metan Error

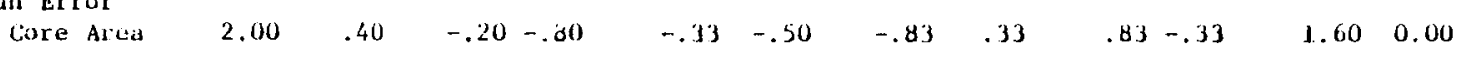

Slandard Devfation

\begin{tabular}{l} 
of Errur \\
Core Alea \\
\hline
\end{tabular}

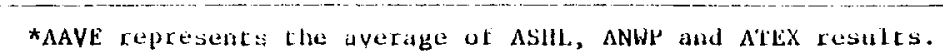


Table 22. Fraction of the Predicted Values Within a Given Number of Compass Points or Meters per Second of observed Values for February 12, 1975 Simulation.

$\begin{array}{llllll}0300 & 0700 & 1100 & 1500 & 1900 & 2300\end{array}$

\section{Al1 Stations}

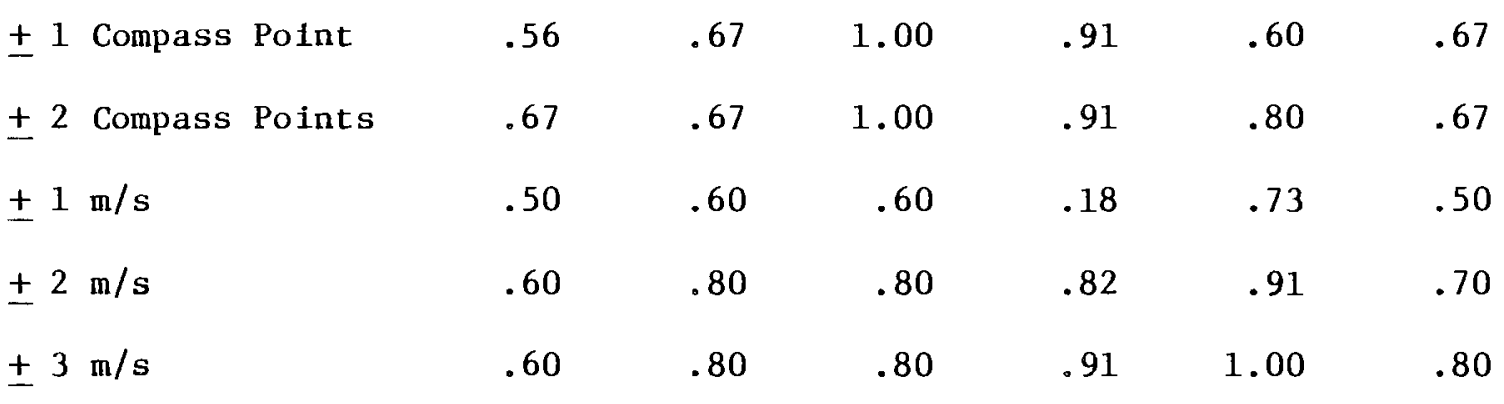

"Core" Stations

\begin{tabular}{|c|c|c|c|c|c|c|c|}
\hline \pm 1 Compass & Point & .25 & .80 & .83 & .83 & .83 & .80 \\
\hline \pm 2 Compass & Points & .50 & .80 & .83 & .83 & .83 & .80 \\
\hline $\pm 1 \mathrm{~m} / \mathrm{s}$ & & .60 & .80 & .83 & .18 & .83 & .67 \\
\hline $\pm 2 \mathrm{~m} / \mathrm{s}$ & & .60 & 1.00 & 1.00 & .83 & .83 & .83 \\
\hline $\pm 3 \mathrm{~m} / \mathrm{s}$ & & .60 & 1.00 & 1.00 & 1.00 & 1.00 & 1.00 \\
\hline
\end{tabular}


Table 23. Analysis of Simulation Accuracy for Each Station for A11 February 12, 1975 Cases.

\begin{tabular}{lcccc}
\hline \multirow{2}{*}{ Station Code } & \multicolumn{2}{c}{ Mean } & Error & \multicolumn{2}{c}{ Standard Deviation of Error } \\
\cline { 2 - 3 } Direction & .75 & -1.00 & 6.70 & Speed \\
\hline FARC & -8.00 & 1.83 & 1.30 & 1.41 \\
FMBL & .67 & -.67 & 2.25 & 1.83 \\
BELA & .67 & .17 & .52 & .82 \\
BELC & 1.60 & .50 & 1.95 & 1.17 \\
BELT & -.33 & -2.00 & 1.03 & 1.64 \\
AAVE & 2.33 & -4.50 & 1.51 & 1.41 \\
BHIL & -.40 & .33 & .55 & 2.59 \\
WHID & -1.50 & -1.00 & 2.38 & 1.03 \\
SMTH & -2.60 & -.20 & 3.78 & 1.41 \\
VICA & .33 & -2.83 & 2.42 & 4.89 \\
VICH & & & & 2.85 \\
\hline
\end{tabular}

Table 24. Comparison of Ferndale Stations and $850 \mathrm{mb}$. Wind Flow From Quillayute Soundings with Background Flow Used as Final Input to WINDS Model (Feb. 12, 1975 Simulation). Direction rounded to nearest compass point (16-point compass).

\begin{tabular}{|c|c|c|c|c|c|c|}
\hline & $\begin{array}{c}0300 \\
\text { Dir/Spd }\end{array}$ & $\begin{array}{c}0700 \\
\text { Dir/Spd }\end{array}$ & $\begin{array}{c}1100 \\
\text { Dir/Spd }\end{array}$ & $\begin{array}{c}1500 \\
\text { Dir/Spd }\end{array}$ & $\begin{array}{c}1900 \\
\text { Dir/Spd }\end{array}$ & $\begin{array}{c}2300 \\
\text { Dir/Spd }\end{array}$ \\
\hline \multicolumn{7}{|l|}{ Background } \\
\hline Flow WINDS & 1125.0 & 1576.0 & 1806.5 & 2475.5 & 1572.5 & 1571.5 \\
\hline FARC Obs. & 905.0 & 1004.0 & 906.0 & 1004.0 & 906.0 & 808.0 \\
\hline FMBL Obs. & 1103.0 & 1104.0 & 1104.0 & 1104.0 & 1106.0 & 1007.0 \\
\hline $\begin{array}{l}\text { QUIL } 850 \mathrm{mb} \text {. } \\
\text { Observation }\end{array}$ & 2206.0 & - & - & 2459.0 & - & - \\
\hline
\end{tabular}


January 3,1975

The comparison tables (Tables 9,10 and 11) show a fairly accurate simulation of the observed meteorology for January 3, 1975. The largest mean error occurred with the wind speed simulation at 1900 hours -- the underprediction of velocity evident here was influenced by large discrepancies at the two Victoria locations (VICA and VICH). The station analysis shows that the VICH and SMTH sites observed higher wind velocities than predicted for all the January 3 cases and VICA speeds were underpredicted during the final three hours. The station analysis also indicates that estimates of wind direction at the BHIL station were the least reliable (largest standard deviation) and that the accuracy of wind speed calculations at this station was also variable.

The background flow directions used (Table 12) shifted counterclockwise through the 24 hours simulated, following the trend in direction indicated by the $850 \mathrm{mb}$. sounding data. The differences between the 850 mb. data and the inputs used, however, are not consistent enough to have made the $850 \mathrm{mb}$. direction a good indicator of background flow over the entire grid. Likewise, the wind velocities at $850 \mathrm{mb}$. show little relation to the surface patterns evident from the data collected.

The Ferndale stations, on the other hand, would have been fairly good indicators of background flow on January 3, 1975. One station or the other showed the same direction as was used for the background flow for each case except $2300 \mathrm{PST}$, and the remaining station differed by no more than one compass point from the input direction. For the exception, 2300 PST, the input direction used $\left(22.5^{\circ}\right)$ lay between the directions indicated by FARC and FMBL $\left(360^{\circ}\right.$ and $45^{\circ}$, respectively). Similarly, one station or the other was always within one meter per second of the 
inputs used. Since the predicted wind speeds averaged slightly below the observed values for all the January 3 cases, using an average of FARC and FMBL observations for wind speed inputs, rather than the inputs actually used, would most likely have resulted in similar or improved overall model performance for the January 3 cases.

January 24,1975

As with the January 3 cases, the overall simulation of observed conditions was reasonably accurate, although the direction errors exhibited a higher degree of variance in the January 24 simulation. Some difficulties were encountered in reproducing observed wind directions on January 24 due to the low wind velocities ( $2 \mathrm{~m} / \mathrm{s}$ or less) encountered over much of the simulation period. The lack of a dominating background flow means that local thermal influences and small scale perturbations in flow become important, introducing a fair amount of random deviation into the observed flow characteristics. This, in turn, means that any individual observation is more difficult to duplicate in the simulation modeling than would be so under conditions of stronger flow. The large number of calm observations on January 24 adds to the wind speed prediction error figures as the WINDS model rarely, if ever, estimates such a low wind speed $(\leq .1 \mathrm{~m} / \mathrm{s})$ that a zero wind speed prediction results.

The mean error figures in the station analysis table (Table 15) indicate that none of the stations were consistantly misrepresented by the model -- the errors are instead distributed in either direction from the observed values, resulting in fairly large standard deviations for some locations but small mean error figures. Stations at BELC, BHIL and VICA were particularly variable with respect to direction errors. 
The $850 \mathrm{mb}$. flow measurements from Quillayute do not show a predictable relationship to the input values used and would not have made effective estimators of the flow prevailing over the northern Puget Sound study area. The flow directions measured at FMBL are closest to the input values -- at 0700 and 2300 PST the observations were two compass points different from the input direction, while the discrepancy was one compass point or less for the other three hours where comparison was possible (1900 PST observations at both FARC and FMBL were calm). FMBL observations also closely approximate the input wind speeds -within one meter per second for all cases but 2300 PST, when the difference was two meters per second.

The meteorological data collected for January 24, 1975 (Table B6 in Appendix B) shows a significant amount of variation from hour to hour at each station and also between stations at each time period, particularly during the latter half of the day. With this situation, the background flow direction used is really a compromise figure, an average of the directions indicated at the various station locations. Consequently, the input flow characteristics do not match observed conditions at any one location consistently. Under conditions of light and/or variable wind speeds and directions, even fairly "open" stations like FARC and FMBL make relatively unreliable indicators of prevailing flow over the entire grid area, if indeed a prevalling flow can be defined under such conditions. 
January 30,1975

The simulations of the January 30,1975 cases show a significant amount of disagreement with measured conditions at some of the observation stations. Difficulties were encountered due to the fact that meteorological conditions over the entire grid area were not really at a "sub-synoptic" level. Synoptic conditions remained fairly uniform throughout the day, with surface level flow over the study area from an easterly to southeasterly direction, toward the depression centered off the west coast of Washington. The grid area is large enough, however, that observed prevailing flow differed by one or two compass points between the northern portions of the study area and the southern boundary. Thus, measured flow direction at the northernmost station (FARC) was regularly one compass point counterclockwise from the simulated value, while the Anacortes and Victoria stations erred in the opposite direction (a "compromise" input direction of $112.5^{\circ}$ was used as prevailing flow over the entire grid for all cases).

The two Bellingham stations for which meteorological observations were obtained show north to northeasterly flow much of the day, while the prevailing background flow over the rest of the grid appears more southeasterly. The wind flow simulation predicted directions for these stations more in line with the overall pattern, resulting in large directional error statistics for these stations.

SMTH, VICA and VICH were consistantly under-predicted with respect to wind velocity. Wind speeds as high as $18 \mathrm{~m} / \mathrm{s}$ were recorded at VICH and SMTH during several of the simulation periods. Velocities in excess of the background flow were input at the "observation" points in Puget Sound and the Strait of Juan de Fuca in an attempt to duplicate the 
strong winds apparent in the straits on January 30 . It should be noted that wind speeds predicted by the model are somewhat more conservative than the range seen in the data collected from the northern Puget Sound area -- calm situations are not duplicated and the highest wind speeds are under-predicted to some extent. Under-prediction of extreme wind speeds is also, in part, due to the fact that input winds were chosen to err towards slower speeds to insure that the air quality information was conservative.

Comparison of the Ferndale stations (FARC and FMBL) to the input background flow once again shows good correlation with respect to both direction and wind speed. FMBL is the better choice for wind direction estimation here, while the two stations appear equally useful in formulating input speeds. The $850 \mathrm{mb}$. data show a more consistent relation to input direction than was seen in either of the days simulated previously but are still less useful predictors than the surface observations at Ferndale.

February 12, 1975

February 12, 1975 again presented a situation where the conditions to be modeled were not on a "sub-synoptic" scale and, consequently, were very difficult to duplicate. An occluded front lay across the northem Puget Sound region during the entire simulation period and, from the meteorological observations, appears to have intersected the surface of the study area around the Ferndale stations in the northern section of the grid. The result was a very complex situation with erratic speed and direction observations at any particular station over the course of the 24 hour simulation and quite different "background" 
flows apparent over various portions of the grid at any one time. The FARC station, apparently lying to the north of the frontal region, had observed wind directions up to $180^{\circ}$ different from the nearest stations to the south and was, for the most part, ignored in formulating input data for the simulation cases.

The comparison tables (Tables 21 and 22) show reasonably good mean error values for most of the cases but large standard deviations. This indicates the background flow used was really a compromise, with the errors in direction and speed at different locations cancelling out to produce a small mean error. The station analysis shows a similar effect with significant mean errors and fairly large standard deviations at many of the stations for the February 12 simulations. The FARC station shows particularly large deviations between predicted and observed wind direction values.

Because of the synoptic situation in the vicinity of the Ferndale locations, neither station is a good indicator of overall background flow. Similarly, the $850 \mathrm{mb}$. sounding data have little relationship to the background flows input for the individual cases.

\section{AIR QUALITY EMISSION LIMITATION ANALYSES}

PI-Matrix calculations expressing air quality constraints on pollutant emissions are also presented. Maps of the PI-Matrix results for the entire studyarea are included in Appendix B. A one hour standard of .40 Ppm $\left(1047 \mu \mathrm{g} / \mathrm{m}^{3}\right) \mathrm{SO}_{2}$ (not to be exceeded more than once per year (PSAPCA, 1977)) was used in calculating allowable emissions for each hour simulated. A 24 hour PI-Matrix was also calculated using a standard of .10 ppm (260 $\mu \mathrm{g} / \mathrm{m}^{3}$ ) (not to be exceeded more than once per year (PSAPCA, 1977)). The 
0300 and 1500 PST runs for each day were used to represent night and day situations, respectively, to calculate the long-term PI-Matrices.

Tables showing emission restrictions at particular locations for each case simulated are presented in this section. These grid locations correspond to existing or projected source locations in the core area, including the largest area sources of contaminants (see Figure 3). Table 25 is a list of the locations used and the corresponding grid coordinates. Figure 12 is a map of the grid points indicated as source locations.

Table 25. Major $\mathrm{SO}_{2}$ Source Grid Locations for Northern Puget Sound Study Area.

\begin{tabular}{lc}
\hline Source & Grid Coordinates (row, column) \\
\hline Source A, Ferndale & 10,48 \\
Source B, Ferndale & 9,47 \\
Source C, Ferndale & 7,46 \\
Source D, Ferndale & 8,46 \\
Source E, Ferndale & 11,47 \\
Source F, Ferndale & 8,45 \\
Source G, Ferndale & 9,46 \\
Source H, Bellingham & 13,54 \\
Source I, Anacortes & 28,52 \\
Source J, Anacortes & 28,53 \\
Source K, Anacortes & 29,53 \\
Bellingham & $12-17,53-58$ \\
Anacortes & $27-29,49-51$ \\
\hline
\end{tabular}




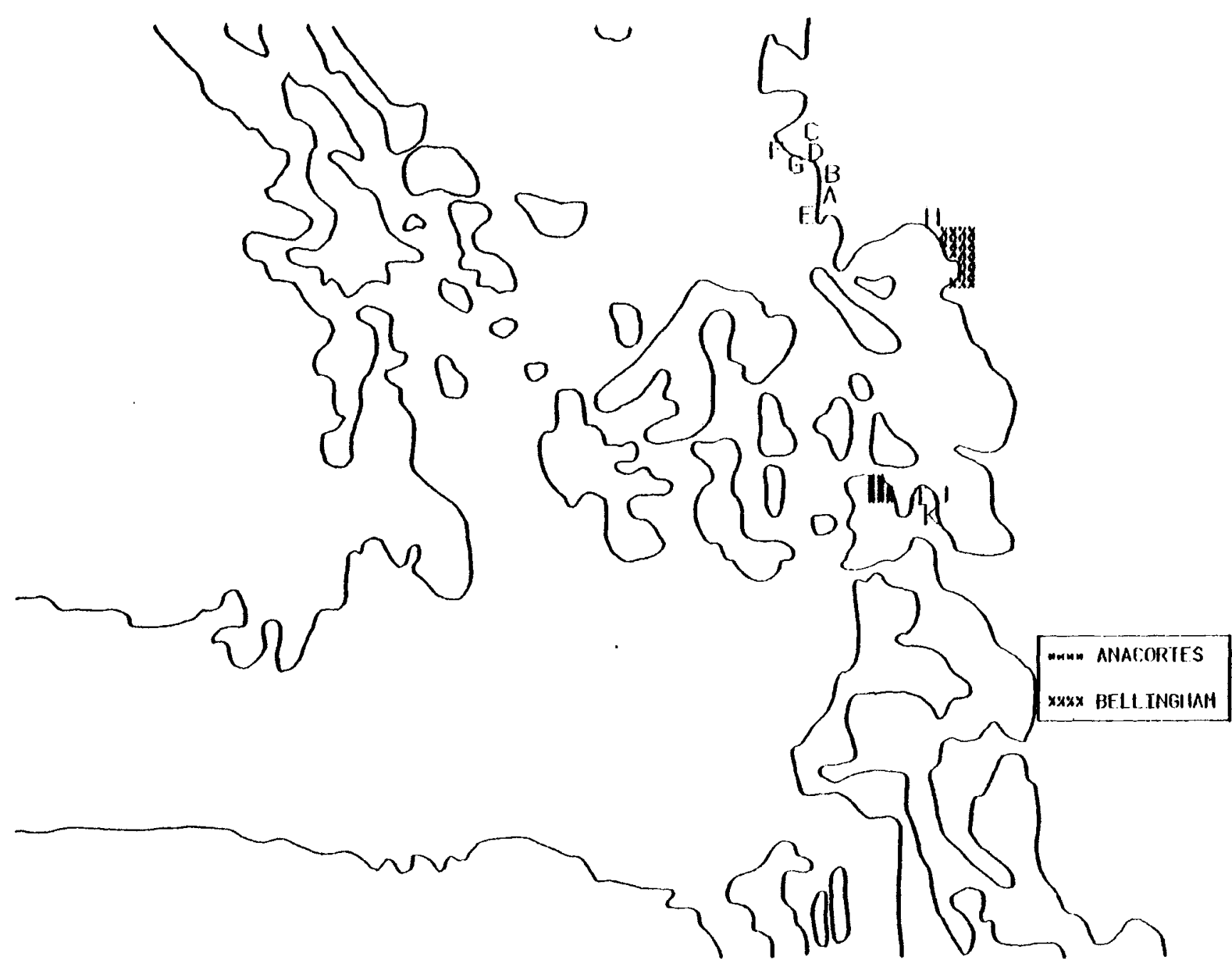

Figure 12. Location of Major $\mathrm{SO}_{2}$ Sources for Northern Puget Sound.

(See Tab1e 25) 
Tables showing total emissions allowable for each case simulated in the core area as a whole $\left(3118 \mathrm{~km}^{2}\right)$ are also included. These tables were computed by adding together the allowable emissions at each grid cell in the core area. They represent emissions into the available mixing volume over the entire core area that could be tolerated under conditions of complete mixing and, consequently, uniform concentration over the region as a whole.

\section{Daily Cases}

Tables $26,27,28$ and 29 show hourly emission rates that would result in $\mathrm{SO}_{2}$ concentrations equal to the standard limitations at the 10cations corresponding to proposed or existing pollutant sources. These tables also show the total pollutant mass that would need to be emitted over the 24-hour period represented to maintain an ambient concentration equivalent to the 24-hour standard limit. The Bellingham area emission figures are for a total of $36 \mathrm{grid}$ cells (approximately $148 \mathrm{~km}^{2}$ ) and the City of Anacortes represents a nine grid cell area (approximately $37 \mathrm{~km}^{2}$ ).

Tables $30,31,32$ and 33 show the total emissions into the core area mixing volume which would result in ambient concentrations of $\mathrm{SO}_{2}$ equal to indicated standard limitations if complete mixing and, therefore, uniform concentrations were attained. 
Table 26. Emission Strength That Would Result in Ambient $\mathrm{SO}_{2}$ Concentrations Equal to Short and Long Term Standard Limitations (.40 ppm and $.10 \mathrm{ppm}$, respectively) at Locations Indicated -- January 3, 1975 Simulation. Units are grams per second for hourly cases and kilograms for 24-hour cases.

\begin{tabular}{lccccccc}
\hline Source & 0300 & 0700 & 1100 & 1500 & 1900 & 2300 & $24-$ Hour \\
\hline Source A, Ferndale & $30-59$ & $<30$ & $<30$ & $30-59$ & $30-59$ & $30-59$ & $20.30-20.39$ \\
Source B, Ferndale & $30-59$ & $30-59$ & $<30$ & $<30$ & $<30$ & $30-59$ & $20.30-20.39$ \\
Source C, Ferndale & $30-59$ & $<30$ & $<30$ & $<30$ & $30-59$ & $30-59$ & $20.30-20.39$ \\
Source D, Ferndale & $30-59$ & $<30$ & $<30$ & $<30$ & $<30$ & $<30$ & $20.30-20.39$ \\
Source E, Ferndale & $30-59$ & $<30$ & $<30$ & $<30$ & $30-59$ & $<30$ & $20.30-20.39$ \\
Source F, Ferndale & $30-59$ & $<30$ & $<30$ & $<30$ & $<30$ & $30-59$ & $20.30-20.39$ \\
Source G, Ferndale & $30-59$ & $<30$ & $<30$ & $<30$ & $<30$ & $30-59$ & $20.30-20.39$ \\
Source H, Bellingham & $30-59$ & $<30$ & $<30$ & $<30$ & $<30$ & $30-59$ & $20.30-20.39$ \\
Source I, Anacortes & $30-59$ & $<30$ & $<30$ & $<30$ & $30-59$ & $30-59$ & $20.30-20.39$ \\
Source J, Anacortes & $30-59$ & $<30$ & $<30$ & $<30$ & $30-59$ & $30-59$ & $20.30-20.39$ \\
Source K, Anacortes & $30-59$ & $<30$ & $<30$ & $30-59$ & $30-59$ & $30-59$ & $20.30-20.39$ \\
Bellingham & $1140-2184$ & $270-1314$ & $90-1134$ & $300-1344$ & $300-1344$ & $1110-2214$ & $730.90-734.46$ \\
Anacortes & $210-471$ & $60-321$ & $30-291$ & $150-411$ & $210-471$ & $270-531$ & $182.60-183.49$ \\
\hline
\end{tabular}


Table 27. Enission Strength That Would Result in Ambient $\mathrm{SO}_{2}$ Concentrations Equal to Short and Long Term Standard Limitations (.40 ppm and .10 ppm, respectively) at Locations Indicated -- January 24, 1975 Simulation. Units are grams per second for hourly cases and kilograms for 24-hour cases.

\begin{tabular}{|c|c|c|c|c|c|c|c|}
\hline Source & 0300 & 0700 & 1100 & 1500 & 1900 & 2300 & 24-Hour \\
\hline Source A, Ferndale & $30-59$ & $<30$ & $<30$ & $<30$ & $<30$ & $<30$ & $20.30-20.39$ \\
\hline Source B, Ferndale & $30-59$ & $<30$ & $<30$ & $<30$ & $<30$ & $<30$ & $20.30-20.39$ \\
\hline Source C, Ferndale & $30-59$ & $<30$ & $<30$ & $<30$ & $<30$ & $<30$ & $20.30-20.39$ \\
\hline Source D, Ferndale & $30-59$ & $<30$ & $<30$ & $<30$ & $<30$ & $<30$ & $20.30-20.39$ \\
\hline Source E, Ferndale & $30-59$ & $<30$ & $<30$ & $<30$ & $<30$ & $<30$ & $20.30-20.39$ \\
\hline Source F, Ferndale & $30-59$ & $<30$ & $<30$ & $<30$ & $<30$ & $<30$ & $20.30-20.39$ \\
\hline Source G, Ferndale & $<30$ & $<30$ & $<30$ & $<30$ & $<30$ & $<30$ & $20.30-20.39$ \\
\hline Source H, Bellingham & $30-59$ & $<30$ & $<30$ & $<30$ & $<30$ & $<30$ & $20.30-20.39$ \\
\hline Source I, Anacortes & $<30$ & $<30$ & $<30$ & $<30$ & $<30$ & $<30$ & $20.30-20.39$ \\
\hline Source $J$, Anacortes & $<30$ & $<30$ & $<30$ & $<30$ & $<30$ & $<30$ & $20.30-20.39$ \\
\hline Source K, Anacortes & $<30$ & $<30$ & $<30$ & $<30$ & $<30$ & $<30$ & $20.30-20.39$ \\
\hline Bellingham & $720-1677$ & $90-1134$ & $180-1224$ & $60-1104$ & $90-1134$ & $120-1164$ & $730.90-734.46$ \\
\hline Anacortes & $0-300$ & $0-234$ & $0-234$ & $0-234$ & $0-234$ & $0-234$ & $182.60-183.49$ \\
\hline
\end{tabular}


Table 28. Emission Strength That Would Result in Ambient $\mathrm{SO}_{2}$ Concentrations Equal to Short and Long Term Standard Limitations (.40 ppm and .10 ppm, respectively) at Locations Indicated -- January 30, 1975 Simulation. Units are grams per second for hourly cases and kilograms for 24-hour cases.

\begin{tabular}{lccccccc}
\hline Source & 0300 & 0700 & 1100 & 1500 & 1900 & 2300 & $24-$ Hour \\
\hline Source A, Ferndale & $30-59$ & $30-59$ & $30-59$ & $30-59$ & $30-59$ & $30-59$ & $20.30-20.39$ \\
Source B, Ferndale & $30-59$ & $30-59$ & $30-59$ & $30-59$ & $30-59$ & $30-59$ & $20.30-20.39$ \\
Source C, Ferndale & $30-59$ & $30-59$ & $30-59$ & $30-59$ & $30-59$ & $30-50$ & $20.30-20.39$ \\
Source D, Ferndale & $<30$ & $<30$ & $30-59$ & $<30$ & $30-59$ & $30-59$ & $20.30-20.39$ \\
Source E, Ferndale & $30-59$ & $30-59$ & $30-59$ & $30-59$ & $30-59$ & $30-59$ & $20.30-20.39$ \\
Source F, Ferndale & $30-59$ & $30-59$ & $30-59$ & $30-59$ & $30-59$ & $30-59$ & $20.30-20.39$ \\
Source G, Ferndale & $<30$ & $<30$ & $30-59$ & $<30$ & $30-59$ & $30-59$ & $20.30-20.39$ \\
Source H, Bellingham & $<30$ & $<30$ & $30-59$ & $<30$ & $30-59$ & $30-59$ & $20.30-20.39$ \\
Source I, Anacortes & $30-59$ & $30-59$ & $30-59$ & $30-59$ & $30-59$ & $30-59$ & $20.30-20.39$ \\
Source J, Anacortes & $30-59$ & $30-59$ & $30-59$ & $30-59$ & $30-59$ & $30-59$ & $20.30-20.39$ \\
Source K, Anacortes & $30-59$ & $30-59$ & $30-59$ & $30-59$ & $30-59$ & $30-59$ & $20.30-20.39$ \\
Bellingham & $270-1314$ & $270-1314$ & $900-1944$ & $270-1314$ & $1260-2304$ & $1110-2154$ & $710.80-714.27$ \\
Anacortes & $180-441$ & $180-441$ & $300-561$ & $180-441$ & $360-621$ & $330-591$ & $182.60-183.49$ \\
\hline
\end{tabular}


Table 29. Emission Strength That Would Result in Ambient $\mathrm{SO}_{2}$ Concentrations Equal to Short and Long Term Standard Limitations (.40 ppm and . $10 \mathrm{ppm}$, respectively) at Locations Indicated -- February 12, 1975 Simulation. Units are grams per second for hourly cases and kilograms for 24-hour casts.

\begin{tabular}{|c|c|c|c|c|c|c|c|}
\hline Source & 0300 & 0700 & 1100 & 1500 & 1900 & 2300 & 24-Hour \\
\hline Source A, Ferndale & $30-59$ & $30-59$ & $60-89$ & $30-59$ & $<30$ & $<30$ & $20.30-20.39$ \\
\hline Source B, Ferndale & $30-59$ & $30-59$ & $60-89$ & $30-59$ & $<30$ & $<30$ & $20.30-20.39$ \\
\hline Source C, Ferndale & $30-59$ & $30-59$ & $30-59$ & $30-59$ & $<30$ & $<30$ & $20.30-20.39$ \\
\hline Source D, Ferndale & $30-59$ & $30-59$ & $60-89$ & $30-59$ & $<30$ & $<30$ & $20.30-20.39$ \\
\hline Source E, Ferndale & $30-59$ & $30-59$ & $60-89$ & $30-59$ & $<30$ & $<30$ & $20.30-20.39$ \\
\hline Source F, Ferndale & $30-59$ & $30-59$ & $60-89$ & $30-59$ & $<30$ & $<30$ & $20.30-20.39$ \\
\hline Source G, Ferndale & $30-59$ & $30-59$ & $60-89$ & $30-59$ & $<30$ & $<30$ & $20.30-20.39$ \\
\hline Source H, Bellingham & $30-59$ & $30-59$ & $60-89$ & $30-59$ & $<30$ & $<30$ & $20.30-20.39$ \\
\hline Source I, Anacortes & $30-59$ & $30-59$ & $30-59$ & $30-59$ & $<30$ & $<30$ & $20.30-20.39$ \\
\hline Source J, Anacortes & $30-59$ & $30-59$ & $30-59$ & $30-59$ & $<30$ & $<30$ & $20.30-20.39$ \\
\hline Source K, Anacortes & $30-59$ & $30-59$ & $30-59$ & $30-59$ & $<30$ & $<30$ & $20.30-20.39$ \\
\hline Bel1ingham & $7 ? 0-1764$ & $1320-2364$ & $1860-2904$ & $1110-2154$ & $210-1254$ & $60-1104$ & $730.90-734.46$ \\
\hline Anacortes & $270-531$ & $270-531$ & $300-561$ & $270-531$ & $0-261$ & $0-261$ & $182.60-183.49$ \\
\hline
\end{tabular}


Table 30. Emission Rate or Total Emission Into Core Area Mixing Volume That Would Result in Ambient Concentrations Equal to Standard Limitations (.40 ppm SO 2 , 1-Hour standard; .10 ppm $\mathrm{SO}_{2}$, 24Hour standard) for January 3, 1975 Simulation. Units are kilograms per second for each hourly case and kilograms for 24-Hour simulation.

\begin{tabular}{lr}
\hline Time & Emisssion Rate $(\mathrm{kg} / \mathrm{s})$ or Emission $(\mathrm{kg})$ \\
\hline 0300 & 42.4 \\
0700 & 27.7 \\
1100 & 18.9 \\
1500 & 28.7 \\
1900 & 31.8 \\
2300 & 34.6 \\
24 Hour & $19,054.9$
\end{tabular}

Table 31. Emission Rate or Total Emission Into Core Area Mixing Volume That Would Result in Ambient Concentrations Equal to Standard Limitations (.40 ppm $\mathrm{SO}_{2}$, 1-Hour standard; .10 ppm $\mathrm{SO}_{2}$, 24Hour standard) for January 24, 1975 Simulation. Units are kilograms per second for each hourly case and kilograms for 24-Hour simulation.

\begin{tabular}{|c|c|c|}
\hline Time & Emission Rate & $(\mathrm{kg} / \mathrm{s})$ or Emission $(\mathrm{kg})$ \\
\hline 0300 & & 28.7 \\
\hline 0700 & & 19.7 \\
\hline 1100 & & 20.6 \\
\hline 1500 & & 14.1 \\
\hline 1900 & & 13.7 \\
\hline 2300 & & 13.0 \\
\hline 24 Hour & & $19,055.0$ \\
\hline
\end{tabular}


Table 32. Emission Rate or Total Emission Into Core Area Mixing Volume That Would Result in Ambient Concentrations Equal to Standard Limitations (.40 ppm SO 2,1 -Hour standard; .10 ppm $\mathrm{SO}_{2}, 24-$ Hour standard) for January 30, 1975 Simulation. Units are kilograms per second for each hourly case and kilograms for 24-hour simulation.

\begin{tabular}{|c|c|c|c|}
\hline Time & Enission Rate $(\mathrm{kg} / \mathrm{s})$ & or Emissien & $(\mathrm{kg})_{0}$ \\
\hline 0300 & & 32.4 & \\
\hline 0700 & & 32.4 & \\
\hline 1100 & & 42.9 & \\
\hline 1500 & & 32.4 & \\
\hline 1900 & & 50.1 & \\
\hline 2300 & & 46.6 & \\
\hline 24 Hour & 19,0 & 054.1 & \\
\hline
\end{tabular}

Table 33. Emission Rate or Total Emission Into Core Area Mixing Volume That Would Result in Ambient Concentrations Equal to Standard Limitations (.40 ppm $\mathrm{SO}_{2}, 1$-Hour standard; .10 ppm SO $2,24-$ Hour standard) for February 12, 1975 Simulation. Units are kilograms per second for each hourly case and kilograms for 24-hour simulation.

\begin{tabular}{lc}
\hline Time & Emission Rate $(\mathrm{kg} / \mathrm{s})$ or Emission $(\mathrm{kg})$ \\
\hline 0300 & 39.8 \\
0700 & 51.3 \\
1100 & 56.8 \\
1500 & 45.2 \\
1900 & 24.2 \\
2300 & 17.1 \\
$24-$ Hour & $19,054.9$ \\
\hline
\end{tabular}


In general, the hours or days simulated with low wind speed inputs (i.e. 0700 or 1100 PST, January 3; much of January 24, etc.) show more severe restrictions on pollutant emissions than periods of increased ventilation. The long-term (24-hour) PI-Matrices (Appendix B) show areas of lower pollution potential in the upland areas west of Bellingham, the higher interior portions of Vancouver Island and the section of the Olympic Peninsula included in the analysis, and over some of the San Juan Islands, with higher potential for pollutant buildup over flat terrain and ocean areas. These pollution potential patterns generally correspond to wind speed variations seen in the wind flow modeling results -- high wind speeds at scattered upland locations (i.e. exposed ridges, etc.) with lower wind speeds, similar to the background flow inputs, in areas of less complex terrain.

Emission restrictions for all the January 24 cases are more severe than on January 3,1975 . This can be attributed to the fact that wind speeds were generally lower on January 24 ; pollutants would consequently disperse more slowly than under conditions similar to the January 3 cases and the potential for pollution accumulation over the grid area as a whole is therefore greater. Conversely, emission are less constrained for the January 30 cases due to the greater wind velocities relative to either of the previous days. Higher wind speeds increase ventilation, causing pollutants to disperse more efficiently, thereby reducing the pollution potential.

The February 12 cases show less restrictive emission strengths during the early part of the day than during the last two simulation periods. This corresponds to a change in the background wind flow velocity used as input; between 5 and $6.5 \mathrm{~m} / \mathrm{s}$ early in the day and 1.5 to $2.5 \mathrm{~m} / \mathrm{s}$ for the 1900 and 2300 PST cases. 
The variation in pollution potential and emission limitations with meteorology is evident from the source location summary tables (Tables 26, 27, 28 and 29). Emission rates at the indicated grid locations are generally less than $60 \mathrm{~g} / \mathrm{s}$ for the January 3 cases, consistently less than $30 \mathrm{~g} / \mathrm{s}$ for most of January 24 , generally between 30 and $60 \mathrm{~g} / \mathrm{s}$ on January 30 and as high as $90 \mathrm{~g} / \mathrm{s}$ during the first part of February 12 , dropping to under $30 \mathrm{~g} / \mathrm{s}$ during the last two time periods of that day. The longer-term (24-hour) calculation smooths out these time period fluctuations such that the daily total emission for individual source location grid cells is always between 20,300 and 20,390 grams. The area source figures show similar patterns between the various cases.

\section{$\underline{\text { Annual Case }}$}

The WINDS model and TAPAS system were also used to calculate emission constraints on an annual basis. Annual wind rose data from Bellingham was used for the annual situation (Figure 7). The average speed $(5.5 \mathrm{~m} / \mathrm{s})$ indicated by the Bellingham data was used to generate wind speed and direction arrays for background flow over the northern Puget Sound region from eight directions (N, NE, E, SE, S, SW, W and NW). Mixing volume index arrays associated with each of the eight situations described above were also calculated -- these indices express the relative "pollution potential" at each grid cell. Enissions into the atmosphere over the study area would tend to concentrate in areas where stagnation or convergence would occur under the input flow conditions -hence, these locations have a high potential for pollutant buildup. Further information about wind flow conditions and pollution potential 
arrays associated with each of the eight situations may be found in Appendix B .

An annual pollution potential (mixing volume index) array was generated by weighting the index values associated with each input flow direction according to the percentage of annual flow occurring from that direction (as indicated by the Bellingham wind rose data) and averaging the eight cases. Four PI-Matrix arrays were calculated using the annual mixing volume index. The PI-Matrices represent emission restrictions necessary on an annual basis to achieve concentrations of $80,60,40$ and $20 \mathrm{\mu g} / \mathrm{m}^{3}(.03, .02, .015$ and $.0076 \mathrm{ppm}) \mathrm{SO}_{2}$. These figures represent federal annual average standards for $\mathrm{SO}_{2}$, Washington annual average standards and federal incremental (PSD) $\mathrm{SO}_{2}$ standards for Class III and Class II areas, respectively. The annual PI-Matrix maps appear as Figures 13 through 16.

The annual PI-Matrices were calculated as an emission rate and are indicative of the allowable source strength assuming a 24 hour a day, 365 day per year operation. These have been converted to total mass allowable on a yearly basis. Table 34 shows annual emission constraints at the grid cells representing present or future point source locations and for the two area source locations (Anacortes and Bellingham). Also included are the 1976 NEDS estimates of pollutant emission for the major point sources in the study area. Table 35 shows core area total emission limitations for the annual case.

The mixing volume indices generated for the eight background flow directions show that relative pollution potential at any particular grid location depends upon the direction of flow occurring (mixing volume index maps are included in Appendix B). Different areas of the grid will be sheltered from strong flow under each different background 


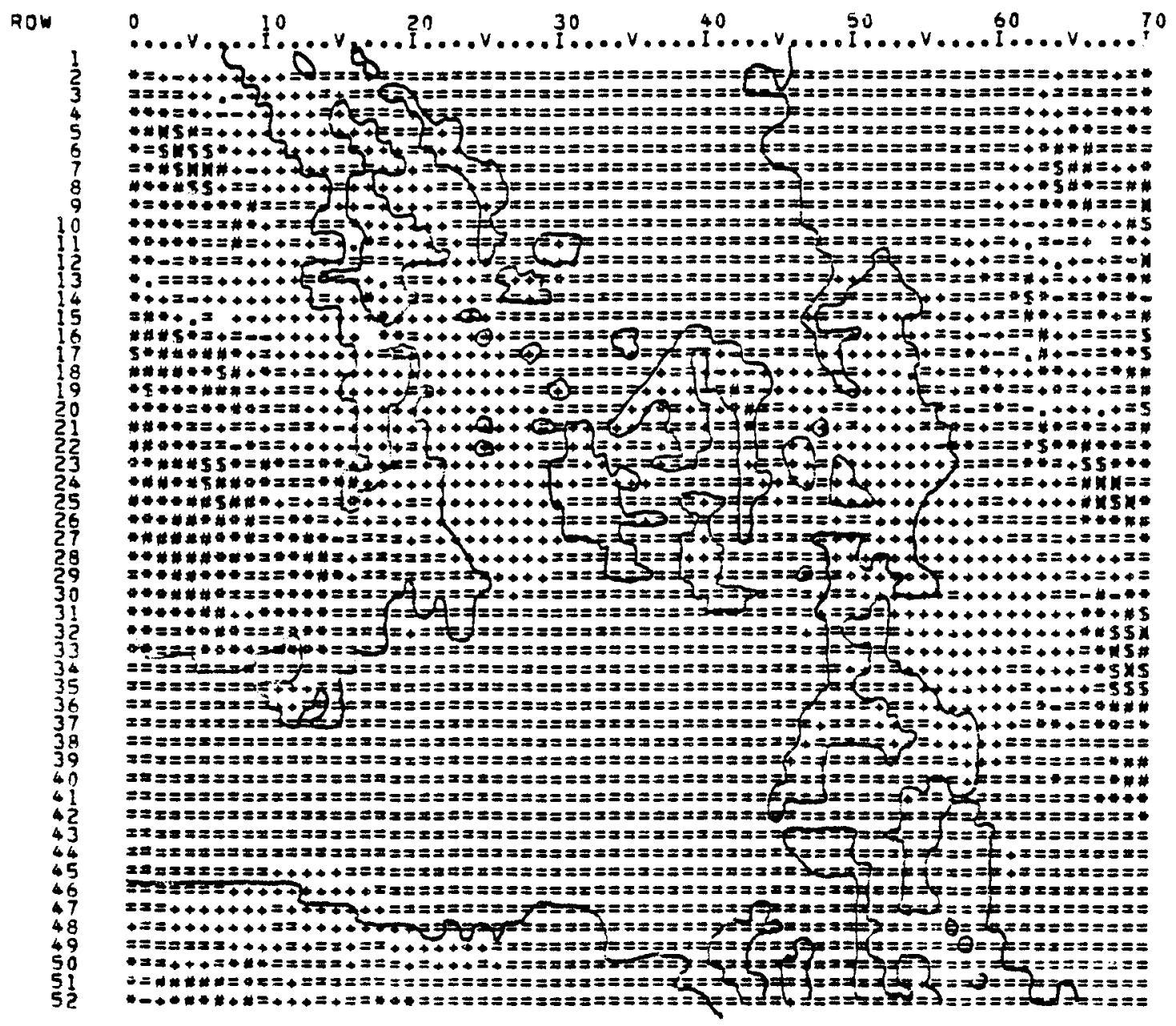

\begin{tabular}{|c|c|c|c|c|c|c|c|c|c|}
\hline rall: & Mlown & . & - & + & * & • & 1 & $s$ & r \\
\hline BHISSAUW RATR (KB X II') & $=4$ & $\cdot-111$ & $11-17$ & $18-24$ & $25-11$ & $B 2-34$ & $14-65$ & $26-52$ & si-sy \\
\hline
\end{tabular}

Figure 13. Combined PI( $\pi)$-Hatrix For the Annual Case; $\mathrm{SO}_{2}$ Standards are $20 \mu \mathrm{g} / \mathrm{m}^{3}$. 


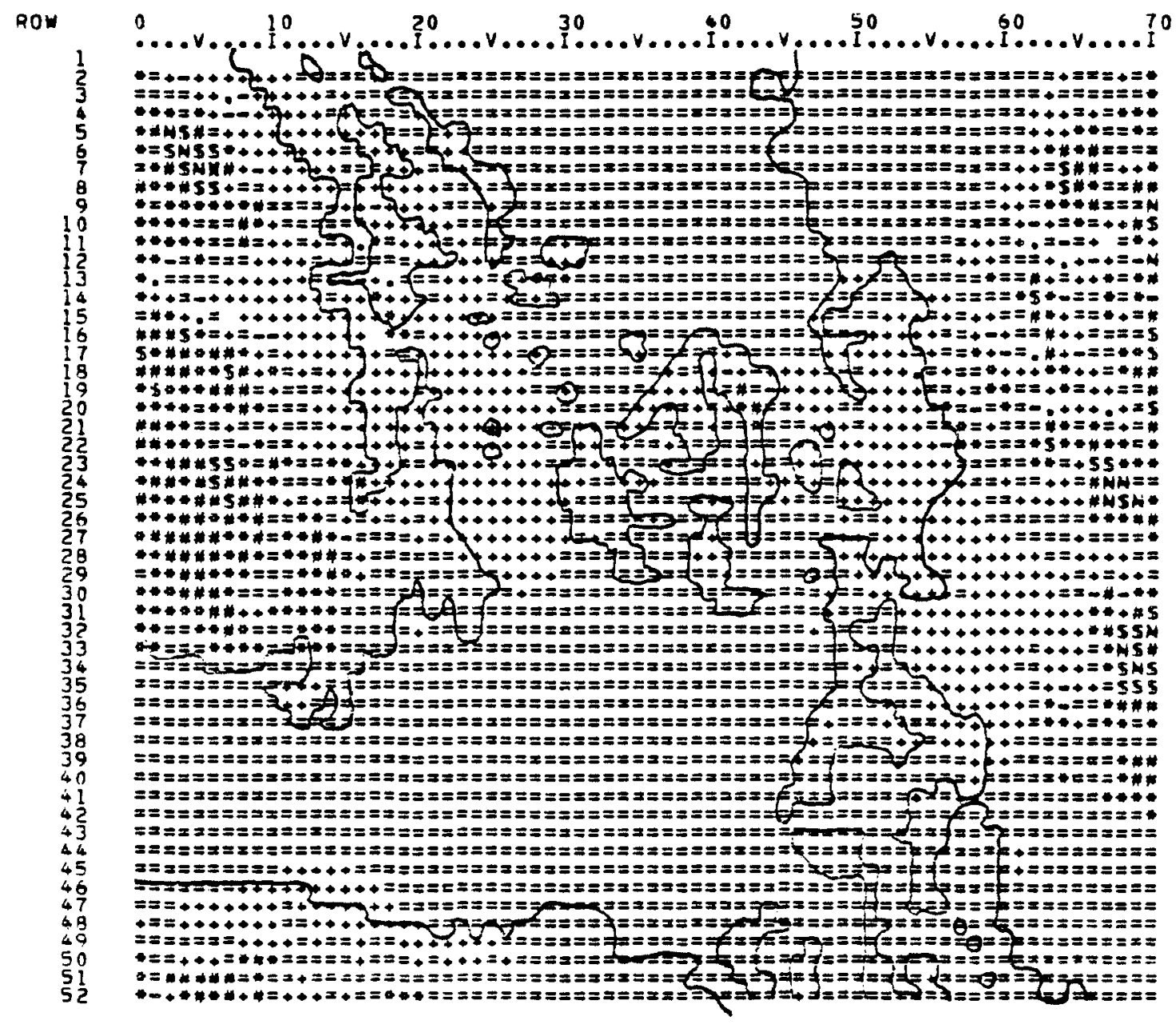

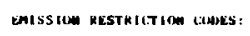

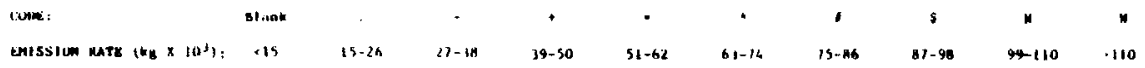

Figure 14. Combined PI( $\pi)$-Matrix for the Annual Case; $\mathrm{SO}_{2}$ Standards are $40 \mathrm{\mu g} / \mathrm{m}^{3}$. 


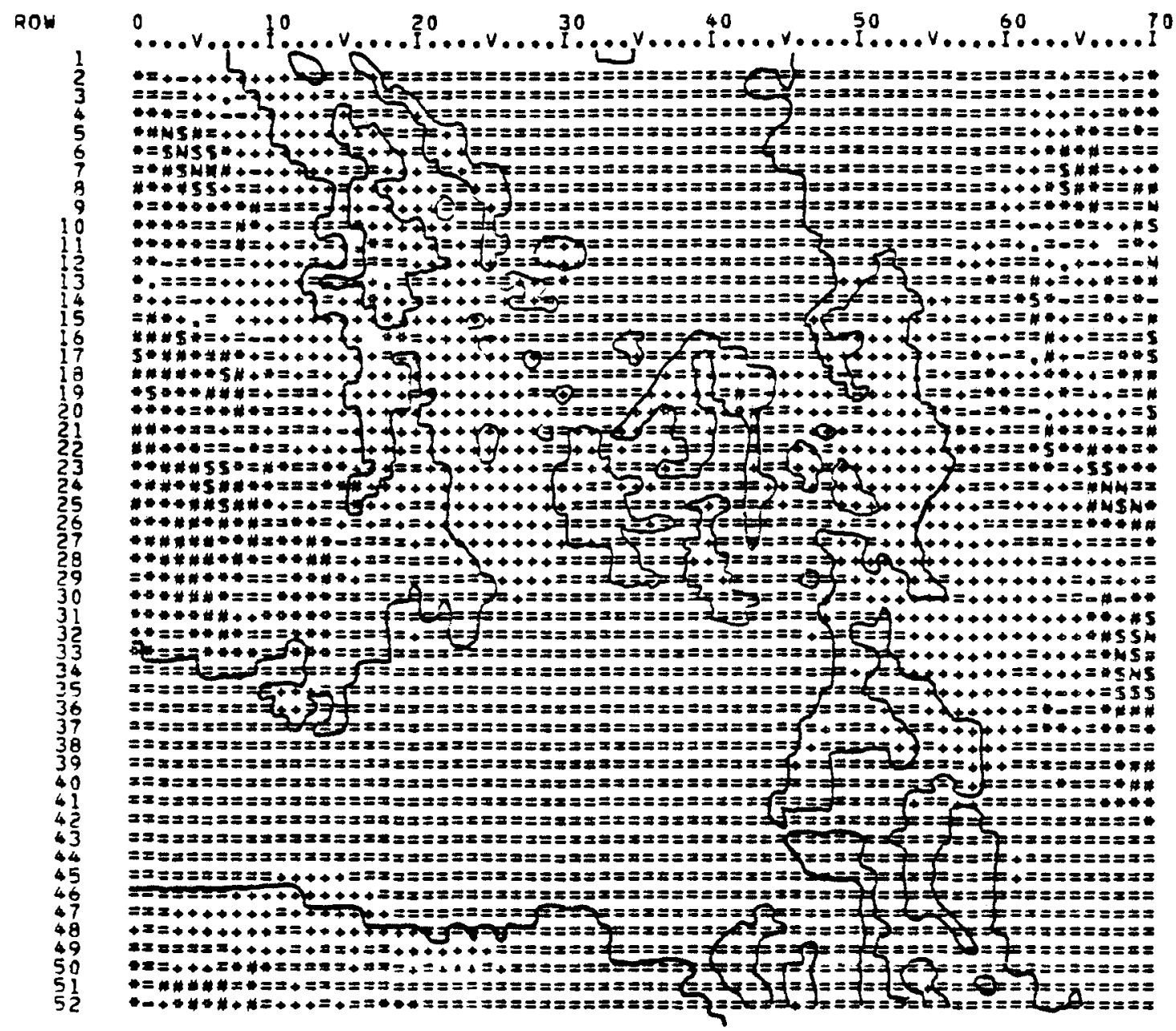
EMISS IIM RESTRHLTLW LAHES:
Wowt:
Itank
EMSSBII MATE (Ke X 10'):
19-1)
is-s6
si-n
$16-94$
45-111 $116-172$
11-151
$152-1 / 0$
$\checkmark$ 


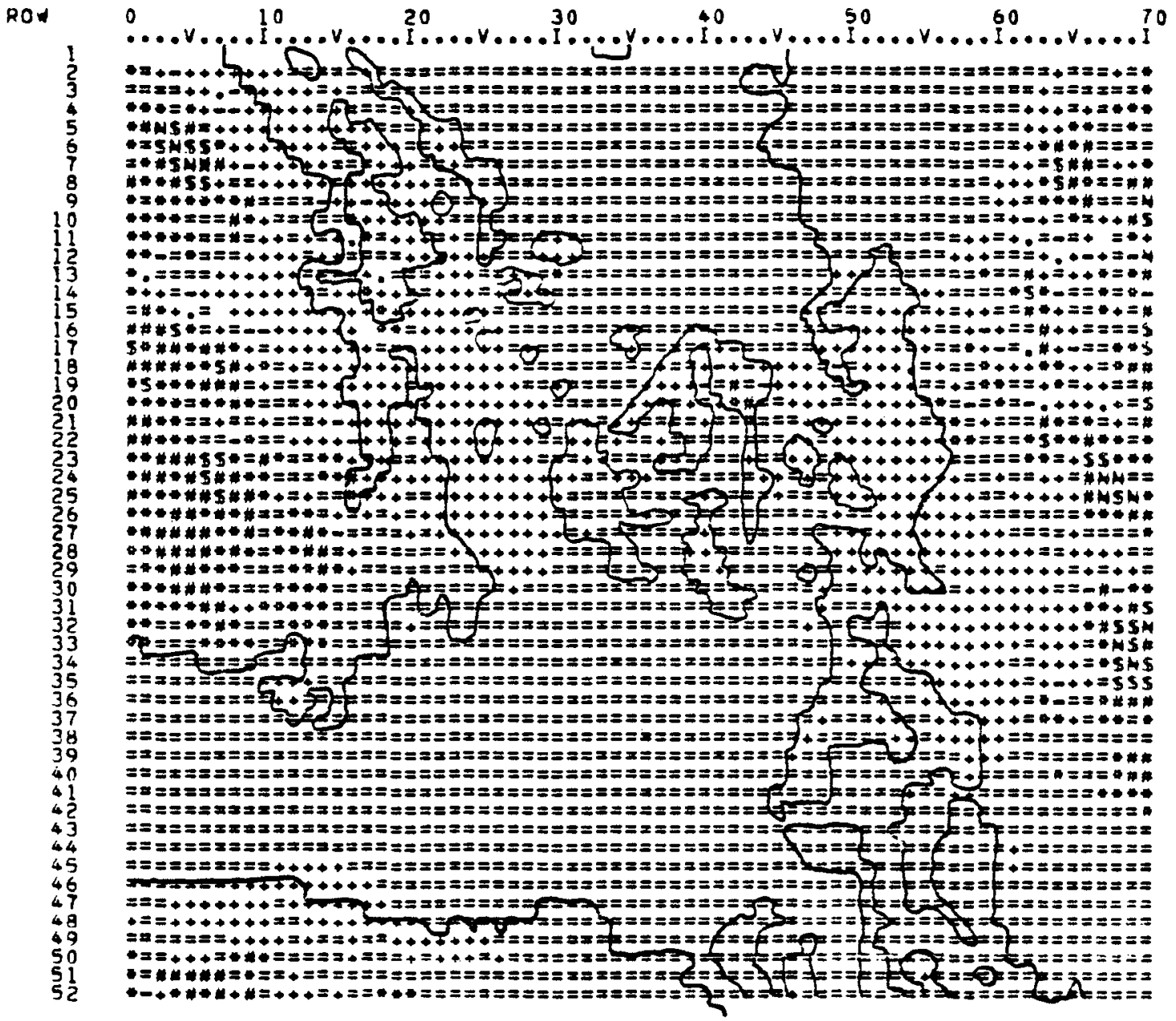

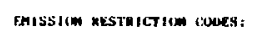

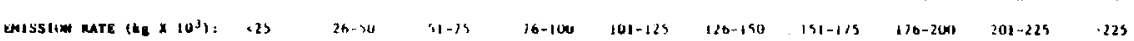

Figure 16. Combined PI( $\pi)$-Matrix for the Annual Case; $\mathrm{SO}_{2}$ Standards are $80 \mu \mathrm{g} / \mathrm{m}^{3}$. 
Table 34. Annual Total Emissions of $\mathrm{SO}_{2}$ That Would Result in Ambient Concentrations of $80,60,40$ or $20 \mu \mathrm{g} / \mathrm{m}^{3}$ at Present or Projected Source Location Grid Cells. Emission estimates for current sources for 1976 are also 1isted (EPA, NEDS, 1976). Units are $10^{3}$ kilograms $\mathrm{SO}_{2}$.

\begin{tabular}{|c|c|c|c|c|c|}
\hline Source and Location & $80_{\mu \mathrm{g} / \mathrm{m}^{3}}$ & $60 \mu \mathrm{g} / \mathrm{m}^{3}$ & $40 \mu \mathrm{g} / \mathrm{m}^{3}$ & $20 \mu \mathrm{g} / \mathrm{m}^{3}$ & $\begin{array}{l}\mathrm{SO}_{2} \text { Emission } \\
\text { Estimates } 1976\end{array}$ \\
\hline Source B, Ferndale & $101-125$ & $76-94$ & $51-62$ & $25-31$ & 3821 \\
\hline Source C, Ferndale & $101-125$ & $76-94$ & $51-62$ & $25-31$ & 4225 \\
\hline Source D, Ferndale & $101-125$ & $76-94$ & $51-62$ & $25-31$ & - \\
\hline Source E, Ferndale & $101-125$ & $76-94$ & $51-62$ & $25-31$ & - \\
\hline Source F, Ferndale & $101-125$ & $76-94$ & $51-62$ & $25-31$ & - \\
\hline Source G, Ferndale & $101-125$ & $76-94$ & $51-62$ & $25-31$ & - \\
\hline Source H, Bellingham & $101-125$ & $76-94$ & $51-62$ & $25-31$ & 1124 \\
\hline Source I, Anacortes & $76-100$ & $57-75$ & $39-50$ & $18-24$ & 12609 \\
\hline Source J, Anacortes & $76-100$ & $57-75$ & $39-50$ & $18-24$ & - \\
\hline Source K, Anacortes & $76-100$ & $57-75$ & $39-50$ & $18-24$ & - \\
\hline Bellingham & $3235-4110$ & $2432-3061$ & $1628-2026$ & $804-999$ & - \\
\hline Anacortes & $734-959$ & $551-713$ & $368-474$ & $183-320$ & - \\
\hline
\end{tabular}


Table 35. Total Allowable Emissions within Core Area on an Annual Basis to Achieve Ambient Concentrations of $80,60,40$ and $20 \mu \mathrm{g} / \mathrm{m}^{3} \mathrm{SO}_{2}$. Units are $10^{6}$ kilograms $\mathrm{SO}_{2}$.

\begin{tabular}{cc}
\hline $\mathrm{SO}_{2}$ Concentration & Emission Total \\
\hline $80 \mu \mathrm{g} / \mathrm{m}^{3}$ & 95.58 \\
$60 \mu \mathrm{g} / \mathrm{m}^{3}$ & 71.68 \\
$40 \mu \mathrm{g} / \mathrm{m}^{3}$ & 47.79 \\
$20 \mu \mathrm{g} / \mathrm{m}^{3}$ & 23.89 \\
1976 Point Source Estimated Emissions $=27.29$ \\
(total core area)
\end{tabular}

direction. Consequentiy, a particular location may have a high potential for pollutant buildup under some short-term episodes but will be relatively free from high contaminant concentrations under other situations.

The annual index shows the areas with poor dispersion characteristics under the most prevalent flow conditions. The PI-Matrix maps translate this average pollution potential into emission restriction numbers for each grid cell for the various air quality standard limitations. In general, areas with good ventilation occur at scattered locations within the upland areas on Vancouver Island, the Olympic Peninsula and in the foothills of the Cascades in the eastern portions of the study area. These areas correspond to localized windy sites such as ridge top locations. Areas of reduced dispersion occur from Port Angeles west along the northern edge of the Olympic Peninsula, along the eastern edge of Vancouver Island and from the Bellingham Bay area south to Mt. Vernon and Camano Island and north along the edge of the higher terrain to the west. Table 34 shows that in 1976 the estimated emissions of $\mathrm{SO}_{2}$ at the major point source locations were in excess of the emission constraints 
indicated by the TAPAS system. This coincides with air quality monitoring data which indicate occasional violations of $\mathrm{SO}_{2}$ standards in the northern Puget Sound region. However, the emission rate indicated for each location represents emissions into the mixing volume above a single $2 \mathrm{~km}^{2}$ grid cell on an instantaneous basis -- dispersion outside this volume is not taken into account. Since pollutants emitted into the northern Puget Sound airshed will normally disperse over a fairly large area, the core area totals are perhaps more appropriate figures for comparison. The total $1976 \mathrm{SO}_{2}$ load from major point sources in the core area $\left(27.29 \times 10^{6} \mathrm{~kg}\right)$ represents approximately 28 percent of the total emission load that could be tolerated without exceeding federal primary annual average $\mathrm{SO}_{2}$ standards or 38 percent of the total load that would result in ambient air quality equal to Washington State annual average $\mathrm{SO}_{2}$ standards. Since these figures assume complete mixing and uniform concentrations over the entire core area mixing volume they represent upper limits to emission loads that could be tolerated while maintaining acceptable levels of air quality.

The standards that may be of particular interest in reference to new industrial development in the northern Puget Sound region are the federal incremental standards for Class II and Class III areas (PSD). These represent allowable increments of $\mathrm{SO}_{2}$ that may be added to baseline concentrations, provided that these additional emissions do not result in violations of ambient air quality standards. The emission levels representing these incremental amounts are shown in Tables 34 and 35 under concentrations of $40 \mu \mathrm{g} / \mathrm{m}^{3}$ (Class III) and $20 \mu \mathrm{g} / \mathrm{m}^{3}$ (Class II). As before, the individual grid cell restrictions are conservative while the core area totals represent upper limits to the tolerable emissions. 


\section{DISCUSSION OF RESULTS}

Wind Flow Simulation

Some of the difficulties encountered in simulating wind flow patterns in the northern Puget Sound region were detailed in previous sections of this report. Channelization of flow coming into the grid presents a problem which can be partially dealt with by setting some grid locations to the observed or inferred flow direction or speed. The wind flow maps (Appendix B) show a somewhat awkward transition of the affected regions into the background flow conditions -- however, the overall result was a more effective duplication of observed conditions than was possible using a single flow as initial conditions over the entire grid. Other problems encountered related to the size of the region modeled. The wind flow simulation model is designed to handle sub-synoptic level situations where a single, characteristic prevailing flow can be used to model the entire grid area. The January 30, 1975 cases provided a situation where background flow was easterly in the northern grid sections but southeasterly along the southern grid boundary. The compromise direction used resulted in consistent direction errors at both extremes. The February 12, 1975 cases showed another synoptic level complication -this time a frontal region passing through the grid area. The wind speed and direction shifts associated with the presence of a front are impossible to duplicate with a simple flow model and any input flow chosen would thus be characteristic of only a portion of the affected area.

Light wind speed and variable wind direction conditions also proved difficult to duplicate in terms of direction, although the wind speeds 
were simulated with reasonable accuracy (January 24,1975 cases). For pollution potential analyses, the wind speed at each grid cell is a major determinant of mixing volume -- hence, when the wind flow model is used for this purpose, discrepancies in flow direction that occur under light wind conditions are relatively less important than the wind speed prediction accuracies. Wind speeds at some locations will be regularly over-predicted under similar conditions due to the infrequent occurrence of zero wind speed predictions. This is in contrast to strong wind situations where the wind speeds extremes will be under-predicted.

In general, the simplest situations were the ones most successfully duplicated. January 3, 1975 represented a relatively uncomplicated situation and was reproduced fairly well. January 24,1975 was well simulated with respect to wind speed but direction variances were greater than in the January 3 cases. The January 30, 1975 cases show still more variation in prediction errors -- several portions of the grid (Bellingham area stations and Smith and Vancouver Islands locations) are consistently in error in either speed or direction estimates. The tendency of the model to under-predict extreme wind speeds is noticeable in severval of the January 30 cases. February 12,1975 proved a nearly impossible situation to duplicate -- wind patterns in various portions of the northern Puget Sound region differed from one another to such an extent that the entire study area could not be modeled as a single situation with much accuracy.

The station analyses show a few stations where simulation errors were regularly encountered. Three of the stations (SMTH, VICA and VICH) were consistently under-predicted with respect to wind speed. These three stations showed the highest wind speeds of any locations and are 
apparently well exposed sites subject to windy conditions under certain meteorological situations. VICH and VICA (and to a lesser extent, SMTH) were also quite variable in speed prediction errors, with relatively high standard deviations overall. The wind flow model also proved to be a poor predictor of the BHIL station observations and showed large standard deviations over the four days simulated with respect to both speed and direction at this location.

The validation situation was somewhat artificial in that the wind flow model simulates a smoothed approximation of the actual terrain -information is entered at $2 \mathrm{~km}$ intervals and smaller scale features which may influence actual wind patterns at station locations may be missed entirely by the grid point network. This was undoubtedly the case at several of the validation points and probably accounts for many of the discrepancies between simulated and observed flow conditions. Because of the simplification of the terrain represented by the grid point network, the wind flow and TAPAS predictions are more useful to identify clusters of grid cells where particular air quality constraints are necessary rather than single points.

Comparison of input flows utilized with the $850 \mathrm{mb}$. sounding data from Quillayute show the Quillayute data to be unrepresentative of flow situations in the study area. This is not surprising since the Quillayute station is located some distance from the study area, on the other side of a major mountain range. Unfortunately, it is the nearest location for which upper air data are available. In such a situation, temperature and pressure data needed to define $\mathrm{T} 70, \mathrm{~T} 80, \mathrm{H} 70$ and $\mathrm{H} 80$ input variables can be taken from an upper air station adjacent to the study area but wind flow inputs should be derived from a location within the 
area to be modeled (preferably toward the center of the grid area). The FARC and FMBL stations were, in most cases, representative of the prevailing background flow estimates used. The lack of similarity between observations at these two locations and most of the other stations indicates that "open" locations, relatively free from local topographic influences, are necessary to estimate prevailing flow conditions for model input.

\section{TAPAS Results}

The hourly PI-Matrix results for each of the four days simulated show little difference in overall pollution potential among the present or projected source locations. The expansion of the averaging period to 24 hours involves calculating the allowable emissions based on Gaussian dispersion and ventilated box model concepts. The net result in this analysis was that day to day differences in meteorology were "smoothed" for the individual grid cell locations and the 24 hour emission totals were the same for each of the source grid points and for each of the four days simulated. The 24 hour emission cotals also tend to be more restrictive than the instantaneous emission rates since the acceptable average concentration over a 24 hour period is less than could be tolerated for a shorter time (i.e. .10 ppm versus .40 ppm). Differences in meteorology resulted in various levels of constraint for each hour and day over the core area as a whole. In general, periods with slower input wind velocities resulted in lower allowable emission rates and dayto-day differences tended to disappear over the longer averaging period (24 hours).

The annual average PI-Matrices indicate that currently operating point sources emit pollutants at a greater rate than the effluent can be 
dispersed to acceptable levels of air quality at the source location, under average meteorological conditions. Most of the $\mathrm{SO}_{2}$ emitted into the northern Puget Sound airshed comes from these large point sources. With such a source configuration, annual standard violations could be expected in the vicinity of the contaminant sources even when the total emission into the core area mixing volume is within the constraints indicated by the TAPAS system. This situation will occur because complete and uniform mixing over the entire core area is unlikely, especially with most of the pollutant emissions coming from a few locations. The effective mixing volume available for dilution is therefore considerably reduced from that used in the core area total calculations. Short-term standard violations may also be expected in the area immediately "downwind" from the major emitters under episodes of reduced dispersion. This situation is currently applicable to the northern Puget Sound region -- total $\mathrm{SO}_{2}$ emissions in the core area are apparently below the upper limits defined by the TAPAS system but short-term standard violations do occur under some meteorological conditions in the vicinity of the "overloaded" grid cells.

In contrast, the individual grid cell emission levels may be considered a conservative "lower level" for pollutant emission constraints. The short-term PI-Matrices represent maximum allowable emission rates under episodic conditions. The four days simulated some of the differing meteorological conditions under which high groundlevel concentrations of pollutants can result -- allowable emission rates vary from one grid cell to another and depend upon the conditions of prevailing flow. The short-term PI-Matrix constraints are particularly applicable to the type of situation that occurred on January 24, 1975 (light wind conditions 
and poor dispersion) since contaminants emitted into the northern Puget Sound airshed under such conditions will tend to disperse from the points of emission at a fairly slow rate. Emission rate constraints are therefore more restrictive over most of the January 24, 1975 simulations than for the other three sets of meteorological conditions modeled. 

CHAPTER V

SUMMARY AND CONCLUSIONS

The wind flow simulation analyses performed for this study provided valuable information regarding the model's utility for subsequent analyses of a similar nature. In using the WINDS model to simulate wind flow patterns, the following points should be addressed:

1) Upper air data at some location within or near the study area is needed to define pressure and temperature profiles during the simulation period.

2) If a particular situation is to be modeled, wind flow data must be obtained from some location within the study area which is relatively free from local topographic influences to provide critical background flow estimates. The data station used would preferably be near the center of the area to be analyzed.

3) In any area of complex terrain as large as the study area used for this report, prior channelization of flow entering the grid area must be considered. Inferred flow tendencies that differ in either speed or direction from the estimated prevailing background flow may have to be input at some of the grid locations.

4) The grid size used for this analysis ( $110 \mathrm{~km}$ by $146 \mathrm{~km}$ ) is probably as large an area as can be successfully modeled with this procedure under many meteorological conditions. For instance, the January 30, 1975 and February 12, 1975 cases were not entirely "sub-synoptic" situations, which reduced simulation accuracy over parts of the grid area. 
5) In complex terrain, a smaller grid scale would have picked up more of the local topographic features that influence flow at some of the station locations.

The TAPAS system was used to identify areas with high or low relative pollution potential under average meteorological conditions. The relative potential for contaminant accumulation was also identified for each grid cell under eight different directions of flow that occur with varying frequency in the northern Puget Sound region. Such information could be of use in siting studies for potential industrial development in the northern Puget Sound region.

Estimates of allowable emission rates or total mass emitted at each grid cell location were also calculated for a variety of meteorological conditions. These rates or amounts indicate pollutant emissions that would result in ambient concentrations equal to the Washington State one hour standard of $0.4 \mathrm{ppm} \mathrm{SO}_{2}$ or to the 24-hour standard of $.10 \mathrm{ppm} \mathrm{\textrm {SO } _ { 2 }}$ (not to be exceeded more than once per year (PSAPCA, 1977)). The simulated flow conditions represent episodes of poor dispersion or other meteorological situations that have a demonstrated potential to interact with contaminant emissions in such a way that high groundlevel concentrations could result.

Emission limitations necessary to meet annual average $\mathrm{SO}_{2}$ standard limitations at each grid cell were also calculated. The emission rates and summed total yearly emission amounts are related to federal and state ambient $\mathrm{SO}_{2}$ concentration restrictions as well as to the increments of air quality degradation allowable under federal Class II and Class III PSD standards. 
The "carrying capacity" of the entire northern Puget Sound core area may be defined in reference to either federal or state ambient air quality standards or to the federal PSD standards. Short-term "episodic" carrying capacity figures are represented by the core area totals under the various hours or days simulated. Annual carrying capacities for the entire core area are approximately 96 million kilograms per year under federal annual $\mathrm{SO}_{2}$ standards or 72 million kilograms per year under the equivalent state limitations. Incremental limits for Class II standards are 24 million kilograms per year and Class III conditions allow 48 million kilograms per year.

These estimates are necessarily upper limits to the emissions that could be tolerated in the northern Puget Sound region since topographic considerations and the configuration of major sources result in a much smaller effective mixing volume than is indicated above. Consequently, although major $\mathrm{SO}_{2}$ sources currently operating in the core area released less than half of the 72 million kilograns indicated above with respect to state annual $\mathrm{SO}_{2}$ standards in 1976 , comparison of source characteristics with source location and cell emission restrictions indicates that several areas of the northern Puget Sound region are already far in excess of the $\mathrm{SO}_{2}$ "carrying capacity". This conclusion is supported by air quality monitoring data which indicate state standard violations at several receptor locations within the study area -- in some cases at points many grid cells "downwind" from the source of contaminant emissions.

The results indicate that siting of future $\mathrm{SO}_{2}$ emission sources should be made in reference to the location of major sources already present. A larger effective "carrying capacity" for $\mathrm{SO}_{2}$ emissions would be obtained for the northern Puget Sound region if the points of 
emission were more evenly dispersed throughout the study area. Analysis of current or projected source locations with conventional air quality modeling techniques may indicate how many grid cells are at or above the $\mathrm{SO}_{2}$ carrying capacity, under annual or episodic conditions, due to advection of pollutants emitted by the major sources into the adjacent grid cell space. Future sources should be sited away from these air spaces which are currently at or above carrying capacity. If possible, future industrial development should be sited in areas with the least potential for pollutant concentration as indicated by the mixing volume index maps (Appendix B).

Alternatively, the TAPAS results may be used to indicate the amounts by which current emission levels must be reduced to insure acceptable air quality at all locations within the northern Puget Sound region. Future sources of $\mathrm{SO}_{2}$ emission could be limited to the emission rates or total emission amounts the location can tolerate under average annual and episodic meteorological conditions. The mass of $\mathrm{SO}_{2}$ currently entering grid cell locations due to advection from upwind sources must also be considered.

The TAPAS system may also be used for land use planning considerations in other locations. The accuracy of the mixing volume and, hence, emission constraint estimates will depend upon the accuracy of wind flow modeling achievable, as explained above. Mixing volume index maps may be used to indicate areas of relative "pollution potential" and a smaller grid scale may be used in studies requiring finer spatial resolution. The PI-Matrix calculations may then be used to set allowable emission rates or amounts for each grid cell or for clusters of grid cells with reference to any standard of air quality desired. The TAPAS 
system may also prove useful in preparing attainment plans for areas where contaminant concentrations are currently in excess of standard limitations, by defining levels to which emissions must be reduced to insure acceptable air quality at a11 locations. 

CHAPTER VI

REFERENCES

Battelle Pacific Northwest Laboratories. 1977. Work Statement: Assessing Limitations on Energy Development in Northern Puget Sound Due to Air Quality Regulations. Richland, Washington.

Childs, J. E. and W. E. Marlatt. 1978. Estimating Mesoscale Wind Flow Patterns in Complex Terrain From Topographic Data and Potential Temperature: Guidelines for Model Users. Earth Resources Dept., Colorado State University, Fort Collins, CO.

Danard, Maurice. 1977. A Simple Model for Mesoscale Effects of Topography on Surface Winds. Monthly weather Review, Vo1. 105, pp. 572-581

Environmental Protection Agency. 1972. Oceanography of the Nearshore Coastal Waters of the Pacific Northwest Relating to Possible Pollution, Vol. I. Water Pollution Control Research Series, Grant 非16070 EOK, Pp. 29-38.

Environmental Protection Agency. 1976. National Emissions Data Systems.

Environmental Protection Agency. 1977. National Emissions Data Systems.

Fosberg, Michael A., William E. Marlatt and Lawrence Krupnak. 1976. Estimating Airflow Patterns Over Complex Terrain. U.S.D.A. Forest Service Research Paper RM-162. Rocky Mountain Forest and Range Experiment Station, Forest Service, Fort Collins, Colorado. March.

Fosberg, Michael A. and Douglas G. Fox. 1977. A Topographic Air Pollution Analysis System. Atmospheric Environment Paper, Rocky Mountain Forest and Range Experiment Station, U. S. Forest Service, Fort Collins, CO.

Fox, D. G. 1975. Impact of Concentrated Recreational Development on Air Quality. Man, Leisure and Wildlands: A Complex Interaction. Eisenhower Consortium Bull. 1, Rocky Mountain Forest and Range Experiment Station, Fort Collins, Colorado, pp. 259-273.

Jensen, Joan V. and Maurice B. Danard. 1975. A Numerical Model for Surface Winds in Juan de Fuca, Haro and Georgia Straits. Prepared for Atmospheric Environment Service, Meteorological Services Research Branch, Downsview, Ontario. March, 73 pp.

Northwest Air Pollution Authority. 1977. Personal Communication.

Puget Sound Air Pollution Control Agency. 1977. Air Quality Data Summary for Counties of King, Kitsap, Fierce, Snohomish, 1976. Measured and Compiled by the Technical Services Division, Seattle, Washington. $44 \mathrm{pp}$. 
Public Law 95-95, 95th Congress. 1977. Clean Air Act Amendments of 1977. August 7, 1977.

Thom, B. C. and W. R. Ott. 1976. A Proposed Uniform Air Pollution Index. Atmos. Env. 10:261-274.

U. S. Department ö Agriculture, Forest Service. 1976. TOPographic Analysis System: User's Guide, Second Edition (TOPAS). Washington, D. C. 20250, January.

Washington State University. 1972. Washington Climate for These Counties: Challum, Grays Harbor, Jefferson, Pacific, Wahkiakum. Cooperative Extension Serv., Coll. of Ag., Washington St. Univ., Pullman, Washington.

Welch, Robert E. 1975. Intermittent Control of Sulfur Dioxide Emissions From a Copper Smelter. For presentation at the Annual Meeting of the Pacific Northwest International Section, Air Pollution Control Association, Vancouver, British Columbia, November 19-21. 
APPENDIX A

Estimating Airflow Patterns Over Complex Terrain 
USDA Forest Service

Research Paper RM-162

March 1976

\section{Estimating Airflow Patterns Over Complex Terrain}

Michael A. Fosberg. ${ }^{1}$ William E. Marlatt, ${ }^{2}$ and Lawrence Krupnak ${ }^{3}$

1Principal Meteorologist, Rocky Mountain Forest and Range Experiment Station, with central headquarters maintainet at Fort Collins, Colorado, in cooperation with Colorado State University.

2protessor, Department of Earth Resources, Colorado State University, Fort Collins, Colorado.

${ }^{3}$ Graduate Research Assistant, Department of Earth Resources, Coloiado State University, Fort Collins, Colorado. 


\section{Contents}

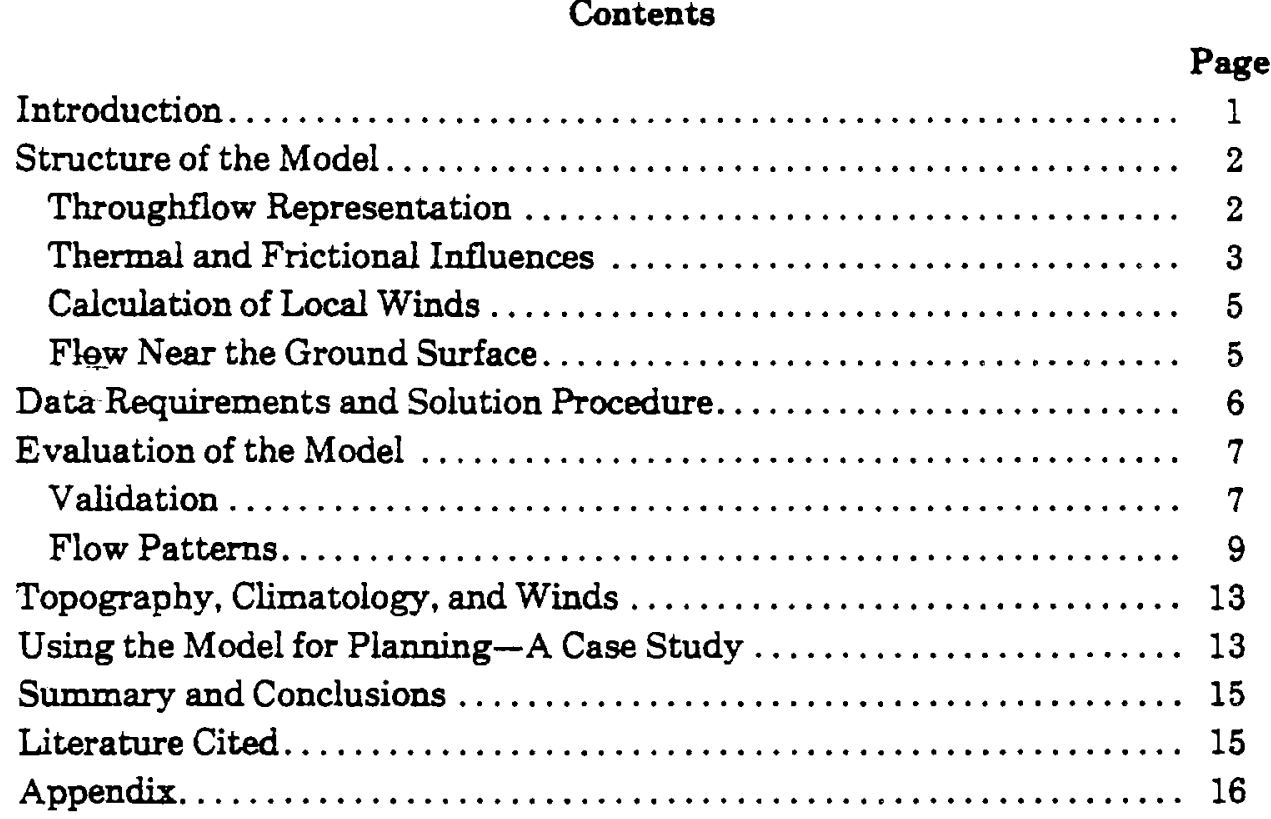




\title{
Estimating Airflow Patterns Over Complex Terrain
}

\author{
Michael A. Fosberg, William E. Marlatt, \\ and Lawrence Krupnak
}

\section{Introduction}

Land managers are increasingly faced with problems requiring quantitative estimates of airflow patterns over complex terrain. Use of the wind information may range from potential air quality analysis for land use planning to predicting spread of actual wildfires. Areas in which such analysis is required are typically mountainous. Nearly always, evaluation is severely hampered by a single problem-lack of data, particularly wind data.

For these reasons, a method of determining boundary layer flow patterns is needed as an alternative to expensive data networks.

Boundary layer flow over complex terrain cannot currently be numerically modeled with complete fluid and thermodynamic equations. Accuracy and quantity of the initial data fields required for complete solution oniy shift the problem back to need for expensive data networks (Fosberg 1967, 1969). While these costs and efforts may be worthwhile in special locations and for specific problems, flexibility in geographic location and timeliness of solutions are sacrificed. We feel that an alternative procedure is required for wind pattern analysis which provides geographic flexibility and does not require sophisticated, expensive data networks. Such solutions cannot include all atmospheric processes, however. The governing equations must be simplified and some important effects must be represented by parameterized analogs.

A model of this type is based on tradeoffs between simplified solutions and preservation of realistic results. Several models of thermal and terrain-induced perturbation of near-ground flow have been developed recently. Anderson (1971) and Lantz developed single layer models of twodimensional potential flow over terrain features. Anderson (1971), Fosberg et al. (1972), and Fosberg $^{5}$ examined the thermally driven flow disturbances. These studies were all based on the philosophical argument that major simplification of the equations was acceptable, and that the disturbed flow field could be superimposed on a mean flow field. While superficially attractive, these preliminary models did not evaluate the consequences of the simplifications, nor did they provide quantitative arguments supporting neglect of specific processes.

Each of the models describes a single layer of finite depth, depicts a quasi-horizontal slab, and treats body forces as an impulse acceleration. The models also require the flow to be statically stable, either as an incompressible fluid or through the hydrostatic assumption. Superposition of disturbed flows on the riean flow also implies limitations on the processes that can be modeled. Impulse accelerations tacitly imply that a model is intended as a diagnostic tool rather than a timedependent predictive model. Also, these models generally neglect or highly linearize the advection terms in the dynamic equations.

The highly idealized treatment of the advection term is probably the most serious of the assumptions. Justification is provided partially by examination of the energy integrals in Fos-

¿Lantz, R. 8. 1972. Application of a three dimensional numerical model io air pollution calculations. Preprint of paper presented at 65th Annu. Meet., Air Pollution Control Assoc., Miami Beach. Fla., June 18-22. 22 p.

${ }^{5}$ Fosberg. Micnael A. (in press). New tecnnology for ceter. mining atmospneric influencs on smcke concentrations. Proc. Int. Symo. on Air Quality and Smoke trom Urban and For. Fires. 1973. 110. 
berg's $(1967,1969)$ analysis of flow over single ridges. Conversion of potential energy to kinetic energy dominates the kinetic energy budget. The advective redistribution of kinetic energy and the viscous dissipation are about an order of magnitude smaller. While these energy budget comparisons do not fully justify neglect of the advective processes, they do show that the assumption does not invalidate the models, but only restricts interpretation of the results.

In general, this class of airflow models excludes the small-scale physical situations in which transient or highly interactive processes such as gravity waves or unstable thermal convection dominate the flow field. Also, because of the restrictions imposed on the advection terms. downwind wake effects are not properly represented.

Use of these simplified models-rather than complex and complete solutions or as a substitute for extensive field studies-becomes attractive when extensive data are not available, or in land use planning where alternatives may be great, the time span for planning short, and the accuracy or costs of more physically precise solutions and studies unwarranted.

The model described in this paper is intended to meet these applied objectives. This model is based on simplified solutions of the complete equations. It is philosophically identical to the preliminary models, but combines more of the physical processes.

\section{Structure of the Model}

The mathematical model is based on the terrain-induced flow processes derived by Anderson (1971) and the thermally induced flows described by Fosberg et al. (1972). In addition, the frictional terms are included in the model. Anderson's model was based only on a form of the diver. gence equation, while the Fosberg et al. (1972) model was based only on the vorticity equation. The model developed here is developed from both equations.

The advection terms were neglected in both previous equations. The consequences of this assumption are that the equations were simplified at the expense of losing a complete physical description of the dynamic processes. Thermally, frictionally and terrain-induced flows were superimposed on a beckground potential flow across the computational boundaries. Each of these disturbances was assumed to take place only within the computational area. Superposition of disturbances on the background flow, after ne- glecting advection terms, is physically analagous to an impulse acceleration. The diagnostic nature of the model was achieved by allowing the impulse to act over a small, finite time interval defined by phase velocities of disturbances. Flow following the ground surface was obtained by a coordinate transform. A rigid upper surface above the terrain was assumed in order to define the top of the atmospheric slab.

Procedures for solving the equations involve serial approximations which superimpose a new physical effect on the previous solution. The first step in the solution was to transfer the large-scale background wind into a terrain-induced modifica. tion of the throughflow. This step provided a local throughflow wind.

Next, thermal and frictional modification of the vorticity and divergence were introduced. These changes were superimposed on the terraininduced flow. Finally, stream functions and velocity potentials were calculated so that the windspeed and direction could be defined at all interior points.

\section{Throughflow Representation}

Total airflow through the computation field was required to satisfy conservation of mass. Since the lower surface of the layer was mountainous, provisions had to be made for local channeling and deflection. Throughflow characteristics were calculated from the steady-state mass continuity equation as outlined by Anderson (1971). Vertical integration and use of the anelastic approxima. tion permitted local velocities to be approximated from the overall wind field and terrain features. The steady-state continuity equation is: ${ }^{6}$

$$
\nabla \cdot \rho \underline{v}=-\frac{\partial(\rho w)}{\partial z}
$$

In this equation, $\nabla$ is the horizontal gradient operator, $\rho$ is density, $v$ is the velocity vector, and $w$ is the vertical motion, positive upward.

Vector coordinates used in the mathematics are west to east in the $x$ direction, south to north in the $y$ direction, and vertically as $z$.

The anelastic approximation, where kinematic influences on density are negligible compared to thermodynamic influences, was first applied to the equation:

$$
\vec{\rho} \nabla \cdot \underline{v}=-\frac{\partial(p w)}{\partial z}
$$

6See appendix for a complete description of notations used in the equations. 
Bars over a variable indicate a mean value for the slab. The equation was then integrated over the depth of the layer between the ground (h) and the top of the slab (H).

$$
\bar{\rho} \int_{h}^{\mathrm{H}} \nabla \cdot v \mathrm{dz}=-\int_{\mathrm{h}}^{\mathrm{H}} \frac{\partial(\rho w)}{\partial z}
$$

Horizontal divergence was defined as $\delta=\nabla \cdot \underline{y}$ so that the integral equation was solved as:

$$
\bar{\rho} \bar{\delta}(\mathrm{H}-\mathrm{h})=-\rho w(H)+\rho w(h)
$$

through the mean value theorem. A rigid lid was assumed, so $\rho w(\mathrm{H})$ became zero. Vertical motion near the ground was assumed to be dominated by orographic lifting. The horizontal divergence of the mean wind field then became:

$$
\bar{\delta}=\frac{\rho}{\rho} \mathrm{v} \cdot \frac{\nabla \mathrm{h}}{(\mathrm{H}-\mathrm{h})}
$$

Terrain modification of the large-scale flow could then be calculated from the channeling and deflection of this flow, and could provide a first approximation of the local wind.

\section{Thermal and Frictional Influences}

Modification of the wind field produced by thermal acceleration and retardation required invoking the assumptions associated with the advection terms of the complete equations. Frictional influences were included as spatial variations of ideslized and parameterized solutions relating surface characteristics to the wind profile. The fundamental procedure was to: (1) derive the complete divergence and vorticity equations, (2) judiciously neglect the advective terms, (3) allow full influence of the thermal terms, and (4) utilize classic frictional solutions.

The procedure required for inclusion of these body forces was to define the thermal and frictional solutions in terms of the Navier-Stokes equations. The first step was to express the divergence equation utilizing these assumptions. Divergence was obtained through:

$$
\nabla \cdot \frac{d v}{d t}=\frac{\partial \delta}{\partial t}=\nabla \cdot\left(-c_{p} \theta \nabla p_{*}\right)+\nabla \cdot\left(K \nabla^{2} \underline{v}\right)
$$

where $\theta$ is the potential temperature, $p_{*}$ is the pressure variable defined as $p_{*}=T / \theta$, and $K$ is the turbulent viscosity. The friction term was simplified to:

$$
\nabla \cdot K\left(\frac{\partial^{2} v}{\partial z^{2}}\right)=K\left(\frac{\partial^{3} u}{\partial x \partial z^{2}}+\frac{\partial^{3} v}{\partial y \partial z^{2}}\right)
$$

since the horizontal dissipation terms are small compared to the vertical terms when expressed in finite-difference mesoscale models. A parameterized form of the dissipation was obtained from the logarithmic wind profile near the ground. The wind protile for an adiabatic surface layer (Sutton 1953) is:

$$
\frac{\partial u}{\partial z}=\frac{u_{*}}{k z^{2}} \text { and } \frac{\partial v}{\partial z}=\frac{v_{*}}{k z^{2}}
$$

where $u_{*}$ and $v_{*}$ are the friction velocities and $k$ is the von Karman constant. These two functions were differentiated with respect to $z$ to produce the second derivative. The terms

$$
\frac{\partial^{2} u}{\partial z^{2}}=-\frac{u_{\star}}{k z^{2}} \text { and } \frac{\partial^{2} v}{\partial z^{2}}=-\frac{v_{\star}}{k z^{2}}
$$

were then substituted into the divergence equation. The divergence equation then became:

$$
\begin{aligned}
\frac{\partial \delta}{\partial t}= & -c_{p} \theta \nabla^{2} p_{*}-c_{p} \nabla \theta \cdot \nabla p_{*} \\
& -\frac{k}{k z^{2}}\left(\frac{\partial^{u} \star}{\partial x}+\frac{\partial^{v} \star}{\partial y}\right)
\end{aligned}
$$

Friction velocity was obtained by integrating the wind profile equation for fully rough surfaces. The friction velocities are then:

$$
\begin{aligned}
& u_{\star}=\frac{|\bar{u}| k}{\ln \left(\frac{z+z_{0}}{z_{0}}\right)} \\
& v_{\star}=\frac{|\bar{v}| k}{\ln \left(\frac{z+z_{0}}{z_{0}}\right)}
\end{aligned}
$$

where $z_{o}$ is the surface roughness length and $Z$ is the height of the wind above ground.

Rotational flow characteristics were introduced through the vorticity equation. Using the same assumptions for this derivation as were 
used in the development of the divergence equation, the vorticity equation became:

$$
\begin{aligned}
\mathrm{k} \cdot \nabla \mathrm{x} \frac{\mathrm{dv}}{\mathrm{dt}}= & \frac{\partial \zeta}{\partial t}=-c_{p} \frac{\partial\left(\Theta, \mathrm{p}_{\star}\right)}{\partial(\mathrm{x}, \mathrm{y})} \\
& -(\zeta+f) \delta-\frac{\mathrm{k}}{\mathrm{k} \mathrm{z}^{2}}\left(\frac{\partial \mathrm{v}_{\star}}{\partial \mathrm{x}}-\frac{\partial \mathrm{u}_{\star}}{\partial \mathrm{y}}\right)
\end{aligned}
$$

The divergence and vorticity equations are time-dependent and therefore must be integrated. Divergence was integrated directly through the mean value theorem over an arbitrary time interval. Since little is known about the rate of change of divergence, a square wave impulse of interval $\Delta t_{\delta}$ was assumed. The integrating time factor then became $1 / 2 \Delta t_{\delta}$ when the magnitude of the acceleration was assumed to be $1 / 2$ the observed or maximum value. Divergence was then:

$$
\begin{aligned}
\partial=\frac{1}{2} \Delta t \delta & {\left[-c_{p} \theta \nabla^{2} p_{\star}-c_{p} \nabla \theta \cdot \nabla_{*}\right.} \\
- & \left.\frac{k}{k z^{2}}\left(\frac{\partial u_{\star}}{\partial x}+\frac{\partial v_{\star}}{\partial y}\right)\right]+\bar{\delta}
\end{aligned}
$$

Given this solution, the vorticity equation became the ordinary differential equation of:

$$
\begin{aligned}
\frac{\partial \zeta}{\partial t}+\zeta \delta=-c_{p} \frac{\partial\left(\theta, p_{*}\right)}{\partial(x, y)}-f \delta & \\
& -\frac{k}{k z^{2}}\left(\frac{\partial v_{\star}}{\partial x}+\frac{\partial u_{\star}}{\partial y}\right)
\end{aligned}
$$

The solution is:

$$
\begin{aligned}
& \zeta= \mathrm{e}^{-\int \delta \mathrm{dt}} \int\left[-c_{\mathrm{p}} \frac{\partial\left(\theta, \mathrm{p}_{*}\right)}{\partial(\mathrm{x}, \mathrm{y})}-f \delta\right. \\
&\left.-\frac{\mathrm{K}}{\mathrm{k} z^{2}}\left(\frac{\partial \mathrm{v}_{\star}}{\partial \mathrm{x}}-\frac{\partial \mathrm{u}_{\star}}{\partial \mathrm{y}}\right)\right] \exp (\delta \delta d t) d t \\
&+c e^{-\int \delta d t}
\end{aligned}
$$

Again, an impulse of finite time interval $\Delta t_{\zeta}$ was assumed, so that the mean value integration gave:

$$
\begin{aligned}
& \zeta=\exp \left(-\frac{1}{2} \delta \Delta t_{\zeta}\right)^{\frac{1}{2} \Delta t_{\zeta}} \\
& {\left[-c_{p} \frac{\partial\left(\theta, p_{\star}\right)}{\partial(x, y)}-f \delta-\frac{k}{k z^{2}}\left(\frac{\partial v_{\star}}{\partial x}-\frac{\partial u_{\star}}{\partial y}\right)\right]} \\
& \exp \left(\frac{1}{2} \delta \Delta t_{\zeta}\right)+c e^{-\delta \Delta t / 2}
\end{aligned}
$$

The arbitrary time intervals for the divergence and vorticity were defined in terms of phase velocities. Because a rigid lid was used as an upper boundary for the calculation and an anelastic approximation was used for compressibility, the phase velocity for divergence was taken as the speed of sound. This gave a time interval of:

$$
\Delta t_{\delta}=\frac{\sec \Delta}{300 \text { m }}
$$

where $\Delta$ is the minimum distance between computational points. Phase velocities for vorticity were taken to be the large-scale windspeed, so that the time interval for vorticity was:

$$
\Delta t_{\zeta}=\frac{\Delta}{\left(u^{2}+v^{2}\right)^{\frac{1}{2}}}
$$

For typical distances of 1 to $10 \mathrm{~km}$ between computational points and windspeeds of 1 to $10 \mathrm{~m}$ per second, the time interval is of the order of 1,000 seconds. Divergence at the mesoscale is typically $10^{-5}$ to $10^{-3}$ per second. These magnitudes suggested the exponential term in the vorticity equation should be approximately one, except under condition of light winds and large grid spacing. The constant of integration was then taken to be the large-scale flow vorticity. The vorticity then became:

$$
\begin{aligned}
\zeta=\frac{1}{2} \Delta t_{\zeta} & {\left[-c_{p} \frac{\partial\left(\theta, p_{\star}\right)}{\partial(x, y)}-f \delta\right.} \\
& \left.-\frac{k}{k z^{2}}\left(\frac{\partial v_{\star}}{\partial x}-\frac{\partial u_{\star}}{\partial y}\right)\right]+\zeta_{0}
\end{aligned}
$$

Equations (1) and (6) for the frictionally, thermally and terrain-induced flow deviations on divergence, and equation (7) for the frictionally and thermally induced flow deviations on rotation. were then used to calculate the wind components. 


\section{Calculation of Local Winds}

Wind components of the flow were calculated in the area by superimposing the terrain, frictional, and thermal deviations on the large-scale wind. The wind deviations were obtained by separating the flow into an irrotational divergent component and a nondivergent rotational component. The divergent flow was described with a velocity potential and the rotational component with a stream function. The deviation velocity was then:

$$
\underline{v}=\underline{k} \nabla \nabla+\nabla \phi
$$

where $\psi$ is the stream function and $\phi$ is the velocity potential. The vorticity is:

$\zeta=\mathrm{k} \cdot \nabla \mathrm{xv}=\underline{\mathrm{k}} \cdot \nabla \mathbf{x} \underset{\sim}{\mathrm{k}} \nabla \psi+\underline{\mathrm{k}} \cdot \nabla \mathrm{x} \nabla \phi=\nabla^{2} \psi$

and the divergence is:

$$
\delta=\nabla \cdot \underset{v}{v}=\nabla \cdot \underset{\sim}{\mathrm{k} x} \nabla \psi+\nabla \cdot \nabla \phi=\nabla^{2} \phi
$$

Since the vorticity and divergence were known from equations (1), (6), and (7), the stream function and velocity potential could be obtained from a numerical solution of the elliptical differential equations (8) and (9). Decomposing the velocity into the west-east component and the south-north component, and superimposing the background flow, the velocities are:

$$
u=u_{0}+\frac{\partial \phi}{\partial x}-\frac{\partial \psi}{\partial y}
$$

and

$$
v=v_{0}+\frac{\partial \phi}{\partial y}+\frac{\partial \psi}{\partial x}
$$

where $u_{0}$ and $v_{0}$ are the background velocities.

\section{Flow Near the Ground Surface}

Airflow along the terrain surface is of much more interest in fire or air-quality studies than flow at some fixed elevation. Since the equations require calculations to be made on a flat horizontal surface, transformations from the horizontal plane to the terrain surface are necessary.

Data required by the model are observed at a fixed height above the ground (generally between 1 and $3 \mathrm{~m}$ ) so the transforms chosen were to re structure the observed data to a flat surface locally, and then to consider the model as being composed of a series of adjacent horizontal surfaces, each of slightly different elevation based on the elevation of the local terrain. The coordinate transform required expressing the derivations of the variables observed on the terrain surface as though they were along a horizontal surface. If vertical variations of the variables are known, the transform for any quantity, $S$, is then:

$$
\left.\left.\left.\frac{\partial S}{\partial x}\right)_{E}=\frac{\partial S}{\partial x}\right)_{T}-\frac{\partial S}{\partial z} \frac{\partial h}{\partial x}\right)_{T}
$$

where the unsatisfied parentheses indicate derivations taken along either constant elevation or along the terrain surface.

Second derivations were obtained similarly as:

$$
\begin{aligned}
\left.\frac{\partial^{2} s}{\partial x^{2}}\right)_{E}= & -2 \frac{\partial^{2} s}{\partial x \partial z} \frac{\partial h}{\partial z}-\frac{\partial S}{\partial z} \frac{\partial^{2} h}{\partial x^{2}} \\
& +\left(\frac{\partial h}{\partial x}\right)^{2} \frac{\partial^{2} s}{\partial z^{2}}
\end{aligned}
$$

The higher order terms involving derivatives of the terrain are small compared to the linear term. Six separate derivatives of the thermal characteristics are required by the vorticity and divergence equations. These are the first derivative of potential temperature and pressure, and the second derivative of pressure in the cardinal directions.

Vertical variations of these thermodynamic variables are required. These were obtained first for potential temperature through static stability for a hydrostatically balanced atmosphere as:

$$
\begin{aligned}
& \left.\left.\left.\frac{\partial \theta}{\partial x}\right)_{E}=\frac{\partial \theta}{\partial x}\right)_{T}-\theta \sigma \frac{\partial h}{\partial x}\right)_{T} \\
& \left.\left.\left.\frac{\partial \theta}{\partial y}\right)_{E}=\frac{\partial \theta}{\partial y}\right)_{T}-\theta \sigma \frac{\partial h}{\partial y}\right)_{T}
\end{aligned}
$$

where $\sigma=\frac{1}{\theta} \frac{\partial \theta}{\partial z}$.

Vertical decrease of pressure was represented by hydrostatic equilibrium:

$$
\frac{\partial p_{\star}}{\partial z}=-\frac{g}{c_{p} \theta}
$$


Utilizing these relationships, the pressure derivatives are:

$$
\begin{aligned}
& \left.\left.\left.\frac{\partial p_{\star}}{\partial x}\right)_{E}=\frac{\partial p_{\star}}{\partial x}\right)_{T}+\frac{g}{c_{p} \theta} \frac{\partial h}{\partial x}\right)_{T} \\
& \left.\left.\left.\frac{\partial p_{\star}}{\partial y}\right)_{E}=\frac{\partial p_{\star}}{\partial y}\right)_{T}+\frac{g}{c_{p} \theta} \frac{\partial h}{\partial x}\right)_{T} \\
& \left.\left.\left.\frac{\partial^{2} p_{\star}}{\partial x^{2}}\right)_{E}=\frac{\partial^{2} p_{\star}}{\partial x^{2}}\right)_{T}-2 \frac{g}{c_{P} \theta^{2}} \frac{\partial h}{\partial x} \frac{\partial \theta}{\partial x}\right)_{T} \\
& \left.\left.\left.\frac{\partial^{2} p_{\star}}{\partial y^{2}}\right)_{E}=\frac{\partial^{2} p_{\star}}{\partial y^{2}}\right)_{T}-2 \frac{g}{c_{P} \theta^{2}} \frac{\partial h}{\partial y} \frac{\partial \theta}{\partial y}\right)_{T}
\end{aligned}
$$

Horizontal derivatives of the friction velocities remain unchanged, since the model is designed for use in the constant flux layer where they are constant in the vertical. This shallow layer assumption then gives:

$$
\begin{aligned}
& \left.\left.\frac{\partial u_{\star}}{\partial x}\right)_{E}=\frac{\partial u_{\star}}{\partial x}\right)_{T} \\
& \left.\left.\frac{\partial u_{\star}}{\partial y}\right)_{E}=\frac{\partial u_{\star}}{\partial y}\right)_{T} \\
& \left.\left.\frac{\partial v_{\star}}{\partial x}\right)_{E}=\frac{\partial v_{\star}}{\partial x}\right)_{T}
\end{aligned}
$$

$$
\left.\left.\frac{\partial \mathrm{v}_{\star}}{\partial \mathrm{y}}\right)_{E}=\frac{\partial \mathrm{v}_{\star}}{\partial \mathrm{y}}\right)_{T}
$$

These linear coordinate transforms neglect the small changes in the grid spacing due to slope distances projected to the horizontal grid.

\section{Data Requirements and Solution Procedure}

Specific data required by the model are temperature, pressure, elevation, and roughness length of the underiying surface for all computational points. Temperature and pressure data are transformed thermodynamically to model variables by:

$$
\theta=\frac{T}{P_{*}}
$$

and

$$
P_{*}=\left(\frac{p}{p_{0}}\right)^{\frac{R}{c_{p}}}=\left(\frac{p}{p_{0}}\right)^{0.2857}
$$

where $p_{0}$ is a reference pressure and $T$ is expressed in an absolute temperature scale. While any consistent set of units may be used, the appendix list of symbols gives constants only in metric units.

Roughness lengths are a reasonably constant

\begin{tabular}{|c|c|}
\hline $\begin{array}{l}\text { Classification of } \\
\text { underlying surface }\end{array}$ & $\begin{array}{l}\text { Typical value of } \\
\text { roughness length (z.) }\end{array}$ \\
\hline $\begin{array}{l}\text { Mud flats, ice } \\
\text { Smooth snow } \\
\text { Snow, prairle } \\
\text { Thin grass up to } 50 \mathrm{~cm} \mathrm{high} \\
\text { Thick grass up to } 50 \mathrm{~cm} \text { high } \\
\text { Wheat } \\
\text { City } \\
\text { Orehard } \\
\text { Fir forest }\end{array}$ & $\begin{array}{cc} & c m \\
0.001 & \text { (Sutton 1953) } \\
0.005 & \text { (Priestiey 1959) } \\
0.01 & \text { "1 } \\
5 & \text { (Sutton 1953) } \\
9 & \text { " } \\
22 & \text { (Seilers 1965) } \\
165 & \text { "1" } \\
198 & " 1 \\
283 & \end{array}$ \\
\hline
\end{tabular}
value describing the height at which the mean wind flow is zero. These values are conveniently describable to acceptable accuracy by classifying the surface into such categories as (but not limited to) grass, brush, boulder field, and so forth. These data are tabulated in numerous texts (Sutton 1953, Priestley 1959, and Sellers 1965); a sample listing is provided in table 1 for convenience and completeness. 
Since the model was designed for use in moderateiy small areas of finite size, several singlevalued coefficients are required to complete the model. Most important of these are the static stability and the large-scale wind direction and windspeed. The large-scale winds were specified as the geostrophic wind. Less important coefficients are the eddy viscosity, the latitude (if the geographic scale of a particular solution is large so that the earth's rotation is important), and the large-scale vorticity (if strong curvature exists in the large-scale flow).

Wind patterns are calculated from a sequence of approximations, each refining the previous step by introducing a more refined physical process, and superimposing the refinement on the previous solution. The first step was to transfer the largescale background wind into a major terraininduced modification of the throughflow. This step provided a local throughflow wind.

First, the divergence was calculated through equation (1) for a smoothed terrain. This smoothed terrain was obtained by averaging the four surrounding points with each computational point; the rigid lid was placed well above the terrain in this step. Second, the terrain-induced vorticity was calculated by equation (7) with the thermal and frictional terms neglected. Once these vorticities and divergences were calculated, they were transformed to stream functions and velocity potentials through numerical solutions of equations (8) and (9), and subsequently resoived into velocities by equation (10). These first approximations to the wind field represented the influences of the large terrain feature on the throughflow.

The second level of approximation involved recalculating the terrain-induced divergence for a shallow layer. Divergence was calculated by equation (1) for the actual terrain.

The next step required using the large-scale terrain flow to calculate the friction velocities by equations ( $4 a$ and $4 b$ ) and making the coordinate transforms of equation (11). These intermediate steps provided the input to the final level of approximation.

Friction terms, defined by the friction velocity, and the spatial derivations of temperature and pressure after the coordinate transform were included in the solution of equation (6) for divergence and of equation (7) for vorticity. Background divergence came from the second level of approximation, and the background vorticity came from the divergence and vorticity as in the first step through numerical solutions of equations (8) and (9). Velocity components were then calculated through equation (10), where the background velocities were defined by the first step.

\section{Evaluation of the Model}

\section{Validation}

There are several philosophies of model validation, each designed to accomplish specific objectives. Because this model is intended for use in applied meteorology, we have chosen to use actual data sets from mountain mesoscale studies to validate the wind field estimates. In adopting this approach, we have imposed a severe test since the model is limited in spatial resolution by computational filtering to approximately four times the grid distance, while the validation data have small-scale spatial resolutions defined by nearby terrain or vegetation and sensor response characteristics. Also, calculated winds represent an average wind over an area defined by the grid mesh. Such a test is desirable, however, because it defines operational uncertainty rather than model potential, and represents actual resolution attainable.

Seven data sets were used in the model validation. Six came from studies conducted by Cramer ${ }^{7}$ in the Oregon Cascade Mountains. One data set came from central California (Fosberg and Schroeder 1966). All seven depict daytime conditions: five for midafternoon, and two for morning. One morning case occurred under overcast skies and rain. The other six occurred with strong solar heating. Background winds ranged from 2 to 5 m per second. The Oregon winds were calculated on a $6 \mathrm{~km}$ square grid, the California case on a $16 \mathrm{~km}$ square grid. Air temperatures were extrapolated to grid points from the field network weather stations.

Evaluation of the errors in both windspeed (fig. 1) and wind direction (fig. 2) demonstrates the utility of the model. The root mean square windspeed error was $2.0 \mathrm{~m}$ per second. Forty-five percent of the calculated windspeeds fell within $1 \mathrm{~m}$ per second of the observed wind, and 75 percent fell within $2 \mathrm{~m}$ per second. These comparisons were made over an observed range of 0.5 to 10.5 $m$ per second. Observed wind directions were reported on a 16-point compass basis. Therefore, the error analysis is presented as a compasspoint error rather than a more precise evaluation

${ }^{7}$ We thank Owen P. Cramer of the U.S. Department oi Agriculture, Forest Service, Pacific Northwest Forest and Range Experiment Station. Portland. Oregon for unqualified use of original data for the model validation and for permission to reproduce unpublished results in this paper. 


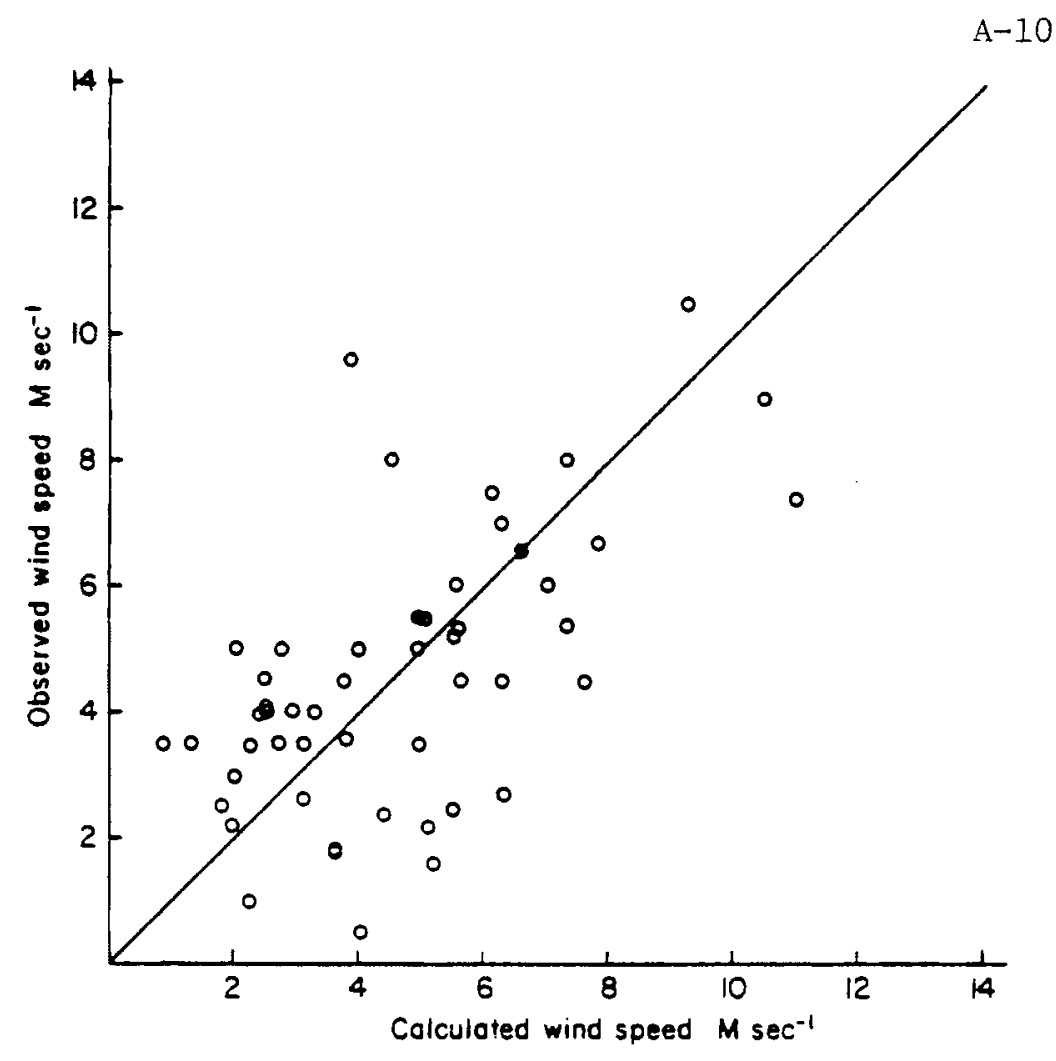

Figure 1.-Comparison of calculated and observed windspeeds. Bold diagonal line defines perfect fit. Forty-five percent of predictions tall within $1 \mathrm{~m} \mathrm{sec}^{-1}$ of the observed windspeed and 75 percent fall within $2 \mathrm{~m} \mathrm{sec}^{-1}$

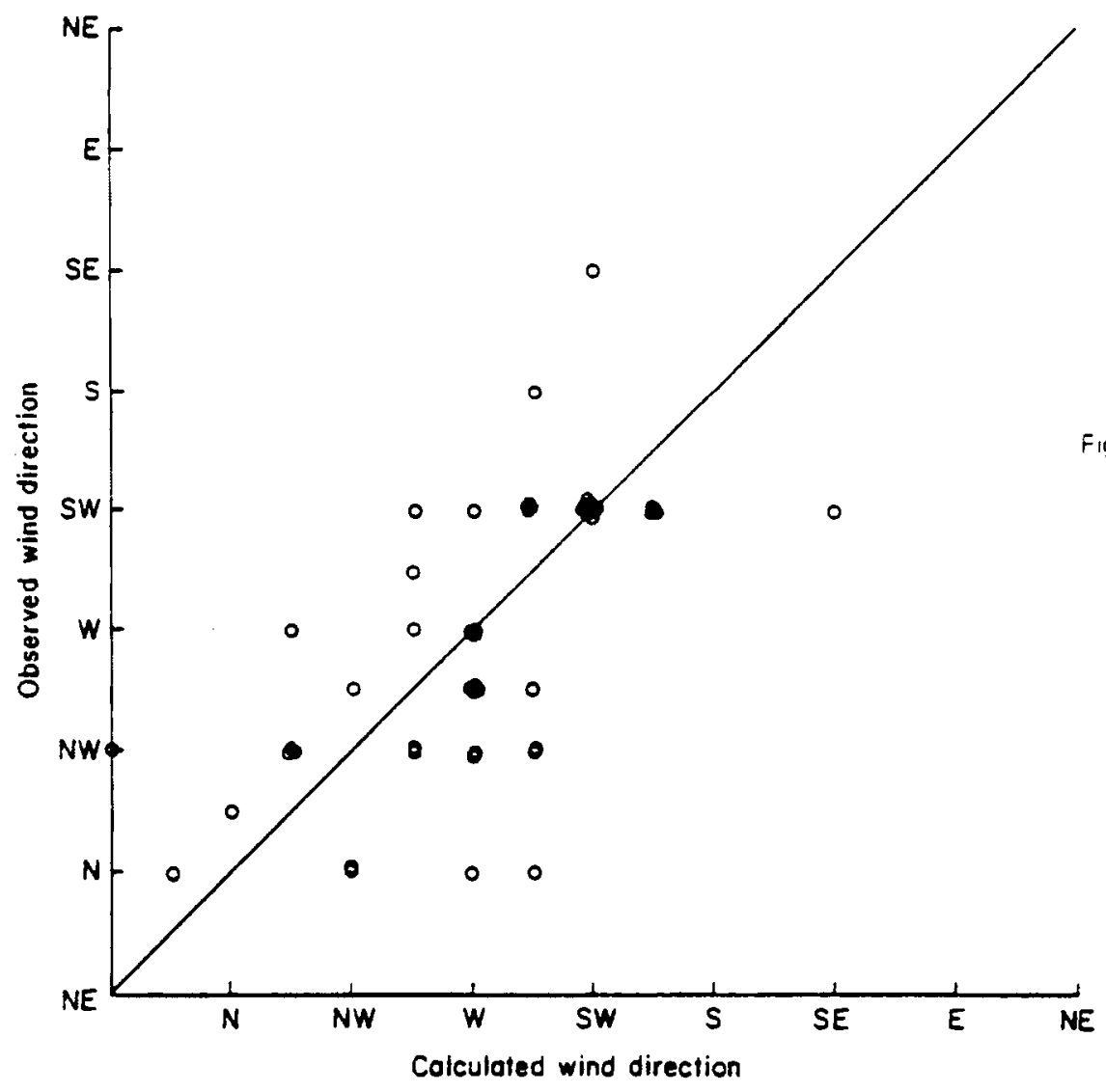

Figure 2.-Comparison of calculated ano observed wind direction. Comoarison is on a 16-point compass basis. Sixty per. cent of calculated directions tall within 11 degrees and 92 percent fall within 22 degrees of observed directions. 
of angle. The root mean square direction error was 1.9 points. Sixty percent of the calculated directions fell within 1 compass point of the observed direction, and 92 percent fell within a 2-point envelope of the observed direction. Observed directions were from all compass points except for the east and northeast sections.

Models of this type implicitly require a num. ber of specifiable coefficients which tune the model to particular geographic regions. While we have attempted to specify these coefficients through physical arguments, some are still at the discretion of the user. The integration coefficients were totally predetermined. The rigid lid coefficients for potential flow were selected through several criteria. The first was that the terrain could not penetrate the rigid lid. The second was based on a large number of solutions where the lid was systematically varied until a high level of sensitivity was reached. Lid height is based on elevation differences across the grid field. The user guidelines in the climatology section of this paper describe the techniques for determining layer thickness. They are cited here because the consequences are reflected in the error analysis.

\section{Flow Patterns}

Each of the seven cases showed similar dynamic processes (table 2). Thermally and terrain. produced divergence dominated the dynamics of the flow. Divergencegenerated flow perturbations were 2.5 to 4.3 times as large as vortically generated deviations. Vortex flow accounted for 11 to 24 percent of total kinetic energy, while 32 to 69 percent was accounted for by the potential flow. Strongest local wind energetics were ob- served under conditions of light background winds and strong surface heating. As background winds increased, or the solar heating decreased, the local winds became a weaker component and less important in the total kinetic energy budget. Tests of background windspeeds for several hypothetical cases beyond the range of data used in validation show that at $0.5 \mathrm{~m}$ per second, the wind patterns are controlled completely by the thermal patterns. At $10 \mathrm{~m}$ per second, thermal control vanished and terrain influences were weak.

Mesoscale flow characteristics are best illustrated through a detailed examination of one of the case studies. The area is in the Oregon Cascades, south of Mt. Hood, north of Mt. Jefferson, and runs from the Willamette Valley across the crest of the Cascade Range to the edge of the eastern Oregon plateau. The mountains are penetrated by a major drainage on the west side, the Clackamas River. The particular case selected, August 8,1963 at 1600 PST, was chosen as representing a typical or intermediate situation. The synoptic weather pattern was characterized by a weak upper-level, high-pressure area, and west to west-north-west geostrophic surface flow over the Cascades. Details of the observation and analysis procedures were presented in Cramer (1972) and Cramer and Lynott $(1961,1970)$. Discussion here is limited to resultant computations and estimation of the wind field from the analysis. Only salient features of Cramer's analysis are presented here for completeness.

Cramer's data analysis of temperature and $850 \mathrm{mb}$ surface heights were interpolated to $6-\mathrm{km}$ grid points. These data were converted to poten. tial temperature and normalized station pressures for each grid point. Terrain elevation at each grid point was interpolated from a topographic map.

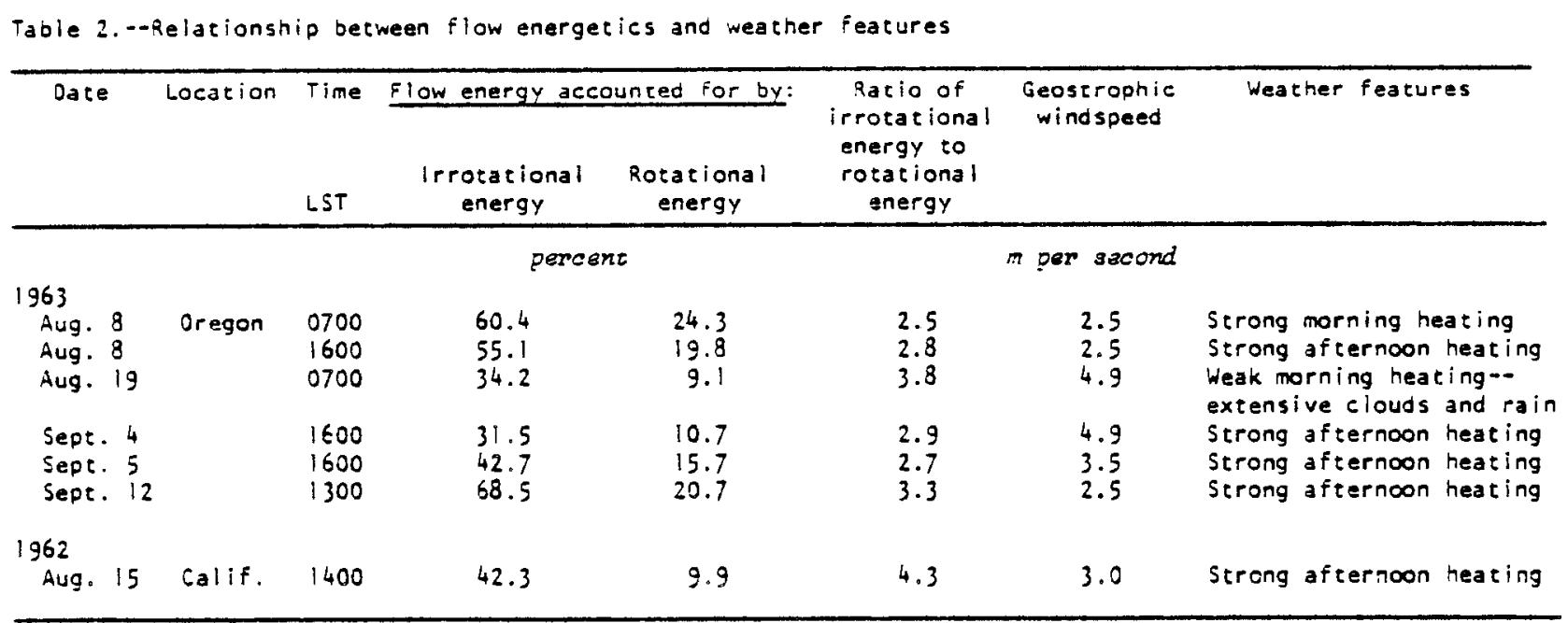


The underlying surface was almost exclusively characterized by conifer forest, so the roughness length was taken as a constant $283 \mathrm{~cm}$.

Temperature distribution (fig. 3) over this terrain generally showed a slight decrease with elevation. West-facing slopes were warmer than east-facing slopes. The major river valley was much warmer than surrounding areas, and provided strong local temperature gradients. In general, the area east of the Cascades was warmest as a result of morning heating and topographic blocking of cool marine air flowing off the Pacific Ocean.

Height of the $850 \mathrm{mb}$ pressure surface (fig. 4) generally reflected the high-pressure area over northwest Oregon. Surface heating of the mountain slopes produced the "thermal low" frequently observed along the West Coast. This "thermal low" appeared as a mesoscale low-pressure area through the higher terrain and modified the largescale pressure pattern.

These observed data were transformed to the thermodynamic variables of potential temperature and normalized nondimensional surface pressure used in the model equations. The $850 \mathrm{mb}$ surface height was reduced to the terrain surface through the hydrostatic equation using the static stability from the Salem, Oregon rawinsonde observation. Once this pressure calculation was made, the potential temperatures were calculated with these pressures and the interpolated temperatures.

Since the dynamic influences due to pressure gradients are completely reflected in the $850 \mathrm{mb}$ pressure surface height, only the potential temperature patterns will be described here (fig. 5). Potential temperature showed a highly organized pattern. Higher elevations had higher potential temperatures. Valleys where cooler marine air could penetrate from the coast were clearly marked by low potential temperature. East-slope highlands had highest potential temperatures.

These observed fieids of temperature and pressure were used to calculate the fundamental kinematic characteristics of divergence and vorticity. Divergence patterns were strongly cor. related with terrain. Even small topographic features such as narrow ridges and valleys stand out in the divergence patterns (fig. 6). Flow divergence was confined to lowlands and valley bottoms. Convergence zones were on ridgetops and followed the terrain contours. Gentle slopes and large continuous upland areas on the east slopes typically had weak gradients. Magnitudes of the divergence were typically $\pm 10^{-3}$ per second. The convergence areas in regions of rugged terrain were much smaller than divergence areas, and maximum values were two to three times as in- tense as the compensating divergent areas. The intense gradients generated in the rugged terrain were most noticeable between the Claciramas River valley and the surrounding ridges. Areas of moderately changing terrain had divergent and convergent patterns of roughiy equal area and intensity.

Vorticity patterns (fig. 7) did not show either the response to smail terrain features or the large magnitude found in the divergence fields. Vorticity was typically $\pm 10^{-5}$ per second with the strongest areas only $\pm 10^{-4}$ per second. Ridgetops tended to have anticyclonic relative vorticity of $10^{-4}$ per second while valley bottoms had cyclonic vorticities of $-10^{-4}$ per second. Areas with slowly changing elevation did not show a consistent terrain-vorticity correlation. In general, vorticity tended to have a much longer wave length than divergence. The only generalizations we can make about the patterns are that major ridges tend to favor anticyclonic vorticity, while lowlands and major valleys tend to favor cyclonic vorticity. This characteristic was probably due to the dominant influence of the divergence in flow properties.

Resultant wind fields (fig. 8) showed strong topographic and thermal control of the wind patterns. Airflow in the Clackamas River valley and the other two west-slope watersheds penetrating into the Cascades showed strong topographic channeling up the rivers and the influence of slope winds angling away from the valley axis. Westslope areas away from these major drainages generally showed upslope flow, even on the lee sides. Strongest winds were calculated on high ridgetops and at the heads of canyons as they reached the highlands. Lee effects were most noticeable along the eastern slope. Winds flowing across the ridge in the northeast portion of the area were reduced from $5 \mathrm{~m}$ per second to $1 \mathrm{~m}$ per second by the upslope influences on the lee side. Strong, thermally driven winds were calculated in the south-central and southeastern portion of the area, where prevailing winds were reduced and influences of the penetrating west-slope canyons were negligible. Those variables which had greatest impact on model reliability were determined by sensitivity analysis. Both processes and variables were systematically excluded and error analy sis conducted on all seven cases. This analysis indicated that frictional influences and the vorticity equation affected the resultant wind field only slightly. Terrain and thermal influences totally dominated the calculations. Neglect of either created large errors in the wind field in the test cases. Further analysis of these processes indicated that pressure variation could be excluded with introduction of a small error, but that 


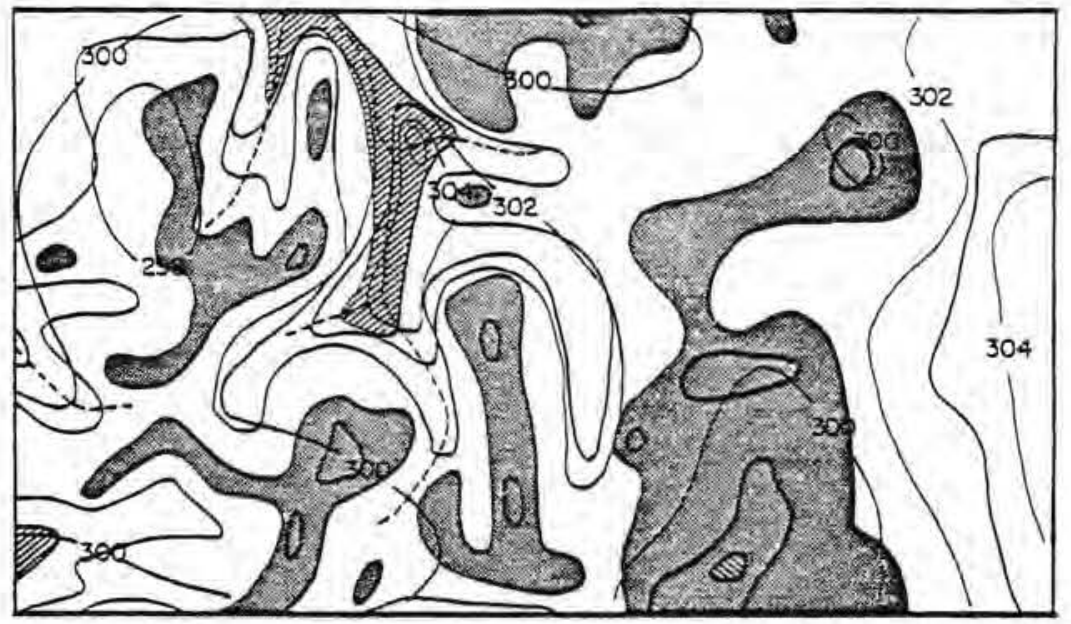

Figure 3.-Temperature field for 1600 PST, August 8, 1963. Temperatures are in degrees k: warmest areas are on the east slopes of the Cascade Mountains.

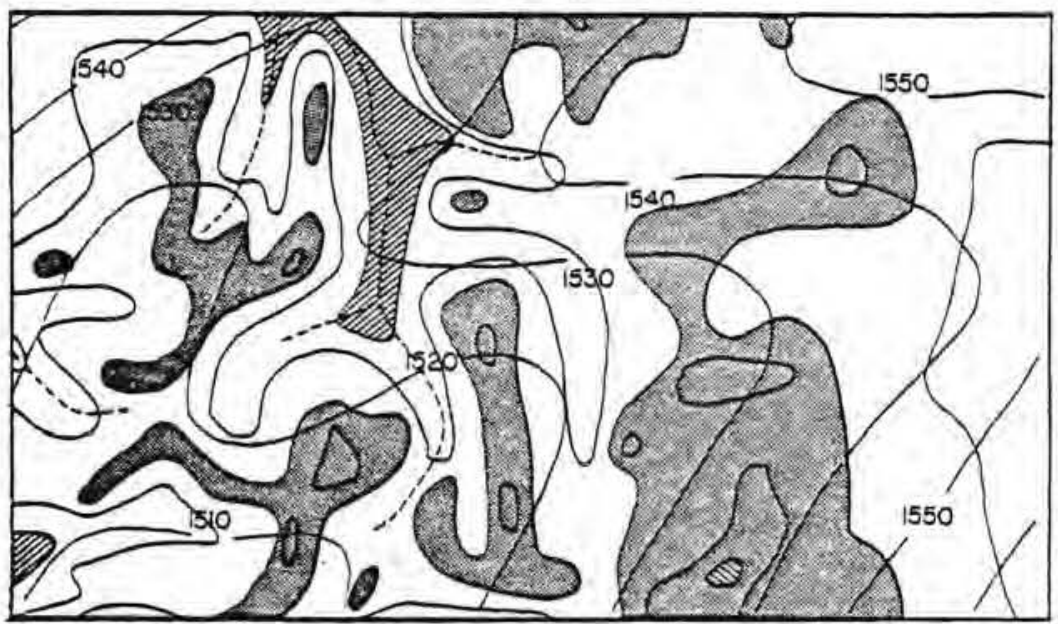

Figure 4.- Height of 350 mo surface tor 1600 PST. August 8. 1963. Height is in meters above m.s.l. Thermai trougn is oriented southwest to northeast.

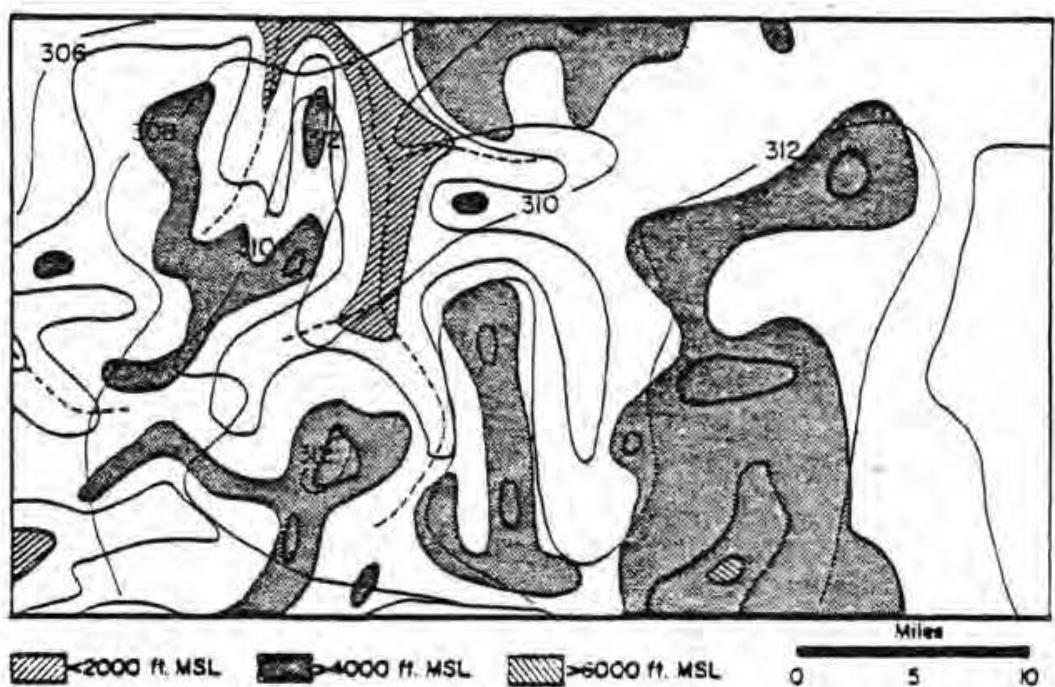

Figure 5.-Distribution of potential temperature for 1600 PST. August 8,1963 . Temperatures are in degrees $k$, Inversion of marine ait into the mountains is confined to the Clackamas River valley. 


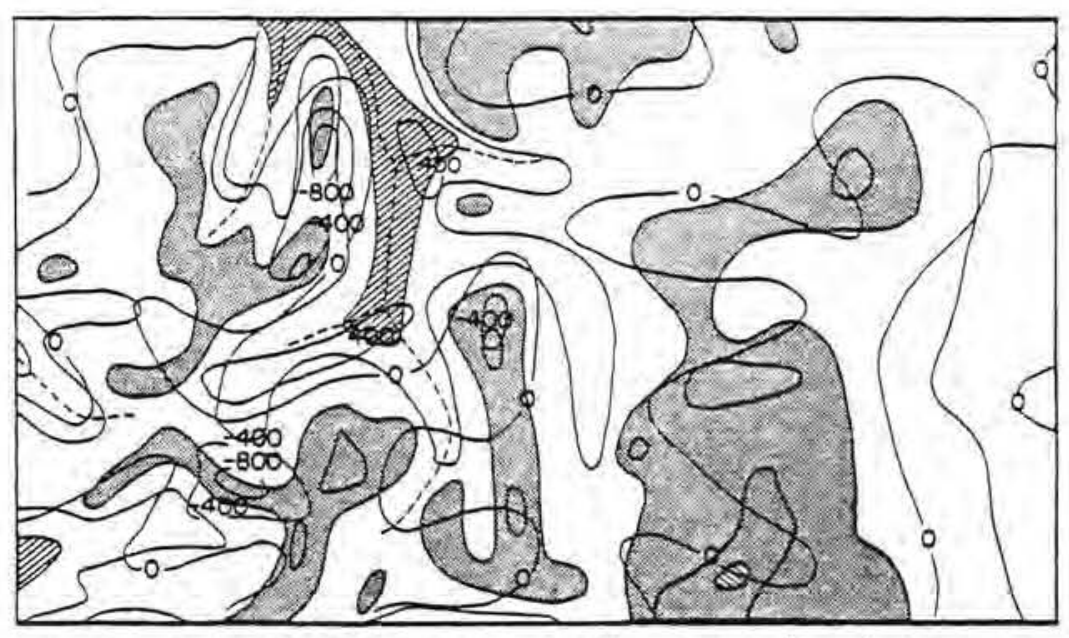

Figure 6.-Horizontal divergence field for 1600 PST, August 8,1963 . (Units are $10^{-5} \mathrm{sec}^{-1}$ ) Strongest gradients of divergence are between the west side drainages and the surrounding ridges.

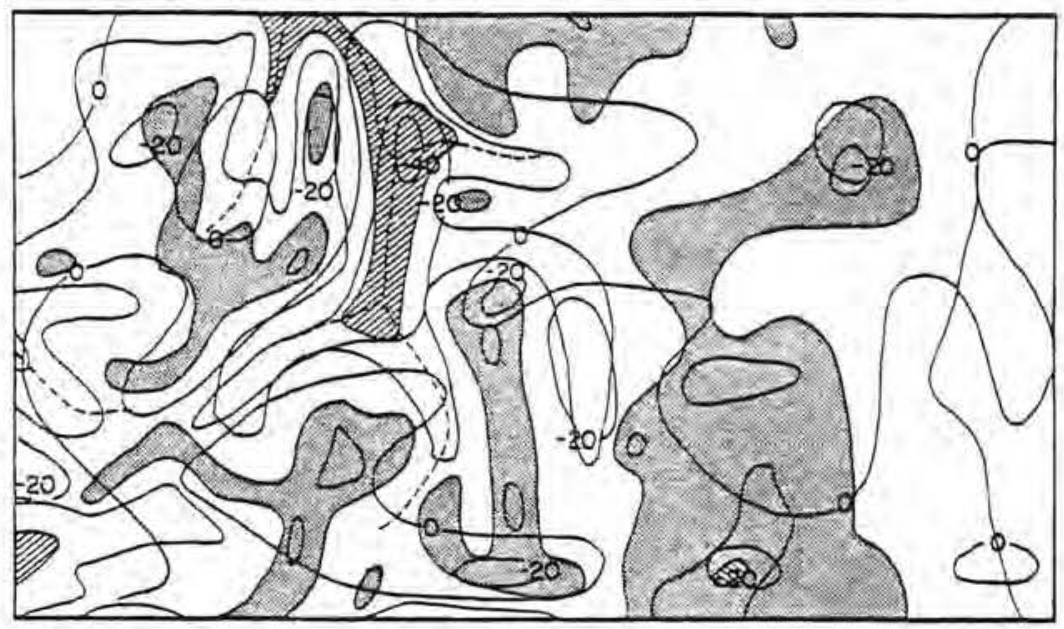

Figure 7.-Vertical component of vorticity for 1600 PST. August 8, 1963. (Units are $10^{-5} \mathrm{sec}^{-1}$ ) Anticycionic vorticity is on ridgetops and cycionic vorticity is in valieys.

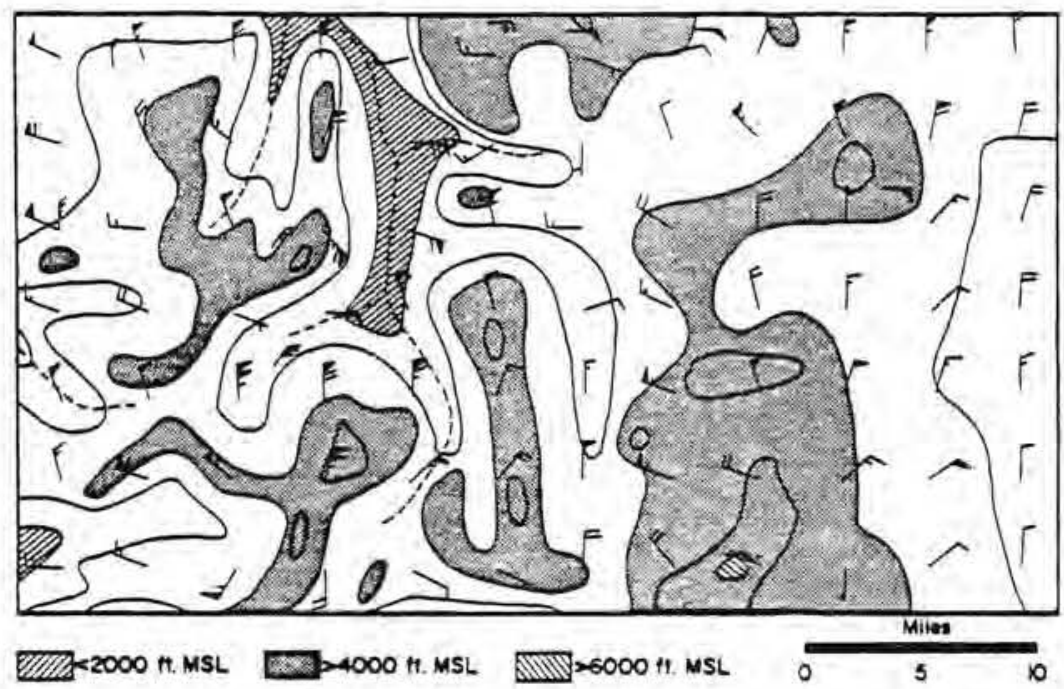

Figure 8.-Calculated and observed wind fieid for 1600 PST, August 8, 1963 . Arrows indicate direction of wind. Speeds are given by sum of barbs and pennants on arrow tails. Pennants indicate $5 \mathrm{~m} \mathrm{sec}^{-1}$; a full barb. $2 \mathrm{~m} \mathrm{sec}^{-1}$ a half barb. $1 \mathrm{~m}$ sec ${ }^{-1}$ Calculated wind field is in red, observed wind field is in black, 
the topographic and temperature field must be represented in complete detail for the model to provide realistic and verifiable results.

\section{Topography, Climatology, and Winds}

Two parameters are required if the model is to define the topographic influence on throughflow. The first of these, the depth of the rigid lid at the top of the slab, should normally be expected to be 1,500 to $2,000 \mathrm{~m}$ above the spatially smoothed terrain. The second parameter, the thickness of the shallow layer, refines the first estimate of terrain influences. It is 1.25 times the difference between the smoothed terrain and the actual ter. rain. These guidelines for the relationship between throughflow and terrain were determined from a large number of test cases where the coefficients were varied systematically. The final choice was a judgment of which relationships produced the most acceptable resultant wind field.

Grid point spacing is also related to topographic variations. In rugged terrain, a closely spaced grid is required to resolve the local winds, while in gently rolling terrain, a low-resolution grid will give acceptable wind fields. Guidelines for selecting appropriate grid spacing were developed from procedures similar to those used in evaluating layer thicknesses.

Numerous analyses were carried out with the same data sets so that performance could be evaluated by overall resultant wind field errors. Grid spacings between 1 and $6 \mathrm{~km}$ were evaluated for maximum terrain differences of 300 to $1,500 \mathrm{~m}$. These analyses indicated that grid spacing should be reduced by $1 \mathrm{~km}$ for every increase in terrain range of $300 \mathrm{~m}$. If the terrain within an area $10,000 \mathrm{~km}$ square varies by $300 \mathrm{~m}$ or less between the highest and lowest spatially averaged elevations, then a $6 . \mathrm{km}$ grid would be appropriate. If, on the other hand, the terrain variation were $1,500 \mathrm{~m}$ a $1-\mathrm{km}$ grid would be appropriate. These spacings must be modified to the smallest grid spacing if the wind field is intended to be used for fine-scale analysis of pollution patterns or fire spread, or to larger spacing if broad geographic coverage is required. Grid spacing of $500 \mathrm{~m}$ is probably the minimum the model will treat properly. Close grid spacing does not permit a 50 - by 50-point grid to include major terrain features. These two nonmodel dependent factors (terrain complexity and computer resources) must be considered in selecting the grid interval The spacings specified here represent maximum spacing-not optimum for any particular application.
The seven cases in this analysis all came from detailed mesometeorological research studies. Such intensive data would not normally be available. Detailed temperature patterns can be estimated from climatological relationships developed for slope temperatures. Super and Grainger (1969) and Hayes (1941) made detailed studies of slope temperatures by aspect and time of dav. Baker (1944), using maximum and minimum temperatures, characterized the slope temperatures for 20 mountainous areas of the Pacific and Rocky Mountain States. These climatological relationships will approximate the temperature distribu. tion when the available observations and topog. raphy are used to fine tune the slope functions.

\section{Using the Model for Planning-A Case Study}

Consider a case where engineers and architects are designing a new plant at a mine mouth. The mineral extraction process will create a large volume of tailings over a period of years. These tailings could be hot. In the design study the questions are asked: (1) What will be the effect of changing the terrain in the immediate area by filling the gulches and small valleys with tailings on the downwind dispersal of stack contaminants?, and (2) What will be the effect on downwind dispersal if the tailings are approximately $70^{\circ} \mathrm{C}$ warmer than the surrounding areas?

The wind model may be used to estimate probable consequences of the mining activity on pollution dispersal patterns. To illustrate this use, we assumed a light wind of $0.45 \mathrm{~m}$ per second (1 mile per hour) from the southwest; temperature distribution was given by climatology for nighttime conditions. Wind patterns before construction showed well-organized drainage flow into and down the valleys and gulches (fig. 9).

Two cases of land change were simulated to anticipate influence on the local wind patterns. In the first case, the valleys and gulches were filled with heated tailings. Changes in the flow were calculated near the modified landform. Heated areas increased the windspeeds and changed the directions to a uniform south-southwest flow down the major gulch (fig. 10). Airflow in the large river drainage to the north was turned and flowed up canyon. The second case considered was that the same gullies and canyons were filled with tailings, but the tailings were not heated. This modification also increased and organized the drainage flow (fig. i1). Winds were from the southwest over the tailings for this case. How- 


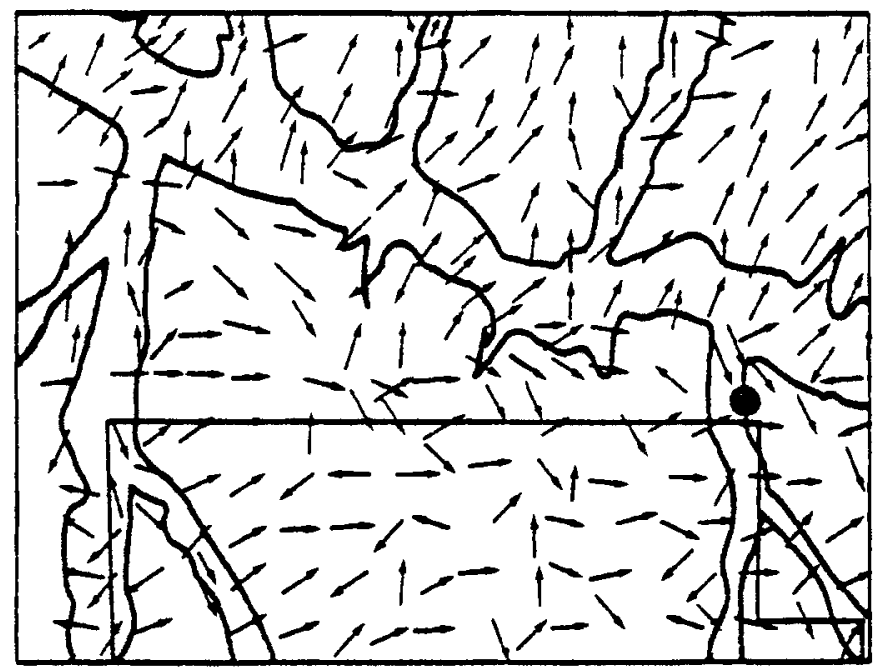

Figure 9.-Local wind pattern before construction of a hypothetical plant and mine tailings.

Figure 10.-Simulated wind field for a case with neated tailings.

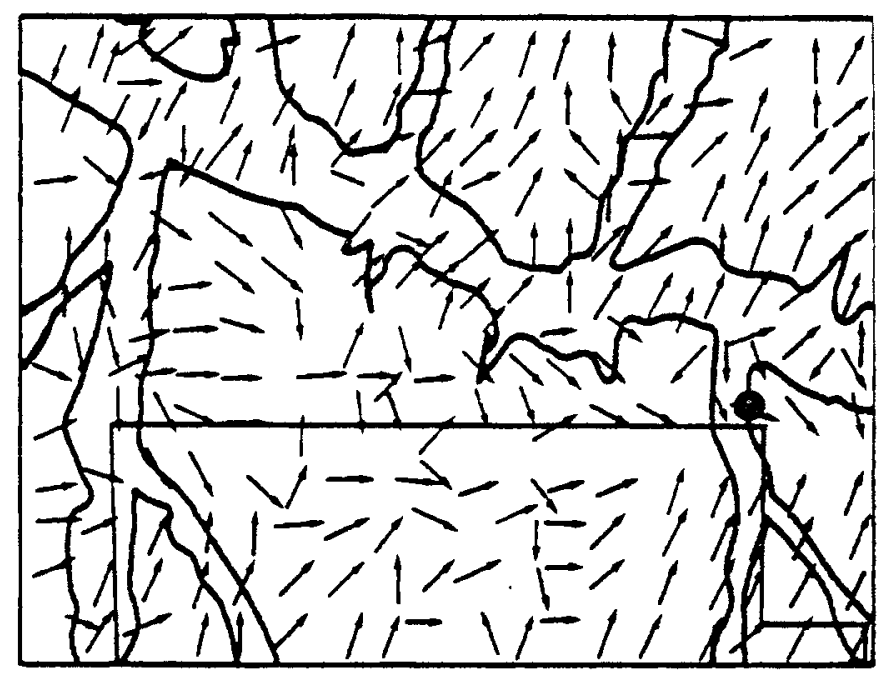

indicates plant and mine site.

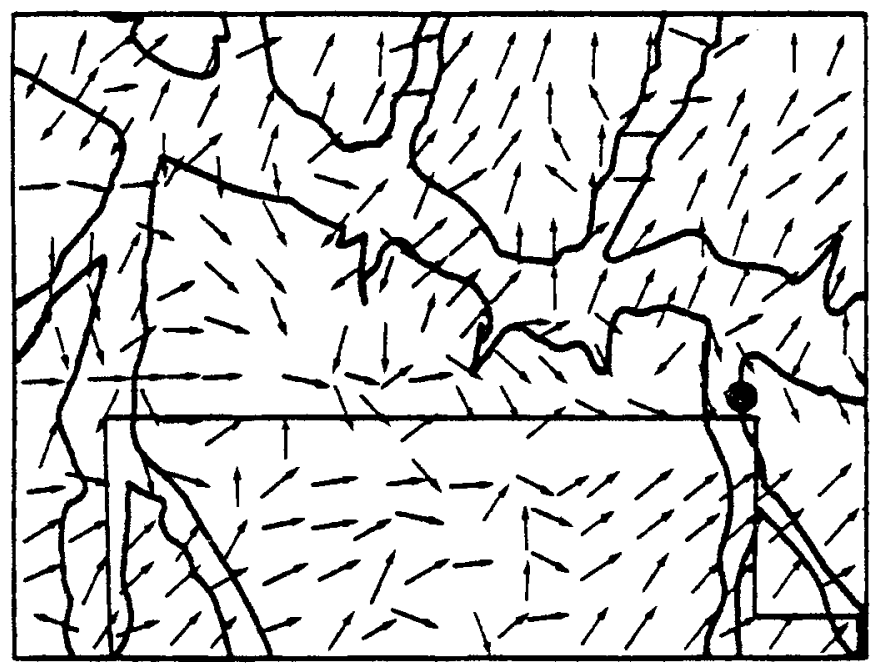

Figure 11. - Simulated wind field for a case with cold tailings. 
$A-17$

ever, air did not flow up the canyon from the major river drainage. The two cases predicted increases in windspeed of as much as $1 \mathrm{~m}$ per second and direction changes of 180 degrees near the planned mine activities.

\section{Summary and Conclusions}

Local thermally driven flows such as moun. tain and valley winds are simulated reasonably well. Interactions of these local flows, and influence of the larger scale potential flow on these local winds, are also included in the resuitant flow. These thermally driven flows are determined properly primarily because the thermal forcing processes are stationary and locked to the terrain features. Also, these thermal features are in a quasi-steady state for several hours. Thus, neglect of the advective process is permissible, and the impulse integration to steady state approximates the flow field.

Thermal modification of potential flow across the terrain features dominates the driving forces. Potential flow was three times as important as rotational flow.

Error analysis of seven case studies and the application example indicated that the model could be used as an aid in fire and air-quality programs because of the low data requirements and the reduced computer effort required.

The wind model developed here was based on simplifications of the complete set of equations governing atmospheric flow over complex terrain. These simplifications imposed several limitations on the use of the model, both in types of flow that can be depicted and in spatial coverage of the solutions. Moving flow systems and flows which are characterized by intermittency or by separa. tion are explicitly excluded, because (1) advection was eliminated, and (2) the model is intended to depict quasi-steady state processes. Coefficients describing static stability and overall wind throughflow place spatial limits on the model because (1) constant static stability prohibits larger scale baroclinicity, and (2) the single-valued wind vector probibits large-scale flow discontinuity. Also, the neglect of advective processes excludes strong interaction of the mesoscale with larger scales, and does not allow for modification of the downwind environgent.

\section{Literature Cited}

Anderson, Gerald E.

1971. Mesoscale influences on wind fields. J. Appl. Meteorol. 10:377-386.

Baker, Fredrick S.

1944. Mountain climates of the western United States. Ecol. Monogr. 14:223-258.

Cramer, Owen P.

1972. Potential temperature analysis for mountainous terrain. J. Appl. Meteorol. 11:44-50.

Cramer, Owen P., and Robert E. Lynott.

1961. Cross section analysis in the study of windflow over mountainous terrain. Bull. Am. Meteorol. Soc. 42:693-702.

Cramer, Owen P., and Robert E. Lynott.

1970. Mesoscale analysis of a heat wave in western Oregon. J. Appl. Meteorol. 9:740-759.

Fosberg, Michael A.

1967. Numerical analysis of convective motions over a mountain ridge. J. Appl. Meteorol. 6:889-904.

Fosberg, Michael A.

1969. Airflow over a heated coastal mountain. J. Appl. Meteorol. 8:436-442.

Fosberg, Michael A., and Mark J. Schroeder.

1966. Marine air penetration in central California. J. Appl. Meceorol. 5:573-589.

Fosberg, Michael A., Albert Rango, and William E. Marlatt.

1972. Wind computations from the temperature field in an urban area. Proc. Conf. on Urban Environ. and second Conf. on Biometeorol., Oct. 31-Nov. 2, Philadelphia, Pa. p. 5-7.

Hayes, G. Lloyd.

1941. Influence of altitude and aspect on daily variations in factors of forest fire danger. U.S. Dep. Agric., Circ. 591, 37 p.

Priestley, C. H. B.

1959. Turbulent transfer in the lower atmosphere. 130 p. Univ. Chicago Press.

Sellers, William D.

1965. Physical climatology. 272 p. Univ. Chicago Press.

Super, Arlin B., and Anthony C. Grainger.

1969. Studies of the diurnal wind and temperature regimes above mountain slopes. Dep. Earth Resour., Mont. State Univ., Bozeman. Mont. Final rep. to U.S. Dep. Comm., Environ. Sci. Serv. Admin. 77 p.

Sutton, O. G.

1953. Micrometeorology. 333 p. McGraw-Hill, N.Y. 


\section{APPENDIX}

\section{List of Symbols}

Symbol

$$
\begin{aligned}
& \mathrm{f}_{\mathrm{p}} \\
& \mathrm{g} \\
& \mathrm{H} \\
& \mathrm{h} \\
& \mathrm{k} \\
& \mathrm{k} \\
& \tilde{\mathrm{K}} \\
& \mathrm{p} \\
& \mathrm{p}_{\mathrm{o}}
\end{aligned}
$$

$\mathrm{R}$

$\mathrm{T}$

$\mathrm{t}$

$u, \bar{u}, u_{0}$

$\mathrm{u}_{*}$

$\stackrel{v}{v}, \bar{v}, v_{0}$

$v *$

$w_{\mathbf{w}}$

$\mathrm{x}$

$\mathrm{y}$

$z$

$z_{0}$

$\delta, \delta$

$\xi, \vec{\zeta}$

$\theta$

$\rho$

$\phi$

$\phi$

$\nabla$

$\nabla^{2}$

$\Delta$

$\Delta \mathrm{t}_{\zeta}$

$\Delta t_{\delta}^{\zeta}$
Definition

Specific heat at constant pressure

Coriolis parameter

Acceleration of gravity

Thickness of rigid lid layer

Thickness of second approximation to flow von Karman constant

Unit vector in vertical direction

Turbulent viscosity

Pressure

Non-dimensional pressure $\left(\frac{p}{p_{0}}\right)^{\frac{R}{c_{p}}}$

Ideal gas content

Temperature

Time

$W$ indspeed in west to east direction

Friction velocity in west-east direction

Horizontal velocity vector

Windspeed in south-north direction

Friction velocity in south-north direction

Vertical windspeed

Horizontal coordinate in west east direction

Horizontal coordinate in south-north direction

Vertical coordinate

Surface roughness length

Horizontal divergence

Vertical component of vorticity

Potential temperature $\theta=\frac{T}{P}$.

Density of air

Static stability $\sigma=\frac{1}{\theta} \frac{\partial \theta}{\partial z}$

Velocity potential

Stream function

Horizontal vector gradient operator

Horizontal laplacian operator

Horizontal grid spacing

Impulse time for vorticity

Impulse time for divergence

\section{Units}

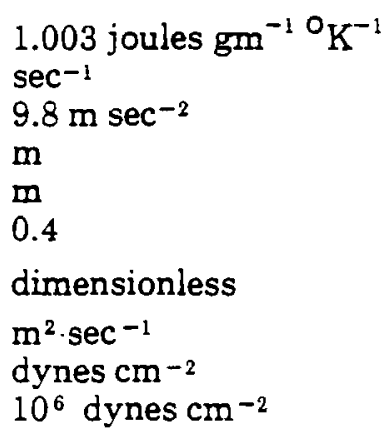

dimensionless

$\mathrm{m}^{2} \cdot \mathrm{sec}^{-1}$

dynes $\mathrm{cm}^{-2}$

$10^{6}$ dynes $\mathrm{cm}^{-2}$

0.287 joules $\mathrm{gm}^{-1} 0_{\mathrm{K}}^{-1}$

Deg K

$\mathrm{Sec}$

$\mathrm{m} \mathrm{sec}-1$

$\mathrm{m} \mathrm{sec}^{-1}$

$\mathrm{m} \mathrm{sec}-1$

m sec-1

$m \sec ^{-1}$

$\mathrm{m} \mathrm{sec}^{-1}$

$\mathrm{m}$

m

m

m

sec-1

$\sec ^{-1}$

Deg $\mathrm{K}$

$\mathrm{kg} \mathrm{m}^{-3}$

$\mathrm{m}^{-1}$

$\mathrm{m}^{2} \mathrm{sec}^{-1}$

$\mathrm{m}^{2} \mathrm{sec}^{-1}$

m-1

$\mathrm{m}^{-2}$

$\mathrm{m}$

sec

sec 
APPENDIX $B$

Topographic Analysis System (TOPAS) 
TOPOGRAPHIC ANALYSIS SYSTEM USER'S GUIDE SECOND EDITION
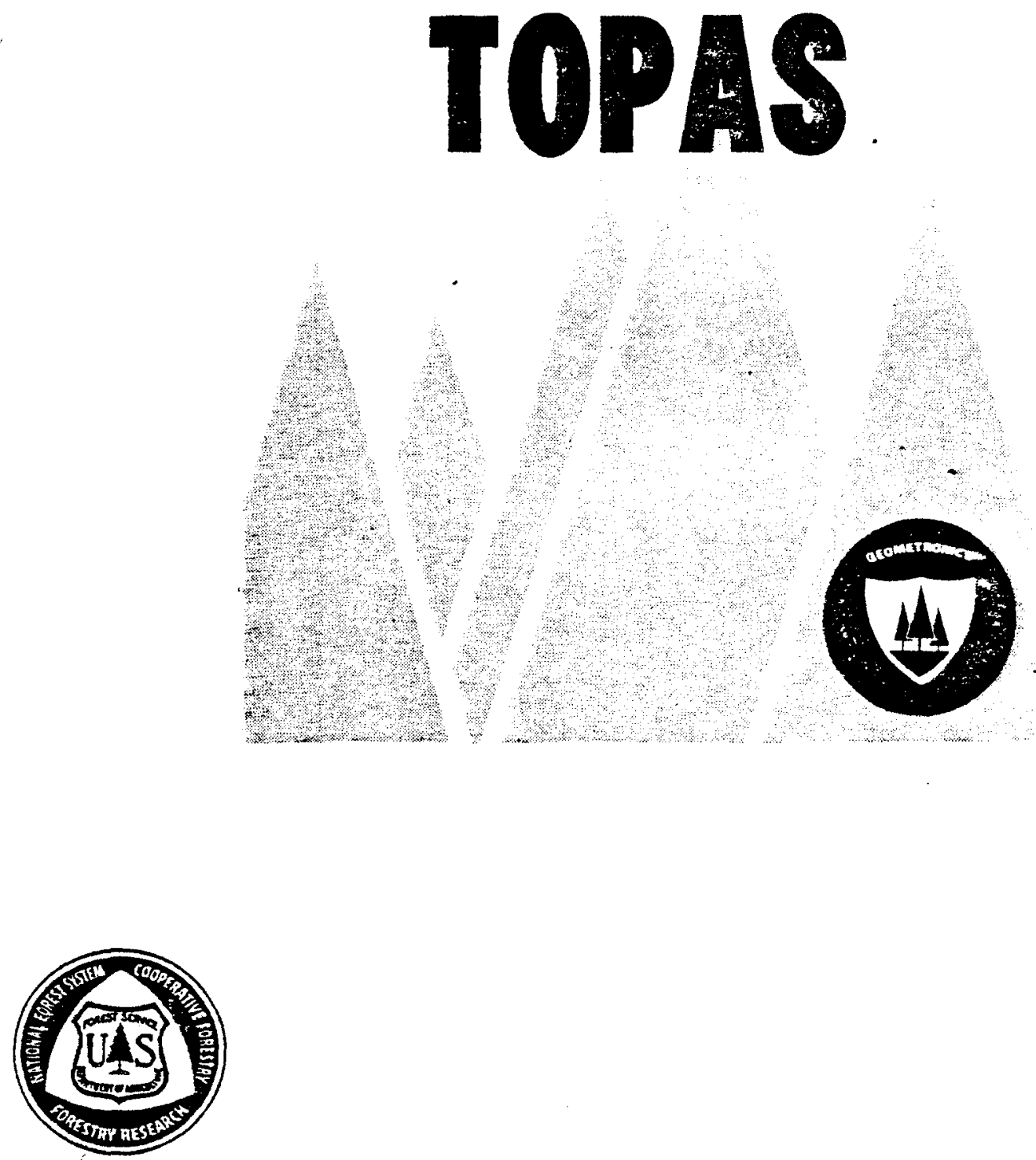

Forest Service U.S. Department of Agriculture Washington, D.C. 20250 


\section{PREFACE}

The TOPAS system of programs and associated User's Guide has been in use for over a year. However, just as any computer program needs "debugging" to function properly. so does a user's instruction of this scope. This second edition, the TOPAS User's Guide, has been published to clarify, correct, update, and add features which will make the system easier to use and understand.

This new edition has been professionally edited to standardize chapter format and insure consistency of terminology throughout the text. All programs, segments and functions which were included in the original manual have been edited and are included in this edition with the exception of the program VIEWIT. This program has been renamed SEEN to distinguish it from PSW's VIEWIT program. The program is otherwise unchanged.

Several additions to the original TOPAS system have been incorporated into this edition. They are programs ITOPAS, ARRAY, COPYNCIC, and FLIGHT. A section is devoted to each of these programs. In addition, function CAT has been included in the MANUv segment of DTIS.

A glossary of commonly used acronyms, abbreviations and technical terms has also been added for easy user reference. 



\section{TABLE OF CONTENTS}

Chapter

Page

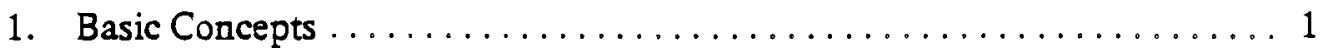

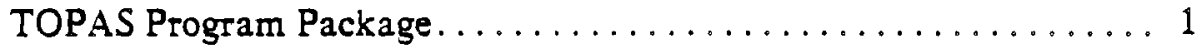

Output Products from TOPAS . ......................

Generation of Digital Terrain Data ...................

Gridded and Non-Gridded Digital Data ............... 2

Project Data File .............................. 2

2. TOPAS Glossary ............................ 8

II PROCUREMENT OF DMH DATA TAPES $\ldots \ldots \ldots \ldots \ldots \ldots \ldots \ldots$

Description of DMA Tapes ......................... 15

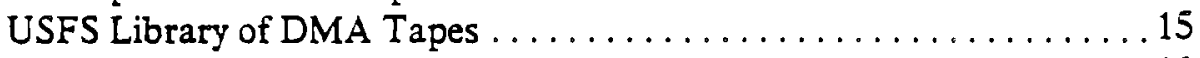

NCIC Digitized Terrain Data Computer Tapes $\ldots \ldots \ldots \ldots \ldots \ldots$

How to Order DMA Tapes . . . . . . . . . . . . . . . . . 16

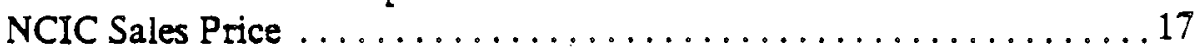

How to Create a Permanent Data File for a New DMA Tape......... 17

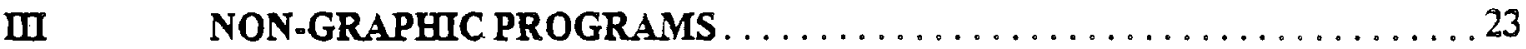

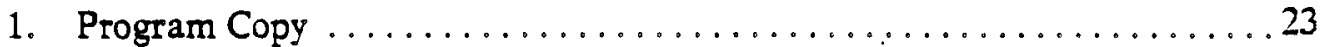

Create a Data File Tape with Program Copy ................ 23

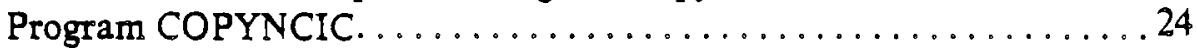

To Make a New File Permanent ................... 25

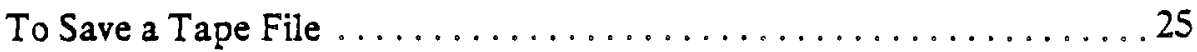

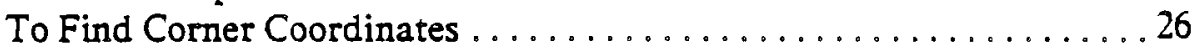

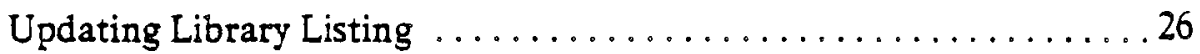

Creating a Temporary Data File for a $7.1 / 2$ or

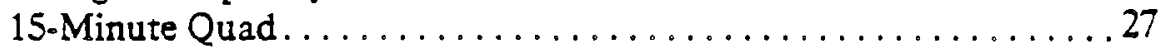

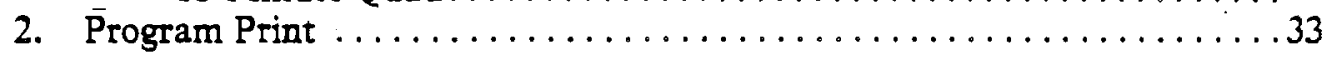

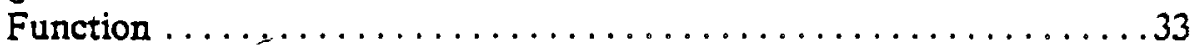

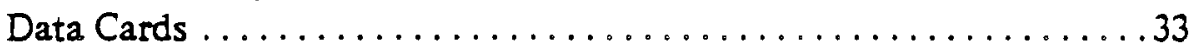

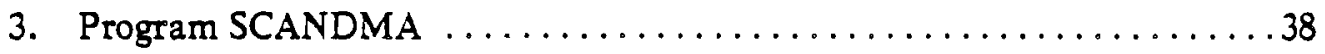

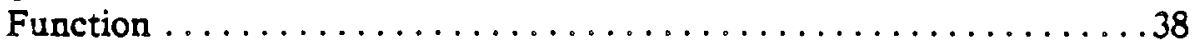

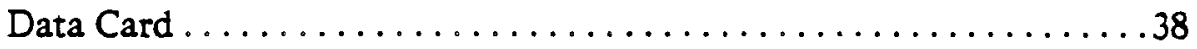

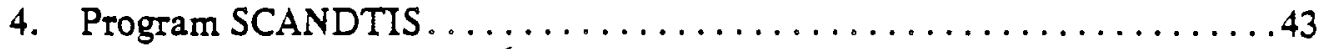

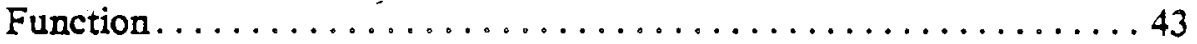

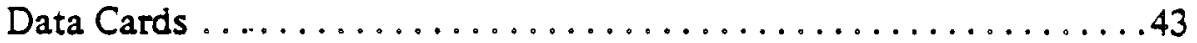

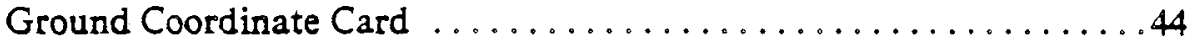

Map Coordinates Card .......................44

IV DIGITAL TERRAIN MODEL PROGRAMS $\ldots \ldots \ldots \ldots \ldots \ldots \ldots \ldots$

1. Digital Terrain Information System. . . . . . . . . . . . . . . . . . . . 49

Program Segments in DTIS . . . . . . . . . . . . . . . . 49

Segment Call Card .............................. 
TABLE OF CONTENTS CONT.

Chapter

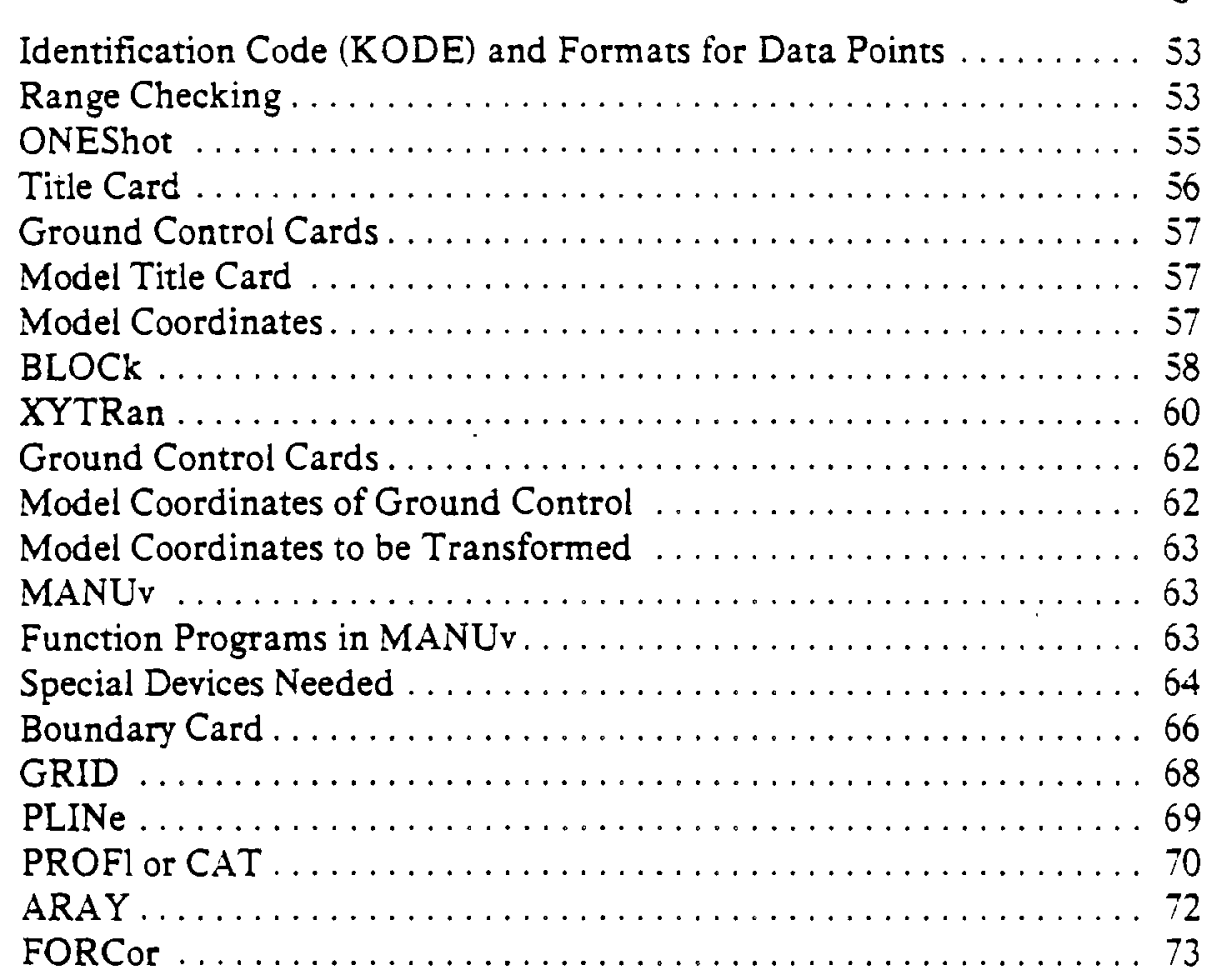

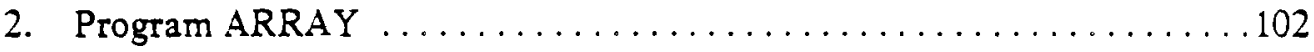

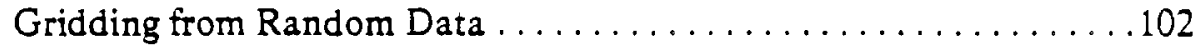

3. Program BLOCKBACK ............................. 104

4. Gridding of Irregular Terrain Data with Program FSGPCP . . . . . . . . 106

SLOPE AND ASPECT PROGRAM (SLOPE)

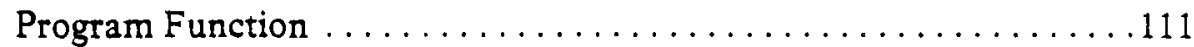

Data Card Formats ............................

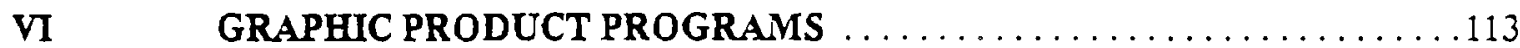

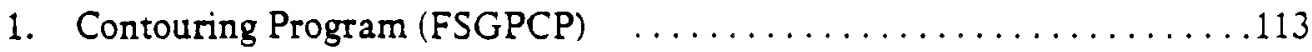

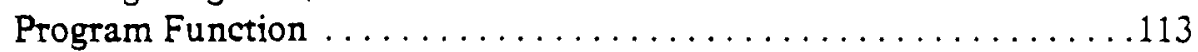

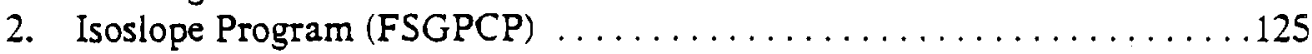

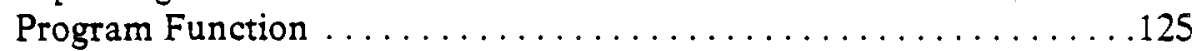

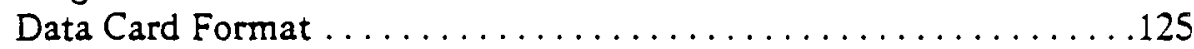

Command Card NWPN . . . . . . . . . . . . . . . . . . 125

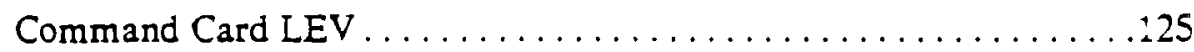

Input Deck for Plotting Isoslope Lines. . . . . . . . . . . . . . . 127

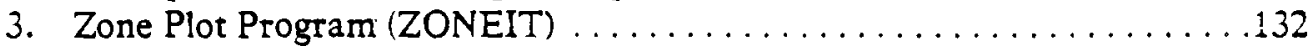

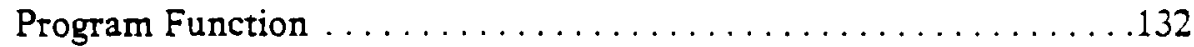

Data Card Furmat . . . . . . . . . . . . . . . . . . . . . 132 
TABLE OF CONTENTS CONT.

Chapter

Page

4. View Area Program $($ SEEN) . . . . . . . . . . . . . . . . . . . 141

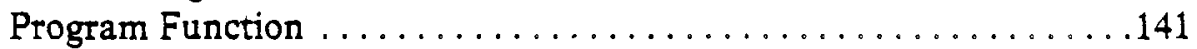

Data Card Format . . . . . . . . . . . . . . . . . . . . 141

Parameter Card Format .......................... 142

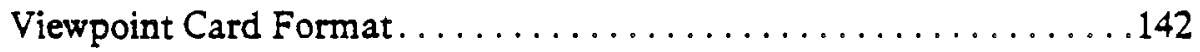

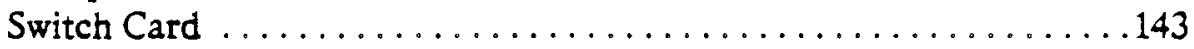

Input Deck Assembly $\ldots \ldots \ldots \ldots \ldots \ldots \ldots \ldots \ldots \ldots \ldots \ldots \ldots \ldots$

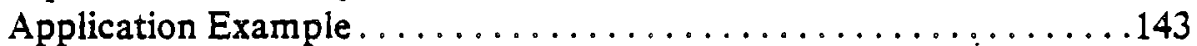

5. Perspective Program (THREED or PREP3D) $\ldots \ldots \ldots \ldots \ldots \ldots \ldots . \ldots 148$

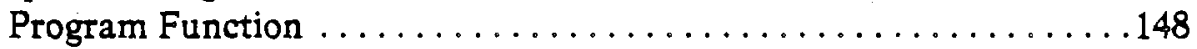

Data Card Format ............................ 148

Input Deck for Drawing Perspective with Program

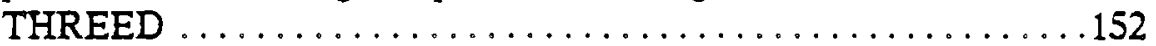

Input Deck for Drawing Perspective with Program

PREP3D . . . . . . . . . . . . . . . . . . . . . . . . . . 152

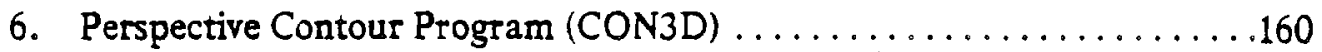

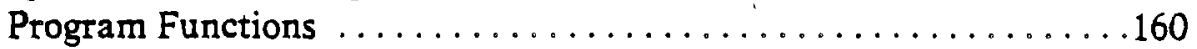

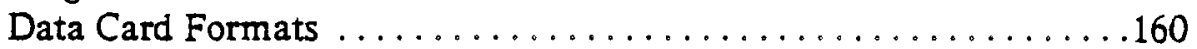

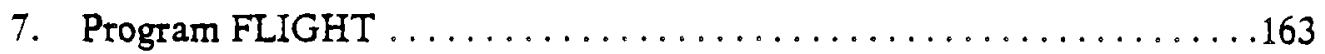

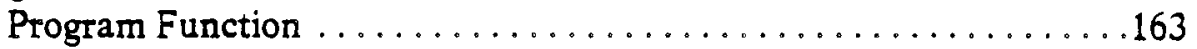

Data Card Format . . . . . . . . . . . . . . . . . . . . 163

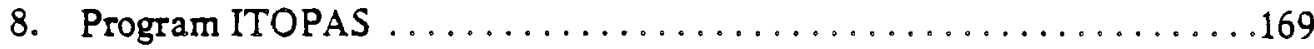

APPENDLX A . . . . . . . . . . . . . . . . . . . . . . . 183

Access to TOPAS through USFS Univac 1108 Computer . . . . . . . . 183

Location of TOPAS Computer Program Package. ..............183

Job Control Cards . . . . . . . . . . . . . . . . . . . . . . . . . . . . 183

Job Routing . . . . . . . . . . . . . . . . . . . . . . . . 183

Procedure for Using a Teletype Demand Terminal ............184

Brief Description of UNIVAC @ ED Processor .................185

APPENDIX B . . . . . . . . . . . . . . . . . . . . . . . . . . . . 193

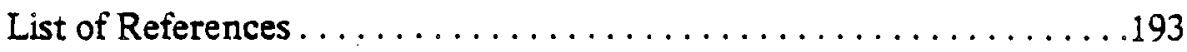




\section{CHAPTER I}

INTRODUCTION

\section{SECTION 1. BASIC CONCEPTS}

\section{TOPAS Program Package}

TOPAS is the acronym for TOPographic Analysis System. It is the code name given to a package of computer programs that have been developed for the collection, manipulation, and analysis of digital terrain data.

Exhibit 1.1 illustrates the basic concept of TOPAS and all the computer programs within the system. Exhibit 1.2 lists these programs in an alphabetical order, and gives brief descriptions of the major functions of each program.

The sub-set of programs, which are used in the generation of digital terrain data directly from existing topographic maps or from airphotos, have been code-named DTIS (DIGITAL Terrain Information System). This system of programs may be used to produce scan profiles for ortho photo production or to generate cross-section elevations for road design analysis.

\section{Output Products from TOPAS}

The following types of output products can now be generated with TOPAS:

1. Contour map

2. Isoslope plots

3. Drawing of slope zones

4. Drawing of aspect zones

5. Perspective view

6. Delineation of visable area from view points

7. Scan profiles for ortho-photo production

8. Cross-section elevations for road design applications

9. Flight planning plot using DMA tapes

10. Perspective contour view

11. Skyline logging ground profile data

12. Slope or aspect zone data for input to program GELO

\section{Generation of Digital Terrain Data}

There are three basic methods by which digital terrain data of a given area can be obtained. These are:

1. Existing terrain data, such as the digital terrain data tapes that the Defense Mapping Agency (DMA) has generated from the USGS 1:250,000 scale maps.

2. Existing topographic maps, such as the 1:24,000 and 1:62,500 USGS maps. These maps can be easily digitized to provide numerical data of the terrain.

3. Air photos. Conventional photogrammetric mapping procedures can be used to create a three-dimensional optical model of the terrain, which can, in tum, be digitized to provide numerica! data. 


\section{Gridded and Non-Gridded Digital Data}

Depending on the method used in the digitization process, the digital terrain data can be stored in either a gridded or non-gridded (also referred to as random or irregular) pattern. If the data is digitized in a rectangular pattern, as shown in Exhibit 1.3, the grid spacing remains constant, and only the elevation of data points need to be recorded. Thus, the digital terrain data tape from a gridded digitization procedure will consist of a string of elevation data. Knowing the grid spacing and the number of rows and columns in the grid, the exacr $x$ and $y$ coordinates of each elevation point can be easily derived.

An irregular digitization procedure, such as that shown in Exhibit 1.4. will result in an irregular (random or non-gridded) distribution of data points. In order to define the spatial positions of each data point, the $x, y$, and $z$ coordinates of each point must be recorded in the data file.

The non-gridded approach is most commonly used in the manual digitization process because it reduces the number of data points that need to be recorded. For example, digitization of a 10" x 10" area of a 1:24,000 map sheet in a gridded pattern with a grid size of .01 inch $\times .01$ inch, will result in about 1,000,000 data points. However, by following a non-gridded approach and digitizing only terrain points which show sharp change in topography, the number of data points may be reduced by a factor of 100 or higher.

On the other hand, gridded data are more convenient in computation. Hence. most application programs either require gridded data as input or have the capability of converting non-gridded data into gridded format before entering the computation phase. Programs MANUy, FSGPCP, or ARRAY in the TOPAS package may be used to perform the conversion.

\section{Project Data File}

A digital terrain data tape or file usually contains digital data for a large area. For example, a DMA digital terrain data tape stores approximately 2,000,000 data points for one-half of a 1:250,000 sheet. However, none of the application programs within TOPAS can accept a data file containing more than 14,641 data points. Therefore, it is necessary to extract a subset of data from an existing data file. This subset of data will be referred to as project data file in this manual. 
TOPOGRAPHIC ANALYSIS SYSIEM (IOPAS)

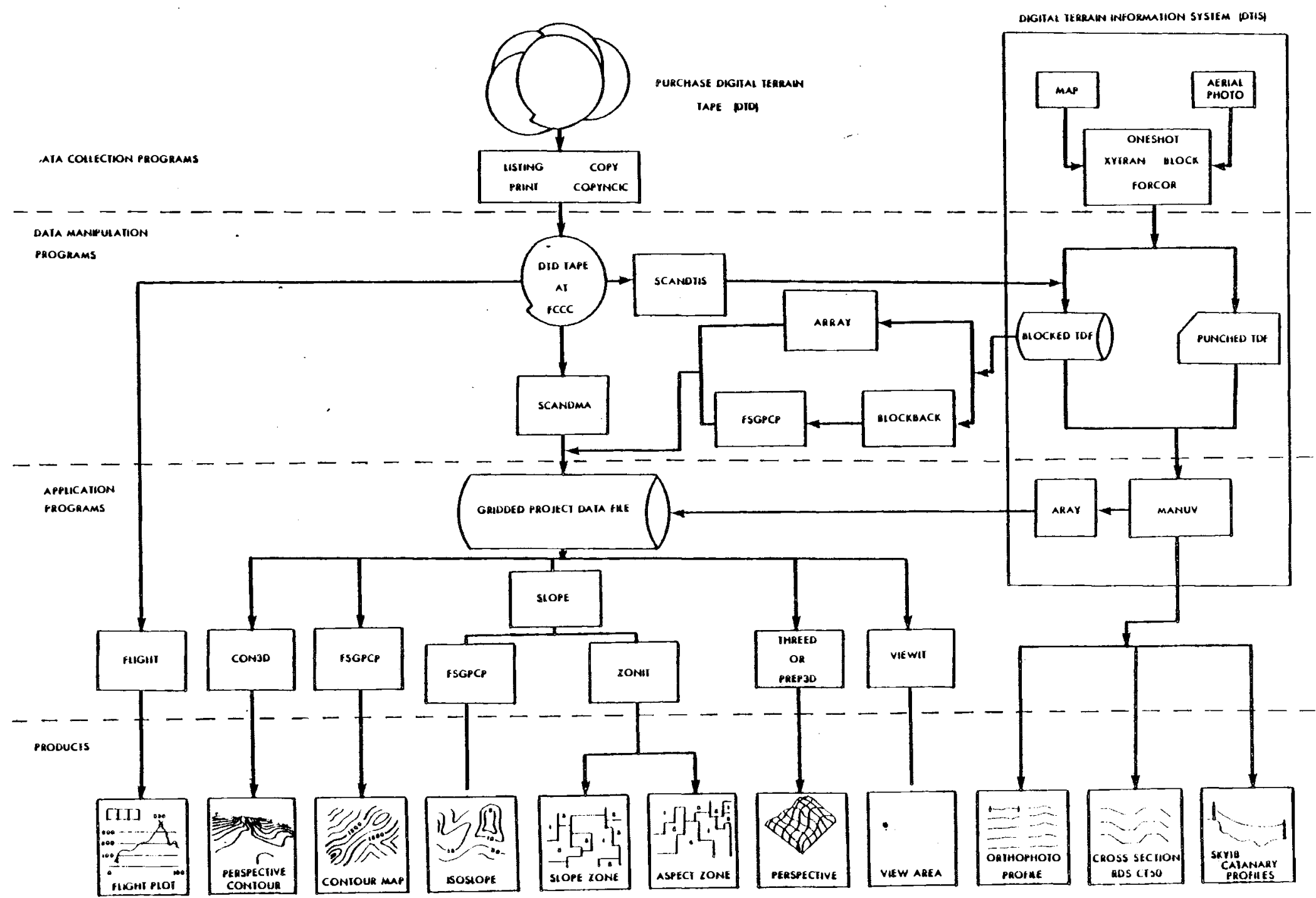


Programs

1. ARAY

2. BLOCK

3. BLOCK. BACK

4. CON3D

5. COPY

6. FLIGHT

7. FORCOR

8. FSGPCP

\section{Functions}

Converts MANUV generated grid data to Calcomp GPCP ARAY formats.

Collect up to 500 points of UNITrecord data or RESOurce data into a single record with Min-Max, North-East for the included data.

Reformat Blocked Terrain Data File to card image.

Produce a perspective contour or contour plot from Digital Terrain Data. Copy a DMA tape to a USFS tape to establish a permanent data file. Produce a Profile and Flight Planned Data from Digital Terrain Data Tape.

Convert State Plane coordinates to or from Geodetic Positions or from one State-zone to another.

1. Convert random data into gridded data.

2. Plot contour map.

3. Plot isoslope lines.

9. LISTING Produces a listing of all DTD tapes available to FCCC.

10. MANUV Operate on the Digital Terrain Data base. (see MANUV functions)

11. ONESHOT Transform spatial coordinates of a controlled model to ground. May also be called with a 'SPAT' Segment call card.

12. PREP3D Draw perspective view using every second data point in a gridded data file.

13. PRINT List the $x$ and $y$ coordinates of the corners of all the $71 / 2$-minute quads contained in a DMA tape.

14. SCANDMA Scan a DMA tape and extract the elevation data within some local area to build a project data file.

15. SCANDTIS Produce a random file formatted for use in DTIS from a DMA type terrain tape.

16. SEEN Identifies all data points that are directly visible from a given viewpoint. The visible area is denoted by a Calcomp and/or line-printer plot. 
Programs

Functions

17. SLOPE Computes the slope and aspect at each elevation point, and stores the results in a new data file.

18. THREED Draw a perspective view of the elevation grid which represents the terrain surface.

19. XYTRAN Transform map coordinates to ground.

20. ZONEIT Provides a graphic plot of slope or aspect zones. 


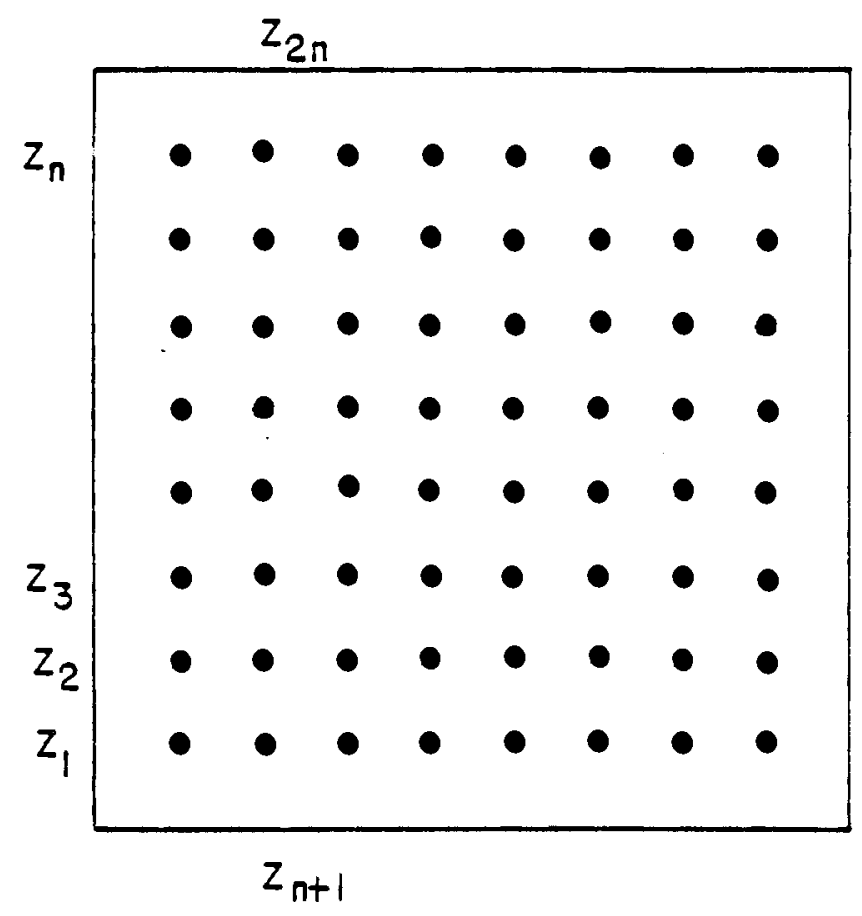

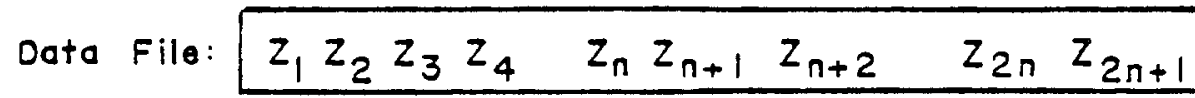




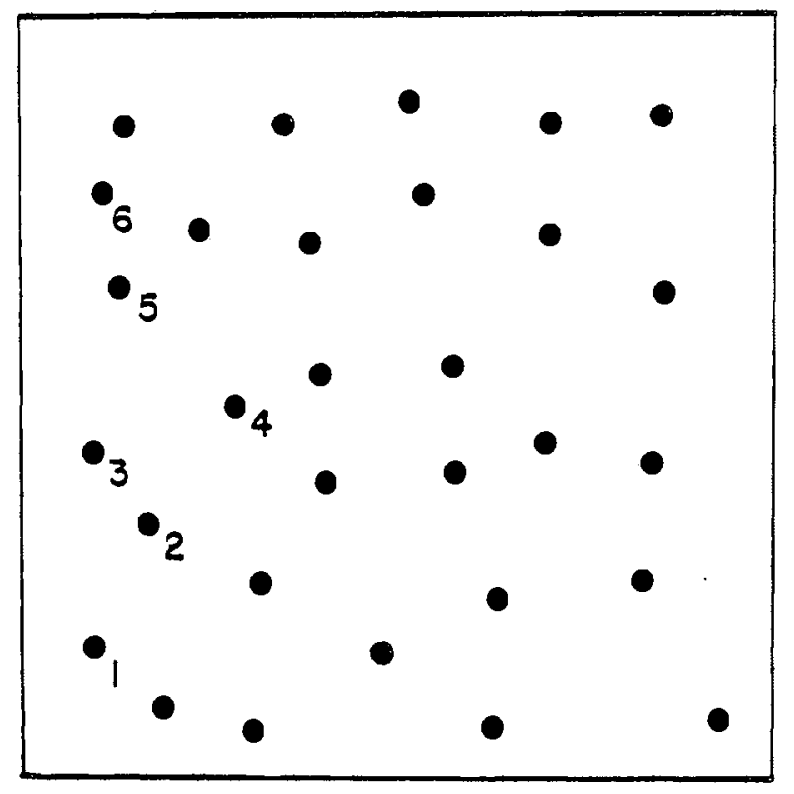

DATA FILE $X_{1} Y_{1} Z_{1} X_{2} Y_{2} Z_{2} X_{3} Y_{3} Z_{3} \cdots \cdots \cdots$ 


\section{SECTION 2. TOPAS GLOSSARY}

ARAY

ARAY block

ARRAY

BLOCk

BLOCk

BLOCKBACK

Blocked TDF

Boxed data

CI

CON3D

COPY

COPYDMA

COPYNCIC

CT50

DE

Defined File

DELDE

DELDN
DTIS segment. Converts MANUv gridded data to Calcomp ARAY block format.

Calcomp GPCP formatted gridded data file.

TOPAS program to generate a gridded data file in an ARAY block from random or pattern data.

DTIS segment. Converts punched TDF to blocked TDF.

DTIS input/output option to generate or enter data from a blocked TDF. TOPAS program to retrieve terrain from a blocked TDF and generate a punched TDF.

Terrain data file where each record in the file contains up to 500 positions with the area covered by these positions described (Min-Max, North-East).

MANUv function STOF1 collects terrain data into regular areas. This method (boxing) generates a "boxed data" file.

Abbreviation, Contour Interval

TOPAS program to generate a perspective contour plot.

TOPAS program to create a copy of a DMA Tape.

Same as copy.

TOPAS program to create a copy of a NCIC terrain data tape. The result of this copy procedure is in the DMA tape format.

Forest Service Road Design System Card type used to input road crosssection information.

MANUv segment variable. Describes the east-west size of the work area.

Mass storage file which can be accessed either randomly or sequentially.

MANUv segment variable. Describes the east distance to the work area from the Project southwest corner.

MANUv segment variable. Describes the north distance to the work area from the Project southwest corner. 
DIF

DMA

DMA Tapes

DTD

DTIS

EOF

FCCC

FILL

FLIGHT

FORCor

FORCor

FSGPCP
MANUv segment variable. Describes the size of "boxes" when collecting and operating on boxed data.

Abbreviation, Defense Mapping Agency.

Elevation data collected by the DMA from USGS 1:250,000 Maps at .01 inch interval. These tapes, or versions of them, are available to the government and to the public. They are adequate for area planning, but generally not accurate enough for use in planning on smail areas (sites).

Abbreviation, Digital Terrain Data. A collection of positional information $(x, y, z)$ dense enough to describe the terrain covered by them.

A TOPAS Program, Digital Terrain Information System. This program is a group of functions for transforming spatial terrain data from stereo model or map coordinates to ground positions, and, to grid, profile, and/or reformat the data for use by other programs in the TOPAS system.

Abbreviation, End of File.

Abbreviation, Fort Collins Computer Center.

A MANUv function which creates the Work Area Data File from the Project Data File.

A TOPAS program which generates ground profiles and determines flying elevation and degradation of side lap at maximum terrain elevation. It generates a graphic of the ground profile. The source terrain data for program FLIGHT is a DMA Tape.

A MANUv function which converts either geographic positions to State Plane positions, or vice versa. It can also convert from one State Plane zone to another.

A MANUv input/output option which enables the user to enter control points for function XYTRan in geographic positions. These are converted to State Plane coordinates.

Abbreviation, Forest Service General Purpose Contouring Program. It generates contour plots from Digital Terrain Data (DTD), or from gridded data (ARAY blocks). This program was purchased from Calcomp and was slightly modified for TOPAS. It cannot be copied, given away, or sold by the Forest Service.

Abbreviation, General Purpose Contouring Program. This was purchased by the Forest Service from Calcomp for use only at FCCC. This program cannot be copied, moved, sold, or given away by the Forest Service. 
GRID A MANUv function which interpolates elevations at a regular interval along the profiles generated by ORTHO. (Grids the ORTHO profiles.)

GRID

IBOT

ILEFT

INC

IN80

IRIGHT

IRITE

Isoline

Isoslope

ITOP

ITOPAS

IXSW

IYSW

JCL

KODE
A MANUv Input/Output option which generates grid lines at a specified interval on the Ortho photo overlay generated by MANUv function SETUp.

A variable describing the southern boundary of an area to be retrieved from a DMA tape. (Distance in .01 inch units from bottom of Quad.)

A variable describing the western boundary of an area to be retrieved from a DMA tape. (Distance in .01" units from left edge of Quad.)

A variable, Increment of collection of data from a DMA tape. For example, when INC $=2$, alternate elevations are collected from alternate columns.

MANUv Input/Output option which enables user to input control points (80 series points) in Standard SPATL format, while DTD is entered from blocked TDF.

A variable, eastern boundary of area to be retrieved from a DMA tape (see ILEFT).

A variable, same as IRIGHT.

A line joining points of equal value. For example, a contour is an isoline joining points of equal elevation.

A line joining points of equal slope.

A variable, northern boundary of area to be retrieved from a DMA tape (see IBOT).

A TOPAS program to generate and submit for execution, runstreams to produce graphic products where the source data is from DMA tapes.

A variable, map location of the southwest corner $(X)$ of the grid data on a DMA tape.

A variable, map location of the southwest corner $(\mathrm{Y})$ of the grid data on a DMA tape.

Abbreviation, Job Control Language. Used here to refer to the Univac 1100 Series Executive Command Language.

A variable, the first two characters of a DTD position, consisting of KODE, ID, and position. When KODE is from 80 to 89 , the point is a control point. When it is less than 80 , the point is a terrain point, and when it is 90 to 99 , the point is an "EOF" indicator. 
MANUv

NECE

NECN

NEEL

NOBLock

NOPLot

ONEShot

ORTHo

Ortho Photo

Pattern Data

PLINe

PLOT

PREP3D

PRINT

Project Data File

PRORt
DTIS segment. MANUv is the calling routine for the DTD handling functions.

A variable, east value for northeast corner of the Project Data File.

A variable, north value for northeast corner of the Project Data File.

DTIS Input/Output Option. The order of ordinates on the input DTD is North, East, Elevation.

DTIS Input/Output option. DTD generated by ONEShot or XYTRan is to be output as a Punched TDF. Input DTD to MANUv is Punched TDF.

MANUv Input/Output option specified when MANUv function SETUp is not to generate an Ortho photo overlay.

DTIS segment. Transforms controlled stereo model spatial coordinates to ground positions. Outputs DTD in blocked or, optionally, in punched TDF.

A MANUv function which generates north running profiles across the Work Area for use by functions PRORt and/or GRID.

Scale corrected aerial photo image.

A method of collecting non-gridded digital terrain data. Ridges, drains, and major breaks are digitized (collected), then additional data is collected by digitizing along "Form lines" or contour lines.

A MANUv function which generates cross-section data in RDS CT-50 format for input to the RDS program.

MANUv Input/Output option. Causes MANUv option SETUp to generate an Ortho photo overlay.

A TOPAS program which generates and submits for execution, a runstream, to prepare a perspective plot using Calcomp program THREED.

A TOPAS program. From the 4 sets of corner coordinates of a 1 degree quad, the corner coordinates for the $647-1 / 2$ minute quads are generated and graphically printed. This graphic is very useful when running programs SCANDTIS or SCANDMA.

An area of digital terrain selected by MANUv function STOF1 for division into one or more work areas by MANUv function FILL.

A MANUv function which generates a plotted Ortho photo storage plate (profile plate), to drive the Zeiss Ortho photo projector. 
PSW

Punched TDF

Random Data

RDS

RESOurce

SCALE

SCANDMA

SCANDTIS

$\mathrm{SCN}$

SEEN

SETUp

SIZE

SLOPE

SPATL

SPATL Digitizer format
Abbreviation, U. S. Forest Service Pacific Southwest Forest and Range Experiment Station.

Card image terrain data in SPATL packed format (2 positions per card image).

See Pattern Data.

Abbreviation, a program called Forest Service Road Design System.

DTIS Input/Output option. Terrain Data Input into DTIS segments ONEShot or MANUv is in SPATL Resource format.

A variable, generally scale denominator (SCALE $=24000$ for a scale of 1:24000). Except in MANUv function FILL, SCALE is the feet per inch value. (SCALE $=2000$ for a scale of $1: 24000$ ).

A TOPAS program which retrieves from a DMA tape, a selected area, and outputs it in an ARAY Block.

A TOPAS program which retrieves from a DMA tape, a selected area or areas, and outputs it as a blocked TDF.

A variable. In MANUv function FILL, it describes the Ortho photo scan width and/or grid size.

A TOPAS program (formerly TOPAS version of VIEWIT) which determines what terrain can be seen from 1 or a number of viewpoints. It produces a plot of the seen areas at any scale, and optionally produces a printer map of each seen area (this printer map is not to scale).

A MANUv function which produces a table of Zeiss Ortho photo projector setup coordinates, and optionally plots an Ortho photo overiay.

Card type in program FSGPCP which describes the area to be contoured.

A TOPAS program which generates slope and aspect files in the ARAY block format from an elevation ARAY block.

An aerotriangulation program developed by $R$. O. Mahan, USFS, Division of Engineering, to bridge strips and blocks, and perform ground adjustment of terrain data. This program was extensively modified and made into MANUv segment ONEShot. Note: ONEShot is not an aerotriangulation program.

A card image format containing 3 spatial positions per card. 
SPATL Packed format

SPATL Resource format

SPATL Standard format

STOF1

SWCE

SWCN

TDF

TOPAS

THREED

TYPE50

UNITrecord

UPERIN

USGS

VGRID

VIEWIT
A card image format containing 2 ground terrain positions per card.

A tape image format containing 1 ground or spatial position, and 72 characters of resource descriptors per 112 character tape record.

A card image format containing 1 ground position per card.

A MANUv function which generates a Project data file (boxed data) from either punched or blocked TDF.

A variable. Describes the east value for the southwest corner of the Project data file.

A variable. Describes the north value for the southwest corner of the Project data file.

Abbreviation, Terrain Data File. This usually refers to blocked Terrain Data File.

Acronym for TOPographic Analysis System. A collection of programs to produce a variety of graphics from gridded or non-gridded (pattern) terrain data.

TOPAS program. Generates perspective (warped grid) plot from gridded data. This program was purchased from Calcomp, and cannot be copied, moved, sold, or given away by the Forest Service.

MANUv Input/Output option. It causes road design cross-sections to be output in the RDS CTSO format.

DTIS Input/Output option. It indicates that DTD input to one of the DTIS segments will be in 1 of the 3 SPATL card image formats; standard, packed, or digitizer.

A variable. In program ZONEIT, the number of grid Units PER INch at plot scale.

Abbreviation, United States Geological Survey.

MANUv function. Generates gridded data in the printer output format required by PSW's program, VIEWIT.

A PSW program for generating seen areas, slope and aspect. A user's guide is available from PSW. 
Viewpoint

Work Area

Data File

XYTRan

$X Y Z$
A viewing station or location, and an input to program SEEN.

The area, and boxed data associated with it, as described for, and collected by, MANUv function FILL.

A DTIS segment which transforms digitized map coordinates, digitized in any unit of measure, to ground positions.

A DTIS Input/Output option which indicates that the punched DTD input to any of the DTIS segments will be in the order X (East), Y (North). Z (Elevation). Note that the east and north values are in reverse orcer from those of I/O option NEEL. 


\section{CHAPTER I}

\section{PROCUREMENT OF DMA DATA TAPES}

\section{Description of DMA Tapes}

The Defense Mapping Agency (DMA), formerly the U. S. Topographic Command (TOPOCOM), has produced digital tapes of the entire 1:250,000 USGS map series covering the United States. These tapes contain only elevation data. Each 1:250,000 map sheet is subdivided into an East half and a West half, with the data from each half being contained on a separate tape.

The DMA tapes store the elevation data in a rectangular grid pattern. Exhibit 2.1 shows the subdivision of a 1:250,000 sheet into East half and West half, and Exhibit 2.2 shows the grid pattern and the rectangular coordinate system for the two halves. The distance between two adjacent data points, in both the $x$ and the $y$ directions, was 0.01 inch on the 1:250,000 map sheet, which is equivalent to $208-1 / 3 \mathrm{ft}$ on the ground. Thus, each DMA tape contains approximately 2,000,000 data (elevation) points.

Exhibits 2.3 and 2.4 are index maps of the 1:250,000 USGS maps for the western and eastern parts of the United States, respectively.

\section{USFS Library of DMA Tapes}

A library of DMA tapes available in the USFS, is stored in the Fort Collins Computer Center $(F C C C)$. These tapes are kept in permanent storage, and can be directly accessed by any region.

An up-to-date listing of all the DMA tapes at FCCC may be obtained by the execution of the following simple program:

@ RUN

@ ASG,A GEOM*DMATAPES

@ PRT,S GEOM*DMATAPES.LISTING

@ FIN map sheet:

Exhibit 2.5 is an example output listing. It includes the following data for each $1: 250,000$

Data Type

Sheet Name

DMA Sheet Number

East or West Half

FCCC Tape No.
Example

ALTURAS

NK-10-9

WEST

FO2710 
Data Type (cont)

State

Latitude of SW Comer of sheet

Longitude of SW Corner

FCCC Tape Label

$X$-coordinate of SW Corner

$Y$-coordinate of SW Corner

$X$-coordinate of SE Corner

$Y$-coordinate of SE Corner

$X$-coordinate of NW Corner

$\mathrm{Y}$-coordinate of NW Corner

$X$-coordinate of NE Corner

$Y$-coordinate of NE Corner

Latitude of SW Corner

Longitude of SW Corner
Examples (cont)

CALF

41

122

GEOM*K1009W

276

225

1601

218

296

1975

1601

1968

41

122

This listing provides all the information and data that is needed to access any tape stored in the FCCC file.

\section{NCIC Digitized Terrain Data Computer Tapes}

The National Cartographic Information Center (NCIC) is now acquiring the Department of Defense, Defense Mapping Agency's library of digital terrain tapes for sale to civilian users.

These tapes were prepared from the USGS 1:250,000-scale topographic quadrangle map series for the contiguous 48 states. All contour lines falling within a one-degree block of latitude and longitude were first digitized. Next, a matrix of elevations was generated, with one elevation for every .01 inch on the map, or about 200 feet on the ground. As 1:250,000 quads generally cover one degree by two degrees, two one-degree matrices were prepared for each quad; generally, an East and a West portion, each on one 7-track computer tape produced on a UNIVAC 1108 computer system.

NCIC will store eight one-degree quadrangles of latitude-longitude on each 9-track 1600 byte-per-inch tape ( 4 at 800 bytes/inch). For this reason, NCIC uses a flexible pricing approach which results in the cost of each one-degree block decreasing as more blocks are ordered.

\section{How to Order DMA Tapes}

Requisitions for DMA tapes contain the following:

Latitude and longitude of one-degree block or 1:250,000 quad name and number. East or West half.

Example:

Digital Topographic tape as follows:

DMATC Tape, Ashton sheet. NL-12-11-E 1 ea. $\$ 15.00$

DMATC Tape, Ashton sheet, NL-12-11-W 1 ea. $\$ 15.0 n$ 
The Digital Terrain Data tapes are available from the following two sources:

1. Director - DMA-TC

6500 Brooks Lane

Washington, D.C. 20315

Attn: Code 40320

Cost $-\$ 15.00$ for loan of each $1^{\circ}$ by $1^{\circ}$ quad.

2. National Cartographic Information Center

U. S. Geological Survey

National Center, Mailstop 507

Reston, Virginia 22092

Attn: Information Unit/Digital Terrain Data Tapes

NCIC Sales Price

Regular Processing Priority Processing*

$\begin{array}{lll}\text { Per tape } & \$ 15 & \$ 15\end{array}$

$\begin{array}{lll}\text { Per one-degree block } & \$ 6 & \$ 10\end{array}$

Example:

An order for 8 one-degree blocks, regular processing, will cost:

On 1600 bytes-per-inch tape: $\$ 15$ for one tape plus 8 times $\$ 6=\$ 63$

On 800 bytes-per-inch tape: $\$ 30$ for two tapes plus 8 times $\$ 6=\$ 78$

* Priority processing guarantees 2 day turnaround on the computer, but NCIC can make no guarantee on shipping time. Capacity may not be available for priority processing of large orders (over 10 tapes).

If you wish to order tapes from NCIC, identify the one-degree blocks you need by latitude-longitude, or by quad name or number, and East and West half, or simply by marking " $X$ 's" in the areas needed on the enclosed index map. Unless you state a preference between 800 or 1600 bytes-per-inch, we will send you a 1600 byte-per-inch tape. Prepayment is required. Make check or purchase order payable to U. S. Geological Survey. Delivery time should not exceed 15 days.

\section{How to Create a Permanent Data File Tape for a New DMA Tape}

After a new DMA digital data tape has been purchased from the appropriate source, the following steps should be taken to store the new tape in a permanent data file at FCCC:

1. Send the tape to FCCC.

2. Create a data file tape at FCCC by executing program COPY, which copies the original DMA tape into a data file tape. Where tapes were purchased from NCIC, execute program COPYNCIC.

3. Make the data file tape permanent by executing a save tape program. 
4. Determine the $x$ and $y$ coordinates of the SW corner of that half $1: 250.000$ sheet from the output listing of program COPY.

5. Send the necessary information to WO for updating the library listing of DMA tapes.

Transmitting DMA Digital Terrain Data Tapes to Fort Collins Computer Center. The new DMA digital terrain data tapes must be sent to the Fort Collins Computer Center for processing. Send to:

\author{
Mr. Ken Lini \\ Department of Agriculture \\ Fort Collins Computer Center \\ Fort Collins, Colorado 80521
}

A speed-memo should accompany the tape, with the wording similar to the following:

"Enclosed is a 7 track-556 BPI ( 9 track 1600 BPI if from NCIC) unlabeled tape. Please call me concerning reel number at (703) 235-8006."

Thanks,

\title{
Harold Greenlee
}

When the tape librarian has received the tape, you will be notified and given the user reel number (i.e. UR0123), which you can then use to create a permanent data file tape by using program COPY. 


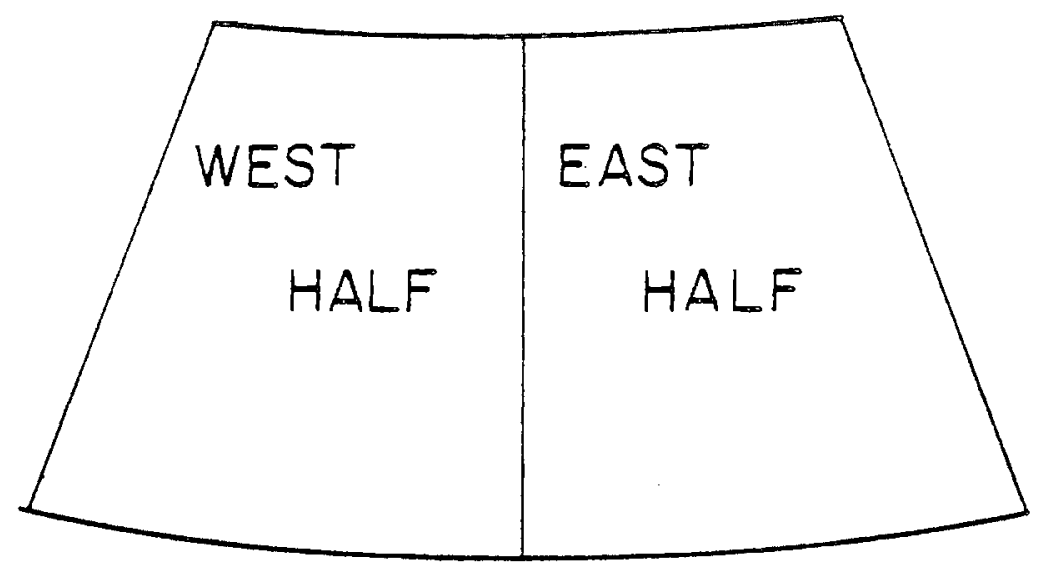

Exhibit 2.1 - Subdivision of a 1:250,000 Sheet
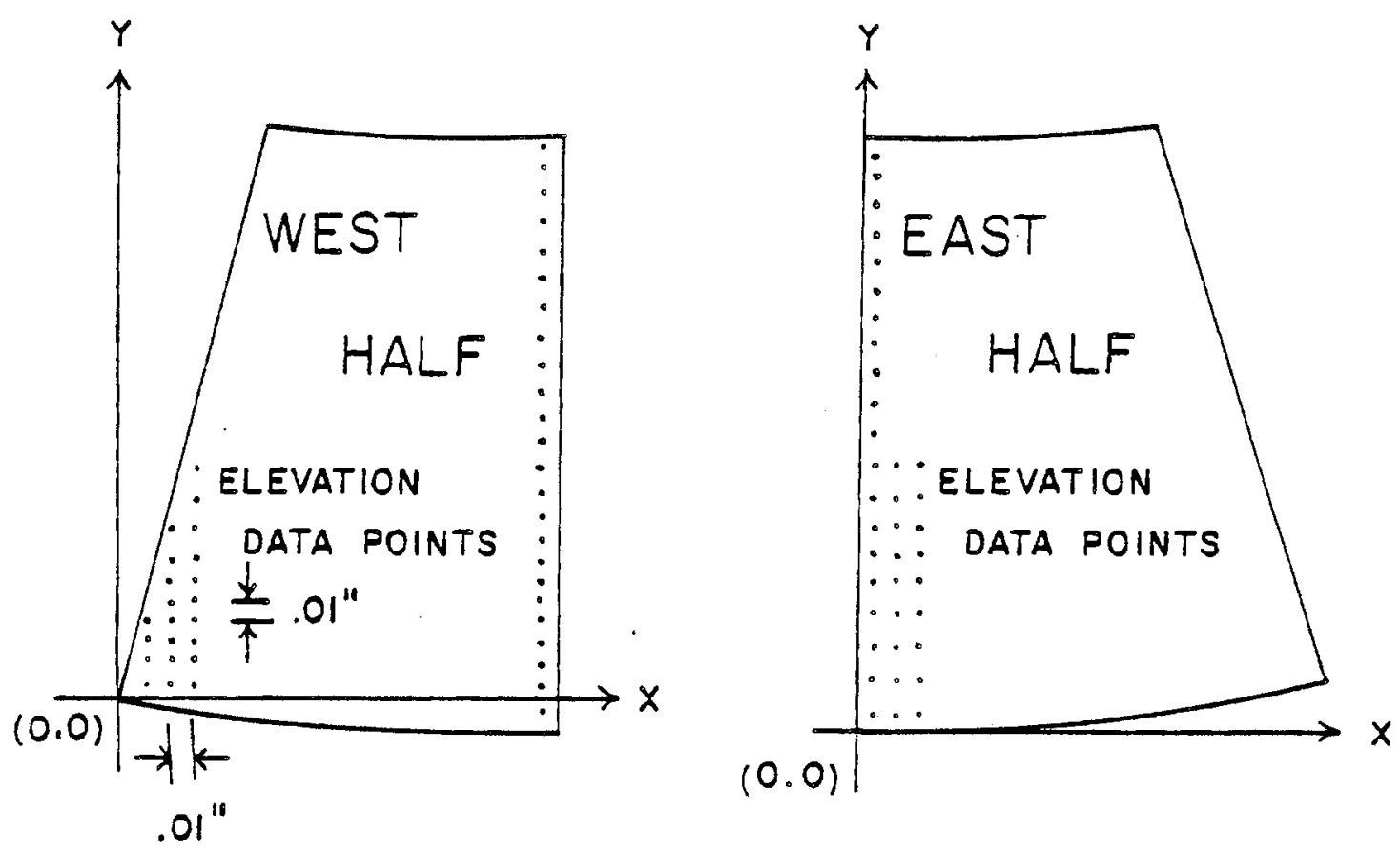

Exhibit 2.2 - Rectangular Coordinate System of Grid Pattern 


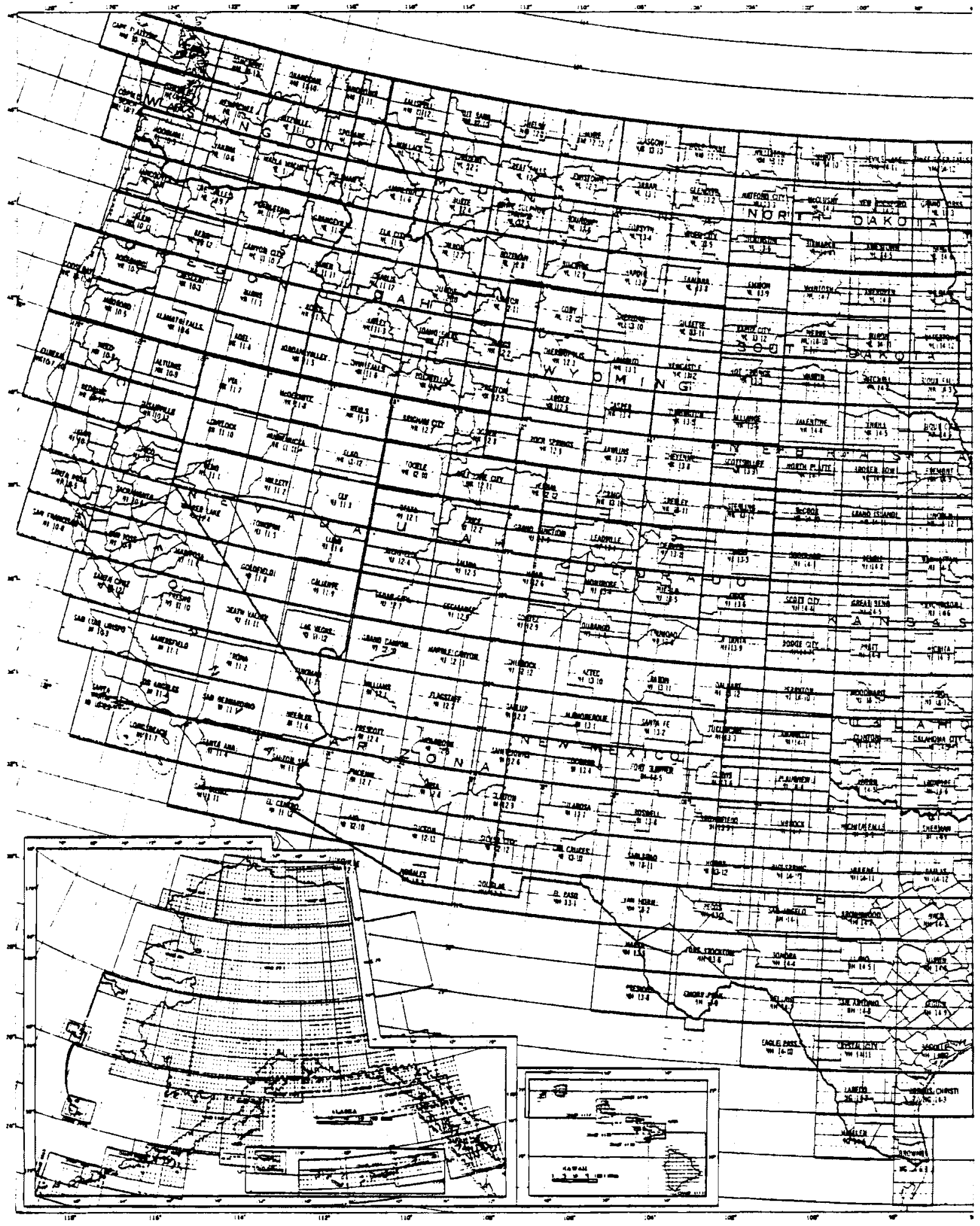

Exhibit 2.3 - Index Map for Western United States 


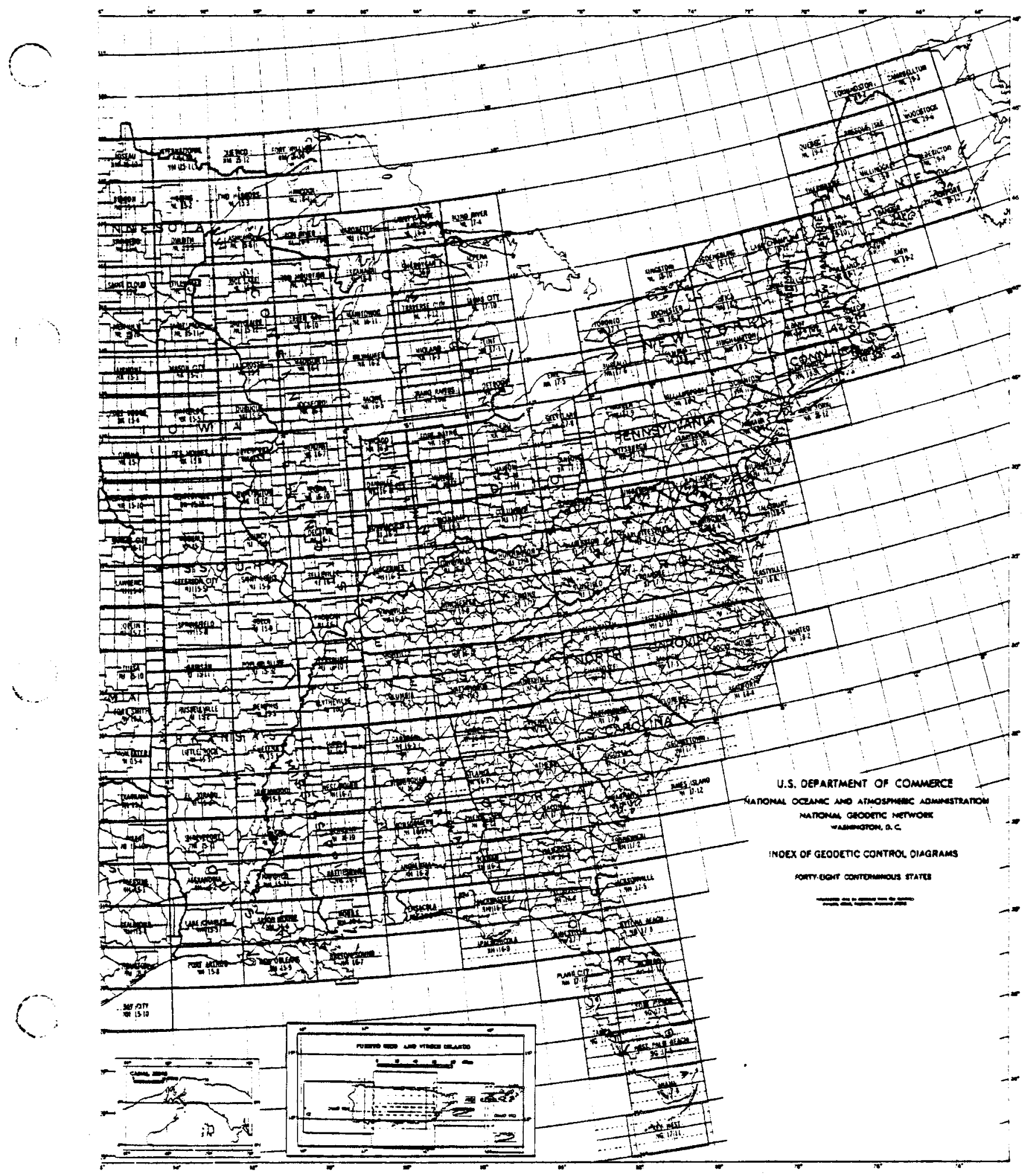

Exhibit 2.4 - Index Map for Eastern United States 


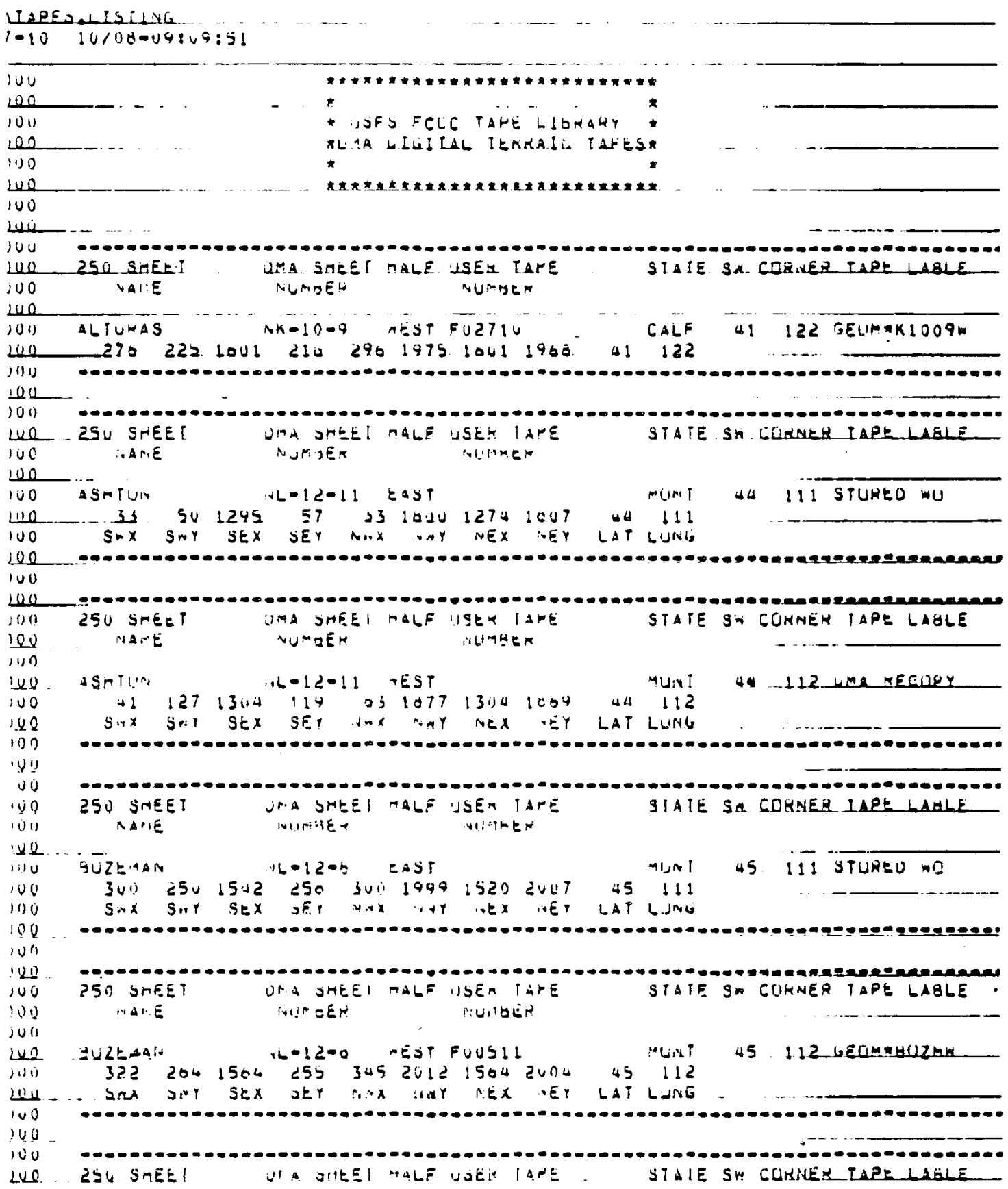

Exhibit 2.5 - Example Listing of DMA Tapes Available in USFC FCCC Tape Library 


\section{CHAPTER III}

\section{NON-GRAPEIC PROGRAMS}

\section{SECTION 1. PROGRAM COPY}

\section{Create 1 Data File Tape with Program COPY}

rrogram: COPY

Data Source: $\quad$ DMA digital Terrain Data (DTD) Tape

Function: $\quad$ Copy DMA Tape to a USFS tape to establish a permanent data file.

Data Card: Only one data card is needed for this program. This card contains, in columns 1 to 2 , the latitude (in degrees) of the south boundary of the $1: 250,000$ sheet half. Format (I2).

Naming New In order to identify the tape in the FCCC library, a label name must be Tape: assigned to it at this time. A standardized method of naming tapes will insure that all tapes have a unique label.

Each 1:250,000 sheet has a name and an alphanumeric code number.

Example:

$\begin{array}{ll}\text { Name } & \text { Code Number } \\ \text { WEED } & \text { NK } 10-8 \\ \text { REDDING } & \text { NK } 10-10\end{array}$

Each 250 sheet has two tapes per sheer (one for the east half and one for the west half).

By using the alphanumeric code number, we can label each tape half with a unique name. All alphanumeric codes start with an $\mathrm{N}$. Therefore, we can drop the $\mathrm{N}$ in our label and add an $\mathrm{E}$ or $\mathrm{W}$ at the end to distinguish between the east and west halves of the sheet.

Examples:

$\mathrm{K} 1008 \mathrm{E}$ or $\mathrm{K} 1008 \mathrm{~W}$

K1011E or K1011W

This will be the name used in the COPY program in the two following JCL statements:

@ ASG,T GEOM*K1008W,U9V

@ USE 9.,GEOM*K1008W See Input Deck Assembly 
This name will also appear in the system $\log$ at the end of the run with the qualifier making up the complete tape label name. Example:

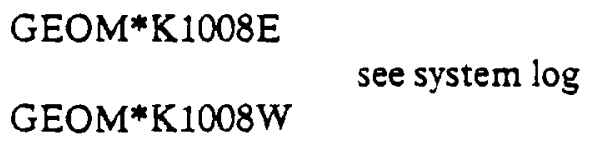

Input Deck See Exhibit 3.1 for a schematic diagram of the input deck.

Assembly:

Sample

Input:

Sample

See Exhibit 3.3.

Output:

Program

There are two messages printed on the Printour:

Diagnostics:

1. AN ERROR HAS OCCURRED ON RECORD NUMBER (OCTAL NUMBER). Run COPY again. If this error persists, contact FCCC.

2. TAPE HAS BEEN COPIED. This indicates a normal end of job.

Program

Output:

The output is used to find the $\mathrm{x}$ and $\mathrm{y}$ coordinates of the four corners of the half $1: 250,000$ map sheet. The output is graphic as well as digital, and shows the convergence of the meridians.

\section{Program COPYNCIC}

Program COPYNCIC converts Digital Terrain Data (DTD) files available from the U. S. Geological Survey (NCIC) to the DMA DTD tape format.

The program operates without any control cards. The map location of the 4 corners of the Quad is available in the NCIC DTD.

The NCIC DTD tape can contain up to 8 DTD files. Before execution of COPYNCIC, it is necessary to put the tape at the beginning of the file that contains the terrain to be converted. This is done by use of the MOVE command. If the selected file is the first on the NCIC DTD tape, go directly to execution. If the file selected is not the first on the NCIC DTD tape, use @ MOVE, and pass all files preceeding the selected file.

Example:

The selected file is file 4 on the NCIC DTD tape. It is necessary to move past 3 files to get to the beginning of the selected file. The @MOVE command is: 
@MOVE 7.,3

Where 7 is the use attached to the NCIC DTD tape.

Sample Input for COPYNCIC

@RUN

@ASG,A DTIS*ABS

@ASG,T 7.,U9V,UR _ _ _user reel number of NCIC DTD tape

@ASG,I GEOM*K1008E,U9V

@USE 8.,GEOM*K1008E.

@MOVE 7.,(N-1) where $\mathrm{N}$ is the file number of the selected file

NOTE: If the file selected is first on the tape do not use the @ MOVE command.

\section{@XQT DTIS*ABS.COPYNCIC $@$ @IN}

To Make the New Flle Permanent. This procedure is outlined in the FCCC user's manual on System Bulletin 75-010, dated 6/04/75. This run should be made for each tape copied to make sure the tape is retained in the library for an extended period. The standard purge date will be $12 / 30 / 80$.

To Save a Tape File.

@RUN,M RUNID,ACCOUNT\#,PROJECT QUALIFIER

@SAVE,T QUAL*FILE(1).,751231,F01000,01,01.

XXXXXXXXXXXXXXXXXXXXXXXXXX $@$ FIN

@@

@@

Where: QUAL*FIIE(1). is the file's complete name. The qualifier must be explicitly stated. If an absolute cycle number is not explicitly identified, it is assumed that the user intends to save the most recently created cycle of the file.

751231 is the purge date for the file in YYMMDD format.

F01000

is the reel number of the tape file being saved. Note that this parameter is only valid in saving a tape file.

01,01

is the sequence of the reel if the tape file is multi-reel. These can be omitted, and 01,01 will be used as default values. Note that these parameters are only valid in saving a tape file. 
$\mathrm{XXXXXXXXXX}$ is a separate card image or line providing a 50 character description of the file which is being saved. This card (or line) must be submitted or the entire save transaction will be rejected.

Note: Tape activity data is posted on the save files on a nightly basis. Do not attempt to save a tape file immediately after creation. Instead, allow overnight posting of tape transactions before a save is run. This limitation does not apply to mass storage files. which can be saved immediately upon creation.

To Find Corner Coordinates. As shown in Exhibit 2.2, the corners on the east and west of the 250 sheet are not due north and south of each other. Due to meridional convergence, the corner coordinates have to be obtained from the output of program COPY.

The convergence will appear in the first fifty scan lines on the west half, and in approximately the last fifty scan lines on the east half.

To find the corner coordinates for the west half, scan the $x$-bottom, $y$-bottom and $y$-top printout from COPY. Usually, the first $x$-bottom and $y$-bottom will be the S.W. corner coordinates. If these values are more than three (3) units higher or lower than the next scan line limits, use the second set of values. Search down the first fifty scan line values until you reach a point where $y$-top stops increasing, and the remainder of the values are within \pm two (2) units. At this point, using the $\mathrm{x}$-bottom and $\mathrm{y}$-top values, you have the northwest corner coordinates. The southeast and northeast corners are found in the last fifty scan lines. These two corners are the last scan line values. The southeast comer coordinates are the $x$-bottom and $y$-bottom values. The northeast corner coordinates are the $y$-bottom and $y$-top values.

The east half corner coordinates may be obtained by using the same approach as above, except the convergence will occur in the last fifty scan lines for the half.

The method of finding corner coordinates is illustrated in Exhibit 3.3.

\section{Updating Library Listing}

Washington Office will be responsible for keeping an up-to-date listing of all the DMA digital terrain data tapes available in FCCC. Therefore. whenever a permanent file is created for a new DMA tape at a given Region, that Region should send the following information to WO for updating purposes.

1:250,000 sheet name:

DMA sheet number:

East or West half:

FCCC Tape no.:

State:

FCCC Tape Label Name:

$\mathrm{X}$-coordinate of SW Corner: (e.g. ALTURAS)

(e.g. NK-10.9)

(from output of COPY, see Exhibit 3.3)

(e.g. GEOM*K1009W) 
Y-coordinate of SW Corner:

$X$-coordinate of SE Corner:

NOTE: $x$ and $y$ coordinates from

$Y$-coordinate of SE Corner:

$X$-coordinate of NW Corner:

Y.coordinate of NW Corner:

$X$-coordinate of NE Corner:

$Y$-coordinate of NE Corner:

Latitude of SW Corner:

Longitude of SW Corner: output of Program COPY.

\section{Creating a Temporary Data File for a 7.1/2 or 15-Minute Quad}

A 1:250,000 map sheet measures approximately 17.5 inches $\times 23.5$ inches. As the DMA tapes stored the elevation data in a .01" $\mathrm{x} .01$ " grid pattern, each DMA tape of one-half of a $1: 250,000$ sheet will contain approximately $2,000,000$ data points.

At present all the application programs, such as FSGPCP for contouring, and SLOPE for computing slopes and aspects, can handle only a maximum of about 14,000 data points in each computer run. If every data point in the original DMA tape is to be used in the computation, the existing application programs can only operate on a map area containing 3-3/4 minutes of longitude and 3.3/4 minutes of latitude. If every second data point is used, these programs can operate on a $7-1 / 2$ minute quad, and if every fourth data point is used, they can operate on a 15-minute quad. However, as the spacing between adjacent data points increases, the digital data presents a decreasingly accurate representation of the terrain surface.

The following two computer programs have been developed to generate a small file of digital terrain data for direct input to the application programs:

1. Program PRINT - to list the $x$ and $y$ coordinates of the comers of all the $7 \cdot 1 / 2$-minute quads contained on a DMA tape.

2. Program SCANDMA - to scan a DMA tape and extract the elevation data within a certain area of interest.

These two programs are documented in the next two sections. 


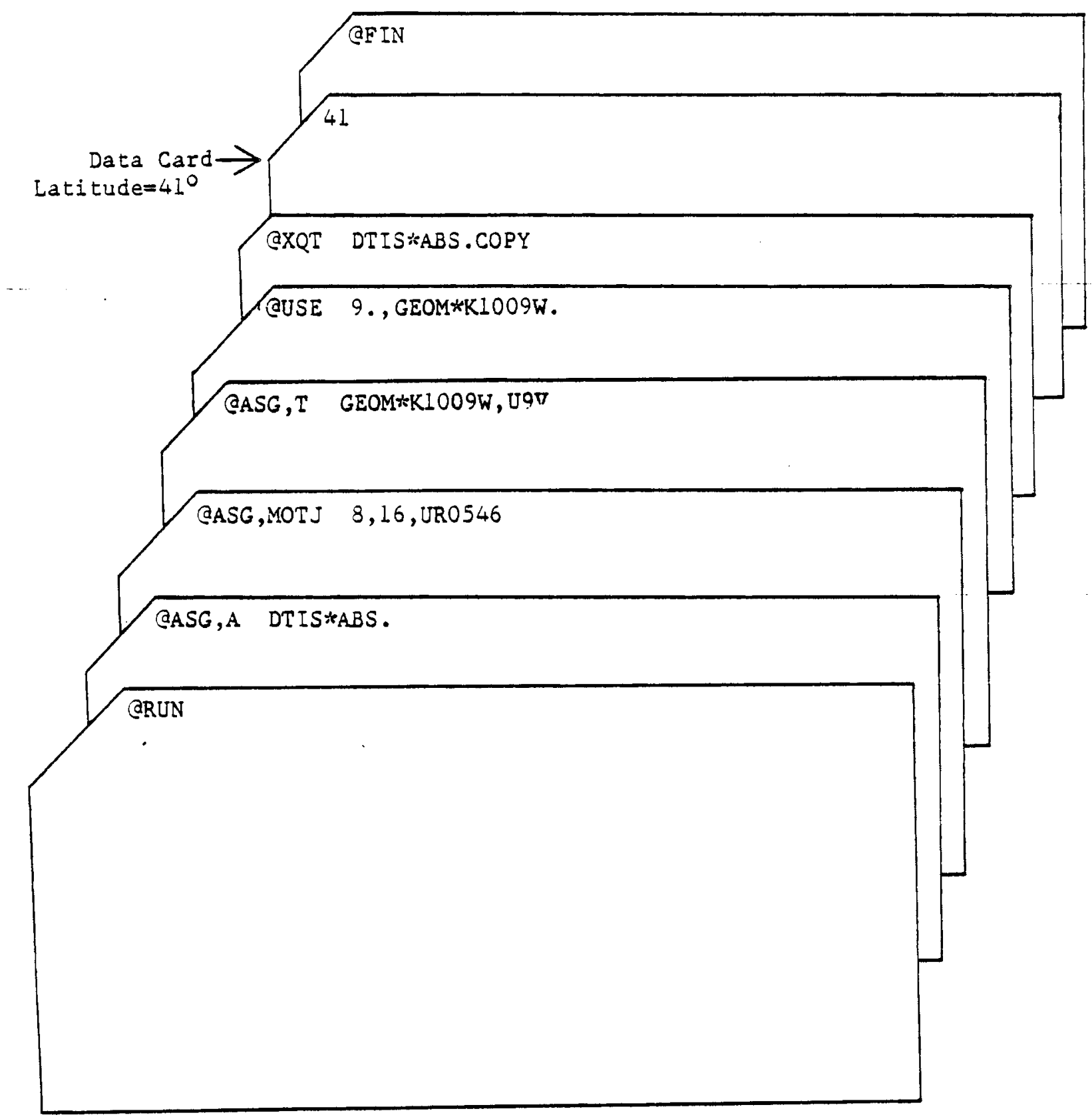

Exhibit 3.1 - Input Deck Assembly for Program Copy 


\section{r}

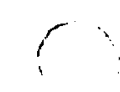

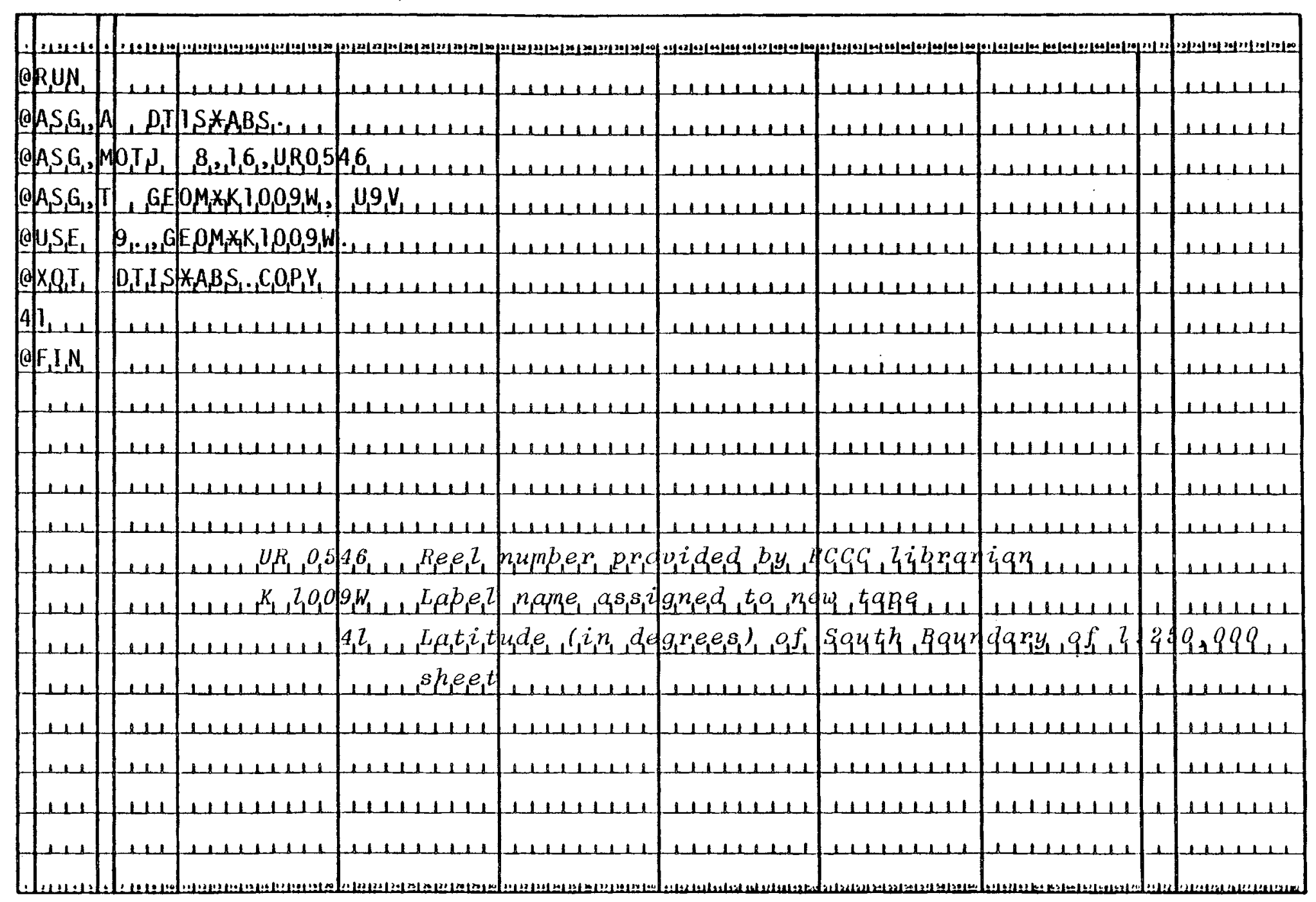




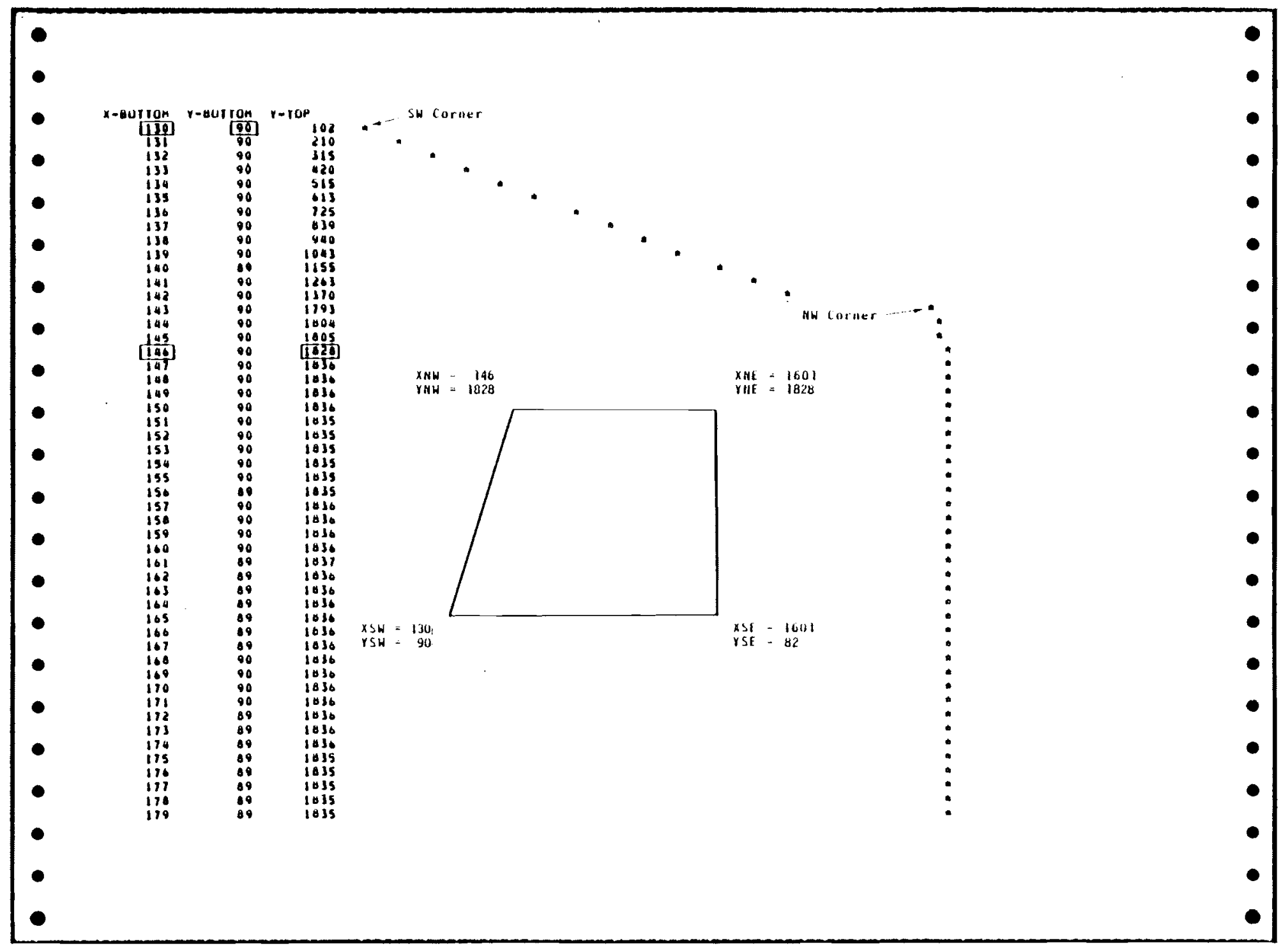

Exhibit 3.3 - Sample Output trom Copy 


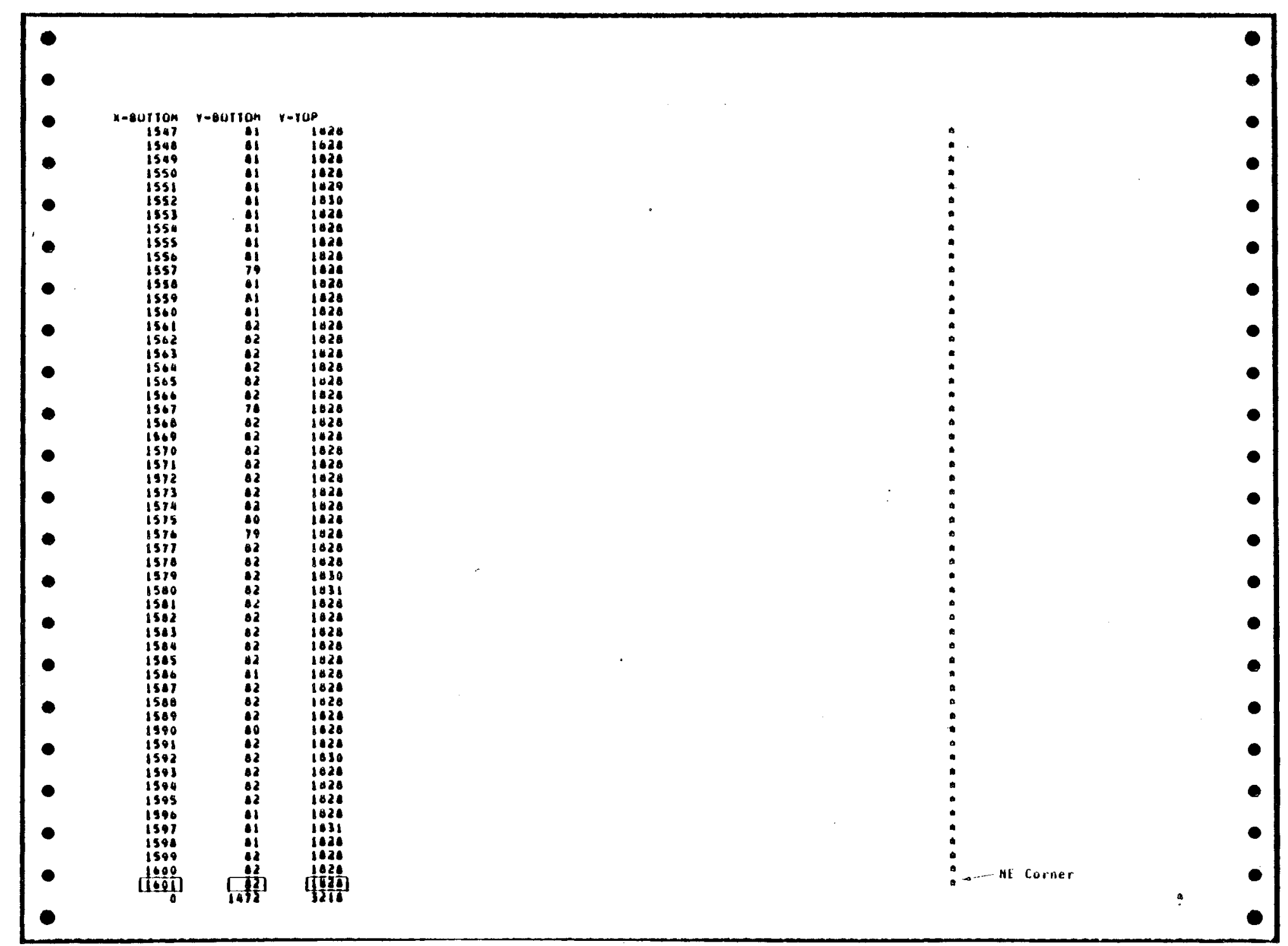

Exhibit 3.3 - Sample Output from Copy (Continued) 


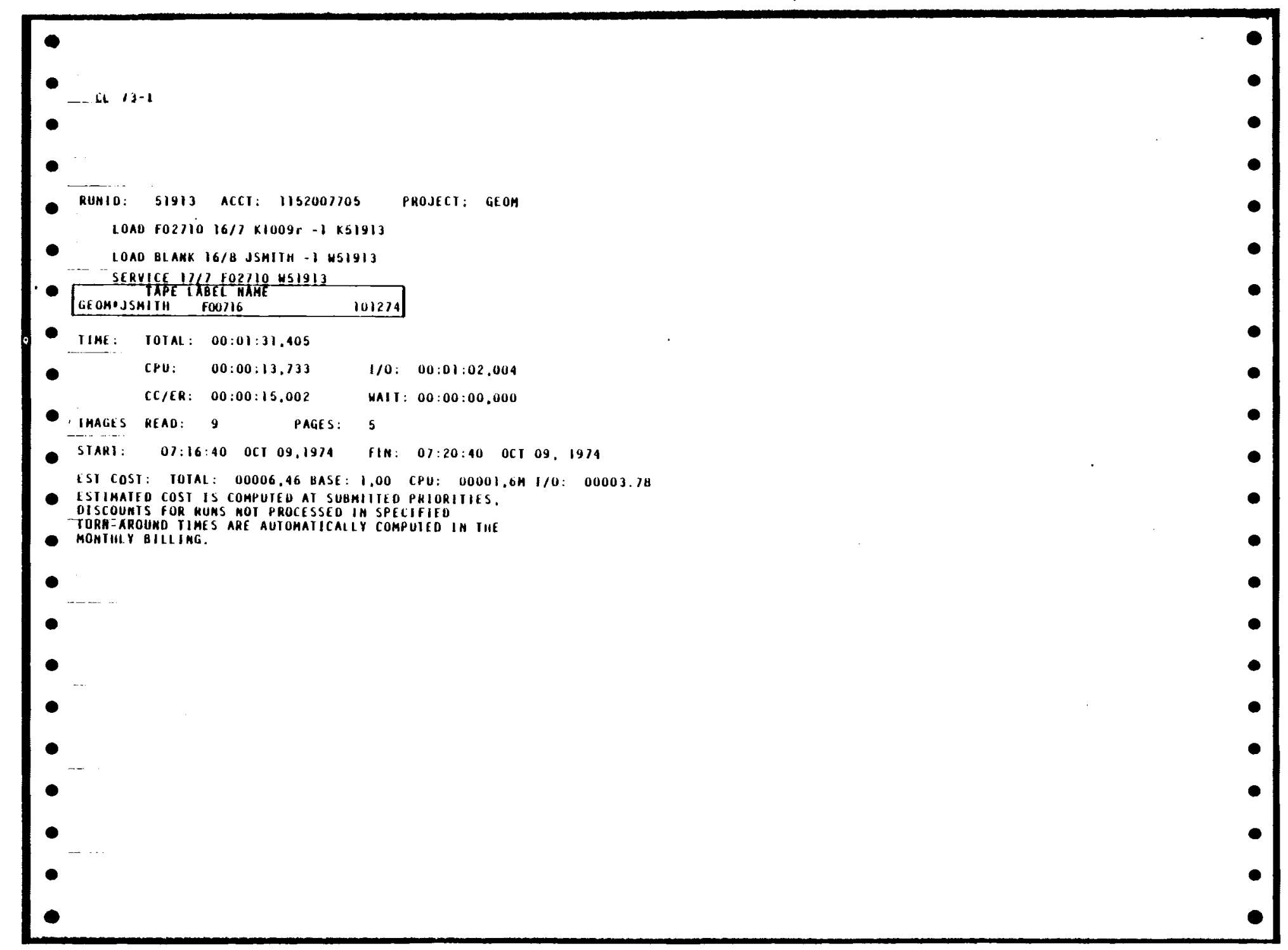

Exhibit 3.3 - Sample Ouput from Copy (Contimued) 


\section{SECTION 2. PROGRAM PRINT}

\section{Function}

To list the $x, y$ coordinates of the corners of all the $7.1 / 2$ - minute quads contained in a DMA digital terrain data tape. These coordinates can be used by the user to define the area of interest within the DMA tape.

\section{Data Cards}

There are two types of data cards to be included in the data deck. The formats of these two types of data cards are explained in Exhibits 3.4, 3.5, and 3.6. Note that all the information needed for these data cards can be obtained directly from the listing of FCCC library, see Chapter 2.

\section{Input Deck}

Assembly

See schematic diagram of an input deck in Exhibit 3.6.

\section{Sample Input}

See Exhibit 3.7.

\section{Sample Output}

See Exhibit 3.8. 


\section{DATA 1 CARD}

PURPOSE: Identifies DMA Sheet information and Forest Service tape number that will be incorporated in page header.

FORMAT: $\quad$ cc

$\begin{array}{ll}1-30 & \text { Name of DMA } 1: 250.000 \text { Sheet } \\ 31-42 & \text { DMA Sheet number } \\ 43-50 & \text { (Blank) } \\ 51-62 & \text { DMA Sheet Half (East or West) } \\ 63-68 & \text { (Blank) } \\ 69-80 & \text { USFS Tape number }\end{array}$

format

\section{ALL VALUES ARE LEFT JUSTIFIED}

Exhibit 3.4 - Data 1 Card

\section{DATA 2 CARD}

PURPOSE: This card is used to input the machine $x$ and $y$ limits for the DMA Sheet Half involved. The SW latitude and longitude are also put on this card to be placed in the page header with the DMA Sheet information.

FORMAT: $\quad$ cc

use

format

$1-5$

6-10

$11-15$

16-20

$21-25$

26-30

$31-35$

$36-40$

4145

$46-50$
DMA SW X

DMA SW Y

DMA SE X

DMA SE $Y$

DMA NW X

DMA NW Y

DMA NE $X$

DMA NE Y

SW Latitude of Sheet Half

SW Longitude of Sheet Half

ALL VALUES ARE RIGHT JUSTIFIED

Exhibit 3.5 - Data 2 Card 


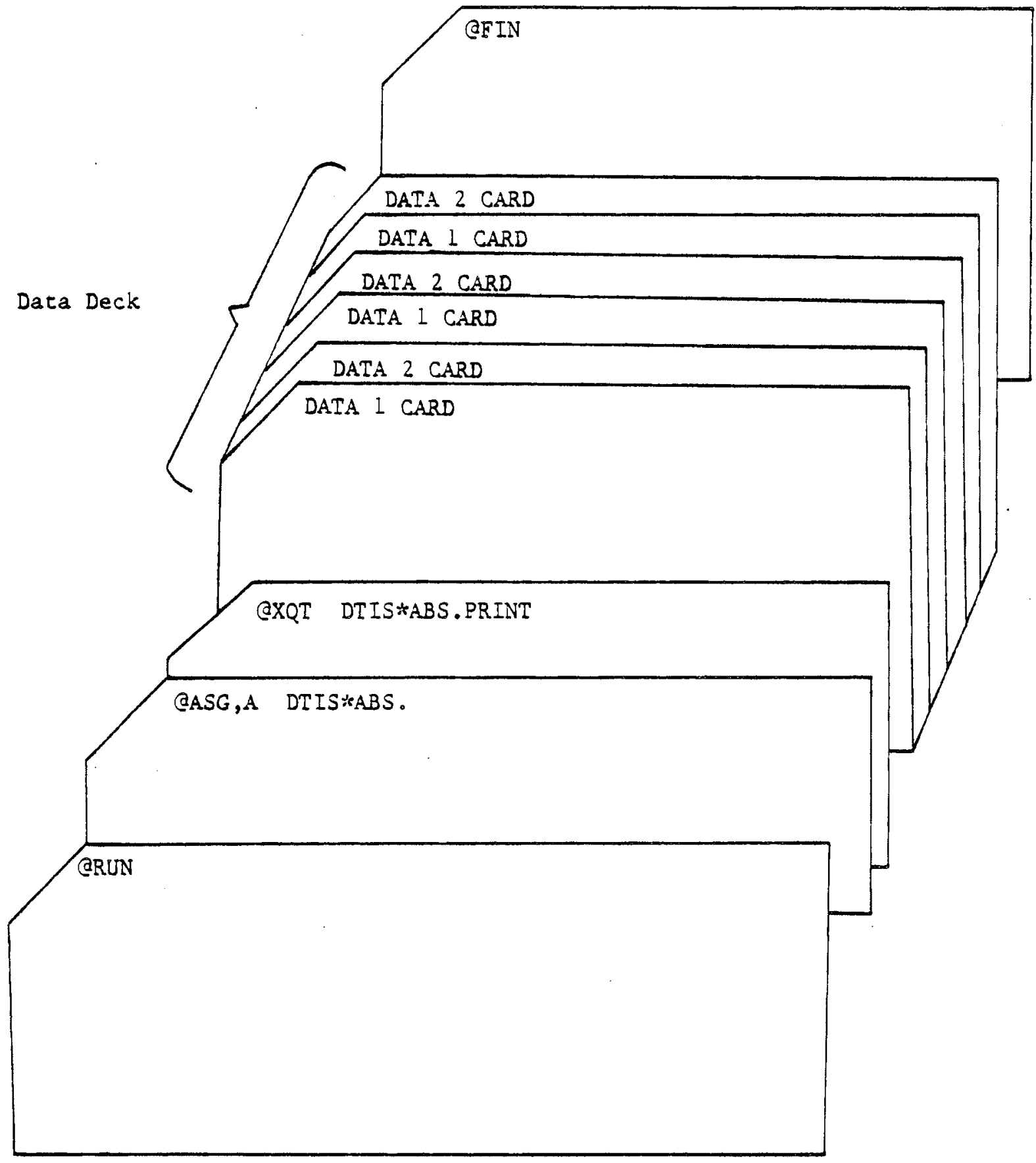




\begin{tabular}{|c|c|c|c|c|c|c|c|c|c|}
\hline 14 & \multicolumn{8}{|c|}{ 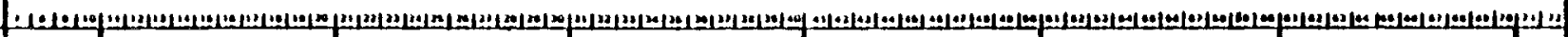 } & \multirow{2}{*}{ 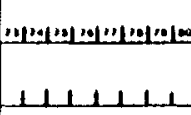 } \\
\hline & & $1+1,11,11$ & $41,1+11$ & $111,111+1$ & $1,1,1+1,1,1$ & 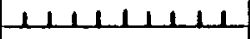 & $211+111112$ & & \\
\hline (o) $A_{1} S_{1} G_{1}$ & DI & $I S_{1} * A_{1} S_{1} \cdot 1$ & $1,1,1,111$ & $1+11111$, & $1,1+1,1,1,1$ & 111111111 & $1,1+1,111$ & 1. & $111+112$ \\
\hline$\times \Omega_{1} T_{2}$ & $D_{1} I_{1} S$ & $X_{1} A_{1} B S_{1}, P_{\perp} R_{1} I_{1} N T$ & $21,1,1,12$ & $1,1111+11$ & $11+1,1+1$ & $1+1+1,1,1$, & $11,1 L 1+1$ & 1 & $11+1111$ \\
\hline 111 & 111 & $\perp \perp \perp \perp \perp A A_{1} I$ & $A_{1}, C_{1} A_{1} R_{1} D_{2} S_{1}, \mathrm{~S}$ & $e_{1} e_{1} f_{1} o_{1} r_{1} m_{1} a_{1} s$ & ${ }_{1} b_{1} e_{1} l_{2} q 4 l_{1} \ldots$ & 11111111 & 11111111 & 1 & 1111111 \\
\hline$Q F_{1} I N_{1}$ & 1.1 & $\perp 1 \perp \perp \perp 111$ & 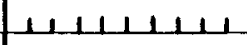 & 111111111 & $\perp \perp|\perp| \perp|\perp| \alpha$ & 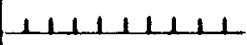 & $\perp \perp \perp \perp \perp 111$ & 1 & $\perp \perp \perp \perp \perp$ \\
\hline 11,1 & 111 & 1111111111 & $\mu 1111111$ & $111+11111$ & $\mid 1111,111$ & $\perp 1111111 L$ & 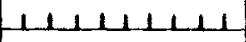 & 1 & 1111111 \\
\hline$A+1$ & 11 & $1 / 1+1111$ & $1+1 / 1+1 / 1$ & $\perp \perp \perp \perp \perp \Lambda 1$ & 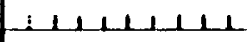 & 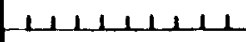 & 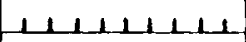 & 1 & $1,1<1,1$ \\
\hline & & & & & & & & & \\
\hline
\end{tabular}

\section{SAMPLE DATA CARDS}

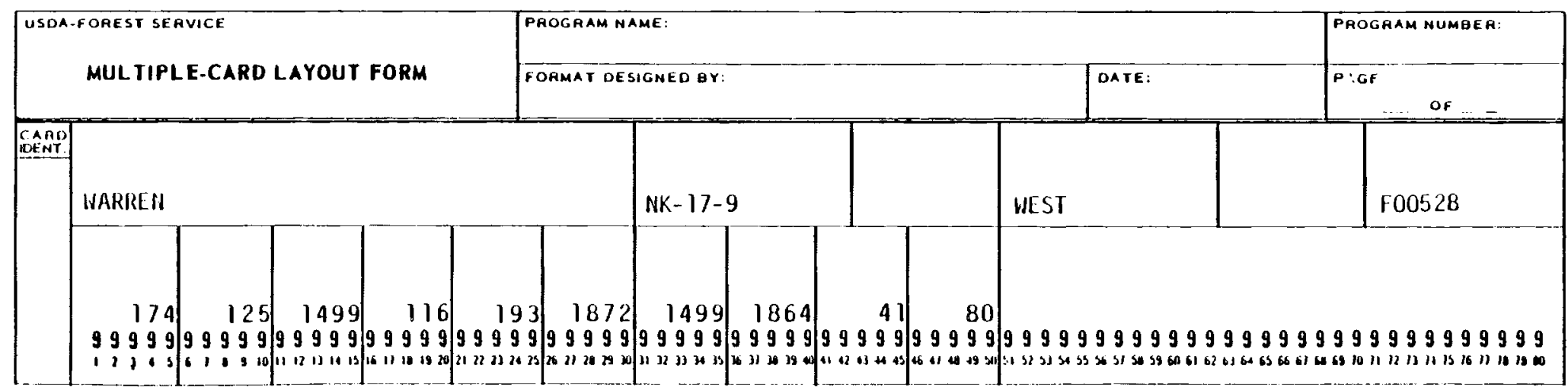


- crallis easi

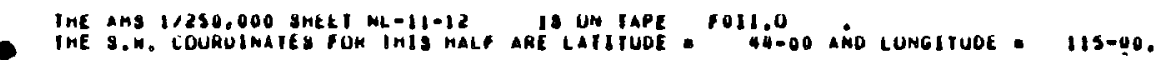

- insm a soo irsm. 211

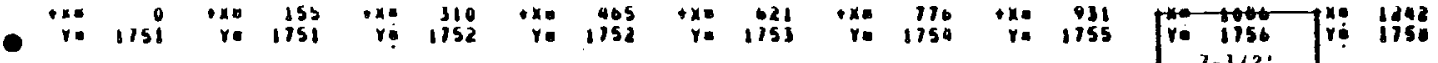

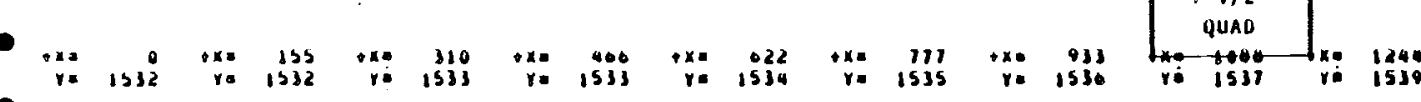

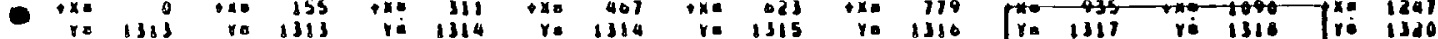

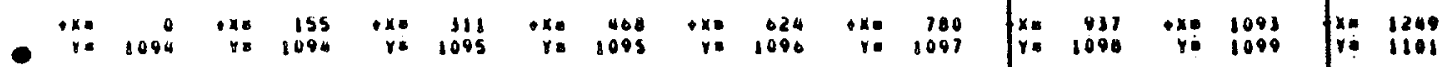

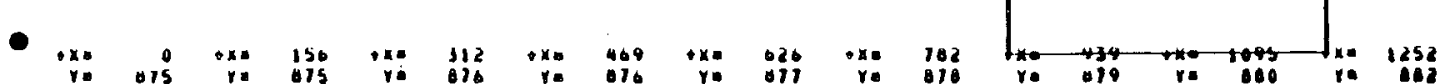

-

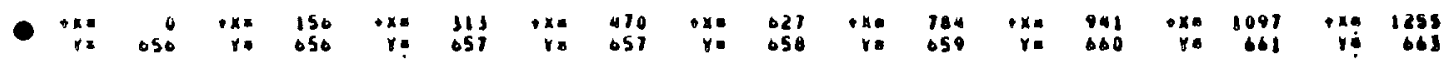

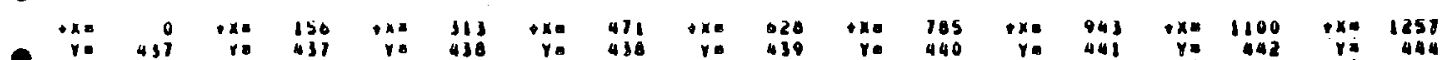

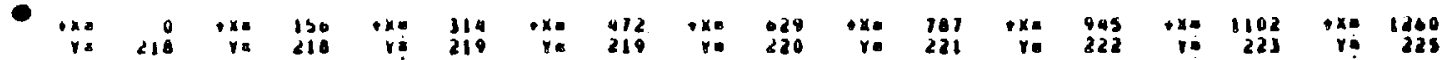

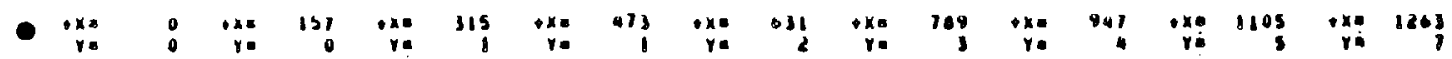

-

of

$0^{.01 m}$

$\bullet$
$\bullet$
$\bullet$
$\bullet$
$\bullet$
$\bullet$
$\bullet$
$\bullet$
$\bullet$
$\bullet$
$\bullet$
$\bullet$
$\bullet$
$\bullet$
$\bullet$
$\bullet$
$\bullet$
$\bullet$
$\bullet$




\section{SECTION 3. PROGRAM SCANDMA}

\section{Function}

To scan a DMA tape and extract the elevation data within a certain area defined in the input data cards. The extracted elevation data are stored in a Fastran file under the name assigned on the @ ASG,UP card; example, GEOM*JSMITH. This Fastran file can then be used as direct input to the application programs.

\section{Data Card}

There is only one type of data card in the input deck. Each data card defines the $\mathrm{x}$ and $\mathrm{y}$ limits, grid increment, and southwest corner of the quad for the map area desired.

Exhibits 3.9 and 3.10 explain the format in the data card.

Input Deck

Assembly

See Exhibit 3.11.

\section{Sample Input}

Deck

Exhibit 3.12 shows a sample input deck for extracting data of a 15 -minute quad. 


\section{DATA CARD}

PURPOSE: This card defines the limits of $x$ and $y$ for the AREA to be searched.

FORMAT: ALL VALUES RIGHT JUSTIFIED

\begin{tabular}{|c|c|}
\hline$c c$ & use \\
\hline $1-5$ & $\begin{array}{l}\text { ILEFT - The lowest } x \text { value to } \\
\text { be scanned decreased by INC. }\end{array}$ \\
\hline $6-10$ & $\begin{array}{l}\text { I.RIGHT - The highest } x \text { value } \\
\text { to be scanned increased by } \\
\text { INC.* }\end{array}$ \\
\hline 11.15 & $\begin{array}{l}\text { IBOT - The lowest y value to } \\
\text { be scanned decreased by INC.* }\end{array}$ \\
\hline $16-20$ & $\begin{array}{l}\text { ITOP - The highest y value to } \\
\text { be scanned increased by INC.* }\end{array}$ \\
\hline $21-25$ & $\begin{array}{l}\text { INC - Scan interval. For } \\
\text { example, if INC }=3 \text {, every } \\
\text { third elevation point will be } \\
\text { copied from a DMA tape. }\end{array}$ \\
\hline $26-30$ & $\begin{array}{l}\text { IXSW-X value of SW corner } \\
\text { obtained from PRINT. }\end{array}$ \\
\hline $31-35$ & $\begin{array}{l}\text { IYSW-Y value of SW corner } \\
\text { obtained from PRINT. }\end{array}$ \\
\hline
\end{tabular}

format

*This value is increased and decreased to insure overlap of your 7-1/2 minute quads. THIS PROCEDURE SHOULD NOT BE USED FOR QUADS AROUND THE BORDER OF THE $1 / 250,000 \mathrm{HALF}$. In this case, take the highest value of ILEFT or IBOT, and the lowest value of ITOP or IRITE that is divisible by INC. 


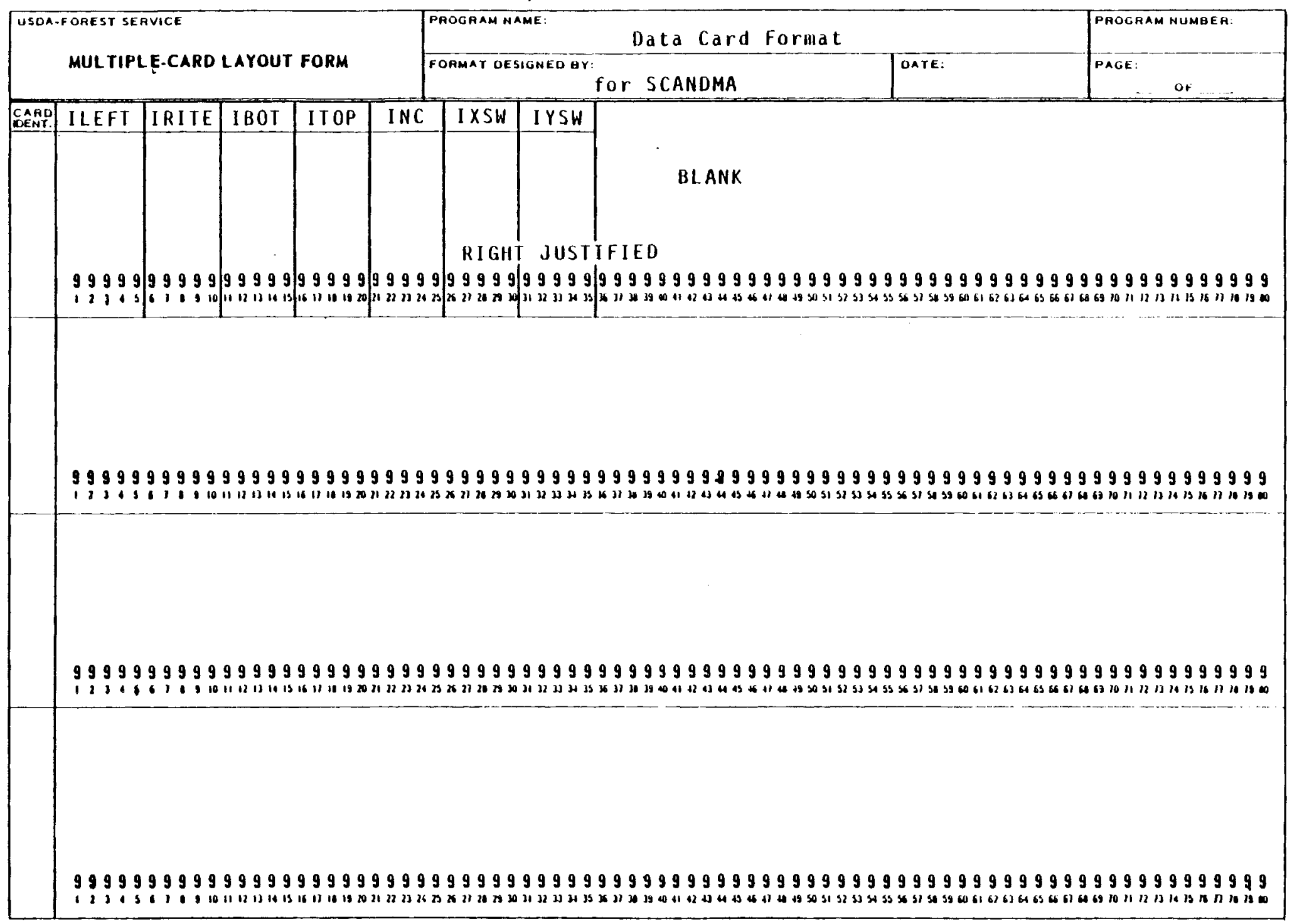

Exhibit 3.10 - Data Card Format for SCANDMA 
QXQT DTIS*ABS.SCANDMA

QUSE 8., GEOM*JSMITH

QASG, UP GEOM*JSMITH, F 24

QUSE $7 .$, GEOM*WARNW.

QASG, I GEOM*WARNW, U9V, F00101

1.

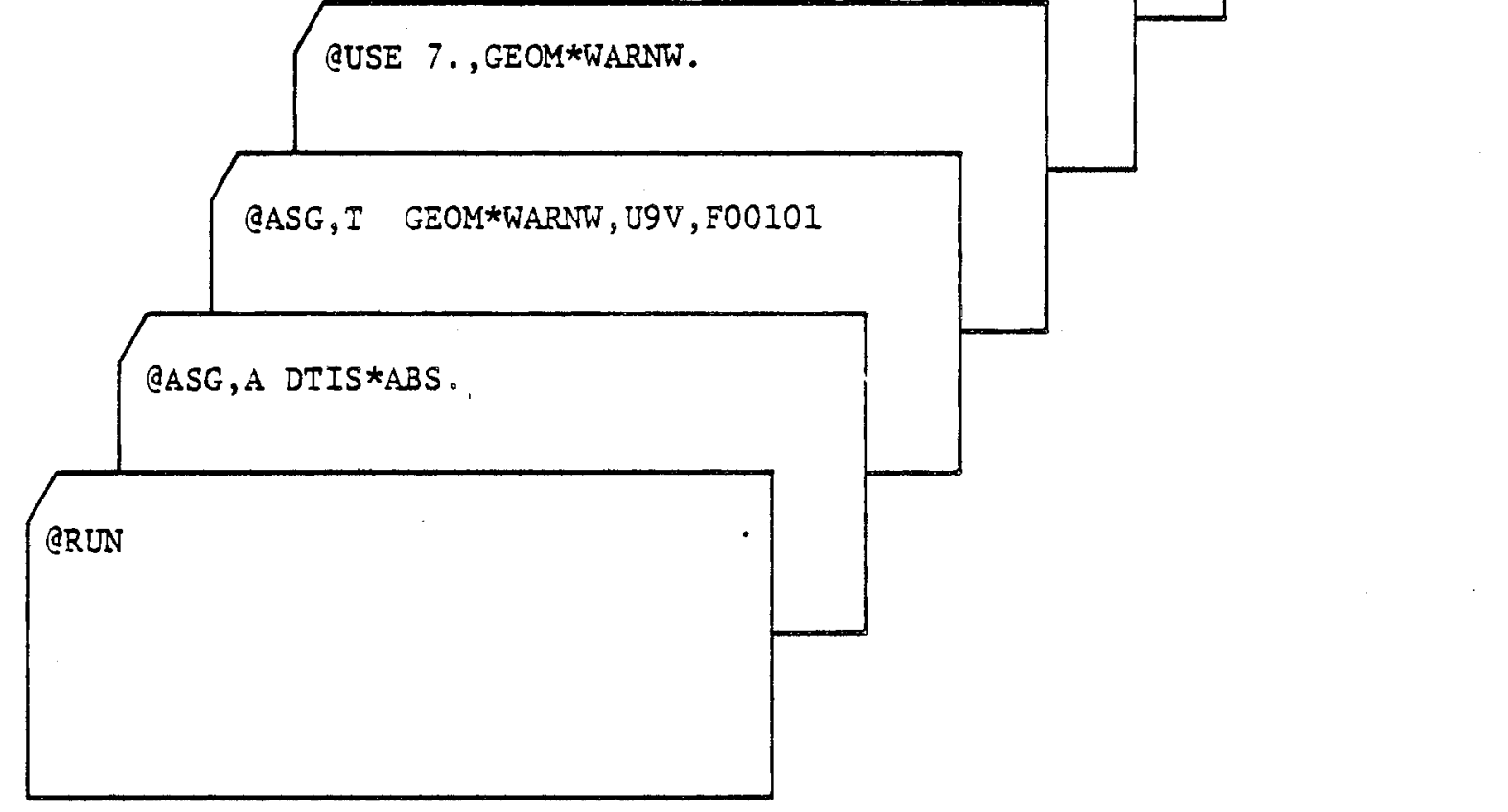

Exhibit 3.11 - Input Deck Assembly for Program SCANDMA 


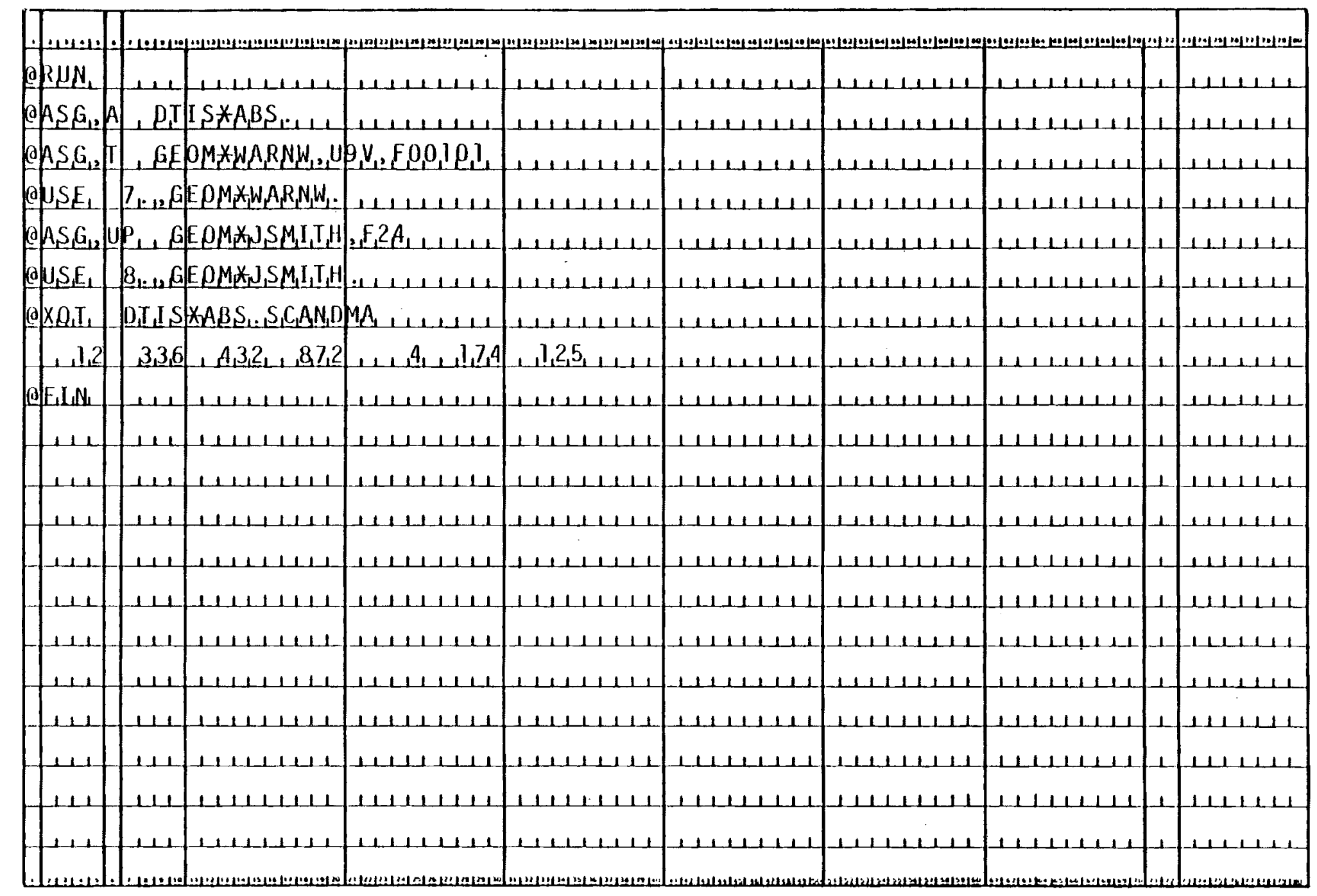




\section{SECTION 4. PROGRAM SCANDTIS}

\section{Function}

The function of SCANDTIS is to extract the elevation data from a DMA tape (or tapes) to build a Blocked TDF (see Chapter IV). The program is selective and will only extract those points which were originally digitized and ignores those points which were mathematically interpolated. The Blocked TDF can then be used for any of the DTIS functions.

\section{Data Cards}

A minimum of four data cards are required as follows (Exhibit 3.13):

1. Title card

2. Ground Coordinates of the four corners of the one degree by one degree quadrangle

3. Map Coordinates from "PRINT" (see Section 2) for the same four corners

4. Limits of area to be scanned - this card may be repeated

Input

See Exhibits 3.14 and 3.15 for a sample runstream. An explanation of the cards in these runstreams follow:

@RUN

Time - allow at least 5 minutes if the entire tape is to be converted.

Pages - allow 20

@ASG,A DTIS*ABS.

Assign the program file.

@ASG,T GEOM*K1008W,U9V,F00346

Assign the DMA tape to a nine track drive.

@USE 7,GEOM*K1008W

Assign Fortran logical unit 7 for the input of the DMA tape.

New

Blocked

TDF

@ASG,T GEOM*KLAMATH,U9V

or

Assign a tape to collect the Blocked terrain. 
Old @MSG,W PLACE WRITE RING IN F12345

Blocked

TDF

@ASG,T GEOM*KLAMATH,U9V,F12345

Assign an existing Blocked TDF tape labeled GEOM*K1008W and a tape number of $\mathrm{F} 12345$

@USE 9,GEOM*KLAMATH

$@ X Q T$ DTIS*ABS.SCANDTIS

Execute the program.

Title Card:

$\mathrm{cc}$

$1-60$

$60-69$

70 use

Alphanumeric Title Blank

Block Status Old $=0$ format

$10 \mathrm{~A} 6$

$9 \mathrm{X}$

II

Ground Coordinate Card. The following values are generally State Plane coordinates for the four corners of the 1 degree quadrangle:

$\begin{array}{llr}c c & \text { use } & \text { format } \\ 1-10 & \text { SW Corner of } 1^{\circ} \text { quad }-x \text { value } & F 10.0 \\ 11-20 & \text { SW Corner of } 1^{\circ} \text { quad }-y \text { value } & F 10.0 \\ 21-30 & \text { SE Corner of } 1^{\circ} \text { quad }-x \text { value } & F 10.0 \\ 31-40 & \text { SE Corner of } 1^{\circ} \text { quad }-y \text { value } & F 10.0 \\ 41-50 & \text { NW Corner of } 1^{\circ} \text { quad }-x \text { value } & F 10.0 \\ 51-60 & \text { NW Corner of } 1^{\circ} \text { quad }-y \text { value } & F 10.0 \\ 61-70 & \text { NE Corner of } 1^{\circ} \text { quad }-x \text { value } & F 10.0 \\ 71-80 & \text { NE Corner of } 1^{\circ} \text { quad }-y \text { value } & \text { F } 10.0\end{array}$

Map Coordinates Card. The following values are obtained from the output of program "PRINT". They are the map coordinate values for the four corners of the $I$ degree quadrangle.

\begin{tabular}{llc}
$c c$ & \multicolumn{1}{c}{ use } & format \\
$1-5$ & SW Comer of $1^{\circ}$ quad $-X$ map value & $F 5.0$ \\
$6-10$ & SW Comer of $1^{\circ}$ quad $-Y$ map value & $F 5.0$ \\
$11-15$ & SE Corner of $1^{\circ}$ quad $-X$ map value & $F 5.0$ \\
$16-20$ & SE Comer of $1^{\circ}$ quad $-Y$ map value & $F 5.0$ \\
$21-25$ & NW Corner of $1^{\circ}$ quad $-X$ map value & $F 5.0$ \\
$26-30$ & NW Corner of $1^{\circ}$ quad $-Y$ map value & F5.0 \\
$31-35$ & NE Comer of $1^{\circ}$ quad $-X$ map value & $F 5.0$ \\
$36-40$ & NE Comer of $1^{\circ}$ quad $-Y$ map value & F5.0 \\
$41-45$ & IXSW - from print & \\
$46-50$ & IYSW - from print &
\end{tabular}


Limit Cards. The limit cards define the area to be extracted from the DMA tapes. Those limits are expressed in map coordinates as follows:

cc

$1 \cdot 5$

6-10

$11-15$

$16-20$

21.25 use

ILEFT - left limit

IRIGHT - right limit

IBOT - bottom limit

ITOP - top limit

INC - use every INCth scan format

I5

I5

15

15

I5

Limit card may be repeated as needed.

$@$ @IN

On a new tape, the tape number must be saved if you need to keep it. 


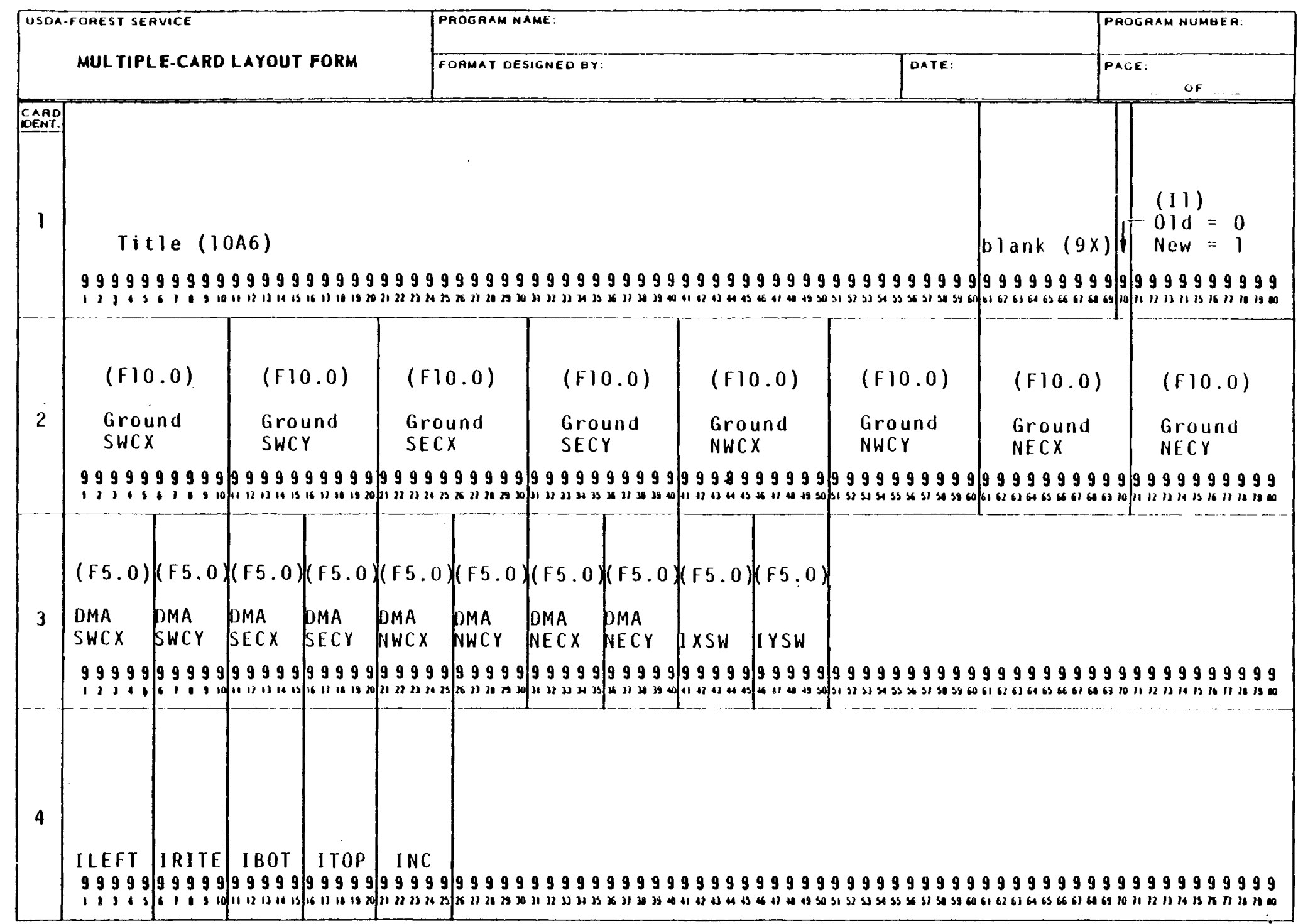

Exhibit 3.13 - Card Layout for Program SCANDMA 


\begin{tabular}{|c|c|c|c|c|c|c|c|c|c|}
\hline & 1.1 & & & & & & & and? & \\
\hline & & 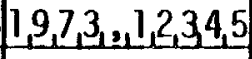 & $6,7,8,9,0, \ldots, E_{1}, M$ & ${ }_{21}, 0_{2}, 2,0, a_{12}$ & ALBEECE & GLNIEIELNAG & 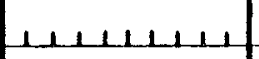 & 1 & 111111 \\
\hline & A. & $I_{1} S_{1}^{*} A_{1} S_{1 \cdot 1}$ & $\rightarrow A_{1} S_{1} S_{1} I G_{1} N_{\Lambda}$ & $\underline{P}_{1} H_{1} E_{1} P_{1} R_{1} Q_{9} R_{1} A$ & $\left.M, F_{1} I_{1} L_{1} E_{1}\right)_{1}$ & 111111111 & $1 \perp|1| 1|1| 1$ & $L$ & $111<111$ \\
\hline OAS & T. & $0 M_{1}^{*} K_{1}, 0,0,8 W_{1}$ & $0,9, V_{1,1}, 0,0,3,4,6$ & Leelelel & $1+1+1+1,1$ & Leldelele & $1,|l| l|l| l \mid$ & $\perp$ & $11+1111$ \\
\hline US & & G,E,OM* $K_{1} I_{1}, 0, W$ & $11|1| 1 \mid 11$ & $11111 \mid 111$ & $|1| 1 L|| l \mid$ & $|1| ,1|\perp| 1$ & $\perp \perp \perp \perp \perp \perp \perp$ & 1 & $1+11+111$ \\
\hline OAS, G & $I, G E$ & $O M_{1}^{*} K_{1} L_{1} A_{M} M_{1} T_{1}$ & $u_{1} v_{1}, \ldots$ & $1121,11+1$ & 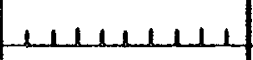 & $\perp 111111$ & $1+1|1| 1] 1$ & 1. & 111112 \\
\hline gus. $E_{1}$ & & GEOM*KLAMA & {$\left[H_{1,1,1,1}\right.$} & $1 / 1|1| 1 \mid 1$ & $\perp \perp, 1,1, L$ & $11,1 / 1,111$ & $1,1+11111$ & 1. & $\mu 111$ \\
\hline$O \times \mathrm{Bi}_{1}$ & $\& 1$ & $I S_{1}^{*} A B_{1} S_{1:} S_{1} C_{1} A$ & NDII $S_{1}, \ldots$ & 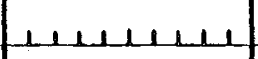 & Llelelul & 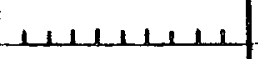 & $N O_{1} l_{1}^{i} E_{L_{1}} \perp_{1}$ & 工. & 1111111 \\
\hline$B U_{1} I L$ & $\mid A_{1} B L$ & $O \subset K E D_{1} I D_{1} F_{1}$ & $F Q_{1} \perp \perp A M A I$ & He NiE $r i$ NiEd & FULELAL & 11111111 & ] & $\Lambda$ & $1 \perp \perp \perp \perp L$ \\
\hline 1448 & 21.92 & $6,1,3,4,48_{1}, 3,9$ & 1723992:98 & $608,72,3,6,8$ & $1,4,5,6,3,3,7,18]$, & $.97,721.9 .98$ & $\left.\left.1,7,28_{1}\right]_{1}\right]_{1: 1}, 0$ & 9 & $7,3,966,14,5$ \\
\hline & 舟 & $1326,-7$ &, $2 P_{1}>50$ & $1326,17,43$ & $\perp 17,6,1,4$ & (1) & 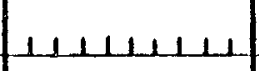 & 1 & $\| \perp \perp \perp \perp$ \\
\hline 0 & 290 & $+10 \Omega_{1}, 500$ & $111 R_{1}+11$ & $\{1,1+1+1,4$ & $\left.E_{1} P_{1} A_{1}\right]_{1} A_{1}$ & $A N \times, A_{1} S, N, E L$ & $Q_{1} E_{1} D_{1} \perp 1,11$ & 1. & $111+111$ \\
\hline 500 & $8 D \rho$ & $, 9 \rho \rho_{1}, 1200$ & $11|2| 1 \mid 1$ & 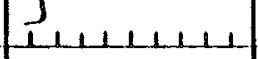 & $\perp|1| 1\left|L_{1}\right|$ & 111111111 & 111111111 & 1 & 1111111 \\
\hline d $I N$ & Int & $1 \perp \perp \perp \perp \perp 1 L$ & $\perp \perp|1| 1 \mid 11$ & $-1,1<|\perp| \perp \mid$ & 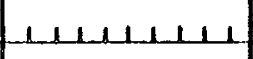 & 1 & $|\perp||L||1|$ & 1 & $\mu \mu_{1} \mu \mu_{1}$ \\
\hline-111 & 111 & $111 / 11111$ & 11111111 & $1111 \mid 1111$ & $1|1| 1 \mid 111$ & 111111111 & 111111111 & 1 & 1111111 \\
\hline thes & L11 & 1111111 & $|l| \perp|l| l \mid$ & $1+11111111$ & 111,11111 & $\mid 1,1 / 1,11$ & $\mid 11111+111$ & 1 & $111+111$ \\
\hline veqti & LBR & sure, te, & $t t_{1} t a p$ & HBer,eti, GE & DM*KLAMATH & $\mu_{1} t_{1}$ & llevis & $4, p ?$ & 11 \\
\hline 1111 & 111 & 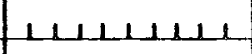 & $11111,1,1$ & $\mid 11111111$ & $1111|1| 1 \mid 1$ & 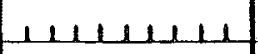 & 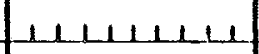 & 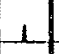 & $1 / 1,111$ \\
\hline 111 & 1111 & 11111111 & 111111111 & $|1| \perp 111 \perp 11$ & $\mid 1,1111+1$ & $1111+1111$ & $11+1121 L 1$ & 1. & $1 \perp \perp|1| 1$ \\
\hline Llel & | & 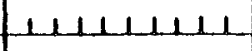 & $\perp 1111 \perp \perp$ & $|1| 1|1| 11$ & $|1| 111111$ & $111 / 11111$ & $\perp \perp|1| \perp \mid 1$ & 1 & 1111111 \\
\hline Hel & Les & Leldelden & $\left|L_{1}\right| L_{1} \mid L_{1}$ & Leneles & Helnenes & Leteldele & $1<1+1<1<1$ & 1 & ene $1+1$, \\
\hline & & & & & & & & & \\
\hline
\end{tabular}




\begin{tabular}{|c|c|c|c|c|c|c|c|c|c|}
\hline & f. & & & & 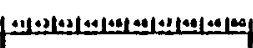 & 1 & | & & 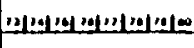 \\
\hline $0 R N_{n}$ & $M 1$ & $5 \mid 9,7 A_{121} 12,3,4,5$ & $6,7,89 \varrho_{4}, \mathrm{GE}, \mathrm{M}$ & $=1,0_{121}, 0,0_{1,1}$ & $\perp A L_{1} B_{1} E_{1} E_{1}, E$ & $N G I, N E_{1} E_{1} R I, N G$ & $1|1| 1|1| 1$ & 1 & 111111 \\
\hline ohs & A. & $I S_{1}^{*} A B_{1} S_{11}$ & $11+1,1112$ & 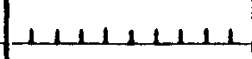 & $11+111 / 11$ & $1+111+111$ & 11111,111 & 1 & $11+1111$ \\
\hline ofs, $G_{12}$ & T.1 & $=0 M_{1}^{*} K_{1}, 00,8, W_{1}$ & $\mu, 9, V_{1}, F, 00,3,4,6$ & $\mid 1,1,1 / 1,11$ & $\perp 1+1+1+11$ & 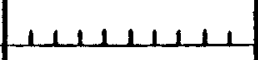 & $11,1,1,1,1$ & 1 & $1+1+111$ \\
\hline dUSE & & $\left.G E O M_{1}^{*} K_{1}\right] O_{1} O_{1}$ & $3 W_{1,1,1,1,1}$ & $1+1+1+111$ & 111 & 111111 & $11+111111$ & $\perp$ & 11111 \\
\hline (2) MSR & $W$ & $A C E_{1} W_{1} R_{1} I_{1} I_{1} E_{1}$ & $R_{1} I_{1} N G_{1} I N_{\perp} F_{1} I$ & $2,3,4,5,1,1$, & $1+1+1+111$ & $|1| 1|1| 1 \mid 1$ & $111 / 11111$ & -1 & 1111111 \\
\hline$\Leftrightarrow A_{1} S_{1} G_{2}$ & $\mathrm{~T}, \mathrm{C}$ & $=0 M_{1}^{*} K_{1} L A M A T_{1}$ & $\left.1, U_{1}, V_{1}, F_{1}\right] 2,3,4$ & $51+111111$ & Lleldelde & $1,1,11,111$ & $\perp 11111111$ & 1 & 111111 \\
\hline OUSWEL & 1, & $G E O M^{*} K_{\perp} \perp M A$ & $f\left[H_{1}, 1,1,1\right.$ & 11,111111 & I I L & 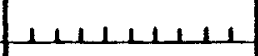 & $1+1+1+111$ & 1 & 1 \\
\hline a $x_{1}, T_{1}$ & LDI & $I S_{1} \star A_{1} B_{1} S_{1 .} S_{1} C_{1} A$ & $N_{1} D_{1} I_{1} S_{1}, \ldots$ & 111,11111 & $1+1,1,1,1$ & 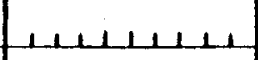 & $\perp \perp 1+1+1 N_{1} O$ & Ine & $1111+11$ \\
\hline 4 IIIL, & (A $E_{1}^{2} X_{2} I$ & $A, I_{1} I_{1} G, B I_{1} Q 0$ & $K_{1} E_{1} a_{1}, R_{1} L_{1} E_{1}, E_{1} C$ & $A R, K L A M A T H$ & $N E_{1 \perp 1 L 1 L_{1}}$ & $\perp L 1|L| \perp \mid L$ & $\perp 1+1,1, \alpha$ & 1 & $11 \perp 111$ \\
\hline$L_{1} A_{1} l$ & $A+D E$ & $C_{1} K_{1 \perp \perp 11}$ & $11+11111$ & $\mid$ & $|1| L_{1}|1| 1 \mid$ & $\perp \perp \perp \perp \perp \perp \perp \mid \perp$ & $\perp \perp \perp 11<L_{1}$ & $L$ & $\perp \perp 1 \perp \perp 1$ \\
\hline L & 4 & $h_{1} i b_{1} i_{1} t_{1}+3_{1}{ }_{1}$ & $P_{1} \perp \perp \perp \perp, 1$, & 111111111 & 11,111111 & 111111111 & $|1| 1|1| 1|1|$ & 1 & 111111 \\
\hline L.1. & lecek. & $i_{1} s_{1} i_{1} d_{1} e_{2} t_{1} i_{1} c$ & $a_{1} L_{1}+1,1,1$ & 11,111111 & Lelelele & $+1+1+1,12$ & $1+1+1 \mid 1+1$ & 1 & 1111.11 \\
\hline 111 & 111 & 111111111 & $11+1111$ & $\mid 1,1<11,111$ & $111+11111$ & $11+1 \perp 11,1$ & $\mid \perp \perp 1+11 \perp 1$ & 1 & LـL \\
\hline (a) FllN & 111 & $|1 \perp 11| 1 \mid 1$ & $11|1| 1 \mid 1$ & $\perp \perp \perp \perp \perp \perp \perp \perp \perp$ & 111111111 & 111111111 & 111111111 & $L$ & 1111111 \\
\hline .111 & 111 & 111111111 & $\perp 1111111$ & 111111111 & 111111111 & LLLLLL & $1 / 1+1,11$ & 1 & $-1 L_{1} \perp \perp_{1}$ \\
\hline fen & 111 & $f+1,1+1,1$ & $111+1+11$ & 1,11111111 & enentele & $1,1+1,1,1$ & $\perp \perp 1+1+1+1$ & 1 & 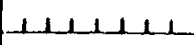 \\
\hline 1112 & 111 & $111+1+111$ & $11111 \| 1<1$ & 111111111 & LAldelle & $\perp \perp \perp \perp 1 \| L L$ & $\perp 1 \perp \perp|1| 1$ & $L$ & $11+111$ \\
\hline 1,1 & -111 & $|+|+\mid 1,1,1$ & $1,111+111$ & 111111111 & 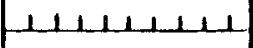 & & 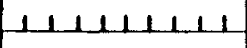 & 1 & 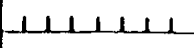 \\
\hline 111 & 111 & 111111111 & 11111111 & $11 \perp 111111$ & 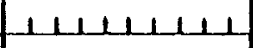 & $1 \perp 1111111$ & 111111111 & 1 & 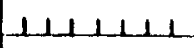 \\
\hline$+1,1$ & $H+11$ & 11121 & 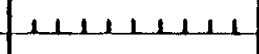 & $\mid 1,111,11$ & $1|1| 1|1| 1 \mid$ & $|1| 1|1| 1 \mid$ & $\perp 1111111$ & 1 & 1111111 \\
\hline & & & & & & & & & \\
\hline
\end{tabular}

Exhibit 3.15 - Input Deck for Proyram SCANDTIS (Adding to an Existing TDF) 


\section{CHAPTERTV \\ DIGITAL TERRAIN MODEL PROGRAMS}

\section{SECTION 1. DIGITAL TERRAIN INFORMATION SYSTEM}

The U. S. Forest Service Digital Terrain Information System (DTIS) is a computer program package that has been developed for the manipulation and analysis of digital terrain data. The data has been collected directly from topographic maps or from a photogrammetric stereoplotter. The program package can perform two basic functions:

1. Transformation of the new digital data into an appropriate ground coordinate system, and then store the transformed data in a format which is compatible with the requirements of the application programs. Characteristically, an efficient digitizing operation requires that the data points be selected at the break points in the topography, resulting in a set of nongridded (or basically, randomly spaced) data points. DTIS was designed to provide efficient handling and storage of large files of random digital topographic data. For example, with the DTIS package, it is possible to store 1-1/2 million data points (which may constitute a complete data file for an entire National Forest) on a single computer tape.

2. Generation of four types of application products from an existing data file:

a. scan profiles and set-up information for the production of ortho photos;

b. cross-section elevations along a center-line in the standard U. S. Forest Service card type 50 (CT50) format;

c. a new data file with the data points being arranged in a gridded patern; and

d. a ground profile for input to skyline logging analysis programs.

\section{Program Segments in DTIS}

The DTIS program package consists of six major program segments: ONEShot, XYTRan, BLOCk, MANUv, ARAY, and FORCor. Exhibit 4.1 illustrates the functions of these program segments and their inter-relationship.

When digitizing is performed directly in a stereoplotter. the spatial position of the model points may be defined in an arbitrary, rectangular coordinate system. Program segment ONEShot accepts these model coordinates as input, and transforms them into a ground reference coordinate system through the use of ground control points. ONEShot is basically a coordinate transformation program, and is not intended for analytical aerotriangulation. At the option of the user, ONEShot may output the transformed data as either "punched" terrain data file (TDF), or blocked TDF.

In a "punched" TDF, the data points are arranged in the same order as the original input data to ONEShot. The spatial position of each data point is defined by its horizontal coordinates $\mathrm{X}$ and $\mathrm{Y}$, and vertical coordinate $\mathrm{Z}$. Since the data points do not follow a gridded partern, tine entire data file must be searched whenever it is necessary to identify all the data points that are located within a certain boundary. 
In a blocked TDF, the data points are collected into groups containing up to 500 points. The lower and upper limits in X- (or easting) and $Y$. (or northing) coordinates of each data group are determined and stored in the file. The blocked format reduces the computing time required to search a file. By checking the boundary limits, it is possible to eliminate, at one time. an entire group of data points from the search process.

When rectangular digitizers are used to digitize terrain data from existing topographic maps, the spatial position of each data point is usually defined by a pair of rectangular coordinates $(x, y)$, and its elevation above mean sea level. The rectangular coordinates are usually in the same scale as the map, and are arbitrarily oriented. Program segment XYTRan accepts this type of coordinates as input, and transforms the horizontal coordinates from the digitizer system into a ground reference coordinate system, such as the State Plane coordinates. The output from XYTRan may also be in either the "punched" TDF or blocked TDF format.

The Calma Digitizing System may be used to digitize topographic maps, and produce digital terrain data directly in a ground reference coordinate system. Further coordinate transformation is not needed. However, program segment BLOCk may be used to collect the data points and output a block TDF.

Program segment MANUv is the application program within DTIS. It is used to produce ortho photo scan profile plates, cross-section elevation data, or a gridded terrain data file (TDF).

Program segment FORCor is a utility program that is not directly related to other program segments within DTIS. It may be used to convert State Plane coordinates to geographic coordinates (longitude, latitude, and elevation) or vice versa. It may also be used to convert State Plane coordinates from one State-zone to another.

Program segment ARAY provides a link between the DTIS program package and the contouring program FSGPCP within TOPAS. ARAY is used to rearrange a gridded TDF, which has been generated from MANUv into the proper format for input to FSGPCP.

Two other programs provide additional links between DTIS and other portions of TOPAS. Program SCANDTIS, as documented in Chapter III, is used to scan a DMA tape and to generate a block TDF for input to program segment MANUv. Program BLOCKBACK. as documented in Section 3. is used to rearrange the data in a block TDF for input to the application programs in TOPAS. Through the use of these data manipulation programs, DTIS becomes an integral part of TOPAS. The inter-relationship between DTIS and the remaining part of TOPAS has already been illustrated in Exhibit 1.1.

\section{Segment Call Card}

Any of the program segments in DTIS can be brought into execution by the use of a segment call card. The call card may consist of eight fields of the following format: 8(A4.A6). The first four characters of each field constitute the key word of the command, and must not contain an erroneous entry. The remaining 6 characters are not tested by the program and are used primarily to provide convenient command words in the segment call cards. The field specifications for the segment call card are as follows: 
$\mathrm{cc}$

$$
1-10
$$

$11 \cdot 20$

$21 \cdot 30$

3140

$41 \cdot 50$

$51-60$

61.70

$71 \cdot 80$ use

Name of program segment to be called: ONEShot, XYTRan. BLOCk. MANUv, ARAY, or FORCor. A blank field would signal the normal end of job.

I/O option

I/O option

I/O option

I/O option

I/O option

I/O option

I/O option

The $\mathrm{I} / \mathrm{O}$ options available for each of the program segments will be discussed in conjunction with the data input requirements of that segment. Following is a listing of the $\mathrm{I} / \mathrm{O}$ options with brief explanation of their uses:

$d=$ default parameter. This option is assumed unless the default disabling option is input.

1

option

NEEL

or

$X Y Z$

BLOCK

or

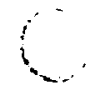

action

d Input is in N, E, EL coordinate order on the INPUT records.

Input is in $X, Y, Z$ order (disables NEEL).

$d$ Output from the transformation segments is to be blocked into 500 positions per record (blocked TDF).

Input to MANUv is in the blocked TDF format (unit 9 contains the blocked data).

NOBLock
Outpu: from the transformation segments is to be left in the same type (UNITrecord or RESOurce) and the format is either Packed Spatl Format or Resource Spat! Format. Input to MANUv is of the type UVITrecord or RESOurce. format is either Packed Spar! or Resource Spatl Format. segments affected

BLOCK. MANUV. ONEShot. SPATL. XYTRan

ONEShot. XYTRan

M.ANUV

ONEShot. XYTRan 


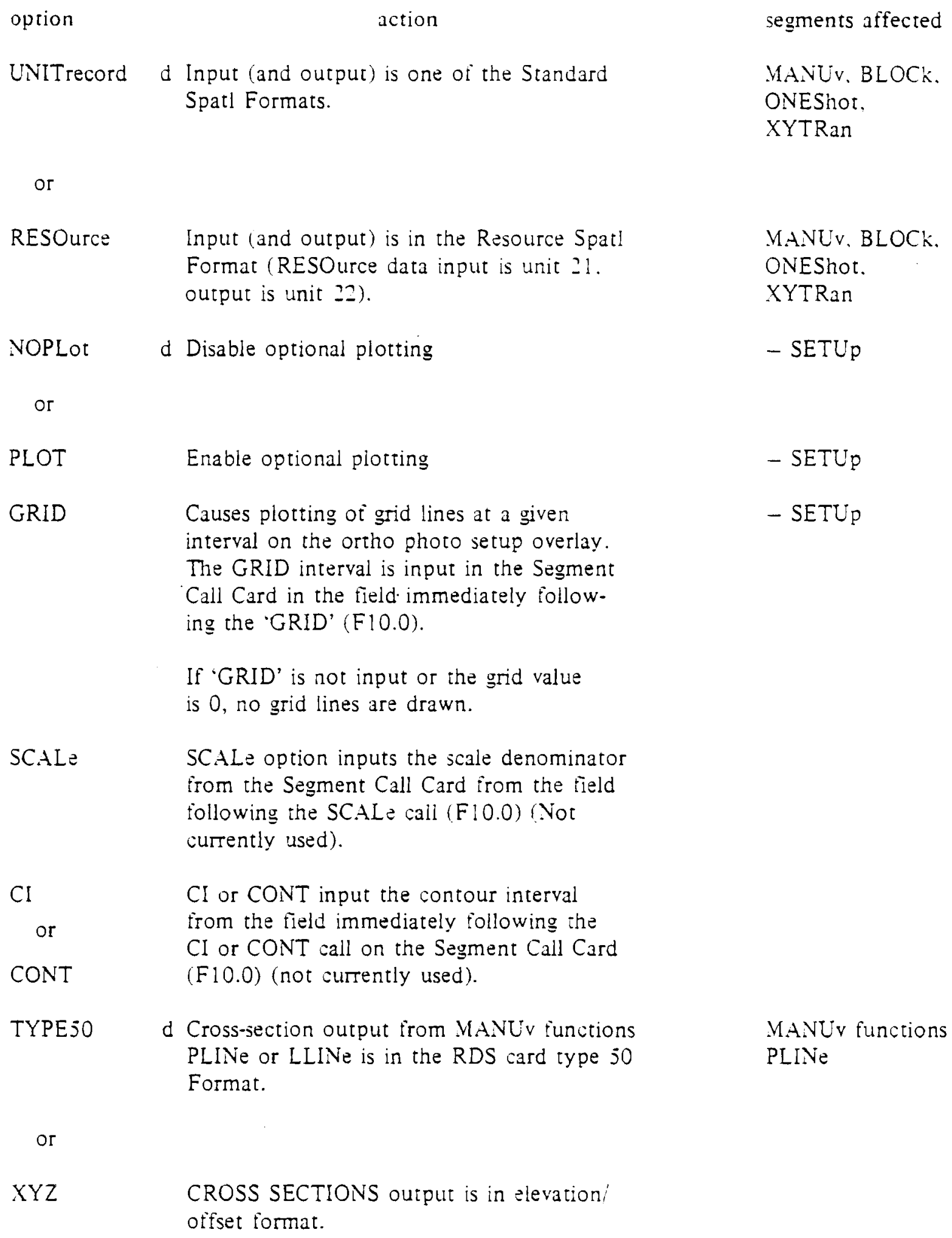

option

action UNITrecord d Input (and output) is one of the Standard
Spatl Formats.

or

RESOurce Input (and output) is in the Resource Spatl Format (RESOurce data input is unit 21 . output is unit 2?).

NOPLot d Disable optional plotting

or

PLOT

GRID

SCAL

$\mathrm{Cl}$

or

CONT

TYPE50

or

$X Y Z$

Enable optional plotting

Causes plotting of grid lines at a given interval on the ortho photo setup overlay. The GRID interval is input in the Segment Call Card in the field immediately following the 'GRID' (F10.0).

If 'GRID' is not input or the grid value is 0 , no grid lines are drawn.

SCALe option inputs the scale denominator from the Segment Call Card from the field following the SCALe cail (FIO.0) (Not currently used).

CI or CONT input the contour interval from the field immediately following the $\mathrm{Cl}$ or CONT call on the Segment Call Card (F10.0) (not currently used).

d Cross-section output from MANUv functions PLINe or LLINe is in the RDS card type 50 Format.

CROSS SECTIONS output is in elevation offset format.

segments affected

MANUV, BLOCk. ONEShot, XYTRan

MANUV, BLOCK. ONEShot. XYTRan

- SETUp

- SETUP

- SETUp

MANUr functions PLINe 
option

action

segments affected

IN80

Input 80 series points through the card reader

MANUV

while terrain data is blocked TDF input. This

option, when used in conjunction with the

default option BLOCk on the Segment Call

Card MANUv, allows input of the 80 series

points in Standard Spatl Format through the

card reader, and the terrain data through

Unit 9 (Blocked TDF) during Function STOF1.

A 98 card signals the end of the 80 series points. If the Option IN80 appeared on the

MANUv Segment Call Card, STOFl expects a deck of 80 series points to follow the Project

Definition Card.

\section{Identification Code (KODE) and Formats for Data Points}

KODE. KODE is used throughout DTIS. It is the first two characters of the terrain point identification number. The values of KODE are assigned as follows:

\section{KODE}

80

$81^{\circ}$

82

83

84

87

86 point identifieation

Tie points between strips of photography

Pass points between models

Vertical control points

Horizontal control points

Horizontal and vertical control points

Points used to determine airplane point

Computed airplane points

Terrain data

Less than 80

Range Checking. Range checking is used throughout DTIS to throw out erroneous records. Any points that do not tall within specified limits are "thrown out." The range limits are determined by the following methods:

range check value

function

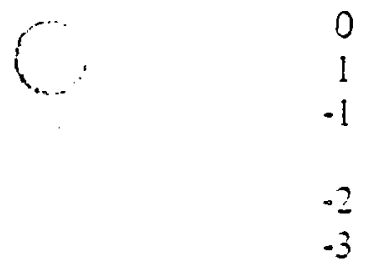

Range limit developed from 12 airplane points (87)

Use the first six cards in the input deck

Range values on following eard (Format 6F10.2 HX, LX. HY, LY, HZ, LZ)

$-2$

Use Preset Values 6000.00 and $\$ 000.00$ as range limits.

Disable range checking 
Card Formats. There are + ard tormats retered io throughout DTIS (Exhibit +2 ). They are are as follows:

\section{Standard Spatl:}

$c c$
$1-2$
$3-10$
$11-20$
$21-30$
$31+0$

Packed Spatl:

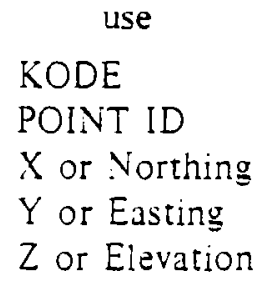

KODE

POINT ID

$\mathrm{X}$ or Northing

$Y$ or Easting

$Z$ or Elevation

KODE

POINT ID

$X$ or Northing

$Y$ or Easting

$Z$ or Elevation format

12

18

F10.2

F10.2

F10.2

Digitizer Spatl:

$c c$
$1-2$
$3-8$
$9-14$
$15-20$
$21-26$
27
$28-29$
$30-35$
$36-41$
$42-47$
$48-53$
54
$55-56$
$57-62$
$63-68$
$69-74$
$75-50$

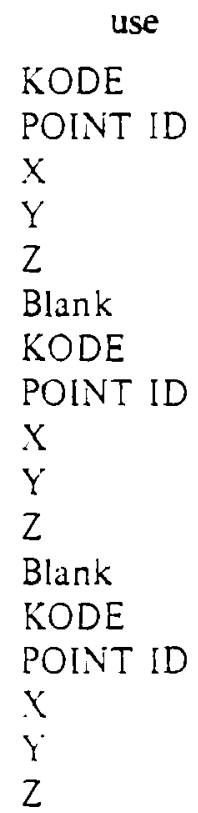

format

I

I6

F6. 2

F6.2

F6.2

I. $\mathrm{X}$

I2

16

F6.2

F6.2

F6.2

IX

I2

16

F6.2

F6.2

F5.2

8

10.2

F10.2

10.2

2

10.2

F10.2

F10.2 
Resource Spatl: (R-5)

cc

$1 \cdot 2$

$3 \cdot 10$

$11-20$

$21-30$

3140

$41-112$ use

format

KODE

POINT ID

12

I8

$X$ or Northing

F10.2

$Y$ or Easting

$Z$ or Elevation

Resource Data
F10.2

F 10.2

$12 \mathrm{~A} 6$

\section{ONEShot}

ONEShot is a simplified version of R. O. Mahan's program SPATL. It is used to transform model coordinates measured in a stereoplotter to a ground coordinate system. It is not an aerotriangulation program.

\section{INPUT}

Refer to Exhibits 4.3 and 4.4.

\section{$\cdot$ RUN}

Time -15 seconds per model -1 minute minimum

Pages -10 per model -100 minimum

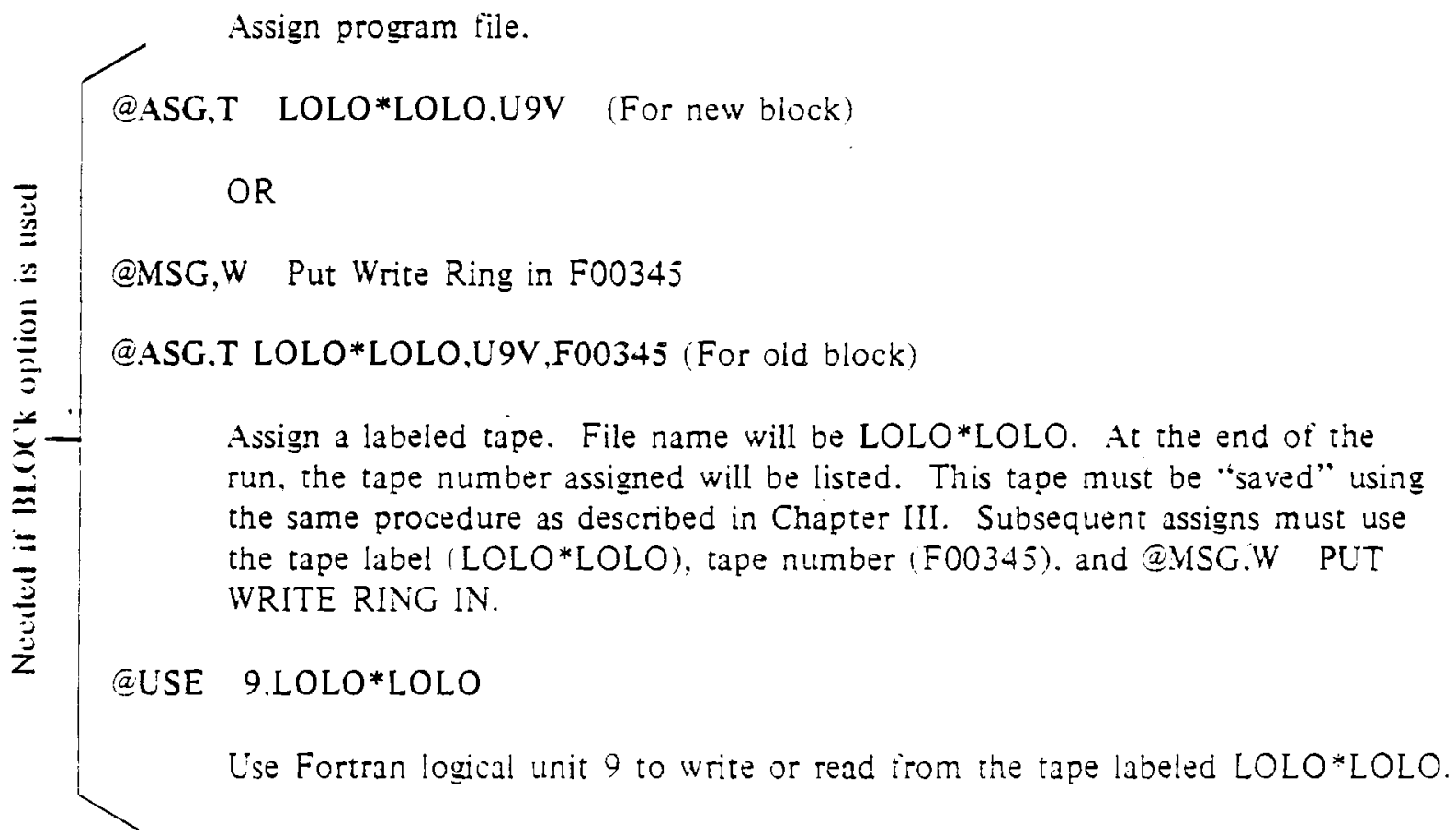


@XQT DTIS*ABS.DTIS

$B-56$

Execute the mainline program:

$\begin{array}{llll} & (\mathrm{XYZ}) & \text { (NOBLock) } & \text { (RESOurce) } \\ \text { ONEShot } & \text { NEEL } & \text { BLOCK } & \text { UNITrecord }\end{array}$

Directs program to the ONEShot segment. NEEL, BLOCk. and UNITrecord are default options: XYZ. NOBLock (card image), and RESOurce are default disabling options and must be specified if desired.

Title Card: (see Exhibit 4.5)

cc

use

format

$1-60$

Title

$10 \mathrm{~A} 6$

$61-69$

Blank

$9 x$

70

Block file status

I1

$1=$ new

$0=$ old

(Exercise caution as your data tile created in a previous run can be wiped out with a 1 in cc70)

$71-80$

Rejection limit (standard error of the

transformed model coordinates above this

value will cause segment termination.

There will be no output for this model.

but all previous model output will be preserved). This valve should be approximately 1 part in 2500 of the flight height. 
Ground Control Cards: (up to 500)

Input is in Standard Spatl Format.

cc

use

format

$1-2$

KODE

I

$3-10$

ID

18

$11-10$

Coordinates of Control point

3F 10.2

(X, Y.\& Z if XYZ option. North.

East and Elevation if NEEL option)

98 Card:

A "98" card tells the program that it has completed reading ground control

points.

Model Title Card: (see Exhibit 4.5)

ce

1

$\checkmark i$

$\therefore \quad 56-78$

$79-80$ use

format

Record type of model coordinates

II $1=$ Standard Spatl Format

2=Packed Spatl Format

3=Digitizer Spatl Format

$4=$ Resource Spatl Format

Left Plate Number

13

Right Plate Number

Strip Number

I4

14

Model Title (Alphanumeric) $3 A 6$

Blank

Switches

$9 \mathrm{X}$

1611

(In general, leave blank. For further

information, see R.O. Mahan documentation

on SPATL.)

Blank

$23 \mathrm{X}$

Range Checking

\section{Model Coordinates (Unlimited Number)}

Model coordinates are in one of the four Spatl Formats which has been selected in column 1 of the "Model Title Card." This deck contains model coordinates for all points - pass. tie. airplane. control, and terrain points. The model coordinates with KODE values of $80-87$ must be at the fromt of the deck. The coordinates are in X, Y. and $Z$ (north, east, and elevation is not a valid input for model coordinates). 
98:

This "98" card indicates the last model coordinates have been read in.

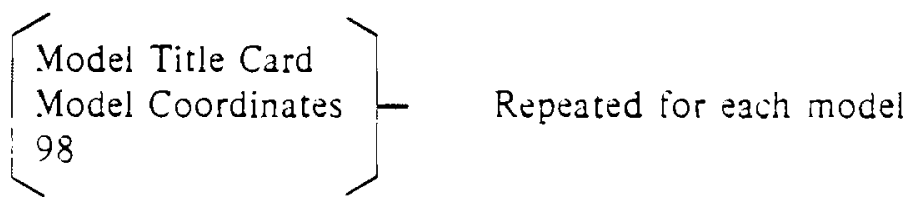

Blank Card

A blank card signifies the end of the segment ONEShot and returns control to the mainline program.

END:

Segment call to END makes a normal exit from DTIS.

$@ F I N:$

System end of run.

If Resource Spatl Format data is to be entered, it must already be in a catalogued file at FCCC; i.e., data could be entered using a @DATA,I SIXRIVERS*SIX. The file is read from logical unit 21 and written on logical unit 22.

Sample:

@ASG,A SIX*SIX
$@$ USE 21,SIX*SIX
@ASG,UP SIX*SIXGRND,U9V
@USE 22,SIX*SIXGRND

\section{OUTPUT}

There are three possible outputs from ONEShot.

1. Packed Spatl Format output to logical unit 8.

2. Blocked TDF output to logical unit 9 . This can be a new blocked file or an addition to an existing blocked TDF.

3. Resource Spatl Formatted data to logical unit 22.

\section{BLOCk}

BLOCK is a program used to build a random terrain data file from ground coordinates entered through the card reader, or RESOurce data entered through logical unit 21. BLOCk collects input records into groups of up to 500 points, determines the Northing and Easting limits of the group, and outputs the block (group) on logical unit 9 . The program can be used very effectively with the Calma Digitizing System. The program can be used to build a new file or add to an existing file. 


\title{
INPUT
}

See Exhibit 4.6 for Sample Card Setup, 4.7 for Input Deck

$@ R U N$

$$
\begin{aligned}
& \text { Time - one minute per } 20,000 \text { points entered } \\
& \text { Pages - } 50 \text { pages }
\end{aligned}
$$

@ASG,A DTIS*ABS.

Assign program file

@ASG,T LOLO*LOLO.U9V (For new block)

OR

@MSG,W Put Write Ring in F00345

@ASG,T LOLO*LOLO,U9V.F00345 (For old block)

Assign a labeled tape. File name will be LOLO*LOLO. At the end of the run, the tape number will be listed. This tape must be "saved" using the procedure as outlined in Section 1 of Chapter III. Subsequent assigns must use the tape label (LOLO*LOLO) and tape number (F00345).

\section{@USE 9,LOLO*LOLO}

Use Fortran logical unit 9 to read or write from the tape labeled LOLO*LOLO:

\section{$@ X Q T$ DTIS*ABS.DTIS}

\author{
Execute the program \\ (XYZ) (RESOurce) \\ BLOCk NEEL UNITrecord
}

Directs the program to the BLOCk segment of the program. The input is defaulted to North, East, and Elevation. $X, Y, Z$ ground coordinate input must have an $X Y Z$ option in the BLOCk card. 
Title Card:

cc

$1-60$

$60-69$

70

71.80 use

format

Alphanumeric Title $10 \mathrm{A6}$

Blank

Block file status

$1=$ new

$0=$ old

Blank
$9 \mathrm{X}$

I1

$10 \mathrm{X}$

Data Cards:

Data cards in Packed Spatl Format.

98:

"98" terminates BLOCk segment of program.

END:

Normal exit from DTIS.

@FIN:

Normal end of job.

\section{OUTPUT}

Output is Fortran Unformatted records 2005 words long. The output Unit is unit 9. The record structure is:

word

1

$2-5$

$6-505$

$506-2005$ use

Number of positions this record

Max-Min North-East for the positions in this record 500 combined KODE-ID's 500 positions (N.E.EL)

\section{XYTRan}

XYTRan is used to transform $X$ and $Y$ coordinates in digitizer units to a ground coordinate system. An option within the prouram allows entry of control positions in geographic coordinates (latitude-tongitude) which are converted to state plane coordinates. (See Exhibits +.9 and +.10 tor sample setup.) 
$@$ @UN

Time - 15 seconds per 5000 points -1 minute minimum

Pages - 20 per model - 100 minimum

@ASG,A DTIS*ABS.

Assign program file.

@ASG,T LOLO*LOLO (new BLOCked data tape)

OR

@MSG,W PUT WRITE RING IN F00345

@ASG,T LOLO*LOLO,U9V,F00345 (old BLOCked data tape)

Assign a labeled tape. The file name will be LOLO*LOLO. At the end of the run, the tape number assigned will be listed. This tape must be "saved" using the same procedure as described in Chapter III. Subsequent assigns must use tape label (LOLO*LOLO) and tape number (F00345).

@USE 9,LOLO*LOLO

Use FORTRAN logical unit 9 to write or read from the tape labeled LOLO*LOLO.

\section{$@ X Q T$ DTIS*ABS.DTIS}

Execute the mainline program

$\begin{array}{cccccc}\text { XYTRan } & \text { XYZ } & \text { NOBLock } & \text { RESOurce } & \text { FORCor } & 0502 \\ & \text { NEEL } & \text { BLOCk } & \text { UNITrecord } & \text { (BLANK) } & \text { (BLANK) }\end{array}$

This directs program to XYTRan segment. Options NEEL. BLOCk. and UNITrecord are default options - XYZ and RESOurce must be specified if desired. If control file (described later) is to be input in geographic position, FORCor must be specified, and if specified, the state plane zone number (see Exhibit 4.8) must be left registered in the next field. 
Title Card:

cc

$1-68$

$69-77$

$78-79$

80 use

Alphanumeric Title

Blank

Range Checking Switch

Block file status

$$
\begin{aligned}
& 1=\text { New } \\
& 0=\text { Old }
\end{aligned}
$$

format

$11.46 .+2$

$9 \times$

12

[1

Ground Control Cards: (map identifiable ground coordinates or grid ticks)

$$
\begin{gathered}
c c \\
1-2 \\
3-10 \\
11-40
\end{gathered}
$$

use

format

KODE

ID

Position

a. If FORCor option is not specified position is in X. Y.Z. or North. East. Elevation (Standard SPATL format)

b. If FORCor option is specified. position is in:

Degrees latirude

Minutes latitude

Seconds latitude

Degrees longitude

Minutes longitude

Seconds longitude
12

18

3F 10.2

F3.0

F. 0

F5.3

F3.0

F 2.0

$\mathrm{F} 5.3$

98

End of Ground Control Cards

Model Coordinates of Ground Control

Input can be in "Standard SPATL" or "RESOurce SPATL" tormats. Coordinates must be in digitizer units. These coordinates are not output.

98

End of Model Coordinates of Ground Controi 
Model Coordinates to be Transformed.

Input can be in "Standard SPATL" or "RESOurce SPATL" format. The X and $Y$ coordinates are in digitizer units, and the $Z$ is not transformed. and therefore, must be in ground units.

98:

End of the model coordinates to be transformed.

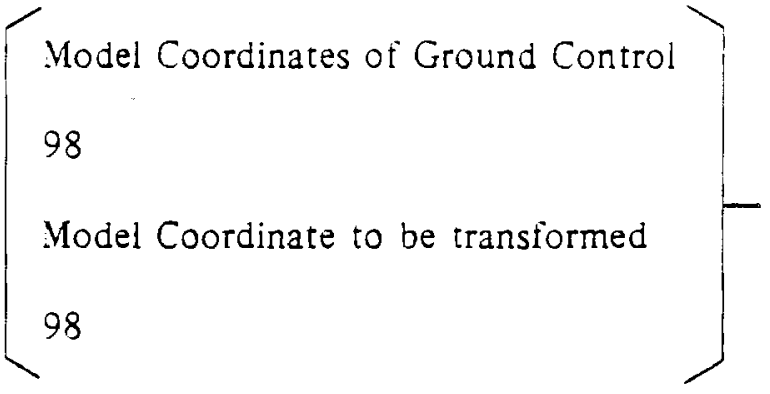

Repeat as needed

98:

Two "98" cards make a normal exit from XYTRan segment.

END:

Normal exit from DTIS

$@$ @IN:

Normal end of job

\section{OUTPUT}

The output from XYTRan is identical with ONEShot.

\section{MANUv}

\section{Function Programs in MANUv.}

1. STOF1 - the function program STOF1 is used to collect digital terrain data into "boxes" of a given size and store them for use by other MANUv function programs. A maximum of $\$ 0$ terrain data points may be stored in one box. STOF1 is used to extract a small set of terrain data from a large data file, and to box the data according to geographical regions for efficient processing. These files are to be further subdivided into Work Areas by function program FILL.

2. FILI - the function program FILI enters the direct access files created by STOF 1 and collects a still smaller area (called the Work Area) of "boxed" data for use by orher function programs in MANUv. Exhibit 4.11 illustrates the application of STOF1 and FILL. A large existing file could contain all the random digital terrain data for an entire narional forest. STOF 1 
is used to extract from this file, a small set of the data and create a "Project data file", and FILL is used to extract a smaller subset of the "project data file" and store it as a "work area data file".

3. SETUp - this function program accesses the " 80 " series file created by function program STOFl and outputs a table of point coordinates that are needed to set up the GZ-1 for ortho photo production. Optionally, it can also be used to produce a plot tape to make the ortho photo overiay.

4. ORTHo - generates north-running profiles from the "Work Area data file" at a specified interval.

5. PRORt - inputs from the direct acces file generated by ORTHo. and generates a plot tape for the preparation of an ortho photo storage plate for the GZ.1.

6. GRID - inputs from the direct access file generated by ORTHo and converts the profiled data to gridded data. The ourput product is a gridded data file for the work area described by the input to function FILI.

7. VGRId - inputs from the direct access file generated by ORTHo, and converts the profiles data to the properly spaced formatted grid for input into PSW's version of VIEWIT.

8. PLINe - produces P.Line cross-section elevations for road design applications. The output will be cross-section elevations in the standard RDS CT50 format.

9. CAT or PROFile - produces ground profiles for the skyline logging program analysis $\mathrm{Sky} 18$.

MANUv Segment Call Card. Program segment MANUv is called into execution by the use of the following segment cail card:

\section{MANUv $\begin{array}{ccc}\text { (NOBLock) } & \text { (XYZ) } & \text { (RESOurce) } \\ \text { BLOCk } & \text { NEEL } & \text { UNITrecord }\end{array}$}

The default options are BLOCk, NEEL, and UNITrecord; which means that the input data are BLOCked and are defined in Northing, Easting, and Elevation coordinates. The default option UNITrecord means that the data input is to be in the UNITrecord format when the NOBLock option is used.

The MANUv call card is followed by cards calling for the function programs. The call cards for each function program have different requirements and are described separately in the following sections.

\section{SPECLAL DEVICES NEEDED}

MANUv always references unit 20 which contains a backup file either before or after the first function (STOFl) is called. If plotting is to be done (Functions PRORt or SETUp), unit 7 must be assigned. RESOurce input is on unit 21, or BLOCked input is on unit 9, or UNITrecord 
(Packed Spatl Formatted) data is on the card reader. Card image output (CAT, GRID. VGRID, PLINE) is to unit 8.

STOF1. This function collects digital terrain data into 'boxes' of a given size and stores them for use by the other MANUv functions. It creates a backup file of the boxed data. Optionally, it can restore from an old backup.

STOF1 uses temporarily (dynamically) assigned DEFINED FILES as direct access files. It creates these files and stores the data in them for the other functions to use. STOF1 inputs the terrain data, sorts and 'boxes' it, and stores the 'boxed' data to a maximum of $\$ 0$ points per box on a direct access file. It also inputs ' 80 ' series points and stores them on another dynamicallyassigned, sequentially-accessed file. When all the data has been stored, STOFl backs these files up by writing them to unit 20 . During a restore function. it reads unit 20 and restores the files.

\section{INPUT}

STOFl requires a call card, a control card, and a file of terrain data.

Call Card:

cc

entry

$1-5$

\section{STOF 1}

The call card contains no input/output options. All options pertaining to input data must have been input on the MANUv Segment call card.

Control Card: (see Exhibits 4.12 and 4.13 for graphic representation of variables)

\begin{tabular}{ccc}
$c c$ & \multicolumn{1}{c}{ use } & format \\
$1-10$ & 'Box' biock size DIF & F10.0 \\
$11-20$ & Project SW Cor. (N) SWCN & F10.0 \\
$21-30$ & Project SW Cor. (E) SWCE & F10.0 \\
$31-40$ & $\begin{array}{l}\text { Angle of rotation (dec. degrees) } \\
\text { (See Exhibit 4.12) }\end{array}$ & F10.0 \\
$41-50$ & Project size or NE Cor. (N) NEC. & F10.0 \\
$51-60$ & Project size or NE Cor. (E) NECE & F10.0
\end{tabular}

If the block size (DIF) is 0 , the restore function is enabled. If DIF is non-zero, the collect and store function is enabled. 
If the Project size is 0 , the Project size defaults to 1.000 .000 . A file must be assigned to the backup data unit (20) whenever MANUv is called. because this unit is referenced either to create the backup, or to restore from the backup. Function STOFI must always be the first function called.

If the input terrain is Resource Spatl Format, it is assigned to unit 21. If the input terrain is UNITrecord (Packet Spatl Format) it is input through the card reader. terrain data.

In the case of UNITrecord (Packed SPATL) input. an EOF card (98) must follow the

If the input is blocked TDF, it is input througn unit 9.

\section{OUTPUT}

During a store function, a file is written to unit 20.

FUL. This function accesses the files created by STOF1 and collects a 'Work Area' of 'boxed' data for use by other functions. Function STOFI must have been previously called.

\section{INPUT}

Call Card:

$c c \quad$ entry

$1+\quad$ FILL

Boundary Card: (see Exhibit 4.12 for graphic representation of variables)

\begin{tabular}{|c|c|c|}
\hline$c c$ & use & format \\
\hline $1-10$ & $\begin{array}{l}\text { Distance } . v \text { irom the Project SW } \\
\text { Corner DELDN }\end{array}$ & F10.0 \\
\hline $11-20$ & $\begin{array}{l}\text { Distance E trom the Project SW } \\
\text { Corner DELDE }\end{array}$ & F 10.0 \\
\hline $21-30$ & SCALE expressed in FT $=1 \mathrm{INCH}$ & F 10.0 \\
\hline $31+0$ & Size $N$ of the 'Work trea' $D Y$ & $F 10.0$ \\
\hline $41-50$ & Size $E$ of the 'Work trea' DE & $\mathrm{F} 10.0$ \\
\hline $51-60$ & $\begin{array}{l}\text { Distance between protilles SCV. } \\
\text { If SCV is less tian } 10 \text { it is } \mathrm{mm} \\
\text { measured at SCALE. it it is } 10 \text { or } \\
\text { geater. it is in ground units. }\end{array}$ & F 10.0 \\
\hline
\end{tabular}




\section{OUTPUT}

FILI produces a 'printer map' showing the number of positions in each 'box' in the local area. If the number of positions is 50 in a large number of boxes, the value of DIF was too large, and data is being lost. If the number of positions is 0 in a large number of boxes, the area was not fully digitized, or the value of DIF is too small. Try to average 25 in all boxes.

SETUp. This function accesses the ' 80 ' series file created by function STOFl, and produces a table of coordinates needed to set up the GZ.1 to produce an ortho photo. Optionally, it can aiso produce a plot tape to make the ortho photo overlay.

\section{INPUT}

\section{Call Card:}

cc

use

format

$1 \cdot 5$

SETUp - Call function program.

$11 \cdot 20$

PLOT (optional) - Produce a plot tape to make the ortho photo overlay. The graphical plot would include the data points to be used for setting up the GZ-1. If this field is left blank, no plot tape would be produced.

$21 \cdot 30$

GRID (optional) - The plot tape will also include grid lines, and the grid size is specified in the next field.

$31-40$

GRID size

F10.0

Function programs STOF1 and FILL must have been previously called.

Identify Airplane Point Number:

cc

use

format

$1 \cdot 2$

KODE

ID (the airplane point number)

I2

18

\section{OUTPUT}

The table of setup coordinates for the ortho photo projector. The piot tape is optional.

ORTHo. ORTHo produces North-running profiles from the bottom to the top of the Work Area and progresses East, at 1 SCN at a time, across the area. This data is stored for gridding and or storage plate output by other MANUv functions. 
Functions STOF1 and FILI must have been previously called (Exhibit +.14 ).

\section{INPUT}

Call Card:
$\mathrm{cc}$
entry
$1 \cdot 5$
ORTHO

The call card contains no Input/Output options.

\section{OUTPUT}

The sets of coordinates describing the profiles are stored on a direct access file for use by functions PRORt and/or GRID.

PRORt. This function inputs from the direct access file generated by function ORTHo and generates a plot tape for the preparation of an ortho photo storage plate for the GZ-1.

Functions STOF1, FILL, SETUp, and ORTHo must have been previously called.

\section{INPUT}

Call Card:

$\begin{array}{ll}c c & \text { entry } \\ 1-5 & \text { PRORt }\end{array}$

This call card contains no input/output options.

\section{OUTPUT}

PRORt generates a plot tape.

GRID. GRID is called with the Segment Call Card 'GRID'. This function inputs from the direct access file generated by function ORTHo and converts the profiled data to gridded data. It then accesses the ' 80 ' series file and extracts all the points which fall inside the Work Area, and produces a card image file containing all the pertinent information about the Work Area, the ' 80 ' series points, and the gridded data deck. and 4.16).

Functions STOFl, FILL, and ORTHo must have been previously called (Exhibits 4.15

\section{INPUT}

None 


\section{OUTPUT} FIII.

GRID produces a gridded dara deck for the Work Area described by the input to function

\section{VALID INPUT-OUTPUT OPTION}

\section{None}

PLINe. PLINe is called with the Segment Call Card 'PLIN'. This call will read from the card reader the coordinates of points of intersection (PI's), the length, and the distance between the desired cross-sections. Using this information, the terrain data selected by functions STOF1 and FILL are interrogated to generate the elevations on breaks along the cross-sections.

\section{INPUT}

Call Card:

$\begin{array}{ll}c c & \text { entry } \\ 1-5 & \text { PLINe }\end{array}$

This vall card contains no input/output options.

Road Number Card:

cc

$1-10$ use

Road number for RDS format

Beginning Station Card:

Beginning Stationing i.e..

$$
\begin{aligned}
& 0+00.00=0.0 \\
& 1+23.22=123.22
\end{aligned}
$$

PI Cards: (as many as needed up to 50)

$\begin{array}{cl}1-2 & \text { Line Number } \\ 3-4 & \text { PI Number } \\ 5-10 & \text { Blank } \\ 11-20 & \text { Northing } \\ 21-30 & \text { Easting }\end{array}$

I2

12

$6 \mathrm{X}$

F 10.1

F 10.1

98:

End of entry of PI's 
Cross-section Spacing Card:

cc

use

format

$1-10$

Cross-section Spacing

F 10.0

Cross-section Length Card:

cc

use

format

$1-10$

Cross-section Length Each Side

(Maximum of 999 Feet)

F 10.0

\section{OUTPUT}

8.

Output from PLINe is cross-sections formatted for use in RDS. Output is on logical unit

VGRId. VGRId inputs from the direct access file, generated by function ORTHo, and converts the profiled data to the properiy spaced and formatted grid to go into PSW's version of VIEWIT. This gridded data is output to a file on logical unit 8 , which should be catalogued.

Functions STOF1. FILL, and ORTHo must have been previously called.

\section{INPUT}

Call Card:

$\begin{array}{ll}\text { cc } & \text { entry } \\ 1-5 & \text { VGRId }\end{array}$

The call card contains no Input/Output options.

\section{OUTPUT}

VGRId produces a file which can be read into PSW's VIEWIT with the following options: BY COLS, FORMAT = '(16F5.0)'.

PROF1 or CAT. This function accesses the "Work Area data file" of "boxed" data collected by functions STOF1 and FILL to produce a ground protile between two points. This profile information is output in the format acceptable to the skyline logging analysis program, SKY18.

Functions STOF1 and FILL must have been previously called. 


\section{INPUT}

Call Card:

cc

entry

$1-5$

CAT or PROFl

This call card contains no Input/Output option.

Data Cards:

ce

use

format

KODE must be greater than zero and less than 90 for a data card

Two data cards are required to describe a profile, one for each end. As many profiles as desired may be generated with a single call to this function. Each profile requires 2 position cards. This function is terminated by a blank or 98 card. Controi is returned to MANUv.

\section{OUTPUT}

CAT writes card image data to file 8 in the format acceptable to the skyline logging analysis program, SKY18. Each profile is output in 2 type records. Record type 1 is a partial header record which contains the position along the profile of the 2 ends and their elevations.

$\mathrm{cc}$

$1-10$

$11-15$

$16-20$

$21-25$

$26-30$

$31-35$ use

format

Not used

Distance from beginning of profile $(0)$

Elevation of beginning point

Not used

Distance to end of protile

Elevation of end point
$10 \mathrm{X}$

15

15

$5 \mathrm{X}$

15

15

Record type 2 contains distance and elevation iniormation of all detected terrain breaks on the protile.

Each card contains 8 distance and elevation sets. 
cc

use

format

$1-5(11-15 . \quad$ Distance from beginning oí profile

$21-25)$ etc.

to a terrain break

6-10 (16-20. Elevation of the terrain break

I5

26-30) etc.

ARAY. The program segment ARAY provides a link between the DTIS and the contouring program FSGPCP inside TOPAS. It reformats gridded data deck or decks generated by the function program GRID in MANUv for direct input to the contouring program FSGPCP as a single grid (ARAY block).

\section{INPUT}

Segment Call Card: (see Exhibit 4.18)

$\begin{array}{ll}\text { cc } & \text { entry } \\ 14 & \text { ARAY }\end{array}$

The call card contains no Input/Output options.

Boundary Card:

\begin{tabular}{|c|c|c|}
\hline $\mathrm{cc}$ & entry & format \\
\hline $1-10$ & S.W. Corner $(\mathrm{V})$ of the $A R A Y$ block & $\mathrm{F} 10.0$ \\
\hline $11-20$ & S.W. Comer (E) of the ARAY block & F10.0 \\
\hline $21 \cdot 30$ & $\begin{array}{l}\text { V.E. Corner }(N) \text { of the ARAY block } \\
\text { or size } \quad(N) \text { of the ARAY block }\end{array}$ & $\begin{array}{l}F 10.0 \\
F 10.0\end{array}$ \\
\hline $31+0$ & $\begin{array}{l}\text { N.E. Corner }(\mathrm{N}) \text { of the ARAY block } \\
\text { or size }(\mathrm{N}) \text { of the ARAY block }\end{array}$ & $\begin{array}{l}F 10.0 \\
F 10.0\end{array}$ \\
\hline
\end{tabular}

If the Size (N.E. Cor.) fields are left blank, these values default to 1,000,000. If the S.W. Cor. fields are left blank, the values default to the Work Area S.W. Cor. of the first MANUv gridded data deck input.

\section{Gridded Data Decks from MANUv:} GRID.

The MANUv gridded data decks are unchanged from the o/p of MANUv function 


\section{End of File Card:}

The End of File Card is a blank or a 98 card.

Operation. ARAY inputs the boundary card and the 4 control cards from the first MANUv gridded deck. and from this information, sets the constants for this call of the segment. These constants are: ARAY S.W. Cor., ARAY size, grid size, number of rows and columns in the ARAY block, MANUv Project S.W. Cor., and the row and column offsets from ARAY S.W. Cor. to Project S.W. Cor.

Failure of any MANUv Project S.W. Cor. or Grid Size to compare with these constants causes a segment termination without any ARAY block output.

The grid points which fall within the ARAY block are put in a holding array, and the next MANUv gridded data deck is input and processed.

When an EOF card is encountered, the segment preps and outputs the ARAY block.

\section{OUTPUT}

ARAY outputs ARAY blocks in the Calcomp GPCP ARAY type 2 and type 3 formats, and a BEND card.

FORCor. FORCor converts State Plane Coordinates ( $\mathrm{x}, \mathrm{y}$, elevation) to geographic coordinates (longitude, latitude, and elevation) or vice versa. It can also convert State Plane coordinates from one zone to another.

FORCor is called into exectuion by the following segment call card.

$\mathrm{cc}$

entry

$1 \cdot 6$

FORCor

The call card contains no I/O option. If card image output of the transformed coordinates is desired, it can be specified in the header card as explained in the documentation which follows.

\section{FORCor}

Description. Given Geographic Coordinates or State Plane Coordinates, this program will compute State Plane Coordinates or Geographic Coordinates in the State Lambert or State Transverse Mercator grid coordinate systems. The program will also compute a Zone-io-Zone transformation within a projection system. or from one projection system to another.

Header Card. The header card must signify the type of transformation desired. The Format for the header card is as follows (see Exhibit +.21 ): 
$\mathrm{cc}$

$1-4$

5

$6-9$

10

11

$12-14$

$15-80$ use

State Code of Input (0 if geographic coordinates)

Blank

State Code of Output (0 if geographic coordinates)

CAUTION - BOTH STATE CODE FIELDS CANNOT BE O

Blank

Number of records per card to be punched $(0=$ print only, 1 , or 2$)$

Blank

Alphanumeric identification

Data Card. The data card at this time is formatted for SPATL (Independent Model Bridging) and is as follows (see Exhibit +.22 ): 
cc

$1-$

$5-10$

$11-20$

$11-20$

$21 \cdot 30$

$21-30$

$31-40$

$41-76$

$70-80$ use

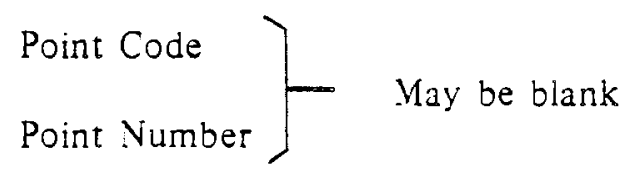

$X=$ Easting (feet to 2 decimals): Decimal understood between cc $18 \&$ cc 19

\section{OR}

Latitude (Deg, Min. Sec to 3 decimals); Decimal understood between cc 17 \& $c 018$

$\mathrm{Y}=$ Northing (feet to 2 decimals); Decimal understood between oc 28 \& cc 29

\section{OR}

Longitude (Deg, Min, Sec to 3 decimals): Decimal understood between cc $27 \&$ cc 28

Elevation (feet to ? decimals); Decimal understood between cc 38 \& $\approx c 39$

Ignored (May be used for alphanumeric identification)

Sequence number if desired

Each data deck must be followed by a 98 (cc 1-2) ard, and after the last 98 card, a @FIN card. (See sample deck set-up, Exhibit 4.23.) 


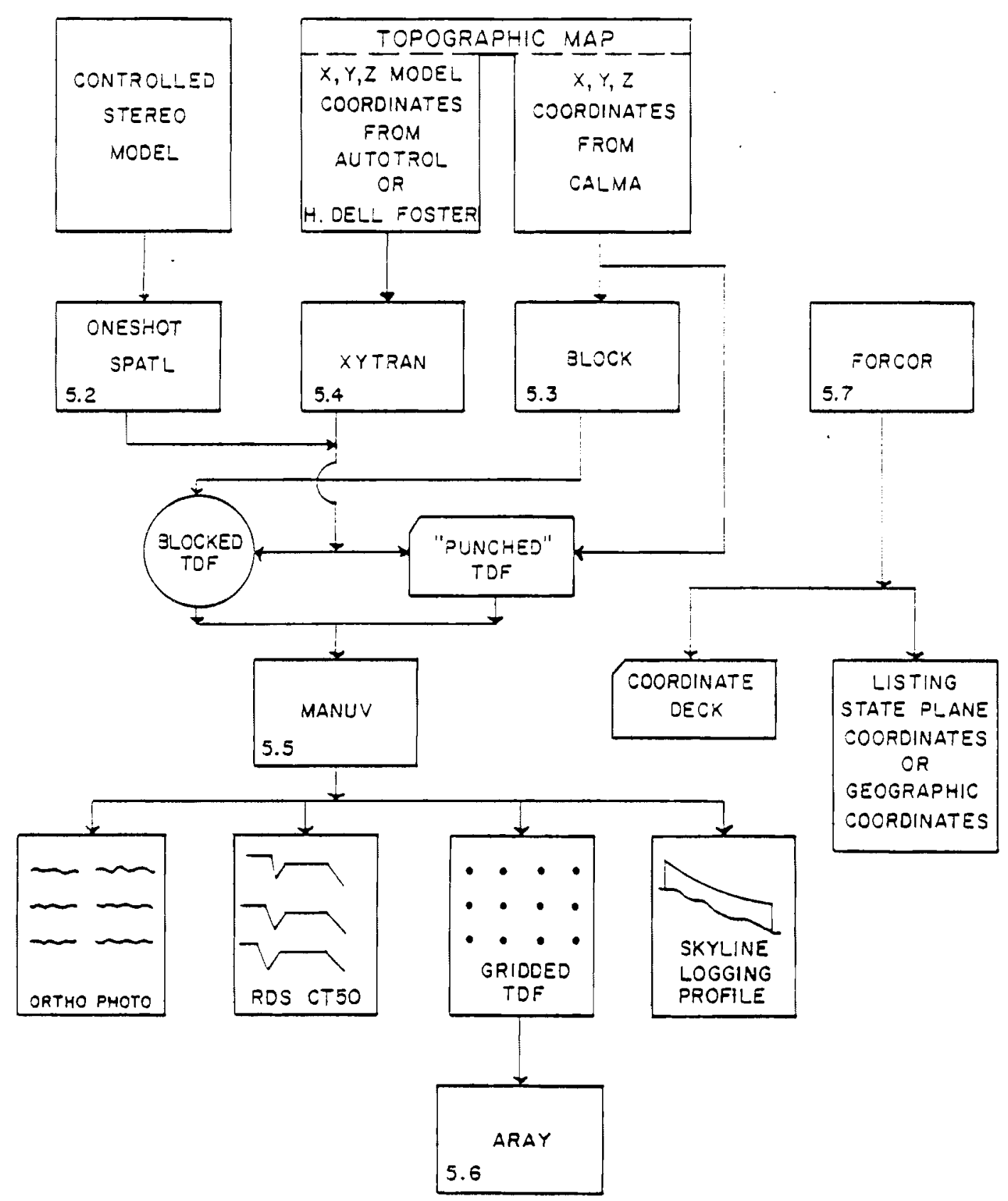

Exhibit 4.1 - Functional Relationsnip of OTIS Program Segments 


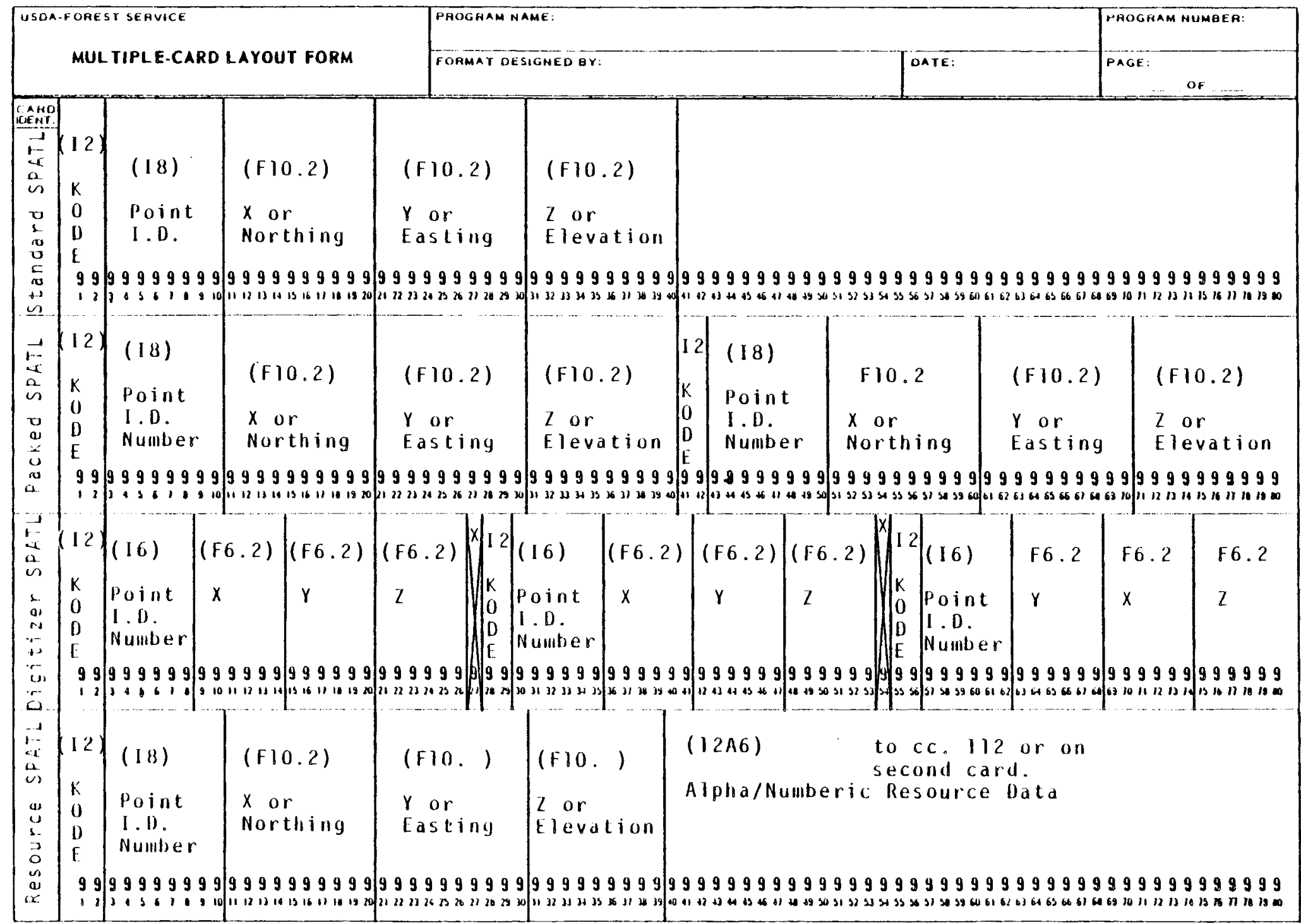


ONESHOT CIRD DECK SETEP

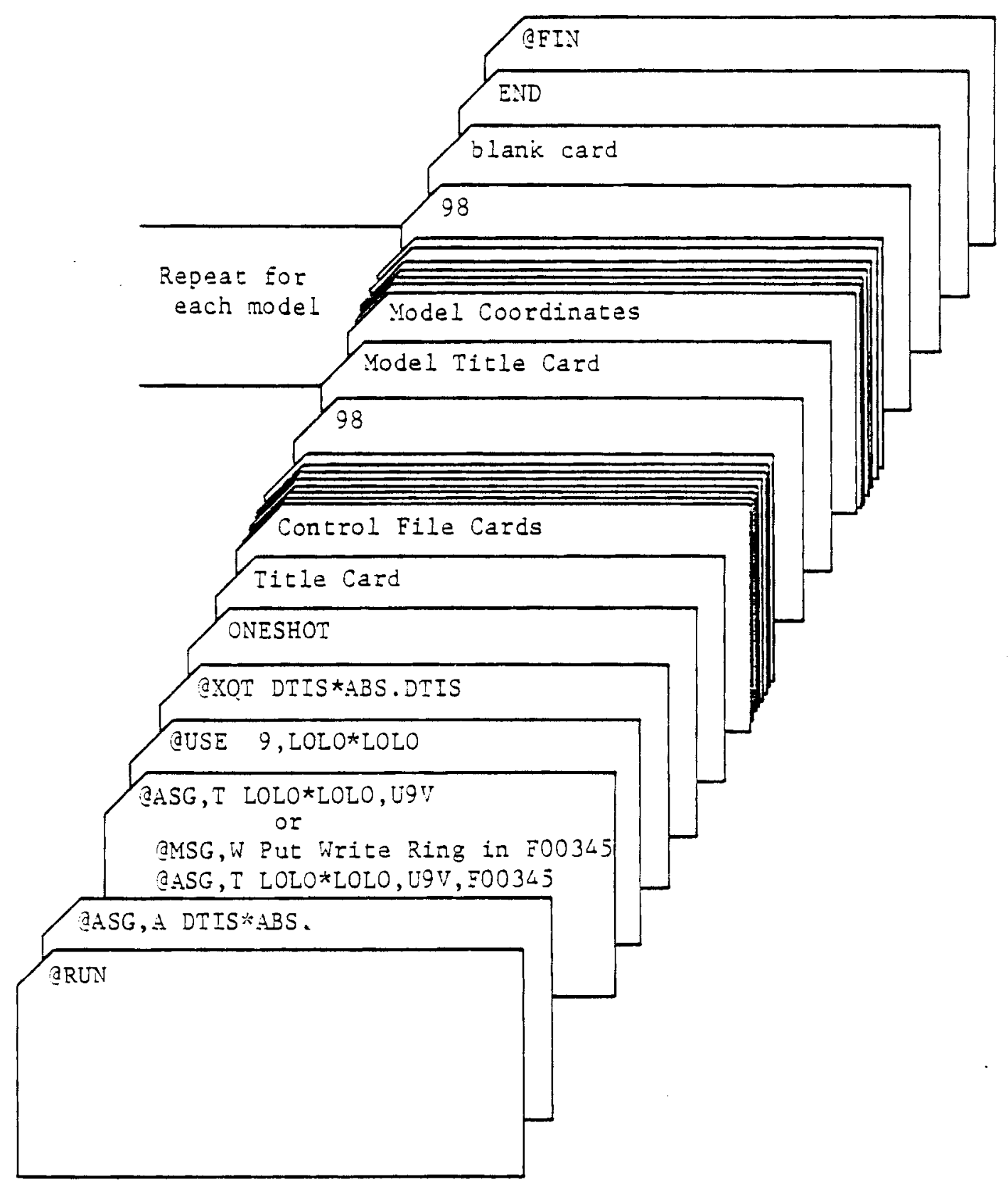

Exhibit 4.3 - ONEShot Card Deck Setup 


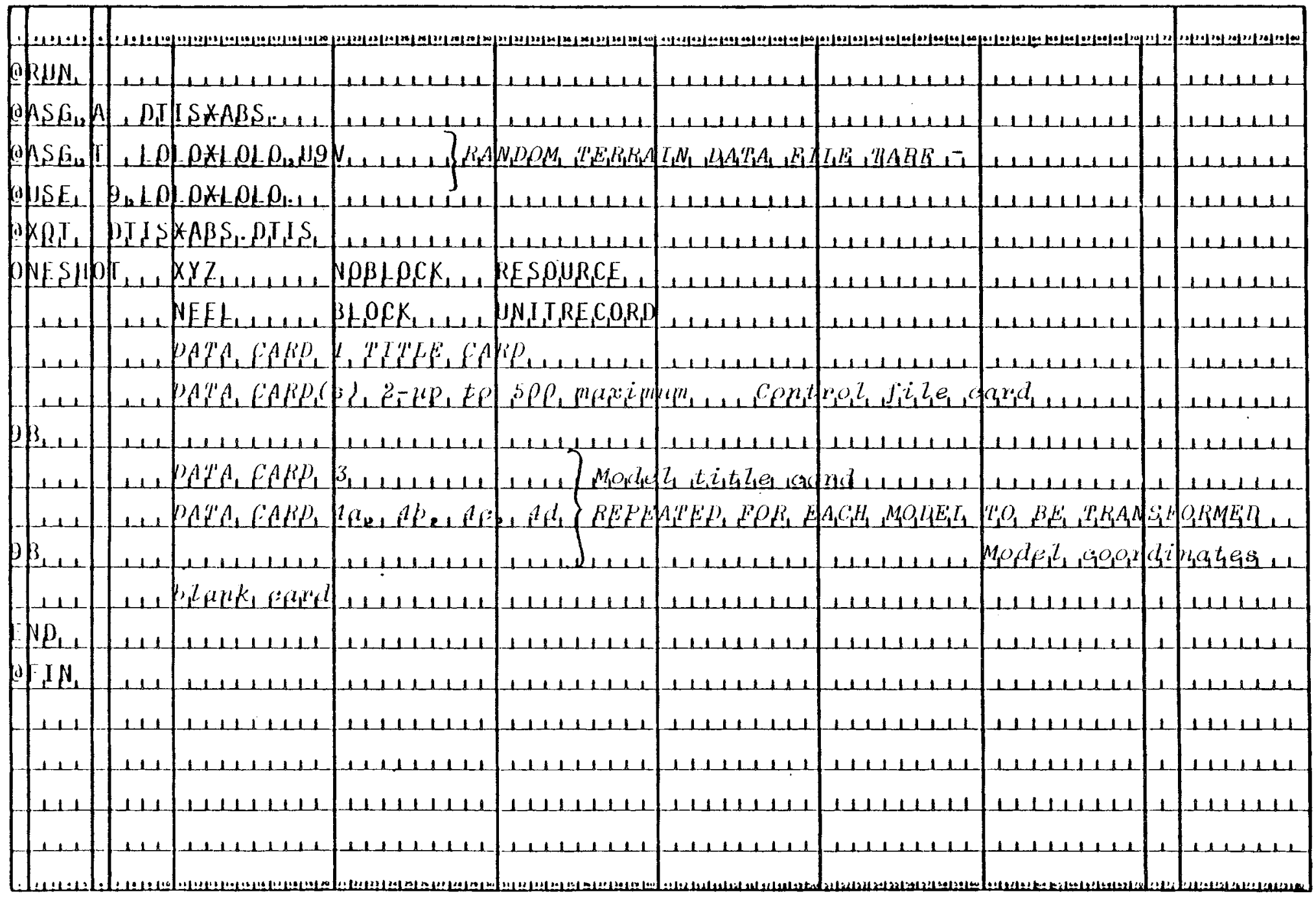




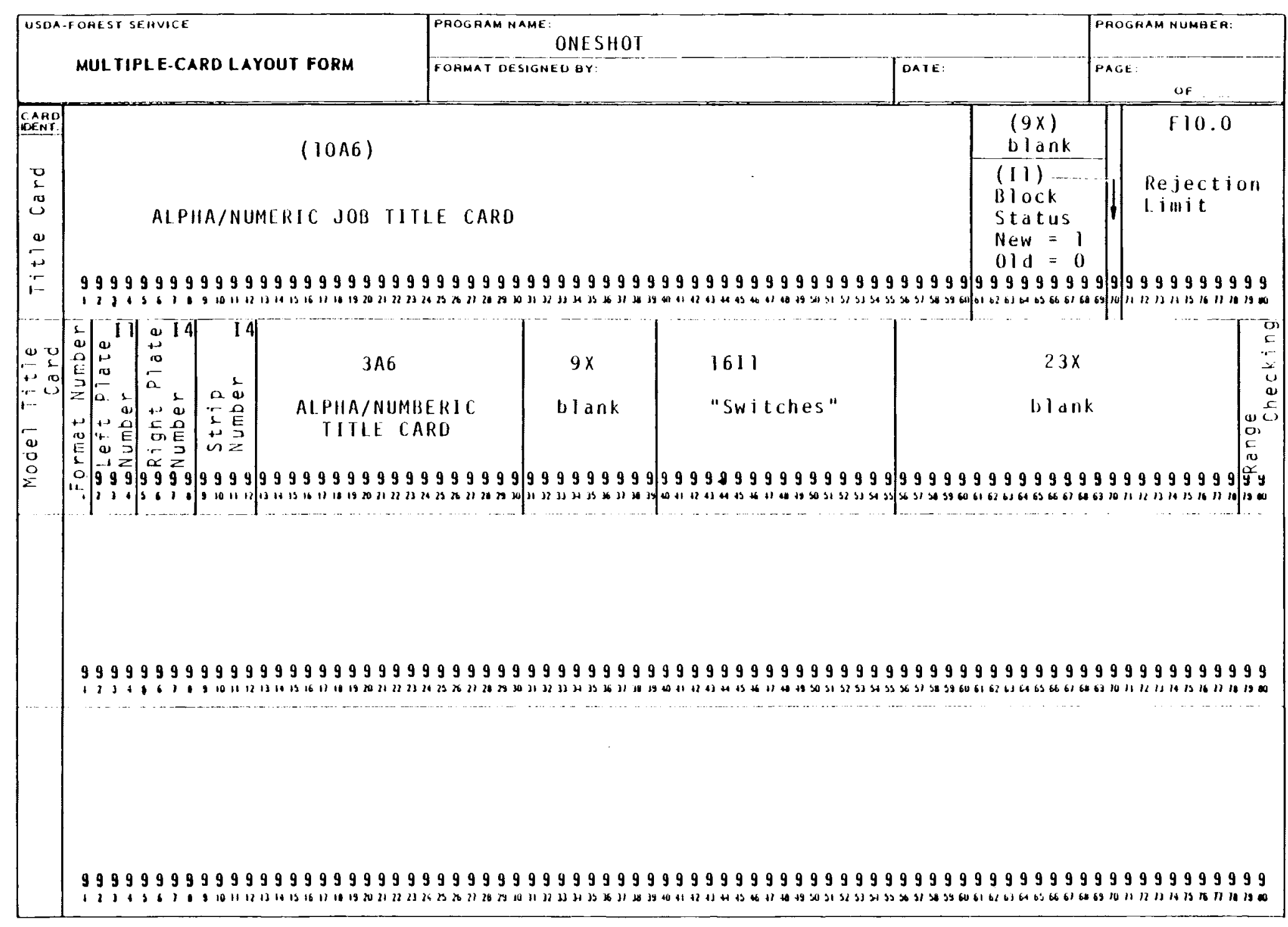




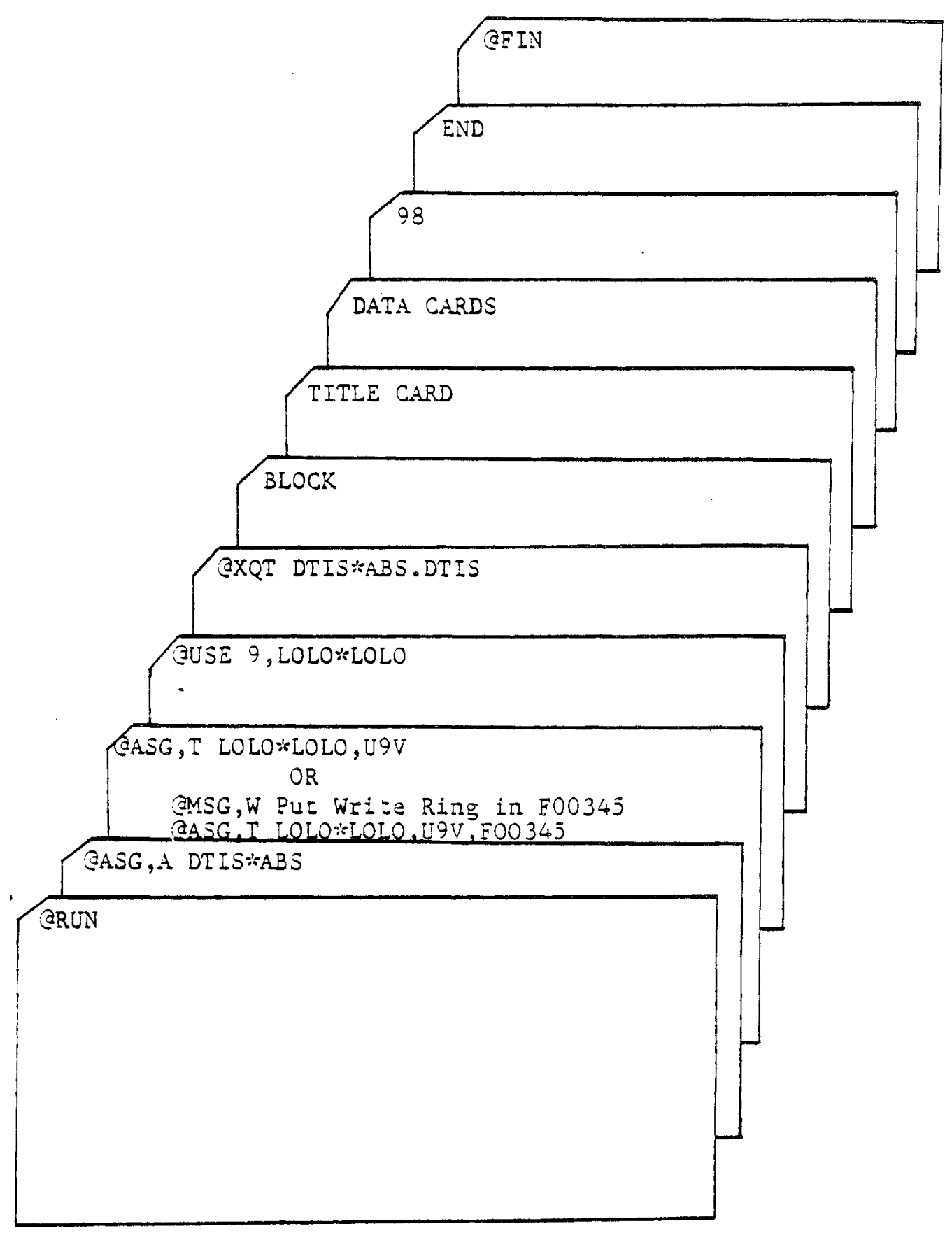

Exhibit 4.6-BLOCK Card Deck Setup 


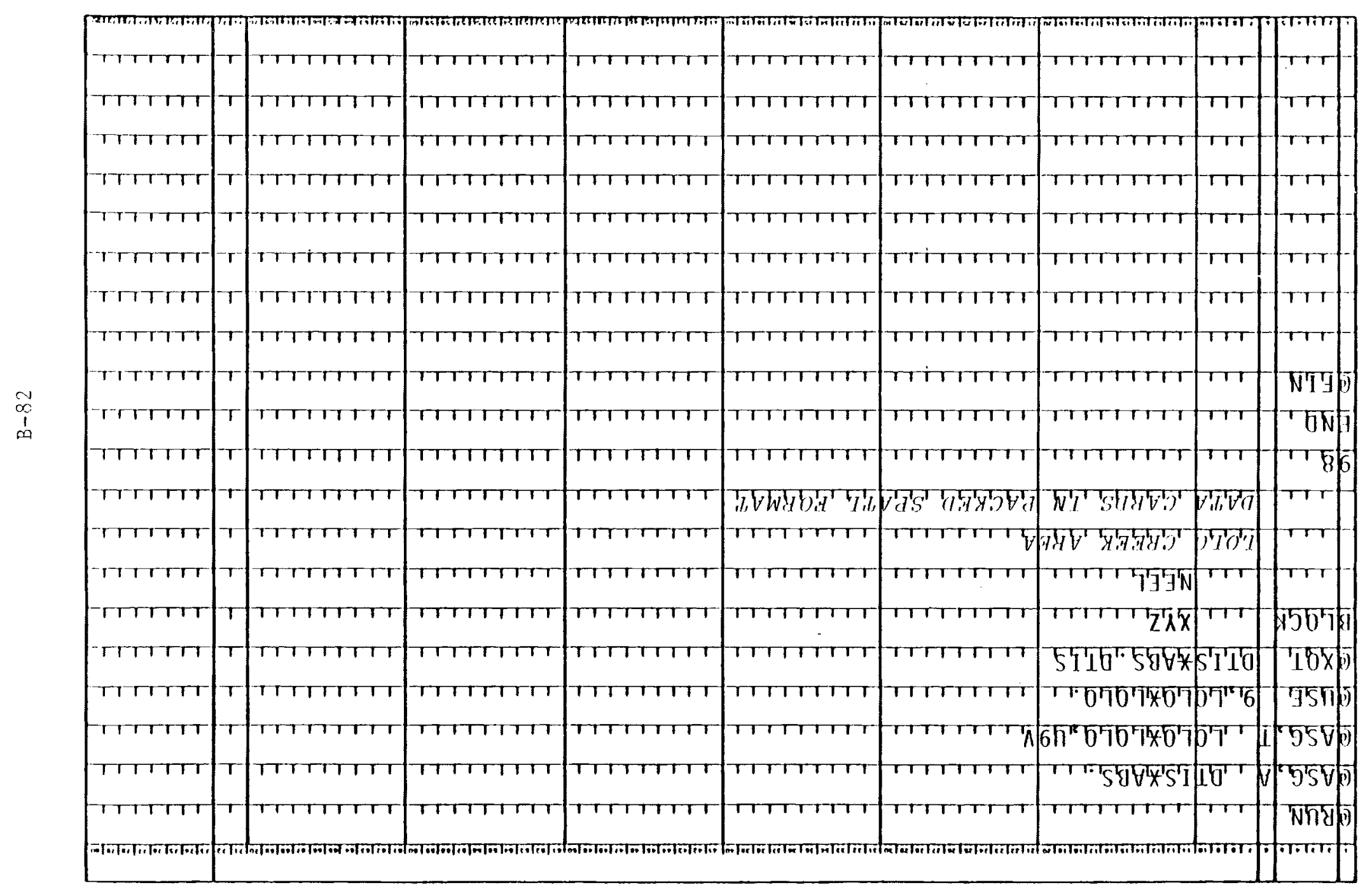




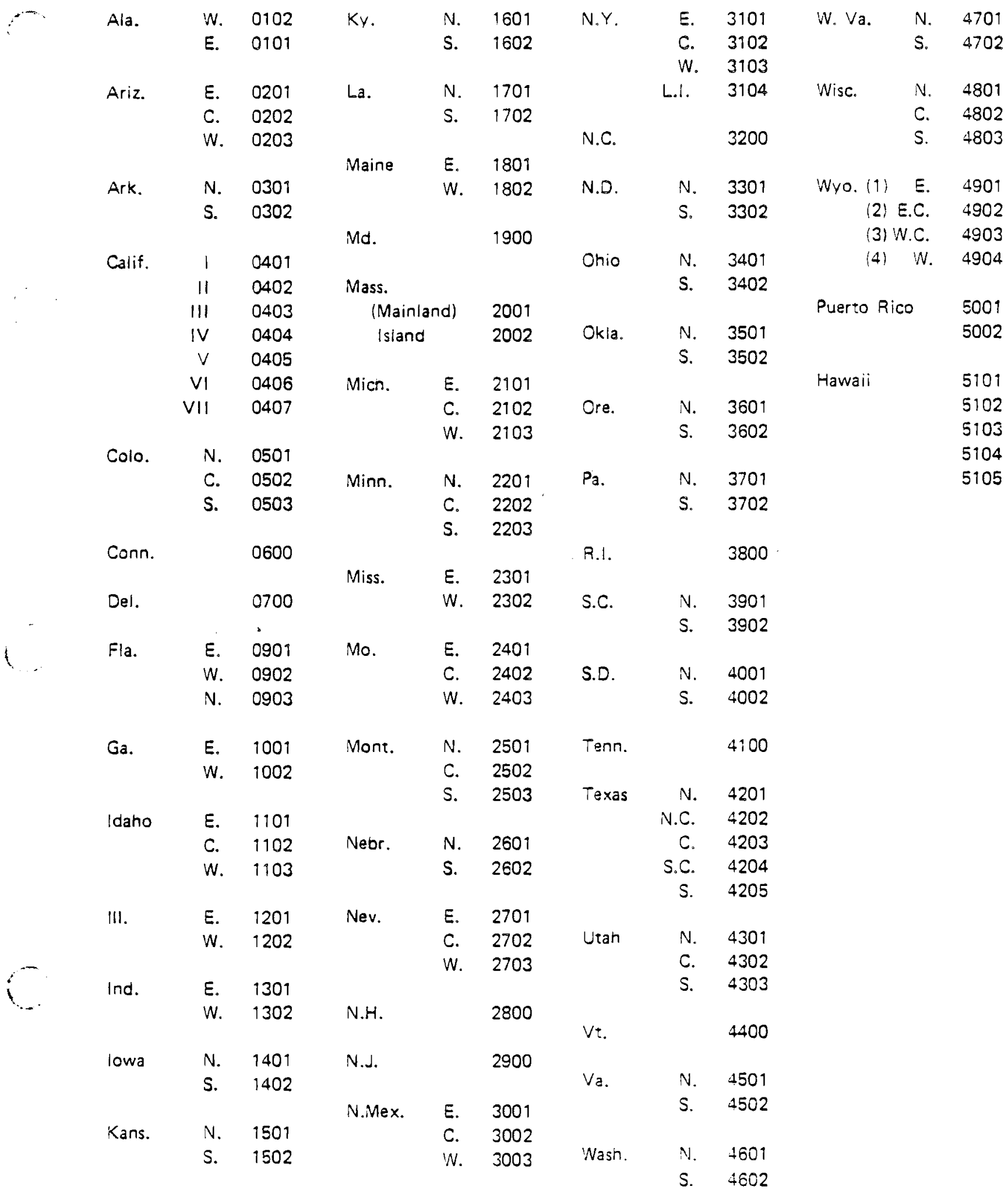




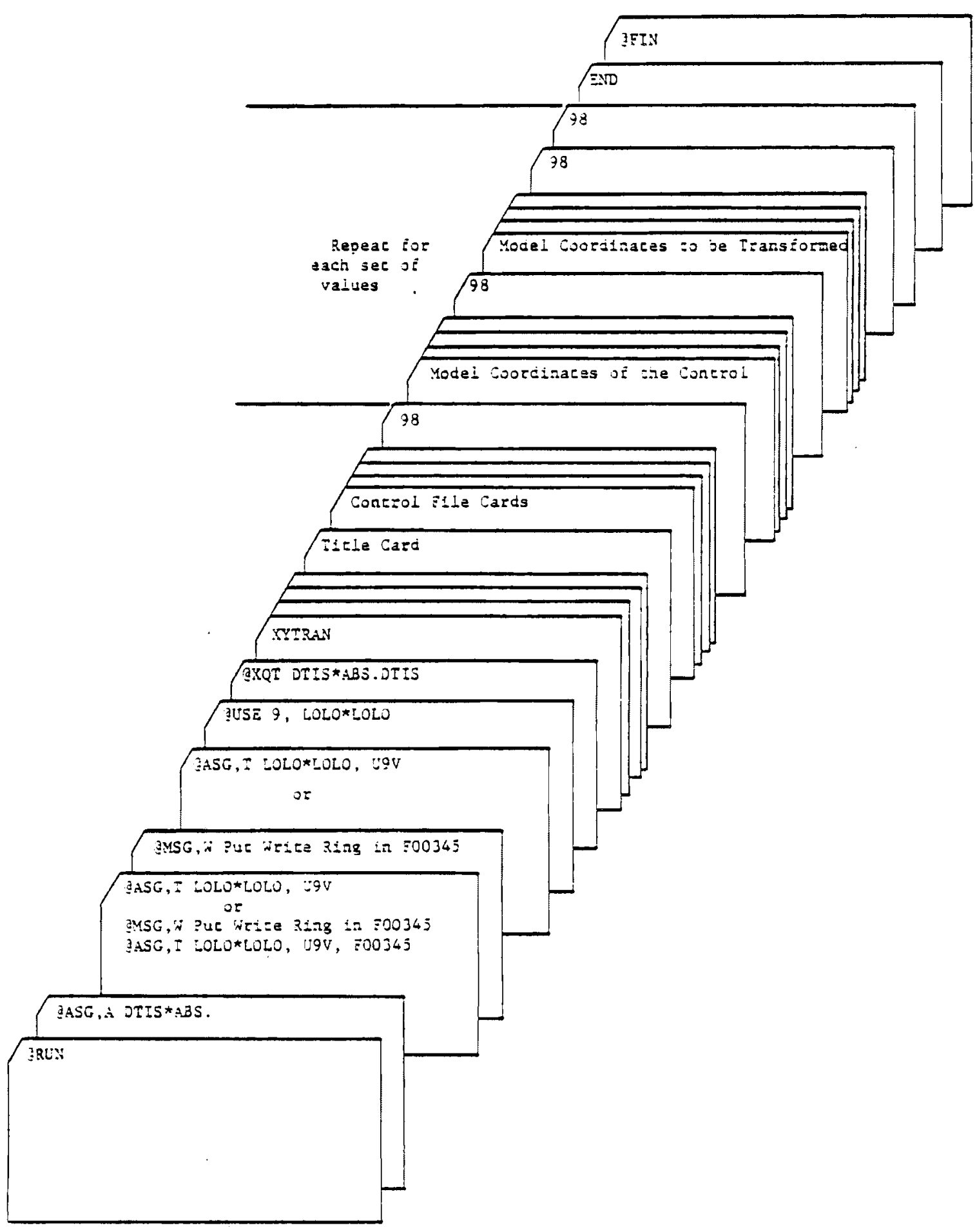

Exhibit 4.9 - XYTRan Card Deck Setup 


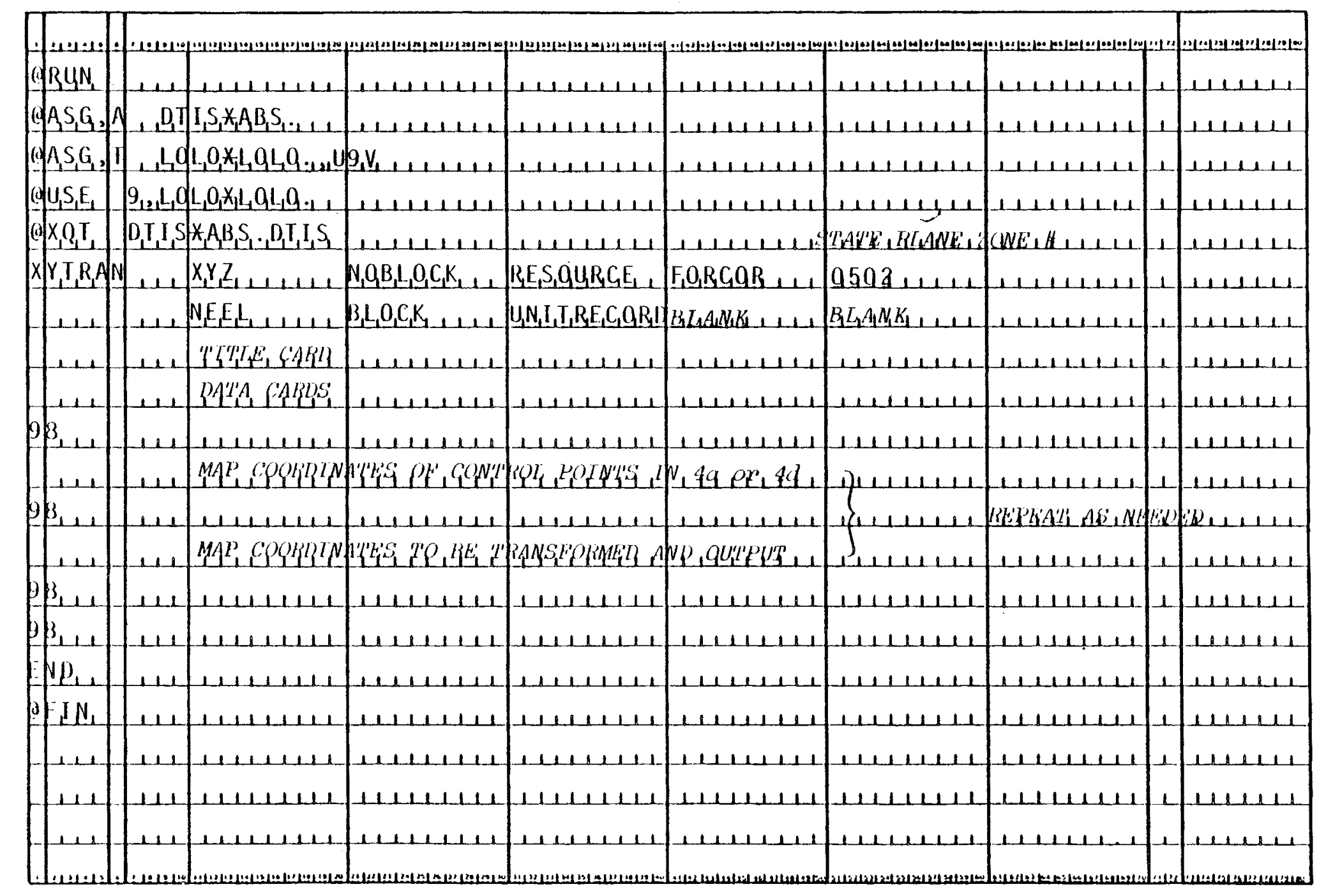




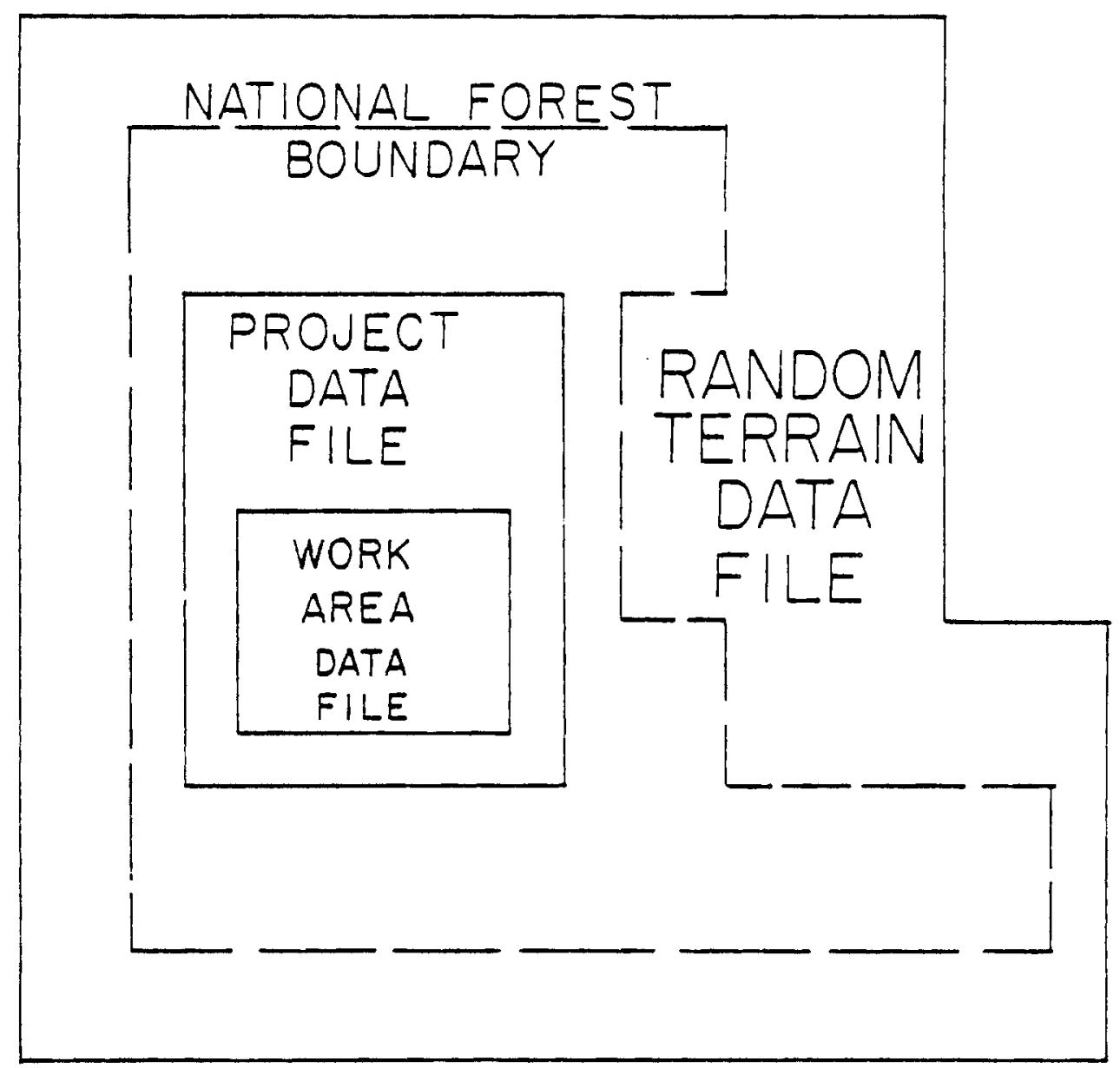

Exhibit 4.11 - Application of STOFl and FILL 


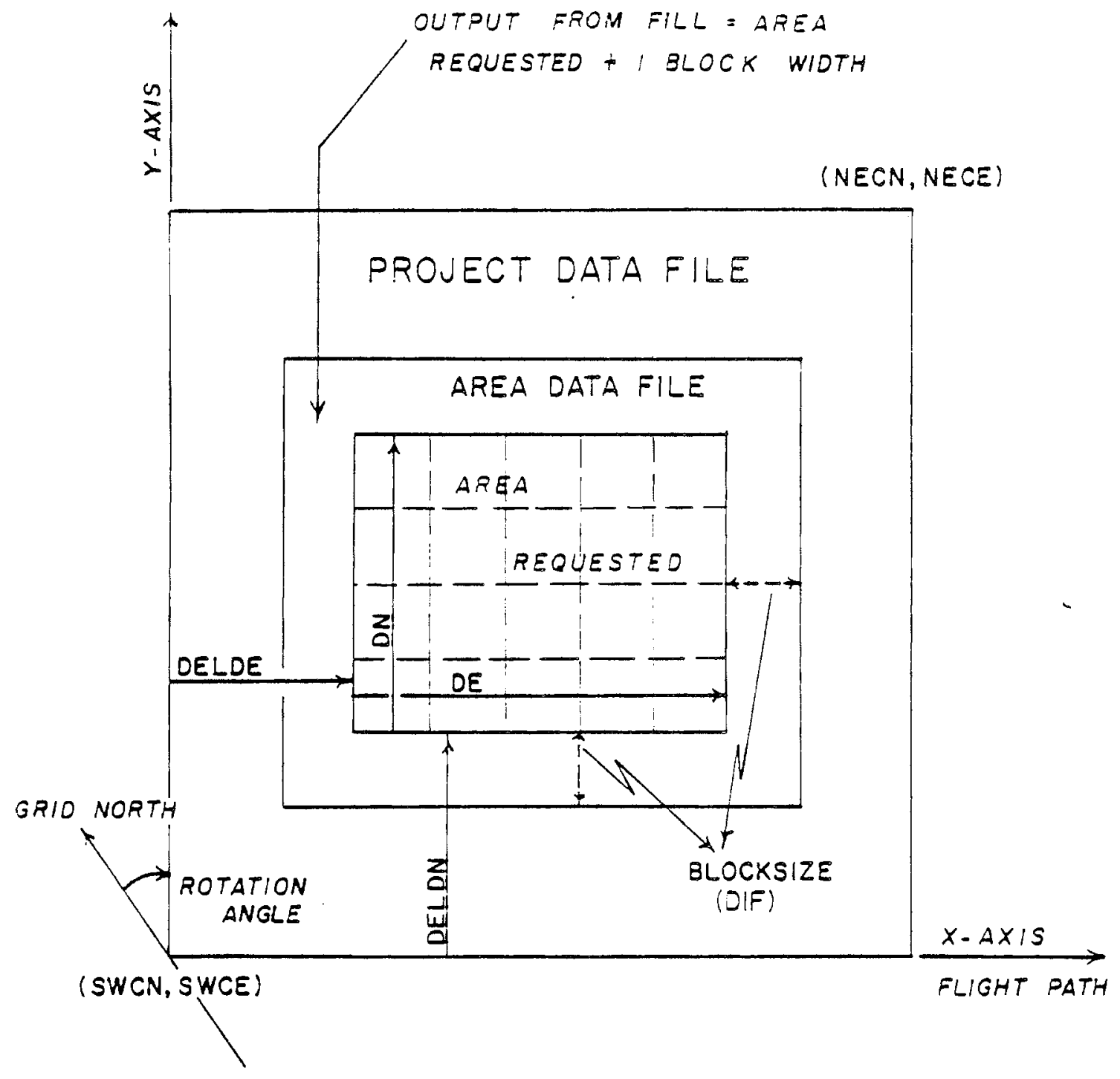


SELECTING S.W. CORNER and ROTATION ANGLE

N, E - COORDINATES OF INPUT DIGITAL TERRAIN

$n, e$ - COORDINATES AFTER ROTATION

SWCN, SWCE - PROJECT SW CORNER. THIS IS THE ONLY PLACE WHERE INPUT \& ROTATED COORDINATES ARE EQUAL (ROTATIONAL PIVOT POINT)

DELDN, DELDE, DN, DE - AS DESCRIBED IN EXHIBIT 4.12

NOTE

THE STRIP STAYS $N$ and E OF SWCN, SWCE IN BOTH COORDINATE SYSTEMS. Dots in the diagrams represent pass points for a strip.

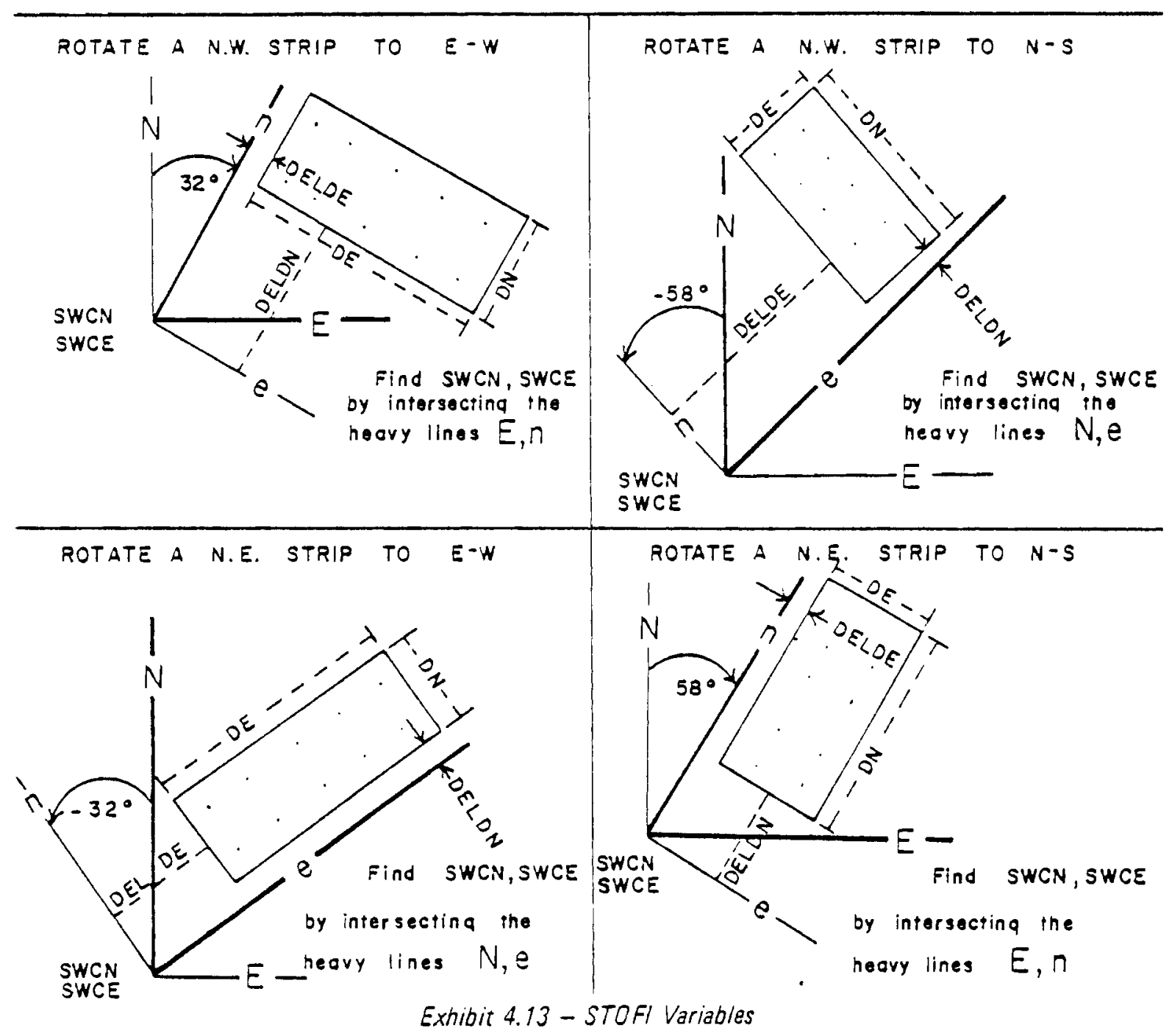




\section{r}

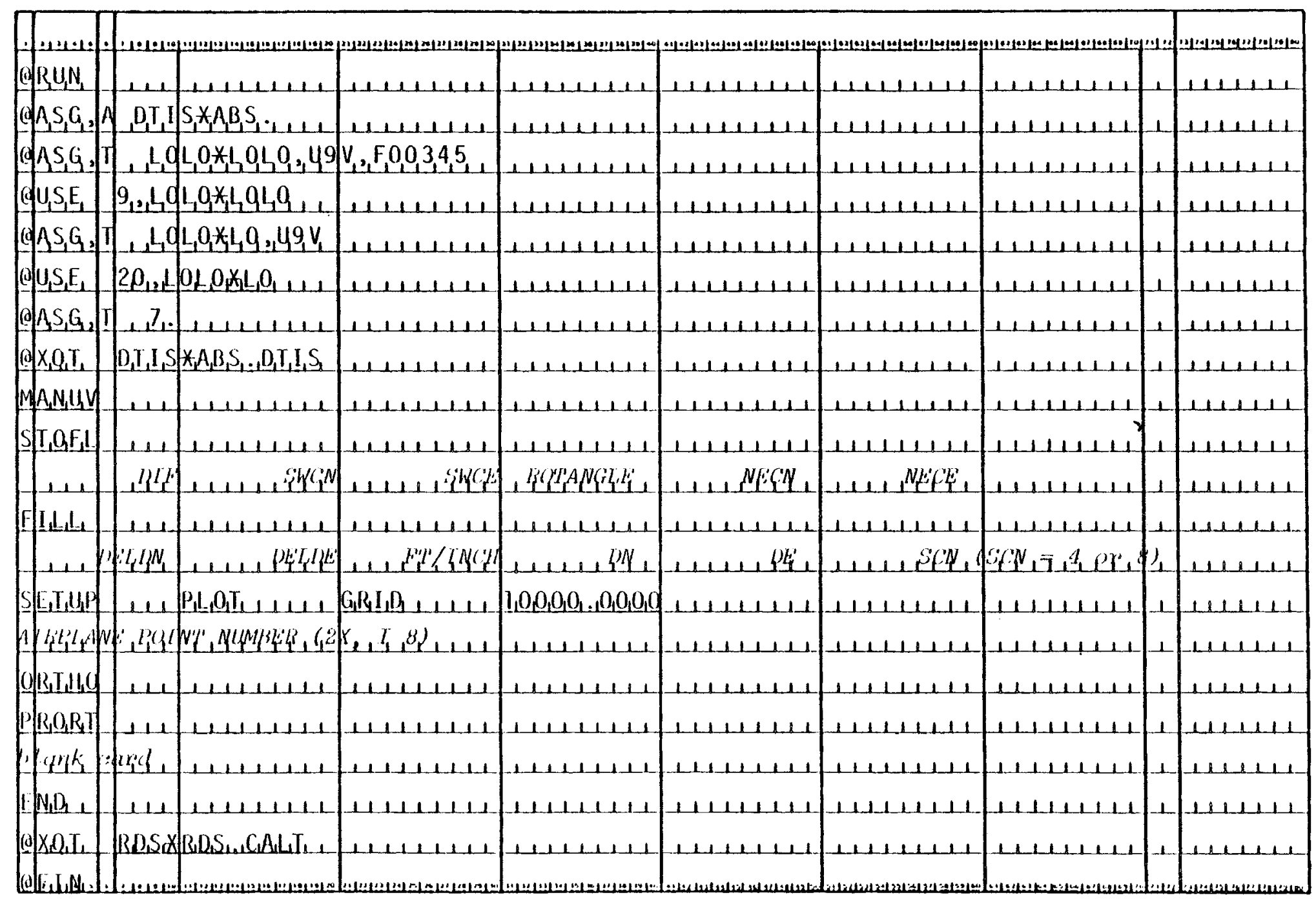




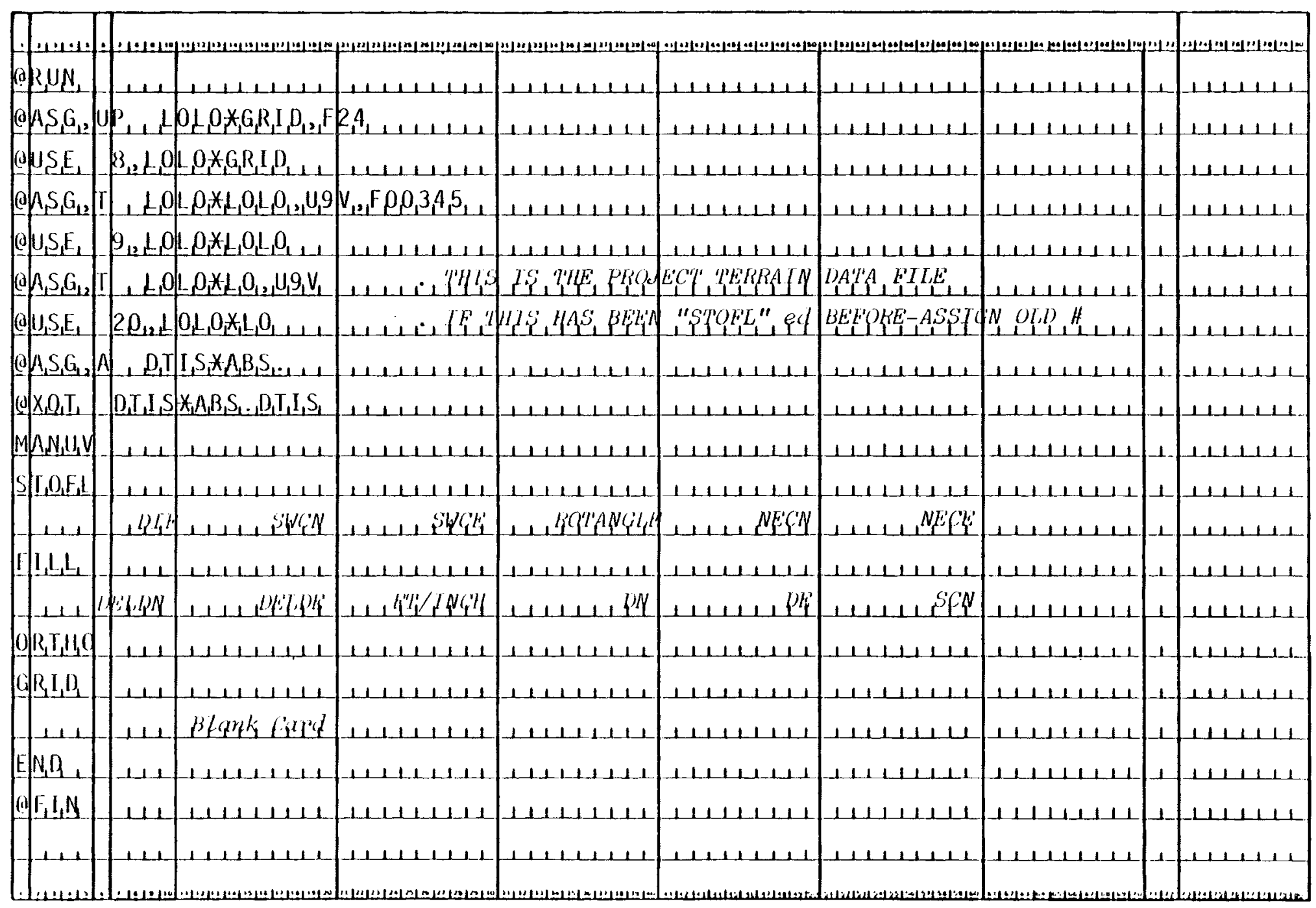




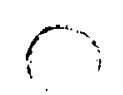

\begin{tabular}{|c|c|c|c|c|c|c|c|c|c|}
\hline $4: 1$ & $\forall$ |1:1:1 & 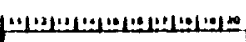 & 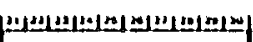 & 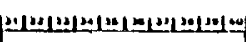 & 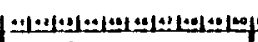 & 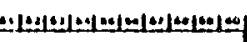 & & 너영 & \\
\hline (d) RUN & & LLes & 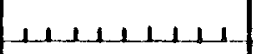 & 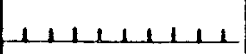 & $\mid 1,1,1,1]$ & $1+11,1212$ & $\perp \perp \perp \perp \perp \perp \perp 11$ & 1 & $1+1<1<11$ \\
\hline & A $10 T$ & $I, S * A B S, \ldots 1$ & 11,111111 & $1 / 1|1| 1,11$ & -111111111 & 111111111 & 111111111 & 1 & $111+111$ \\
\hline & (I) L & $0 L, 0 * G R I, D, F$ & $24,1,1,1$ & $\perp 11 \perp 111+1$ & $\perp+1+1+1 \perp 1$ & $\Lambda \perp 1 \perp 1111$ & 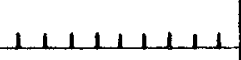 & $\perp$ & $121+11$ \\
\hline$(0)$ & $8,1,0$ & $L_{1} 0_{1} G_{1} R_{1} I_{1} D_{1}$ & 111111111 & $\perp \perp 1|\perp| 1 \mid L$ & $111<1<11$ & $\perp 1 \perp L \perp \perp \Lambda 1$ & 1111111,1 & 1. & $1111+11$ \\
\hline $0 \mu_{1} S_{1}$ & $1, L_{1} 0$ & $L_{1} 0_{1} x_{1} L_{1} Q_{1}, U_{1} Y_{1} V_{1}$ & $\Lambda \perp P R H G_{1}^{\prime} C^{\prime \prime}$ & TEGISAIN $N_{1} A^{\prime}$ & $A_{1} f^{\prime}\left[l_{1} b_{1,1}\right.$ & 1111111111 & 111111111 & 1 & $|\perp| \perp|\perp|$ \\
\hline$(0), u_{1} \mathrm{~S}$ & $2 Q_{121}$ & $0_{L_{1}} 0_{1} X_{1} L_{1} O_{1} \perp_{1}$ & $\Lambda, 14 S E A L$ & $H_{\perp}^{\circ} N U M B E K_{\perp} f F_{1}^{\prime}$ & ;IPFL:S BEF & $H E_{\perp} A N D, I I F_{1}$ & $0,1+11+1$ & 1 & $\perp$ \\
\hline 0 & QLILS & $x_{1} A_{1} B_{1} S_{1}, D_{1} I_{1} I_{1} S_{1}$ & 111111111 & $|1| 1|1| 11$ & $1+1,1+114$ & $111|1| 1|1|$ & $11+111111$ & a & 1111111 \\
\hline$M A_{1} N_{1}$ & & $N_{1} O_{1} B_{1} L_{1}, C_{1} K_{1,1}$ & $X_{1} Y_{1} Z_{1}, \ldots, \ldots$ & Leleldel & 11111111, & $1+1111112$ & $\Lambda \perp 111 \perp \perp$ & 1 & 11 \\
\hline 2150 & 1, & $\mid 1,1+1,1,1$ & $11+1+1,1]$ & Ledelelel & 111111111 & 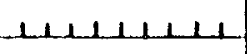 & $\perp \perp \perp \perp \perp \perp \perp \perp$ & 1 & Llelele \\
\hline 1 & , III & $1,1,1,1+6$ & $1,1,1, S W(\%)$ & 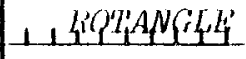 & $\perp 1,1, N E G N$ & $\perp \perp \perp \perp \Lambda E F E^{\prime}$ & 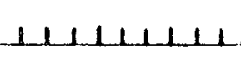 & 1 & LL \\
\hline & He & Astrlisared & 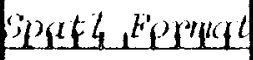 & $e_{1} d_{q} q_{1} P \mathcal{K}_{1}$ & is $z_{1} h g_{1} e_{11}$ & $1 / 1|l| l \mid l$ & $|1| 1|1| 11$ & 1 & $11+1|1|$ \\
\hline $9 g_{1}$ & $-1+1$ & $11+1,1111$ & $11,11+111$ & $\perp l+1|+1| l$ & 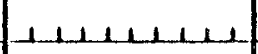 & $1111<11111$ & $\perp|1| 1|1|<1$ & -1 & $|l| l \mid l$ \\
\hline$F \perp L$ & 111 & $\| 1|1| 1|1|$ & $111|1| 1|1|$ & $11+11111$ & $1111<1<1<$ & $|1| 1|1| 1 \mid$ & 111111111 & 1 & $|1| 1|1|$ \\
\hline -1es & $14: 6, p N$ & $\perp \perp \perp \perp \perp x_{1} \int_{x}$ & 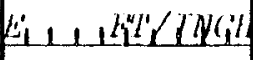 & $1 \perp \perp 1, \perp P N$ & LLuLLPF & $\mu 1 \mu \alpha S C N$ & 111111111 & 1 & 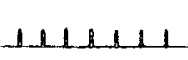 \\
\hline Q $R_{1} T_{1} H_{2}$ & 11 & 111111111 & Intelete & $\perp|1| L 1 \mid l l$ & $|1| l|l| l \mid$ & $11111_{111}$ & $11+141+1$ & $\perp$ & Hetele \\
\hline$G_{1} I$ & 111 & $|1| \perp|| 1 \mid 1$ & $11+111111$ & 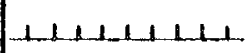 & 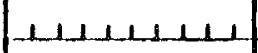 & $1+1+1+1,1]$ & 11 & 1 & 1 \\
\hline 11 & 111 & $B_{1} A N K, C A R D_{1}$ & 111111111 & $\perp \perp_{1} \perp_{1} \perp_{1}$ & $\left|1 \perp_{1}\right| \perp_{1} \mid L_{1}$ & $|\perp \perp \perp| l \mid l$ & 1111111111 & 1 & $|11| 1|1|$ \\
\hline$S I, Q R$ & $1 \mathrm{LL}$ & $1|1| 1 \mid 111$ & 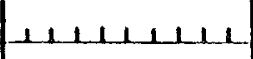 & 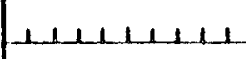 & $|L| 1|L| C \mid$ & $\| 1 / 11111$ & $|1| 1|1| 1|1|$ & 1 & $1+1+11$ \\
\hline$\theta 0][-I, N$ & 111 & $1 / 11 / 1|1|$ & $|1| 1|1| 1 \mid$ & 111111111 & 111111111 & $|\perp| \perp \mid \perp \perp \perp 1$ & $\perp|1| 1|1| 1$ & 1 & Lesele \\
\hline Hen & 1111 & 111111111 & $\perp 11,1111$ & $\perp|\Lambda| \perp \Lambda \perp \mid L$ & $|1,111+1|$ & $111+11111$ & $\perp 11111111$ & 1 & $A \perp 1,1, L$ \\
\hline & & & & & & & & & \\
\hline
\end{tabular}




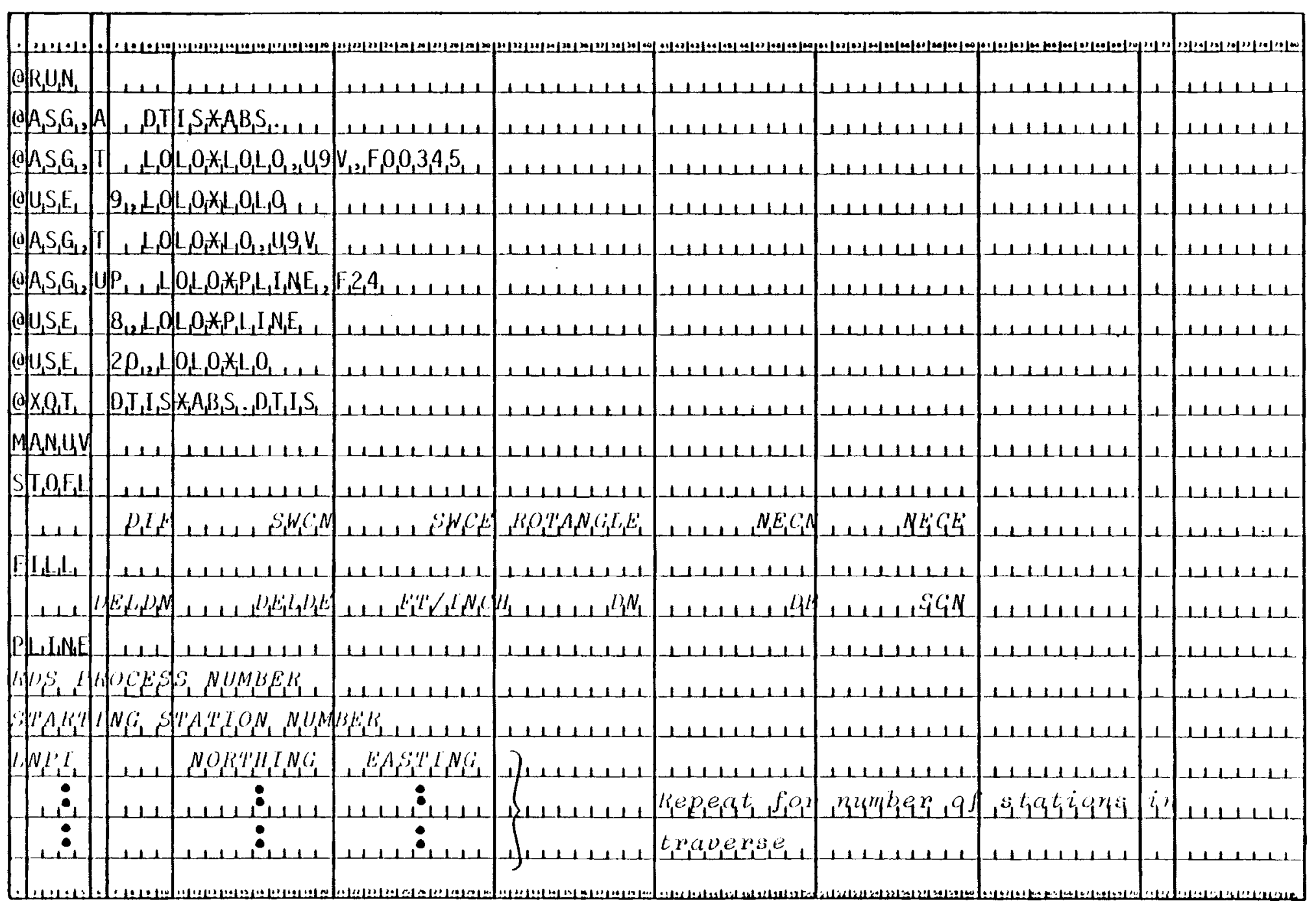




\begin{tabular}{|c|c|c|c|c|c|c|c|c|c|}
\hline t:11:1: & & 60 & 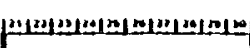 & 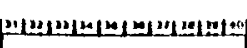 & 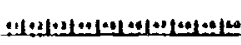 & 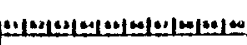 & 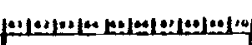 & & $\log l \cdot 1+21$ \\
\hline 8,1, & 11 & $1,1111,11$ & 111111111 & $1+1+1+1,1$ & $11,1+1 L_{1} \mid 1$ & 111111111 & $1+111111$ & $\perp$ & 111111 \\
\hline$X \mid S_{1} E_{1} C_{2}$ & S.P. & $I_{1} N_{1} G_{1,1}\left(F_{1} l_{1}, 0_{1}, 0\right.$ & $1+1+1+1+1$ & $\| 1111111$ & 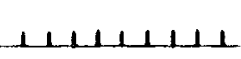 & $|111| 1111$ & $|1| 1|1| 1 \mid$ & 1 & $111+11 L$ \\
\hline$X \mid S_{1} E_{1} C_{a}$ & $L_{1} E, N, G$ & $\mathrm{~T}_{1} \mathrm{H}_{1},\left(\mathrm{~F}_{2} \mathrm{l}_{1}, 0_{1} \mathrm{Q}\right)$ & 1111111,1 & $L 1+11111$ & 111111111 & 111111111 & $11+1111+1$ & 1 & $1<1<11$ \\
\hline 111 & Lel & 111111111 & $B_{1} L_{1} a_{4} k_{1}, C_{a} a_{1}$ & $\perp \perp 111 \perp 11$ & $\perp \perp \perp \perp \perp \perp \perp \mid$ & $\perp \perp \perp \perp \perp \perp 11$ & 111111111 & $\perp$ & 111111 \\
\hline$S\left[, Q P_{1}\right.$ & -1.1 & $\perp 11111111$ & 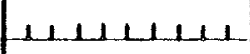 & 111111111 & $1+1+1+112$ & $|1| 1|1| 1,1$ & $|\perp| \perp|1|<1$ & 1 & $111 \perp 111$ \\
\hline$F_{1} N_{1} N$ & 111 & 111111111 & 111111111 & $11\llcorner 111 \perp$ & $\perp 1 L 111111$ & 11111111 & 111111111 & 1 & $1111 \mid 11$ \\
\hline-111 & 111 & 111111111 & $|1| 1|1| 1 \mid$ & $\perp \perp \perp \perp \perp \perp \perp 1$ & $|1+1+1+1|$ & 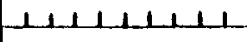 & $\mid L \perp \perp 1 L \perp \perp 1$ & 1 & 1111111 \\
\hline 111 & 111 & $+1|1| 1+11$ & 111111111 & $A+4+L 1$ & -11111111 & 1.1111112 & 111111111 & $\perp$ & $|11+1| 1$ \\
\hline 111 & 111 & 111111111 & 111111111 & 11111111 & 111111111 & $1+1|1| L L 1 L$ & $1 \mid 1,11111$ & 14 & $111 \mid 11$ \\
\hline LLe & 1.1 & $1+111+111$ & $\perp 1 \perp \perp \perp L 1$ & $\perp+1|c| c \mid 1$ & 1111111111 & 111111111 & 111111111 & 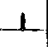 & $11 \perp 1111$ \\
\hline 111 & 111 & $11+121111$ & $111|1| 1 \mid 1$ & 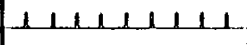 & $\perp 111|1| 1$ & 11111111 & $\mu l|l| l|l|$ & 1. & 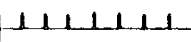 \\
\hline 111 & 111 & 111111111 & $111 \perp \perp 1 \perp 1$ & $\perp \perp \perp \perp \perp L \perp \perp \perp$ & $1+1+1+1+1$ & $1+1,11 / 1$, & $1+1,1+1,1$ & $\perp$ & $\perp \perp \perp \perp \perp \Lambda$ \\
\hline 11 & 111 & 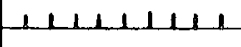 & 111111111 & $A|1| \perp|\perp| l$ & $11111|1| 1$ & 121111112 & 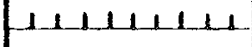 & 1 & $1 \perp 111 \ldots 1$ \\
\hline 111 & 111 & 1111111 & $\angle 1111111$ & 111111111 & $1|\perp| \perp|\perp| 1$ & $\perp \perp|1| 1 \mid 1 L$ & $|1 \perp| 1|1| 1$ & $\perp$ & 1111111 \\
\hline 111 & 111 & $1+1|1| 1 \mid 1$ & $|1| 1|\perp| 1 \mid$ & $\perp 1,1|1| 1 \mid$ & 111111111 & $1+11+1+11$ & 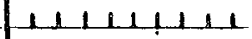 & 1 & 11,1111 \\
\hline 111 & 111 & $11<11111$ & $\perp 11<L L L 1$ & 111111111 & $-1121,111$ & $\perp 1,1,1, L 1$ & $11+111+11$ & 1 & Helelele \\
\hline 111 & 111 & 111111111 & 11111111 & 111111111 & $1 \perp 1 \perp 1111$ & $\perp 1 \perp \perp \perp 1 \perp \perp 1$ & $11 \perp 111111$ & 1 & $\perp \perp 1 \perp \perp \perp L$ \\
\hline 111 & 111 & $111111+11$ & $\perp 1111111$ & $\perp 1111111$ & $1 \mid 1+11 \perp 1$ & $11+111111$ & $\perp \perp \perp 11|\perp| 1$ & 1 & 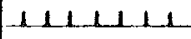 \\
\hline 111 & 111 & 111111111 & $11 \perp 111111$ & $L|1| 1|1| L$ & $1+|1| 1 \mid 1$ & 111111111 & 111111111 & $\perp$ & $|1| \perp|1|$ \\
\hline 111 & Len & $\perp 1,1,111$, & $11,111+1$ & 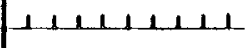 & $-1+1+1,1+1$ & 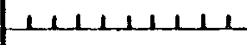 & 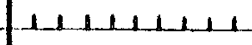 & 1 & 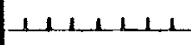 \\
\hline & & & 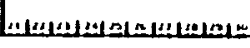 & & & & & & \\
\hline
\end{tabular}




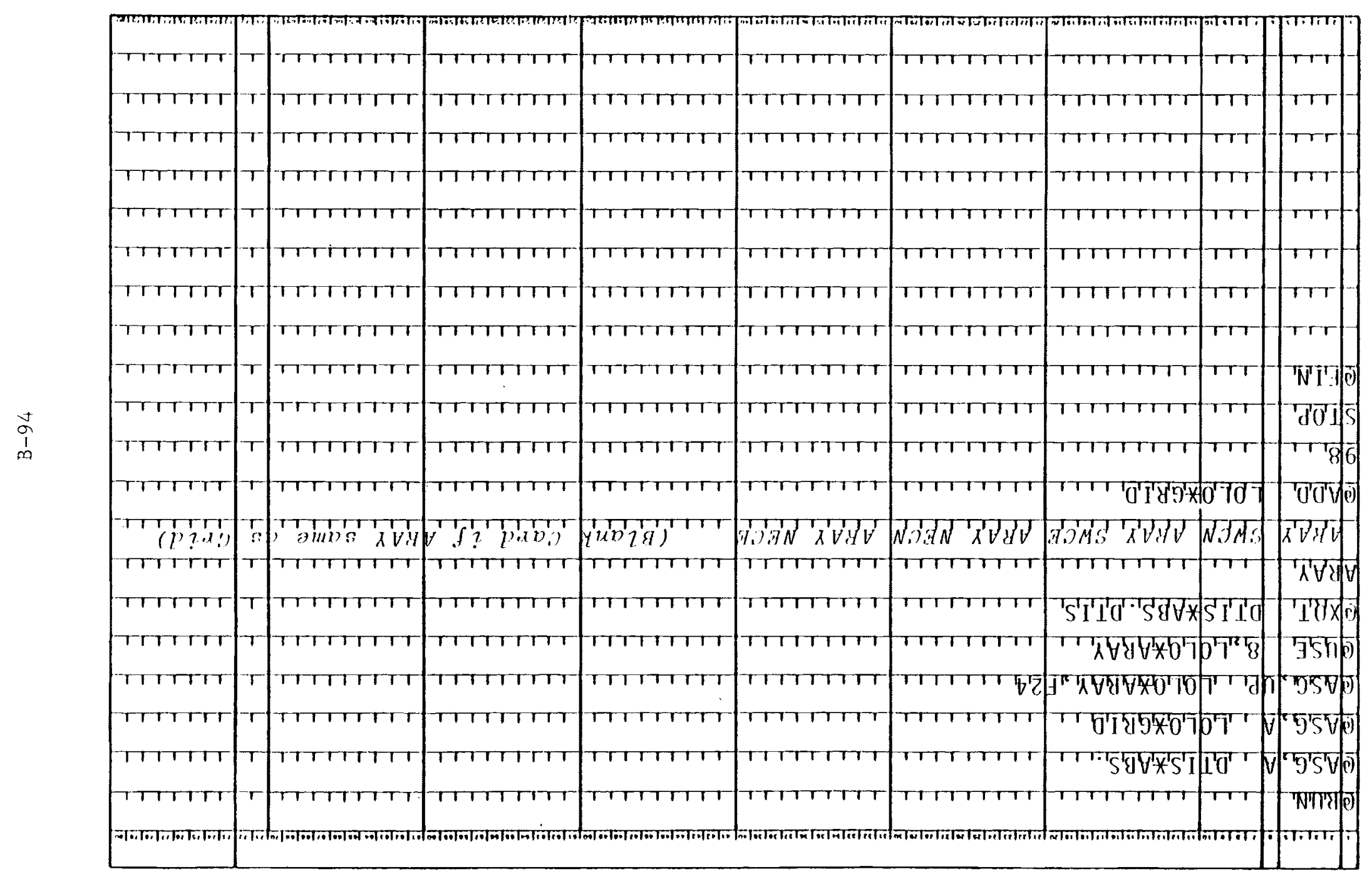




\begin{tabular}{|c|c|c|c|c|c|c|c|c|c|}
\hline it & A.15 & & !! & 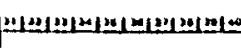 & 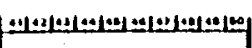 & 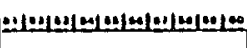 & 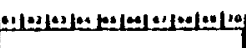 & :14 & 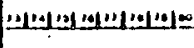 \\
\hline [d] & $M$ & $19,7,5,1,2,3,4,5$ & $6,7,8,90_{1}, G_{1} E_{1}, 0, M$ & $\left.{ }_{21}\right]_{1} 5_{1,1} 20_{1} 0_{1}$ & $\perp A L_{1} B_{1} E_{1}, E_{1} N$ & GINEER LNG & $|1| 1|1| 1 \mid 1$ & -1 & $|\perp| l \mid \perp 1$ \\
\hline $0 M S G_{12}$ & $N$ & $S_{1} R_{1} U_{1} N_{1} S_{1} R_{4}$ & $A_{2} M_{1} W_{1} I_{1} I_{1} L_{1} P_{1} A$ & $q_{1} D_{1} U_{1} E_{i} I_{1} M_{1} P_{1}$ & $i_{1}, k_{1} Q R, R M K$ & YSBWITLL & $1|1| 1+111$ & 1 & $1+1$ \\
\hline of & II & $O M_{1}^{*} L_{1} K A M A_{1} I_{1}$ & $2,4,9, V_{12}, F_{1}, 2,3,4$ & 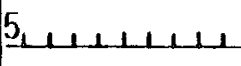 & $\perp \perp|L| \perp|\perp| L$ & $1+1|1| 1 \mid 1$ & $\mid 11111111$ & 1 & $11+1111$ \\
\hline $0, S, 5$ & & $G E D M^{*}{ }_{1} K_{1} L_{1} A_{M} A$ & {$\left[H_{1}+1,1,1\right.$} & $|1| 1|1| 1 \mid$ & 111111111 & $\perp \perp \perp \perp \perp 111$ & 111111111 & $\perp$ & 1111111 \\
\hline$\theta A S G_{12}$ & UP & $O M_{1}^{*}{ }^{*} V_{1} D_{1} A_{1} T A_{21} F$ & $2 A_{1} \perp \perp \perp \perp 1$, & 121111111 & 111111111 & 11111111 & $\perp \perp \perp \perp \perp \perp \perp \Lambda$ & 1 & 1111112 \\
\hline Q $U S, S_{L}$ & - & GEDM*VDATA & $-1,1+1,1]$ & $1+1+1 L_{1}$ & 111111111 & 11111111 & $|\perp| \perp|1| \perp|1|$ & 1 & 111111 \\
\hline ons & & $=\mu 9, V_{1}+1,1$ & $\perp 11|| \backslash 11$ & $\perp 1|1| 1|1|$ & 111,11111 & $|\perp| 1|1| \perp 1$ & 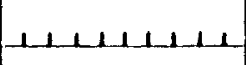 & 1 & Leldel \\
\hline (A.S & $A$ & $I S_{1}^{*} A B S_{1+L L}$ & 111111111 & $1|1+1| \perp|1|$ & $1+1+11+11$ & $\perp 1,\left|L_{1}\right| \perp$ & $1+1+14112$ & 1 & $111+11$ \\
\hline $0 x$ & & $I S_{1}^{*} A B_{1} S_{1} D_{I} T_{1} I$ & $S_{1111+111}$ & 111,11111 & 1111,11111 & $1 \perp 111 \mid 1$ & $-1+1+1+L$ & -1 & $\perp|\Lambda| \perp \mid 1$ \\
\hline MNNUV & 14 & $1111111 \mid 1$ & $|1| 1|\perp| 1 \mid$ & 111111111 & $\mid 111111111$ & $\perp 11|1| 1 \mid 1$ & $111<11 \perp 11$ & 1 & 1111111 \\
\hline 5 & & 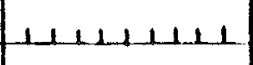 & $\| 1+1 \mid 111$ & $|1| 1|1| 1 \mid$ & $1|111| 1 \mid 1$ & $\perp|||L| \perp \mid l$ & $|1| 1|1| 1 \mid$ & 1 & $\triangle L_{1} \mid L_{1}$ \\
\hline-1 & 2500. & $1,61,7,509$. & $, 1,4,4,7,5,0,0$, & $1<11 \mid 1111$ & $\perp+6 A_{1} 2500_{1}$ & $L_{1}, 1,4,7,2,50,0$. & 111111111 & $\perp$ & $|l| l|l|$ \\
\hline 11 & & $\perp \perp \perp \perp \perp \mid \perp \perp \perp$ & $|\perp| \perp|\perp| \perp \mid$ & $1+1<1+1 \mid 1$ & $H L L L L L L L$ & $1 \perp 11|1| 1 \mid$ & 1111111111 & 1 & 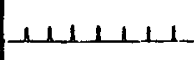 \\
\hline- & 250. & $1+1,2,5,0,0$ & $1,112,000$. & $+1,20,0,0,0$ & $1 \perp 2,0,0,0,0$ & $1,1,1,4,0,04$ & $\|\perp\| 1|1| 1$ & 1 & $|1| 1 \mid 11$ \\
\hline $0 \mathrm{R}_{2} \mathrm{~T} 110$ & 111 & $\perp \perp \perp \perp \perp \perp \perp$ & $\mid \perp \perp 1111 \perp 1$ & $\Lambda \perp \perp \perp \Lambda 1$ & 111111111 & 111111111 & $11+11+11$ & 1 & $-1 \perp \perp 1 \perp 1$ \\
\hline$V G R$ & 111 & $1 \perp 1 \perp 111$ & 11111111 & $11111<1111$ & 111111111 & $L 1+111111$ & $|1| 1|1| 11$ & 1 & $|+1| 1 \mid 1$ \\
\hline & isto & 1,11111111 & $\perp 11111111$ & 111111111 & 11111111 & 11111111 & 111111111 & 1 & $1 \perp 1 \perp 11$ \\
\hline$N[$ & 111 & $1+1<11<1<$ & 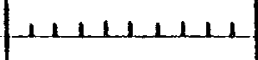 & $\perp 1 \perp \perp \perp \perp \Lambda$ & $1+1+1+111$ & $111111 \mid 11$ & 111111111 & 1 & $1 \perp 1|1| 1$ \\
\hline (d) $I N_{1}$ & 111 & $|1| 1|1| 1|1|$ & $\| \perp|1| \perp|1|$ & $|1| 1|1| 11$ & $1 \perp 1|1| 1 \mid 1$ & 111111111 & $\perp 11111111$ & 1. & 1111111 \\
\hline$-1+1$ & -1.1 & 111111111 & 11111111 & $\perp \perp 1 \perp 11,11$ & $11+1,1124$ & Helldedele & $\perp 1+1|1| 11$ & 1 & L L L L L L L L L \\
\hline
\end{tabular}




\begin{tabular}{|c|c|c|c|c|c|c|c|c|c|}
\hline & H.14: & | & 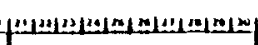 & 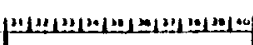 & $4=0.101011$ & & 10 & $\because \because 4$ & \\
\hline 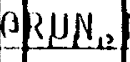 & 4 & $197,5,2,3,5$ & $67890_{12}, \mathrm{GEOM}$ & ${ }_{2}{ }_{1} 0_{121} 200_{1 \perp}$ & $\perp A L B E E-E$ & NGINEERING & 1,1 & & $1<1,11$ \\
\hline & $N+S_{N}$ & $M_{1} P_{1} L_{1} E_{1}, R_{1} U_{1} N_{1}$ & $\left.P_{1} S_{1} W_{1}, V_{1} I_{1} E_{1} W_{1}\right]$ & $I_{1}, L_{1} O M, L M A$ & I $A_{1} E_{1} S_{1 \perp \perp}$ & 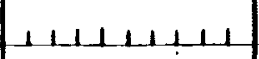 & 111111111 & 1 & $|L||||| \mid$ \\
\hline & $A, G E$ & $O M_{L}^{*} V_{\perp} D A_{\perp} A_{1}$ & $\perp \perp \perp L 111$ & $1+1+1,1$ & $\perp \perp \perp \perp \perp \perp \perp \perp$ & 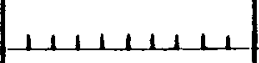 & $1,1,1+1,121$ & $\perp$ & $\perp \perp \perp 11$ \\
\hline & $1 Z D$ & $A_{1} A_{1}, G_{E} \rho M_{1}^{*} V$ & pAT $A_{1,1,1}$ & $\perp \perp 11 \perp \perp \perp 11$ & 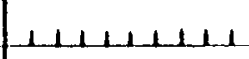 & 111111111 & $\Lambda \perp \perp \perp \perp \perp \perp \Lambda$ & 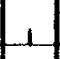 & $111+111$ \\
\hline ohs & A , PS & $W_{1}^{*} V_{I} E W_{1} I_{1}$ & $1111 \mid 1111$ & -111111111 & 111111111 & $\mid 11111111$ & $\perp 1111111$ & 1 & 1111112 \\
\hline & $-1 P S$ & $W_{1}^{*} V_{1} I E W_{1} I_{1-1}$ & $\perp \perp \perp \perp \perp \perp 1$ & $\perp \perp \perp \perp \perp \perp \perp \mid$ & 111111111 & $|\perp \perp \perp 1| 1$ & $\perp 1|1| 1 \mid 1$ & 1 & 111.1111 \\
\hline$N \mid R 0$ & $=61, N$ & 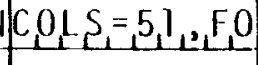 & RMAT $=:(1,6, F$ & $5,0,)_{1}^{\prime}, B_{1}, q$ & $0, S_{1}, R_{1} A_{1} A_{1}, \perp$ & 11111111 & -11111 & 1 & 1111 \\
\hline & $E=1$ & SMALL SCA & $E, E_{L} S_{1} M A$ & $P^{\prime} 1^{\prime}+111+11$ & $\perp \perp 1111111$ & $1 \perp \perp 11 \perp 11$ & 11111111 & 1 & $L L 1 \perp \perp 1$ \\
\hline & $25,2,5$ & S, RADIUS $A_{1}=1$ & $.5, V_{L} I, E W_{1}, M A$ & $P+1 \mid 1,1+1$, & 111111111 & 111111111 & 11111 & $\perp$ & 1111 \\
\hline & 40,40 & $M_{1}, V_{1} L_{1} W_{1}, M A P$ & $2 G M A P_{L \perp \perp L L}$ & $-1 \perp \perp \perp 1 \ldots|\perp| 1$ & $|1 \perp \perp 11| 11$ & $\| \perp \perp \perp \perp 1 \mid \perp$ & $\perp \perp \perp \perp \perp \perp 1$ & 1 & $\perp \perp \perp \perp L L$ \\
\hline & $\because 111$ & $\mid 11111111$ & 11111111 & $\perp 1111111$ & 111111111 & $11|1| 1 \mid 11$ & 111111111 & 1 & 1111111 \\
\hline & 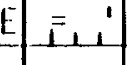 & SILPE MAP,' & $M 11111111$ & $1+1,11114$ & $11+1,1111$ & 111111111 & $\perp 1 \perp \perp L 1 \perp L$ & L & $1+1 \mid 111$ \\
\hline S) $O P I$ & $=\pi A B \mid$ & $E, M A P, G M A P$ & 11111112 & $-1+1+1+1+1$ & $-\perp \perp \perp \perp \perp \perp \perp \perp \perp$ & $\perp \perp \perp \perp L \perp \perp \perp$ & $\perp \perp \perp \perp \perp \perp \perp 11$ & 1 & $\Lambda \perp \perp \perp 1-1$ \\
\hline & $=111$ & 11111111 & LIL111 & 111111111 & 111111111 & 111111111 & $1|1| 1|1| 1$ & 1 & 111111 \\
\hline $1 N_{1}$ & $-1,1$ & $\perp 1 \perp \perp \perp 111$ & 111111111 & 111111111 & 111111111 & 11111111 & $\perp \perp \perp 11 \perp<1$ & 1 & 1111111 \\
\hline$-1+\Lambda$ & $1+1$ & $\perp L 111111$ & 111111112 & $\perp \perp L_{1 \perp \perp L}$ & $+1 \perp L_{1} \mid L_{1}$ & & $\perp \perp \perp \perp \perp \perp L$ & 1 & 1111111 \\
\hline $1+1$ & -111 & $\perp \perp 11 \perp 1 \perp 1$ & 11111111 & $\perp \perp \perp \perp \perp \perp \perp \perp$ & $\perp \perp \perp|1| \perp \mid 1$ & 11111111 & $\perp \perp \perp|1| 1 \mid 1$ & 1 & $\perp \perp 1111$ \\
\hline 111 & 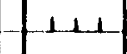 & 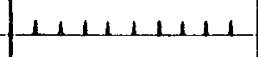 & $\perp \perp 111 \perp L$ & $\perp 111 \perp 111$ & $\lfloor\perp \perp|| \downarrow L$ & 111111111 & 111111111 & 1 & $\perp L 1111$ \\
\hline 111 & 111 & $1111111 L$ & $11+11111$ & $1+1111111$ & $11111 L_{1}$ & $\perp \perp \perp \perp \perp \perp \perp$ & $\perp \perp \perp \perp \perp \perp \perp$ & 1 & $\left|L_{1}\right| L_{1}$ \\
\hline $1 \perp 1$ & -11, & 111 & $\mu 1+111$ & $+1+1+1,1$ & $1111<1$ & 111111111 & $111,1,11$ & + & 121,121 \\
\hline & & & & & & & & & \\
\hline
\end{tabular}




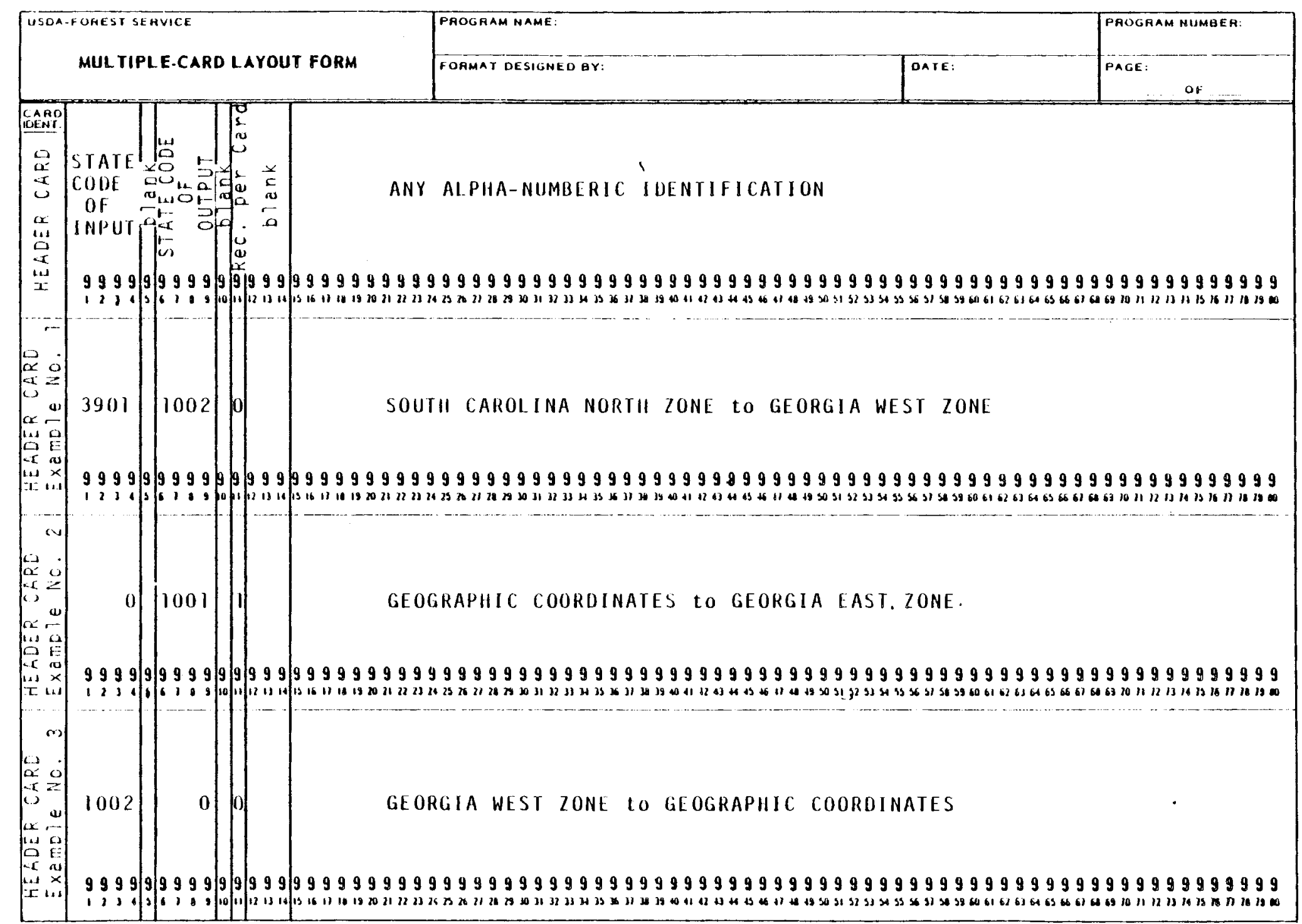




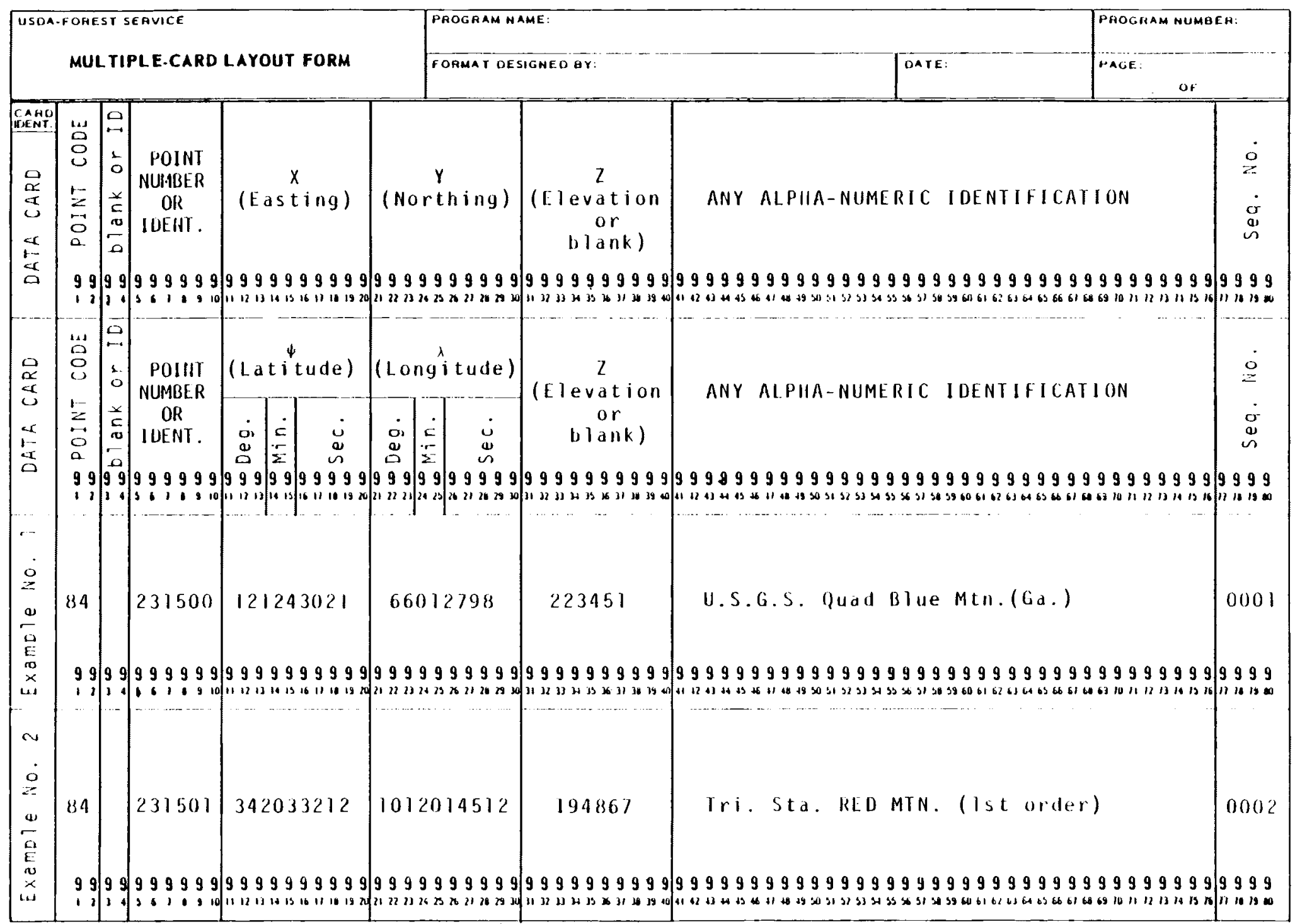




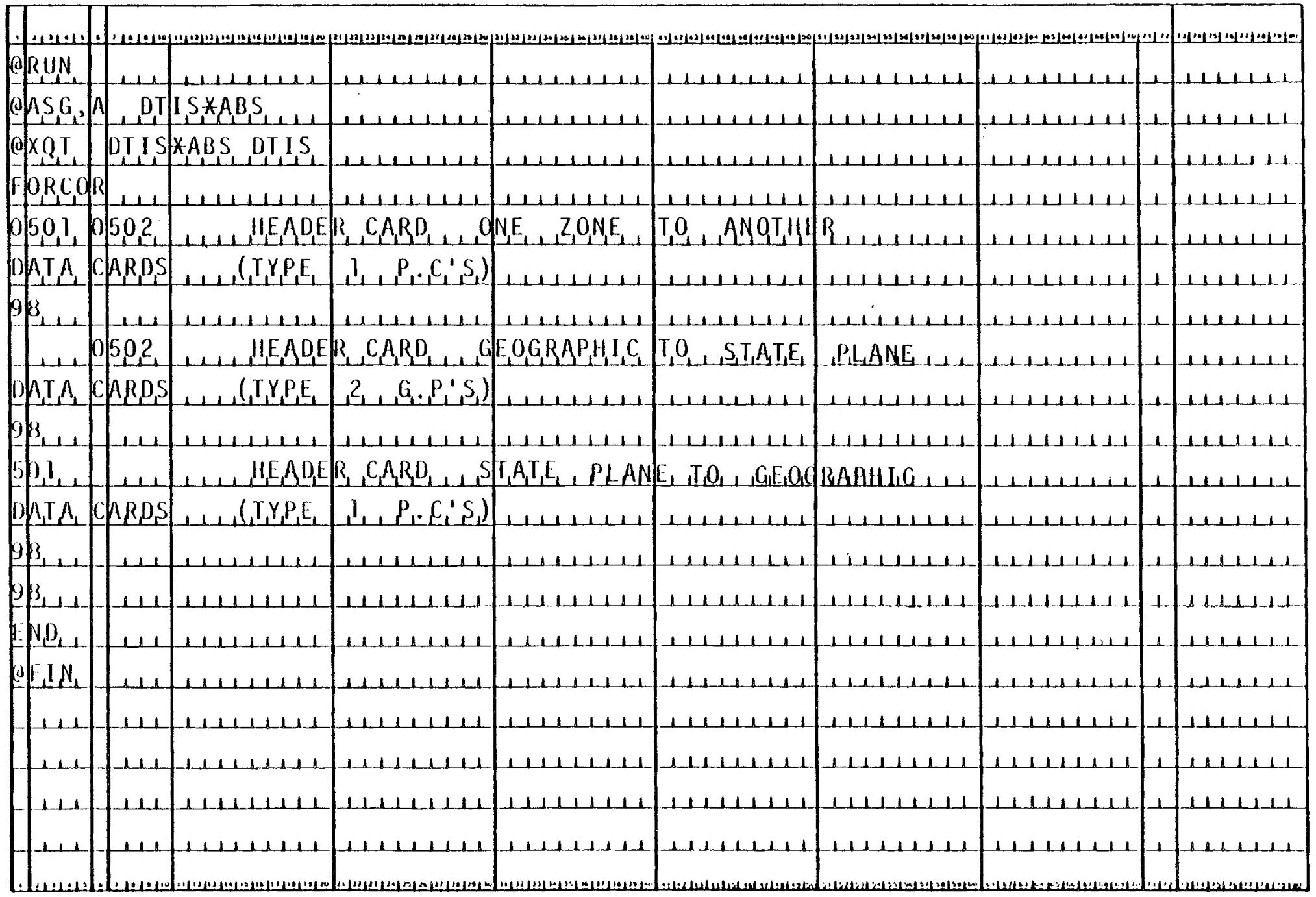




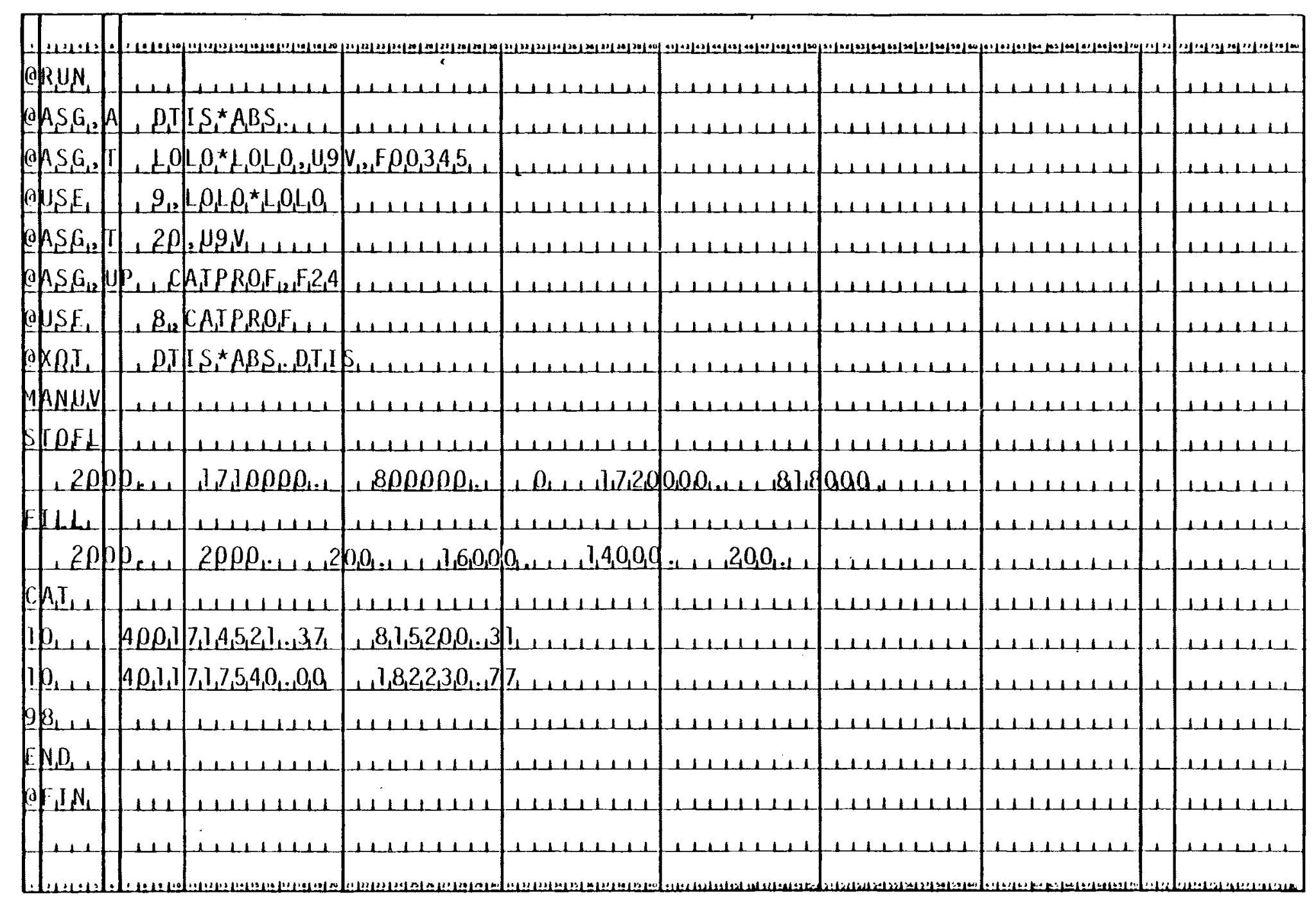




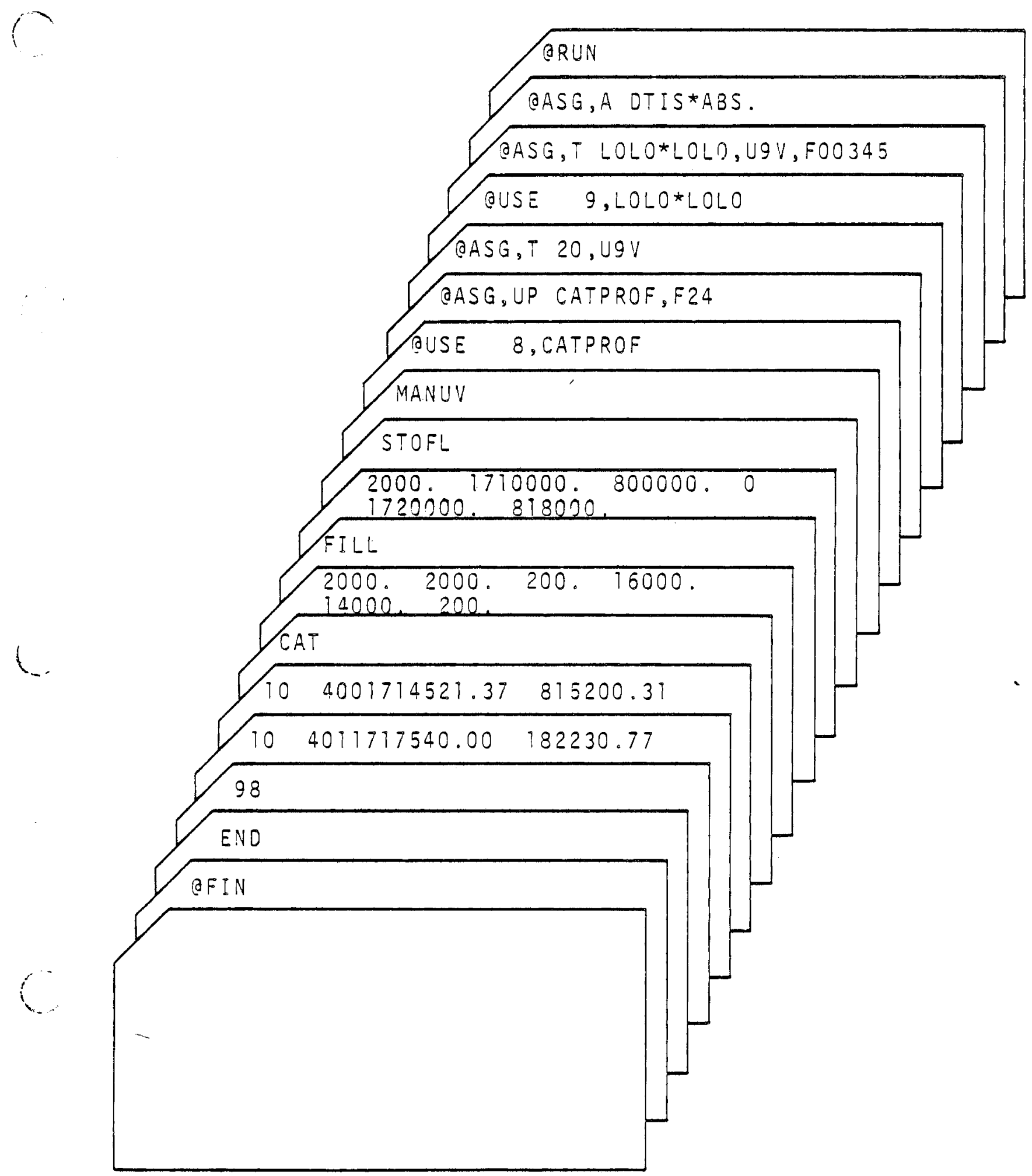

Exhibit 4.25 - CAT Card Deck Setup 


\section{SECTION 2. PROGRAM ARRAY}

\section{Gridding from Random Data}

This program inputs random data for a selected area from the blocked TDF. It then grids this area by the least square fitting of a saddie function $\left(z=A x^{2}+B x y+C y^{2}+D x+E y-F\right)$ onto the nearest 12 to 50 points to the grid point. and outputs the gridded information in Calcomp GPCP ARAY block format. The program also selects and outputs a set of Control. 80 series. points for use in Calcomp GPCP program.

One control card is required.

\begin{tabular}{|c|c|c|c|}
\hline $\mathrm{cc}$ & & use & format \\
\hline $1-10$ & XMIN & Low $\mathrm{X}$ value & F10.0 \\
\hline $11-20$ & XMAX & High $X$ value & $F 10.0$ \\
\hline $21-30$ & XINC & $X$ size of the grid (cell) & $F 10.0$ \\
\hline $31-40$ & YMIN & Low $Y$ value & F10.0 \\
\hline $41 \cdot 50$ & YMAX & High $Y$ value & F10.0 \\
\hline $51-60$ & YINC & $Y$ size of the grid (cell) & $\mathrm{F} 10.0$ \\
\hline $61-65$ & $\begin{array}{l}\text { ISPLIT } \\
\text { file is to } \\
\text { zero or } \\
\text { will be } \\
\text { depende } \\
1500 \text { to } \\
\text { band tor } \\
\text { and grid }\end{array}$ & $\begin{array}{l}\text { Number of times the random } \\
\text { be split into bands. If left } \\
\text { lank, the default value of }+ \\
\text { sed. This value is input data } \\
\text { t, and should be set to obtain } \\
2500 \text { random data points per } \\
\text { optimum program efficiency } \\
\text { point elevation accuracy. }\end{array}$ & I5 \\
\hline
\end{tabular}

Typical Runstream for Program ARRAY:

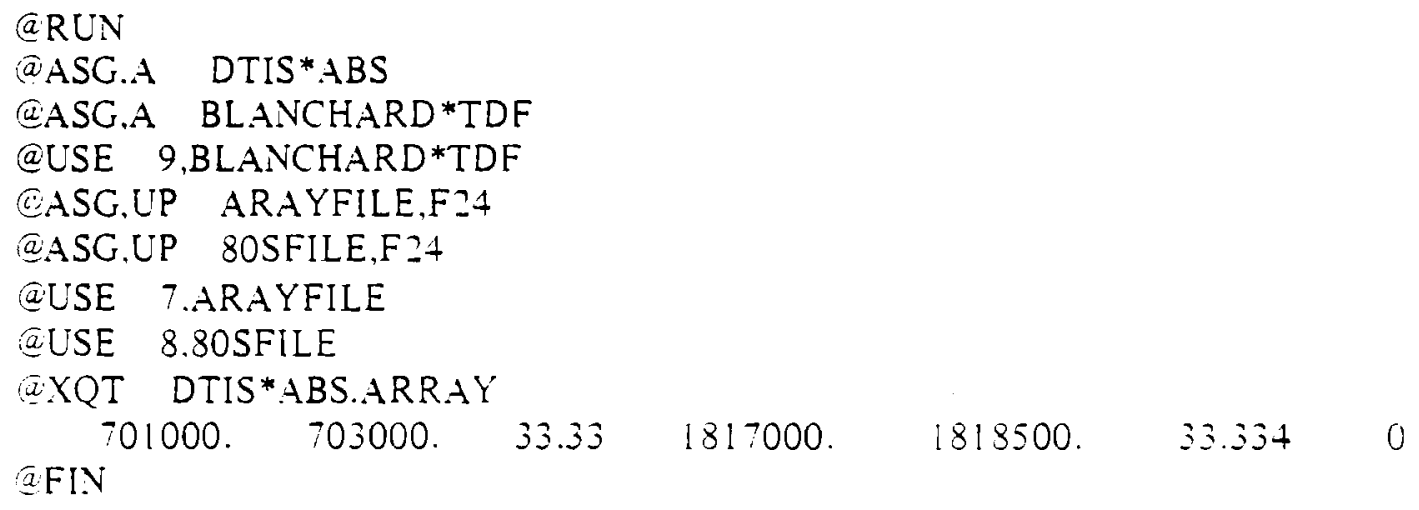


The GPCP ARAY block will be put in tile ARAYFILE, and the Control. 80 series, points $r^{\prime}$ into 80SFILE. 


\section{SECTION 3. PROGRAM BLOCKBACK}

\section{Description}

The program BLOCKBACK recovers data from the blocked TDF in a selected area. It outputs the recovered data in Standard Spatl Format.

The program was written to bypass use of DTIS Segment MANUr as gridder for small areas where contours are desired. Program FSGPCP grids small amounts of random (terrain) data efficiently and can go directly to contouring.

One data card is required to run BLOCKBACK. That card describes the area to be recovered.

\section{Data Card Format:}

$\mathrm{cc}$

use

format

$1-10$

SWCN Southwest Corner (N) of Area

F10.0

$11-20$

SWCE Southwest Corner (E) of Area

F10.0

$21-30$

NECN Northeast Corner (N) of Area

F10.0

3140

NECE Northeast Corner (E) of Area

F 10.0

$41-50$

Upper Elevation limit

F 10.0

Elevations above this valve are ignored

Input Deck Assembly. Exhibit 4.26 is a schematic diagram of an input deck for program BLOCKBACK. 


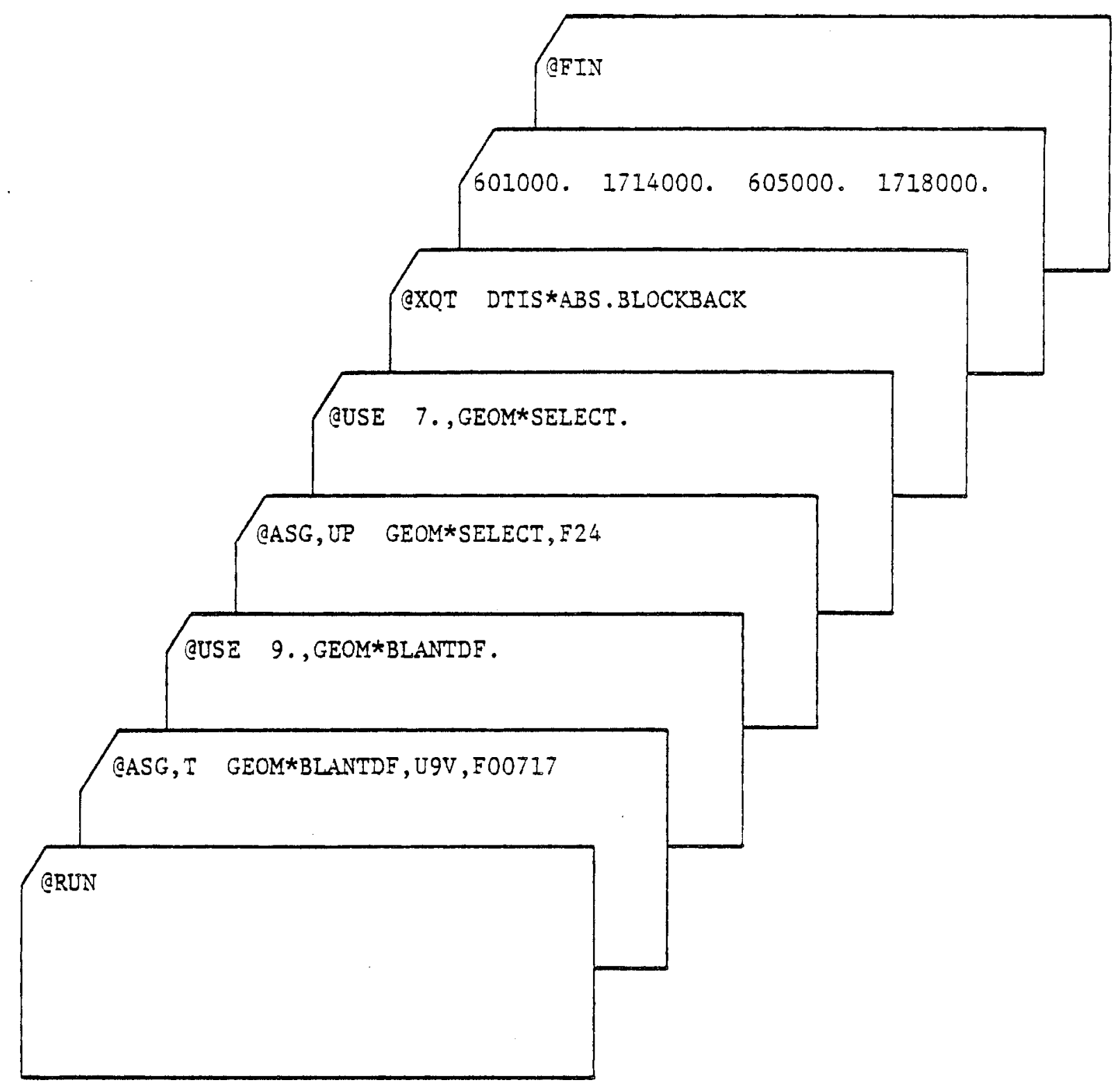

$i^{2}$ 


\section{Gridded and Irregular Data}

Digital terrain data can be generated in either one of two different formats: gridded and non-gridded. In the gridded format, spot elevations are recorded at a regular gridded interval. Since the grid intervals are constant, the data file basically consists of strings of spot elevations. In the non-gridded format. the terrain elevations are digitized in an irregular manner, and each data point must be defined by its three rectangular coordinates, $x, y$, and $z$.

Most of the application programs included in the TOPAS program package require that the terrain data be stored in a gridded partern. If a terrain data file contains irregularly sampled data, a corresponding data file, consisting of gridded elevation data, can be generated using program FSGPCP.

FSGPCP is the acronym for Forest Service General Purpose Contouring Program. It can be used to generate elevation contour plots from either gridded or non-gridded data files. If the input data is non-gridded. FSGPCP first computes the spot elevations along a rectangular grid, and then generates a contour plot. At the discretion of the user, the computed grid elevations may be written onto a data file or punched out on cards.

The application of FSGPCP for plotting contours will be explained in Chapter VI. This section will emphasize the use of this program to build a gridded data file from a set of irregular digital terrain data.

Data Card Formats

SIZE Card.

Purpose: This card introduces the parameters XSCALE, YSCALE, X00, Y00, XMIN, XINC. XMAX, YMIN, YINC, and YMAX, which determine the size, scale, position of the map; and grid size.

Notes: The map will correspond to the Region that has $X$ between XMIN and XMAX, and $Y$ between YMIN and YMAX. The scale will be XSCALE units/ inch in the $X$ direction, and YSCALE units/inch in the $Y$ direction. The point $X=X M I N, Y=Y M I N$ will be plotted at the point (X00, $Y 00$ ) (inches) on the plotter, and other values of $X$ and $Y$ will be plotted relative to this point. Any linear units may be used. But the units used in this card must be the same as those used in the elevation data card. 


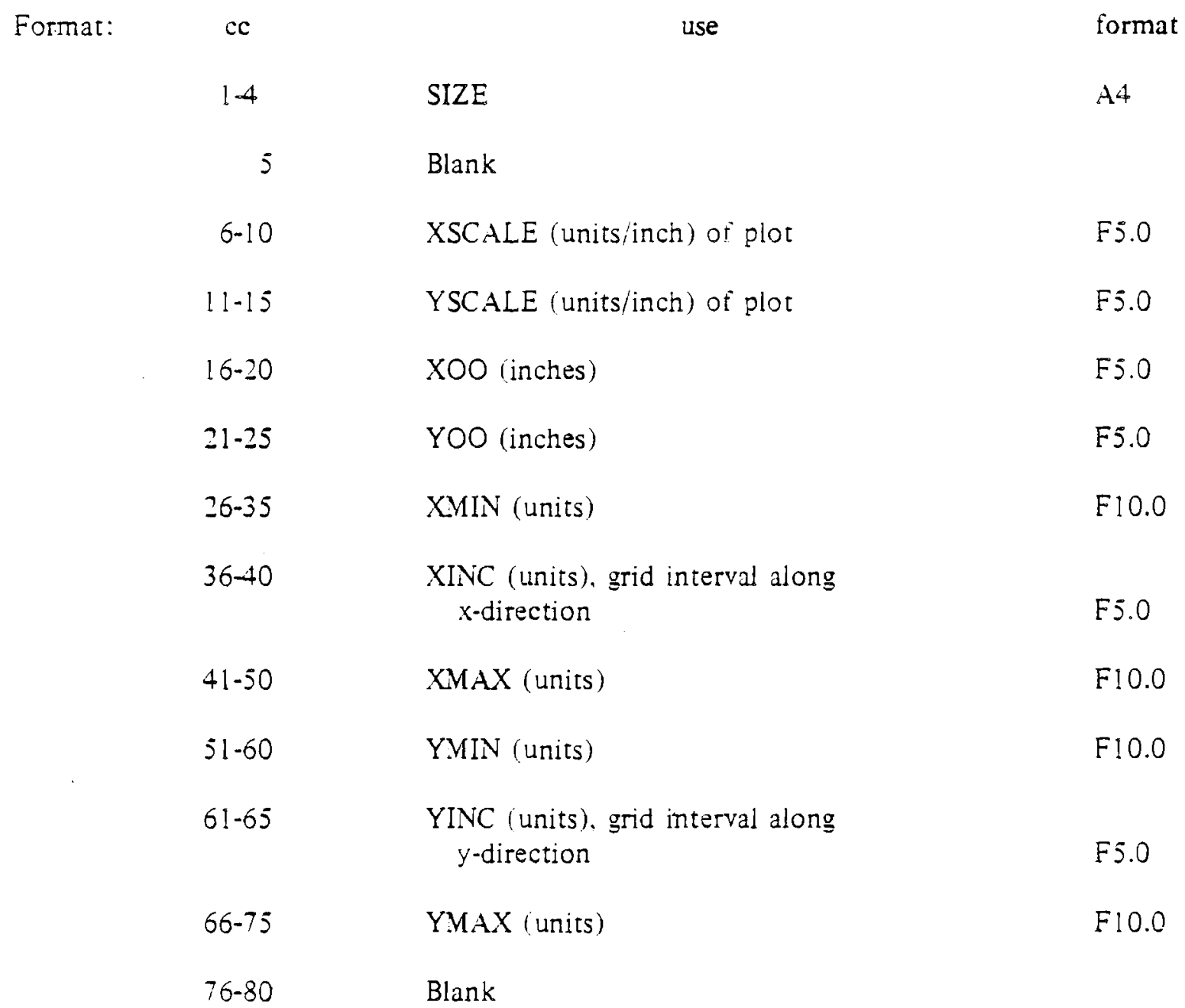

Data Card for Terrain Points: (one card to define each terrain point)

$\begin{array}{ccc}c c & \text { use } & \text { format } \\ 1-10 & \text { Blank } & 10 \mathrm{X} \\ 11-20 & \text { X-coordinate } & \mathrm{F} 10.0 \\ 21-30 & \text { Y-coordinate } & \mathrm{F} 10.0 \\ 31-40 & \text { Elevation } & \mathrm{F} 10.0\end{array}$


BLEV Card: (to specify contour interval)

\begin{tabular}{llc}
\multicolumn{1}{cc}{ use } & format \\
14 & BLEV & A4 \\
5 & Blank & $1 \mathrm{X}$ \\
$6-10$ & Contour interval & F5.0
\end{tabular}

Input Deck Assembly. Exhibit 4.27 illustrates the input deck assembly for generating gridded data from a set of irregular data, and plotting a contour map of the terrain. The gridded terrain data is stored in a file called GEOM*FILE.

For further explanation on the various command cards shown in Exhibit 4.27, the users can refer to Chapter VI. 


\begin{tabular}{|c|c|c|c|c|c|c|c|c|c|}
\hline & o. & 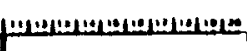 & 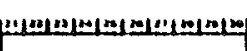 & 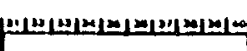 & $10.1004-5$ & & & $\because n$ & 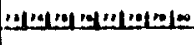 \\
\hline & H & 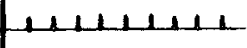 & Helenden & 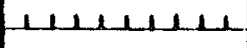 & 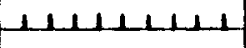 & $1+1111211$ & 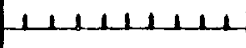 & L & $|1| 1111$ \\
\hline & A $2 \mathrm{~T}$ & $S_{1}^{\star} A B_{1,11}$ & 1121112 & 111111111 & $|1| 1|1| 11$ & 111111111 & $\triangle 1111, L L$ & $L$ & 1111111 \\
\hline$\underline{G}_{12}$ & $1 \perp 1$ & $.1+1+1+1$ & 11.111214 & 12111211 & $|+| 1|||l|$ & 11111111 & LLLLLLL & 1 & $111111 L$ \\
\hline & & -111111111 & 111111111 & $|1| 1|1| 11$ & $|1| 1|1| 1 \mid$ & $|\perp| 1|1| 11$ & 111112111 & 1 & 1111111 \\
\hline$(A) A_{1} G_{12}$ & {$[1]+3$} & $|L| \perp|\perp| 1$ & $\| \perp \perp \perp \perp \mid \perp L$ & $\perp \perp \perp \perp \perp \perp \mid \Lambda$ & 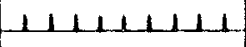 & $-1 \perp \perp L \perp \perp L_{1} \mid \alpha$ & 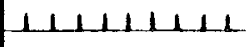 & 1 & 1111111 \\
\hline$A_{1} G_{12}$ & & $.1111|1| 1 \mid$ & $1+11+112$ & $\Lambda \perp \perp \perp \perp \perp L$ & $11+11111$ & $\mid 11 \perp 1 L 11$ & $11 \mid+11111$ & 1 & 111111 \\
\hline$A S G_{12}$ & $U_{-1}$ & $E Q M_{1}^{*} F_{1} L_{1} E_{1,1} F$ & $24,1,11$ & 111111111 & $\perp 1+L 1+111$ & 111111111 & $1+111111$ & -1 & $11-11111$ \\
\hline$d U_{1} E_{1}$ & & $G E \varrho M^{*} F_{L} L_{1} E_{1}$ & 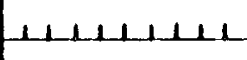 & $\perp \perp \perp \perp \perp \perp$ & L1. & $|1| 1|1| 1 \mid 1$ & $1+L 1+1+1]$ & L & $111111 L$ \\
\hline$\left(\mathrm{d} \times \mathrm{X}_{1} \mathrm{I}\right.$ & $D_{1} \mathrm{I}$ & $I S_{1}^{\star} A B S_{d}$ & $P_{1} P_{1+1}$ & $+1+1+1 \mid L 1$ & $1 \mid 1111111$ & -11111111 & 111111111 & ll & $111+111$ \\
\hline$C B_{1}$ & CDNV & $E, R_{I} I_{1} R_{1} A_{N}, Q \Omega M$ & $\triangle A_{1} I A_{\perp} D_{1} \in$ & $\left.R I, D, E_{1}, 1, D A\right]$ & $A, \ldots+1,1 \ldots$ & $|1| 1|| 1|1|$ & $\perp \perp \perp \perp \perp \perp \perp 1$ & $L$ & 1111111 \\
\hline$E X_{1}$ & 114 & $\mid 12111$ & $|1| L|1| 1 \mid$ & $111+11111$ & 11111111 & 111111111 & 111111111 & 1 & 1111111 \\
\hline oh $g_{1} E_{1}$ & 1,3, & & $\| \perp \Lambda \perp \perp 1$ & $\perp|\perp| \perp|1| 1$ & 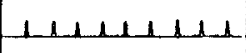 & $\perp \perp \perp 1 \| \perp \perp \perp L$ & $\perp \Lambda \perp \perp L \perp \perp \perp \Omega$ & $\perp$ & LLL $1 \perp L$ \\
\hline SILEL & 111 & $|1| 1|1|$ & 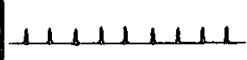 & $A L A|l| l \mid$ & SER "SIZE"L & $q a_{1}^{2} l_{1} q M_{1} G P$ & MameqL, & 1 & (2) \\
\hline CNIL & $=0,5$ & 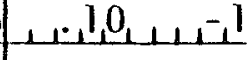 & 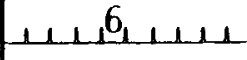 & 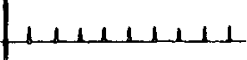 & Cprtyol $\{4$ & bi is sards & 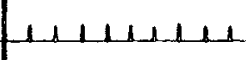 & 1 & Llllull \\
\hline NII & 1 & $x_{1}, 3, F_{1}, 0_{1}, 0_{1}$ & $3 A_{1} \Omega_{1 \perp \perp 1}$ & 111111111 & foxtrgzalu & $9,1, q 9 r \mid, 1$ & 111111111 & 1 & $|1| 1 \mid 11$ \\
\hline CNIL & Lel & $+1+d^{2} u \Lambda 1^{3}$ & 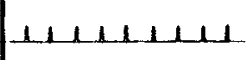 & 111111111 & Epkitral full & $A_{1} A_{1} x_{1} L_{1}$ & 111111111 & 1 & $1111+11$ \\
\hline 111 & 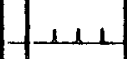 & $1,1 \ldots \ldots x_{1}$ & $\left., \ldots, \ldots, Y_{1}, l_{1}\right)$ & $1,1,1,1$ & LLLLLLL & 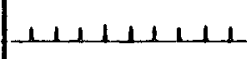 & $1 / 1<1<1<1$ & 1 & 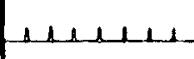 \\
\hline$\perp \perp \perp$ & L1 & 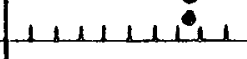 & $\mid \perp \perp 1 \perp$ il $_{1}$ & $1+11111$ & Plg, qarold & y exs:h rangl & m latarapezal & 1 & $1|1| 1 \mid 1$ \\
\hline $1+1$ & -111 & $1,1,1, x_{1}(n)$ & $\perp \perp \perp, Y\left(n_{1}\right)$ & $\ldots+1, Z_{1}\left(n_{1}\right)$ & 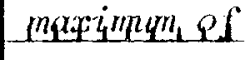 & 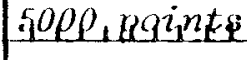 & 1112112121 & 1 & SLLSLL \\
\hline$f+1$ & $-\Lambda \operatorname{B}$ & $\operatorname{lan}_{n} \boldsymbol{k}_{1} \operatorname{cas}_{1} a_{1}$ & $|\perp| \perp|\perp| 1 \mid$ & $\perp \perp \perp 11 \perp \perp 1$ & 111111111 & 111111111 & $\|1\| 11111$ & 1 & HALL1 \\
\hline $3[\mathrm{NO}$ & -111 & $|\perp \perp \perp| \perp|\perp|$ & $1+1+1+11$ & $|1| 1|1| 11$ & $1|1| 1|+| 1$ & $|1111+1| 1$ & $|l| l|1| 11$ & 1 & Hedest \\
\hline & & & & & & & & & \\
\hline
\end{tabular}




\begin{tabular}{|c|c|c|c|c|c|c|c|c|c|}
\hline 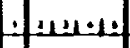 & A. A. & 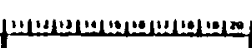 & 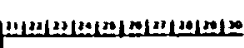 & 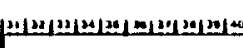 & 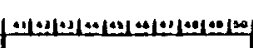 & 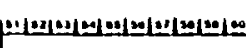 & 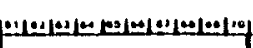 & 果果 & 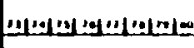 \\
\hline & & 1111 & $1,1,1 L_{1} 11$ & $11,1,1,112$ & 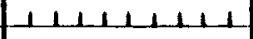 & 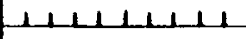 & $\perp 1,111111$ & 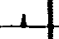 & $1 / 1 / 11$ \\
\hline 154 & 111 & 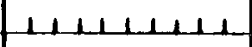 & $L L 1111,11$ & $\triangle 11111111$ & 111111141 & 111111111 & $1+1+1+111$ & 1 & $111+111$ \\
\hline EEV & $5 p_{1 .}$ & 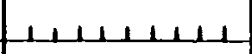 & 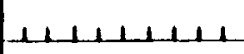 & $\perp 1, \ldots \operatorname{con}_{2}$ & $q u_{1} r_{1} i_{3} \eta_{1} e_{1} r_{2}$ ? & $q l_{1}, \bar{I}, 5 q, q$ & $f e_{1} t_{1}, 1$, & 1 & \\
\hline$\beta_{1} D_{1} R_{1}$ & & $\perp \perp \perp \perp 11$ & $\begin{array}{lllllll}1 & 1 & 1 & 1 & 1 & 1 & 1\end{array}$ & 1111 & 111111112 & $1 \perp 111111$ & $\perp \perp \perp \perp \perp \perp \perp$ & 1 & $\perp$ \\
\hline$N, D$ & & 11111111 & 111111111 & 111111111 & 11111111 & 111111111 & 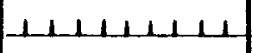 & $\perp$ & $1|1| 1 \mid 1$ \\
\hline TpP & & 111111111 & 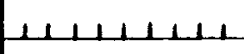 & 111111111 & 111111111 & 1111111111 & 111111111 & 1 & 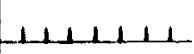 \\
\hline$E D_{12} U$ & & 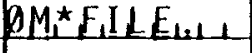 & $1111 / 1111$ & 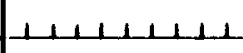 & 11111111 & 111111111 & $1+1,1$ & 1 & 11 \\
\hline L & & 111111111 & 111111111 & 11,111111 & 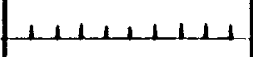 & 111111111 & $1+1111112$ & 1 & $1.1,1.11$ \\
\hline PEEN & & 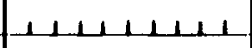 & $1111+1111$ & $11+111111$ & 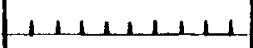 & $\perp \perp \perp \perp L \perp L L$ & 111111111 & 1 & 1111111 \\
\hline SFD & & 111111111 & 11111111 & $\perp 11111111$ & 111111111 & 111111111 & 111111111 & 1 & 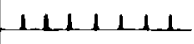 \\
\hline & & 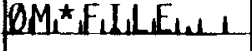 & $\perp \perp \perp \perp \perp \perp$ & $\perp \perp 11 \perp \perp 11$ & 111111111 & $\mid 1+111111$ & 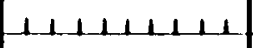 & 1 & $L 1+1+1 / 2$ \\
\hline & & 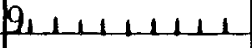 & $11111+111$ & 111111111 & $11+1,1,1,4$ & 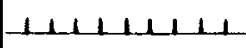 & 111111111 & 1 & $11<1<1]$ \\
\hline & $R D$ & $S_{1}^{*} R_{1} D_{1} S_{1}, C_{1} A_{1} L_{1} I$ & $\perp \perp \perp \perp \perp \mid \perp L$ & $\perp 1 L_{1}\left|L_{1}\right| L$ & 11111111 & $\perp 1111111$ & 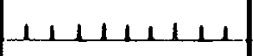 & $\perp$ & $\perp \perp \perp \perp \perp \mid$ \\
\hline$E_{1} N_{1}$ & 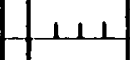 & $\perp \perp \perp \perp \perp \perp \perp$ & $|\perp \perp \perp| \perp \mid \perp$ & $\perp|1||| 1 \mid \perp$ & 111111111 & $\mid 1 / 11111$ & $\perp \perp \perp \perp \perp \perp \perp \mid$ & $\perp$ & 1111111 \\
\hline 111 & 111 & 111111211 & 11111111 & 11111111 & 111111112 & 111111111 & 111111111 & $\perp$ & 1111111 \\
\hline$+1,4$ & 111 & $11 \perp 1 \perp 111$ & $\mid L 1+1+1+1$ & 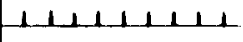 & $\perp 1+1+1+1<$ & 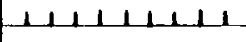 & $-1|+11+1| 1$ & 1 & 1111111 \\
\hline 111 & 111 & 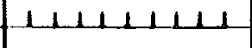 & 111111111 & $1 \perp 1111111$ & 111111111 & 111111111 & $11|1111| 1$ & 1 & 1111111 \\
\hline $11+1$ & fLe & 111111111 & $\perp \perp \perp \perp \perp 11 L$ & $\perp \perp \perp \perp \perp \perp 1$ & 111111111 & $\perp \perp \perp \perp \mid \perp \perp$ & $\perp \perp|\perp| \perp \mid \perp$ & -1 & $|\perp| \perp|\perp|$ \\
\hline 111 & 111 & $\perp 1111111$ & $|1| 1|1| 11$ & 111111111 & $|1111| 11$ & 111111111 & 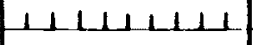 & 1 & 1111111 \\
\hline 14 & |11 & 1.2111111 & 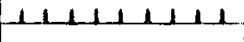 & 121211211 & .12111211 & 1112111121 & $-11+111211$ & 1 & 111111 \\
\hline & & & 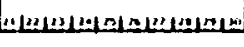 & & & & & & \\
\hline
\end{tabular}




\section{CHAPTER V}

\section{SLOPE AND ASPECT PROGRAM (SLOPE)}

\section{Program Function}

Program SLOPE takes as input a rectangular array of elevation data points which have been stored in a data file. For example, a data file can be created from an existing DMA tape using program SCANDMA.

Program SLOPE computes the slope and aspect as the center of the cell described by 4 elevation points, and stores the output in a new data file. It employs a least square algorithm to determine the best-fitting plane with respect to the four adjacent elevation points. The steepest slope along that plane and its aspect angle are then used as the slope and aspect at that elevation point.

If graphic outputs are desired, isoslope lines may be generated using program FSGPCP (see Chapter VI), and program ZONEIT may be used to plot zones of various slopes and aspects (see Chapter VI).

\section{Data Card Formats}

Only one data card is needed for input to program SLOPE. The card format is as follows:

$$
\text { cc }
$$

$1-10$

$11-20$ use

XINC Ground distance berween adjacent elevation points in the $\mathrm{X}$ direction.

YINC Ground distance between adjacent elevation points in the $Y$ direction. format

F10.2

F10.2

In the case of DMA tapes, the ground distance between adjacent data points in the original data tape is $208.3 \mathrm{ft}$., in both the $\mathrm{x}$ and $\mathrm{y}$ directions. If only every second point is used. then the ground distance between adjacent points becomes $416.7 \mathrm{ft}$.

Input Deek for Generating a Slope and an Aspect Data File. Exhibit 5.1 is a sample input deck for generating a slope and an aspect data file. The elevation data is input from a file called GEOM*JSMITH, and the slopes are stored in a new file called GEOM*JSMITHS. The aspects are stored in a file called GEOM*JSMITHA. Chapter VI will show how the slope file can be used as input to program FSGPCP to produce an isoslope plot. 


\begin{tabular}{|c|c|c|c|c|c|c|c|c|c|}
\hline ULS & | & 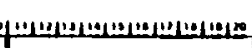 & Hendm & 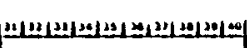 & 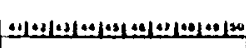 & 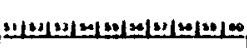 & 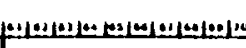 & 止先 & 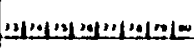 \\
\hline & & (2) & 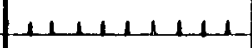 & $111+1111$, & $\perp 11+1 \mid 1+1$ & $\mid 1+111+11$ & $1+1|1+1| 1$ & $a$ & $L \perp \perp \perp L 1$ \\
\hline & & $I, S_{1} x_{1} A B_{1} S_{1,1,1}$ & 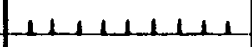 & $\perp \perp \perp \perp L \perp \perp \perp$ & $\Lambda \perp \perp \perp \perp \perp \Lambda$ & 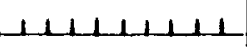 & $\mid\left\|_{1}\right\|_{1} \|_{1}$ & 1 & 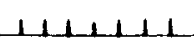 \\
\hline $0, A_{1}$, & & $O_{M} A_{1} J_{1} S_{1} M_{1} I_{1} T_{1} H$ & 111111111 & 111111111 & $|\perp \perp \perp \perp| L L$ & $1 \perp 111+11 L$ & 111111111 & 1 & 1111111 \\
\hline 1115 & & $E_{1} O_{1} M_{1} X_{1} S_{1} M I_{1} T_{1} H$ & 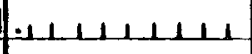 & 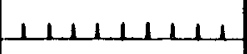 & 111111111 & 111111111 & 111111111 & 1 & $\perp \perp L L 111$ \\
\hline (d) $A_{S}$ & & $E_{1} O M X_{2} J_{S} S_{M} L_{1} T_{1} H$ & $S_{2,1} F_{1} A_{1} A_{1 \ldots}$ & 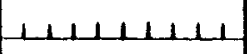 & 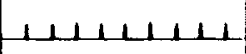 & $11+1+1111$ & 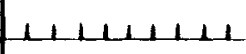 & 1 & $1 \perp 1 \perp 111$ \\
\hline & & 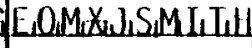 & 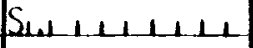 & 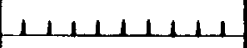 & 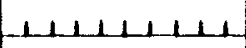 & $1+1 / 11111$ & 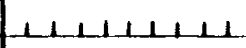 & 1 & $1+1 \mid 1+1$ \\
\hline & & 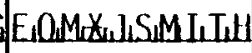 & A, $F_{1} 24,+1$, & 111111111 & $\perp|1| 1|1| 1 \mid$ & 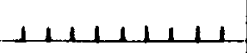 & $1+111$ & -1. & $|1||<| 1$ \\
\hline & & E.MMA政S & $A \cdot 11111111$ & $11,1,1111$, & $\perp 111 \perp 111$ & $\perp \perp 1,1,1,1$ & 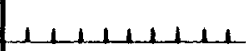 & $\perp$ & $1,1,1,1$ \\
\hline$x_{1} \Omega_{1}$ & DIIS & $X_{1} A_{1} B_{1} S_{1}, S_{1} L_{1} Q P_{1}$ & $\mid 11111111$ & $\not 1,111,11$ & 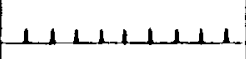 & $-1+111+11$ & 1111,111 & $\perp$. & $1|1|<1$ \\
\hline 1,8 & $3,3 \cdot, 3,3$ & $1, \ldots, 8,3,3,3,33$ & $|L| 1 \mid+L L 1$ & $11111<111$ & $\perp \perp \perp \perp \perp \perp \perp \perp$ & $1+1+111,11$ & $\left|\|_{1}\right| l|l| l$ & 1 & $1<11111$ \\
\hline$g F_{1} I_{\perp}$ & & 111111111 & 111111111 & $1121111 L$ & $|1| 1|1| 1 \mid$ & 111111111 & $\perp L 1 / 1+111$ & 1 & $1 \perp \perp 1 \mid L 1$ \\
\hline 111 & 111 & 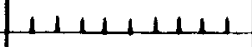 & $|\perp| \perp|1| 1$ & $\triangle \perp \perp L \perp \perp L$ & $11+111,11$ & $\perp \perp \perp 1 L_{1} \perp \Lambda$ & $\perp 11,11111$ & 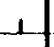 & $\perp \perp \perp \perp \perp$ \\
\hline 111 & 111 & $\| \perp L \perp \perp \perp \perp L \mid$ & 111111111 & $1,1+1,1,1+1$ & $1+1+1,1+1+$ & $1+111+111$ & $|1| 1|1| 1 \mid 1$ & 1. & $|1 \perp| 1|1|$ \\
\hline$\perp \perp$ & -11 & $\mid 1 \perp \perp \perp 111$ & $\perp \perp \perp \perp \perp \perp 11$ & 11111111 & 111111111 & $\|\mu L\| L \Lambda L$ & $\perp \perp \Lambda \perp L \perp L \perp$ & L. & $111 \mid 111$ \\
\hline 111 & $\perp 1$ & 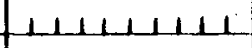 & $1+11+1 \perp \perp L$ & $\perp \mu L L L L L$ & 1,1111111 & $1+1+1+1 L_{1}$ & 11111111 & 1 & $|1| 1|1| 1 \mid$ \\
\hline 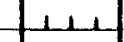 & 1,1 & $\mid 1,1,1,11$ & 111111111 & $1+1,1+1,1$ & 11111,112 & $1 L_{1}\left\|_{1}\right\|_{1}$ & $1111<11111$ & $\perp$ & $-1,1+1<1$ \\
\hline 111 & 112 & $\mu \mu \perp \perp \perp \mu L 1$ & $\perp \perp \perp \perp \perp \perp \perp \perp$ & $|+| \perp|\perp| \perp \mid$ & $\perp \perp+1|\perp| l$ & $\perp \perp \perp \perp \cup \perp \perp$ & $\perp \perp \perp \perp \perp \perp \perp \perp$ & $L$ & $1 \perp 11$ \\
\hline 111 & 111 & Leteldel & $\left|L_{1}\right| L_{1} \mid L_{1}$ & 111111111 & $1 \mid+1+1,11$ & $\perp 11111111$ & $11 \mid 1+1+11$ & 1 & $111 \perp 11$ \\
\hline 111 & 111 & $\| \perp \perp \perp \perp 111$ & 111111111 & 111111111 & $\mid 1111111$ & 11111111 & 111111111 & 1 & 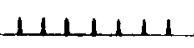 \\
\hline 111 & 1.11 & 111111 & $\perp 1,1+1111$ & 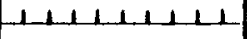 & $11+11+111$ & $1,11,1111$ & 111111111 & 1 & $|1| 1|1|$ \\
\hline & & & & & & & & & \\
\hline
\end{tabular}




\section{CHAPTER VI}

\section{GRAPHIC PRODUCT PROGRAMS}

\section{SECTION 1. CONTOURING PROGRAM (FSGPCP)}

\section{Program Function}

FSGPCP is the acronym for Forest Service General Purpose Contouring Program. This program was developed by California Computer Products, Inc. (Calcomp). It is for general use in displaying, by means of a contour diagram, functions dependent on two independent variables, $x$ and $y$, such as elevation contours and isoslope lines. This computer program determines the contours and presents them in a form suitable for display on any Calcomp graphic system.

The program can accept either gridded or non-gridded elevation data. The user can specify arbitrary regions in which the contours are not to be plotted. and/or regions in which the contours are to be plotted as special lines (dashed, ticked, etc.). Contours can be plotted as heavy lines, lines with cross ticks, lines with ticks on one side, or dashed lines. At the user's option, labels can be printed on contours.

One unique feature of the program is its ability to produce stereoscopic contour maps; that is, maps containing pairs of images of the contour lines which appear to be three dimensional when viewed in a stereoscope.

When the surface is represented by random (non-gridded) data, the data is first converted to gridded format. The values of the function at the mesh points are computed. and the contours are produced from this gridded data. Spacing between grid points is specified by the user.

This chapter will present only a simplified documentation of the program for common application problems in geometronics. For more details in documentation. the users are referred to Calcomp User's Manual for GPCP (Reference 1).

The use of this program for converting random data into gridded format is explained in Chapter IV.

Data Card Formats. The data deck for FSGPCP consists of a series of command cards which guide the program to perform the proper tasks. The most commonly used commands for ploting contours and isoslope maps are explained on the following pages. 
JOB Card

Association: Phase 0.

Purpose: $\quad$ This card initiates a new job.

Category: $\quad$ This is a Parameter Card.

Action Taken: The job identification is plotted on the map. and operation is transferred to Phase 1.

Notes: $\quad$ Every job must begin with a JOB $\quad$ card.

$\begin{array}{lll}14 & \text { JOBb } & \mathrm{A} 4 \\ 5 & \text { Blank } & \\ 6-80 & \begin{array}{l}\text { Any alphameric information (printed } \\ \text { as identification) }\end{array} & 18 \mathrm{~A} 4, \mathrm{~A} 3\end{array}$

\section{PAGE Size Card}

Association: $\quad$ Phase 1.

Purpose: $\quad$ This card introduces the width PAGSIZ of the plotter paper.

Category: $\quad$ This is a Parameter Card.

Action Taken: None.

Notes: If the map is so large that it will not fit on the plotter paper. it is automatically split into two or more pages. If a PAGE card is not present. PAGSIZ is taken to be 28.0 .

\begin{tabular}{|c|c|c|c|}
\hline Format: & cc & use & format \\
\hline & & PAGE & A4 \\
\hline & $\begin{array}{l}5 \\
6-10\end{array}$ & $\begin{array}{l}\text { Blank } \\
P A G S I Z\end{array}$ & $F=0$ \\
\hline & $\begin{array}{r}0-10 \\
11-80\end{array}$ & $\begin{array}{l}\text { PAGSiL linches } \\
\text { Blank }\end{array}$ & \\
\hline
\end{tabular}




\section{SIZE Card}

Association: Phase 1.

Purpose: $\quad$ This card introduces the parameters XSCALE, YSCALE, XOO, YOO, XMIN, XINC, XMAX, YMIN, YINC, and YMAX, which determine the size, scale, and position of the map.

Category: This is a Parameter Card.

Action Taken: This card causes the scale factors to be plotted on the edge of the map, and causes operation to be transferred to Phase 2.

Notes:

The map will correspond to the region that has $X$ between XMIN and XMAX, and $Y$ between YMIN and YMAX. The scale will be XSCALE units/inch in the $X$ direction. and YSCALE units/inch in the $Y$ direction. The point $X=X M I N, Y=Y$ MIN will be plotted at the point $(X O O$. $Y O O)$ (inches) on the plotter, and orher values of $X$ and $Y$ will be plotted relative to this point. The values of XINC and YINC printed (on the printer) will be the ones actually used, and will not necessarily match those on the data cards.

Format: $\quad c c$

use

format

1

$1-4$

SIZE

5

6-10

$11-15$

$16 \cdot 20$

$21 \cdot 25$

$26-35$

$36-40$

$41-50$

$51-60$

$61-65$

$66 \cdot 75$

$76-80$
Blank

XSCALE (units/inch)

YSCALE (units/inch)

XOO (inches)

YOO (incines)

XMIN (units)

XINC (units)

XMAX (units)

YMIN (units)

YINC (units)

YMAX (units)

Blank
$A+$

F5.0

F5.0

F5.0

F5.0

F10.0

F5.0

F10.0

F10.0

F5.0

F10.0

( 
ARrAY Card

Association: $\quad$ Phase 2.

Purpose: This card indicates whether the array points are to be posted on the map, and if so, what format is to be used. It also indicates whether the array is to be cleared bafore the new values are loaded into it.

Category: $\quad$ This is a Parameter Card.

Action Taken: This card begins an array block.

Format: $\quad$ cc $\quad$ use format

1-4 ARAY A4

$5 \quad$ Blank

6-10 Half width of center marks (+) F5.0

$11-15$ Height of symbols for posting array points F5.0

16-20 Number of digits following decimal point in num- I5

21-25 ICTL (posting-control number). If I5

$=1$ or 5 , mark centers but do not post levels of of array points;

$=2$ or 6 , do not mark centers or post arraypoint levels:

$=3$ or 7 , mark centers and post array-point levels;

$=4$ or 8 , draw the grid, but do not post arraypoint levels.

If ICTL $\leq 4$, the array is cleared before the new values are substituted into it: otherwise, the array is not cleared.

26-80 Blank 


\section{Basic (contour) LEVels Card}

Association: $\quad$ Phase 4.

Purpose: This card indicates the contour-level spacing, and causes contour curves th to be generated and plotted.

Category: $\quad$ This is a Parameter Card.

Action Taken: Contours are determined and plotted. Every IBOLDth contour curve is plotted as a heavy line with a label. (See BOLD card.) Contour curves around depressions are plotted with tick marks on the downhill side.

Notes: $\quad$ The maximum and minimum values of the function given on CVTL or ARAY cards are used as limits for the contour levels plotted.

\begin{tabular}{|c|c|c|c|}
\hline Format: & cc & use & format \\
\hline & $\begin{array}{l}14 \\
5\end{array}$ & $\begin{array}{l}\text { BLEV } \\
\text { Blank }\end{array}$ & A4 \\
\hline & $6-10$ & Contour-level spacing & F5.0 \\
\hline & $11-80$ & Blank & \\
\hline
\end{tabular}

\section{BOLD Contours Card}

Association: $\quad$ Phase 4.

Purpose: $\quad$ This card changes the number of light contours between bold contours.

Category: $\quad$ This is a Parameter Card.

Action Taken: See Purpose.

Notes: $\quad$ IBOLD is the number of levels between bold contours. The number of light contours between bold ones is IBOLD-1. (If no BOLD card is present, IBOLD is 5.)

Format:

$c c$
$1-4$
5
$6-10$
$11-80$

use

format

BOLD

$A 4$

Blank

IBOLD

F5.0

Blank 
PLOT Map Card

Association:

Pliase 5.

Purpose: $\quad$ This card causes the Program to transter operation to Phase 5.

Category: This is a Sequence Card.

Action Taken: See Purpose.

$\begin{array}{llll}\text { Format: } & \text { cc } & \text { use } & \text { format } \\ & 1-4 & \text { PLOT } & \text { A4 } \\ & 5-80 & \text { Blank } & \end{array}$


LINE Card

Association: Phase 5.

Purpose: $\quad$ This card causes a line to be drawn trom (X1. Y 1) to (X2, Y2). The line format is determined by the parameters TDIST. THEIT, SHIFT, GAP. and LINE, in the same manner as for a LEVb card. The parameter ICODE determines whether $(\mathrm{X} 1, \mathrm{Y} 1)$ and $\left(\mathrm{X}_{2}, \mathrm{Y}_{2}\right)$ are in inches relative to the plotter origin $O_{p}(I C O D E=0)$, or in units relative to the map origin $($ ICODE $=1)$.

Category: This is a Parameter Card.

Action Taken: See Purpose.

Notes:

If $I C O D E=1$, if $Z 1$ and $Z 2$ have been given on an ELEV card immediately preceding this card. and if non-zero parailax is being used, the line is drawn from the apparent position (X1, Y $1, Z 1)$ to the apparent position (X2, Y2, Z2). The parameter GAP is equal to THEIT if LINE $= \pm 4$.

Format: $\quad c c$ use format

$\begin{array}{lll}1-4 & \text { LINE } & \text { A } \\ 5 & \text { Blank } & \text { I5 } \\ 6-10 & \text { ICODE } & \text { FI. }\end{array}$

$11-15 \quad \mathrm{X} 1$

F5.0

$16-20 \quad Y 1 \quad$ F5.0

21-25 X2

26-30 Y2 F5.0

31-35 TDIST $=$ Distance in inches between tick marks F5.0

36-40 THEIT $=$ Height of tick marks in inches (may be F5.0 negative)

or

GAP $=$ Width of gaps in inches

41-45 SHIFT $=$ Lateral displacement, in inches. in line F5.0

46-50 LINE (integer value determines the line type). If: I5 $=0$, plot a solid bold line of width $2 *$ SHIFT. $= \pm 1$, plot solid line:

$= \pm 2$, plot tick marks on one side of the line (uphill side if THEIT is positive, downhill if it is negative):

$= \pm 3$. plot tick marks that cross the line:

$= \pm 4$, plot a gapped line.

If LINE is positive, the line is not displaced.

If LINE is negative, the line is displaced by SHIFT inches.

$51-80 \quad$ Blank 


\section{Input Deck Plotting Contours from Gridded Data}

Exhibit 6.1 is a schematic diagram of an input deck for plotting elevation contours from gridded data. The elevation data has already been stored in a Fastran computer file called GEOM*JSMITH, which is now used directly as input to the program FSGPCP. When DMA digital terrain tapes are used as the data source, the Fastran file can be built using programs PRINT and SCANDMA, as described in Chapter III.

Exhibit 6.2 is a sample input deck. The area to be contoured is a 15 -minute USGS quad sheet from a DMA tape. Exhibit 6.3 is a sample graphic output from running FSGPCP. 


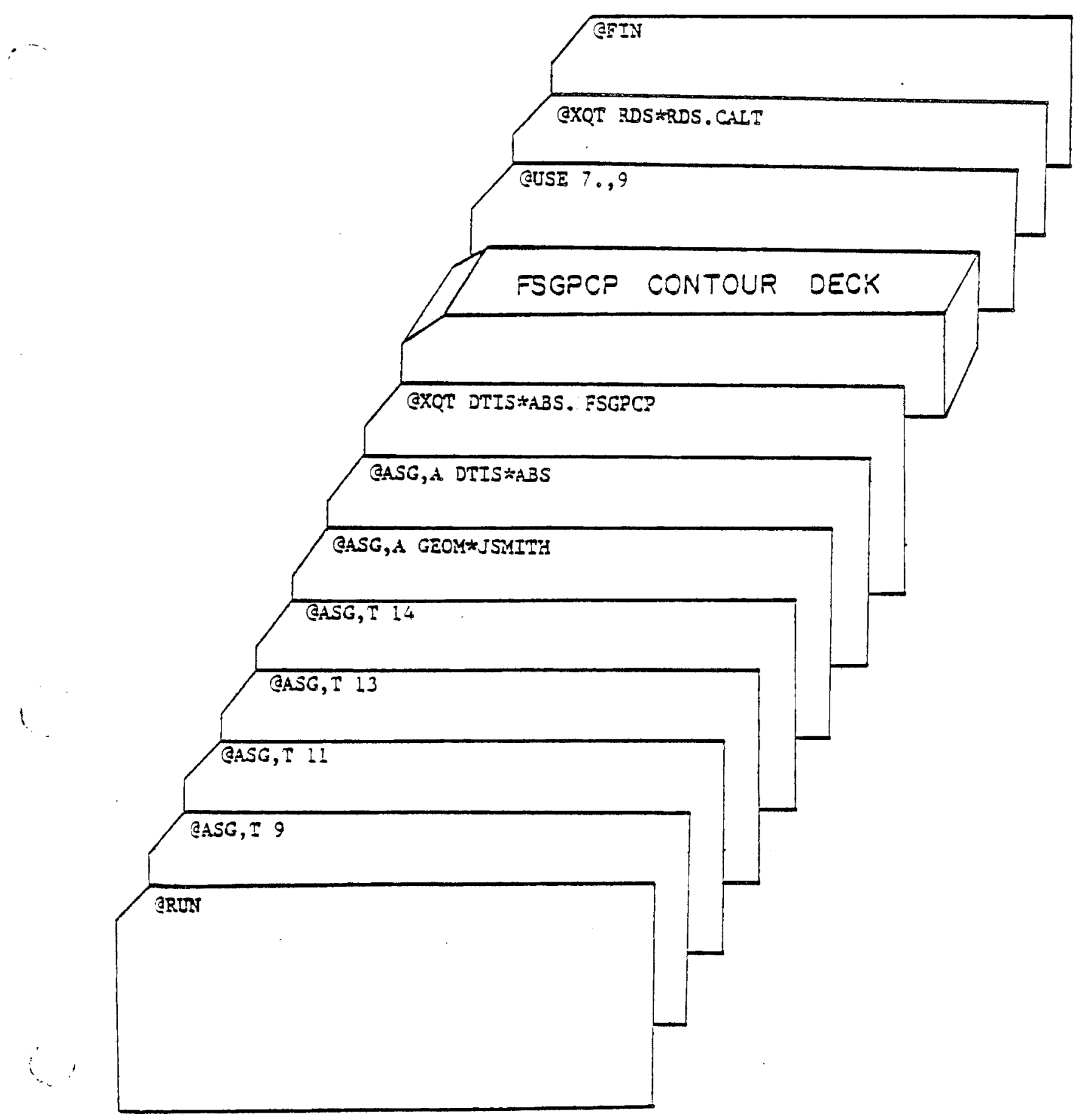

Exhibit 6.1 - Input Deck for Plotting Elevation Contours from Gridder Data with Program FSGPCP 


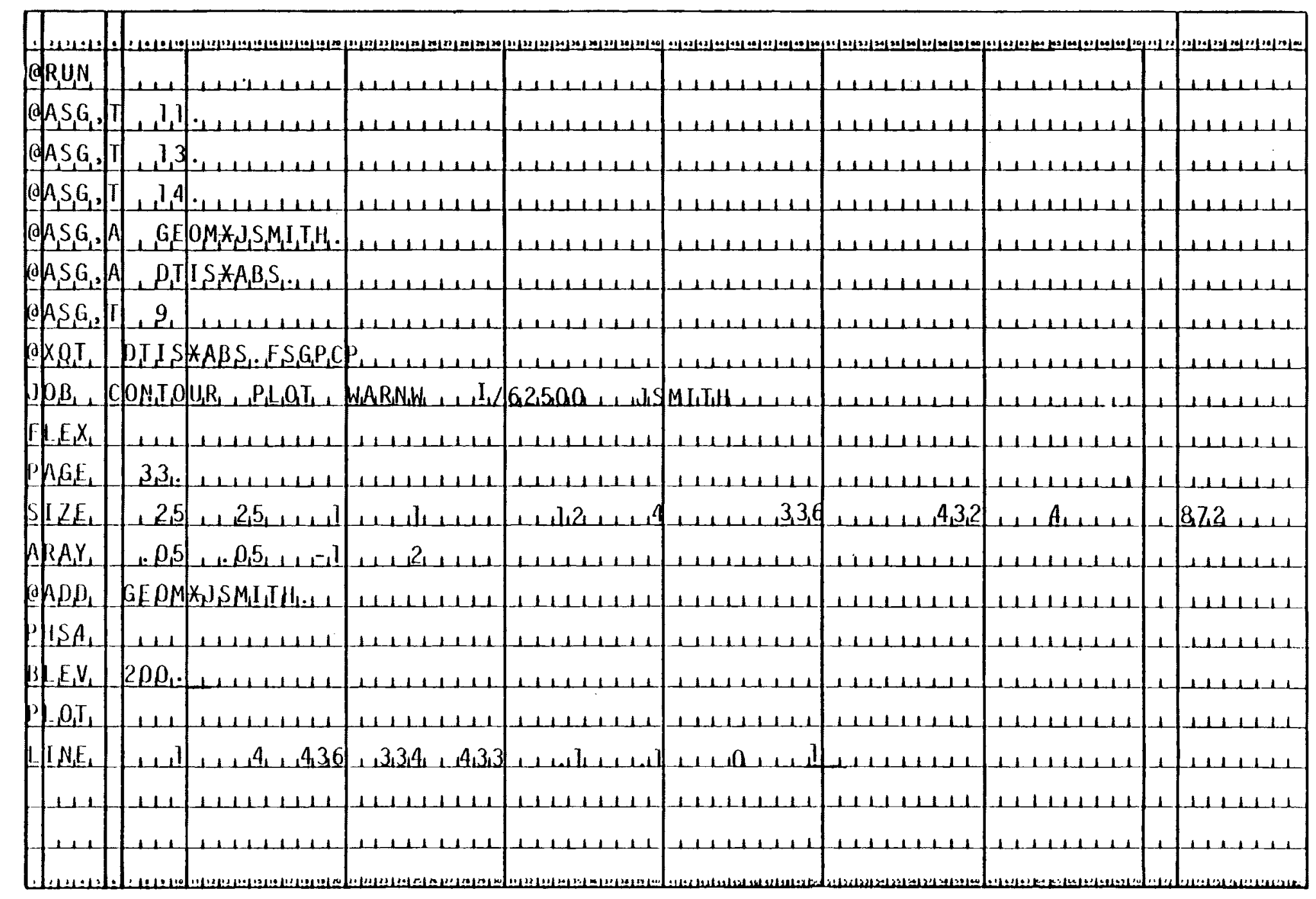




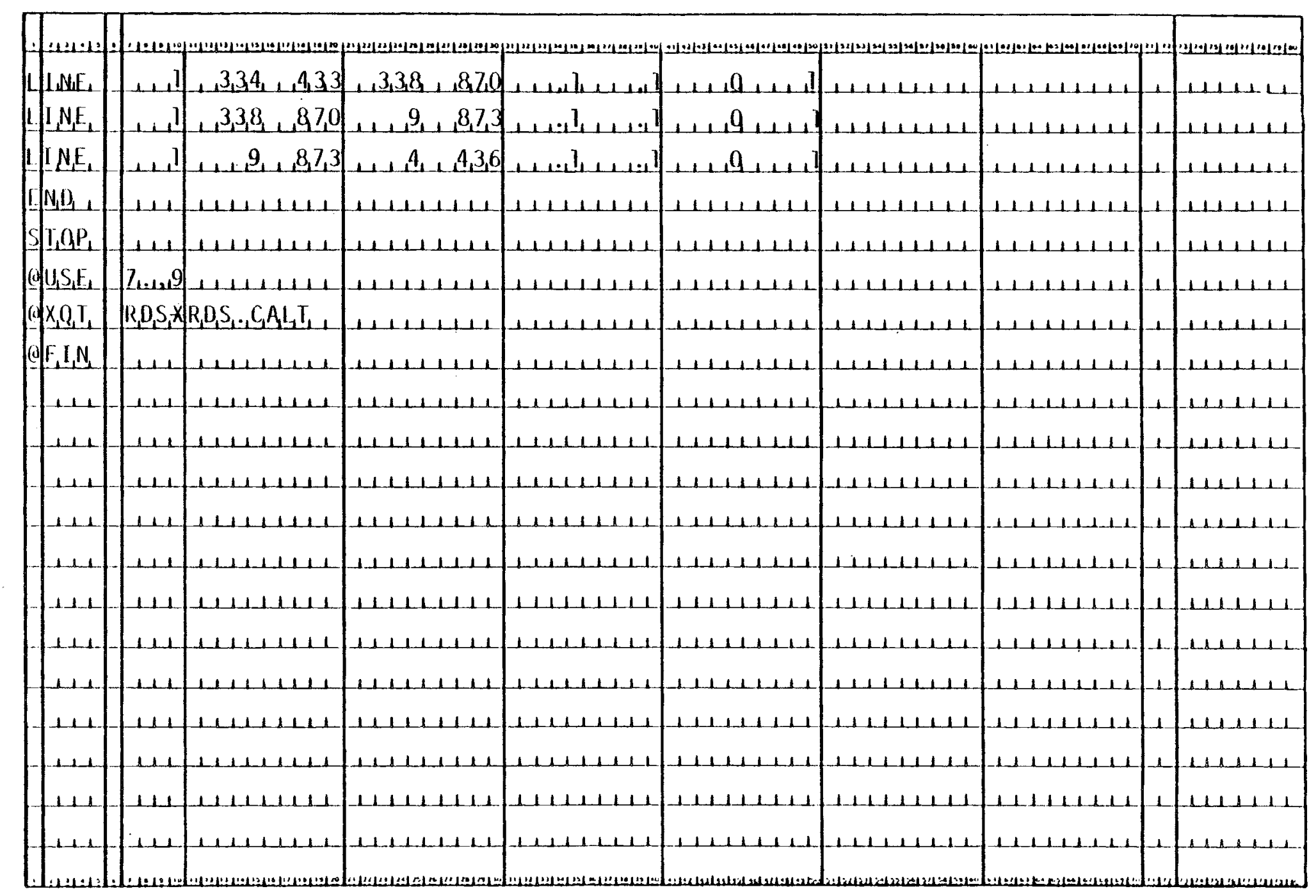

Exhibit 6.2 - Sample Imput Deck for Plothing Elevation Contours from Grithled Data with FSGPCP (Contimted) 
CONTOUA MAP

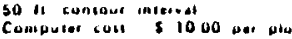

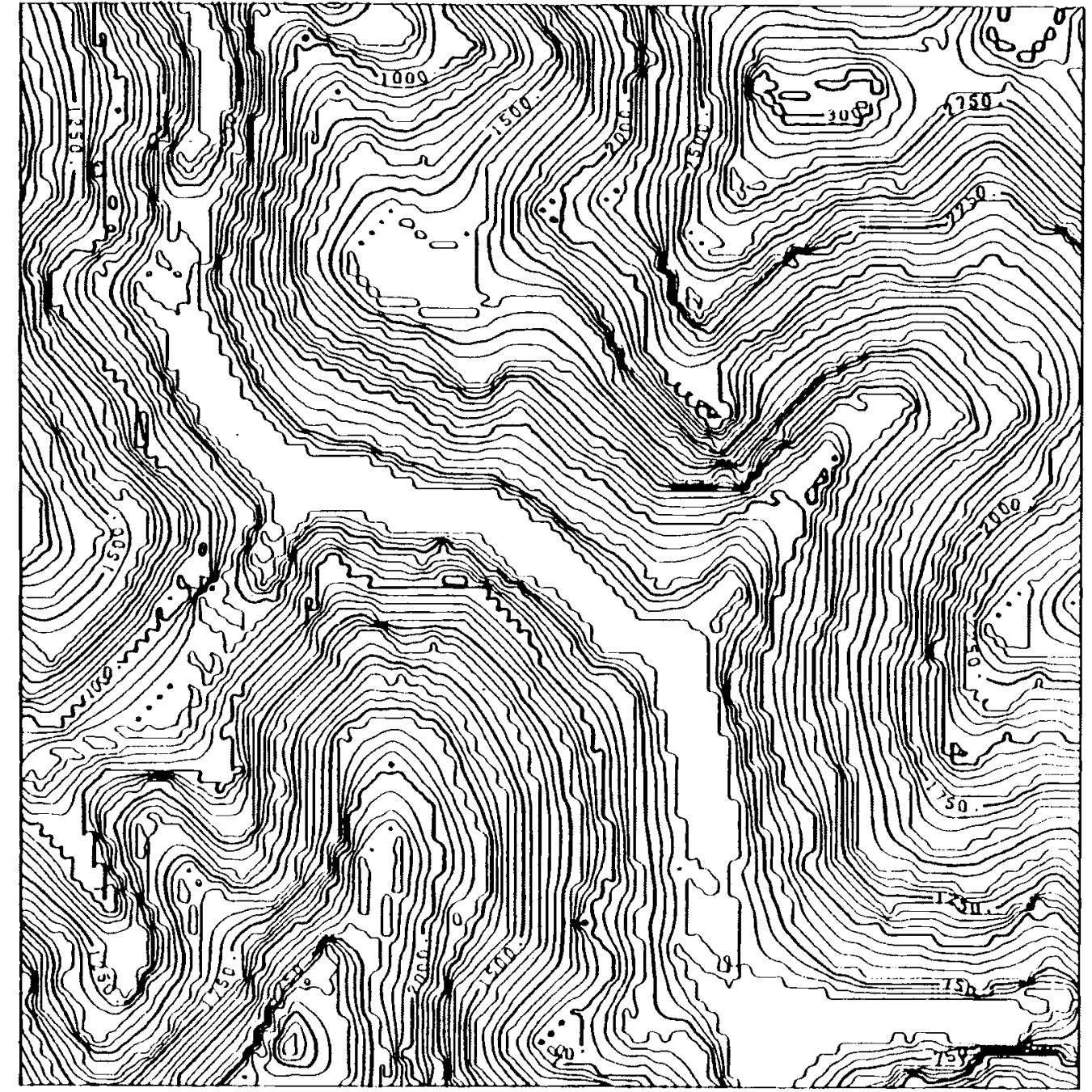

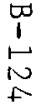

Exhibit 6.3 -- Sample Output from Program FSGPCP 


\section{SECTION 2. ISOSLOPE PROGRAM (FSGPCP)}

\section{Program Function}

The general purpose contouring program FSGPCP may also be used to plot isoslope lines (e.g. lines of equal slopes). The program takes as input slope, data that has been computed through program SLOPE (Chapter V). The user can specify the isoslope lines (such as the $10 \%$. $20 \%$, or $35 \%$ slope lines) to be plotted.

\section{Data Card Format}

In addition to the card types described in Section 1 for plotting elevation contours with FSGPCP, two other command cards are particularly useful for plorting isoslope lines. These command cards are NWPN and LEV.

Command Card NWPN. This card is used to activate a new pen for the next isoline. For example. suppose that the CALCOMP plotter has three pens with pen 1 containing black ink, pen 2, red ink, and pen 3, green ink. By using this command card to activate a particular pen, the user can specify the color for each isoslope line.

\begin{tabular}{lll}
\multicolumn{1}{c}{ use } & format \\
14 & NWPN & A4 \\
5 & Blank & Pen number to be \\
$6-10$ & $\begin{array}{l}\text { used for the next } \\
\text { isoslope line. }\end{array}$
\end{tabular}

Command Card LEV. This card is used to specify the parameters for an isoslope line. 
Blank

$A R C D S T=$ Distance in inches along isoslope line. from line start to label

TDIST $=$ Distance in inches between tick marks or centers of gaps

36-40 THEIT $=$ Height of tick marks in inches (may be negative) $=+1$. plot solid line:

$=+2$. plot tick marks on one side of the line (uphiil side if THEIT is positive. downhill if it is negative): $=+3$. plot tick marks that cross the line:

$=+4$. pior a gapped line.

If LINE is positive, the contour line is not displaced. If LINE is negative. the contour line is displaced by SHIFT inches.

56-60 IFMT $=$ Number of digits after decimal point in the label 


\section{Input Deck for Plotting Isoslope Lines}

Exhibit 6.4 is a schematic diagram of the input deck for plotting isoslope lines. The slope data has already been generated using program SLOPE (see Chapter V) and is stored in a Fastran file called GEOM*ISMITHS, which is now used as input to program FSGPCP.

Exhibit 6.5 is the layout for a sample input deck. Exhibit 6.6 is a sample graphic output. 


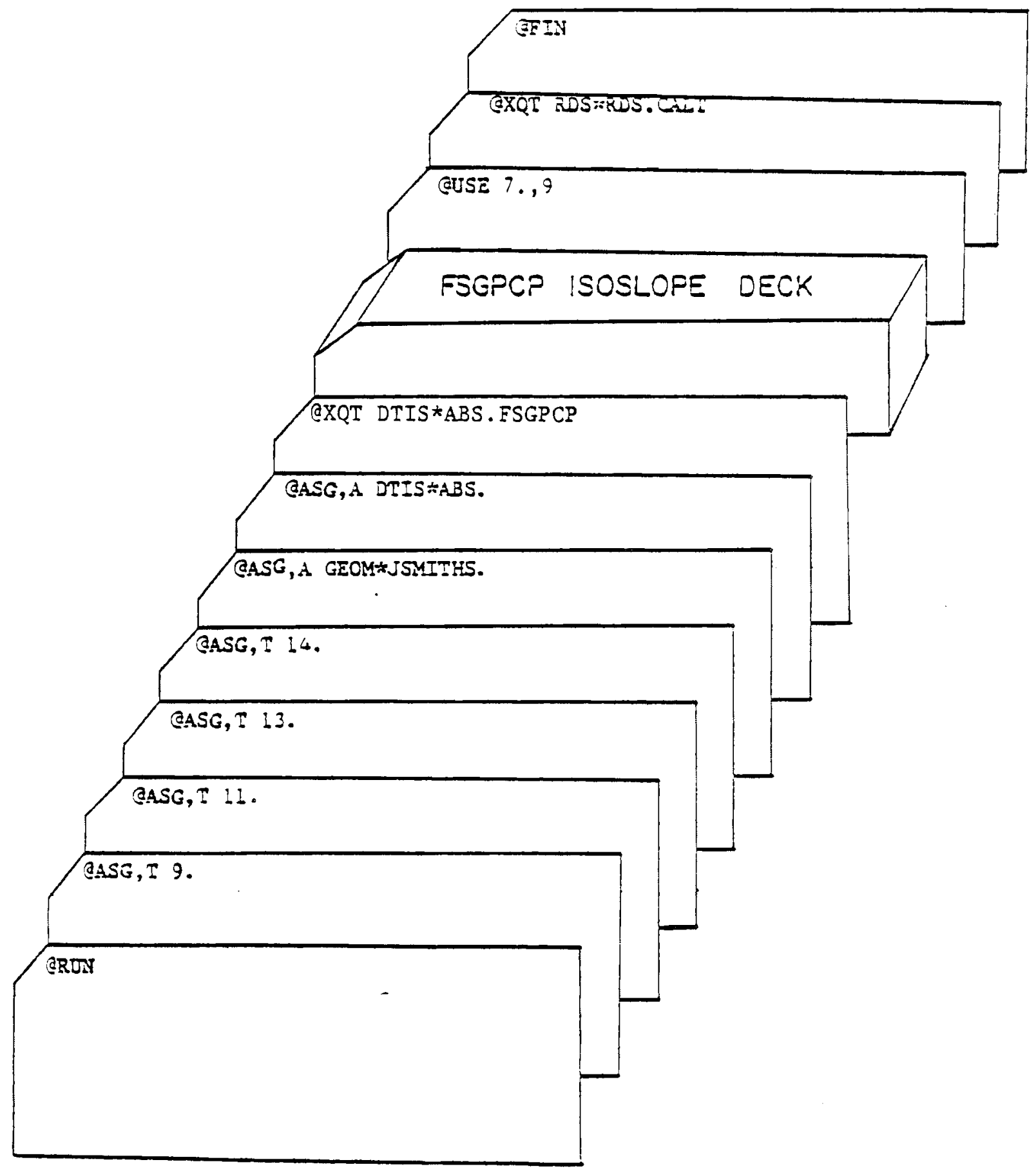

Exhibit 6.4 - Input Deck for Plotting Isosiope Lines 


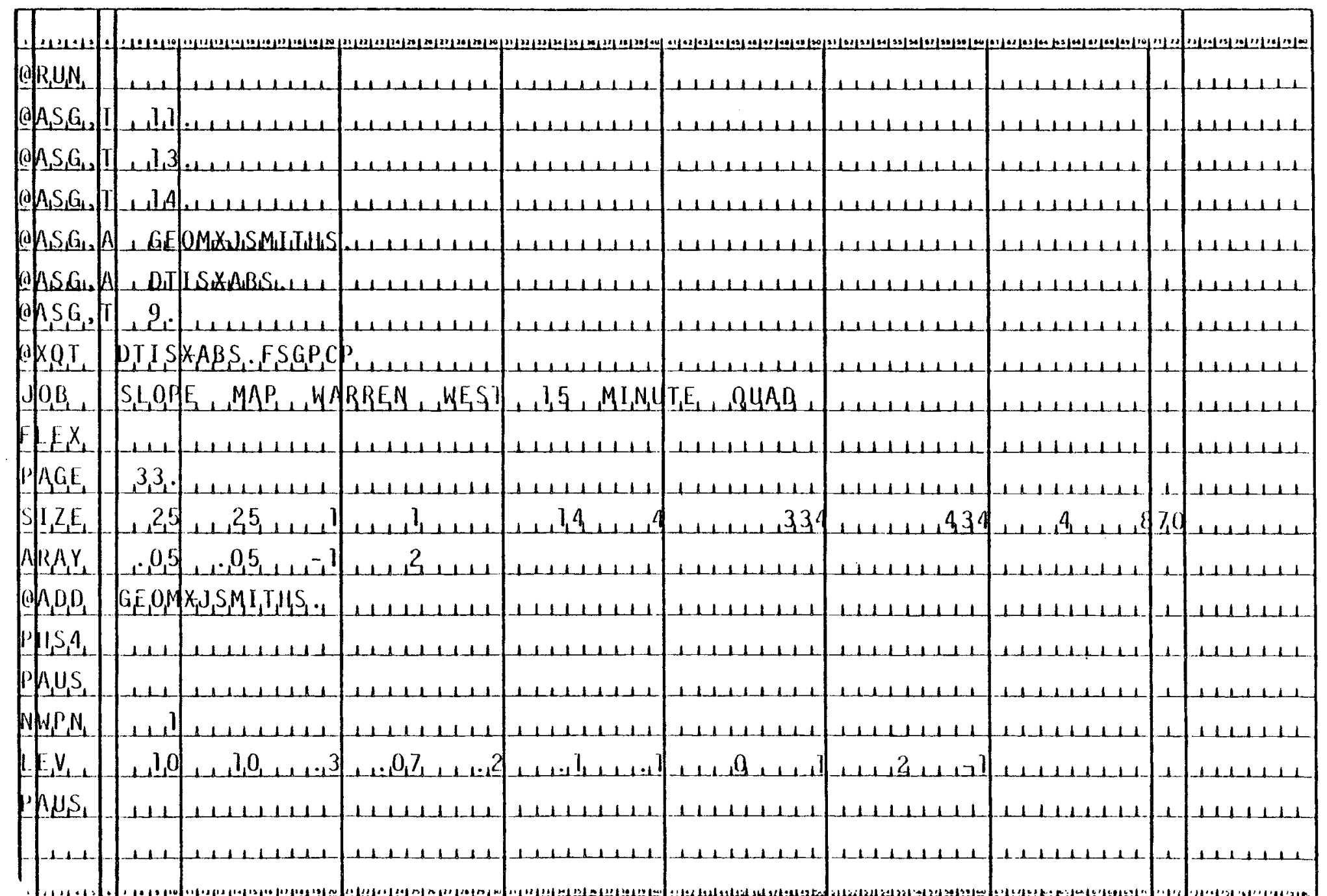




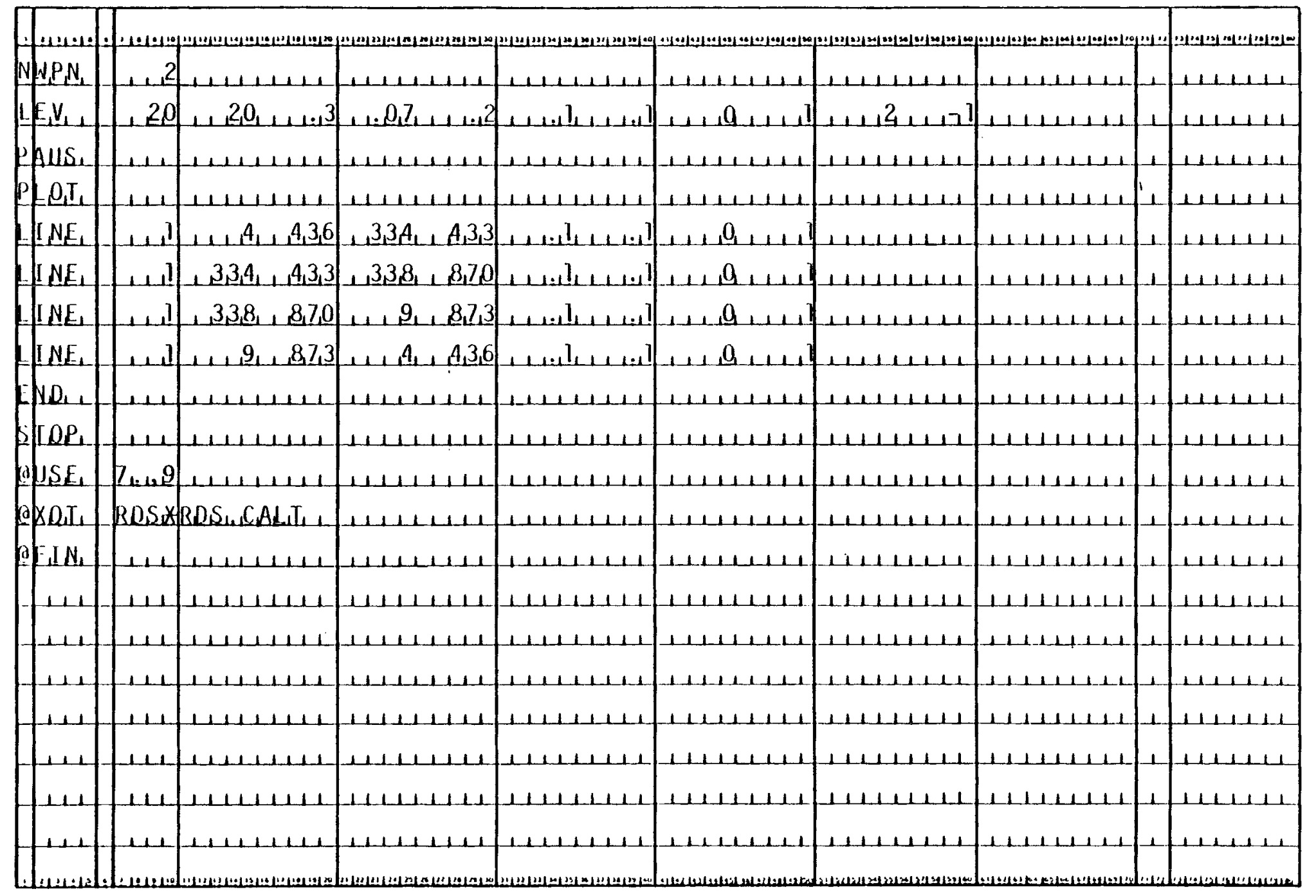




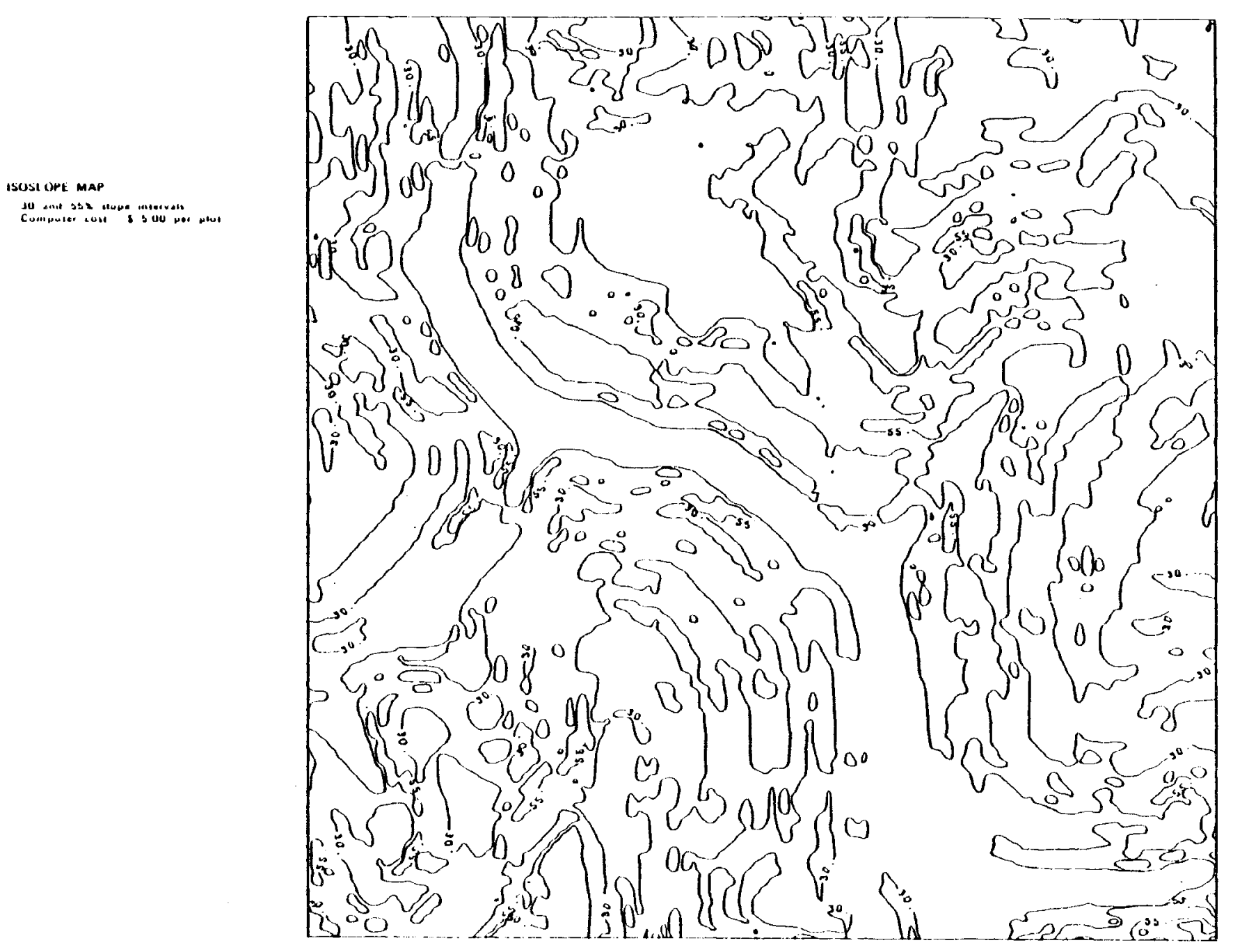


SECTION 3. ZONE PLOT PROGRAM (ZONEIT)

\section{Program Function}

The program ZONEIT takes as input rectangular arrays of slope (or aspect) data, and produces a graphic plot delineating zones of various ranges of slope (or aspect). The slope or aspect zones to be plotted are to be specified by the user in the input deck. A maximum of 20 zones can be included in one plor.

By producing a separate plot of slope zones and aspect zones, and then superimposing the two plots, it will be possible to identify the areas that fall within a certain slope and aspect zone. The program ZONEIT does not have the capability of directly delineating areas of certain slope and aspect combinations.

Data Card Format

Card 1:

cc

use

format

$1-2$

Logical unit for card reader: if left blank. unit 5 is

I2 used as default

3-4 Logical unit for card punch; default $=9$

If set $=99$. no card image output (input to GELO)

I2

is generated.

5-6 Logical unit for line printer, Default $=6$

[2

7-8 Logical unit from which the gridded slope or aspect data is to be read, Default $=8$

$9-10$

Logical unit for plot tape. Default $=7$ 
Card 2: (when ZONEIT is used to plot slope zones)

$\mathrm{cc}$

use

format

14

Upper limit of zone 1 (Example: $=15$ if zone 1 is to be

F 4.0 $0-15 \%$ slope)

$5-8$

Upper limit of zone ?

F 4.0

$9-12$

Upper limit of zone 3

F4.0

$76-80$

Upper limit of zone 20

F4.0

The last zone specified in this card must be either blank or the maximum possible slope in the area.

Card 3: (to Define Boundary limits of Data Array)

$\mathrm{cc}$

use

format

$1-10$

ILEFT $-10=$ lowest value of $X$ in grid units

F 10.0

$11 \cdot 20$

IRIGHT $-10=$ highest value of $\mathrm{X}$ in grid units

F10.0

$21-30$

IBOT $-10=$ lowest value of $Y$ in grid units

F10.0

$31-40$

ITOP $\cdot 10=$ highest value of $Y$ in grid units

F10.0

$41-50$

UPERIN - plot scale to be expressed in grid units per

F10.0 inch; if left blank, the plot will be scaled to fit a format of $30 " \times 30$ " 
In the case of DMA digital terrain data tapes, the grid unit is one hundredth of an inch (.01 inch) on a 1:250,000 map sheet. The value for UPERIN may be computed with the following expression:

$$
\begin{aligned}
& \text { UPERIN }= 0.0004 \times \text { scale factor of the output drawing } \\
& \text { Example: } \begin{aligned}
& \text { Plot scale wanted }=1: 24,000 \\
& \text { Scale of Digital Data }=1: 250,000
\end{aligned} \\
& \text { UPERIN }= 24,000 \times 0.0004=\text { (data grid units } / \text { inch) }
\end{aligned}
$$

\section{Card 4:}

$c c$

use

format

XONE - X-coordinate in grid units of SW corner of

F10.0 array

$21-30$

YONE - Y-coordinates in grid units of SW corner of array array
31-40 YTWO - Y-coordinate in grid units of SE corner of array
$41-50$
XTHREE - X-coordinates in grid units of NW corner
$51-60$ of array
YTHREE - Y-coordinate in grid units of NW comer of array

XTWO - X-coordinate in grid units of SE corner of

F 10.0

F10.0 
Card 5: (coordinates of A Registration Point)
$c c$
use
format
$1-2 \quad$ Latitude in degrees
$3-4 \quad$ Latitude in minutes
5-8 Latitude in hundredths of seconds
9-11 Longitude in degrees
12-13 Longitude in minutes
14-17 Longitude in hundredths of seconds
I4
$18-20$
Blank
$3 \mathrm{X}$
$21 \cdot 30$
$\mathrm{X}$-coordinate of registration point in grid units
F10.0
3140
$Y$-coordinate of registration point in grid units
F10.0
41
$=1$ if this is the last registration point in the data deck
II $=0$ otherwise

There should be one Card.5 for each registration point. The last registration card has a 1 in column 41 . There must be at least one Card.5 (with a 1 in $c c 41$ ) in each ZONEIT run.

\section{Input Deck Assembly}

Exhibit 6.7 is a schematic diagram of an input data deck for program ZONEIT. The slope (or aspect) data has been stored in a file called GEOM*ISMITHS (or GEOM*ISMITHA) which is to be used as input to ZONEIT.

Application Example. Exhibits 6.8 and 6.10 are sample input decks for plotting slope zones and aspect zones respectively.

Exhibits 6.9 and 6.11 are sample outputs of using ZONEIT to plot slope and aspect zones respectively. 


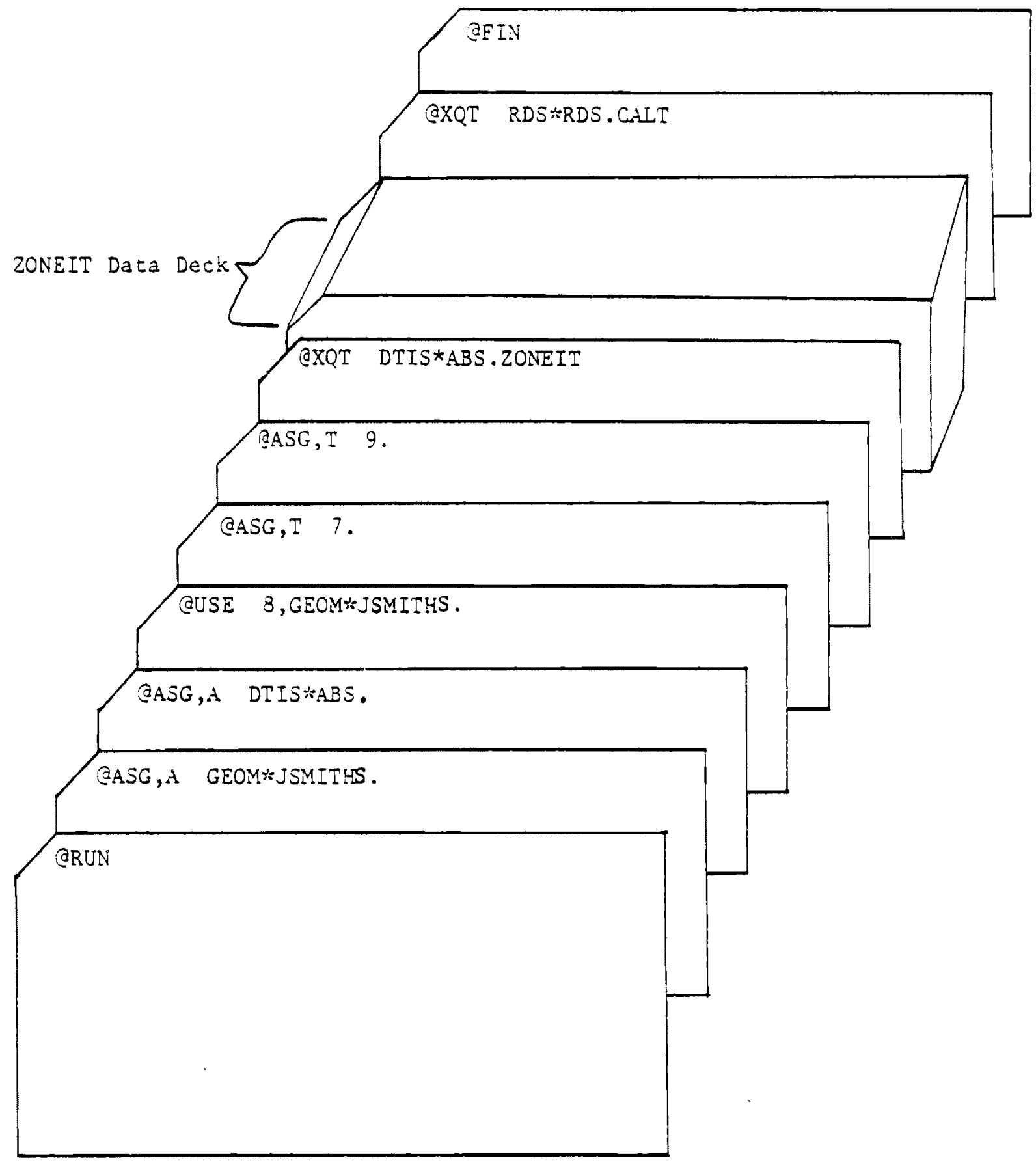

Exhibit 6.7 - Input Deck Assembly for Program ZONEIT 


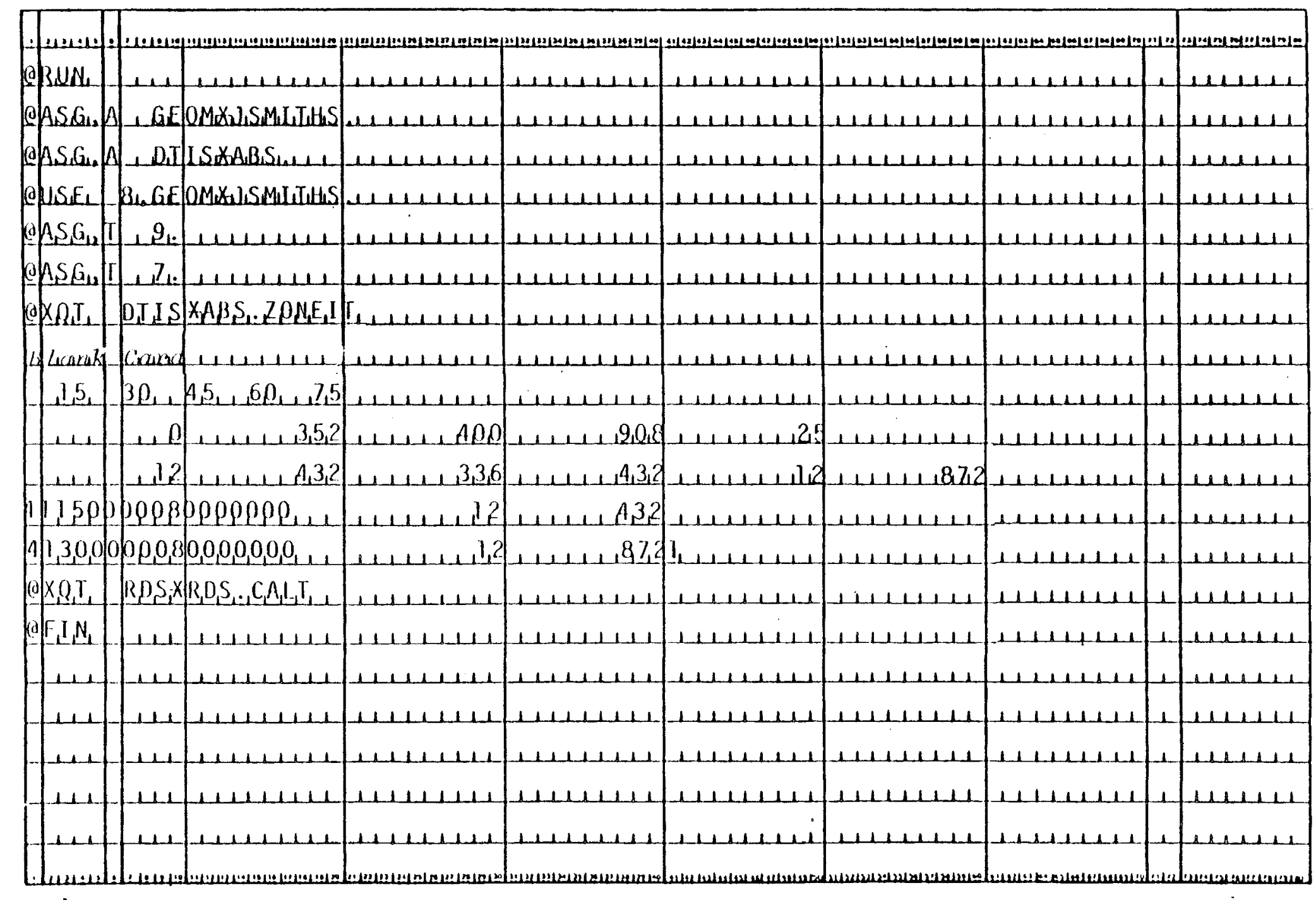




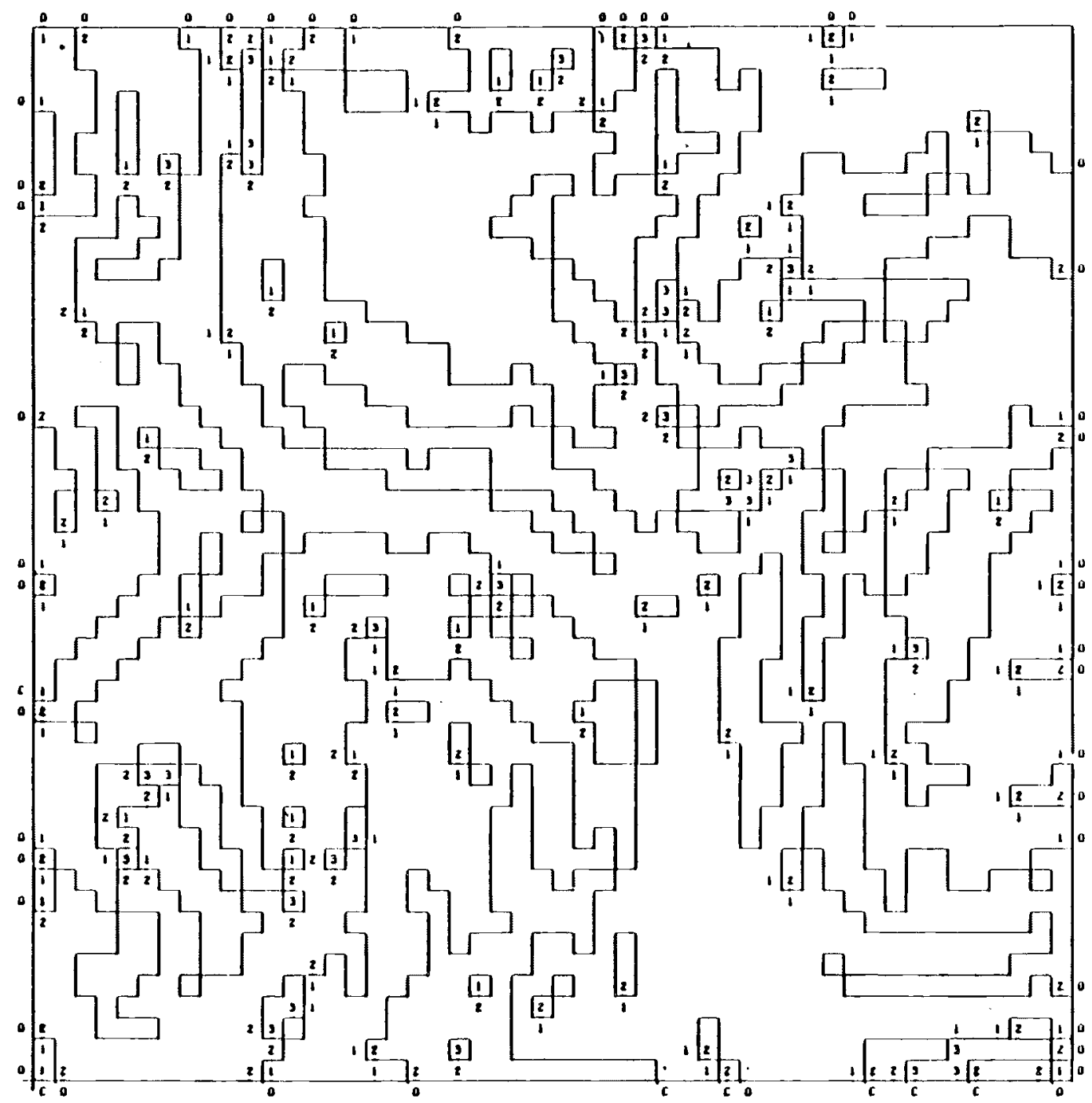




\begin{tabular}{|c|c|c|c|c|c|c|c|c|c|}
\hline & fus $+2+5$ & (1) & 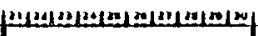 & 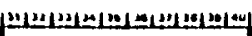 & 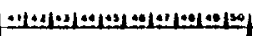 & $3 \pm 1321 \pm 1$ & 4 & 州前 & \\
\hline & & 111 & $\Lambda \perp \perp \perp|\Lambda| \Lambda \mid$ & $\perp \perp 1 \perp \perp 1|1| \perp$ & $\triangle 1 \perp \perp 11114$ & $\mid \perp 1111111$ & 1,111 & 1 & \\
\hline & $A$ & $O O M x_{1} S_{1} M_{1} I_{1} L A$ & aldelese & $|1| L|1| 1 \mid$ & $|1| 1||||||$ & $111+11111$ & $|1| 1|1| 11$ & 1. & $|1| 1 \mid 1$ \\
\hline & $A, D T$ & If $S_{X} \times B_{1} S_{1, L}$ & $1+1 L_{1} \mid L_{1}$ & $111+11+14$ & 1111111114 & $1+1+1,11$ & $\perp 1 \perp \mid 1 \perp<11$ & $-L$ & \\
\hline & Bu, GiE & 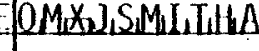 & $|1| 1111111$ & $\perp 1 \perp \perp|1| 1$ & $1+1+11 \perp 11$ & 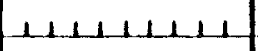 & $1 \perp \perp L_{1} \mid L L$ & $\perp$ & $1+1+1$ \\
\hline & 41 & $1 \perp 11 \perp \perp \perp L 1$ & 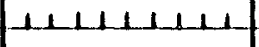 & $|1| 1|\perp| \perp \mid 1$ & 111111111 & 111111111 & 111111111 & 1 & 11111 \\
\hline & alla & $\mid 1111+1111$ & $\perp \perp \perp \perp \perp \perp \mid$ & $\perp \perp \perp \perp \perp \perp \perp \Lambda$ & 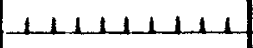 & 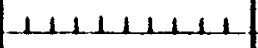 & 111111111 & $\perp$ & Lnden \\
\hline dexaile & Dilles & $\triangle A B S_{L}, Z, O N E I$ & {$[1+1,1,1<$} & 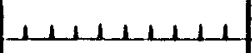 & $L 1+1+1+1 L$ & $1+11+1111$ & $11|1| 1 \mid 11$ & 1 & 11111 \\
\hline LL & $b_{1} l_{1} a_{1}$ & q k carlch, & $\Delta c|c| c|c|$ & 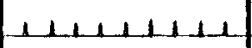 & $1111|1| 11$ & $1+111+1+1$ & $1 \perp \perp \Lambda \Lambda \perp \Lambda \Lambda$ & I & Llessel \\
\hline & Hes & 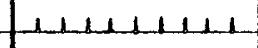 & $11111 \mid 114$ & $\mid+1+1+1+1$ & $-1+1 L 1+1 \perp$ & 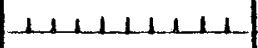 & $\perp \perp \perp \perp \perp \perp \perp \perp l$ & 1. & $11 \mathrm{~L} \perp 11 \mathrm{~L}$ \\
\hline & +10 & $1+<1 \perp 3,5,2$ & $\perp+\perp \perp \perp, 0,0$ & $1+1 \wedge 19,018$ & $1+1111149$ & 111111111 & $|1| 1|1| 11$ & 1 & 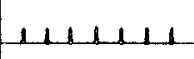 \\
\hline & 12 & $=1,1+1,4,32$ & $(1, L, 1,3,3,6$ & $1,1,1,4,2$ & $1, \ldots+12$ & 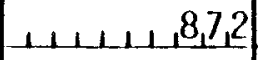 & $\perp 1$ & 1 & $\ln 1$ \\
\hline 41,50 & 9008 & $\rho \rho \rho \rho \rho \rho Q_{\perp \perp}$ & $(1+1+1+12$ & $\ldots, 4,3,2$ & 11 & $\perp$ & LL & 1 & \\
\hline 11300 & $\rho 000 \beta$ & $\rho \rho \rho \rho \rho \rho \rho_{\perp \perp}$ & 4111,12 & $1,1,1,8,7,2$ & $I_{1}+11+1,1$ & 111111111 & $1 \perp 1|1| 1 \mid 1$ & $\perp$ & 141111 \\
\hline$\theta R_{1}$ & $\operatorname{Res} x$ & EDS $S_{1} C A L_{-1} T_{1}$ & $11+1+1111$ & 111111111 & $\perp \perp \perp \perp \perp \perp \perp \Lambda$ & 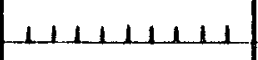 & $\perp 1 \perp \perp_{1} \perp 1$ & $\perp$ & InL \\
\hline$d N_{1}$ & 111 & $111|1| 1 \mid 1$ & $|1| 11|1| 1 \mid$ & 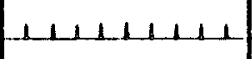 & Let $|1| 1 \mid$ & -11111111 & $1 \perp 1 \perp 1 ! 111$ & 1 & $\operatorname{sel}|\alpha| 1$ \\
\hline$+1,1$ & 111 & $\perp \perp \perp \perp \perp \perp \Lambda$ & $\perp \perp \perp \perp \perp 11$ & $\mid \perp \perp 1 \perp 111$ & $\perp \perp 11+111$ & 111111111 & $1+1,1<1<1$ & 1 & Aleses \\
\hline 111 & 111 & $\perp \perp 11|1| \alpha$ & $|\perp \perp \perp 11 \perp 1|$ & $\perp 1 \perp \perp \perp 1 \perp \perp$ & $\perp \perp L L \perp L \perp \perp 1$ & $|\perp \perp \perp \perp \perp \perp|$ & $1|1| L_{1} \mid 1$ & a. & $\| \perp \perp \perp \perp 1$ \\
\hline 111 & 14 & $1+11+1+11$ & 14111111 & $\mid 111<1111$ & $|\perp 1| 1|1|$ & 111111111 & 11111111 & $L$ & $1|1| 1 \mid 1$ \\
\hline 111 & 111 & $1 \perp 1|1| 111$ & $\perp \perp \perp \perp \perp \perp L \mid$ & $\perp \perp \perp \perp 1 \perp \perp \perp$ & $-1|\perp| \perp|\perp| 1$ & $\perp \perp \perp \perp \perp \perp 11$ & $\| \perp \perp \perp \perp \perp \perp 1$ & 1 & Lledeld \\
\hline $\mid+1+1$ & Pel & $\mid 1+1+1+1+1$ & 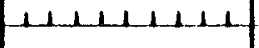 & 111111111 & $111111+11$ & $111+111$ & $11111+11$ & 1 & $\Lambda<1<1<1$ \\
\hline & & & & & & & & & \\
\hline
\end{tabular}




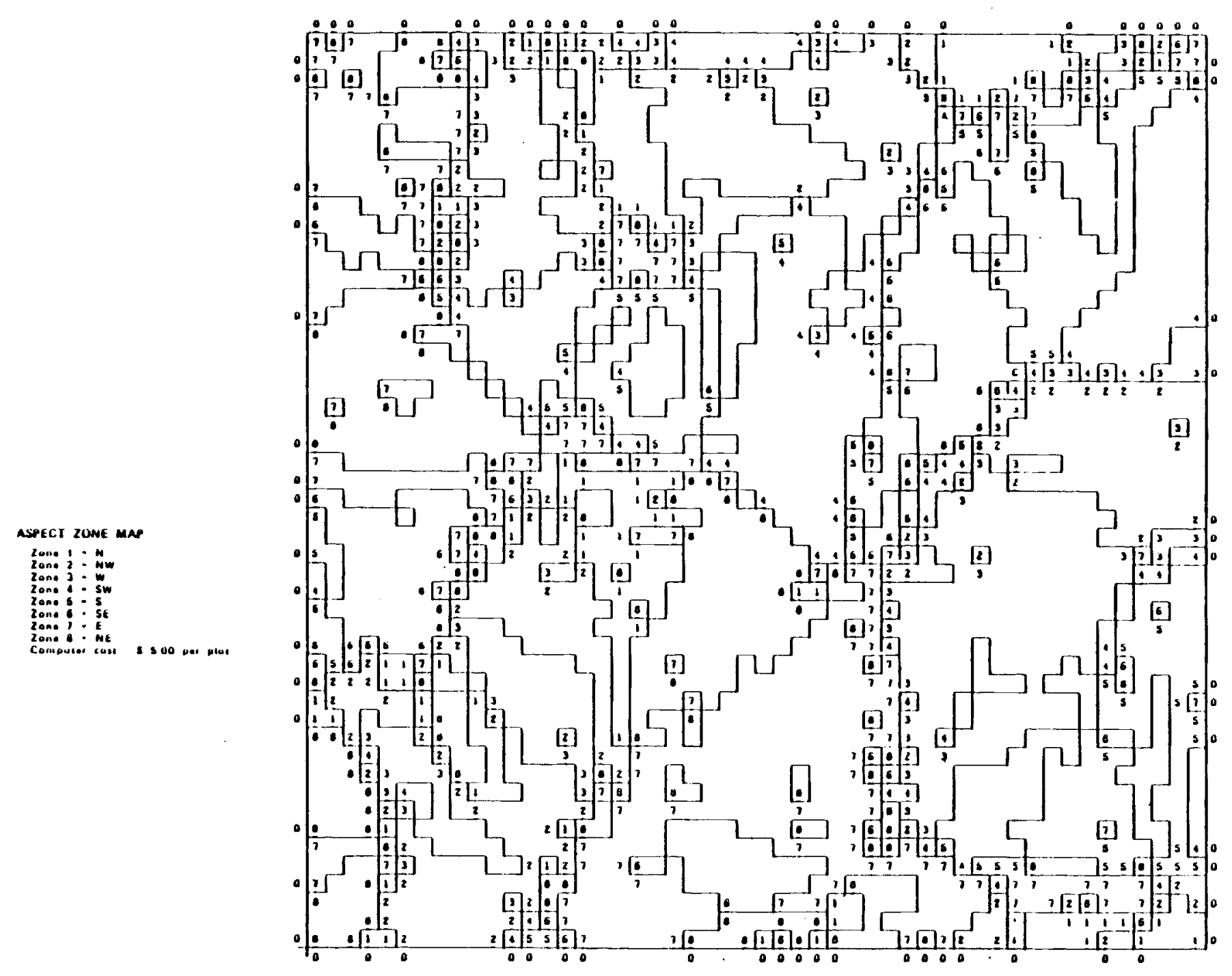




\section{SECTION 4. VIEW AREA PROGRAM (SEEN)}

\section{Program Function}

View area program (SEEN) was formerly the TOPAS version of VIEWIT. Given an array of elevation points and a viewpoint. Program SEEN identifies all the array points that are directly visible from that viewpoint. The program will output a plot tape for the Calcomp 900-1136 drum plotter to show the visible points and the viewpoint in their true location at a specified scale. At the option of the user, the output can also include a map plotted on the line printer. However. this printer map will not be plotted to scale.

The viewpoint need not be located within the area of the elevation array, but the elevation of the viewpoint may be either on or above the actual terrain elevation. If the viewpoint is not within the area, its elevation must be supplied.

More than one viewpoint may be specified for each view plot. The graphic output will show all the array points that can be seen from one or more of the viewing stations. The maximum number of viewing points to be included in a single plot is 50 .

The number of points in the elevation array must not be greater than 14,641 ( $121 \times 121$ ). The maximum viewing distance is currently set at 50 times the grid size.

\section{Data Card Format}

There are four types of data cards included in an input deck for program SEEN:

1. Parameter card

2. Elevation grid data (ARAY block)

3. Viewpoint card

4. Switch card 
Parameter Card Format:

cc

use

format

$1-10$

Scale factor of plot (e.g.. 24000 if desired scale is

F10.2 $1: 24,000)$

Ground distance in feet between adjacent grid points

F 10.2

$21-25$

Number of rows in the elevation array (default is 121)

15

26-30 Number of columns in the elevation array (default is 121) - note that the product of the number of rows and the number of columns must not be greater than $14641(121 \times 121)$.

$31-78$

Blank

$48 X$

79

$=0$ Do not produce a line printer plot

$=1$ Produce a line printer plot

80

$=1$ Elevation array to be input from an existing data file (this option is not recommended)

I1

Viewpoint Card Format:
cc
use
format
$1-34$
Blank
$34 X$
$35-40$
Name of Viewpoint
A6
$41-43$
Column on which the viewpoint is located ( $\mathrm{x}$ -
I3
$44-46$ coordinate in columns)
$47-50$
Row in which the viewpoint is located (y-coordinate)
13
Blank
$4 X$
$51-60$
1. If the elevation field has a " + " elevation $(+100)$, that elevation is added to the array elevation.
F10.0
2. If the elevation field is blank, the array elevation is used.
3. If the elevation field has an elevation (5650), it replaces the array elevation. 
There should be one card for each viewpoint, and the maximum number of viewpoints in a given plot is 50 .

\section{Switch Card}

A switch card has 999999 punched in columns $41-46$.

A switch card must be used to close a viewpoint set; that is, a 999999 switch card must follow that last viewpoint card in a set (combined viewpoint plot).

A second 999999 switch card directly following the first 999999 card will signal the beginning of a new data set which is to be processed in the same execution. SEEN areas can be generated on several sets of terrain (ARAY blocks) in one execution. Another elevation grid and the viewpoints to be processed against it, follow the second 999999 card.

\section{Input Deck Assembly}

Exhibit 6.12 is a schematic diagram of an input deck consisting of only one viewpoint set. Exhibit 6.13 shows a deck consisting of two viewpoint sets.

Application Example. Exhibit 6.14 is a sample input deck. The elevation array has been stored in a file called GEOM*JSMITH, which is now used as input to SEEN.

Exhibit 6.15 is a sample plot from program SEEN. 


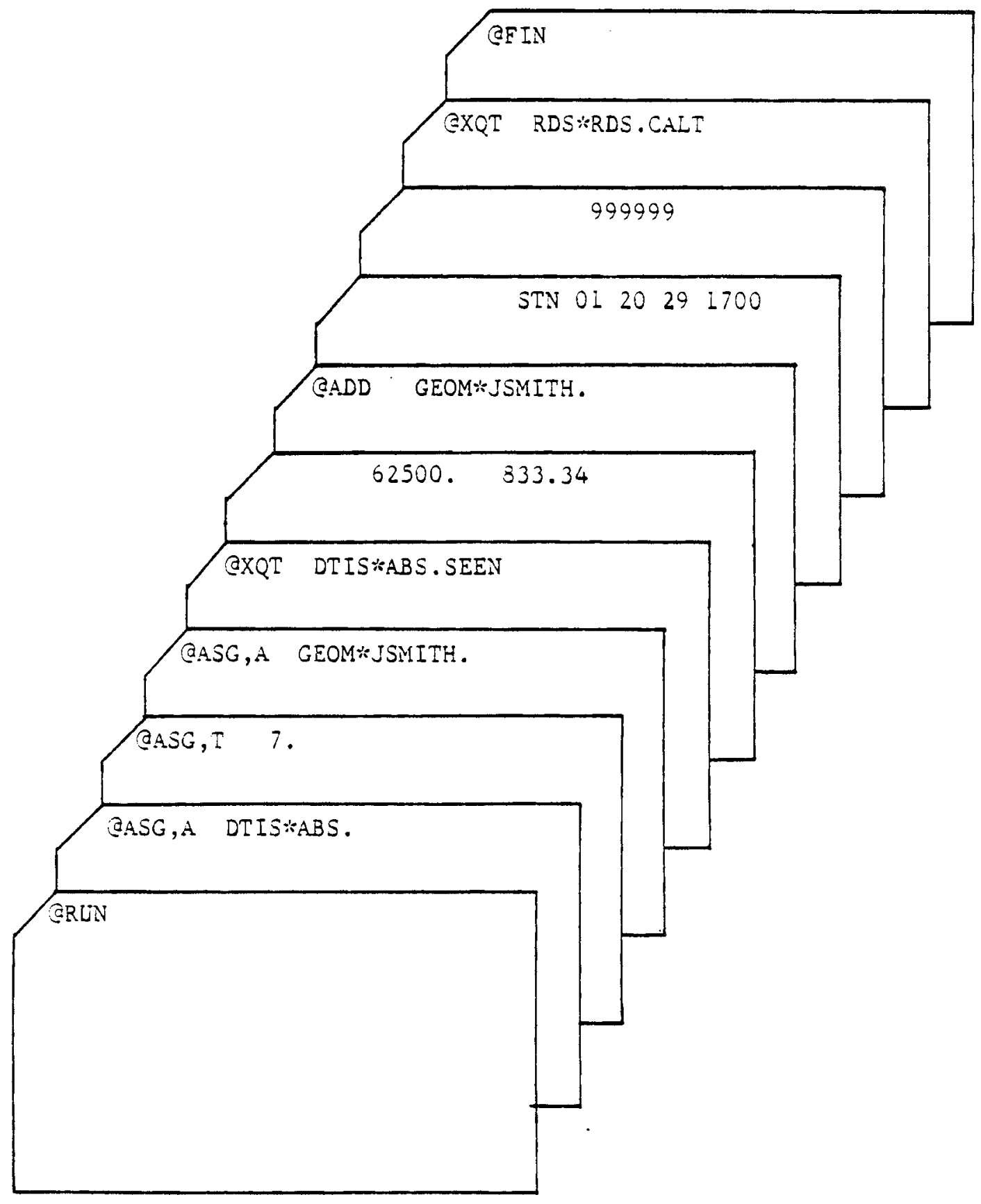

Exhibit 6.12 - Input Deck Setup for Program SEEN Containing One Viewpoint Set 


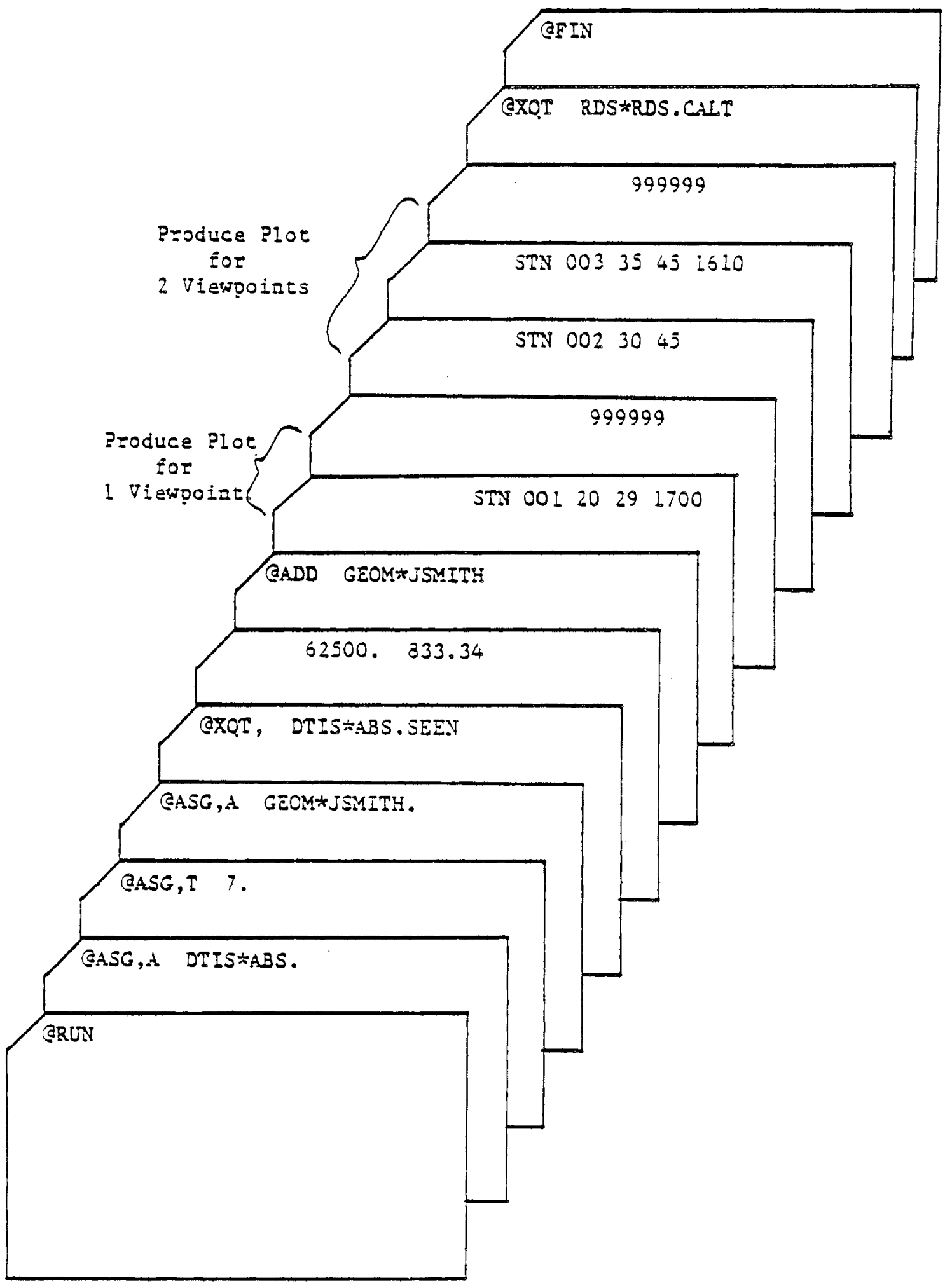

Exhibit 6.13 - Input Deck Setup for irogram SEEN Containing Two Viewpoint Sets 


\begin{tabular}{|c|c|c|c|c|c|c|c|c|c|}
\hline anst & Fi:H & 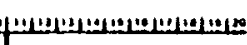 & 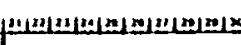 & | & 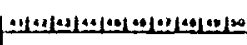 & 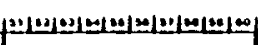 & 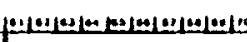 & 证橗 & 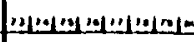 \\
\hline RU & & & 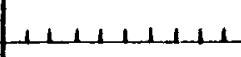 & $11111211 \mathrm{~L}$ & $11 L 1+1+1 \mid 1$ & $\mid 11111111$ & 1111111111 & & $|1| 1|1| L_{1}$ \\
\hline A.S & $A, D T$ & $I_{1} S^{*} A B S_{1}$ & 111111111 & 111111111 & $1 \perp 11111111$ & $1+11+11111$ & $\perp \perp \perp 11 \perp \perp 11$ & -1 & $111+111$ \\
\hline$A S$ & 1 I. & $\left.\right|_{1} L_{1} L_{1} L_{1}$ & $\| 1+11+1,1$ & $-11+1,1,1<1$ & $11+1, L / L 1$ & $\perp \perp \perp \perp \perp \perp \perp \perp$ & $\perp 1+1,1<11$ & 1 & $111+111$ \\
\hline$A, S$ & A $G, 0$ & $M_{1}^{*} J_{1} S_{1} M I_{1} T_{1} H_{1}$ & 111111111 & 111111111 & 111111111 & $1 \perp \perp 11 \perp 111$ & 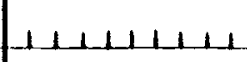 & 1 & L1 \\
\hline d $\mid x_{1} 0$ & DT, S & ${ }^{\star} A, B, S_{1}, S_{1}, E, E N$ & 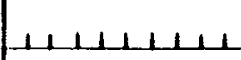 & $\perp i|1| \perp|1|$ & $\perp \perp \perp \perp \perp \perp \perp 1$ & 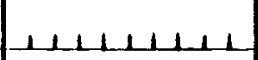 & 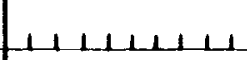 & 1 & $1<1$ \\
\hline 1 & 2500. & $1,1,83,3.34$ & $1 L_{1}|1| 1 \mid 1$ & $|\perp| \perp|l| l \mid l$ & $\perp \perp \perp|L| \perp \mid L$ & 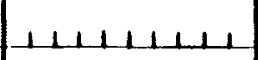 & $|1| 1|1| 1 \mid 1$ & 1 & $1 \perp \perp<1 l_{2}$ \\
\hline (2) ADD & GEPM & ${ }^{k}{ }_{1} S M_{1} I_{1} T H_{1}$ & 111111111 & 111111111 & $\perp \perp \perp \perp \perp \perp \perp$ & 111111112 & $\perp \perp \perp \perp L \perp \perp \perp$ & 1 & $11 \perp \perp 1$ \\
\hline & H.1.1 & 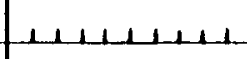 & 111111111 & $\therefore \ldots$ STN99 & $20,29,11$ & $\perp, 1,2,0,0$ & $|1| 1|1| 1 \mid 1$ & 1 & 1111 \\
\hline 11 & 11 & $|1| 1|1| 1|1|$ & $111+1111$ & $|1| 1|1| 11$ & $92,9,999, \ldots$ & $11 \perp 1<L 1$ & 111111111 & 1 & 1111111 \\
\hline $9 \times x_{1} T_{1}$ & $R_{1} D_{1} S_{1} *$ & $R_{1} D_{1} S_{1} C_{1} A_{1} T_{1,}$ & 11111111 & 111111111 & 111121111 & \|\|$\|_{1} \mid L_{1}$ & $\| 1|1| 1|1| 1$ & 1 & $1-1|1| 11$ \\
\hline (f) $I, N$ & 11 & $\perp \perp \perp \perp \perp \perp \perp \perp$ & $1 \perp \perp 111 \perp 11$ & 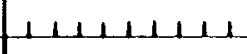 & $\Lambda \perp L \Lambda \Lambda \Lambda \Lambda$ & $\perp \perp \Lambda \perp \perp \perp \perp$ & 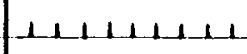 & 1 & $111111_{1}$ \\
\hline-111 & +11 & $|\perp \perp \perp| 1111$ & 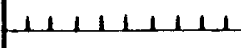 & $\Lambda \perp 11,111$ & 111111111 & $\perp \perp \perp \perp \perp \perp \perp$ & 11111111 & 1 & $-1<1<1<1$ \\
\hline $11 \ldots$ & 111 & $11+11111$ & 111111111 & 111111111 & $|L| L|1|<11$ & 111111111 & 111111111 & 1 & $-111+111$ \\
\hline-11 & 111 & $|\perp \perp| \perp|\perp| 1$ & $\perp \perp \perp \perp \leq 11$ & $\perp \perp \perp|1| 1|1|$ & $111+111111$ & $-1 \Lambda \perp L \perp|L| L$ & $11 \perp \perp 1111$ & 1 & $111|1|$ \\
\hline-111 & 111 & $\perp \perp|\perp \perp \perp| \perp \perp$ & $\perp \perp \perp \perp|\Lambda| 1$ & $\mid \perp \perp \perp \perp \perp \perp L L$ & $\perp \perp \perp \perp \perp \perp \perp 1$ & $|1+1+11+1| 1$ & $\perp \perp \perp \perp 11 \perp 1$ & 1 & $\perp \perp \perp \perp \perp \perp 1$ \\
\hline 111 & 111 & $\mid 1,11+111$ & 11111111 & 11111111 & $11111<L \perp$ & 111111111 & 111111112 & $\perp$ & $1+11+11$ \\
\hline-111 & $-1+1$ & $|\perp| 1|\perp| 1 \mid$ & $\perp \perp \mid \perp \perp \perp \perp \perp$ & $-1 \perp \perp \perp \perp \perp 11$ & 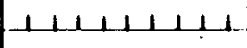 & 111111111 & $-\llcorner L \perp \perp \perp \perp \perp \perp$ & 1 & $1 L \perp 111$ \\
\hline 1.1 & 111 & $1+11 \mid 1111$ & $A 1,1,111$ & $1 \perp \perp+1 \perp \perp 1$ & $1 \perp 1 \perp \perp 111$ & $1+11+111+1$ & 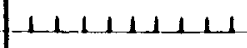 & 1 & 1111111 \\
\hline$H \perp \perp$ & 111 & $\mid 11111111$ & 111111111 & 111111111 & 111111111 & 111111111 & 111111111 & 1 & $1 \perp 1111$ \\
\hline 1.1 & -111 & $\mid 11111111$ & 111111111 & $\mid 1 \perp 111111$ & 111111111 & 111111111 & $\left\lfloor 1 \_\perp \perp \perp \perp l\right.$ & 1 & 11,111, \\
\hline & & & 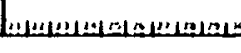 & 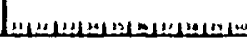 & & & & & \\
\hline
\end{tabular}



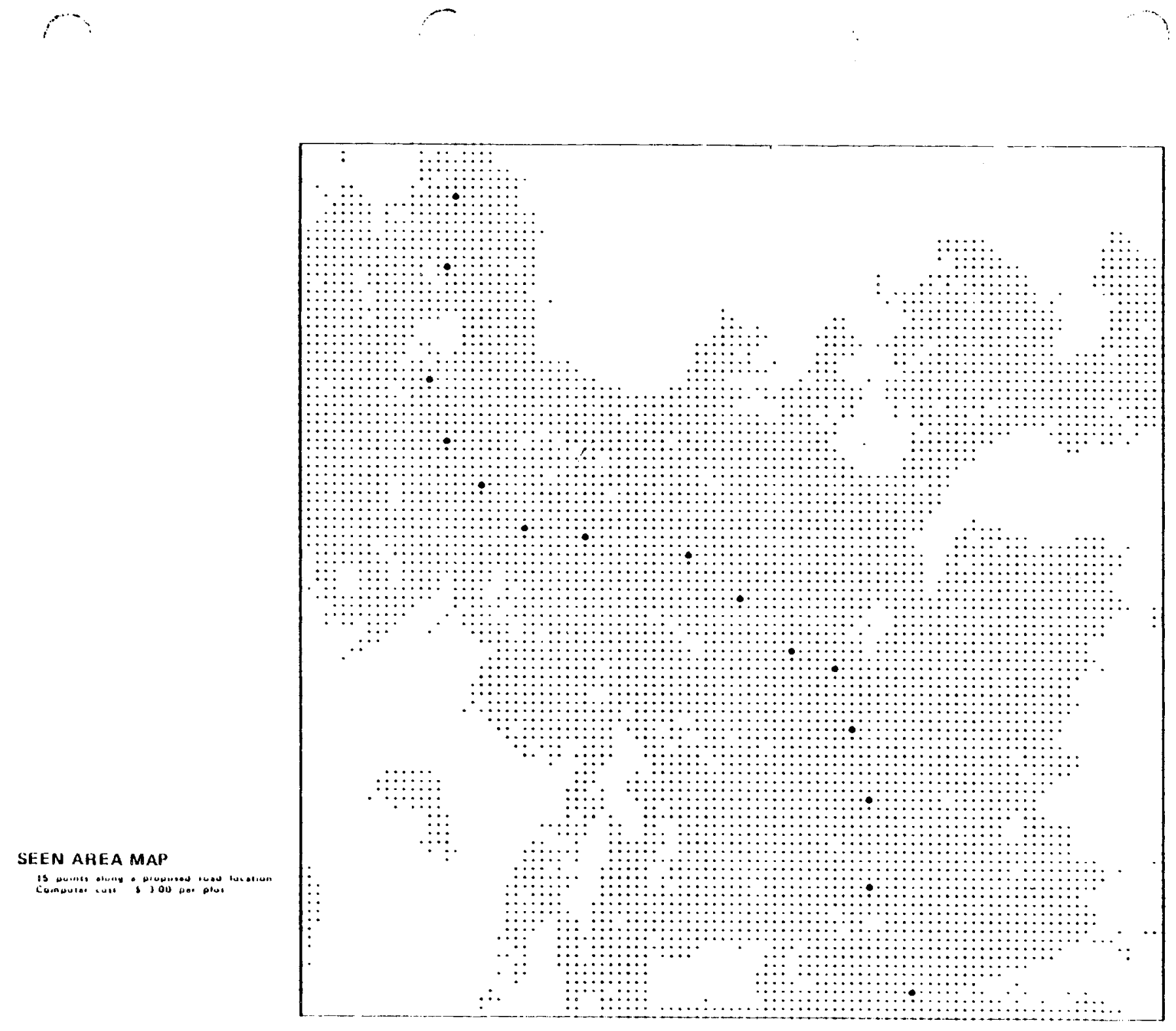
SECTION 5. PERSPECTIVE PROGRAM (THREED OR PREP3D)

\section{Program Function}

Program THREED accepts as input gridded elevation data from a project data file, and produces a perspective view of the grid which represents the terrain surface. The program uses every data point in preparing the perspective drawings. For a perspective plot of a large area, such as that contained in a $7-1 / 2$ - or 15 -minute USGS quad, the computer cost for drawing a perspective view using program THREED can be quite high.

In order to reduce computer cost and to simplify the input data deck, a modified version of Program THREED was specifically developed for drawing terrain perspective. This version is code-named Program PREP3D. Instead of using every data point in the input elevation grid, Program PREP3D uses only every second data point to prepare the perspective. The cost of preparing a perspective drawing using PREP3D is approximately $1 / 6$ of that required by THREED.

This documentation will only explain the use of Programs THREED and PREP3D in drawing terrain perspective. Program THREED may also be used to prepare perspective drawings from non-gridded data, and to prepare perspective drawings for stereoscopic viewing. For a complete documentation of Program THREED, the user may refer to the Calcomp manual (Reference 2).

\section{Data Card Format}

All the card types described below are needed for running program THREED. However, only card types $F, G, H$ and I need to be included in the input deck for program PREP3D.

Card Type A: (parameter card for the entire run)

\begin{tabular}{|c|c|c|c|}
\hline $\mathrm{cc}$ & & use & format \\
\hline 1 & ITYPE & 'A' identifies card type. & $A l$ \\
\hline $2-5$ & NSETS & $\begin{array}{l}\text { The number of data sets to be plotted by } \\
\text { this run }\end{array}$ & I4 \\
\hline $6-10$ & IPRNT & $\begin{array}{l}\text { Message-output logical-device number - if } \\
\text { IPRNT is zero or blank, the program uses } \\
\text { the normal system printer: e.g., } 6 .\end{array}$ & I5 \\
\hline $11-15$ & IPLTP & $\begin{array}{l}\text { The logical unit for the plot tape - if execution } \\
\text { is halted for lack of core after gridding is } \\
\text { completed the gridded data is written onto } \\
\text { this tape (provided that no plotting has } \\
\text { already been done) }\end{array}$ & IS \\
\hline
\end{tabular}


Card Type B: (parameter card for a data ser)

\begin{tabular}{|c|c|c|c|}
\hline cc & & use & format \\
\hline 1 & ITYPE & 'B' identifies card type. & $A]$ \\
\hline $2-5$ & ICNTL & $\begin{aligned} & \text { Switch indicating type of task-if: } \\
&=1-\text { Read } Z \text { values directly into the } \\
& \\
& \text { rectangular array, column by column, } \\
& \text { with no smoothing } \\
&=2-\text { Same as }=1 \text {, except with smoothing } \\
&=3-\text { Read } Z \text { values directly into the } \\
& \text { rectangular array, row by row, with no } \\
& \text { smoothing } \\
&= 4-\text { Same as }=3 \text {, except with smoothing }\end{aligned}$ & 14 \\
\hline $6-10$ & NSTUP & $\begin{array}{l}\text { Number of different perspectives to be drawn } \\
\text { for the data set being processed }\end{array}$ & 15 \\
\hline $11-15$ & $\mathrm{MN}$ & Zero if gridding is not required & Is \\
\hline $16-20$ & IENT & Zero if gridding is not required & 15 \\
\hline $21-25$ & $\mathrm{M}$ & Number of rows in the gridded data, & 15 \\
\hline $26-30$ & $\mathrm{~N}$ & Number of columns in the gridded dara & 15 \\
\hline $31-35$ & IX & $\begin{array}{l}\text { If smoorhing is required, IX is the number } \\
\text { of rows to be inserted between each pair } \\
\text { of already existing rows; zero ocherwise. }\end{array}$ & 15 \\
\hline $36-40$ & IY & Analogous to $\mathrm{IX}$, for columns & I5 \\
\hline 4145 & INLU & $\begin{array}{l}\text { The input-data logical-unit number - if INLU } \\
\text { is zero or blank, the program assumes the } \\
\text { unit to be the card reader }\end{array}$ & 15 \\
\hline $\begin{array}{l}61-70 \\
71-80\end{array}$ & $\begin{array}{l}\text { ZFACT } \\
\text { ZADD }\end{array}$ & $\begin{array}{l}\text { In some situations it may be necessary or } \\
\text { desirable to adjust the input } Z \text { values. The } \\
\text { program adjusts array values as follows: }\end{array}$ & $\begin{array}{l}F 10.0 \\
F 10.0\end{array}$ \\
\hline & & $\begin{array}{l}\qquad Z_{\text {new }}=Z_{\text {input }} * Z F A C T-Z A D D \\
\text { where the default value of } Z F A C T \text { is } 1 \text { and } \\
\text { the default value for } Z A D D \text { is zero. }\end{array}$ & \\
\hline
\end{tabular}


Card Type C: (variable format for input elevation data)

$\mathrm{cc}$

1

$2-80$ use

format

Al

13 A 6

Example: When the project data file is created from DMA digital terrain data filles by using program SCANDMA, the following format should be entered in column 2-80:

Either: (1X./Q(5X,5E14.61), 5X.RE14.6/) if $R \neq 0$

Or: $(1 X, / Q(5 X, 5 E 14.6 /))$ if $R=0$

such that $M=5 Q+R$ and $R$ is less than 5 .

The value of $M$ is stated in Card type $B$.

Card Type F: (parameter card; see Exhibit 6.16 for illustration of the parameters)

$\mathrm{cc}$

1

$2-10$

$11-20$

$21-30$

$31-40$

$41 \cdot 50$

$51-60$

$61-70$

$71 \cdot 75$

$76-80$ use

format

ITYPE ' $F$ ' identifies card type.

Al

XMIN

F9.0

YMIN Define minimum and maximum

$\mathrm{X}, \mathrm{Y}, \mathrm{Z}$ boundaries for view area

ZMIN

XMAX

F10.0

YMAX

F 10.0

ZMAX

F10.0

QS

A scale factor which controls the size of the perspective drawing - see suggested formula below

H Height and width of the perspective window

F5.0

FINE $\quad=0.5,0.25$. or $0.125-$ the fineness with

F5.0

F10.0 which visibility checking is to be pertormed: 0.5 .0 .25 . or 0.125 of distance between two grid points

Normally, 0.5 will be sufficient to provide a satisfactory plot. 
Formula for computing QS from a given focal length (f):

$$
\mathrm{QS}=\frac{1}{\mathrm{H}} \sqrt{\mathrm{H}^{2}+4 \mathrm{f}^{2}}
$$

Card Type G: (parameter sard)

$\mathrm{cc}$

use

format

1

ITYPE ' $G$ 'identifies card type.

A1

NVUES The number of perspective plots to be drawn

I4 from the given data set - each perspective plot is to be defined by one $\mathrm{H}$-Card as described below

Card Type H: (define parameters for a perspective plot, see Exhibit 6.16 for illustration of the parameters)

$\mathrm{cc}$

use

format

1

$2-10$

ITYPE 'H' identifies card type.

$A 1$

IVIS $\quad=1$ position of view point to be defined by

19 angles THETA and GAMMA and distance factor $Q$

$=4$ position of view point to be defined by its $X, Y$, and $Z$ coordinates

$11-20$

THETA Theta angle (see Exhibit 6.16) of viewing

F10.0

or $\mathrm{X}$ axis, if IVIS $=$ ! $\mathrm{X}$-coordinate of view point is IVIS $=4$

$21-30$

GAMMA Gamma Angle (see Exhibit 6.16) of view axis.

F10.0

or $Y$ if IVIS $=$ ! $Y$-coordinate of view point. if IVIS $=4$

3140

$Q$ If IVIS $=1 Q=R a$ tio of the viewing distance

or $Z$ to the radius of the sphere enclosing the three-dimensional Region - $Q$ must be greater than zero (see Exhibit 6.16). If IVIS $=4, Z=$ coordinate of vlew point. 
Card Type I: (title card)

cc

1

$2-51$

$52 \cdot 80$ use

format

ITYPE 'I' identifies card type.

Al

$9 \mathrm{~A} 6$

Input Deck for Drawing Perspective with Program THREED. Exhibit 6.17 illustrates the data deck set-up for running program THREED. Exhibit 6.18 is a sample input data deck for running THREED. The gridded elevation data is stored in a project data file called GEOM*JSMITH, which is used as input to the program. Exhibit 6.19 is a sample graphic output from program THREED.

Input Deck for Drawing Perspective with Program PREP3D. Exhibit 6.20 illustrates the input deck assembly for running program PREP3D. Exhibit 6.21 is a sample input deck. Note that only card types F, G, H, and I are needed in the data deck. The gridded elevation data has been stored in a project data file called GEOM*JSMITH, which is used as input to the program. Exhibit 6.22 is a sample graphic output from program PREP3D. 


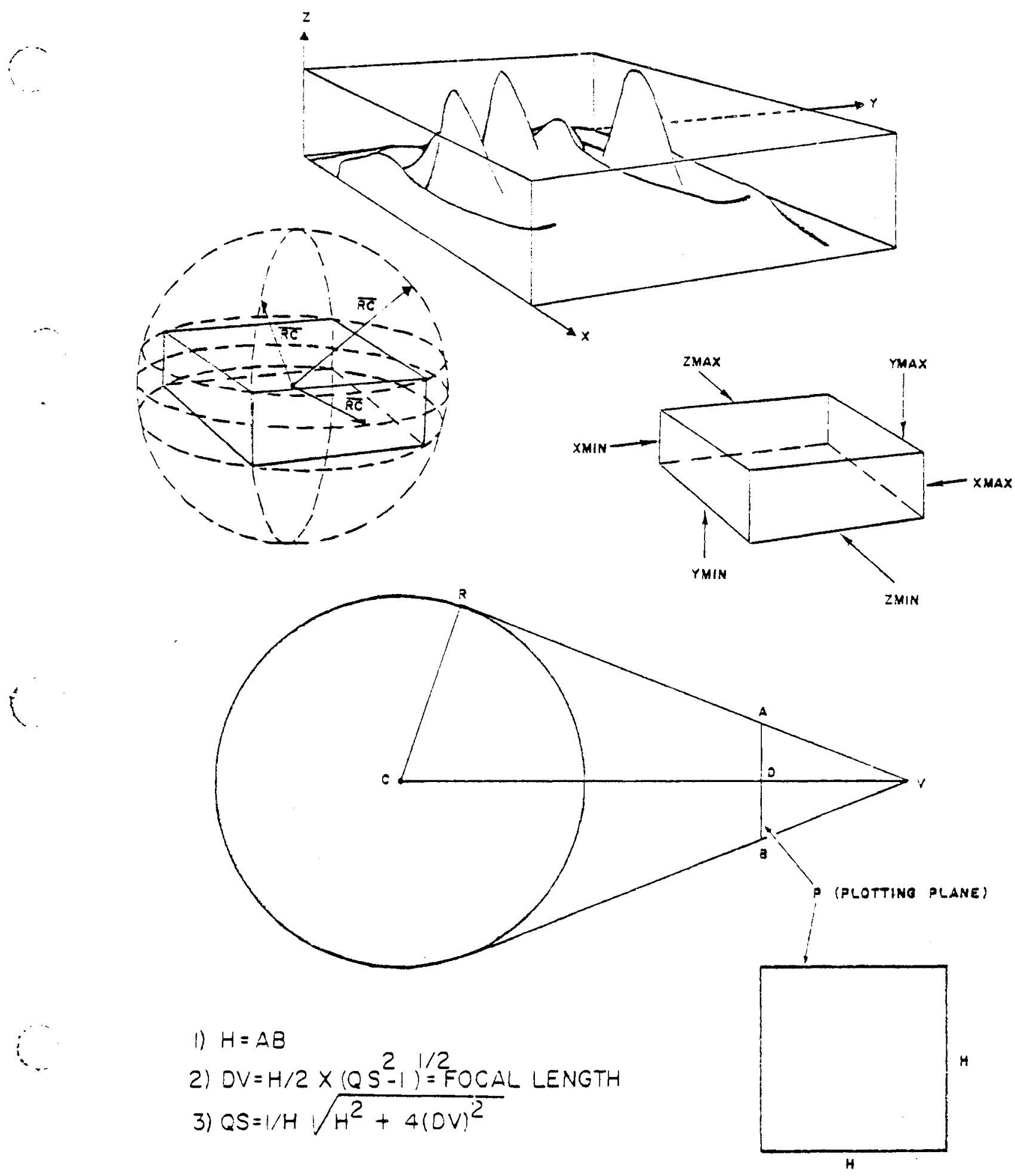

Exhibit 6.16 - Three Dimensional Region and Image Scale Determination 


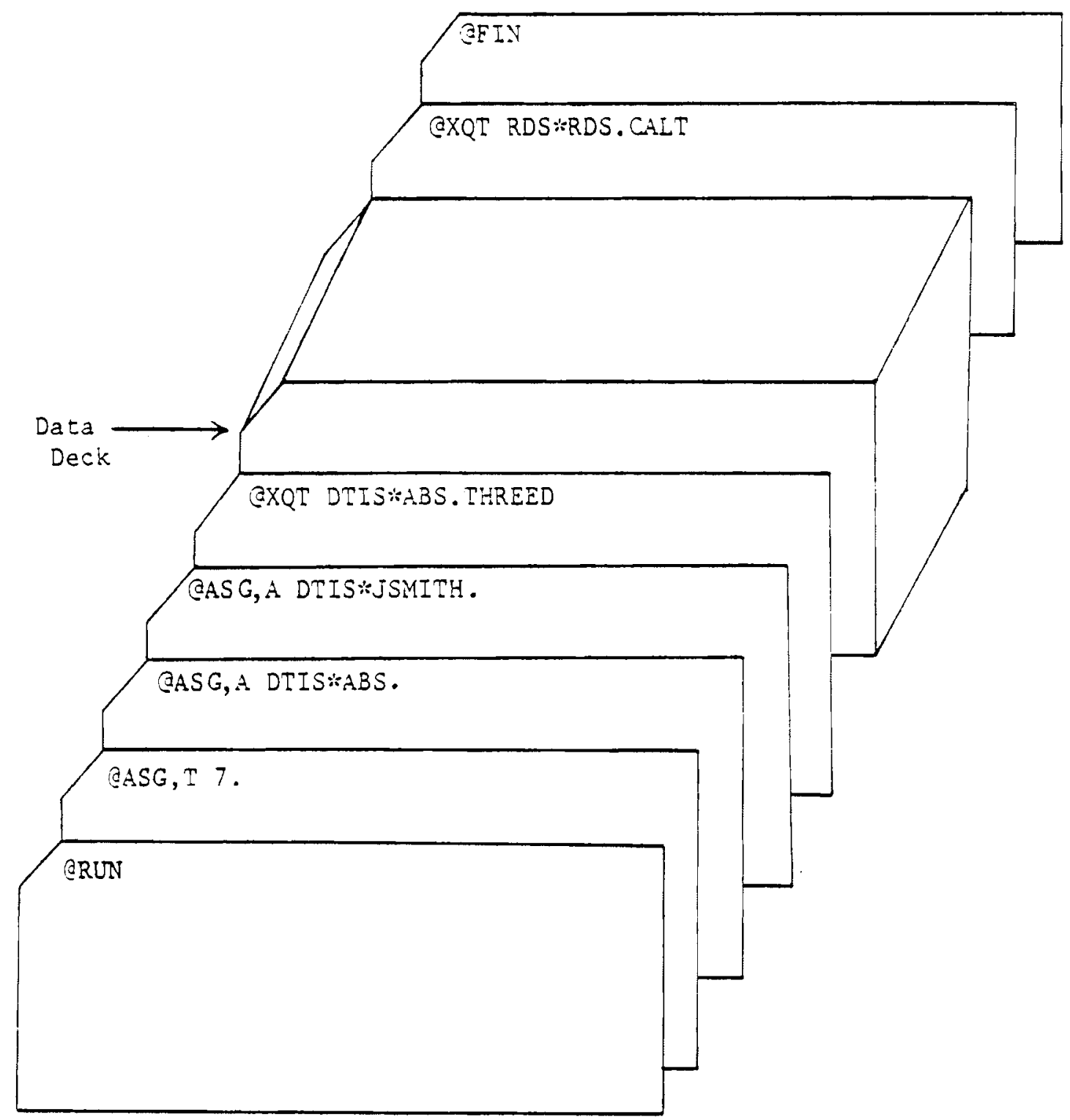

Exhibit 6.17 - Input Deck Assembly for Program THREED 


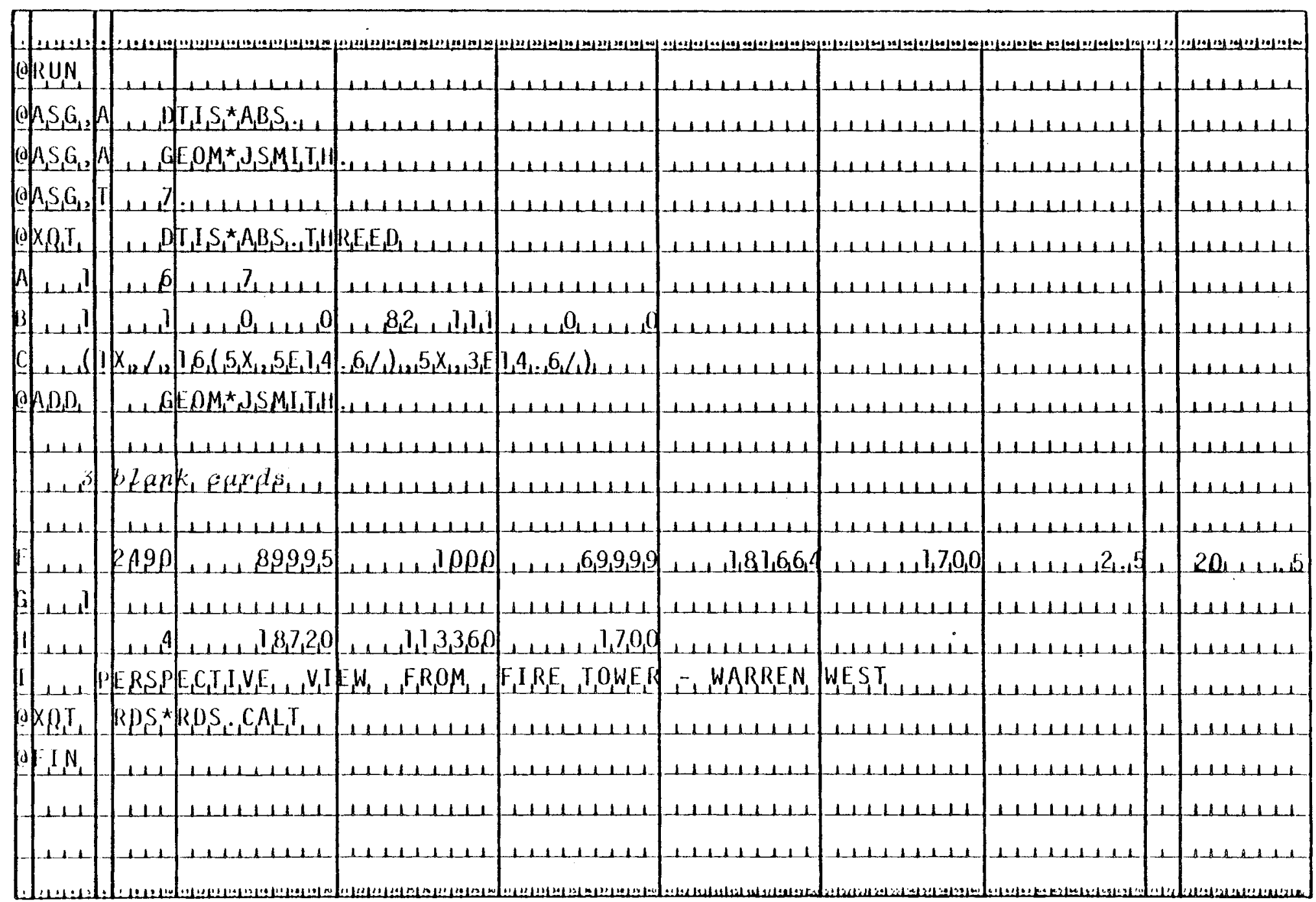




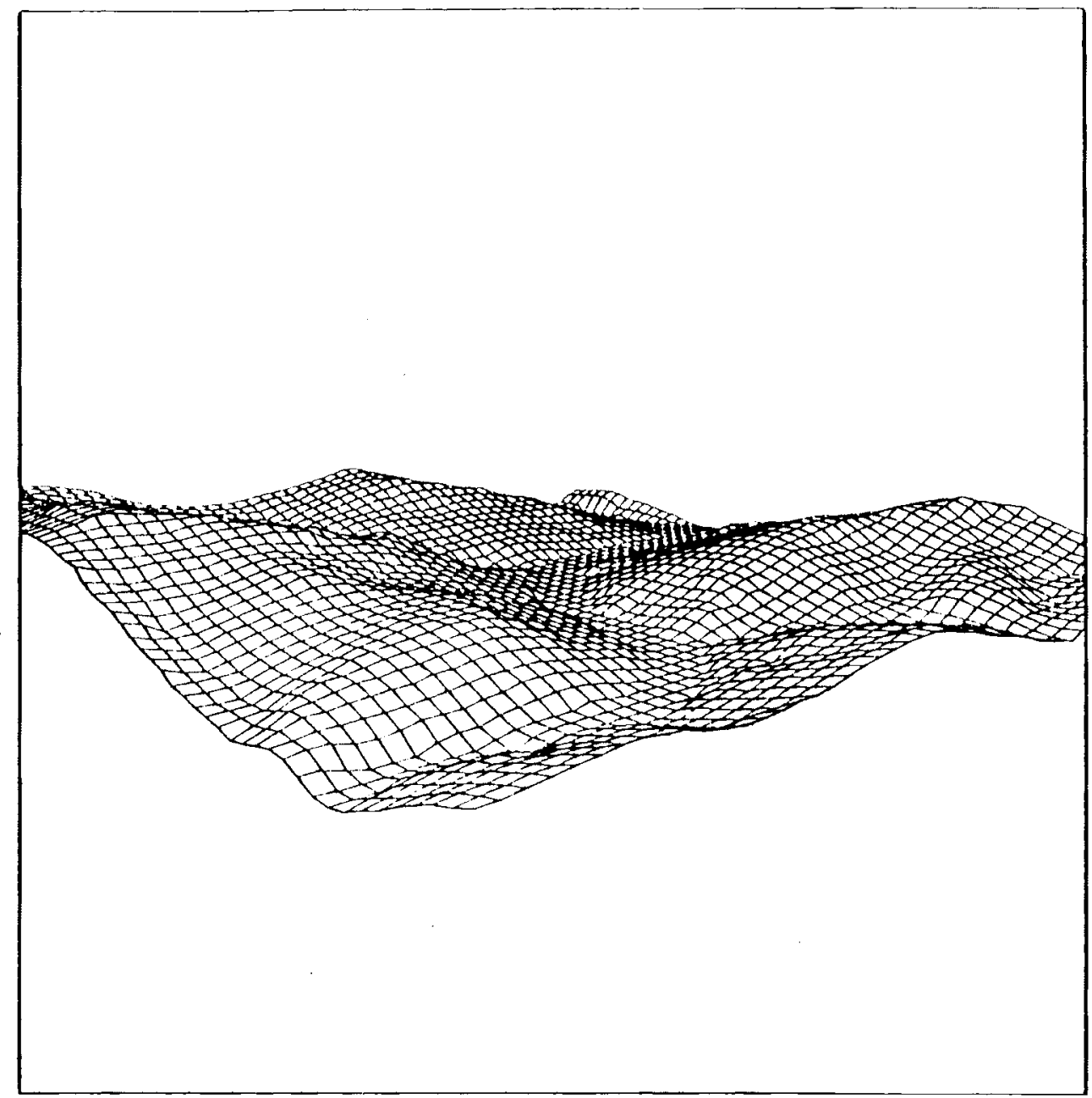

HEASPECTIVE VIEW MAP

compuren con S1000 non alos

Exhibit 6.19 - Sample Graphic Output for Program THREED 


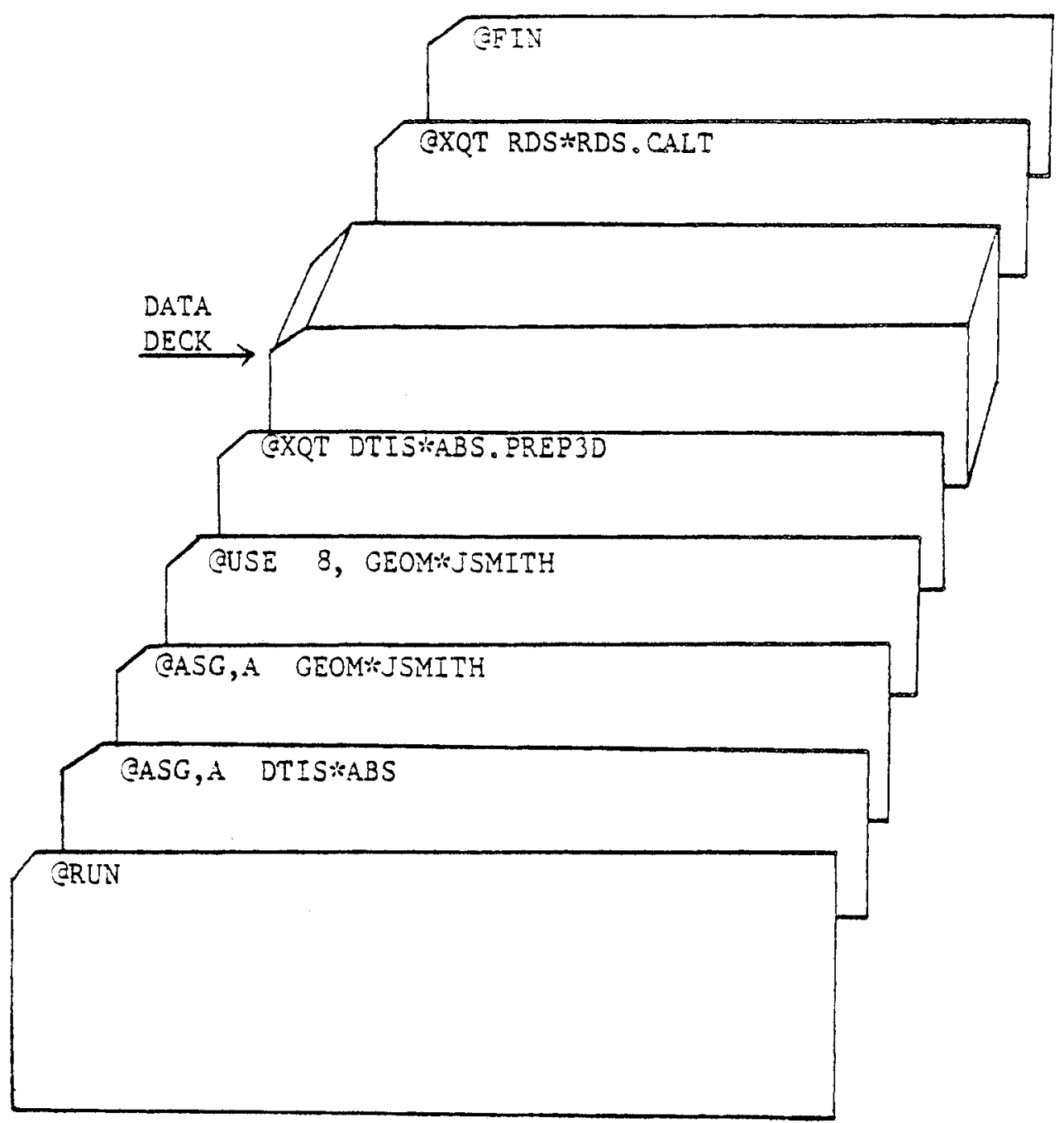

Exhibit 6.20 - Input Deck Assembly for Running Program PREP3D 


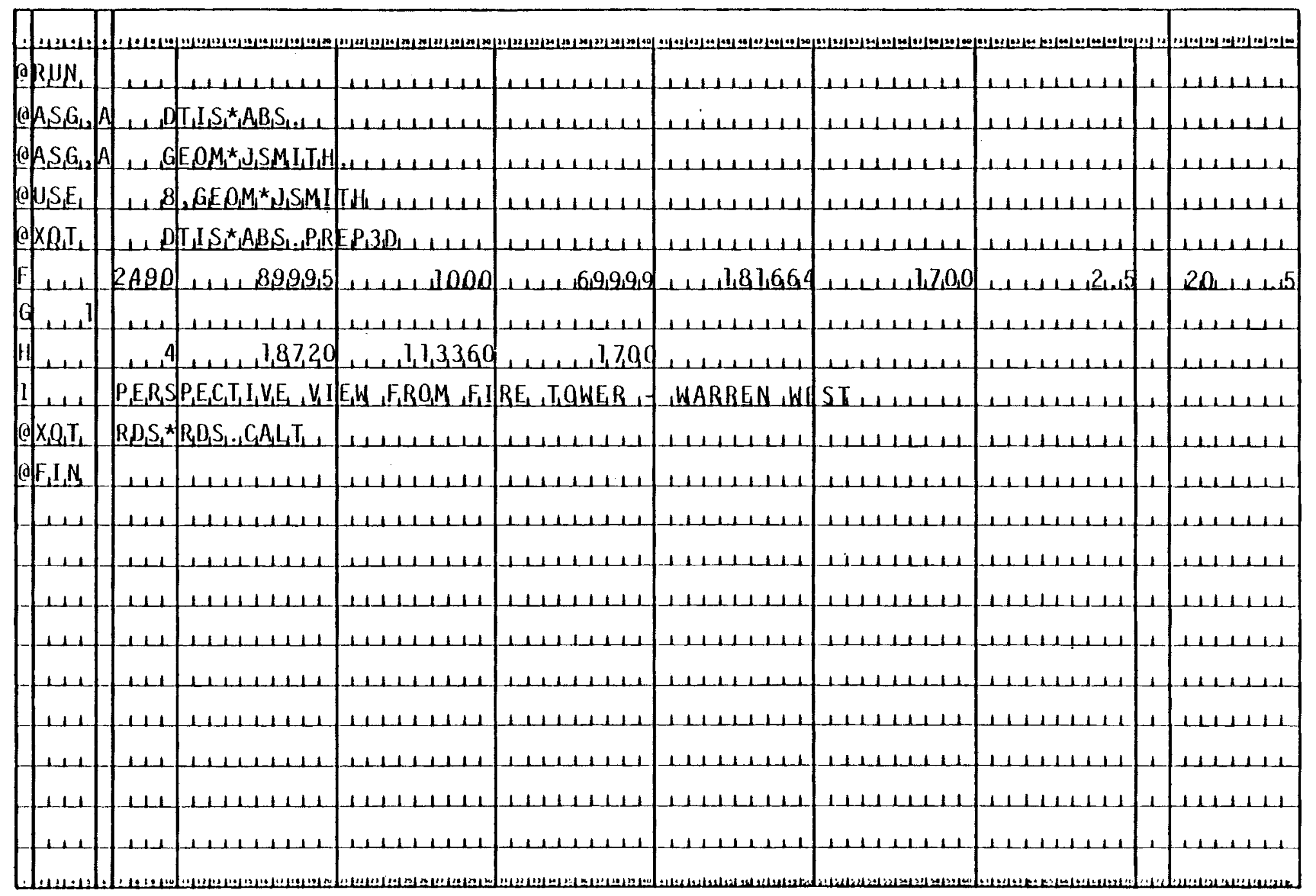




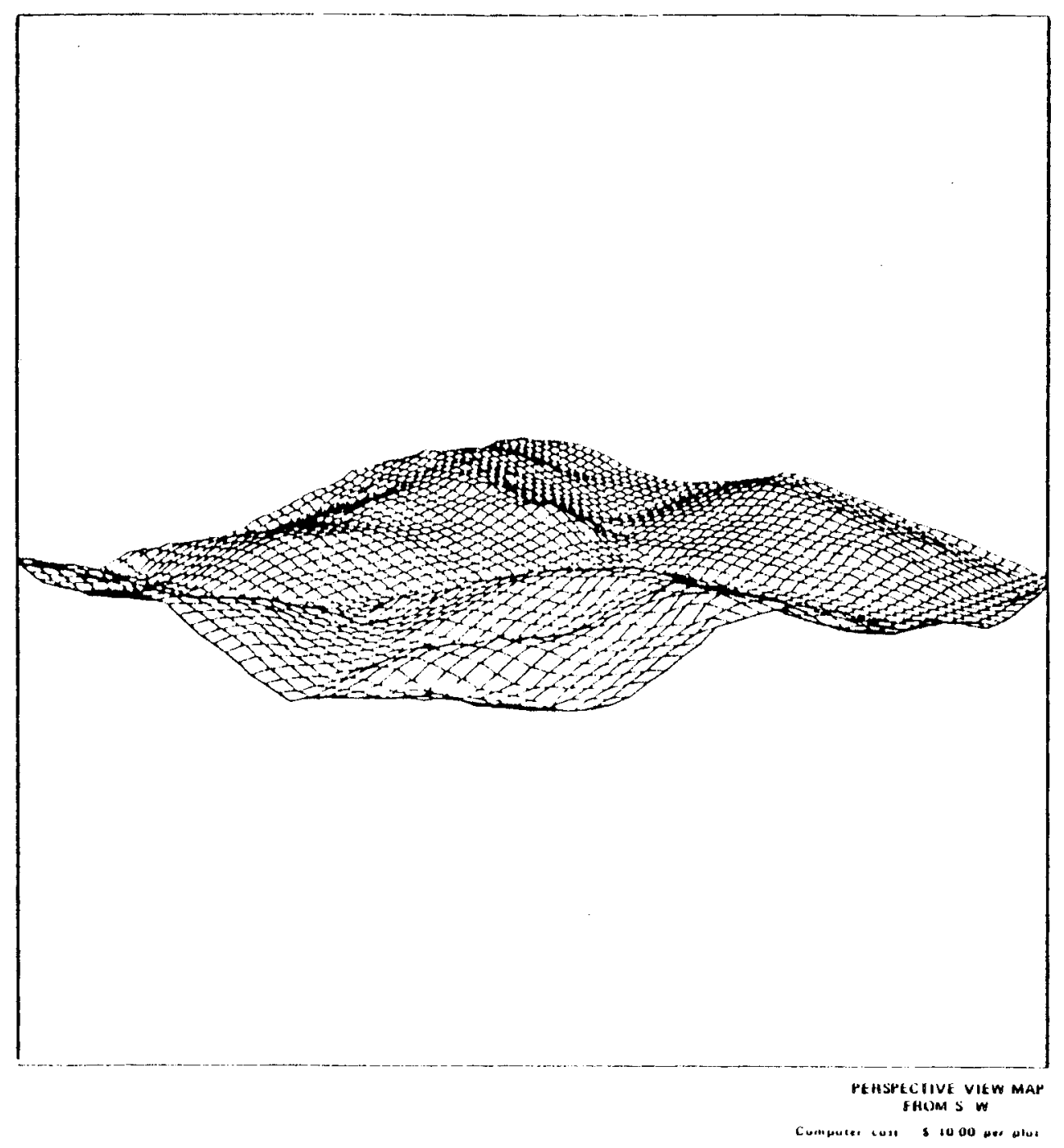




\section{SECTION 6. PERSPECTIVE CONTOUR PROGRAM (CON3D)}

\section{Program Functions}

This program is used to generate perspective contours from gridded data.

The program presently can accept a maximum of 12,000 data points. Output from the SCANDMA program can be used directly as input to this program.

The perspective is generated by the principle of central perspectives. The following parameters can be specified by the user in the input data:

1. $x$ and $y$ dimensions of the view window (i.e. frame size of the photo);

2. focal length of the viewing camera;

3. location from which the view is to be made;

4. the location of the point which is to be at the center of the view window, i.e., photo (this point can be on, below, or above the ground surface);

5. the orientation of the line-of-sight, i.e., optical axis of the view camera.

\section{Data Card Formats}

Assuming that the digital terrain data has already been generated and stored in a file called GEOM*JSMITH, there are only 4 data cards in the input deck to program CON3D.

\section{Card 1}

\begin{tabular}{|c|c|c|}
\hline cc & use & format \\
\hline $1-5$ & NROW - No. of rows of gridded data & 15 \\
\hline $6-10$ & NCOL - No. of columns of gridded data & I5 \\
\hline $11-20$ & Contour Interval in feet & F10.0 \\
\hline $21 \cdot 30$ & $\begin{array}{l}\text { SCALE denominator of the map from which the digital } \\
\text { terrain coordinates ( } x \text { and } y \text { ) are based (for example, } \\
250,000 \text { for all DMA tape data) }\end{array}$ & F10.0 \\
\hline $31-40$ & Spacing (in inches) between adjacent columns & F10.0 \\
\hline $41-50$ & Spacing (in inches) between adjacent rows & F10.0 \\
\hline $51-60$ & $\begin{array}{l}\text { Scale of the contour map to be plotted (omit this } \\
\text { field if perspective contours are to be generated) }\end{array}$ & F 10.0 \\
\hline 61.65 & $\begin{array}{l}\text { I3D - option indicator } \\
=0 \text { generate contour map } \\
=1 \text { generate perspective contours }\end{array}$ & I5 \\
\hline
\end{tabular}


Card 2: @ADD GEOM*JSMITH.

Card 3: (omit this card if the program is used to generate contour map)

cc

$11-15$

$16-20$

$21-25$

$26-30$

3140

$41-50$

$51-60$

$61 \cdot 65$ use

format

XC.AM - Column number of station from which the

55.0 view is to be made

YCAM - Row number of station from which the view

F5.0 is to be made

HCAM - Elevation of the view station

F5.0

$\mathrm{XP}$ - Column number of the object point which

$F 5.0$ will be at the center of the perspective drawing; that is. the point to be looked ar (optional)

YP - Row number of the object point which will be at the center of the perspective drawing (optionai)

HP - Elevation of the object point which will be at the center of the perspective plot (optional)

FRSZX - X-dimension (width) of the perspective plot in

F10.3 inches

FRSZY - Y-dimension (height) of the perspective plot in inches

FL - Focal length of the viewing samera in inches

F 10.3

IORINT - Input option indicator

$=0$ the line-ot-sight is detined by the coordinates of the center picture point (XP, YP, HP)

$=1$ line-of-sight is defined by the orientation angles of the optical axis - these angles are input in the next card
15 
Card 4: (omit this card if either the program is used to generate contour map, or the lineof-sight is defined by coordinates of center picture point)

cc

use

format

1-5 IOPT - Input option indicator

$=1$ line-of-sight to be defined by forward tilt, azimuth measured clockwise from North, and sideward tilt

$=2$ line-of-sight to be defined by the conventional omega $(\omega)$, phi $(\emptyset)$ and kappa $(k)$ angles

ID1 - Degrees forward tilt (or omega)

The sign for the angles of rotation, if negative, must be included in each of the Degrees, Minutes, and Seconds fields. 
SECTION 7. PROGRAM FLIGHT

\section{Function}

Program FLIGHT scans a DMA tape and produces profiles from the elevation data at intervals determined by the scale and sidelap of aerial photography for flight line planning.

Data Card 1. This is the control card on which is specified the scale, sidelap, beginning flight line (strip) number, and a title.

Data Card 2. This is the data card which defines the $x$ and $y$ limits, in grid increments, of the area to be scanned (same format as the data card required by SCANDMA).

\section{Output}

The output of program FLIGHT is a plot of the profiles. The profiles are labeled with the data input on card $\neq 1$. The plots are stacked and each plot shows the strip number, horizontal and vertical scale, and a horizontal and vertical graph (see Exhibit 6.26).

\section{Data Card 1}

Purpose. This card specifies the title, scale, sidelap, and beginning strip number for flight line profiles. (Note: strip number is incremented automatically from West to East.)

Format: (all values right justified)
$\mathrm{cc}$
use
format
$1-10$
SCALE - Denominator of desired aerial photography
I10 scale
$11-15$
SIDELAP - In percent $(15 \%=15)$
15
$16-20$
Beginning STRIP number
Title or description 


\section{Data Card 2}

Purpose. This card defines the limits of $x$ and $y$ for the area to be searched.

Format: (all values right justified)

\begin{tabular}{|c|c|c|}
\hline $\mathfrak{c c}$ & use & format \\
\hline $1-5$ & ILEFT - The lowest $x$ value to be scanned & I5 \\
\hline $6-10$ & IRIGHT - The highest $x$ value to be scanned & I5 \\
\hline $1-15$ & IBOT - The lowest $y$ value to be scanned & 15 \\
\hline $6-20$ & ITOP - The highest $y$ value to be scanned & 15 \\
\hline $21-25$ & $\begin{array}{l}\text { INC - Scan interval. For example, if } I N C=3 \text {, } \\
\text { every third elevation point will be copied from a } \\
\text { DMA tape. }\end{array}$ & 15 \\
\hline $26-30$ & IXSW-X value of SW corner obtained from PRINT & I5 \\
\hline $31-35$ & IYSW-Y value of SW corner obrained from PRINT & 15 \\
\hline
\end{tabular}




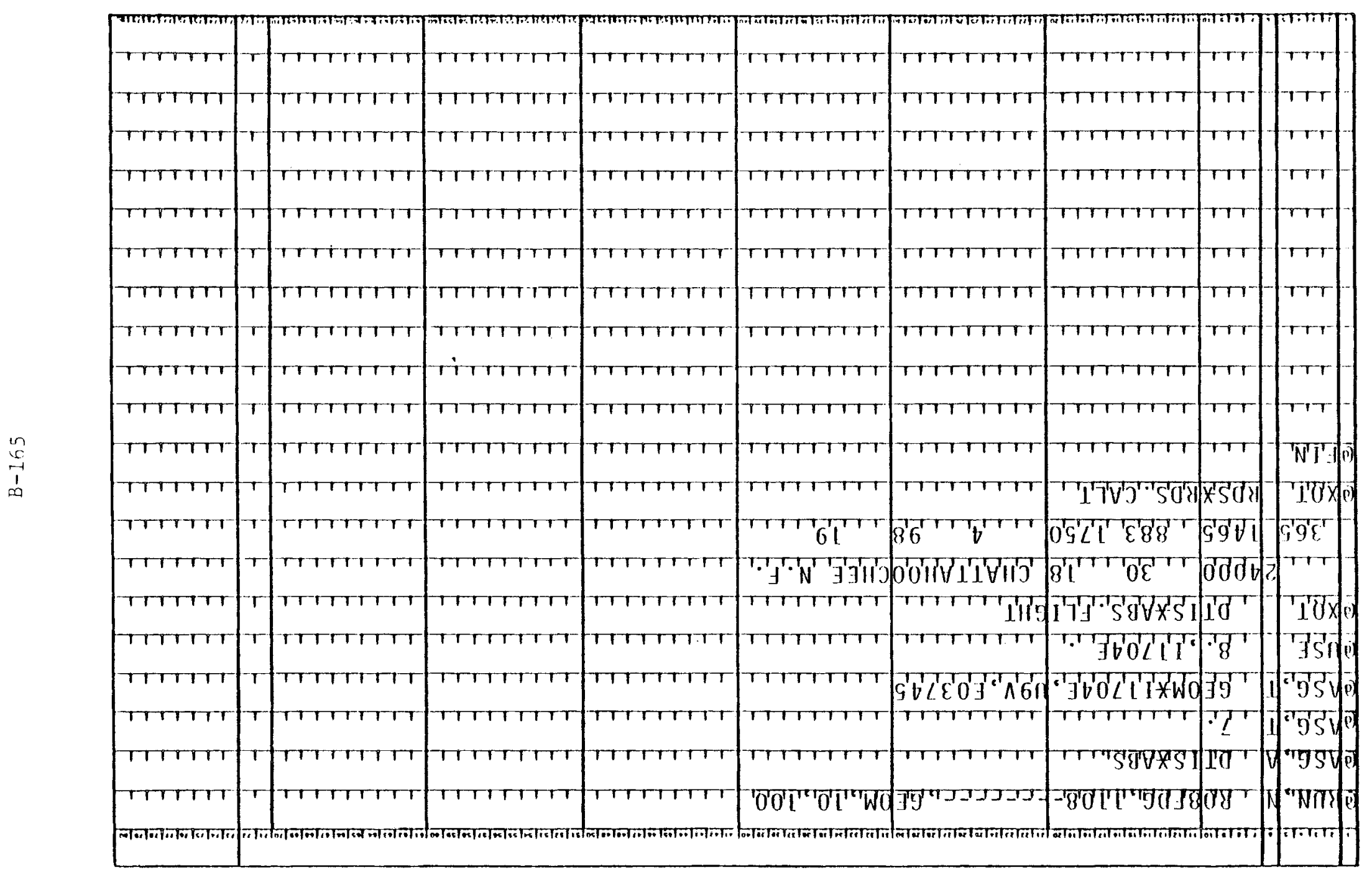




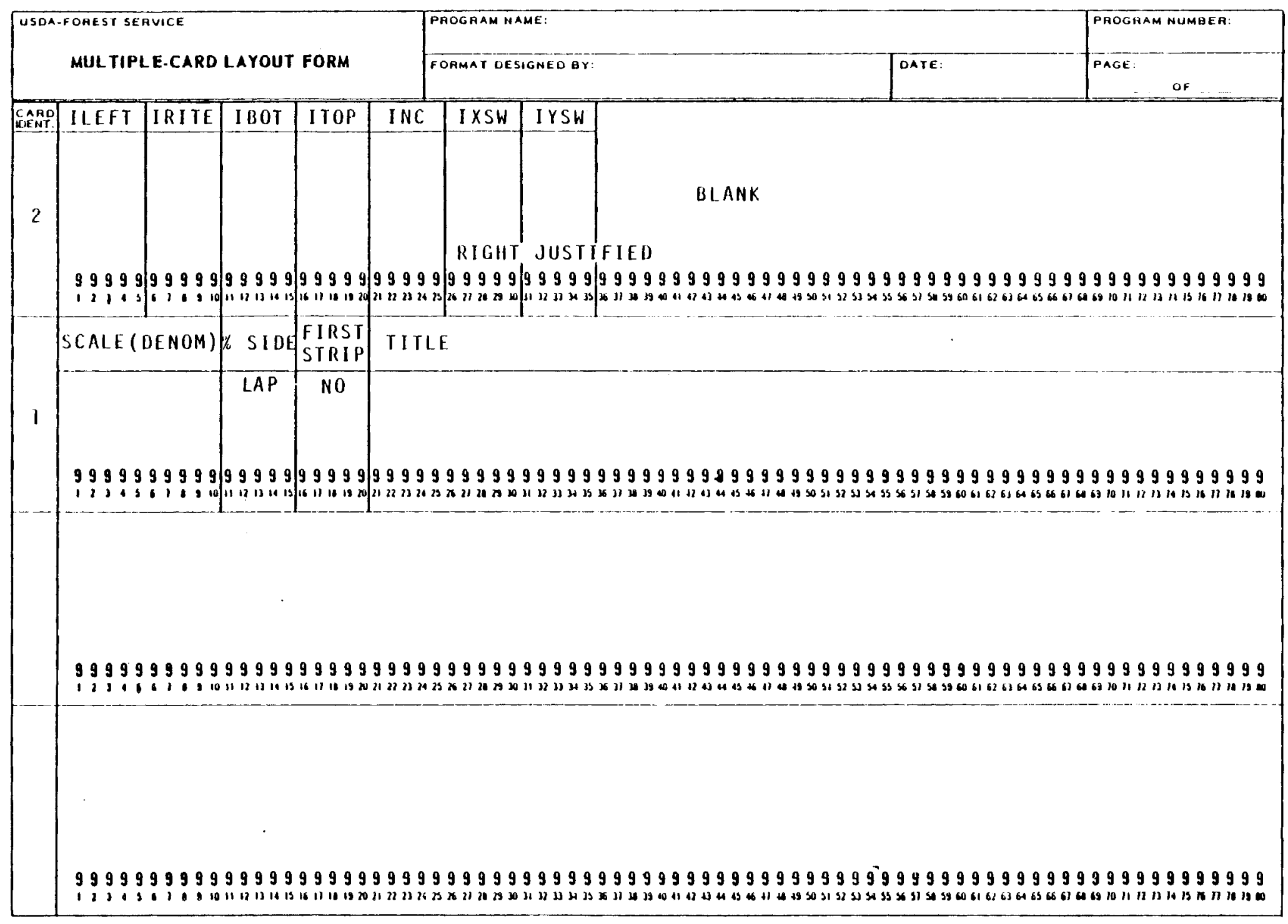

Exhibit 6.24 - Data Cards 1 and 2 for Program FLJGHT

in'




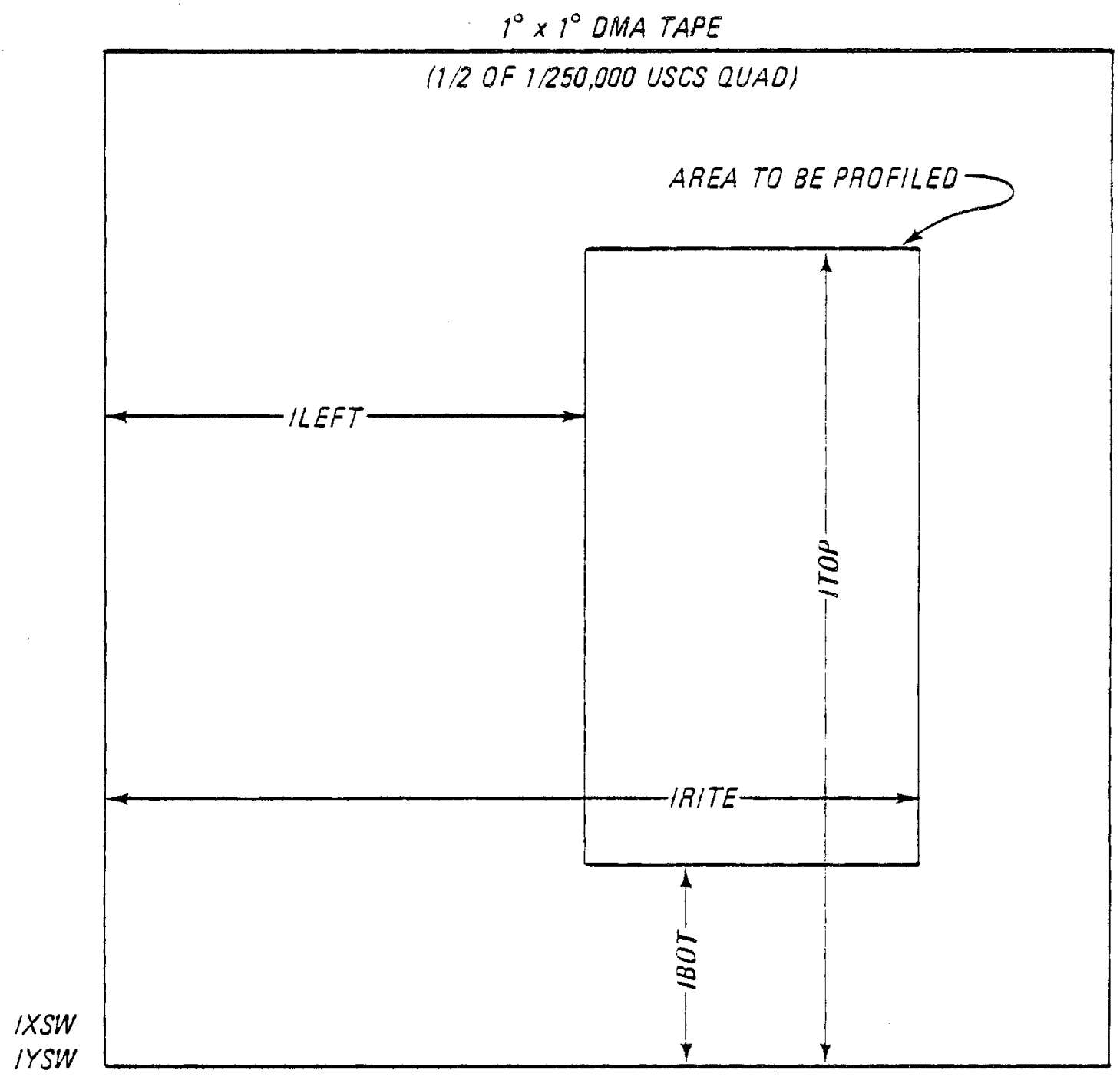

The output from COPY defines IXSW and IYSW. The output from PRINT will assist in determining IIEFT. IRIGHT. IBOT, ITOP.

\section{NOTE}

ILEFT is the location of the centerline of the first flight. 


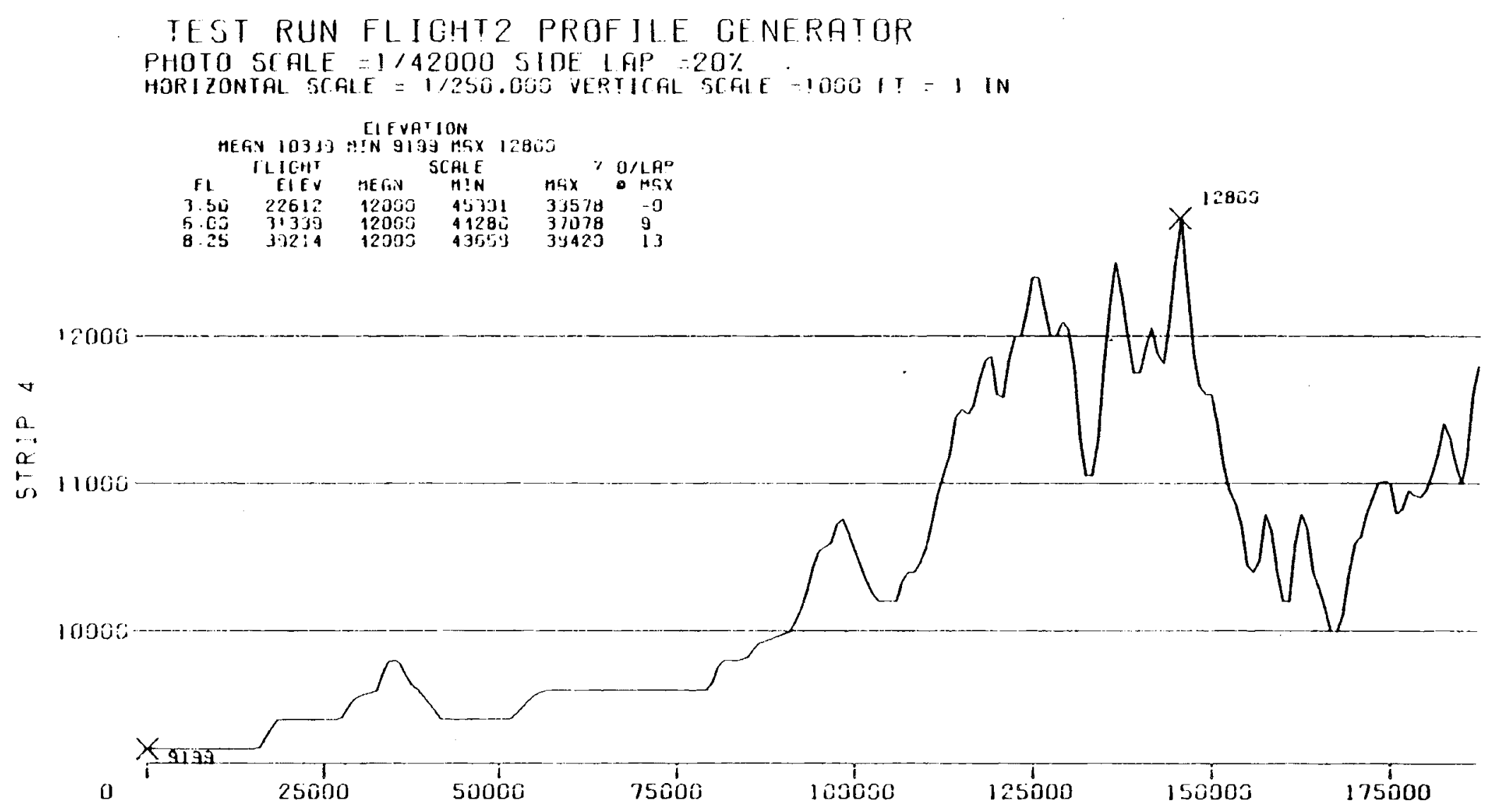


SECTION 8. PROGRAMITOPAS

This program is a self-tutorial program to create and execute runstreams for graphic products where the terrain source is DMA tapes.

The program is executed from DEMAND as follows:

\section{@ RUN \\ @ ASG.A DTIS*ABS \\ @XQT DTIS*ABS.ITOPAS}

The program then responds by asking questions pertaining to required system information, such as account number and RUN number. This is followed by queries about the desired area, tape name and number, location of southwest corner of the tape, and location of the 4 corners of the data to be retrieved. It then requests the type of plot or plots desired. Finally, it schedules the generated runstream for execution.

Optionally, the runstream can be modified and manually scheduled. The annotated runstream on the following pages shows how the program operates, and describes what is being generated for the runstream. 
QRUN RO6SHW, $1106527117 \times 4$, GEOM

DATE: 100675 TIME: 135905

$>$ QASG, A DTIS*ABS

FACILITY WARNING 000200000000

$>$ QXQT DTIS*ABS. ITOPAS

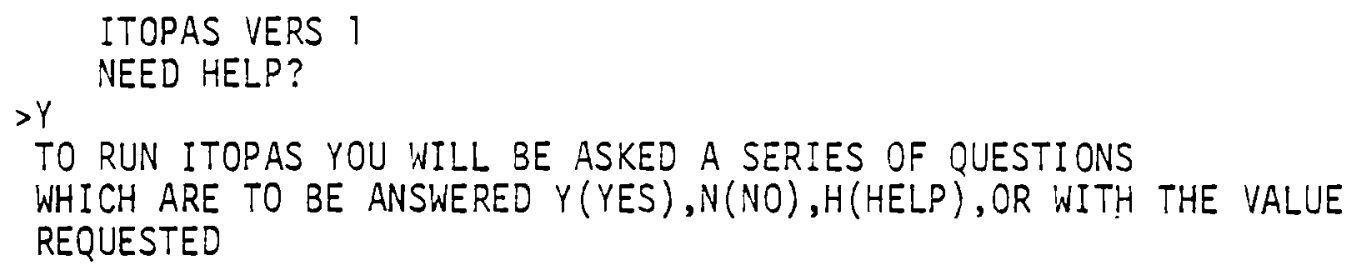

12 DIGIT ACCT. NO.?

$>1106527117 \times U$

ACCOUNT IS $1106527117 \times U$ RIGHT?

$>Y$

YOUR NAME AND SPECIAL INSTRUCTIONS?

>SHERRY FRIESEN PLOT ON PAPER

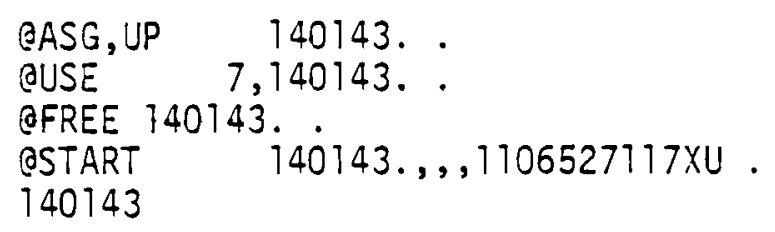

6 DIGIT RUN NUMBER? $>$ R06SAW

PLOT MAILING AND HANDLING INSTRUCTIONS TO WHICH FOREST? >SIUSLAW S.O. MAIL FIRST CLASS 
1. Enter an @ RUN Card.

2. Enter a ASG, A ALB*ALB.

3. After the facility warning, enter $\exists$ XQT ALB*ALB. ITOPAS

If you need help enter a Yes (Y) if you don't, enter $a(N)$ no and you will be asked the next question.

The 12 digit account number used in the ?RUN statement needs to be entered here.

The name of the person who is requesting the plot and any special instructions like paper type, pens, ball point or ink, liquid is standard when not requested up to 60 characters are allowed.

Files are automatically set up by the ITOPAS program.

The 6 digit run number used in the 3 Run statement needs to be entered here.

Enter the name of Forest office or district where you would like your plot or plots nailed to. 60 characters are allowed. 


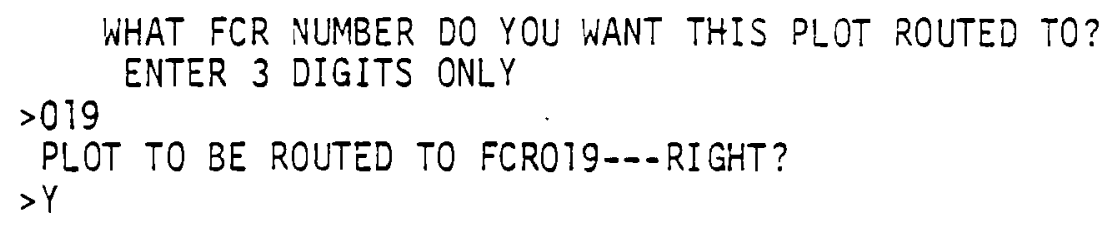

WHAT FCR NUMBER DO YOU WANT THIS PLOT ROUTED TO? $>019$

6 DIGIT TAPE NUMBER?

$>$ F02398

THE FOLLOWING QUESTIONS MUST BE ANSWERED WITHOUT A DECIMAL POINT USE COMMAS TO SEPARATE 2 VALUES WHEN REQUESTED

ENTER VALUES OF IXSW, IYSW AS SHOWN ON TOP OF PRINT SHEET $>201,57$

IXSW $=\quad 201$ IYSW $=\quad 57$ RIGHT?

$>Y$

NOW ENTER CORNER COORDINATES OF THE QUAD SELECTED

SW CORNER $X, Y$ ? $>480,219$ $Y$ SW CORNER $X, Y=480219$ RIGHT? $>Y$

SE CORNER $X, Y$ ?

$>640,220$ $>Y$

SE CORNER $X, Y=640 \quad 220$ RIGHT?

NW CORNER $X, Y$ ?

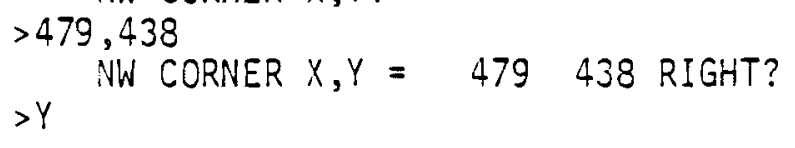


019 is the FCR number used by Region 6 for all plots.

Enter the tape label from the DMA print sneet.

Enter the 6 digit tape number from the DMA print sheet.

Enter the IXSW and IYSW values from the print sheet. Separate the 2 values with a comma. If these values are correct answer either yes $(Y)$ or no $(N)$.

Enter first the $(X)$ coordinate, then the $(Y)$ coordinate, separating them only by a comna. If the values that you have given are correct, answered either Yes ( $Y$ ) or No $(N)$. (Follow this same procedure for each comer of the quad).

Same as above.

Same as above. 
NE CORNER $X, Y$ ?

$>639,439$

$>Y$

NE CORNER $X, Y=639439$ RIGHT?

INCREMENT ? --NORMALLY 2 FOR 7.5 MIN. QUAD.

$>2$

AND 4 FOR A 15 MIN. QUAD

$>Y$ INCREMENT $=2$ RIGHT?

IS THE AREA YOU HAVE SELECTED ON THE LEFT EDGE, RIGHT EDGE, (M).

OR MIDDLE OF THE ONE DEGREE QUADRANGLE ON THIS TAPE? USE $(L),(R)$ OR $>L$

$Z$ DO YOU WANT CONTOUR MAP(C), ISOSLOPE(I), SLOPE ZONE(S) ,ASPECT ZONE (A),VIEWITTV), PERSPECTIVE PLOT(P), PERSPECTIVE CONTOURS(T) OR OONE(D)

$>C$

TITLE TO BE PRINTED ON CONTOUR MAP?

$>$ MT.CLAIRE MAIL TO SIUSLAW S.O.

DESIRED PLOT SCALE IN FT. PER INCH-USE DECIMAL POINT $>2640$.

SCALE REQUESTED IS 2640.00 FEET PER INCH--RIGHT?

$>Y$ $>40$.

ENTER CONTOUR INTERVAL INCLUDING THE DECIMAL POINT

CONTOUR INTERVAL IS 40.00 RIGHT? $>Y$ 
Same as above.

Use a (2) for a $71 / 2$ minute quad, as a (4) for a 15 minute quad.

Enter an (L) for the left edge of the quad.

(R) for the right edge of the quad.

(M) if in the middle of the quad.

Contour example.

I. You are asked what title you would like. It is wise to include the forest nane along with the title. Sixty characters field.

2. The next question now deals with the scale for the plot. Any basic map scale is acceptable.

3. Now you are asked if this is the scale you asked for. Answer $(Y)$ yes or $(N)$ no. 4. You are now asked what contour interval you desire.

5. Now you are asked if this is the contour interval you asked for. Answer $(Y)$ yes $(N)$ no. 
DO YOU WANT CONTOUR MAP(C), ISOSLOPE (I), SLOPE ZONE(S) , ASPECT ZONE(A), VIENIT(V), PERSPECTIVE PLOT(P), PERSPECTIVE CONTOURS (T) OR DONE(D)

$>I$

TITLE TO BE PRINTED ON ISOSLOPE MAP?

$>$ MT.CLAIRE MAIL TO SIUSLAW S.O. $>2640$.

OESIRED PLOT SCALE IN FT. PER INCH-USE DECIMAL POINT

SCALE REQUESTED IS 2640.00 FEET PER INCH--RIGHT? $>Y$

TYPE IN THE SLOPE ZONES DESIRED(UP TO 6) SEPARATED

BY COMMAS, WITH NO DECIMAL PTS, AND IN ASCENDING ORDER FOR EXAMPLE - 5, $15,30,45,65,,,$, , $>5,20,45,60,90,,$, , ZONES ARE

$520 \quad 45 \quad 60 \quad 90 \quad 0$ RIGHT?
$>Y$ . 
Isoslope

1. You are asked what title you would like. It is wise to include the forest name along with the title. Sixty characters field. 2. The next question now deals with the scale for the piot. Any basic map scale is acceptable.

3. Now enter the slope zones you desire (up to 6) in ascending order. After the last value, entar + commas.

4. Now you are asked if this is the Isoslope zones you have asked for. Answer $(Y)$ yes $(V)$ no. 


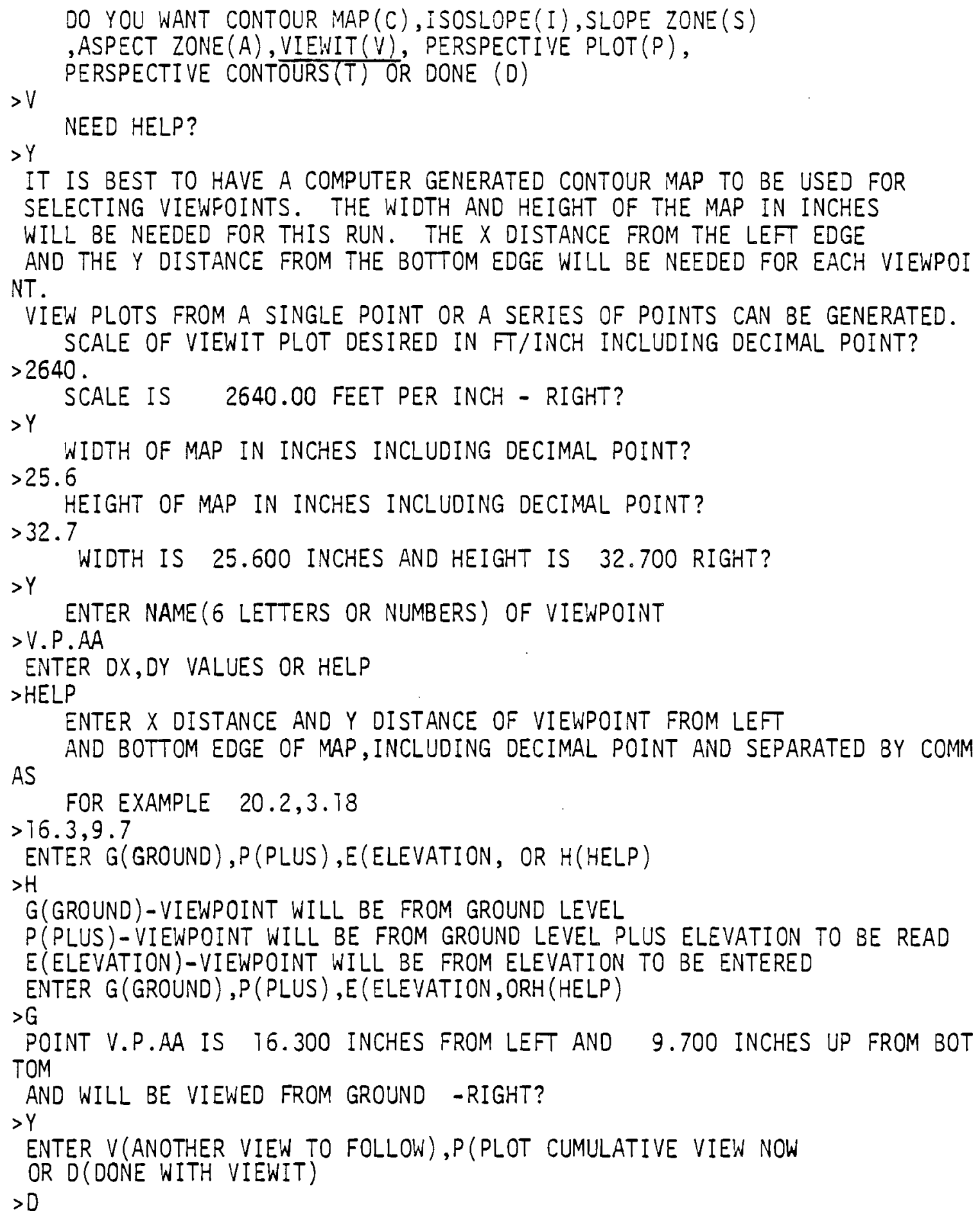




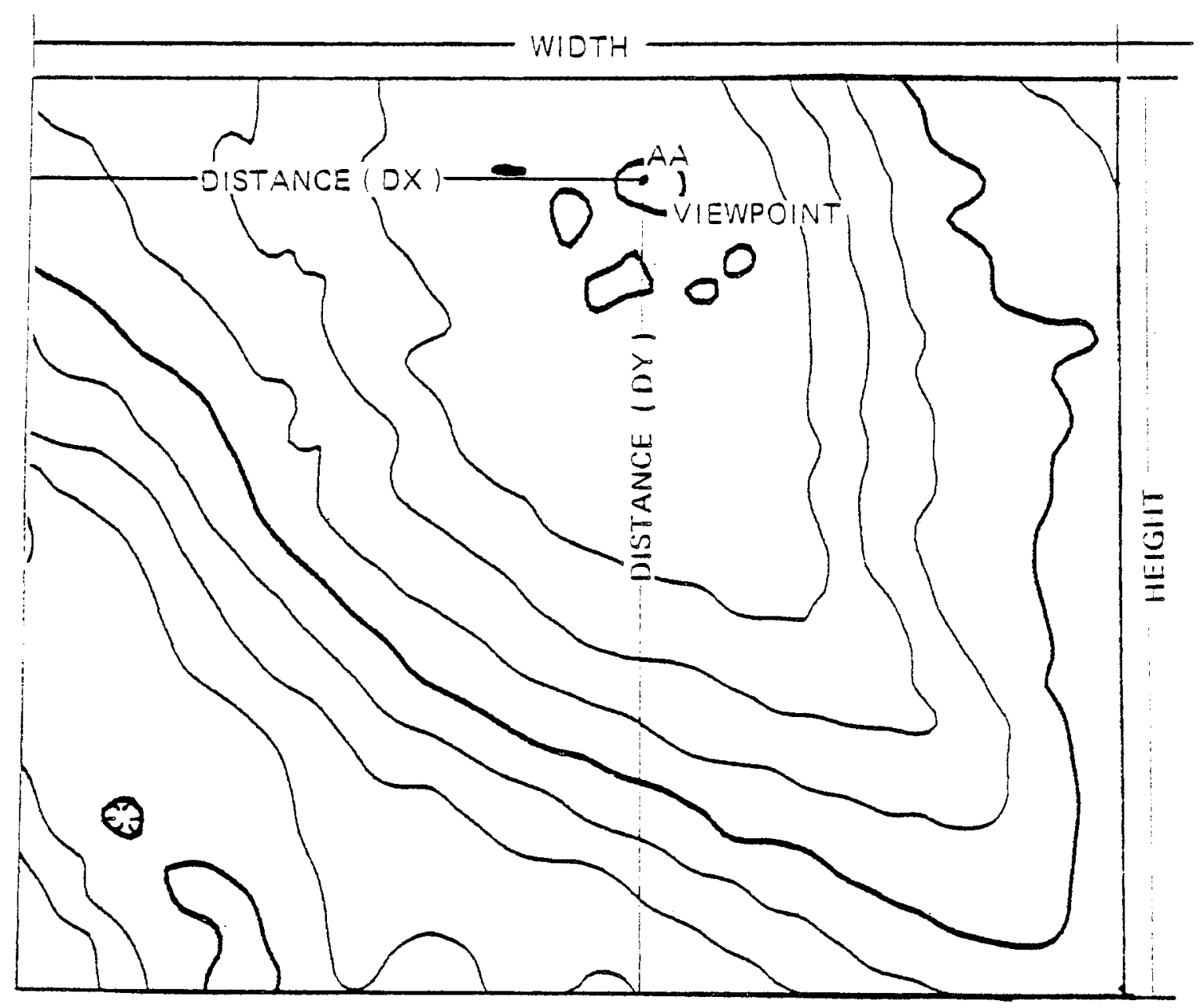




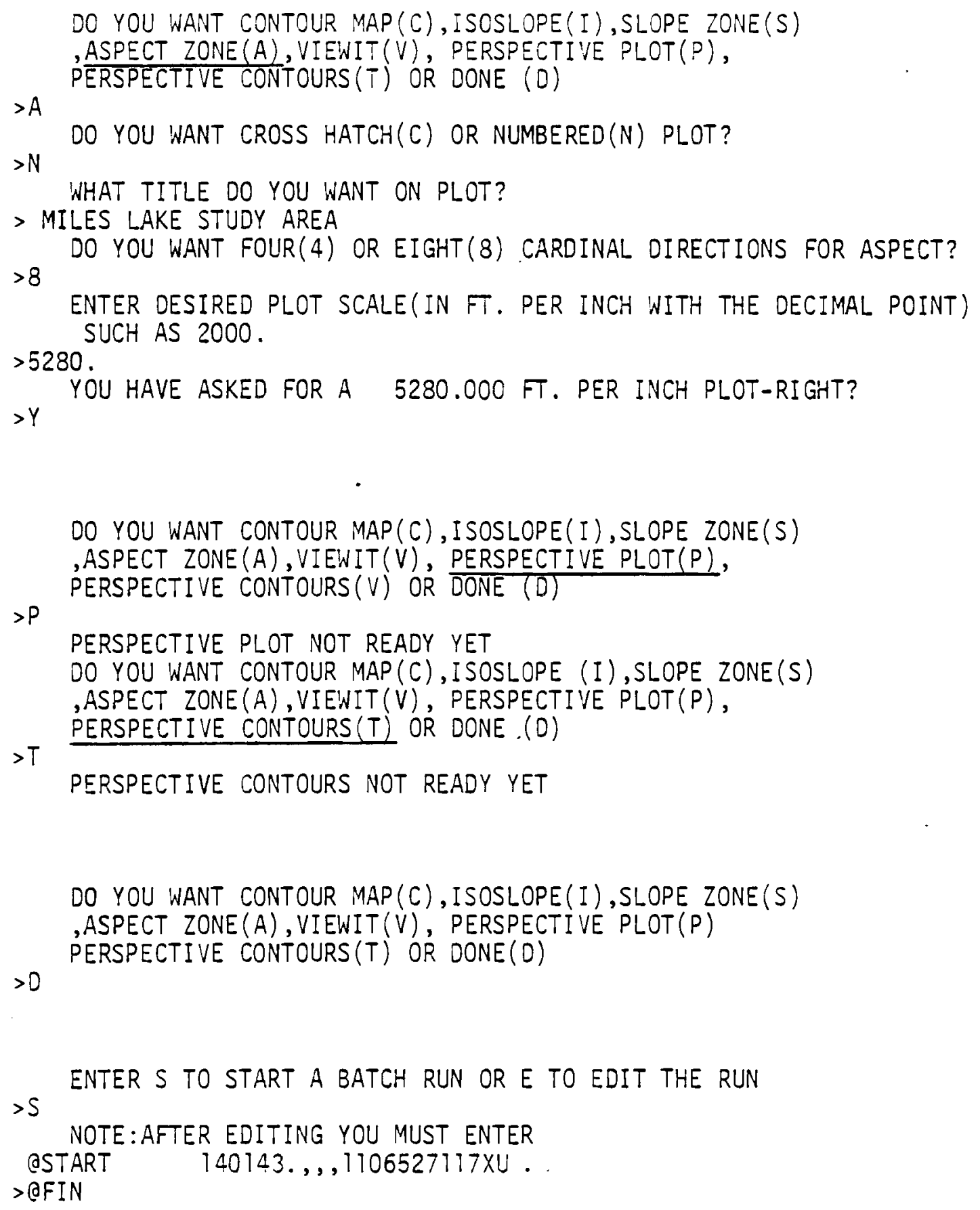

ENTER S TO START A BATCH RUN OR E TO EDIT THE RUN NOTE:AFTER EDITING YOU MUST ENTER QSTART 140143.,,, , 1106527117XU. $>Q F I N$ 


$$
B-181
$$

Aspect

1. Enter a (C) for a cross hatched plot or an (N) for a numbered plot.

2. You are asked what title you would like. It is a good idea to include the forest name along with the title. Sixty character field. 3. Enter either 4 or 8 for cardinal directions of Aspect.

4. The next question now deals with the scale for the plot. Any basic map scale is acceptable.

5. Now you are asked if this is the scale you asked for. Answer $(Y)$ yes or $(N)$ no.

When you are finished enter a (D) for Done.

Enter an (s) to start a batch run of all the plots you have just finished. You have an option to (E) edit the mun. 
RUNID: RO6SAW ACCT: 1106527117 PROJECT: GEOM

TIME: TOTAL: 00:00:04.629

CPU: $\quad 00: 00: 00.714 \quad I / 0: 00: 00: 00.428$

CC/ER: 00:00:03.486 WAIT: 00:18:44.568

START: $13: 59: 04$ OCT 06,1975 FIN: $14: 24: 38$ OCT 06,1975

SIZE: OTOK

EST COST: TOTAL: 00002.91

CPU: 00000.36 I/0: 00000.00 CONNECT: 0002.55

*TERMINAL INACTIVE* 


\section{APPENDIX A}

\section{ACCESS TO TOPAS THROUGH USFS UNTVAC 1108 COMPUTER}

\section{Location of TOPAS Computer Program Package}

The entire package of TOPAS compuipr programs is stored in the UNIVAC 1108 Computer at Fort Collins Computer Center (FCCC). In addition, all the DMA digital terrain data tapes that are presently available within the USFS are also stored and cataloged at FCCC. Access to any of the programs or DMA tapes can be made by following instructions provided in this guide.

In order to make full use of the complete capability of the TOPAS computer program package, a user's terminal should have the following computer equipments:

1 System input device (card reader and/or typagraph)

1 System output (printer and/or typagraph)

1 System output device 9-track tape

19 -track to 7 -track tape routine to convert print image data to a plot tape

1 Calcomp graphic plotter

A Data 100 Level 2 or 3 will meet these requirements.

WO will be responsible for the maintenance and revision of the TOPAS programs. Suggestions for program modifications and improvements should accordingly be forwarded to WO. Users should not make any attempt to modify any of the TOPAS program files.

\section{Job Control Cards}

An input deck to any of the TOPAS programs will consist of two types of cards: job control cards, and data cards. Generally, the job control cards activate the proper program in the TOPAS package, and assign the logical units required for proper execution of the job. The data cards contain the input to the computer program. This guide will outline. for each computer program, the job control cards that are needed to run that particular program. A thorough understanding of the job control language (JCL) for the UNIVAC Computer is desirable. but not essential. However, for the benefits of the users who have no prior knowledge of JCL for the UNIVAC 1108 computer, several commonly-used JCL statements are briefly explained in the following pages.

\section{Job Routing}

A job can be submitted either through a regular batch-run terminal. or through a teletype terminal on demand basis. The following programs involve very little printout. and require short execution time, and therefore, may be run through a teletype terminal:

1. Request listing of DMA library tapes

2. Program COPY

3. Program PRINT

4. Program SCANDMA

5. Program SLOPE 
The following programs usually have graphic output and require longer execution time, and are, therefore, appropriate for batch-run:

1. Program FSGPCP

2. Program ZONEIT

3. Program THREED

4. Program PREP3D

5. Program SEEN

6. Program DTIS

\section{Procedure for Using a Teletype Demand Terminal}

The following procedures may be followed to establish a run with the UNIVAC 1108 through a teletype terminal:

1. Link up telephone and wait for dial tone.

2. Dial the assigned 1108 installation communication system telephone number.

3. The telephone will ring at least once followed by a high pitched tone to indicate a completed connection.

a. If a busy signal is received, all lines are active.

b. Wait a few minutes and re-dial.

4. When the tone is heard, type the assigned SITEID for the Terminal. Six (6) characters are established at system generation time.

a. The SITEID should not be followed by a CR.

5. The 1108 will respond with the message: UNIVAC 1108 TIME/SHARING EXEC

a. The exact response may vary between installations.

b. If no response is received after submission of a SITEID, there may have been noise on the line, parity error or other loss os transmission, or it may be in use by another terminal.

(1). Resubmit SITEID

(2). If there is still no response, type SITEID.

(3). If there is still no response, submit another acceptable SITEID.

(4). If this fails, call the central site operator to verify SITEID.

6. Define page width and characters for delete.

Example: @@TTYW,132,L.],C,I CR

W, 132 means page width will be 132 characters.

,L,] means the character ']' will be used to denote line delete.

,C, $\backslash$ means the character ' $Y$ ' will be used to denote character delete.

7. Enter a RUN Statement in the form:

@ RUN RUNID,Account \#,Project Qualifier

a. @ RUN must be in positions 1-4.

b. RUNID: 1-6 Alpha/Numeric Characters followed by a comma.

c. Account: 12 characters

(1) Followed by comma if project field is used.

d. Project: optional 1-12 characters. This is the implied FILE QUALIFIER.

8. 1108 will respond with a message of the form:

DATE 
The program can now be entered statement by statement, and data card by data card. The run will be executed and the printed output listed.

9. At the completion of the run, terminate the joo with the statements below:

@ FIN

@@TERM

For further details on the use of the demand terminal, the user may consult the UNIVAC User's manual (Reference 4).

\section{Brief Description of UNIVAC@ED Processor}

Under EXEC 8, card images may be maintained on mass storage in symbolic elements. These elements are organized into files. The complete internal name for a symbolic element is of the form:

\section{FILENAME.ELEMENTNAME}

The @ED processor is a text editor which allows the user to create, update, and replace symbolic elements in a cataloged file. Basic editor options are:

C Create new element if old element does not exist (used with option $U$ )

I Initial creation of new symbolic element (file must already be cataloged)

$R \quad$ Read existing symbolic element (no updates allowed)

U Update existing symbolic element

To call the @ED processor from a demand or batch-run enter:

$@$ @D,OPTIONS FILENAME.ELEMENTNAME

The @ED processor operates in two modes: input and edit. In input mode, all lines entered are directly inserted into the text. In edit mode, various commands may be used to modify existing text. To change modes, enter a blank line.

Basic commands to the @ED processor while in edit mode are listed below. Some commands may be abbreviated to 1 or 2 characters as specified. All may be abbreviated to 3 characters. These commands may nor be used in inpur mode.

\section{COMMAND}

CHANGE/S1/S2/ This command searches the current line for the character string S1. The first $\mathrm{C} / 51 / 52$

DELETE N1 N2 DELETE N1 DELETE

\section{DESCRIPTION} occurrence of $S 1$ in the line is changed to $S 2$. Either $S 1$ or $S 2$ may be empry.

This command deletes lines. The first form deletes lines $\mathrm{N} 1$ through $\mathrm{N} 2$. The second form deletes $\mathrm{N} 1$ lines starting with the current line. The third form deletes the current line. DELETE may be abbreviated as D.

EXIT This command applies all corrections, and then exits. 
INSERT S1 This command inserts S1 into a new line following the current line. The new

I S1 line becomes the current line.

LNP

(See PRINT - identical except line numbers are printed.)

LOCATE S1

L S1

This command searches the text. starting with the next line, and stopping

OMIT when $\mathrm{S} 1$ is located.

PRINT N1 N2 PRINT N1

PRINT!

This command exits without applying the corrections made.

N1

$+\mathrm{N} 1$

. $\mathrm{N} 1$
This command prints lines of text. The first form prints lines N1 through N2. The second form prints the next $\mathrm{N} 1$ lines. The third form prints the entire element from the top. Print may be abbreviated as $P$.

These commands position the editor at a desired line. First form directs the editor to line $\mathrm{Nl}$. The second and third forms direct the editor to move N1 lines forward or back from the current line.

For complete information on all editor options and commands, see:

UNIVAC UP 4144, Rev. 2(Level 27 EXEC)

UNIVAC UP 4144,REV. 3(Level 30 EXEC) 


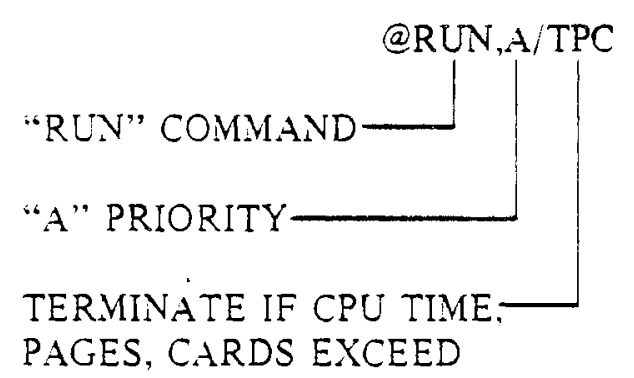

RUN-ID

ACCOUNT NUMBER-MUST BE VAIID

PROJECT-LSED AS IMPLIED FILE QUALIFIER

RUN TIME ESTIMATE(MAX CPU TIME IN MINUTES)

TERMINATE IF OPTION CONTAINS "T"

100 PAGES MAXIMUM. TERMINATE IF OPTION CONTAINS "P"

30 CARDS OUTPUT MAXIMUM-TERMINATE IF OPTION CONTAINS "C" 
@ASG Statement - General Format

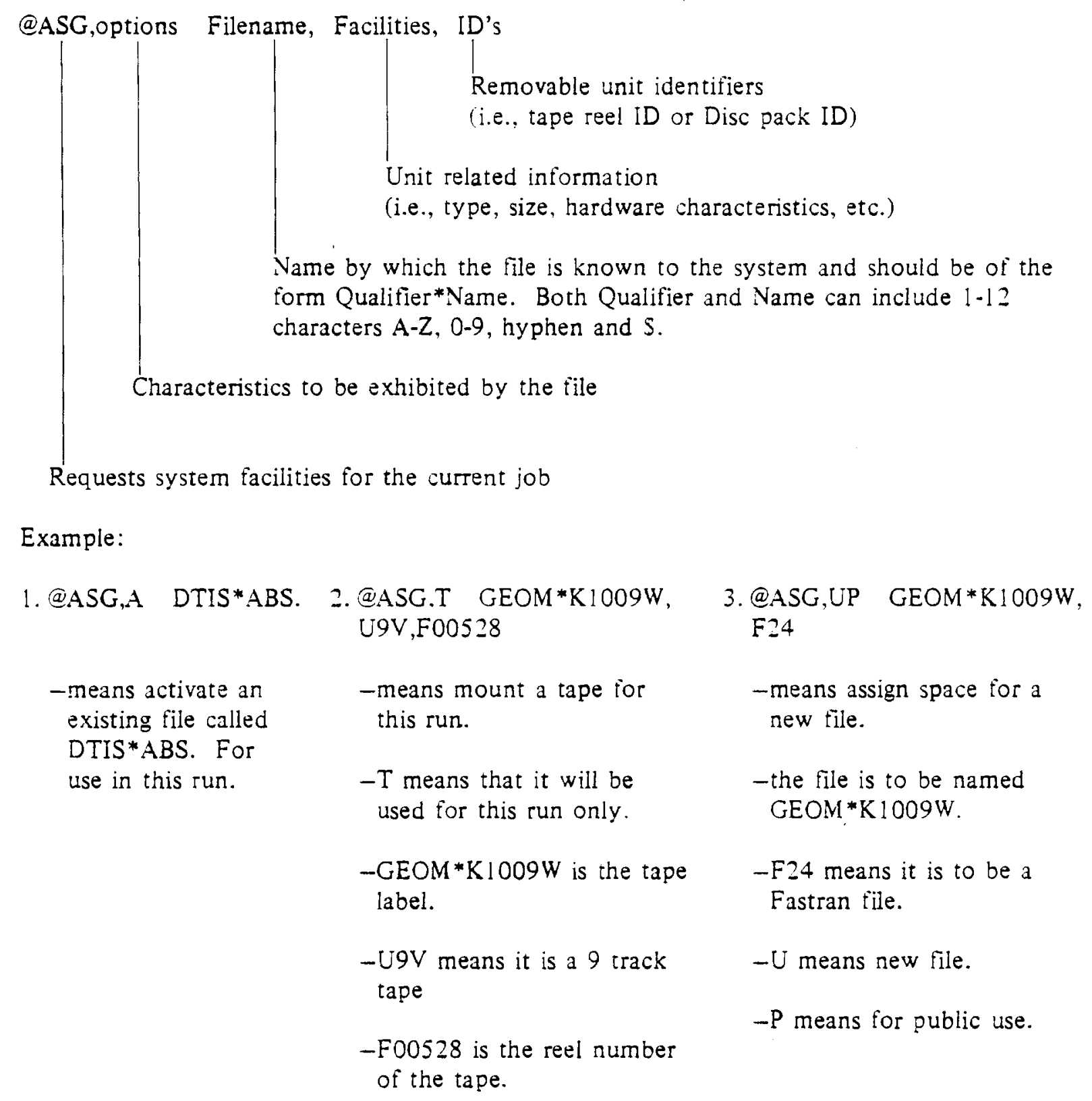




\section{GENERAL aASG STATEMENT OPTIONS}

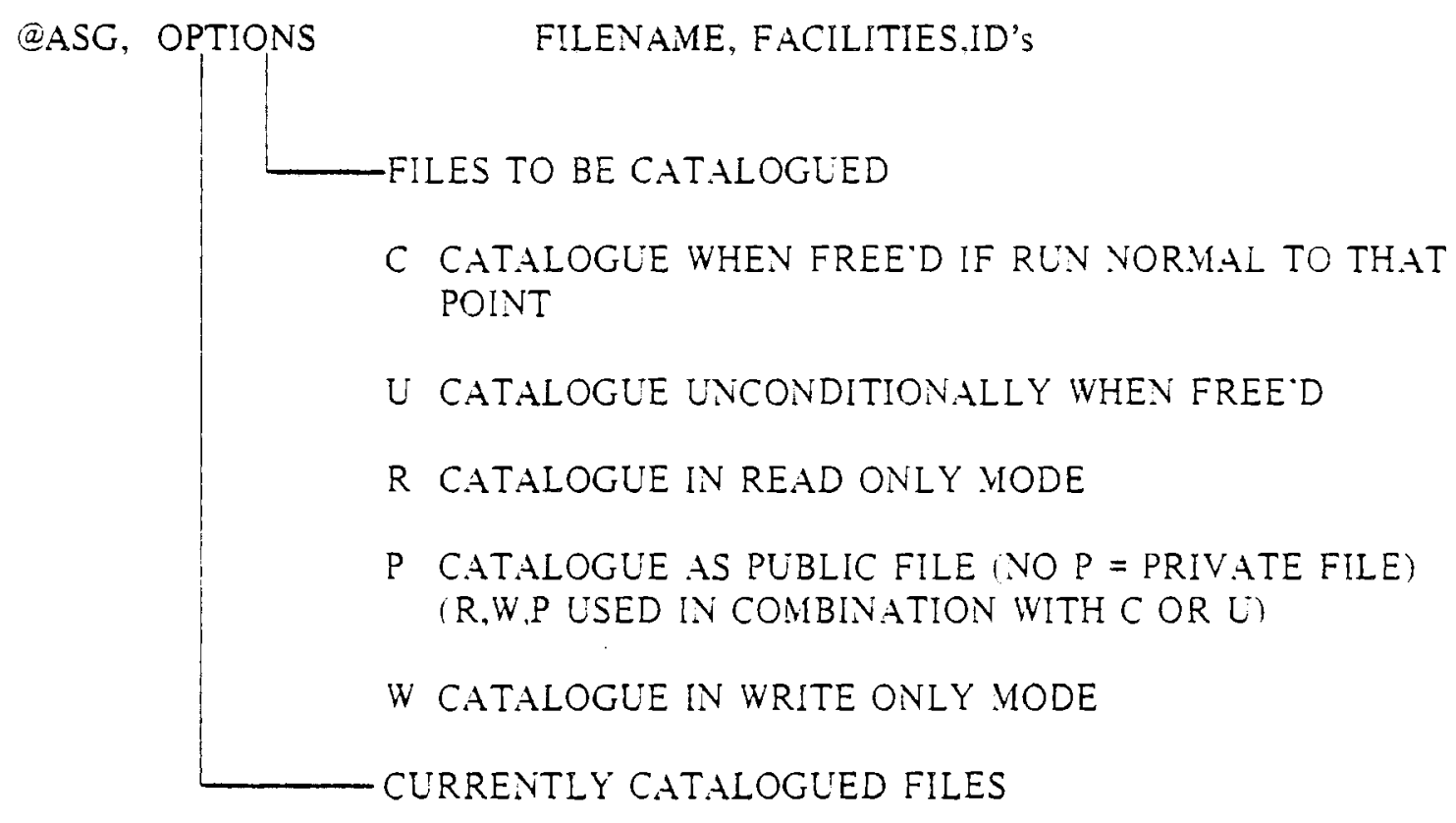

A TERMINATE RLN IF FILE VOT FOUND IN DIRECTORY OF CATALOGUED FILES. SHOULD ALWAYS BE USED WHEN ASSIGNING A CATALOGUED FILE FOR USE IN CURRENT RUN

X ASSIGN CATALOGUED FILE FOR EXCLUSIVE USE BY CLRRENT RUN. (TEMPORARY FILE IMPLIES EXCLUSIVE USE. IN EXISTENCE CURRENT RUN ONLY.)

D DECATALOGUE WHEN FREE'D IF RUN IS NORMAL TO THAT POINT

K DECATALOGUE UNCONDITIONALLY WHEN FREE'D

D OR K ONLY DECATALOGUED AFTER ALL RLNS CLRRENTLY HAVING THE FILE ASSIGNED HAVE FREE'D THE FILE

NOTE: FREE'D IS THE TIME THAT A @FREE FILENAME OR @FIN IS ENCOUNTERED. 
@USE STATEMENT

@USE

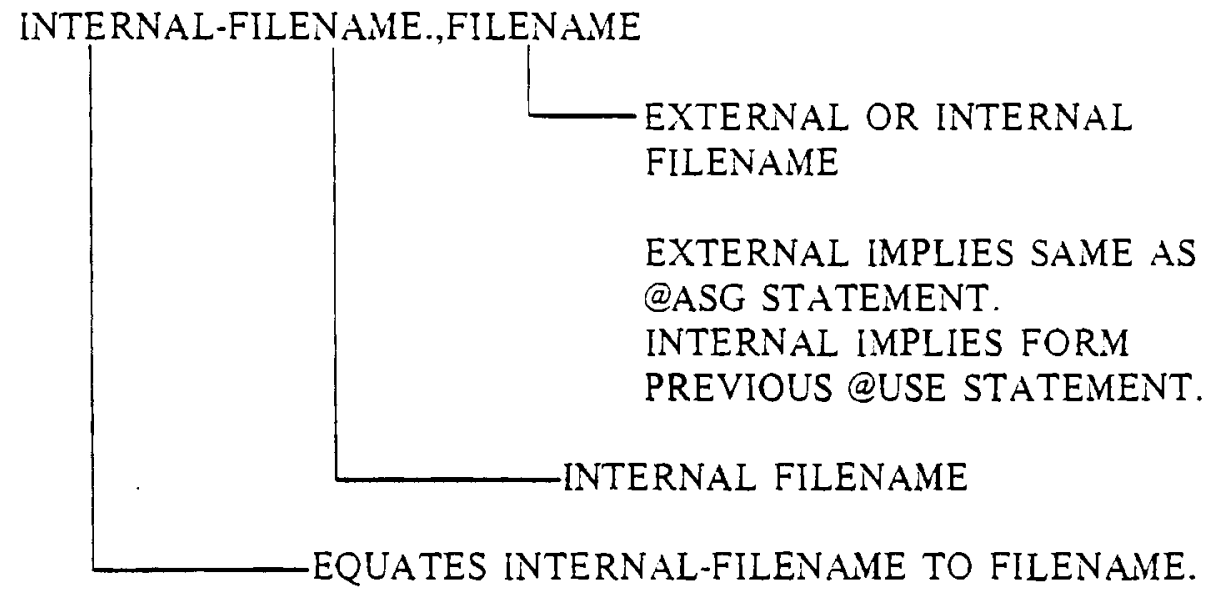

EFFECTIVE IN THIS RUN STREAM ONLY.

EXAMPLES: 1.@USE 7.,9 - Use logical unit 7 for unit 9

2. @USE 7.,GEOM*K1009W - Use logical unit 7 for the file which is labeled GEOM*K1009W 


\title{
@ADD STATEMENT
}

\author{
$@ A D D$ FILENAME. \\ - means insert an existing file called GEOM*K1009W \\ $@ A D D G E O M * K 1009 W$. \\ into the run stream \\ OR
}

@ADD FILENAME.ELTNA.ME - means insert an existing element called A.MY 2 of file @ADD DTIS*DTIS.AMY2 DTIS*DTIS into run stream

@XQT STATEMENT

@XQT PROGRAM ELEMENT NAME

Example: @XQT DTIS*ABS.FSGPCP

- means load and execute a program called FSGPCP which is stored in a program package called DTIS*ABS 


\section{@FIN STATEMENT}

$\stackrel{@ F I N}{\text { COMMAND }}$

COMMENT

MAY NOT BE CONTINUED ON ANOTHER CARD.

1. ALL OPEN FILES ARE CLOSED (TAPES CLOSED AND REWOUND WITH INTERLOCK).

2. ALL USER TEMPORARY FILES ARE RELEASED.

3. ANY FILES REMAINING TO BE CATALOGUED AT RELEASE ARE ENTERED INTO THE MASTER FILE DIRECTORY.

4. ACCOUNTING ROUTINES ARE ENTERED AND SYSTEM LOG UPDATED.

5. PRINTS AND PUNCHS FILES IF ANY ARE QUEUED TO PROPER SYMBIONT. 
APPENDIX B

\section{LIST OF REFERENCES}

1. $\quad \mathrm{GPCP}-\mathrm{A}$ General Purpose Contouring Program

California Computer Products, Inc.

$2411 \mathrm{~W}$. LaPalma Avenue

Anaheim, California 92801 August 1973

2. THREE-D - A Perspective Drawing Software System

California Computer Products, Inc.

2411 W. LaPalma Avenue

Anaheim, California 92801 July 1969 
APPENDIX C

Wind Flow Patterns in Northeastern Washington 
The original intention of this study was to choose a single background speed and direction for the study area for each day and time simulated and then compare the predicted winds at specific grid points with the meteorological data collected. For most situations, however, it is impossible to realistically assume a single prevailing flow over the entire grid area. The area to be modeled was enlarged from the original "core" area in an attempt to include important topographic features that would have a strong influence on the resultant wind field in the proposed refinery development area. The problem still remains, however, that in a real situation the flow over the grid area will have been channelized and perturbed by surrounding terrain features before it reaches the study area.

The northern Puget Sound region seen in Figure Al shows three interconnected low level channels. These are Puget Sound to the southeast, the east-west oriented Strait of Juan de Fuca in the southwestern portion of the study area and the Strait of Georgia, filling the north-central portion of the area and oriented somewhat northwest-southeast. The interior of Vancouver Island, the Olympic Peninsula and the foothills of the Cascades on the eastern edge of the area surround the sea level passages with much higher terrain. A paper dealing with air pollution and meteorology in the southern portions of Puget Sound (Welch, 1975) shows the channelization effect of these low level passages on winds approachin the northern Puget Sound region from various directions. A low level pass through the coastal mountains occurs to the south of the study area near Hoquiam. Winds approaching the coast from a southwesterly direction may be channeled through this low point and enter the study area as a south to southeasterly flow from Puget Sound. At the same time, 


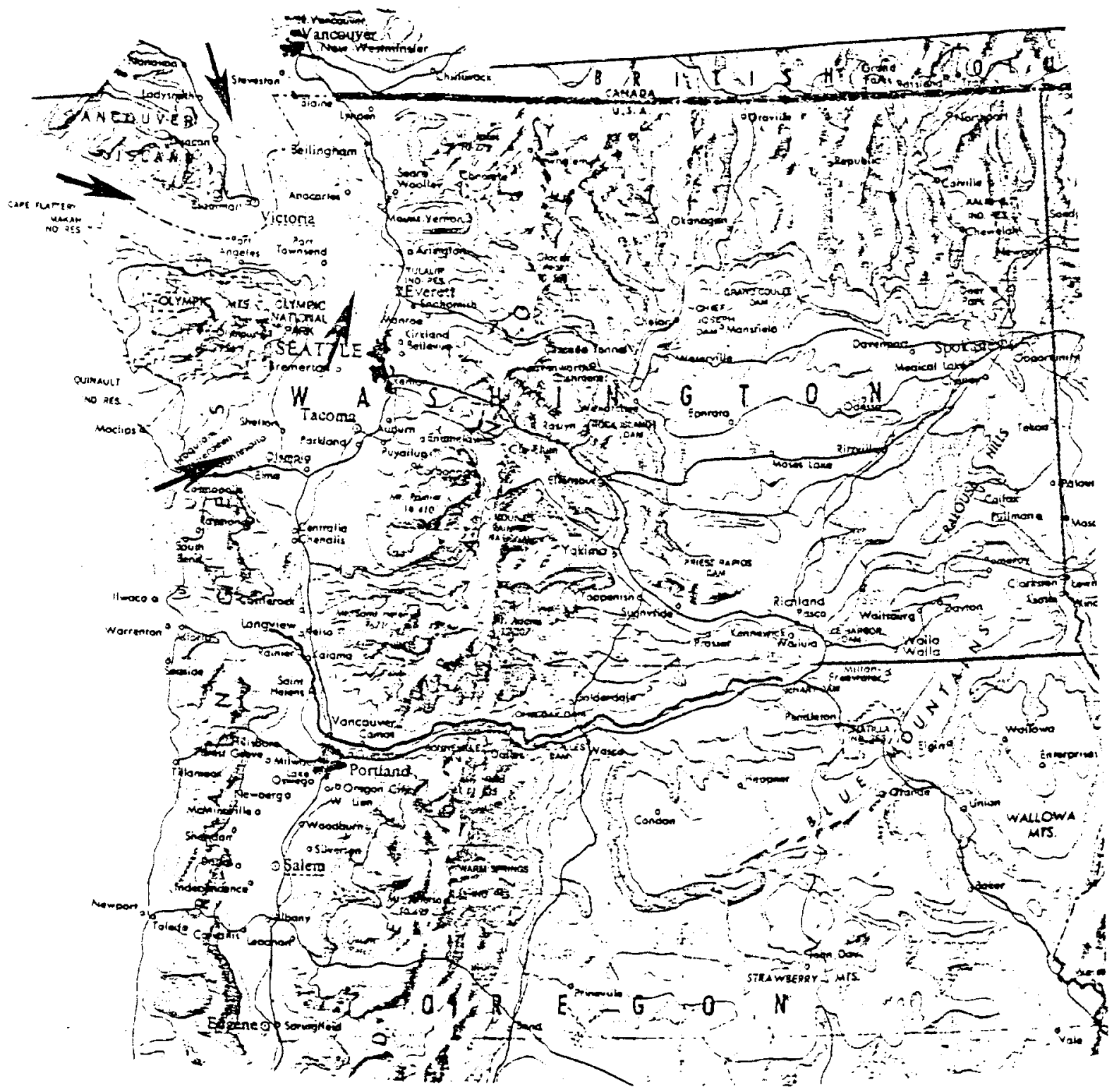

Figure C1. Northern Puget Sound Region Snowing Three Low Level Incerconnecting Channels: Hoquiam-Puge: Sound, Strait of Juan je Euca, Strait of Georgia. 
wind in the Strait of Juan de Fuca is likely to be from the west and flow in the Strait of Georgia southeasterly. A more westerly component may channel the winds from the northwest in Puget Sound while flow is still westerly in Juan de Fuca and southeasterly in the Strait of Georgia. A shift to the northwest may bring about a $180^{\circ}$ reversal in the Strait of Georgia with flow still from the west in Juan de Fuca and northwesterly in Puget Sound. Likewise, winds from other quadrants may result in strikingly different patterns in the three "valley" regions represented by the sea level passages. The patterns described are illustrated as Figures A2 through A7.

Danard (1977) has simulated winds in the same general area but on a somewhat larger scale. His simulations showed several critical angles for the background geostrophic flow around which small changes in the prevailing direction would result in abrupt, $180^{\circ}$ reversals in wind direction in the Straits of Juan de Fuca and Georgia. For geostrophic directions from about $60^{\circ}$ to $220^{\circ}$, the simulated winds are from the southeast in the Strait of Georgia and east to southeasterly in Juan de Fuca Strait. However, when geostrophic directions are in the critical range of approximately $200^{\circ}$ to $250^{\circ}$, winds become west to northwesterly in Juan de Fuca, yet remain southeasterly in the Strait of Georgia. From $240^{\circ}$ to $30^{\circ}$, winds in the Strait of Georgia become northwesterly and continue from the west in Juan de Fuca Strait. Geostrophic directions between $30^{\circ}$ and $60^{\circ}$ resulted in generally northwesterly flow in Georgia Strait and easterly winds in the Strait of Juan de Fuca.

The previous examples serve to illustrate the complex nature of wind flow in the region. Wind speed and direction at the study area boundary will depend on the influence of channelization through the 

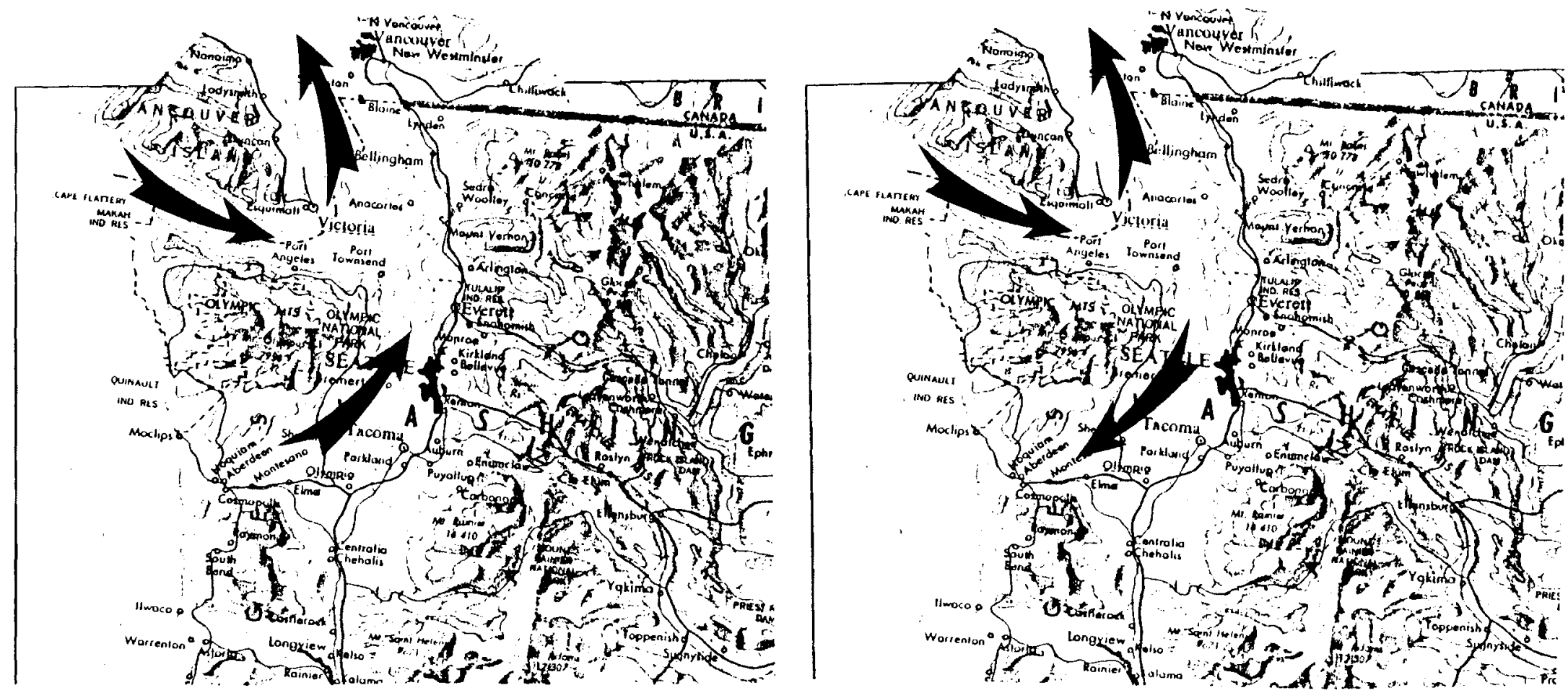

Figure C2 . F1ow Pattern for Background Direction:

Figure C3. Flow Pattern for Background Direction:
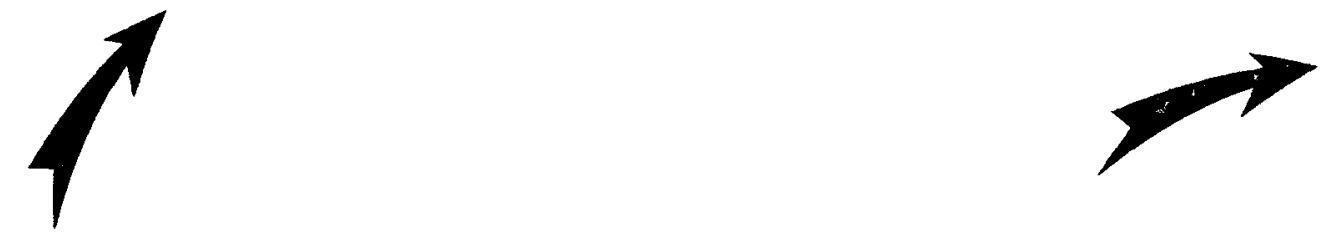

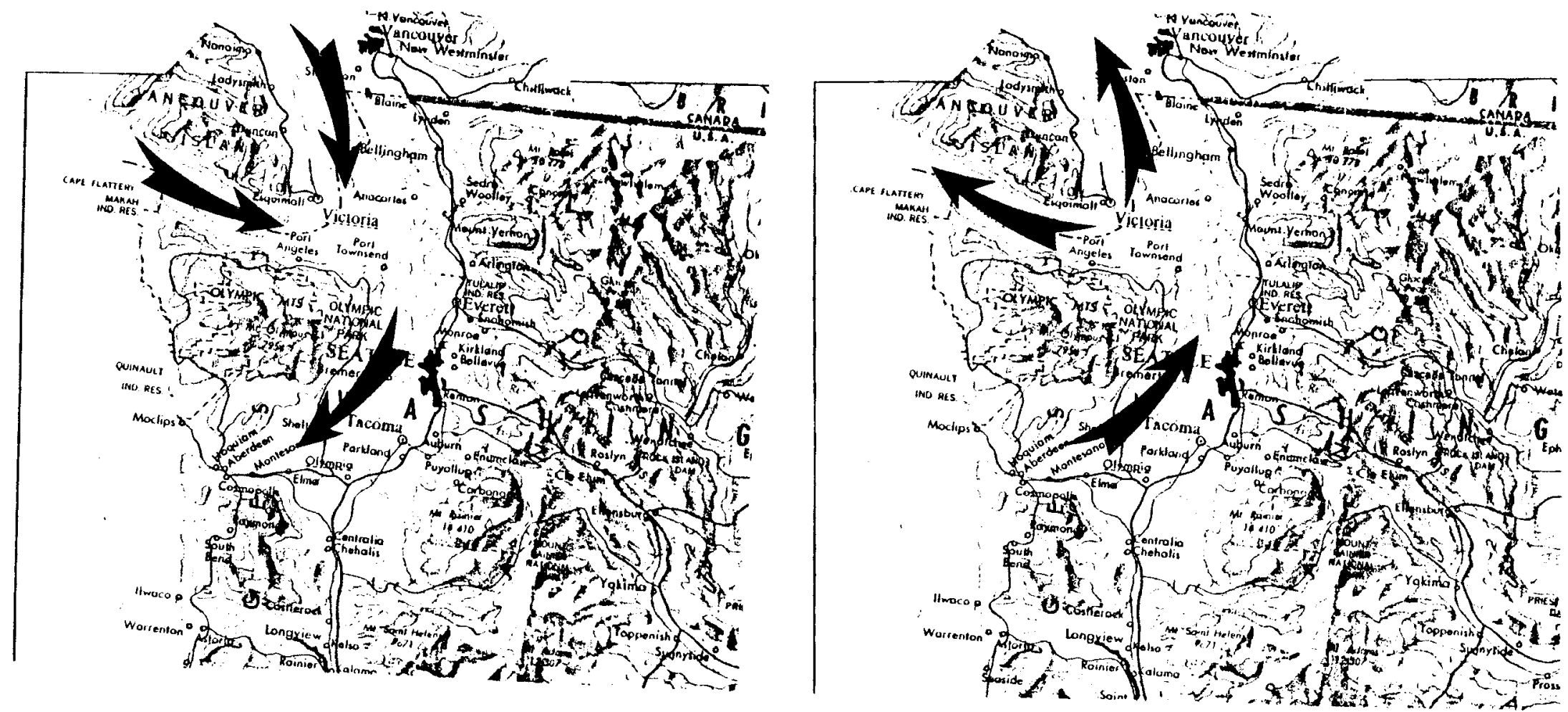

Figure C4. Flow Pattern for Background Direction;

Figure C5 . Iow Pattern for Background Direction:
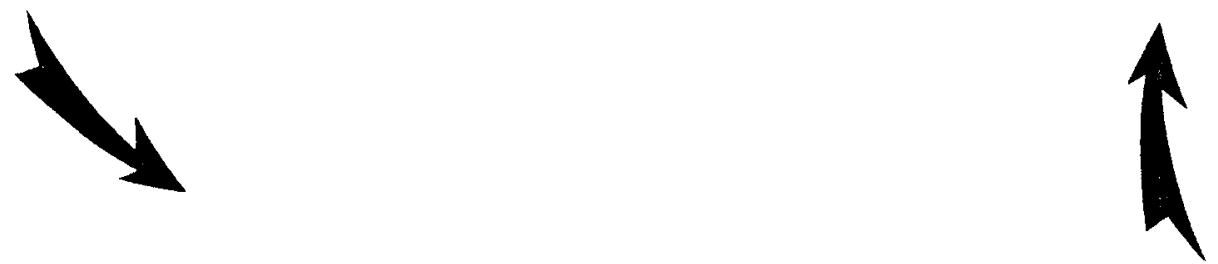


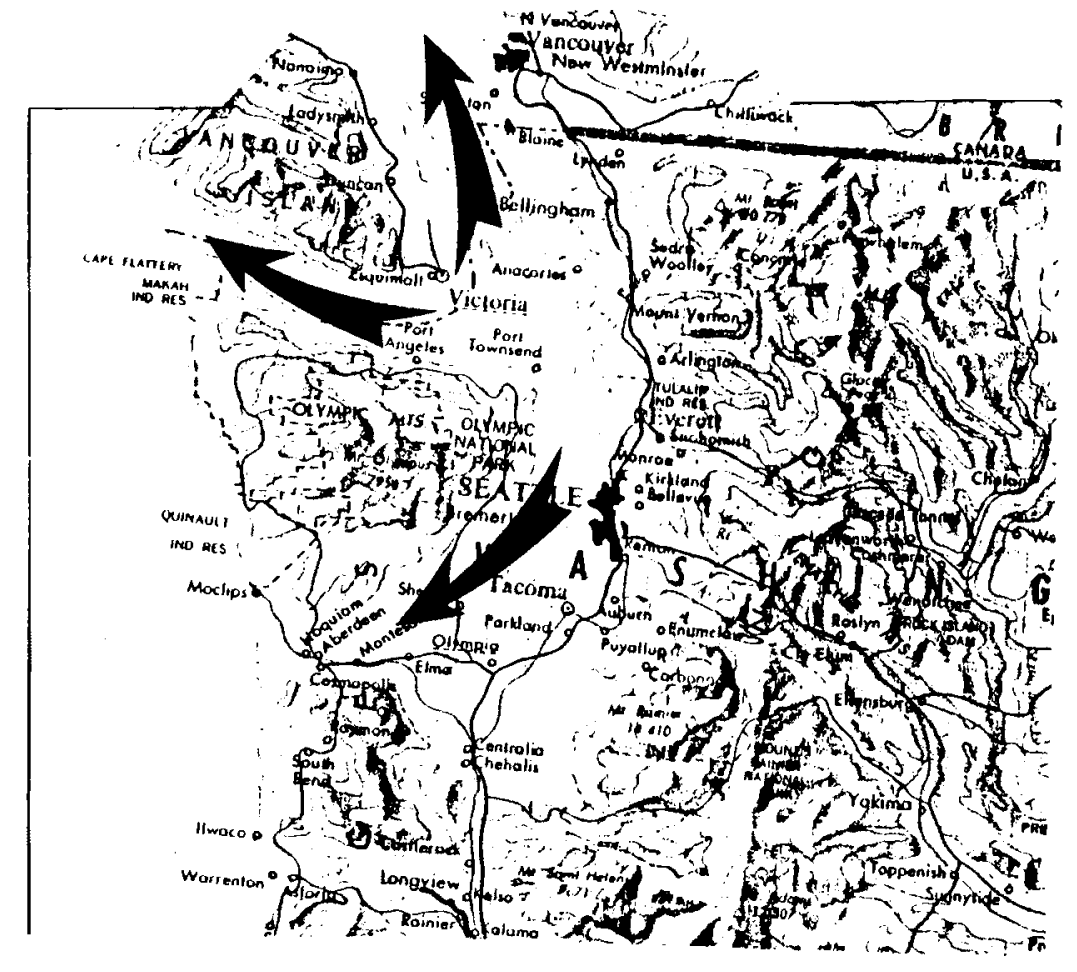

Figure C6. F1ow Pattern for Background Direction:

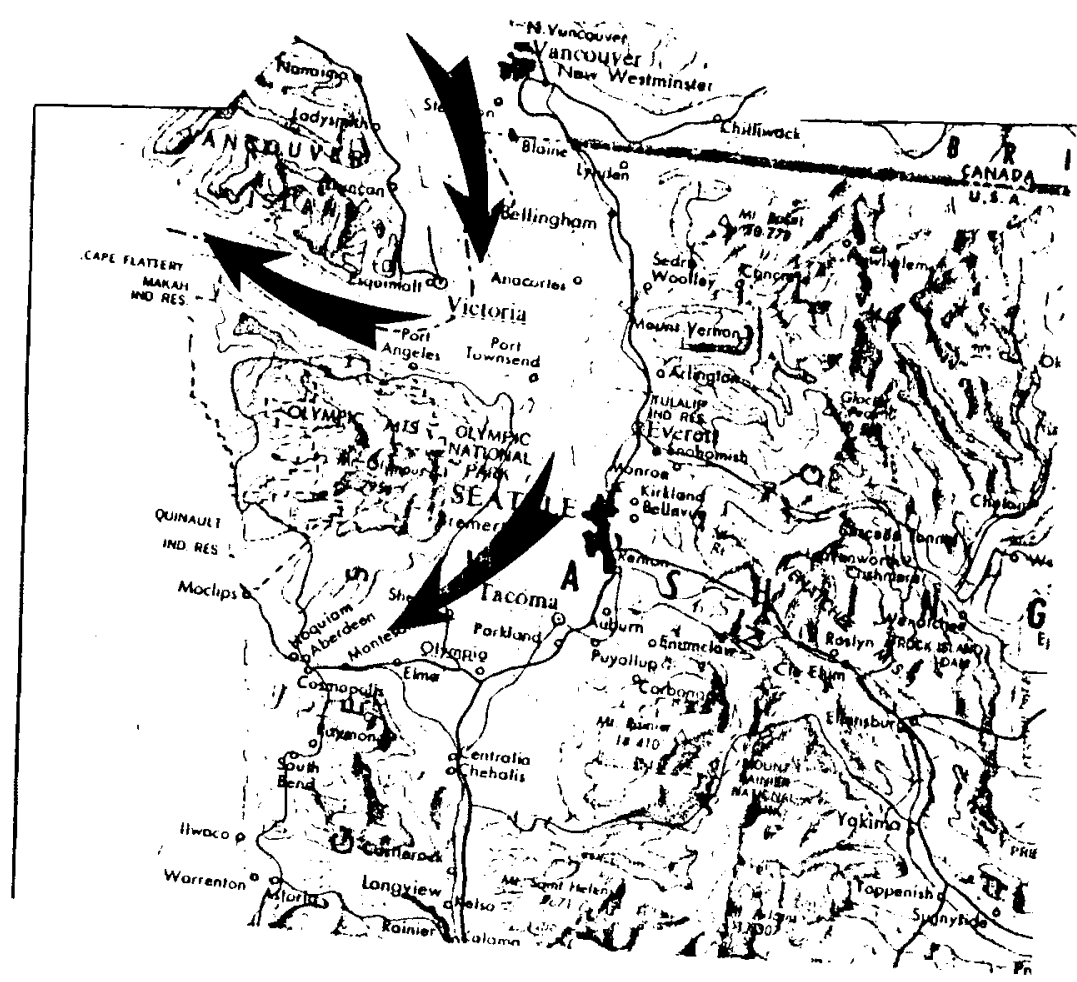

Figure C7 . Flow Pattern for Background Direction:

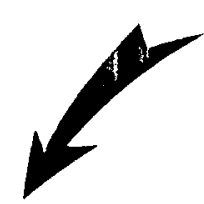


three low level passages on the Pacific coast, with the result that several combinations of flow direction in the three sea level channels can occur. Thus, southeasterly flow from Puget Sound may be accompanied by easterly winds in Juan de Fuca Strait and northwesterly flow in the Strait of Georgia or by easterly winds in the Strait of Juan de Fuca and southeasterly flow in the Strait of Georgia, or a southeast flow in Puget Sound may be coupled with westerly flow in Juan de Fuca and southeasterly flow in Georgia Strait, etc. This can result in quite different prevailing background flow characteristics for various sections of the grid area. 

APPENDIX D

A Topographic Air Pollution Analysis System (TAPAS) 
$D-1$

A TOPOGRAPHIC AIR POLLUTION ANALYSIS SYSTEM

\section{by}

Michael A. Fosberg and Douglas G. Fox Rocky Mountain Forest and Range Experiment Station

U.S. Forest Service, Fort Collins, Colorado 


\begin{abstract}
The Topographic Air Pollution Analysis System (TAPAS) was developed from meteorological and air pollution dispersion models in order to incorporate air quality into land use plans. TAPAS allows planners to introduce the air quality constraints early in the planning activity. A wind field model was combined with dispersion models to determine a mixing volume. This mixing volume, along with the Pollutant Standard Index, provides a means of determining total emission at prespecifled air quality levels. The result and index, the $\pi$ matrix, contains no source information, so planners would use a spatial matrix of the index as an input constraint in land use plans. Allowable emissions are calculated from the $\pi$ index matrix and the ambient air quality standards. These allowable emissions for each pollutant form an atmospheric constraint matrix for air pollution which is compatible with economic, social and other models in land use planning.
\end{abstract}





\section{INTRODUCTION}

There has been a longstanding need to incorporate air quality constraints at an early stage in land use planning. Traditionally, there has been no direct feedback of air quality information in planning until after plans were somewhat formalized. Then, air quality would be evaluated as a residual calculation. Non-degradation requirements set by the Environmental Protection Agency (Code of Federal Regulations 1975) require that all areas of the United States be classified into areas in which no degradation of air quality is permitted (Class I), areas in which some degradation is permitted (Class II), and areas which allow air quality up to the National Ambient Air Quality Standards (Class III). Because of such regulations, an air quality index which defines the carrying capacity defined by an appropriate mixing volume within a geographic region is required. Such an index could be combined with emissions from planned activities in order to project air quality. Generally a palnner can use this index to determine the intenstty and kind of development, compatible with a predefined level of air quality. An index we define as the pollution index is described in this paper. It is shown to accommodate the land use requirements we have described.

\section{THE PLANNING INDEX}

The Topographic Alr Pollution Analysis System outlined in Figure 1 is based on a set of meteorological simulation models 
to calculate the surface temperature and the two-dimensional wind vector over a rectangular grid. Other meteorological factors affecting dispersion, namely stability and the large scale meteorological environment, are required inputs developed from climatology by considering worst-case conditions on a seasonal basis. Dispersion is calculated using a Gaussian plume model with a slight alteration to make it more appropriate for complex topography. The dispersion calculation is output in an index form which represents available mixing volume. This in turn is coupled with the appropriate form of the applicable ambient air quality standard to provide the Pollution Index (PI or $\pi$ ) matrix. The wind and temperature models have been described previously (Fosberg, Marlatt and Krupnak 1976, and Fox et al. 1975) and so will not be detailed here. However, it is significant to note that the calculation of winds is a multistep process. First, a potential flow field is generated from a large-scale driving wind applied over the model domain. The potential flow is then altered by impulse acceleration of the lagrangian forces generated by surface roughness and surface temperature variations. This alteration is done by calculating the vorticity and divergence of the flow field. The divergence calculated is used in the dispersion component. The divergence forms a correction to the classical Gausstan plume model (Fosberg et al. 1976) incorporated in our analysis. 
The essence of TAPAS is a mixing volume index. This index represents the volume that would be incorporated by a plume located within each cell. Basically, this is the air available for mixing the effluent. The theoretical development of this index is based on the Gaussian plume mode1.

The ambient concentration, $x$, can be estimated from dispersion models. For simplicity, consider the mixing volume index as defined by the Gaussian dispersion model for complex terrain (Fosberg, Fox, Howard, Cohen 1976). The concentration is given by

$$
X=\frac{S}{u} G
$$

where

$$
G=\frac{1}{\sqrt{ } 2 \pi \sigma_{y} \sigma_{z}} \exp \left[-\frac{1}{2}\left(\frac{y^{2}}{\sigma_{y}{ }^{2}}+\frac{z^{2}}{\sigma_{z}{ }^{2}}\right)-\delta \Delta t\right]
$$

is the Gaussian distribution,

$X$ Is the ambient concentration

Q Is the emission rate of the pollutant

$u$ is the wind speed

$\sigma_{y}$ and $\sigma_{z}$ are coefficients of plume spread (Turner 1969)

$\delta$ Is the mass divergence in the airshed and

$\Delta t$ is the time increment over which the divergence act.

The expression for the mixing volume index, I, is simply

$$
I=\frac{X}{Q} \times 10^{6}=\frac{G(x)}{u} 10^{6}
$$

where

$10^{6}=$ scaling factor 
The scaling factor, $10^{6}$, was chosen by considering Turner's (1969) maximum value of $X \frac{\mathrm{U}}{\mathrm{Q}}$ from a ground level source with Class F stablity. The maxlmum index value is 1000 although it will generally be well below 100 .

The mixing volume index is coupled with the Pollutant Standards Index (PSI or $\Psi$ ) (Thom and Ott 1976) given by

$$
\Psi=a+b \quad x / x_{s}
$$

where $\chi_{s}$ is the ambient air quality standard for a particular pollutant, and $a$ and $b$ are constants.

The two indices can be combined to yield the Pollution Index by eliminating the ambient concentration and solving for $Q$

$$
Q=\frac{(\Psi-a) x_{s} 10^{6}}{b I}
$$

Emission rates from a mix of sources of a given pollutant are used In the land use model so that $Q=\sum n_{i} Q_{i}$

where

$\mathrm{n}_{1}$ is the number of sources in a particular activity, and $Q_{1}$ is the emission rate for that activity

Total permissible emission within an airshed are

$$
\pi=\sum n_{1} Q_{1}=\frac{(\Psi-a)}{b I} x_{s} 10^{6}
$$

where $\pi$ is the allowable Pollution Index, in units of mass per unit time, and represents the total emission which will result in alr quality of a given $\Psi$ value within each cell. This short-term 
$\pi$ value represents the instantaneous emission rate. Since emissions as well as meteorological conditions do not remain fixed in time, we apply this value only to short-term standards no longer than 3 hours.

For emissions that are highly non-uniform or intermittant and for standards longer than 3 hours, a different $\pi$ is calculated. The long-term index, $\pi_{L}$, is based upon using Gaussian dispersion to define a mixing cross-sectional area, and then using concepts similar to the box model (Fox 1975) to complete the volume. The Gaussian form of the mixing volume index is transformed to a ventilated box model. The Gaussian term, $G$, has the dimensions $\mathrm{m}^{-2}$ and when considering centerline values, $\mathrm{y}=0, z=0$, it describes a half ellipse shaped cross-sectional area. The area swept by the plume, which accounts for $99.87 \%$ of the mass, is simply defined by including 3 times the standard deviation $(\sigma)$ in each direction. This area is $\left(\begin{array}{lll}\pi & 3 \sigma_{y} & 3 \sigma_{z}\end{array}\right)$. Defining an index based upon the flux through the area gives

$$
I \equiv \frac{G}{9 \pi u} 10^{6}
$$

The $\pi$ index for the longer standards or for intermittant sources is then obtained in the same fashion as above and is

$$
\pi_{L}=\frac{(\Psi-a) X_{s} 10^{6}}{b I} \frac{9 X_{L}}{2 U}
$$


where $X_{L}$ is the length of the grid cell. Notice that the dimension of $\pi_{L}$ is mass. An emission rate must be multiplied by the length of time it is operating in order to calculate the allowable pollution loading for a glven cell.

The $\pi$ and $\pi_{L}$ matrices can be calculated without reference to any source information.

\section{APPLICATIONS OF TAPAS TO LAND USE PLANNING}

Output from TAPAS is made directly useful to a planner by providing a technical limitation on development maximums that are commensurate with predefined levels of air quality. Since the information is site specific with good spacial resolution, TAPAS allows identification and evaluation of alternate sites to mitigate air pollution impact. Finally, since TAPAS represents the state of the art in meteorological and air quality model development, it has scientific defensibility far beyond that associated with economically competitive available methods.

The $\pi$ matrix output By TAPAS is conservative. How conservative depends upon how extreme the climatological inputs are. In general, we chose meteorological conditions that are likely to occur as average worst conditions in a season. Air quality violations are often associated with rare event extreme stabllities for which we do not suggest planning. These episodal situations must be handled by restrictions on sources for the episode period. For the vast majority of time, however, our $\pi$ numbers represent conservatively 
the amount of pollution that can be accommodated within the cell. This, we feel represents the proper position for planning. Should anyone propose sources in excess of those allowable under TAPAS outputs, it is incumbent upon the proposer to provide data and analysis to support that position.

From the wildland development planning perspective that we address two major source types emerge, namely transportation and general community development. The pollutants include particulates, carbon monoxide $(\mathrm{CO})$, nitrogen oxides, hydrocarbons and oxidents. Major sources appear to be vehicular traffic and space heating, particularly with wood-burning fireplaces.

The first step in development of a Land Use-Air Quality Analysis is to 1dentify $\Psi$ values, the allowable pollution levels for the area in question. For large areas certain portions will no doubt be associated with different values of $\Psi$. A critical Issue is how to allocate $\Psi$ values. For example, controversy is currently raging in the United States over the issue of non-deterioration. Since ambient air quality standards are set on the basis of protection of human health and welfare, there are many areas, particularly in national trust lands, where air quality is substantially above these standards. Use of the matrix provides an ability to illustrate to the concerned public the restrictions necessary to insure nondegradation of pristine air.

The PI matrix represents maximum allowable emission under eplsode conditions. They therefore, require an assessment of 
current emisstons to be made before planned changes can be evaluated. Emission factors (EPA 1976) multiplied by the number of sources for each activity and summed for a given pollutant cannot exceed the PI matrix value. As an example, consider the PI matrix for $\mathrm{CO}$ based on the 1-hour standard. Assuming a typical mix of vehicles ( $90 \%$ automobiles and light duty vehicles, $10 \%$ heavy duty trucks) operating at slow speeds (30 mph) at $7000 \mathrm{ft}$. above sea level, we find an emission figure of $60 \mathrm{gm}$ per vehicle mlle for 1975, which is doubled for traffic above $5000 \mathrm{ft}$. to give $120 \mathrm{gm}$ per vehicle mile. The number of vehicles permitted through a $3 \mathrm{~km}$ by $3 \mathrm{~km}$ cell with 3 miles of road in a one-hour period without exceeding air quality standards is then

No. of Vehicles $\mathrm{x}$ Emission Factor $=$ PI matrix

or

$$
\text { No. of Vehicles }=\frac{\text { PI matrix }}{\text { Emission Factor }}
$$$$
\frac{\text { No. of Vehicles }}{\text { hour }}=\frac{\left(\pi_{\mathrm{n}}\right) \mu \mathrm{gm} / \mathrm{sec} 3600 \mathrm{sec} / \mathrm{hr}}{120 \mathrm{gm} / \mathrm{veh} \cdot \mathrm{mi} \cdot 10^{6} \mu \mathrm{gm} / \mathrm{gm} 3 \mathrm{miles} \text { of road }}
$$

where $\pi_{n}=$ PI matrix value for each cell.

$$
\frac{\text { No. of Vehicles }}{\text { hour }}=\pi_{n} \times 10^{-5}
$$

Simflarly, the number of vehicles per eight hours would be calculated as 


$$
D-13
$$

$\frac{\text { No. of Vehicles }}{8 \text { hours }}=\frac{\pi_{n} \mu \mathrm{gm} \text { veh. miles gm }}{120 \mathrm{gm} 10^{6} \mu \mathrm{gm} 3 \text { miles of road }}$

$$
\frac{\text { No. of Vehicles }}{8 \text { hours }}=278 \times 10^{-9} \pi_{\mathrm{n}}
$$

To illustrate this, consider a cell with a daytime 1-hour $\pi_{\mathrm{n}}$ value of $46 \times 10^{8}$, a nighttime 1 -hour $\pi_{\mathrm{n}}$ value of $5 \times 10^{8}$, and an 8 -hour $\pi_{n}$ value of $586 \times 10^{13}$. Day and night $\pi$ matrix values are based on diurnal variations in wind speed and stability. The 8 -hour $\pi$ matrix value is based on average of day and night mixing volume. This allows 26,000 vehicles per hour peak traffic load during the daytime, 5000 vehicles per hour at night, and 163,000 vehicles in a given 8 -hour period. Peak traffic volumes during short time periods must be compensated for by light traffic such that the total number of vehicles does not exceed the 8-hour volume.

Calculations involving a mix of sources (i.e. emission factors) are somewhat more complicated. As an example, consider a cell containing 2 miles of secondary road ( $30^{\circ}$ mph traffic) and one mile of primary road (55 mph traffic). This calculation would be set up as follows for the 8-hour standard

$$
\begin{aligned}
& \frac{\text { No. Vehicles (55) } 70 \mathrm{gm} 10^{6} \mathrm{ugm} 1 \text { mile of road }}{\text { vehicle mile gm }}+ \\
& \frac{\text { No. Vehicles (30) } 120 \mathrm{gm} 10^{6} \mathrm{\mu gm} 2 \mathrm{miles} \text { of road }}{\text { vehicle mile } \mathrm{gm}}= \\
& \pi_{\mathrm{n}} \text { gm } \\
& \text { or } \\
& \operatorname{NV}(55) 7 \times 10^{7}+\operatorname{NV}(30) 2.4 \times 10^{8}=\pi_{\mathrm{n}}
\end{aligned}
$$


At this point, some consideration must be given to the intended use of land within the cell. As a hypothetical example of the type of additional information required, assume that the secondary road Is a loop road leading to and from a scenic viewpoint which would attract 75 percent of the vehicles (assumed situations and traffic ratios are purely hypothetical in order to illustrate the calculation). This traffic mix gives 3 vehicles on the secondary road to each vehicle on the primary road. Thus, for each 4 vehicles, $3 / 4$ of the emissions will come from this secondary road and $1 / 4$ from the primary road. These weighting factors allow the total number of vehicles to be calculated as

$$
\begin{aligned}
& \text { NV (al1 speeds) } 3 / 4 \times \text { emission factor }(30)+ \\
& \text { NV (all speeds) } 1 / 4 \times \text { emission factor }(55)=\pi_{n} \\
& \text { No. of Vehicles (a11 speeds }= \\
& \frac{\pi_{n} 10^{11}}{1 / 47 \times 10^{7}+3 / 42.4 \times 10^{8}} \\
& =\frac{\pi_{n} 10^{4}}{7 / 4+72 / 4}=\frac{\pi_{n} 4 \times 10^{4}}{79} \\
& =506 \times 10^{-9} \pi_{n}
\end{aligned}
$$

For the cell used in the first example, this would give 296,516 vehicles each eight hours. 
SUMMARY

A complex system such as TAPAS must undergo extensive validation in order to assure its usefulness. For a mountain air quality analysis system, such a validation is difficult and expensive.

To date, components of TAPAS have been validated. The Winds Model component, for example, has been compared with data from wind studies performed in mountainous terrain. Certainly the Gaussian Diffusion Model needs no validation on our part. The combination of these two models, and the unique manner of their use, however, should be studied.

Toward this end we have undertaken a study in a Colorado mountain valley (Fox et al. 1976) measuring meteorological and air quality parameters. We have used preliminary data to determine, given existing traffic patterns, how many fireplaces could be accomodated within the valley before the applicable air quality standards would be violated. Results indicate that the valley is operating near these standards currently. Measurements support this conclusion.

One of the missing pieces of information in an analysis of mountain air quality is the existing conditions. TAPAS can make an estimate of what local sources might contribute to the ambient air pollution, but the problem of determining background pollution levels, namely pollution received from distant sources, remains. 



\section{REFERENCES}

Fosberg, M.A., W.E. Marlatt and L. Krupnak: Estimating Airflow Patterns Over Complex Terrain. USDA For. Ser. Res. Pap. No. 162, 16p. (1976)

Fosberg, M.A., D.G. Fox, E.A. Howard, and J. Cohen: Nonturbulent Dispersion Processes in Complex Terrain. Atmos. Env. 10 (1976)

Turner, D.B.: Workbook of Atmospheric Dispersion Estimates. Env. Prot. Agency, 84p. (1969)

Thom, B.C. and W.R. Ott: A Proposed Uniform A1r Pollution Index. Atmos. Env. 10:261-27L. (1976)

Fox, D.G.: Impact of Concentrated Recreational Development on Air Quality. USDA For. Ser. Eisenhower Consortium Bulletin 1, 286p. (1975)

Fox, D.G., G. Wooldridge, T. McKee and D. Whiteman. An Experimental Study of Mountain Meteorology. Proc. Third Symposium on Atmospheric Turbulence, Diffusion and Air Quality, Raleigh, North Carolina. (1976)

Code of Federal Regulations. 1975. Title 30, Ch 1, part 52:98-99. EPA (1976). Compilation of Air Pollutant Emission Factors. 2nd edition, AP 42, parts $A$ and $B$. 
Topographic Air Pollution Analysis System (TAPAS)

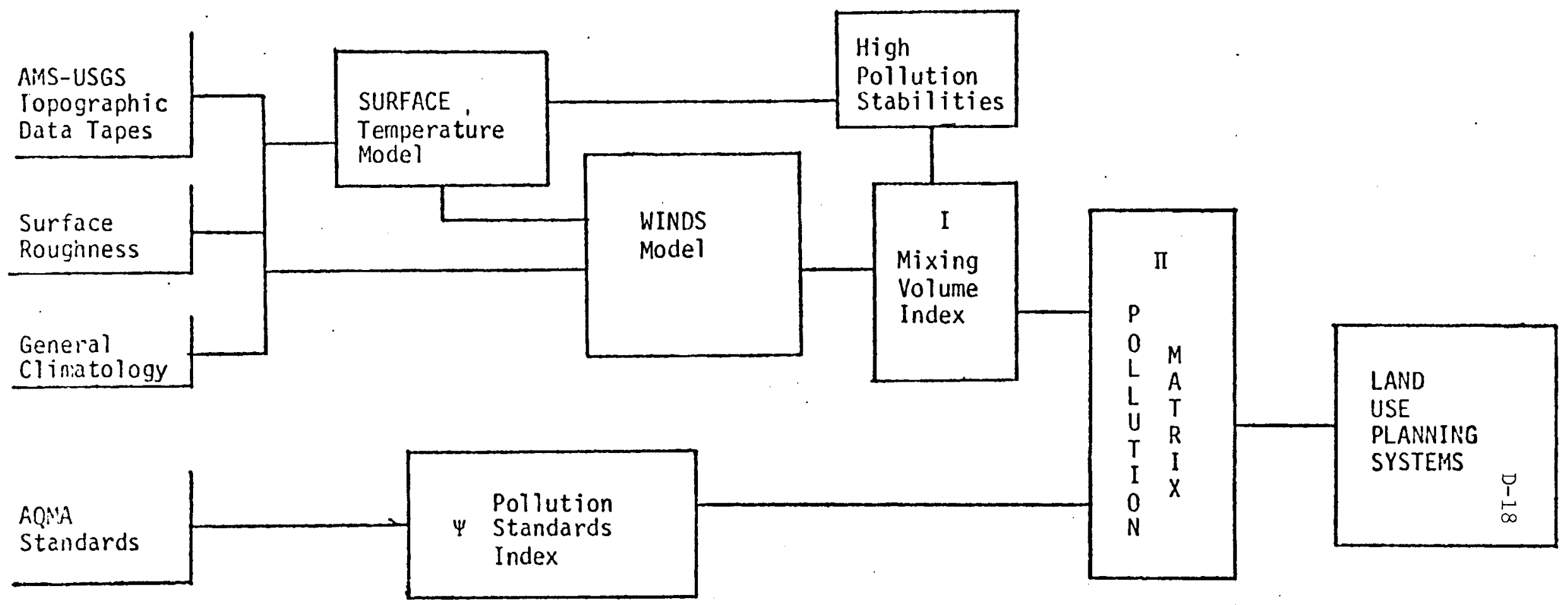

Figure 1 - The conceptual outline of TAPAS, a

combination of complex data files and sophisticated

computer simulation models which provide a useful,

simply applied index of the air pollution carrying

capacity of mountain lands. 
APPENDIX E

Data and Results for Daily and Annual Cases 
This appendix contains data, descriptions and results of the four daily cases and the annual situation simulated in the analysis. Summary tables of the simulation inputs and results, with accompanying discussion, are presented within the body of the report.

Wind flow modeling results are presented in map form. Coasts and islands are outlined and the resultant wind speed and direction at each grid point is shown as a wind flag. The directions predicted define a 16-point compass and wind speeds are presented in meters per second.

In the meteorological data tables, the reported direction, speed in meters per second and the actual time of the observations are given. In some cases the observation times differ from the time simulated by as much as 30 minutes (i.e. Smith Island station). The WINDS results tables are in a similar format but no time is recorded.

PI-Matrix calculations from the TAPAS system are presented in map form. The magnitude of emissions in each grid cell that would result in ambient concentrations corresponding to the pollutant standards described is shown in a series of shaded maps, each representing a separate case. Character codes used for the hourly and 24-hour cases are shown in Table El. Mixing volume index maps are included for the annual case. Character code series for the annual conditions are shown on each of the annual maps.

\section{January 3, 1975}

Air quality data collected for the northern Puget Sound study area showed violations of $\mathrm{SO}_{2}$ standards at Bellingham on January 3, 1975. The three hour average reached a high value of $69 \mathrm{pphm}$ at this location and the 24-hour average a maximum of 21 pphm. 
Table El. Character Codes and Range Data for PI-Matrix Maps

\begin{tabular}{ccc}
\hline & $\begin{array}{c}\text { Emission Rate } \\
\text { for Each Simulation } \\
(\mathrm{g} / \mathrm{s})\end{array}$ & $\begin{array}{c}\text { Total Emission } \\
\text { for } 24\end{array}$ \\
Character & $<30$ & $\begin{array}{c}\text { Hour Period } \\
(\mathrm{kg})\end{array}$ \\
\hline Blank & $30-59$ & $<19.90$ \\
+ & $60-89$ & $19.90-19.99$ \\
- & $90-119$ & $20.00-20.09$ \\
+ & $120-149$ & $20.10-20.19$ \\
$=$ & $150-179$ & $20.20-20.29$ \\
$*$ & $180-209$ & $20.30-20.39$ \\
$\#$ & $210-239$ & $20.40-20.49$ \\
$\$$ & $240-269$ & $20.50-20.59$ \\
$*$ & $\geq 270$ & $\geq 20.60$ \\
$X$ & & $\geq 20.60$ \\
\hline
\end{tabular}

The meteorological information shows a low pressure center at the surface in northern Alberta at the beginning of the simulation period (0300 PST, January 3) and a diffuse high pressure system to the southeast, centered over northern Nevada and Utah. A cold front had passed through the study region sometime in the 24 hours prior to the period of simulation. Twenty-four hours after the first period simulated, the meteorological maps show a low pressure center just east of the Puget Sound area. The high pressure system was over the Four Corners area with an occluded front over Idaho at this time.

At $850 \mathrm{mb}$., a low pressure center developed off the coast to the west of the study area during the first half of the simulation period and then moved through the area during the final eight hours. The 700 mb. maps show westerly flow over the area for the first 12 hours of the simulation, switching to a southerly to southwesterly direction as a 
depression developed to the northwest. The direction of flow switched to northeast by 0300 PST, January 4 as the trough moved through the region. The $500 \mathrm{mb}$. maps show zonal flow at the beginning of the analysis with little warm air advection -- the warm air advection increased through the simulation period.

Temperature and pressure profiles can be seen from the sounding data taken at Quillayute (Table $\Sigma^{2}$ ).

Table E2. Quillayute Sounding Data, January 3 and 4, 1975.

\begin{tabular}{|c|c|c|c|c|}
\hline & $\begin{array}{l}\text { Height (m)/ } \\
\text { Pressure (mb) }\end{array}$ & $\begin{array}{l}\text { Temperature } \\
\left({ }^{\circ} \mathrm{C}\right)\end{array}$ & $\begin{array}{l}\text { Direction } \\
\text { (Degrees) }\end{array}$ & $\begin{array}{l}\text { Speed } \\
\text { (knots) }\end{array}$ \\
\hline & \multicolumn{4}{|c|}{0300 PST January 3, 1975} \\
\hline Surface $(56 \mathrm{~m})$ & $1019.6 \mathrm{mb}$ & 3.9 & 30 & 3 \\
\hline $850 \mathrm{mb}$ & $1511 \mathrm{~m}$ & -5.3 & 255 & 14 \\
\hline $700 \mathrm{mb}$. & $3003 \mathrm{~m}$ & $-14 \cdot 3$ & 280 & 40 \\
\hline \multirow[t]{2}{*}{$500 \mathrm{mb}$. } & $5489 \mathrm{~m}$ & -27.5 & 280 & 54 \\
\hline & \multicolumn{4}{|c|}{1500 PST January 3,1975} \\
\hline Surface $(56 \mathrm{~m})$ & $1003.1 \mathrm{mb}$ & 5.0 & 60 & 7 \\
\hline $850 \mathrm{mb}$ & $1390 \mathrm{~m}$ & -2.9 & 140 & 41 \\
\hline $700 \mathrm{mb}$ & $2909 \mathrm{~m}$ & -10.1 & 180 & 53 \\
\hline \multirow[t]{2}{*}{$500 \mathrm{mb}$. } & $5429 \mathrm{~m}$ & -26.5 & 220 & 53 \\
\hline & \multicolumn{4}{|c|}{0300 PST January 4, 1975} \\
\hline Surface $(56 \mathrm{~m})$ & $1015.1 \mathrm{mb}$ & 3.9 & 135 & - \\
\hline $850 \mathrm{mb}$. & $1365 \mathrm{~m}$ & -5.0 & 315 & 30 \\
\hline $700 \mathrm{mb}$ & $2868 \mathrm{~m}$ & -13.0 & 315 & 35 \\
\hline $500 \mathrm{mb}$ & $5334 \mathrm{~m}$ & -33.0 & 315 & 35 \\
\hline
\end{tabular}


Meteorological data collected for January 3, 1975 is shown as Table E3 and wind flow modeling results for the metecrological station grid points are presented as Table $E^{4}$. Figures $E 1$ through $E 6$ are maps of predicted wind speeds and directions for each hour of the simulation. The PI-Matrix results are shown in Figures $\mathrm{E} 7$ through E13.

January 24,1975

The northern Puget Sound air quality data indicate that particulate concentrations in Mt. Vernon averaged $128 \mu \mathrm{g} / \mathrm{m}^{3}$ for a 24 hour period on January 24 , 1975. While within standard limitations, this is the highest ambient concentration recorded at this receptor site on any sampling day in 1975 or 1976.

The meteorological situation shows a fairly weak low pressure center to the southwest of the study area over the Pacific at the beginning of the simulation period. A high pressure system was located over southwestern Idaho. By 0300 PST on January 25, the depression had moved to the east and was centered along the Washington-Oregon State line, southeast of the northern Puget Sound region. Surface flow over the State of Washington was light and from the southeastern quadrant at the beginning of the simulation period; light and variable to calm conditions prevailed over the state in the early morning hours of January $25,1975$.

Upper level maps for the simulation period all show a strong low pressure center in the Gulf of Alaska. During the simulation period, a low pressure system developed and intensified over the north-central United States and south-central Canadian provinces -- flow at the upper levels appears to have been primarily governed by these two depressions. 
Table E3

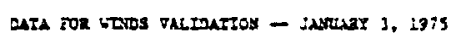

\begin{tabular}{|c|c|c|c|c|c|c|}
\hline \multirow{2}{*}{ Sezcier code } & \multirow{2}{*}{23200} & \multicolumn{4}{|c|}{$x$} & \multirow{2}{*}{2360} \\
\hline & & 3760 & $\$ 100$ & 3300 & $\$ 900$ & \\
\hline \multirow{2}{*}{ Pare } & 220 & 160 & $: 10$ & 30 & 100 & 360 \\
\hline & $0900^{8}$ & 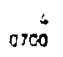 & $1100^{2}$ & $1500^{\circ}$ & $2900^{7}$ & $: 300^{3}$ \\
\hline \multirow{2}{*}{ math } & 220 & 180 & 210 & 90 & $: 0$ & 40 \\
\hline & 0306 & $0700^{3}$ & $2100^{2}$ & $2500^{3}$ & $1700^{3}$ & $: 300^{6}$ \\
\hline \multirow[t]{2}{*}{$32 \mathrm{~s}$} & 220 & 180 & $1+0$ & 30 & io & 360 \\
\hline & 0259 & 0037 & $\cos ^{2}$ & 2035 & $1355^{6}$ & $2225^{4}$ \\
\hline \multirow{2}{*}{ anc } & 200 & 130 & so & 30 & so & 360 \\
\hline & $0500^{6}$ & $0700^{4}$ & $2100^{2}$ & $1500^{2}$ & $1900^{2}$ & $2300^{4}$ \\
\hline \multirow{2}{*}{ ant } & 204 & 158 & 107 & 29 & 26 & 12 \\
\hline & 0300 & $9700^{\circ}$ & 1100 & $1500^{2}$ & 1900 & $2300^{\circ}$ \\
\hline \multirow[t]{2}{*}{. } & 220 & $1+0$ & 130 & 200 & 120 & LOO \\
\hline & $0300^{4}$ & $0700^{\circ}$ & 1100 & $1500^{\circ}$ & 1900 & $2300^{\prime}$ \\
\hline \multirow[t]{2}{*}{ علعi } & 200 & 315 & 310 & 290 & 313 & 300 \\
\hline & $0300^{\circ}$ & $0700^{2}$ & $1100^{3}$ & 1900 & $2900^{\circ}$ & $2330^{2}$ \\
\hline \multirow[t]{2}{*}{ NATP } & 170 & 140 & 130 & 80 & 130 & 100 \\
\hline & $0300^{-}$ & 0700 & $1200^{\circ}$ & $1500^{\circ}$ & $1900^{\circ}$ & $2300^{\circ}$ \\
\hline \multirow[t]{2}{*}{ X } & 210 & 130 & 130 & 30 & 120 & منا \\
\hline & $0300^{\circ}$ & $0700^{3}$ & $2100^{\circ}$ & 1500 & 1900 & $2200^{5}$ \\
\hline \multirow[t]{2}{*}{ 3ant } & 270 & SIS & 68 & 68 & 68 & 68 \\
\hline & $0300^{3}$ & $0700^{2}$ & $1100^{3}$ & $: 500^{5}$ & $1900^{\circ}$ & $2300^{5}$ \\
\hline \multirow{2}{*}{ W } & 280 & 180 & 130 & 100 & 120 & 1.20 \\
\hline & 0255 & 065s & 1093 & $: 453$ & 2855 & 2259 \\
\hline \multirow[t]{2}{*}{ sent } & $: 133$ & 135 & - & I I I & 113 & - \\
\hline & 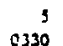 & $\stackrel{4}{\cos 30^{\prime}}$ & $:$ & : & $\begin{array}{r}i \\
1830\end{array}$ & : \\
\hline \multirow{2}{*}{ rics } & 248 & 203 & Las & э0 & 90 & $: 3$ \\
\hline & $0300^{3}$ & $0700^{2}$ & $2100^{2}$ & $1500^{5}$ & $1300^{\circ}$ & 2300 \\
\hline \multirow[t]{2}{*}{$\mathrm{TLO}$} & 235 & 225 & mo & 90 & 90 & 49 \\
\hline & $0300^{3}$ & $0700^{2}$ & 1100 & $1550^{5}$ & $\begin{array}{r}19 \\
1900\end{array}$ & ${ }_{2300}^{3}$ \\
\hline \multirow{2}{*}{ pace } & - & so & - & - & - & - \\
\hline & $=$ & $\begin{array}{r}00 \\
07 c 0\end{array}$ & : & 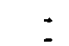 & $:$ & : \\
\hline \multirow{2}{*}{ ?.SPA } & - & 100 & 3 & jo & - & - \\
\hline & : & $3700^{2}$ & $\begin{array}{r}50 \\
1100\end{array}$ & $\begin{array}{r}00 \\
1900\end{array}$ & $=$ & $=$ \\
\hline \multirow{2}{*}{ stris } & : & :59 & $i \neq 7$ & 119 & $: 3.3$ & ist \\
\hline & $2300^{2}$ & $0700^{3}$ & $1500^{\circ}$ & $2560^{2}$ & ${ }_{1}^{3}, 30^{3}$ & $2=00^{3}$ \\
\hline \multirow{2}{*}{ Sourface } & so & 90 & م: & 50 & $\omega$ & $-\infty$ \\
\hline & $.2255^{2}$ & anst & $\begin{array}{r}2 \\
1156\end{array}$ & 1253 & $: 357^{3}$ & 2255 \\
\hline \multirow{2}{*}{${ }_{330}^{201 \pi}=$} & 233 & - & - & 260 & - & - \\
\hline & ons & : & : & isis & : & ! \\
\hline
\end{tabular}

Zey: 220 Fled J1zecsion $(0-360)$

12 iilad spened (keots)

0300 itzo of Jbservactoo 


$$
\text { E-6 }
$$

Table E4 RESULTS FROM WINDS YODELING -- JANUARY 3, 1975

\begin{tabular}{|c|c|c|c|c|c|c|c|}
\hline \multirow[b]{2}{*}{ Station } & \multirow[b]{2}{*}{ Code } & \multicolumn{6}{|c|}{ TIME } \\
\hline & & 0300 & 0700 & 1100 & 1500 & 1900 & 2300 \\
\hline \multirow{2}{*}{\multicolumn{2}{|c|}{ FARC }} & 225 & 158 & 113 & 68 & 90 & 23 \\
\hline & & 5 & 3 & 2 & 4 & 4 & 5 \\
\hline \multirow{2}{*}{\multicolumn{2}{|c|}{ FMBL }} & 225 & 158 & 135 & 68 & 90 & 23 \\
\hline & & 6 & 4 & 2 & 3 & 4 & 4 \\
\hline \multirow{2}{*}{\multicolumn{2}{|c|}{ BEIA }} & 225 & 180 & 135 & 45 & 90 & 23 \\
\hline & & 6 & 4 & 2 & 3 & 3 & 4 \\
\hline \multirow{2}{*}{\multicolumn{2}{|c|}{ BELC }} & 203 & 180 & 135 & 68 & 90 & 23 \\
\hline & & 6 & 4 & 2 & 3 & 3 & 4 \\
\hline \multirow{2}{*}{\multicolumn{2}{|c|}{ BELT }} & 203 & 180 & 135 & 68 & 90 & 23 \\
\hline & & 6 & 4 & 2 & 3 & 3 & 4 \\
\hline \multirow{2}{*}{\multicolumn{2}{|c|}{ ASHL }} & 225 & 158 & 113 & 68 & 90 & 23 \\
\hline & & 4 & 3 & 2 & 4 & 4 & 5 \\
\hline \multirow{2}{*}{\multicolumn{2}{|c|}{ ANWP }} & 225 & 158 & 113 & 58 & 90 & 23 \\
\hline & & 4 & 3 & 2 & 4 & 4 & 5 \\
\hline \multirow{2}{*}{\multicolumn{2}{|c|}{ ATEX }} & 225 & 158 & 113 & 68 & 90 & 23 \\
\hline & & 4 & 3 & 2 & 4 & 4 & 5 \\
\hline \multirow{2}{*}{\multicolumn{2}{|c|}{ BHIL }} & 248 & 158 & 113 & 90 & 90 & 68 \\
\hline & & .7 & 2 & 3 & 5 & 7 & 3 \\
\hline \multirow{2}{*}{\multicolumn{2}{|c|}{ WHID }} & 180 & 135 & 113 & 113 & 113 & 113 \\
\hline & & 2 & 2 & 2 & 3 & 4 & 5 \\
\hline \multirow{2}{*}{\multicolumn{2}{|c|}{ SMTH }} & 158 & 235 & 135 & 135 & 135 & 135 \\
\hline & & 3 & 3 & 3 & 4 & 5 & 7 \\
\hline \multirow{2}{*}{\multicolumn{2}{|c|}{ VICA }} & 225 & 135 & 113 & 68 & 90 & 23 \\
\hline & & 4 & 3 & 2 & 3 & 3 & 4 \\
\hline \multirow{2}{*}{\multicolumn{2}{|c|}{ VICH }} & 225 & 135 & 113 & 68 & 90 & 23 \\
\hline & & 6 & 2 & 2 & 3 & 4 & 4 \\
\hline
\end{tabular}


Flow direction at $850 \mathrm{mb}$, was between $245^{\circ}$ and $325^{\circ}$ during the simulation and westerly at $700 \mathrm{mb}$. and $500 \mathrm{mb}$. for the entire period.

The temperature and pressure profiles from the Quillayute sounding data are shown in Table E5.

Table E5. Quillayute Sounding Data, January 24 and 25, 1975.

\begin{tabular}{|c|c|c|c|c|}
\hline & $\begin{array}{l}\text { Height (m)/ } \\
\text { Pressure (mb.) }\end{array}$ & $\begin{array}{l}\text { Temperature } \\
\left({ }^{\circ} \mathrm{C}\right)\end{array}$ & $\begin{array}{l}\text { Direction } \\
\text { (degrees) }\end{array}$ & $\begin{array}{l}\text { Speed } \\
\text { (knots) }\end{array}$ \\
\hline \multicolumn{5}{|c|}{0300 PST January 24, 1975} \\
\hline Surface $(56 \mathrm{~m})$ & $1016.5 \mathrm{mb}$. & 6.3 & 100 & 4 \\
\hline $850 \mathrm{mb}$ & $1496 \mathrm{~m}$ & -3.0 & 245 & 9 \\
\hline $700 \mathrm{mb}$. & $3017 \mathrm{~m}$ & -5.5 & 270 & 58 \\
\hline $500 \mathrm{mb}$. & $5586 \mathrm{~m}$ & -20.1 & 270 & 75 \\
\hline \multicolumn{5}{|c|}{1500 PST January 24, 1975} \\
\hline Surface $(56 \mathrm{~m})$ & $1012.1 \mathrm{mb}$ & 7.6 & 340 & 4 \\
\hline $850 \mathrm{mb}$ & $1466 \mathrm{~m}$ & -1.9 & 325 & 9 \\
\hline $700 \mathrm{mb}$ & $2996 \mathrm{~m}$ & -7.3 & 265 & 38 \\
\hline $500 \mathrm{mb}$ & $5570 \mathrm{~m}$ & -19.5 & 270 & 77 \\
\hline \multicolumn{5}{|c|}{0300 PST January $25, \quad 1975$} \\
\hline Surface $(56 \mathrm{~m})$ & $1019.3 \mathrm{mb}$ & 2.8 & - & Calm \\
\hline $850 \mathrm{mb}$ & $1461 \mathrm{~m}$ & -4.0 & 293 & 10 \\
\hline $700 \mathrm{mb}$ & $2969 \mathrm{~m}$ & -12.0 & 270 & 30 \\
\hline $500 \mathrm{mb}$. & $5486 \mathrm{~m}$ & -27.0 & 270 & 80 \\
\hline
\end{tabular}

Meteorological data collected for January 24, 1975 is shown as Table E6 and wind flow modeling results for the meteorological station grid points are presented as Table E7. Figures E14 through E19 are maps of predicted winds speeds and directions for each hour of the simulation. The PI-Matrix results are shown in Figures E 20 through E26. 
Table E 6 .

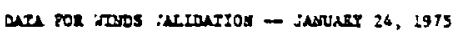

\begin{tabular}{|c|c|c|c|c|c|c|}
\hline \multirow[b]{2}{*}{ Stresso code } & \multicolumn{6}{|c|}{$\operatorname{IIT}$} \\
\hline & 0360 & 0703 & 1100 & 1500 & .000 & $23 C^{20}$ \\
\hline \multirow[t]{2}{*}{ 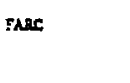 } & 210 & $\$ 70$ & 190 & 270 & so & 300 \\
\hline & 0300 & 0100 & $1100^{3}$ & $1500^{2}$ & $\begin{array}{r}20 \\
: 900\end{array}$ & $2300^{\circ}$ \\
\hline \multirow[t]{2}{*}{ MPL } & 190 & 190 & 190 & 190 & 20 & 310 \\
\hline & $0300^{3}$ & $0700^{j}$ & $1100^{2}$ & $1500^{\frac{1}{1}}$ & $\begin{array}{r}00 \\
1900\end{array}$ & $2300^{3}$ \\
\hline \multirow[t]{2}{*}{ Bers } & 200 & 150 & 190 & 200 & 180 & 30 \\
\hline & $\begin{array}{r}5 \\
0298\end{array}$ & $\begin{array}{r}2 \\
0635\end{array}$ & 1055 & 1657 & $\begin{array}{r}2 \\
1899\end{array}$ & $\begin{array}{r}00 \\
2258\end{array}$ \\
\hline \multirow[t]{2}{*}{ san } & 150 & $\omega$ & 170 & 220 & $\infty$ & 20 \\
\hline & $030^{3}$ & $\begin{array}{r}1 \\
0700\end{array}$ & $1100^{2}$ & 1300 & $\begin{array}{r}20 \\
: 900\end{array}$ & 2300 \\
\hline \multirow[t]{2}{*}{$B E I$} & 256 & 77 & 190 & $\infty 0$ & 233 & $\infty$ \\
\hline & $0300^{2}$ & $0700^{1}$ & 1100 & $\begin{array}{r}00 \\
15 \infty 0\end{array}$ & $\stackrel{\vdots}{1900}$ & $\begin{array}{r}00 \\
2300\end{array}$ \\
\hline \multirow[t]{2}{*}{ ASEII } & 160 & 1.30 & 230 & 160 & $\$ 20$ & 30 \\
\hline & 0300 & $0700^{\circ}$ & 1000 & $1500^{2}$ & $\begin{array}{r}1 \\
1900\end{array}$ & $\begin{array}{r}2 \\
2300\end{array}$ \\
\hline \multirow[t]{2}{*}{ علمد } & $\$ 30$ & 135 & 135 & 120 & 180 & 13 \\
\hline & 0300 & 0700 & $1100^{2}$ & $2500^{1}$ & $\begin{array}{r}1 \\
1900\end{array}$ & $20^{2}$ \\
\hline \multirow[t]{2}{*}{$\Delta E=P$} & 120 & $: 30$ & 110 & 200 & 200 & 350 \\
\hline & $030 \overline{0}$ & $0700^{\circ}$ & $200^{\circ}$ & $1500^{\circ}$ & 1903 & 2300 \\
\hline \multirow[t]{2}{*}{ NTI } & 160 & 320 & 150 & $\infty$ & $\infty$ & 00 \\
\hline & $0300^{3}$ & $0700^{2}$ & $1200^{3}$ & $\begin{array}{r}30 \\
L 500\end{array}$ & $\begin{array}{r}\infty 0 \\
1900\end{array}$ & $\begin{array}{r}00 \\
2300\end{array}$ \\
\hline \multirow[t]{2}{*}{ 3표 } & 293 & 90 & 1013 & 13 & 325 & 225 \\
\hline & 0300 & 0700 & $1100^{3}$ & $: 300^{3}$ & $1900^{\prime}$ & $=300^{\frac{1}{1}}$ \\
\hline \multirow[t]{2}{*}{ DER } & 150 & 100 & $\$ 10$ & 110 & 250 & $\infty$ \\
\hline & 0255 & $065^{2}$ & $\cos ^{2}$ & 1655 & 1853 & $\begin{array}{r}00 \\
2255\end{array}$ \\
\hline \multirow[t]{2}{*}{$\sin 3$} & 190 & :L & - & 00 & 225 & - \\
\hline & $0,330^{5}$ & $0630^{3}$ & $=$ & $\begin{array}{r}10 \\
2530\end{array}$ & $4830^{3}$ & E \\
\hline \multirow[t]{2}{*}{ vica } & 203 & 90 & 270 & 293 & 293 & 270 \\
\hline & $\begin{array}{r}5 \\
0300\end{array}$ & $\begin{array}{r}00 \\
0700\end{array}$ & $1100^{2}$ & $1300^{2}$ & $: 900^{2}$ & $2300^{2}$ \\
\hline \multirow[t]{2}{*}{ ricr } & 225 & 225 & 225 & 223 & $2: 0$ & 35 \\
\hline & $0300^{\circ}$ & $0700^{\circ}$ & 1100 & 2900 & $1900^{3}$ & $2300^{2}$ \\
\hline \multirow[t]{2}{*}{ PACE } & - & $\infty$ & - & 270 & 248 & - \\
\hline & $\because$ & $\begin{array}{r}00 \\
0700\end{array}$ & : & $1500^{2}$ & $1900^{2}$ & : \\
\hline \multirow[t]{2}{*}{ PAPA } & - & to & 30 & 320 & - & - \\
\hline & : & $\begin{array}{r}50 \\
5700\end{array}$ & $\begin{array}{r}60 \\
\$ 100\end{array}$ & $1500^{2}$ & : & : \\
\hline \multirow[t]{2}{*}{ EVRI } & 199 & 200 & 141 & 195 & - & - \\
\hline & $0300^{2}$ & $0700^{\prime}$ & $1100^{2}$ & $2500^{\prime}$ & $=$ & 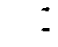 \\
\hline \multirow{2}{*}{ curt. } & 30 & jo & ic & 340 & $\infty 0$ & 20 \\
\hline & 0257 & $\begin{array}{r}20 \\
0630\end{array}$ & $\cos ^{2}$ & $2455^{2}$ & $\begin{array}{r}30 \\
: 358\end{array}$ & $2: 20$ \\
\hline \multirow{2}{*}{ 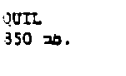 } & $2: 3$ & - & - & $3: 3$ & - & - \\
\hline & 23:3 & $:$ & : & $i s i 3$ & : & : \\
\hline
\end{tabular}

Zay: 220 Had 02race:100 (0-360)

12 ulad speed (icoocs)

0300 Ifan of toservacioa 
Tabie E7. RESUITS FROM WINDS MODELING -- JANUARY 24, 1975

\begin{tabular}{|c|c|c|c|c|c|c|}
\hline \multirow[b]{2}{*}{ Station Code } & \multicolumn{6}{|c|}{ TIME } \\
\hline & 0300 & 0700 & 1100 & 1500 & 1900 & 2300 \\
\hline \multirow{2}{*}{ FARC } & 180 & 135 & 158 & 180 & 225 & 360 \\
\hline & 3 & 2 & 2 & 2 & 2 & 1 \\
\hline \multirow{2}{*}{ ATBL } & 180 & 158 & 180 & 203 & 225 & 315 \\
\hline & 4 & 3 & 3 & 2 & 2 & 1 \\
\hline \multirow{2}{*}{ BELA } & 180 & 158 & 180 & 180 & 203 & 260 \\
\hline & 4 & 2 & 3 & 2 & 2 & 1 \\
\hline \multirow[t]{2}{*}{ BELC } & 180 & 158 & 180 & 180 & 203 & 360 \\
\hline & 4 & 3 & 3 & 2 & 2 & 1 \\
\hline \multirow[t]{2}{*}{ BELT } & 180 & 158 & 180 & 180 & 203 & 360 \\
\hline & 4 & 2 & 3 & 2 & 2 & I \\
\hline \multirow{2}{*}{ ASEL } & 180 & 135 & 158 & 158 & 248 & 360 \\
\hline & 3 & 2 & 2 & 1 & 1 & $=$ \\
\hline \multirow{2}{*}{ ANWP } & 180 & 135 & 158 & 158 & 248 & 360 \\
\hline & 3 & 2 & 2 & 1 & 1 & 2 \\
\hline \multirow{2}{*}{ ATEX } & 180 & 135 & 1.58 & 158 & 248 & 360 \\
\hline & 3 & 2 & 2 & 1 & 1 & 2 \\
\hline \multirow{2}{*}{ BHIL } & 203 & 135 & 158 & 203 & 248 & 225 \\
\hline & 2 & 3 & 2 & 2 & 2 & 1 \\
\hline \multirow[t]{2}{*}{ LaID } & 280 & 135 & 158 & 135 & 270 & 360 \\
\hline & 3 & 2 & 2 & 1 & 1 & 2 \\
\hline \multirow[t]{2}{*}{ SMTE } & 180 & 135 & 135 & 135 & 225 & 360 \\
\hline & 3 & 2 & 2 & $i$ & 1 & 2 \\
\hline \multirow[t]{2}{*}{$\nabla I C A$} & 180 & $\$ 35$ & 158 & 180 & $=25$ & 338 \\
\hline & 3 & 2 & 2 & $i$ & 1 & 2 \\
\hline \multirow[t]{2}{*}{ VICR } & 180 & 225 & 225 & 243 & 248 & 293 \\
\hline & 4 & 2 & 2 & 3 & 3 & $=$ \\
\hline
\end{tabular}

6ay: 220 änd T1Face10n (0-360)

22 Ilad Spand (kooes) 
A similar situation existed at $700 \mathrm{mb}$, with a low pressure center over southern California and a strong depression along the western Canadian coast at 0300 PST January 30. Flow was westerly over Puget Sound. The low pressure center "stacked" to the northwest of the study area intensified and moved south to a point slightly northwest of Puget Sound by 0300 PST January 30, while the southern California low disappeared. Flow over western Washington shifted toward the southwest and was stronger and southerly 24 hours after the beginning of the simulation period.

The low pressure center to the northwest of the study area is also evident on $500 \mathrm{mb}$. maps and moved southward through the analysis period. A depression off the southern California coast weakened and disappeared during the same period as flow over northern Puget Sound moved from west to south-southwest during the simulation.

The pressure and temperature profiles recorded at Quillayute are shown in Table E8.

Meteorological data collected for January 30, 1975 is shown as Table Eg and wind flow modeling results for the meteorological station grid points are presented as Table E10. Figures E27 through E32 are maps of predicted winds speeds and directions for each hour of the simulation. The PI-Matrix results are shown in Figures E33 through E39.

February 12, 1975

Air quality data for 1975 show that violations of the short-term standards for $\mathrm{SO}_{2}$ occurred at the Bellingham site on February 12, 1975. Concentrations of $40 \mathrm{pphm}$ were recorded for a 24 hour average, the highest 24 hour concentration recorded at this location over the two year period examined (1975 and 1976). 
January 30,1975

The air quality data collected indicate that particulate levels reached a concentration of $119 \mu \mathrm{g} / \mathrm{m}^{3}$ for a 24 hour period on January 30 , 1975 at Mt. Vernon. This was the second highest concentration recorded at this location over a two year period.

Surface meteorological data for the beginning of the simulation period show a low pressure system centered to the northwest of the study area, with a warm front just off the western Washington coast and a cold front somewhat further off shore over the Pacific. A high pressure center was evident at the surface over southeastern Idaho and southwestern Montana. By 0300 PST January 31, the low pressure center had moved south and was located directly west of the study area. The warm front had apparently passed through the northern Puget Sound region and lay to the north of the study area, while a cold front extended along the Pacific coast. The high pressure system was still centered over southeastern Idaho. The general direction of flow over western Washington was southeasterly to east-southeasterly at both sounding periods.

The $850 \mathrm{mb}$. maps show a strong depression moving south along the Pacific coast from the Gulf of Alaska early on January 30, 1975. A weak low pressure system was centered over southern California, with a weak high pressure center over southeastern Idaho. During the simulation period, the high pressure center moved east and the southern California trough weakened and disappeared. At the same time, the low to the northwest intensified and continued to move south to a point directly west of the Puget Sound region by the end of the simulation. Flow at this level was light and from the southwest at the beginning of the analysis, shifting to the south and south-southeast and becoming stronger through the following 24 hours. 
Table E8. Millayute Sounding Data, January 30 and 31, 1975.

\begin{tabular}{|c|c|c|c|c|}
\hline & $\begin{array}{l}\text { Height (m)/ } \\
\text { Pressure (mb.) }\end{array}$ & $\begin{array}{l}\text { Temperature } \\
\left({ }^{\circ} \mathrm{C}\right)\end{array}$ & $\begin{array}{l}\text { Direction } \\
\text { (degrees) }\end{array}$ & $\begin{array}{l}\text { Speed } \\
\text { (knots) }\end{array}$ \\
\hline \multicolumn{5}{|c|}{0300 PST January 30,1975} \\
\hline Surface $(56 \mathrm{~m})$ & $1008.8 \mathrm{mb}$ & 1.3 & 160 & 5 \\
\hline $850 \mathrm{mb}$ & $1427 \mathrm{~m}$ & -6.3 & 205 & 17 \\
\hline $700 \mathrm{mb}$ & $2918 \mathrm{~m}$ & -14.6 & 265 & 18 \\
\hline $500 \mathrm{mb}$ & $5403 \mathrm{~m}$ & -28.6 & 290 & 28 \\
\hline \multicolumn{5}{|c|}{1500 PST January $30, \quad 1975$} \\
\hline Surface $(56 \mathrm{~m})$ & $999.0 \mathrm{mb}$ & 2.7 & 140 & 4 \\
\hline $850 \mathrm{mb}$ & $1340 \mathrm{~m}$ & -6.1 & 170 & 38 \\
\hline $700 \mathrm{mb}$. & $2840 \mathrm{~m}$ & -14.5 & 205 & 23 \\
\hline $500 \mathrm{mb}$. & $5302 \mathrm{~m}$ & -32.2 & 210 & 34 \\
\hline \multicolumn{5}{|c|}{0300 PST January 31, 1975} \\
\hline Surface $(56 \mathrm{~m})$ & $998.9 \mathrm{mb}$ & 1.1 & 90 & 15 \\
\hline $850 \mathrm{mb}$ & $1328 \mathrm{~m}$ & -5.0 & 158 & 45 \\
\hline $700 \mathrm{mb}$ & $2785 \mathrm{~m}$ & -13.0 & 180 & 40 \\
\hline $500 \mathrm{mb}$. & $5243 \mathrm{~m}$ & -34.0 & 180 & 50 \\
\hline
\end{tabular}

Surface weather maps for the February 12, 1975 simulation period show a strong low pressure system centered immediately west of the study area at 0300 PST February 12, 1975. An occluded front extended through the northern Puget Sound region. A high pressure center was evident near the Four Corners area over southern Colorado and a second ridge extended across northern Alberta and Saskatchawan. During the 24 hour simulation period, the low pressure center weakened and moved over northern Vancouver Island, with an occluded front across the northern portions of the study region. 
Table E9.

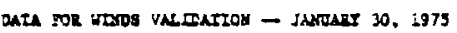

\begin{tabular}{|c|c|c|c|c|c|c|}
\hline \multirow[b]{2}{*}{ Stut100 Codn } & \multicolumn{6}{|c|}{$t \rightarrow E$} \\
\hline & 0300 & 0,00 & 1.00 & 1500 & 1900 & 2700 \\
\hline \multirow{2}{*}{ Faec } & 90 & 100 & 90 & 100 & 90 & 30 \\
\hline & 0300 & 0700 & $\begin{array}{r}5 \\
1130^{5}\end{array}$ & $1500^{4}$ & $\begin{array}{r}6 \\
1900\end{array}$ & $2300^{8}$ \\
\hline \multirow{2}{*}{ FED } & 110 & 110 & 110 & 20 & 110 & 100 \\
\hline & $0300^{3}$ & 0700 & 1200 & 1500 & 1900 & 2300 \\
\hline \multirow{2}{*}{ BEL } & 30 & $\$ 0$ & 20 & 360 & 100 & 30 \\
\hline & 0295 & $\begin{array}{r}2 \\
0656\end{array}$ & 2035 & $1659^{2}$ & 2855 & 2258 \\
\hline \multirow[t]{2}{*}{ عـد3 } & - & - & - & - & - & - \\
\hline & $=$ & $\overline{-}$ & $\overline{-}$ & : & : & $=$ \\
\hline \multirow{2}{*}{ Banz } & 25 & 35 & 369 & 120 & 86 & 37 \\
\hline & $0300^{2}$ & $0700^{2}$ & $1200^{3}$ & $1500^{2}$ & $1900^{3}$ & 22000 \\
\hline \multirow{2}{*}{ ASII } & 160 & 140 & 150 & 140 & Ljo & 120 \\
\hline & $0300^{\circ}$ & $0700^{8}$ & 11000 & $1500^{8}$ & $: 900$ & $2300^{4}$ \\
\hline \multirow{2}{*}{ علس } & 235 & 175 & iso & 135 & L30 & 130 \\
\hline & $0300^{3}$ & $0700^{\circ}$ & $1100^{\circ}$ & $\pm 500^{\circ}$ & 1900 & $: 300^{2}$ \\
\hline \multirow{2}{*}{ Ners } & 140 & 140 & 150 & 240 & 120 & $\therefore 0$ \\
\hline & $0300^{2}$ & $0700^{3}$ & 1100 & $1500^{3}$ & $1900^{2}$ & $2=20^{2}$ \\
\hline \multirow{2}{*}{ TIS } & 230 & 130 & 140 & 230 & 20 & 20 \\
\hline & $0300^{6}$ & $0700^{7}$ & $2100^{3}$ & $1300^{8}$ & $1900^{3}$ & $2300^{4}$ \\
\hline \multirow{2}{*}{ aent } & 68 & 68 & 60 & So & 58 & 53 \\
\hline & $\begin{array}{r}6 \\
2300\end{array}$ & $0700^{6}$ & $1100^{6}$ & $1500^{\circ}$ & $\begin{array}{r}6 \\
1900^{\circ}\end{array}$ & $2300^{6}$ \\
\hline \multirow{2}{*}{ DIE } & 110 & 110 & 120 & 130 & 220 & 120 \\
\hline & 0255 & 0653 & $203^{6}$ & $\begin{array}{r}8 \\
1453\end{array}$ & 1953 & 2293 \\
\hline \multirow{2}{*}{ sent } & $: 35$ & 235 & - & 135 & 123 & - \\
\hline & $0.590^{9}$ & 0630 & $:$ & $\begin{array}{r}12 \\
1530\end{array}$ & $\begin{array}{r}122 \\
1830\end{array}$ & $\overline{-}$ \\
\hline \multirow[t]{2}{*}{ Tea } & 113 & 158 & 1.15 & 1.13 & 135 & $1: 3$ \\
\hline & 0300 & 0;00 & $\begin{array}{r}11 \\
1100\end{array}$ & 15 & 1900 & 2390 \\
\hline \multirow{2}{*}{ VICB } & 135 & 233 & 135 & :3s & 90 & L15 \\
\hline & $0300^{\prime}$ & $0700^{3}$ & $\begin{array}{r}12 \\
1100\end{array}$ & $\begin{array}{r}25 \\
1500\end{array}$ & $\begin{array}{r}18 \\
1900\end{array}$ & $\begin{array}{r}16 \\
2300\end{array}$ \\
\hline \multirow{2}{*}{ ?ACE } & - & - & - & - & - & - \\
\hline & : & $:$ & $=$ & $:$ & $=$ & - \\
\hline \multirow{2}{*}{ ?APA } & - & so & 00 & 00 & - & - \\
\hline & : & $\begin{array}{r}00 \\
0700\end{array}$ & $\begin{array}{r}90 \\
1100\end{array}$ & $\begin{array}{r}00 \\
2500\end{array}$ & $=$ & : \\
\hline \multirow[t]{2}{*}{$378 x$} & 136 & \pm 27 & 136 & $\therefore 29$ & Wi & 203 \\
\hline & $0300^{\prime}$ & $\begin{array}{r}6 \\
\text { ग7co }\end{array}$ & $1100^{\circ}$ & $1500^{\circ}$ & : 7000 & $\begin{array}{r}9 \\
3000^{9}\end{array}$ \\
\hline \multirow{2}{*}{$\begin{array}{l}\text { QuII } \\
\text { Surface }\end{array}$} & $\therefore 0$ & 250 & 150 & 250 & 140 & :ov \\
\hline & 2258 & 0638 & 1096 & 1435 & $: 35 \sigma^{2}$ & $225^{2}$ \\
\hline \multirow{2}{*}{$\begin{array}{l}\text { GOIL } \\
350=b .\end{array}$} & $=05$ & - & - & 270 & - & - \\
\hline & & - & 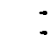 & isis & $\because$ & $=$ \\
\hline
\end{tabular}

Zay: 220 Hind D15aceton (0-360)

12 ines soned (knocs)

0300 ilse of Obserpation 
Table EZO. RESULTS FROM WINDS MODELING -- JANUARY 30, 1975

\begin{tabular}{|c|c|c|c|c|c|c|}
\hline \multirow[b]{2}{*}{ Station Code } & \multicolumn{6}{|c|}{ IIME } \\
\hline & 0300 & 0700 & 1100 & 1500 & 1900 & 2300 \\
\hline \multirow{2}{*}{ FARC } & 113 & 113 & 113 & 113 & 113 & 113 \\
\hline & 4 & 4 & 6 & 4 & 7 & 6 \\
\hline \multirow{2}{*}{ FMBL } & 113 & 113 & 113 & 113 & 113 & 113 \\
\hline & 4 & 4 & 5 & 4 & 6 & 6 \\
\hline \multirow[t]{2}{*}{ BEIA } & 113 & 113 & 113 & 113 & 113 & 113 \\
\hline & 3 & 3 & 4 & 3 & 5 & 6 \\
\hline \multirow[t]{2}{*}{$B E L C$} & 113 & 113 & 113 & 113 & 113 & 113 \\
\hline & 3 & 3 & 4 & 3 & 5 & 6 \\
\hline \multirow[t]{2}{*}{ BELT } & 113 & 113 & 113 & 113 & 113 & 113 \\
\hline & 3 & 3 & 4 & 3 & 5 & 6 \\
\hline \multirow[t]{2}{*}{ ASHZ } & 113 & 113 & 113 & 113 & 113 & 113 \\
\hline & 4 & 4 & 5 & 4 & 6 & 6 \\
\hline \multirow[t]{2}{*}{ ANWP } & 113 & 113 & 113 & 113 & 113 & 113 \\
\hline & 4 & 4 & 5 & 4 & 6 & 6 \\
\hline \multirow[t]{2}{*}{ ATEX } & 113 & 113 & 113 & 113 & 113 & 113 \\
\hline & 4 & 4 & 5 & 4 & 6 & 6 \\
\hline \multirow[t]{2}{*}{ BHIL } & 113 & 113 & 90 & 113 & 90 & 90 \\
\hline & 6 & 6 & 8 & 6 & 10 & 9 \\
\hline \multirow[t]{2}{*}{ WHID } & 135 & 135 & 135 & 135 & 135 & 135 \\
\hline & 5 & 5 & 3 & 8 & 6 & 8 \\
\hline \multirow[t]{2}{*}{ SMTY } & 135 & 135 & 135 & 135 & 135 & 135 \\
\hline & 6 & 6 & 6 & 10 & 6 & 9 \\
\hline \multirow[t]{2}{*}{ VICA } & 113 & 113 & $1: 3$ & 113 & 113 & 113 \\
\hline & 4 & 4 & 5 & 4 & 6 & 6 \\
\hline \multirow{2}{*}{ VICH } & $\ni 0$ & 90 & 90 & 90 & 90 & 90 \\
\hline & 3 & 6 & 10 & 9 & 12 & 11 \\
\hline
\end{tabular}


The $850 \mathrm{mb}$. situation shows a strong low pressure center just west of the northern Puget Sound region at the beginning of the simulation period. Flow over the study area was generally from the southwest. An intense depression was centered northeast of the Newfoundland coast over the Atlantic Ocean, and a ridge of high pressure extended across westcentral Canada and the west-central United States between the two low pressure systems. Flow over the Canadian region and northern United States was governed by these two strong depressions off either coast. Strong warm air advection is evident through the following 24 hours at the $850 \mathrm{mb}$. level. During this period, both low pressure systems weakened. By 0300 PST February 13, the depression off the western Washington coast had weakened appreciably, while a low pressure center in the Gulf of Alaska had intensified. The ridge across the central United States and Canada had moved eastward and also weakened. Overall flow through western Washington remained from the southwestern quadrant through the 24 hour period.

Again, the pattern at $700 \mathrm{mb}$. is similar to that seen at $850 \mathrm{mb}$. with a depression northwest of the study area and a strong low pressure center over northeastern Canada at 0300 PST February 12. The Pacific low pressure center was weaker at $700 \mathrm{mb}$. than the Atlantic depression, with a stronger low pressure system over the Gulf of Alaska. A high pressure ridge extended across the west-central part of the country. During the simulation, the low pressure center to the west of the study area weakened and disappeared while both the Gulf of Alaska depression and low pressure system over north-eastern Canada strengthened and moved south. The associated ridge between the two depressions has shifted somewhat east by 0300 PST February 13. Flow over the northern Puget 
Meteorological data collected for February 12, 1975 is shown as Table E12 and wind flow modeling results for the meteorological station grid points are presented as Table E13. Figures E40 through 545 are maps of predicted wind speeds and directions for each hour of the simulation. The PI-Matrix results are shown in Figures E46 through E52.

\section{Annual Cases}

Figures E53 through E60 are maps of predicted wind speeds and directions for the annual analysis. Figures E61 through E68 show the mixing volume index maps for the eight directions. The mixing volume index is a measure of the potential for pollutant dispersal in each grid cell -- low index values indicate greater dispersion potential than high values. 


$$
\mathrm{E}-16
$$

Sound region was strong and southwesterly at 0300 PST February 12, shifting to somewhat more westerly and then to south-southwesterly over the following 24 hours and becoming lighter.

At $500 \mathrm{mb}$, low pressure centers can be seen over the Gulf of Alaska and the northwestern Atlantic with southwesterly flow over Washington at the beginning of the simulation period. Both depressions intensified during the simulation but little change was apparent in flow direction or speed over the northern Puget Sound region.

The temperature and pressure profiles can be seen from the sounding data taken at Quillayute (Table Ell).

Table E11. Quillayute Sounding Data, February 12 and 13, 1975.

\begin{tabular}{|c|c|c|c|c|}
\hline & $\begin{array}{l}\text { Height }(\mathrm{m}) / \\
\text { Pressure (mb.) }\end{array}$ & $\begin{array}{l}\text { Temperature } \\
\left({ }^{\circ} \mathrm{C}\right)\end{array}$ & $\begin{array}{l}\text { Direction } \\
\text { (degrees) }\end{array}$ & $\begin{array}{l}\text { Speed } \\
\text { (knots) }\end{array}$ \\
\hline \multicolumn{5}{|c|}{0300 PST February 12, 1975} \\
\hline Surface $(56 \mathrm{~m})$ & $980.8 \mathrm{mb}$ & 8.3 & 160 & 15 \\
\hline $850 \mathrm{mb}$. & $1229 \mathrm{~m}$ & 3.0 & 220 & 52 \\
\hline $700 \mathrm{mb}$ & $2777 \mathrm{~m}$ & $-4 \cdot 4$ & 220 & 44 \\
\hline $500 \mathrm{mb}$. & $5338 \mathrm{~m}$ & -22.6 & 220 & 51 \\
\hline
\end{tabular}

1500 PST February 12,1975

$\begin{array}{lrrrr}\text { Surface }(56 \mathrm{~m}) & 990.4 \mathrm{mb} & 6.8 & 240 & 7 \\ 850 \mathrm{mb} . & 1290 \mathrm{~m} & -2.2 & 245 & 19 \\ 700 \mathrm{mb} . & 2806 \mathrm{~m} & -11.8 & 255 & 36 \\ 500 \mathrm{mb} . & 5306 \mathrm{~m} & -27.4 & 235 & 45\end{array}$

0300 PST February 13, 1975

$\begin{array}{llrrr}\text { Surface }(56 \mathrm{~m}) & 1004.7 \mathrm{mb} & 3.9 & 290 & 5 \\ 850 \mathrm{mb} . & 1334 \mathrm{~m} & -3.0 & 248 & 15 \\ 700 \mathrm{mb} . & 2851 \mathrm{~m} & -11.0 & 203 & 30 \\ 500 \mathrm{mb} . & 5334 \mathrm{~m} & -29.0 & 225 & 35\end{array}$


Table El2.

SATA FOR WCOOS TAS:DATIOX - TZ3REARY 12, 1979

\begin{tabular}{|c|c|c|c|c|c|c|}
\hline \multirow[b]{2}{*}{ Seecioo code } & \multicolumn{6}{|c|}{$\pi$} \\
\hline & 0300 & 0505 & $\$ 100$ & 1500 & $\$ 900$ & $\sqrt{300}$ \\
\hline \multirow[t]{2}{*}{ FARC } & - & - & 360 & 30 & 30 & 350 \\
\hline & - & $=$ & $1100^{5}$ & $2500^{5}$ & $1900^{6}$ & $2300^{\circ}$ \\
\hline \multirow{2}{*}{ FMBL } & 00 & 100 & 180 & $2: 0$ & 130 & 170 \\
\hline & $\begin{array}{r}00 \\
0300\end{array}$ & 0700 & $1100^{6}$ & $2500^{3}$ & $1900^{3}$ & $2300^{\circ}$ \\
\hline \multirow{2}{*}{38} & 10 & 280 & 170 & 200 & 170 & 200 \\
\hline & 0259 & $0654^{7}$ & $\begin{array}{r}8 \\
: 095\end{array}$ & $\begin{array}{r}8 \\
1495\end{array}$ & $\begin{array}{r}4 \\
1853\end{array}$ & $223^{2}$ \\
\hline \multirow[t]{2}{*}{3} & 120 & 150 & 160 & 200 & 150 & $: 80$ \\
\hline & 0300 & 0700 & ספט & 1500 & $1900^{2}$ & $2300^{2}$ \\
\hline \multirow[t]{2}{*}{ 3.0.5 } & 358 & 256 & 158 & 201 & 165 & 20 \\
\hline & 0300 & $\begin{array}{r}8 \\
0700\end{array}$ & 1100 & 1500 & $: 900^{2}$ & $\begin{array}{r}x \\
3300\end{array}$ \\
\hline \multirow[t]{2}{*}{ SWII } & 150 & 170 & 170 & 270 & \pm 0 & $1 \omega$ \\
\hline & $\begin{array}{r}10 \\
0300\end{array}$ & $\begin{array}{r}9 \\
0700\end{array}$ & $\begin{array}{r}10 \\
1100\end{array}$ & L500 & 19000 & $2300^{5}$ \\
\hline \multirow[t]{2}{*}{ على } & 320 & 330 & 350 & so & 135 & 145 \\
\hline & 0300 & 3700 & 100 & 1500 & 1900 & $230 c^{3}$ \\
\hline \multirow[t]{2}{*}{ Nis? } & $: 60$ & 160 & 170 & 280 & $: 60$ & $\$ 60$ \\
\hline & 0300 & 0700 & $1: 00^{4}$ & $1300^{3}$ & $1900^{2}$ & $2300^{3}$ \\
\hline \multirow[t]{2}{*}{ חע } & 140 & 160 & 170 & 260 & $: 00$ & :46 \\
\hline & $\frac{12}{0300}$ & 0700 & $\begin{array}{r}16 \\
160\end{array}$ & $\begin{array}{r}10 \\
100\end{array}$ & $: 900^{3}$ & 22006 \\
\hline \multirow[t]{2}{*}{$38 \pi$} & 68 & 68 & 290 & 223 & 38 & 50 \\
\hline & 0300 & 0700 & 1100 & LoO & $1930^{3}$ & 2300 \\
\hline \multirow[t]{2}{*}{ SEID } & LJo & מעL & wo & 270 & 20 & 130 \\
\hline & $\begin{array}{r}3 \\
0235^{3}\end{array}$ & o6ss' & $1055^{9}$ & $\begin{array}{r}65 \\
1455^{6}\end{array}$ & $\begin{array}{r}60 \\
1335\end{array}$ & $2253^{3}$ \\
\hline \multirow[t]{2}{*}{ strTR } & 139 & 135 & - & 270 & 270 & - \\
\hline & 0330 & $\begin{array}{r}9 \\
0630\end{array}$ & : & פ39 & $2830^{4}$ & : \\
\hline \multirow[t]{2}{*}{$\mathrm{ras}$} & 27 & $\infty$ & $: 58$ & 229 & $=03$ & 293 \\
\hline & 0300 & $\begin{array}{r}00 \\
0700\end{array}$ & 1100 & $\begin{array}{r}12 \\
1500\end{array}$ & $: 900^{2}$ & $2300^{\circ}$ \\
\hline \multirow[t]{2}{*}{$\mathrm{TzCH}$} & 225 & 45 & 135 & 299 & 270 & $: 70$ \\
\hline & $\begin{array}{r}30 \\
0300\end{array}$ & $0700^{2}$ & $1100^{\circ}$ & $\begin{array}{r}11 \\
1500\end{array}$ & 1900 & $2300^{\circ}$ \\
\hline \multirow[t]{2}{*}{ PACG } & - & 00 & - & - & - & - \\
\hline & $\overline{-}$ & $\begin{array}{r}80 \\
0700\end{array}$ & : & - & $:$ & : \\
\hline \multirow[t]{2}{*}{ PAPA } & - & 30 & 30 & 230 & - & - \\
\hline & : & $0700^{\circ}$ & $: 100^{2}$ & $\begin{array}{r}5 \\
1500\end{array}$ & : & : \\
\hline \multirow[t]{2}{*}{ EVRT } & 361 & $: 68$ & 265 & 191 & ;0 & $1 \%$ \\
\hline & 0300 & & $\$ 100^{3}$ & $: 300^{9}$ & $: 900$ & $:=0$ \\
\hline \multirow{2}{*}{$\begin{array}{l}\text { ant } \\
\text { Surface }\end{array}$} & $: 60$ & 190 & 230 & $: 10$ & $2 \leq 0$ & $\because: 0$ \\
\hline & 0257 & ${ }_{0658}^{5}$ & $\cos \frac{6}{7}$ & $1+35$ & 2357 & $2093^{2}$ \\
\hline \multirow{2}{*}{. } & $: 20$ & - & - & 243 & - & - \\
\hline & $\begin{array}{r}35 \\
\text { o3is }\end{array}$ & $\because$ & - & 2.315 & : & : \\
\hline
\end{tabular}

\footnotetext{
Zay: 220 ilad J1receson (0-360)

12 Hind speed (knors)

.0300 ita oi Obsarmactoo
} 
Table E13. RESULTS FROM WINDS MODELING - FEBRUARY 12, 1975

\begin{tabular}{|c|c|c|c|c|c|c|}
\hline \multirow[b]{2}{*}{ Station Code } & \multicolumn{6}{|c|}{ TIME } \\
\hline & 0300 & 0700 & 1100 & 1500 & 1900 & 2300 \\
\hline \multirow{2}{*}{ EARC } & 113 & 158 & 180 & 248 & 158 & 158 \\
\hline & 5 & 6 & 6 & 5 & 3 & 2 \\
\hline \multirow{2}{*}{ FIBL } & 113 & 158 & 180 & 248 & 180 & 180 \\
\hline & 5 & 6 & 7 & 6 & 3 & 2 \\
\hline \multirow{2}{*}{ BELA } & 113 & 180 & 180 & 225 & 158 & 180 \\
\hline & 4 & 6 & 8 & 6 & 3 & 2 \\
\hline \multirow{2}{*}{$B E L C$} & 113 & 180 & 180 & 225 & 158 & 180 \\
\hline & 4 & 7 & 8 & 6 & 3 & 2 \\
\hline \multirow[t]{2}{*}{ BEII } & 113 & 180 & 180 & 225 & 158 & 190 \\
\hline & 4 & 6 & 8 & 6 & 3 & 2 \\
\hline \multirow{2}{*}{ ASHL } & 113 & 158 & 180 & 243 & 158 & 158 \\
\hline & 5 & 6 & 7 & $j$ & 3 & 2 \\
\hline \multirow{2}{*}{ ANWP } & 113 & 158 & 180 & 248 & 158 & 158 \\
\hline & 5 & 6 & 7 & 5 & 3 & 2 \\
\hline \multirow{2}{*}{ ATEX } & 113 & 158 & 180 & 248 & 158 & 158 \\
\hline & 5 & 6 & 7 & 5 & 3 & 2 \\
\hline \multirow{2}{*}{ BHIL } & 90 & 135 & 203 & 248 & 158 & 158 \\
\hline & 7 & 4 & 4 & 9 & 2 & 2 \\
\hline \multirow[t]{2}{*}{ WHID } & 135 & 135 & 158 & 248 & 158 & 135 \\
\hline & 8 & 9 & 7 & 6 & 2 & 2 \\
\hline \multirow[t]{2}{*}{ Sirt } & 135 & 135 & 135 & 248 & 158 & 135 \\
\hline & 10 & 10 & 9 & 6 & 3 & 2 \\
\hline \multirow[t]{2}{*}{ VICA } & 113 & 158 & 130 & 248 & 158 & 158 \\
\hline & 5 & 6 & 6 & 5 & 3 & 2 \\
\hline \multirow[t]{2}{*}{ VICH } & 113 & 158 & 158 & 248 & 248 & 248 \\
\hline & 4 & 4 & 4 & 9 & 4 & 3 \\
\hline
\end{tabular}

Kay: 220 filad Dreection $(0-360)$

12 Hiad speed (kates) 
Figure E1. Windfield Analysis of Northern Puget Sound Study Area for January 3, 1975 at 0300 PST. Background flow input: 225.0 degrees at 5.0 meters per second ( $\sigma$ ).

$$
\text { Wind Flag codes: } \begin{array}{r}
\frac{\mathrm{m} / \mathrm{s}}{5.0} \\
2.0 \\
7 \begin{array}{r}
1.0 \\
\end{array} \quad<1.0
\end{array}
$$




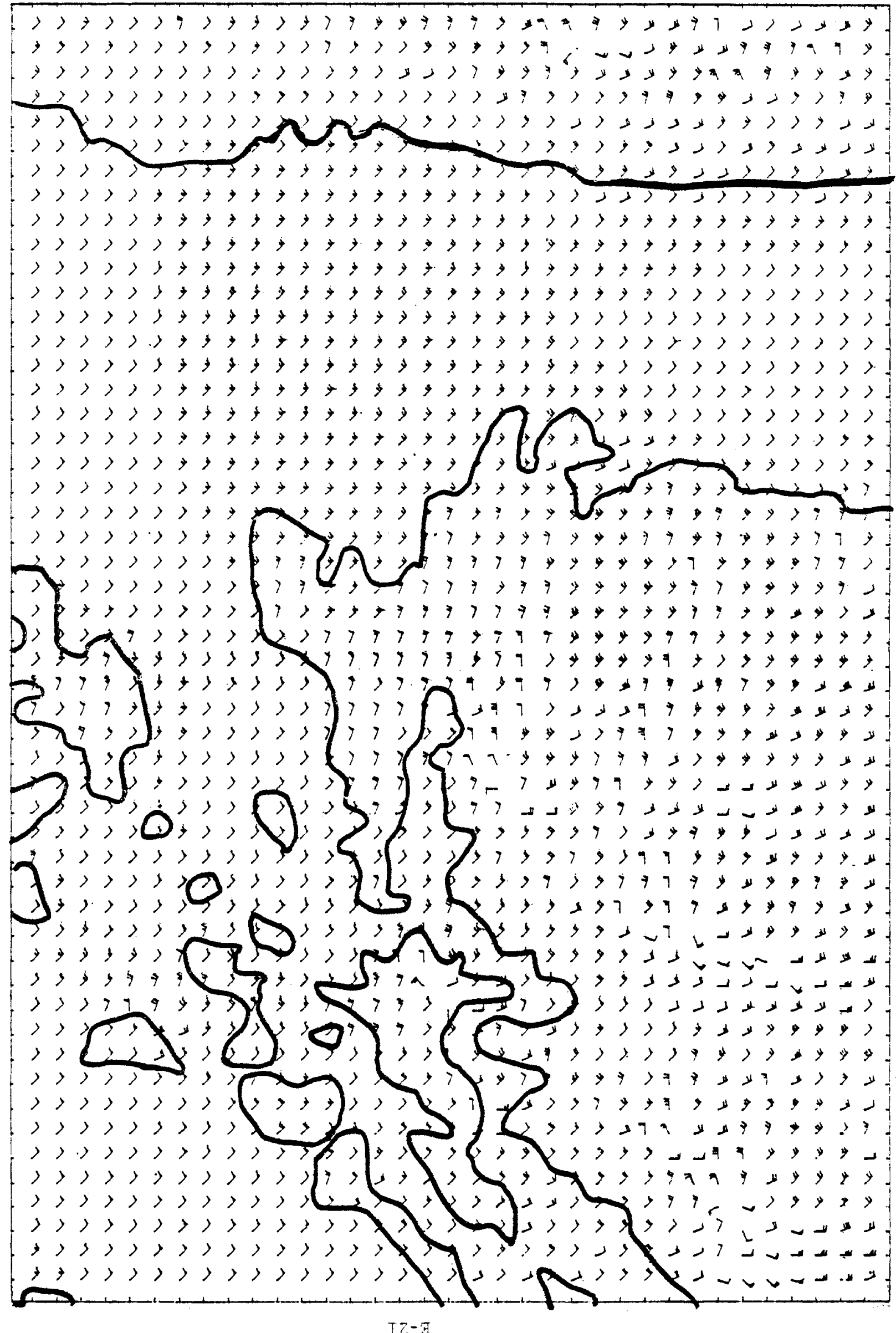




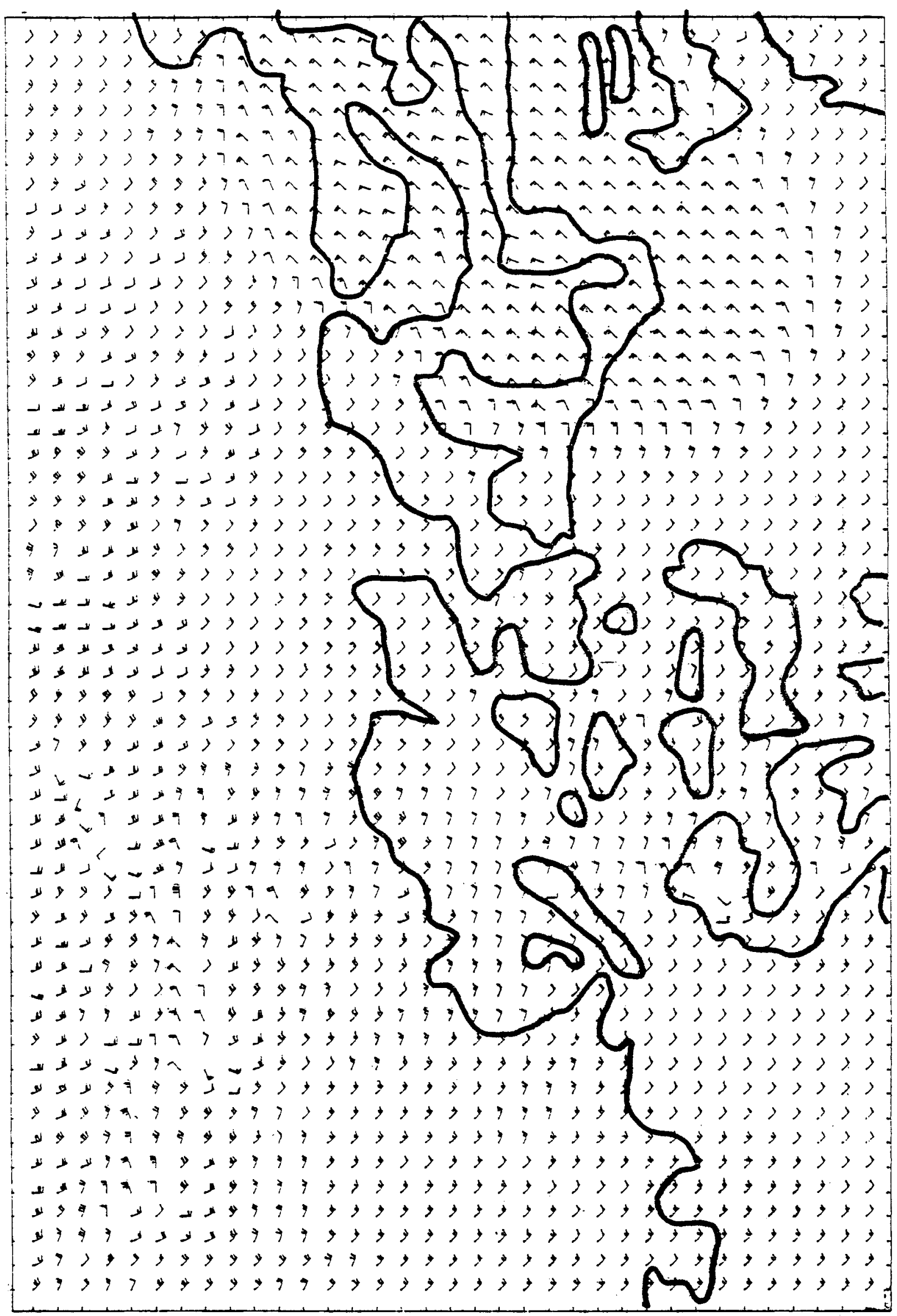


Figure E2. Windfield Analysis of Northern Puget Sound Study Area for January 3, 1975 at 0700 PST. Background flow input: 157.5 degrees at 3.0 meters per second $(k)$.

$$
\text { Wind Flag codes: } \begin{array}{r}
\frac{\mathrm{m} / \mathrm{s}}{5.0} \\
2.0 \\
7 \quad \begin{array}{l}
1.0 \\
! \quad<0
\end{array}
\end{array}
$$




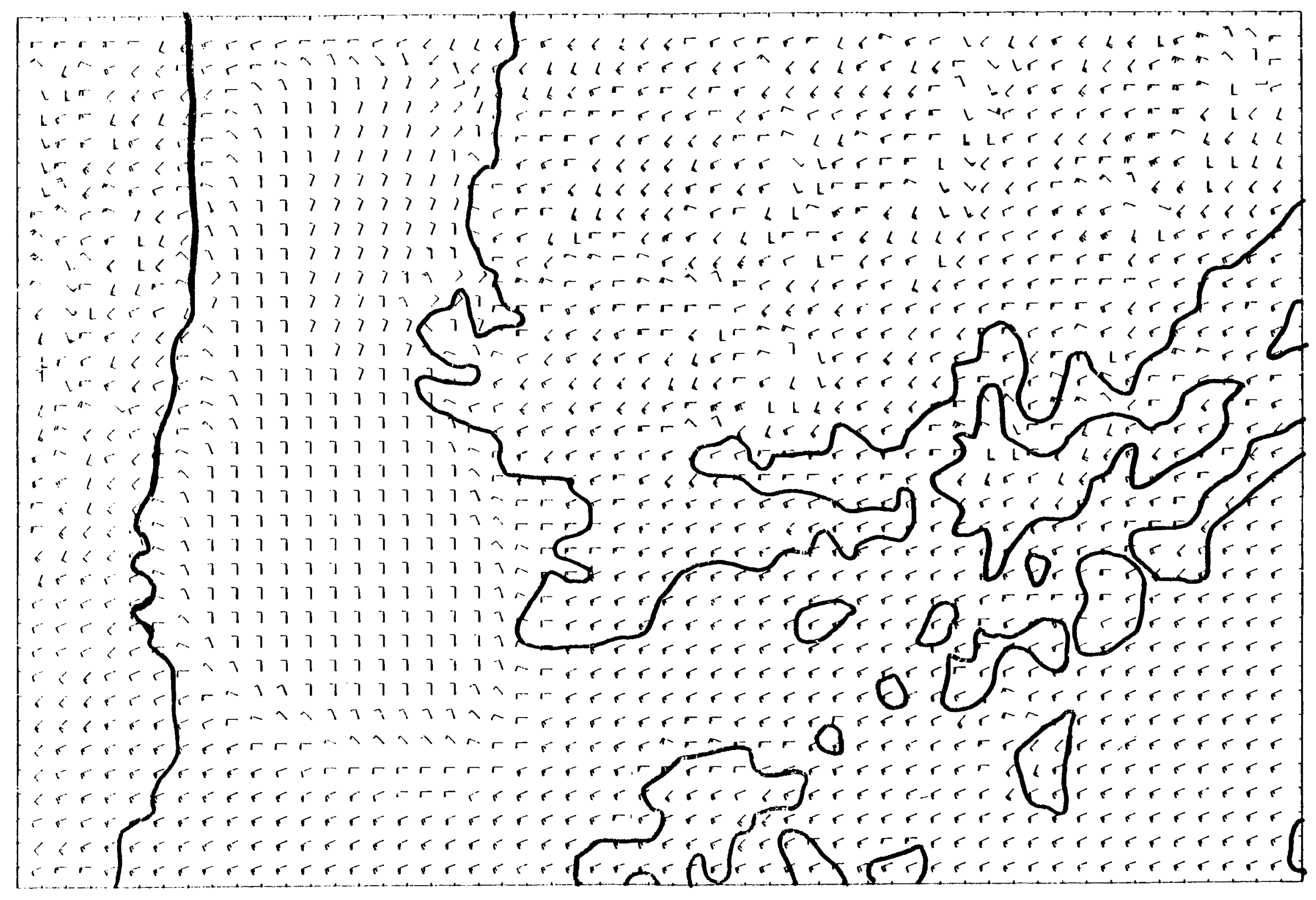




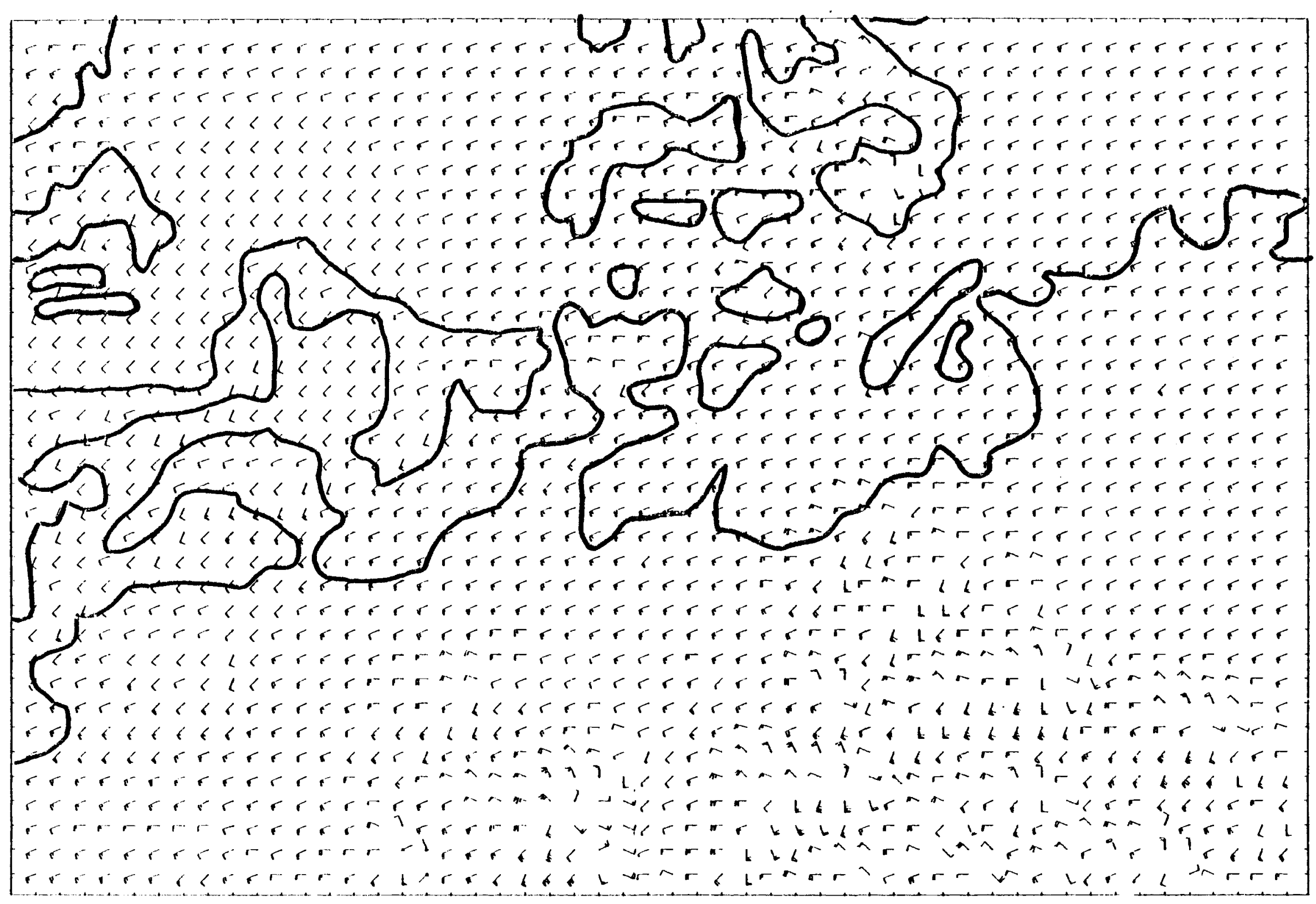


Figure E3. Windfield Analysis of Northern Puget Sound Study Area for January 3, 1975 at 1100 PST. Background flow input: 112.5 degrees at 2.0 meters per second $(\rightarrow)$.

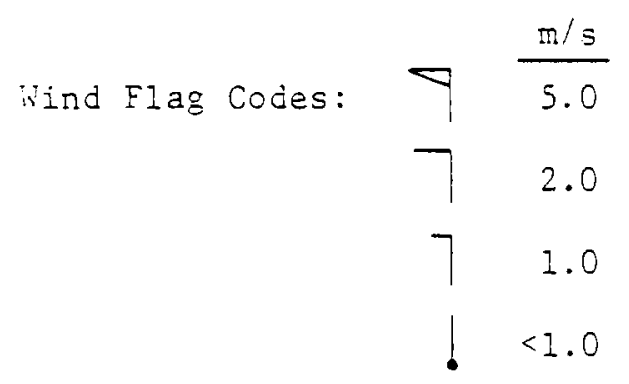




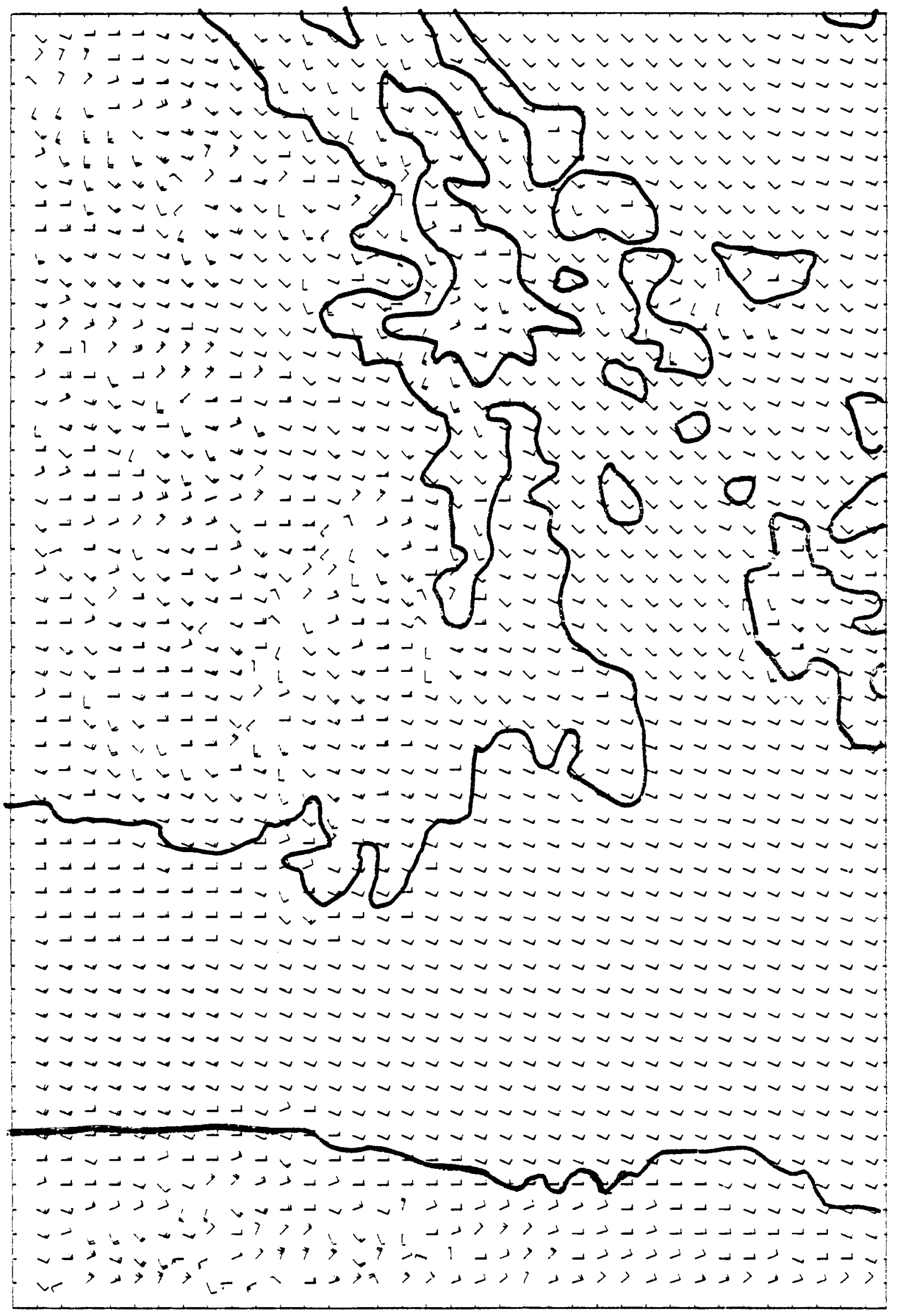




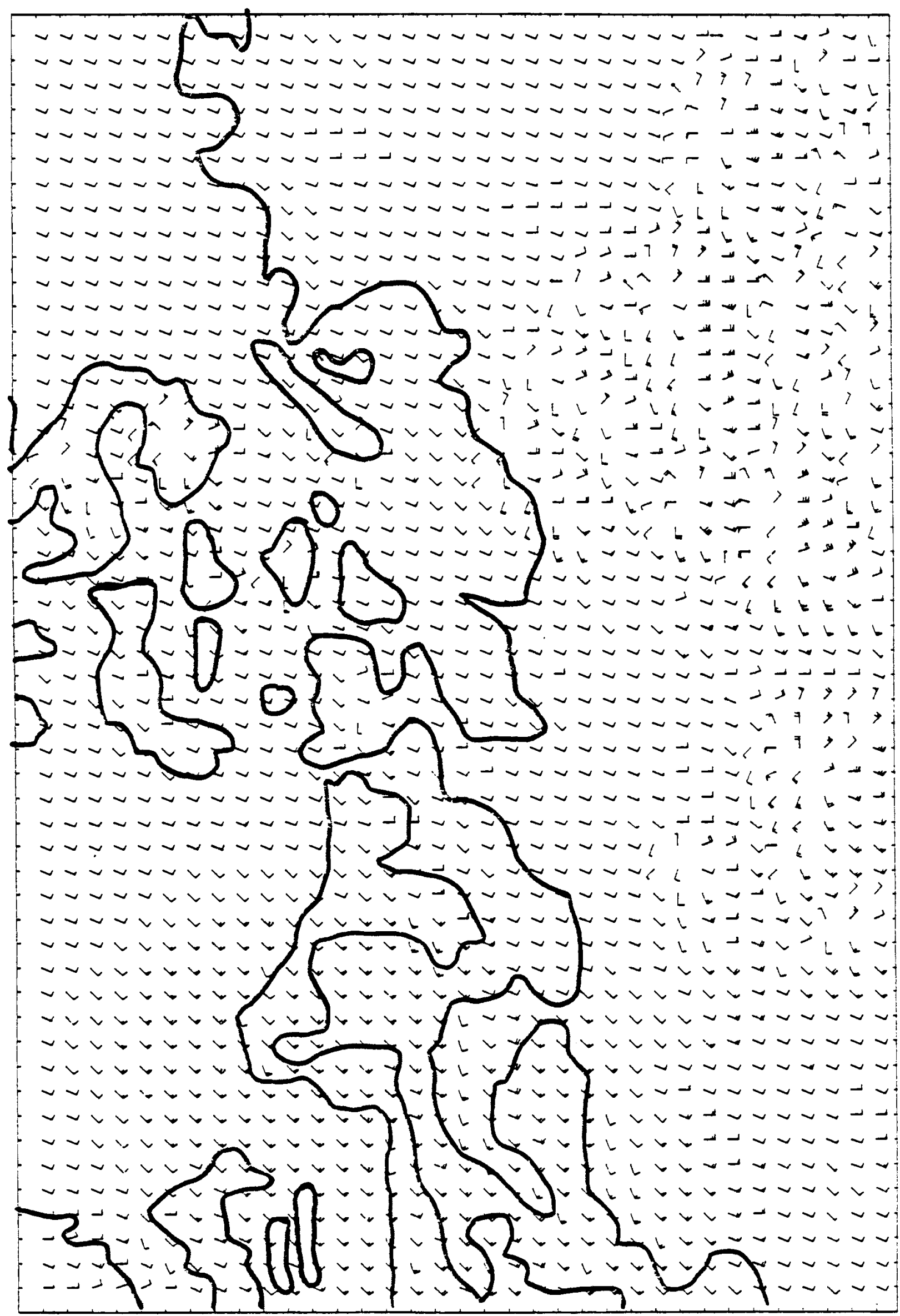


Figure E4. Windfield Analysis of Northern Puget Sound Study Area for January 3,1975 at 1500 PST. Background flow input: 67.5 degrees at 3.5 meters per second (\rangle$)$.

$$
\text { Wind Flag codes: } \begin{array}{r}
\frac{\mathrm{m} / \mathrm{s}}{5.0} \\
2.0 \\
7 \begin{array}{l}
1.0 \\
<1.0
\end{array}
\end{array}
$$




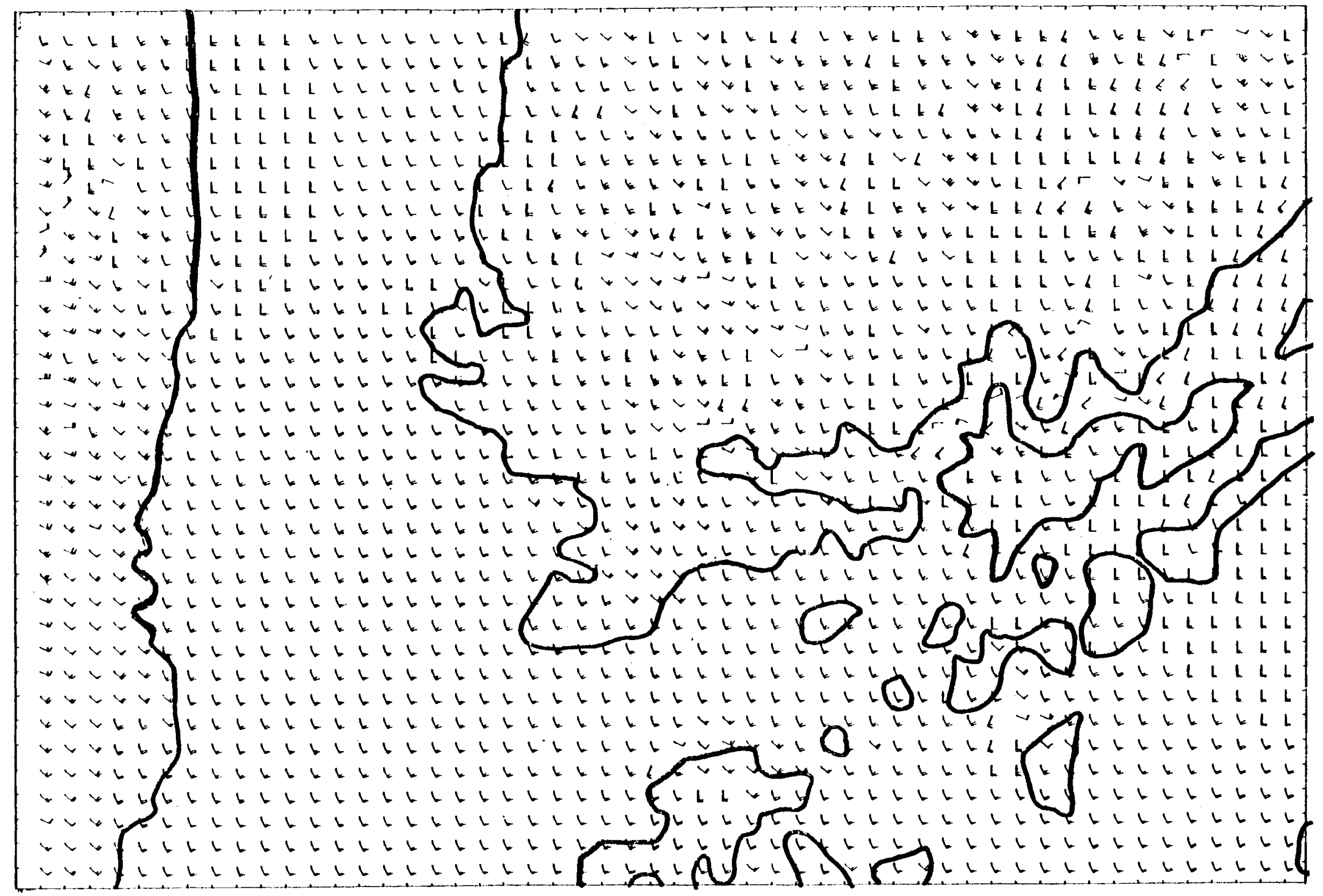




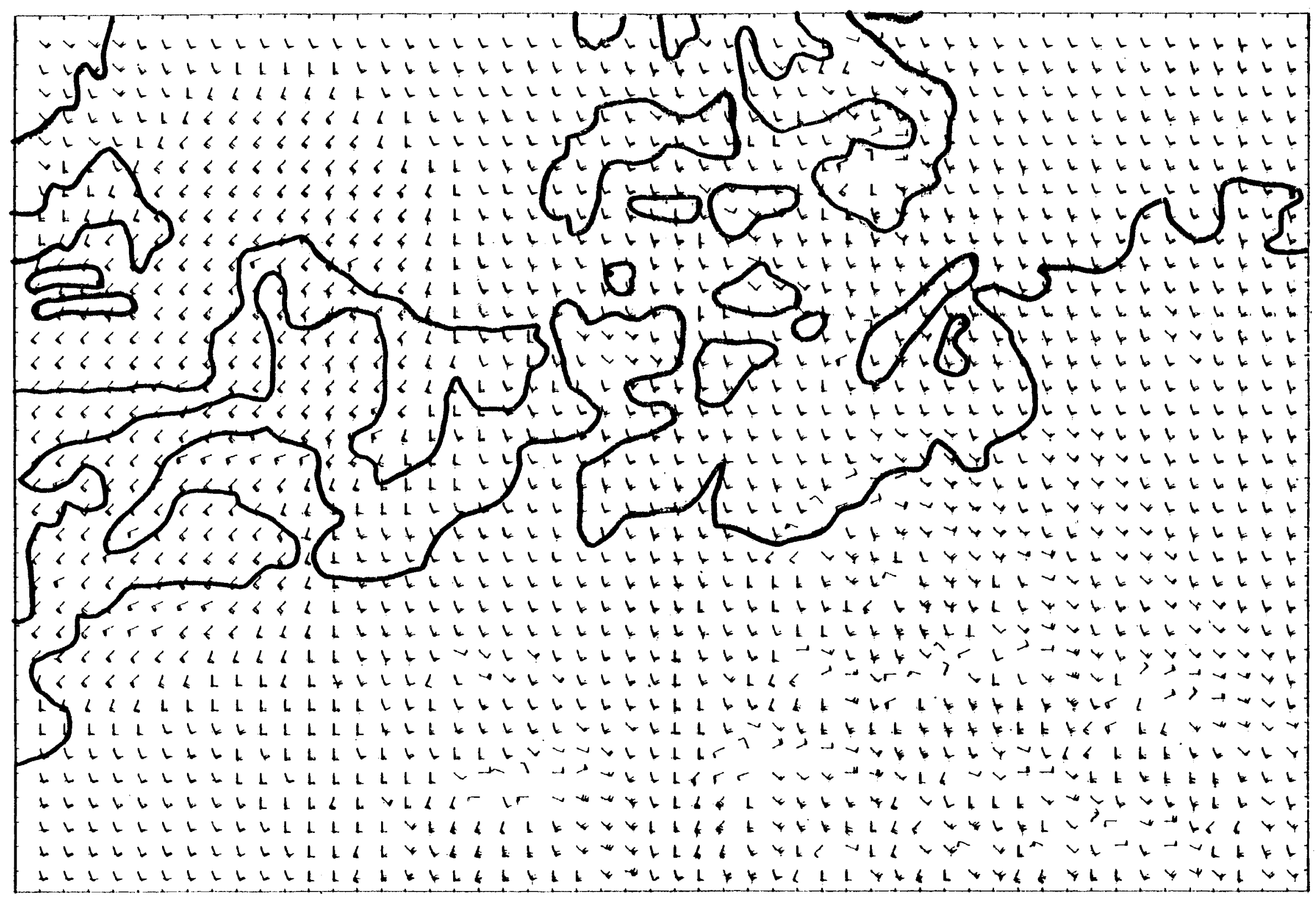




\section{$E-32$}

Figure E5. Windfield Analysis of Northern Puget Sound Study Area for January 3, 1975 at 1900 PST. Background flow input: 90.0 degrees at 4.0 meters per second $(-b)$.

$$
\text { Wind Flag Codes: } \begin{array}{r}
\frac{\mathrm{m} / \mathrm{s}}{5.0} \\
2.0 \\
7.0 \\
\downarrow \quad<1.0
\end{array}
$$




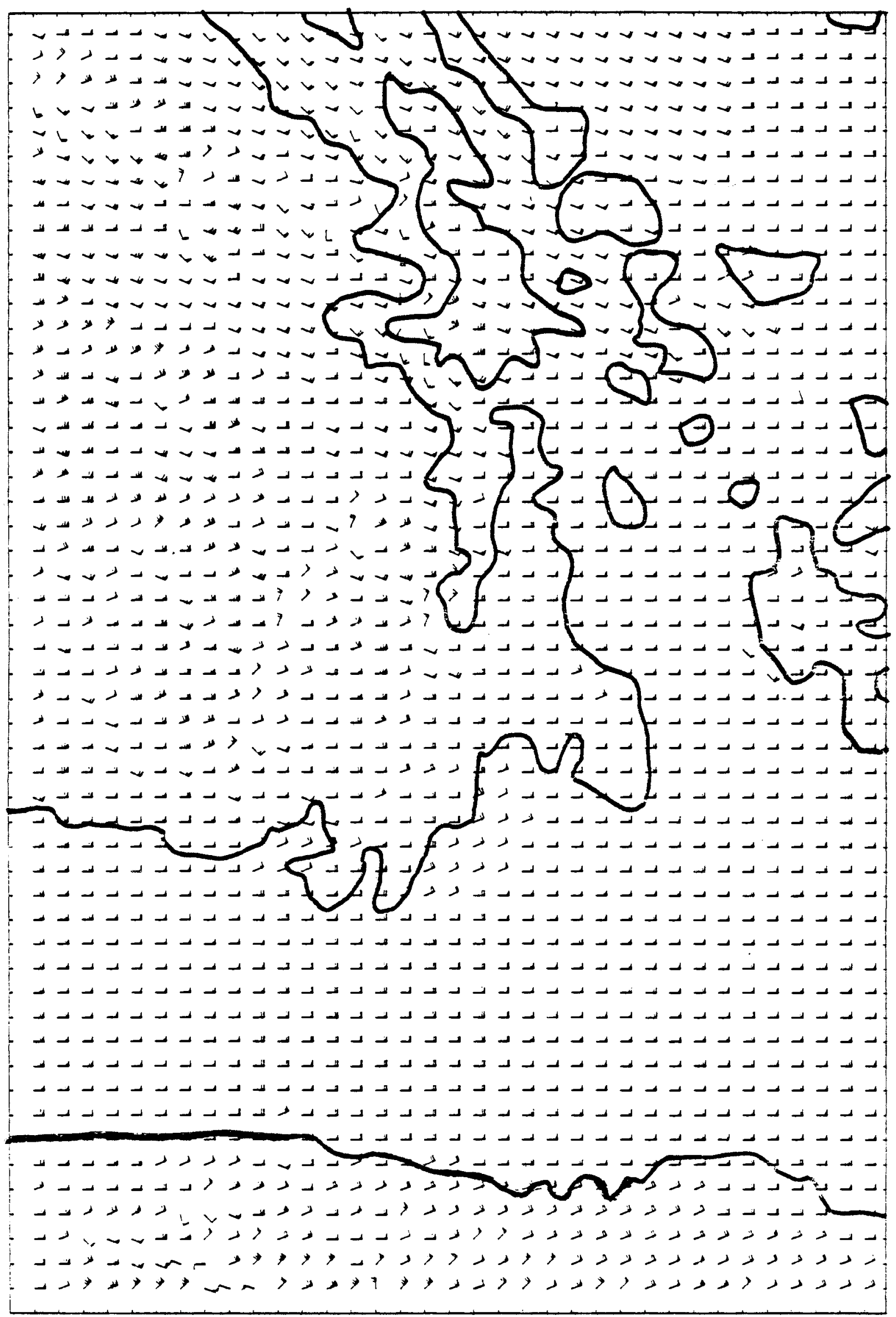




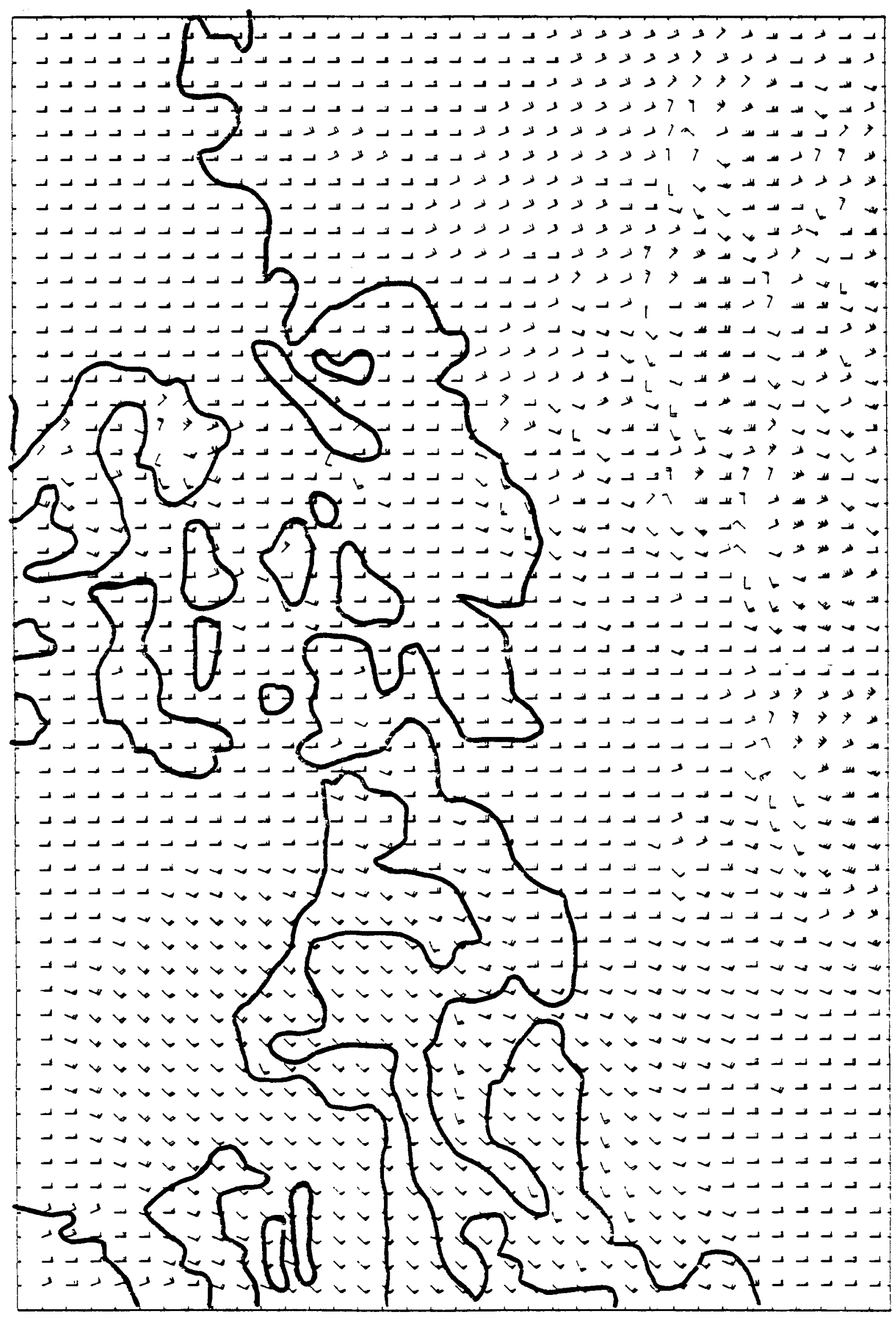


Figure E6. Windfield Analysis of Northern Puget Sound Study Area for January 3, 1975 at 2300 PST. Background flow input: 22.5 degrees at 4.0 meters per second $(7)$.

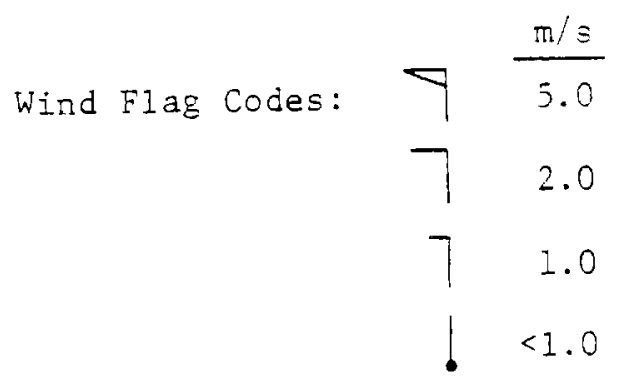




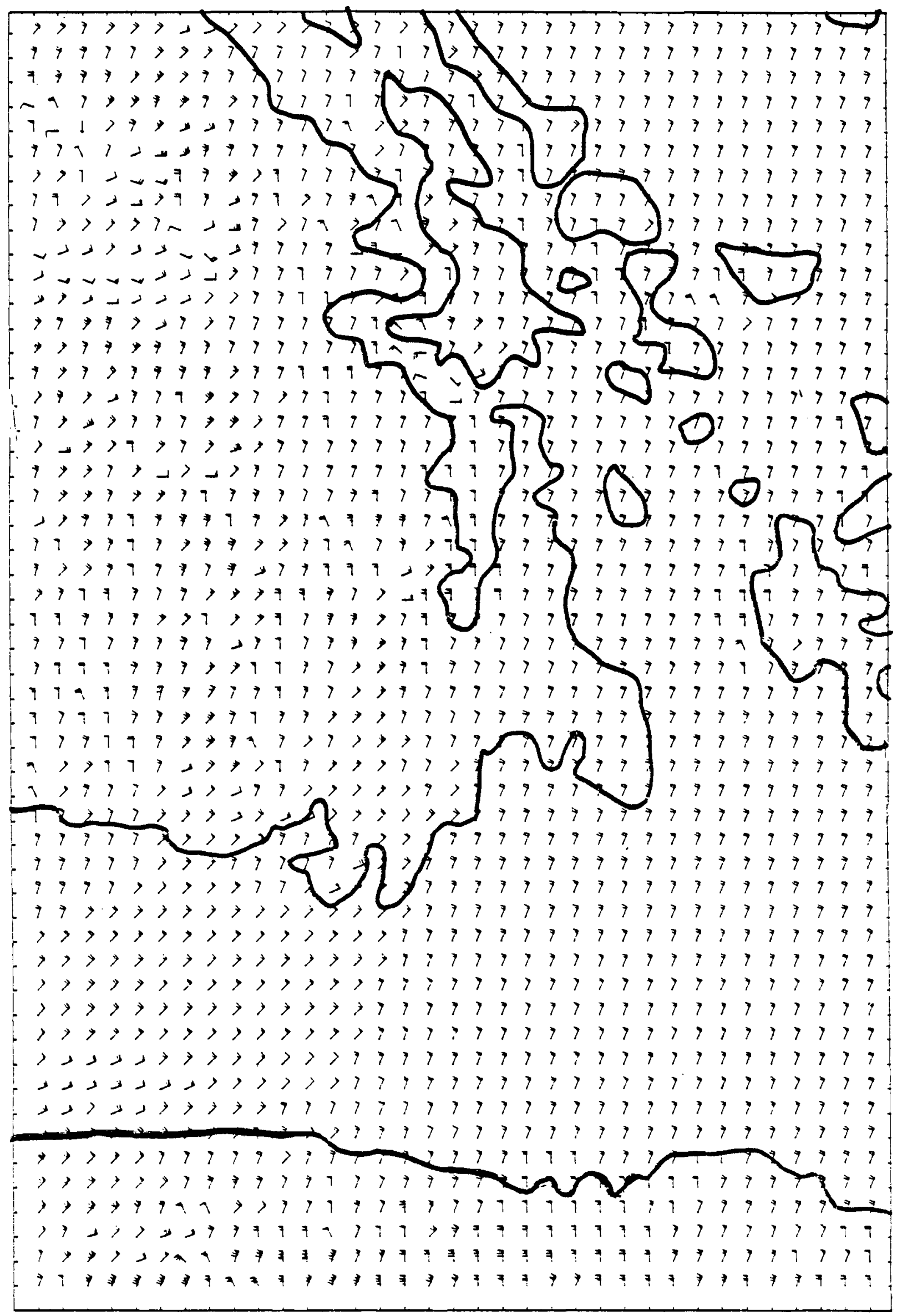




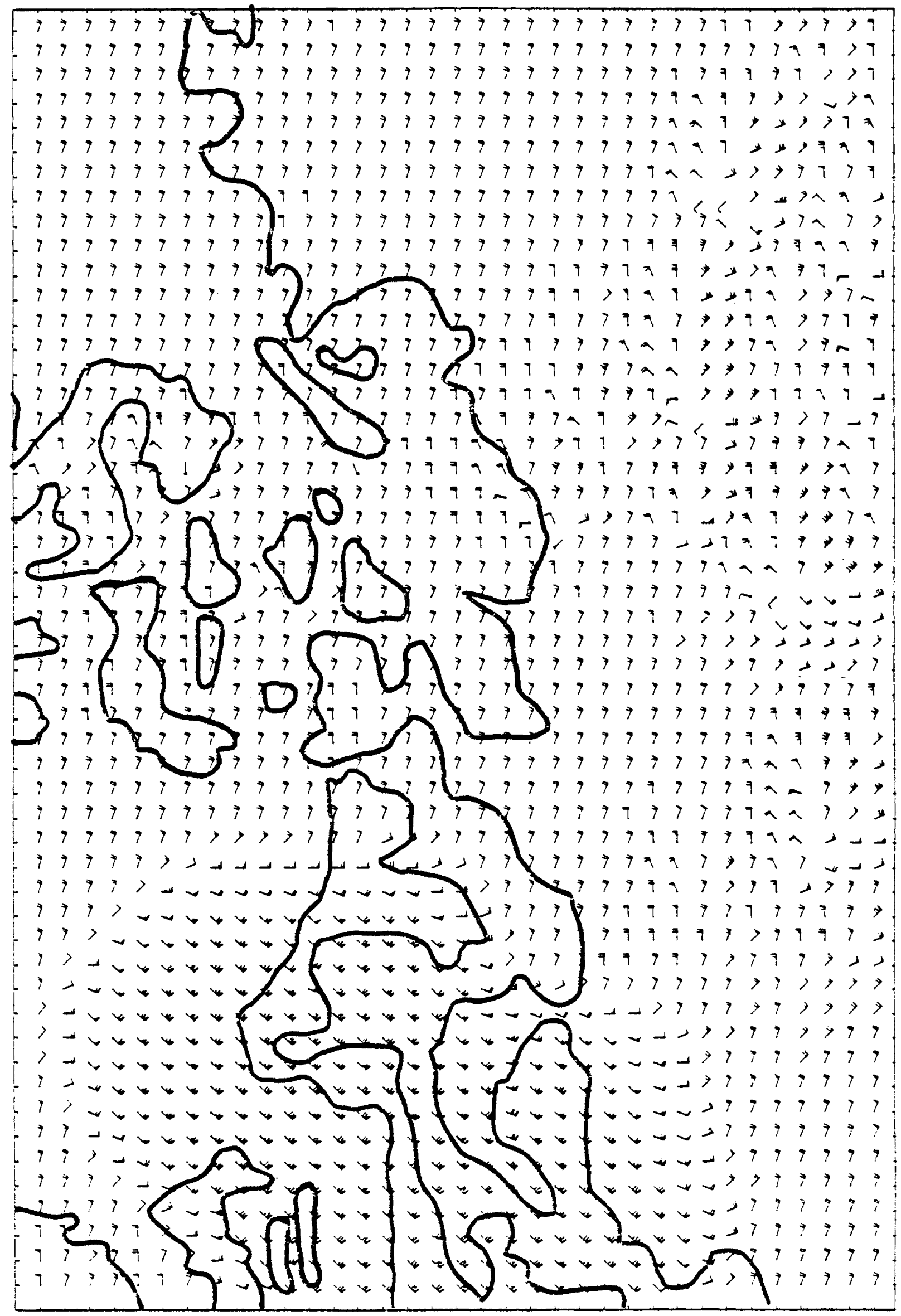




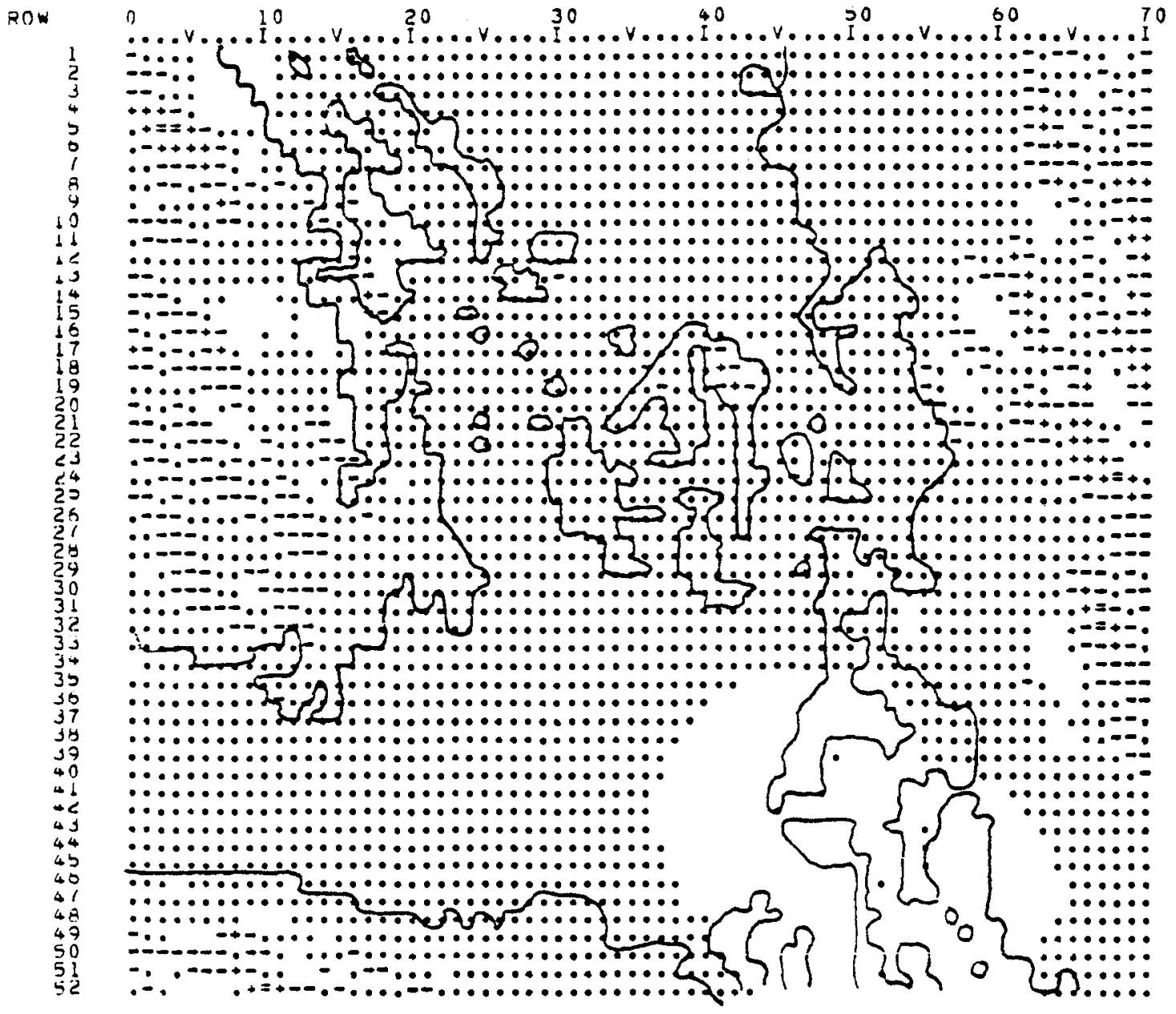

BMSSION RESTRICTION CODES:

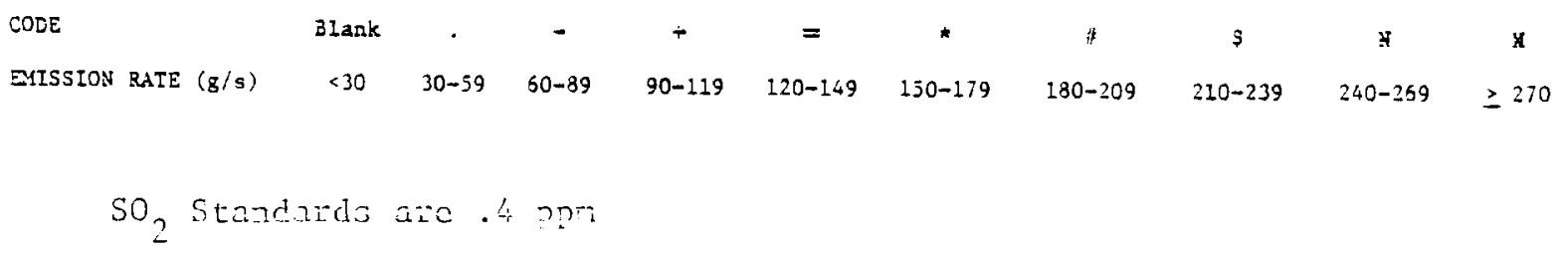


FIGURE ES. PI ( $\pi$ ) YAARIY FOR JANUARY 3, 1975 AT 07:00

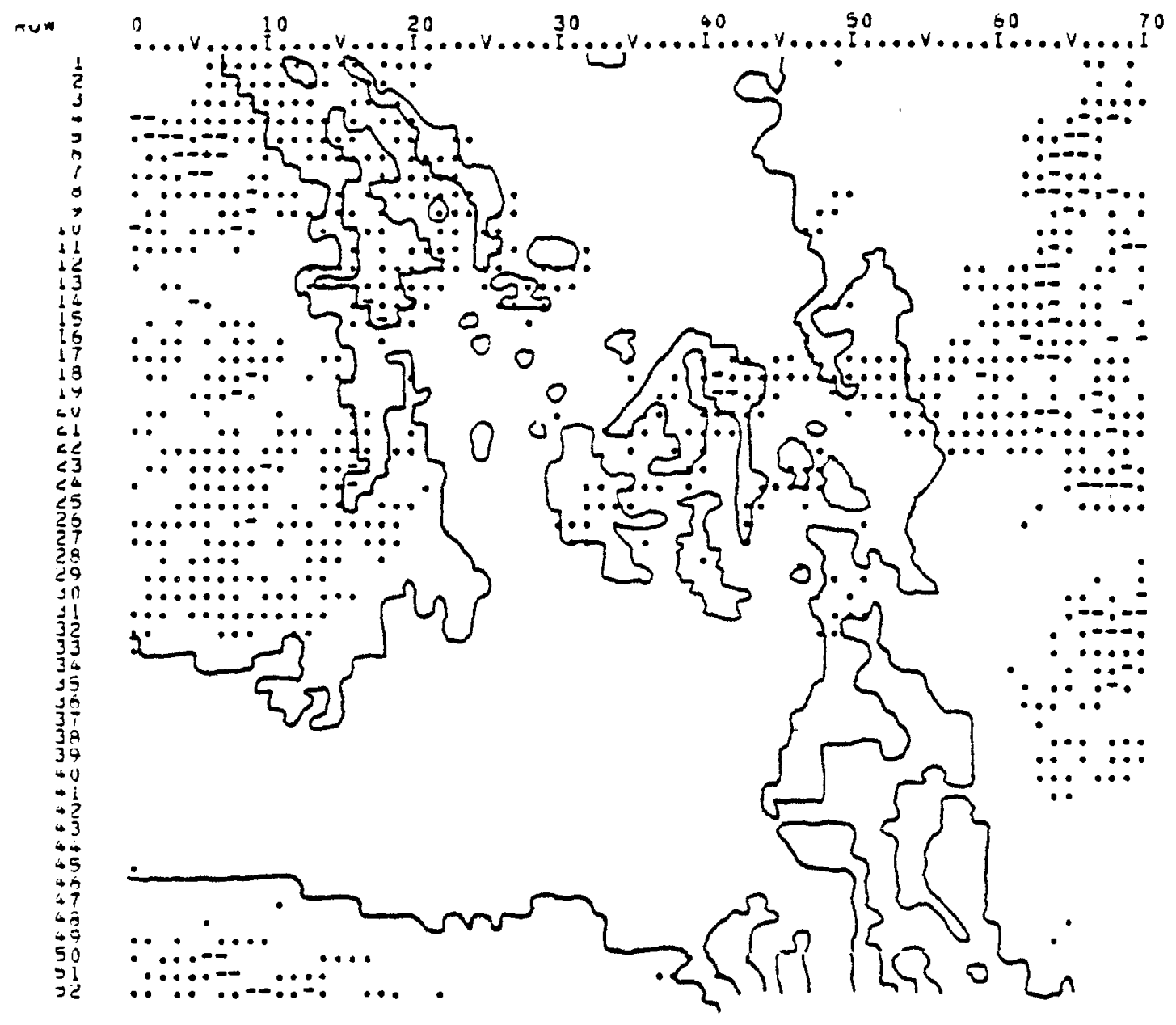

BMTSION RESTRICTION CODES:

CODE

31 anix

zuTSTON $3 A T E(g / s)$

$<30 \quad 30-59 \quad 50-39$

$90-1: 9 \quad: 20-149 \quad 150-1 ; 9$

$130-200$

3

$3 x$

$\mathrm{SO}_{2}$ Standards are $.4 \mathrm{ppm}$ 
FIGURE $89 . \quad$ PI ( $\pi$ ) MATRIX FOR JANUARY 3, 1975 AT 11:00

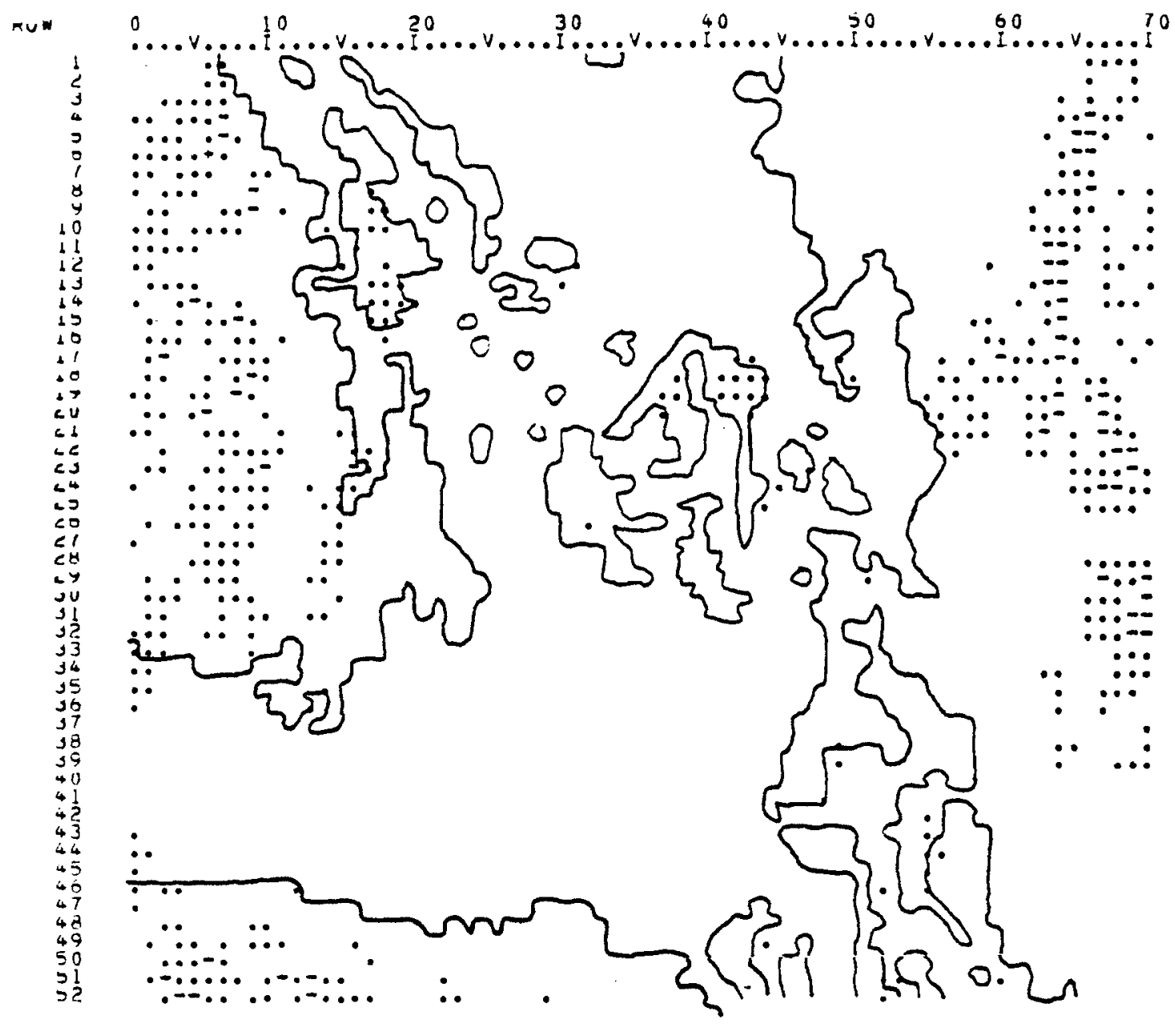

EAISSION RESTRICTION CODES:

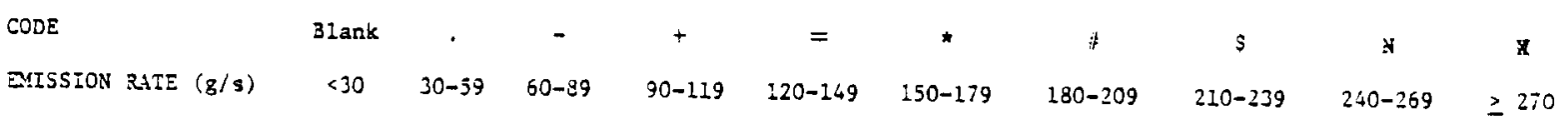


FIGURE E10. PI ( $\pi$ ) MATRIX FOR JANUARY 3, 1975 AT 15:00

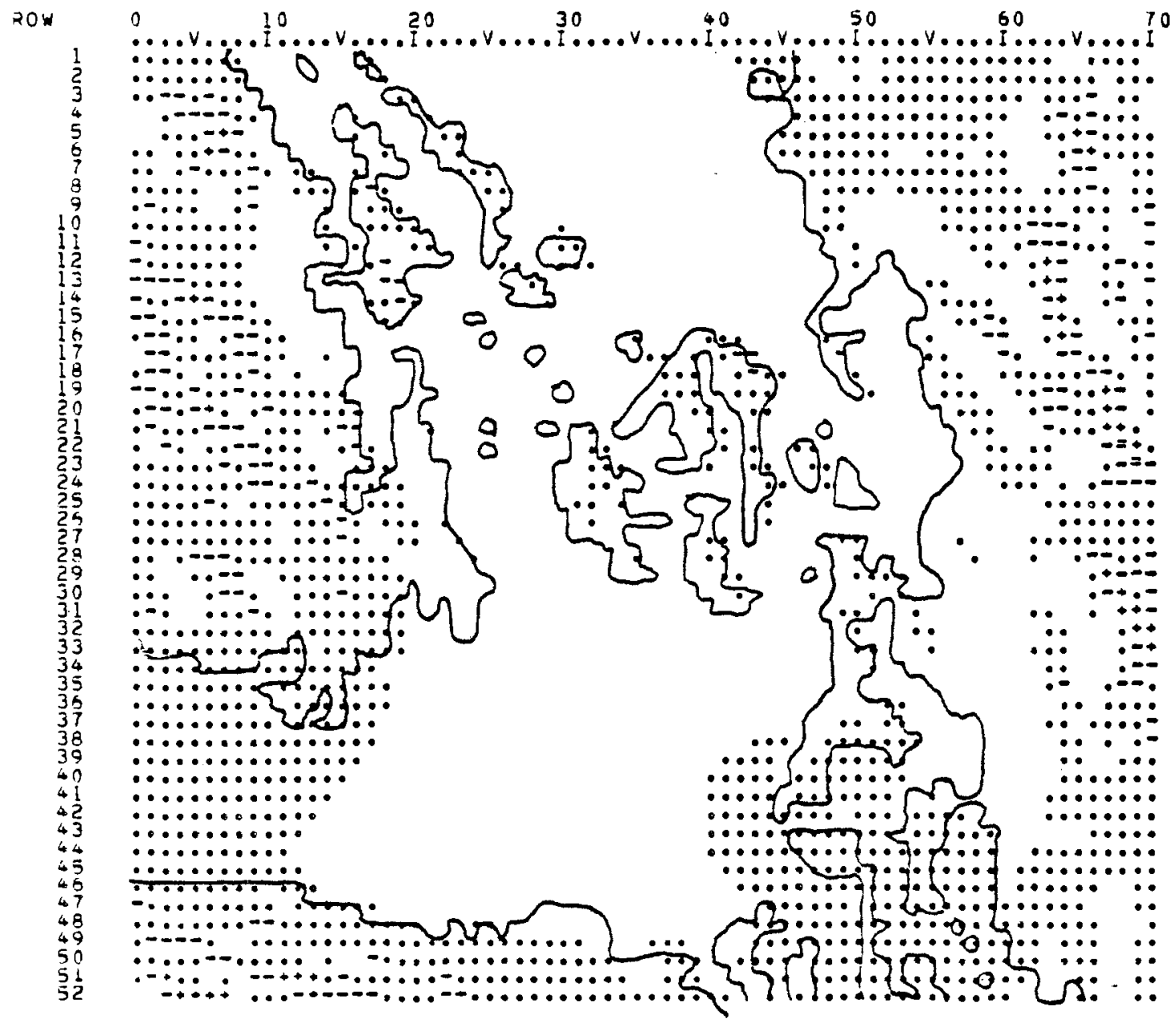

EMISSION RESTRICTION CODES:

\begin{tabular}{|c|c|c|c|c|c|c|c|c|c|c|}
\hline CODE & Blank & . & - & + & $=$ & * & * & s & $\forall$ & $\mathrm{x}$ \\
\hline ZIISSION RATE (gis) & $<30$ & $30-59$ & $60-89$ & $90-119$ & $120-149$ & $15 c-179$ & $180-209$ & $210-239$ & $240-269$ & $\geq 270$ \\
\hline
\end{tabular}

$\mathrm{SO}_{2}$ Standards are $.4 \mathrm{ppm}$ 
FIGURE E11. PI ( $\pi$ ) MATRIX FOR JANUARY 3, 1975 AT 19:00

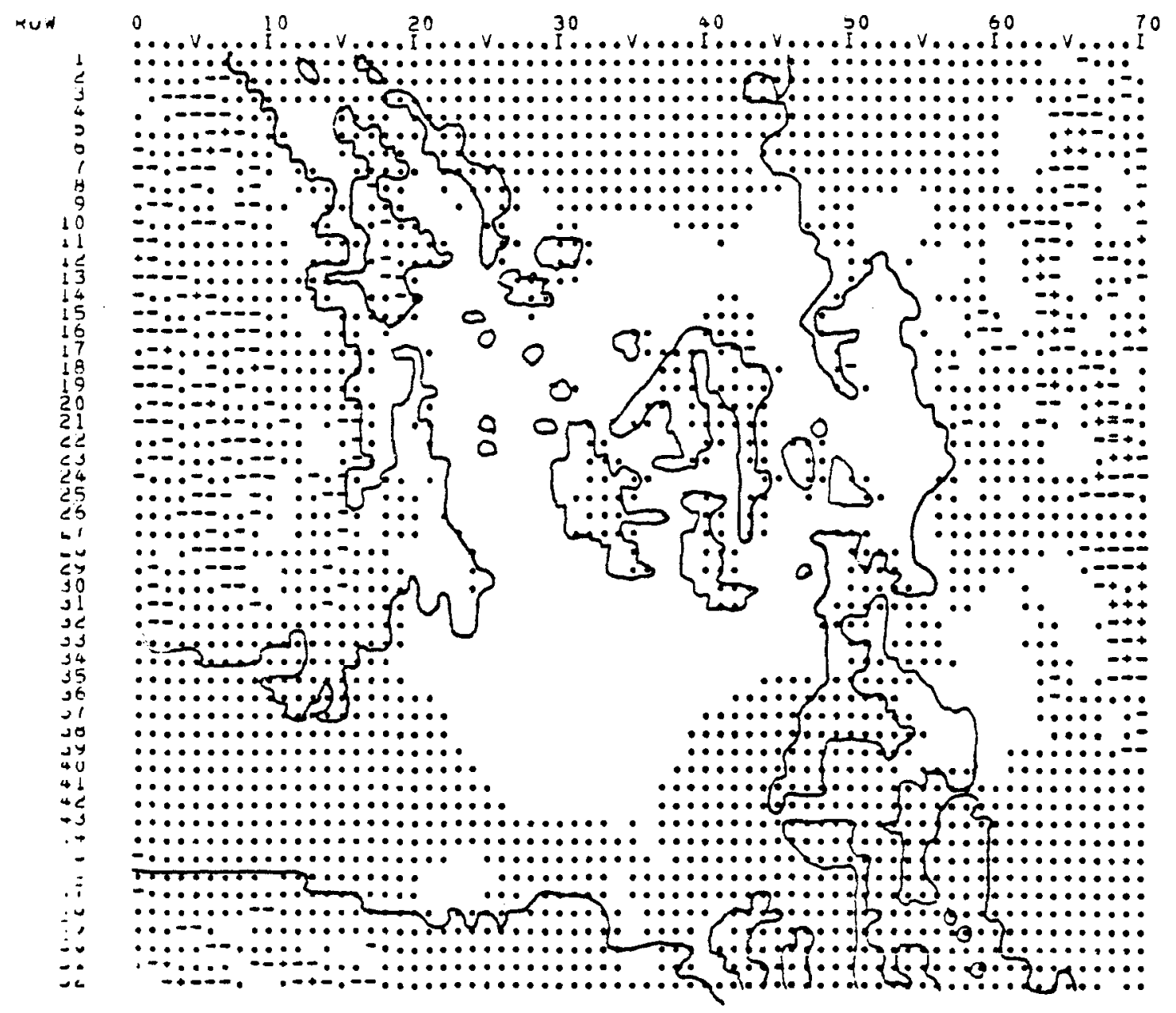

EMISSION RESTRICTION CODES:

\begin{tabular}{|c|c|c|c|c|c|c|c|c|c|c|}
\hline CODE & BIank & . & - & + & $=$ & $\star$ & $;$ & $s$ & 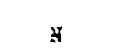 & $x$ \\
\hline ZIISSION RATE $(\mathrm{g} / \mathrm{s})$ & $<30$ & $30-59$ & $60-89$ & $90-119$ & $120-149$ & $150-179$ & $180-209$ & $210-239$ & $240-269$ & \\
\hline
\end{tabular}

$\mathrm{SO}_{2}$ Standards are $.4 \mathrm{ppm}$ 


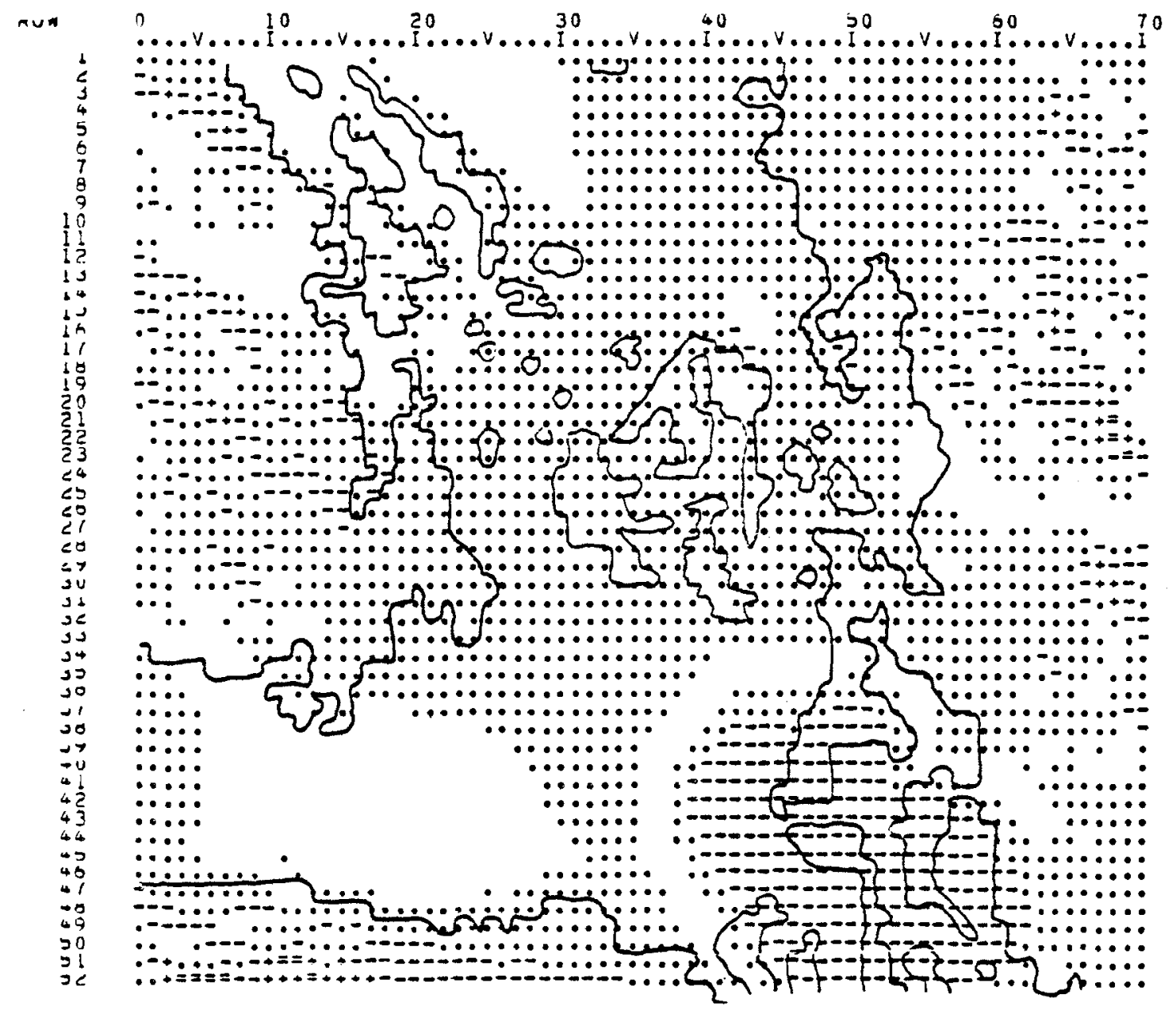

EMISSION RESTRICTION CODES:

\begin{tabular}{|c|c|c|c|c|c|c|c|c|c|c|}
\hline$C O D E$ & Blank & . & - & + & $=$ & * & $*$ & $s$ & $\sharp$ & $x$ \\
\hline IISSION RATE $(\mathrm{g} / \mathrm{s})$ & $<30$ & $30-59$ & $50-89$ & $90-119$ & $120-149$ & $150-179$ & $180-209$ & $210-239$ & $240-269$ & $\geq 270$ \\
\hline
\end{tabular}

$\mathrm{SO}_{2}$ Standards are .4 ppm 


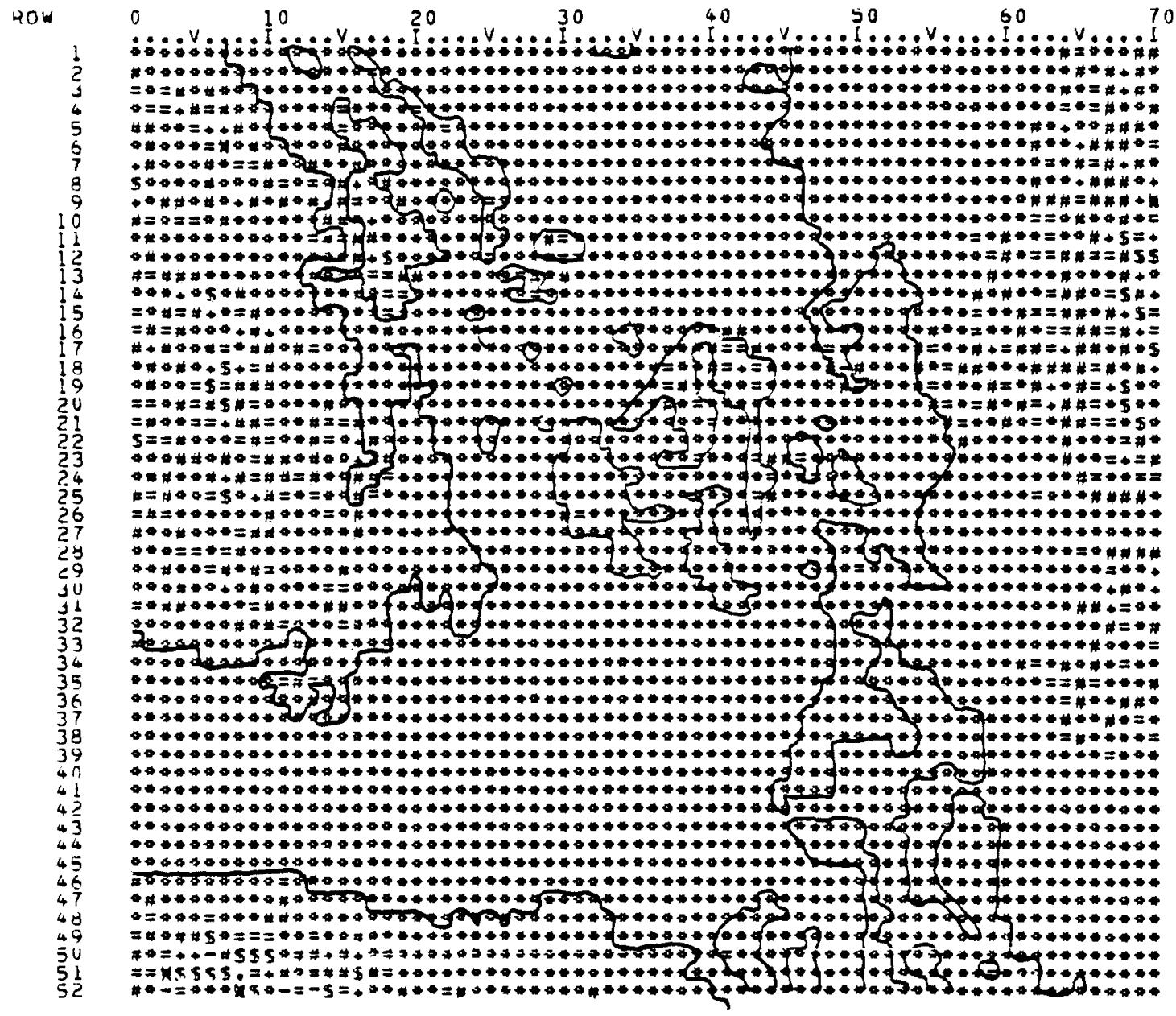

EMISSION RESTRICTTON CODES:

CODE

Blank

$+$

$=$

$\#$

$\$$

x

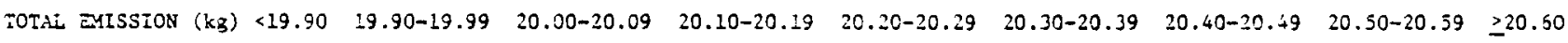

$\mathrm{SO}_{2}$ Standards are $.1 \mathrm{ppm}$ 
Figure E14. Windfield Analysis of Northern Puget Sound Study Area for January 24,1975 at 0300 PST. Background flow input: 180.0 degrees at 3.0 meters per second $(t)$.

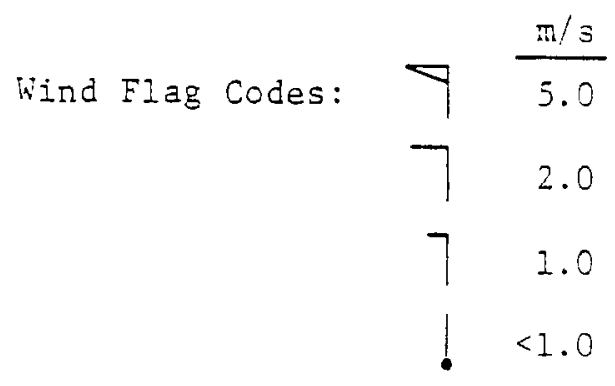




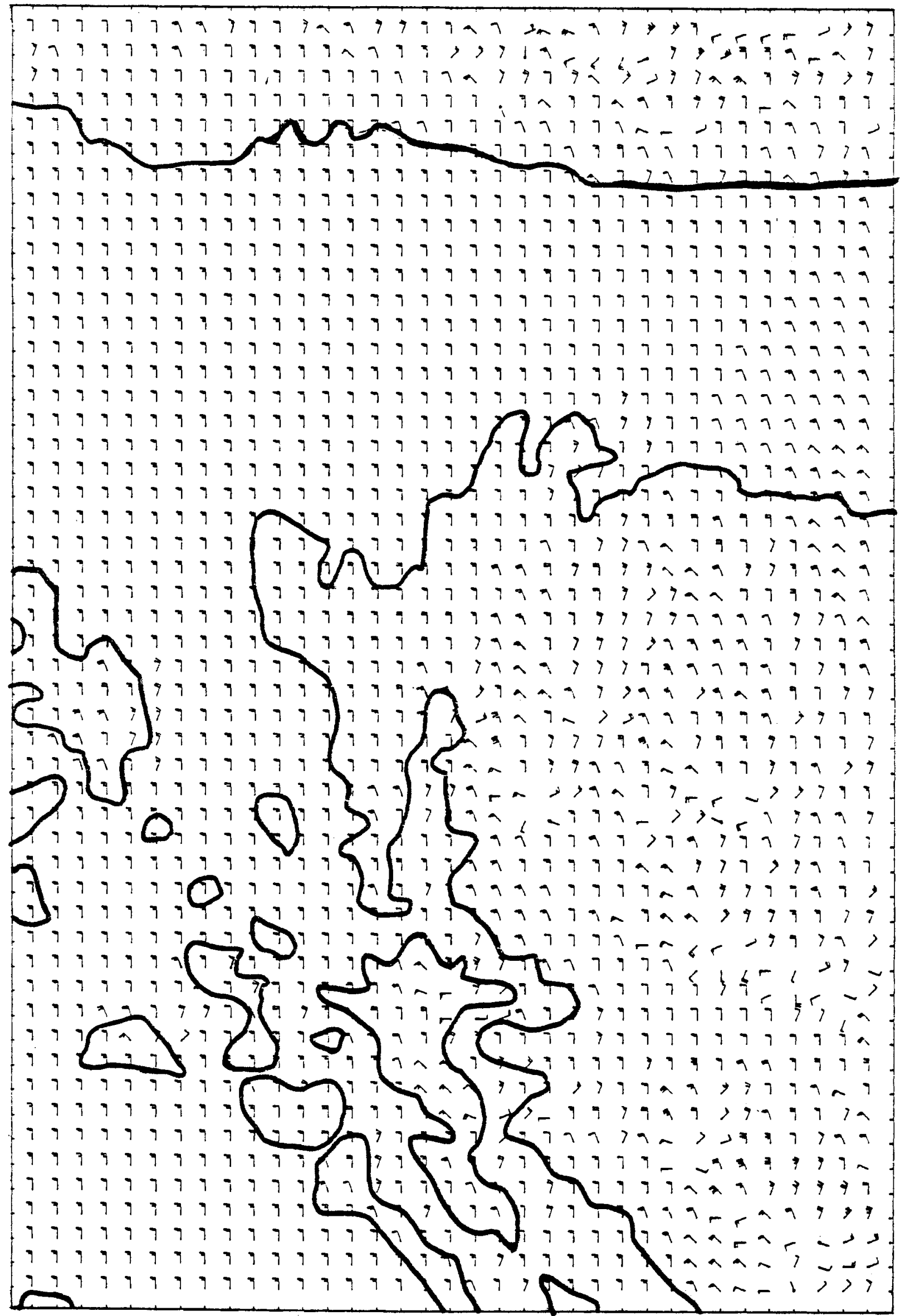

$9+7$ 


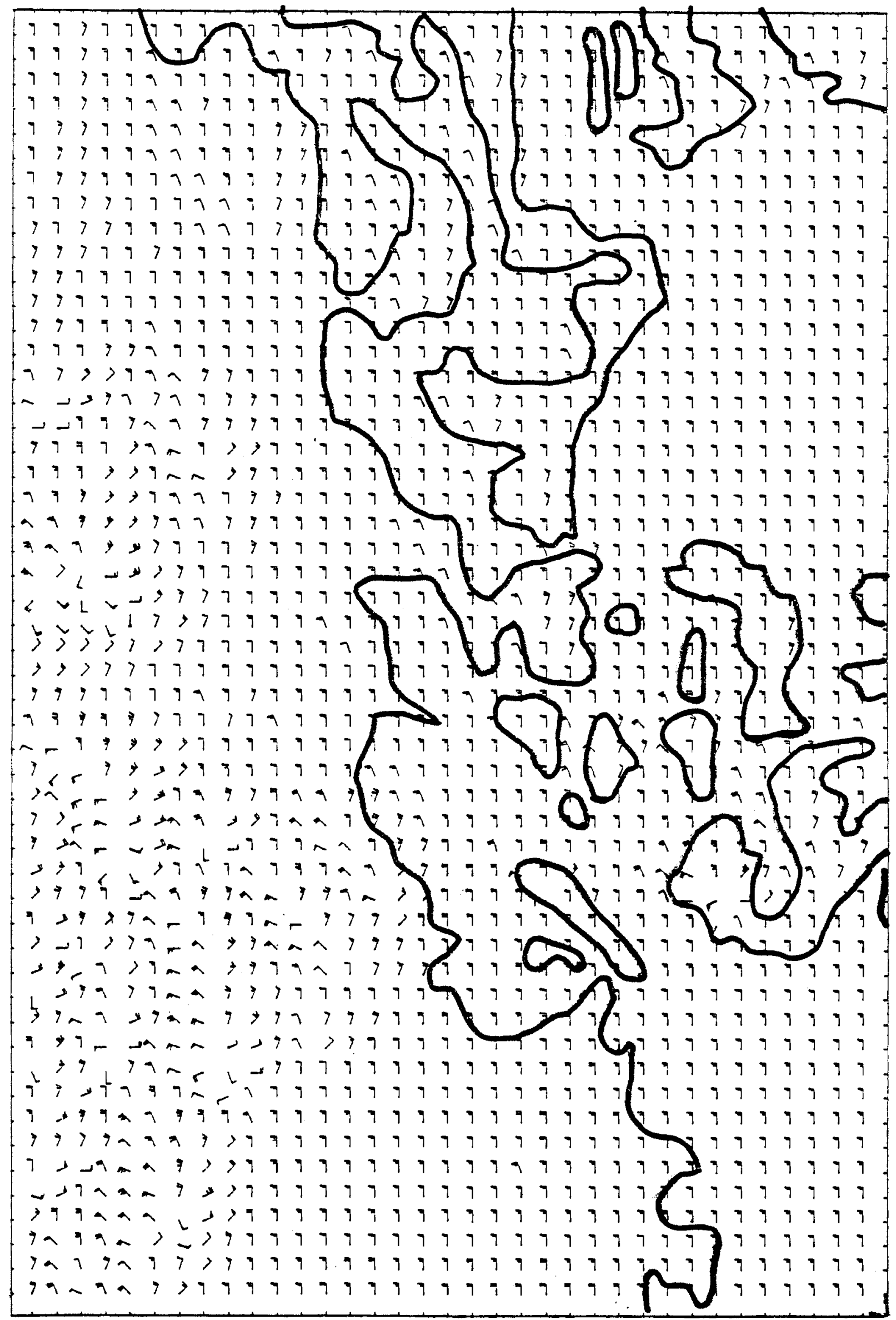


Figure 515. Windfield Analysis of Northern Puget Sound Study Area for January 24, 1975 at 0700 PST. Background flow input: 135.0 degrees at 2.0 meters per second $(V)$.

$$
\text { Wind Flag codes: } \begin{array}{r}
\frac{\mathrm{m} / \mathrm{s}}{5.0} \\
2.0 \\
7.0 \\
\\
\downarrow
\end{array}
$$




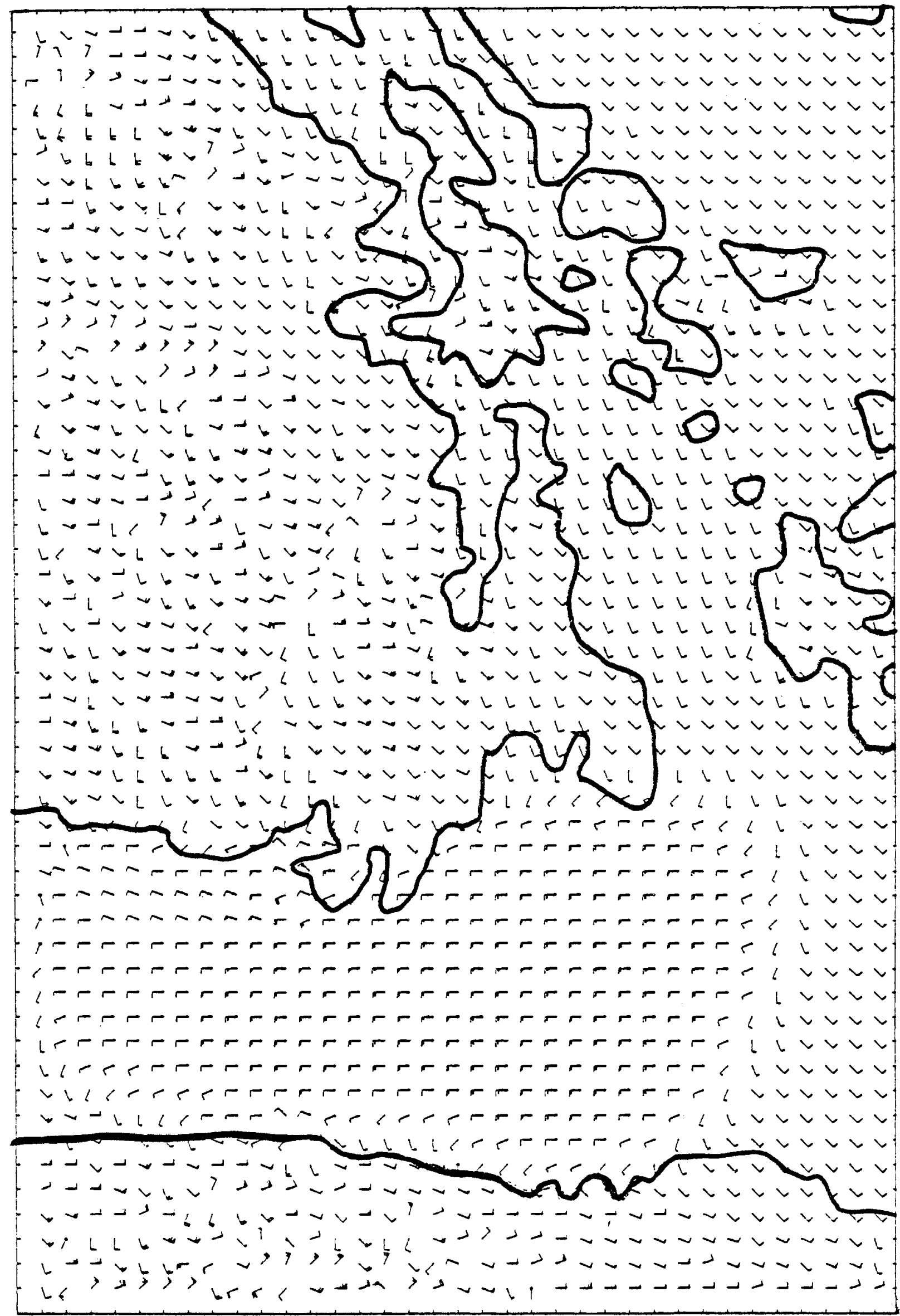




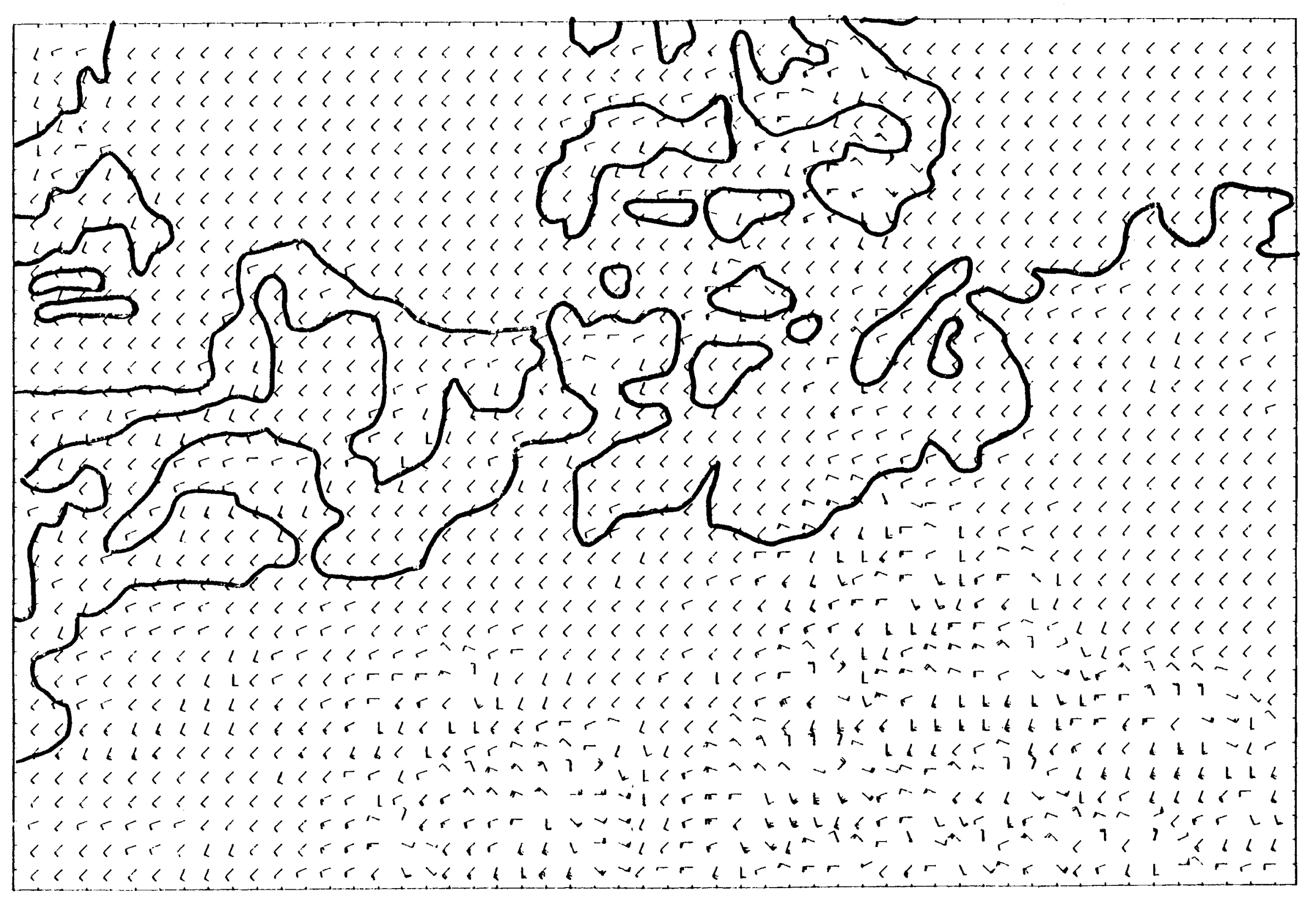


Figure E16. Windfield Analysis of Northern Puget Sound Study Area for January 24, 1975 at 1100 PST. Background flow input: 157.5 degrees at 2.0 meters per second $(L)$.

$$
\text { Wind Flag Codes: } \begin{array}{r}
\frac{\mathrm{m} / \mathrm{s}}{5.0} \\
2.0 \\
1.0 \\
! \quad<1.0
\end{array}
$$




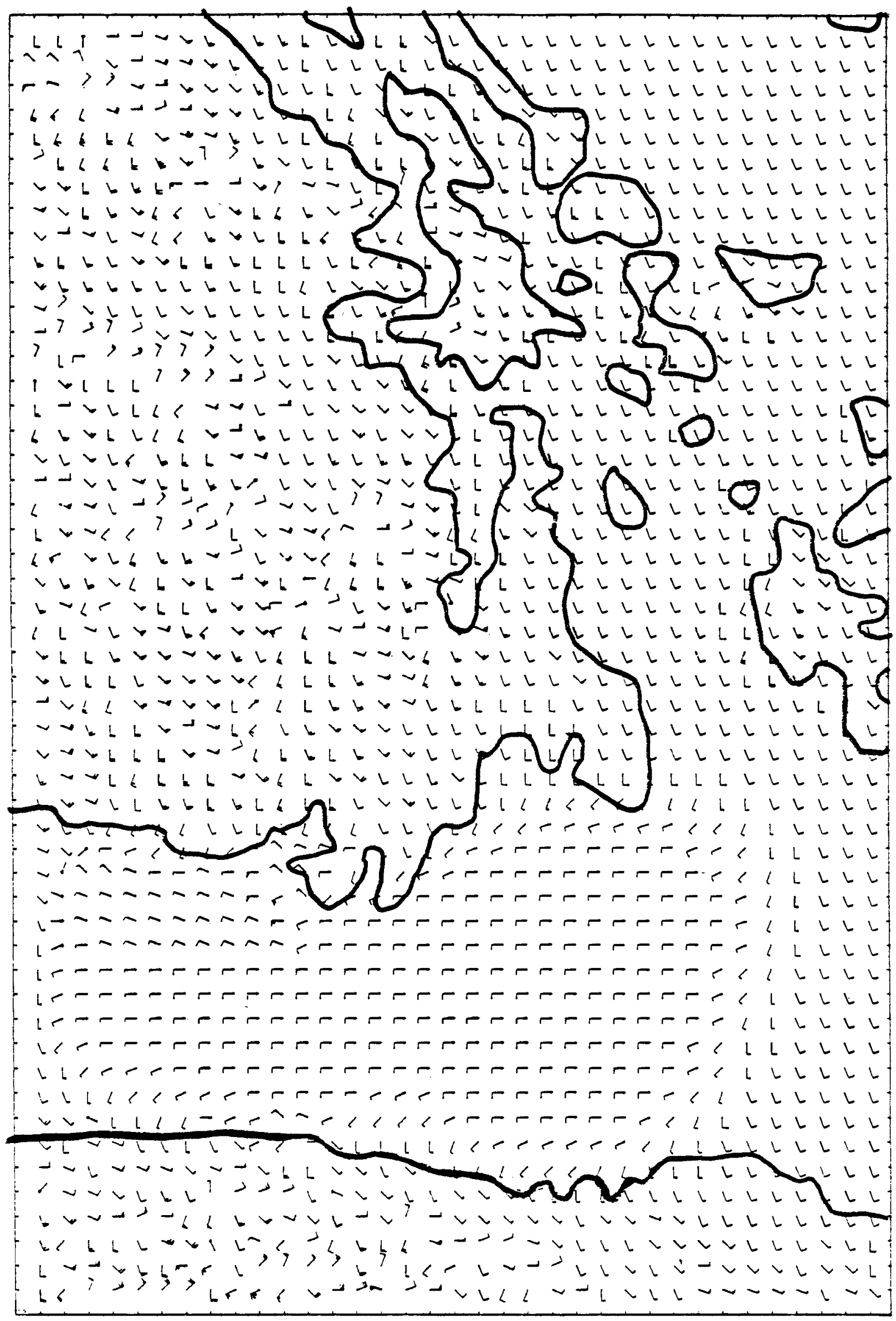


Figure E17. Windfield Analysis of Northern Puget Sound Study Area for January 24, 1975 at 1500 PST. Background flow input: 180.0 degrees at 1.0 meters per second $(L)$.

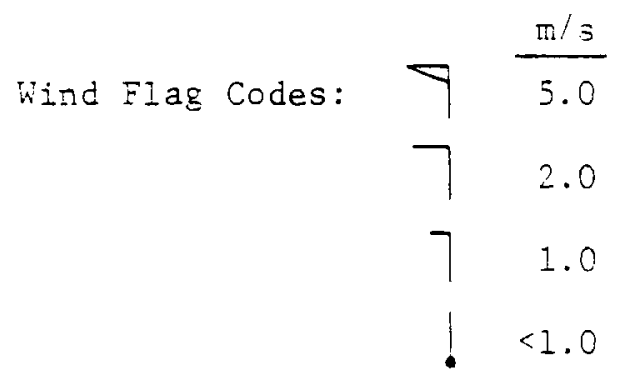




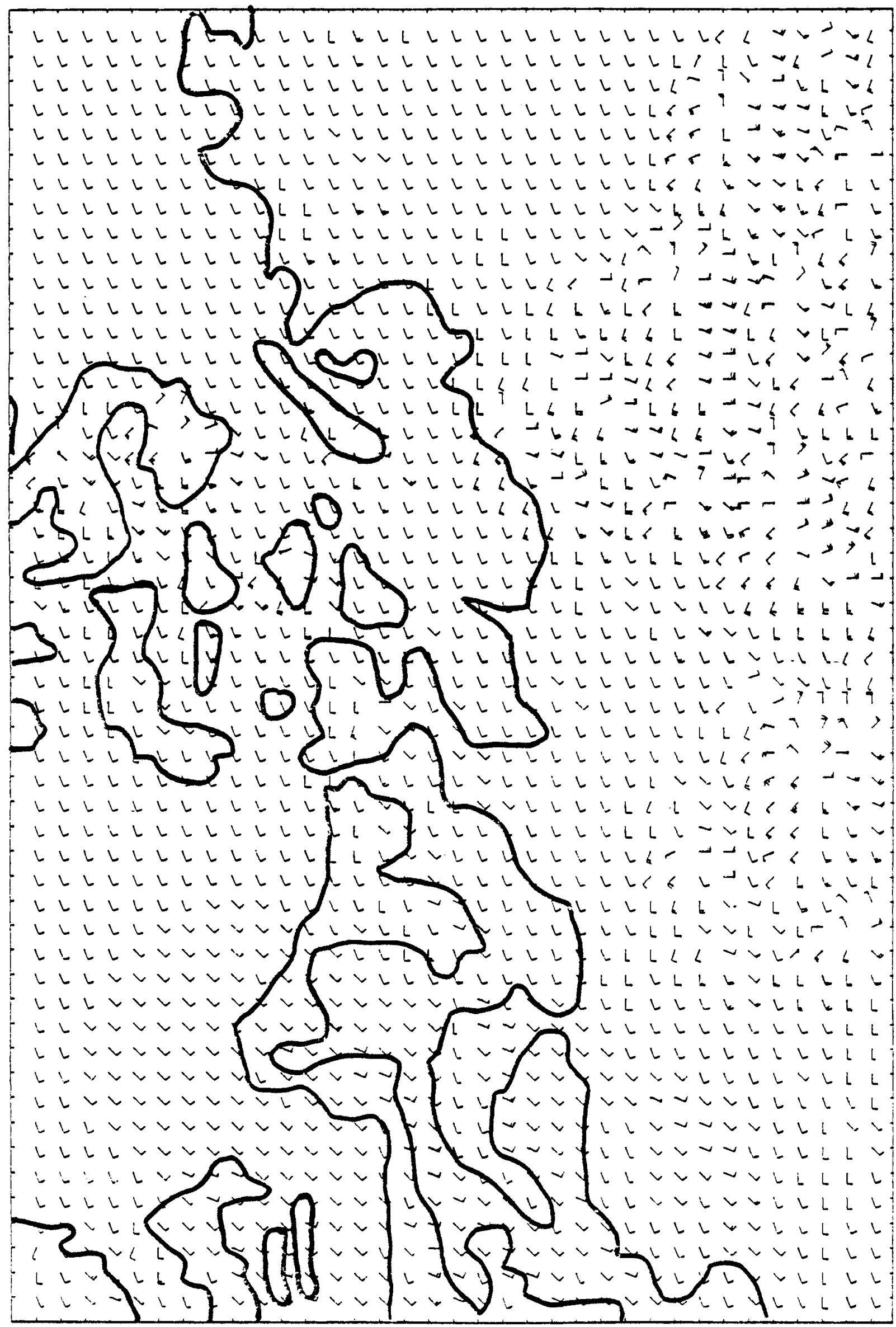




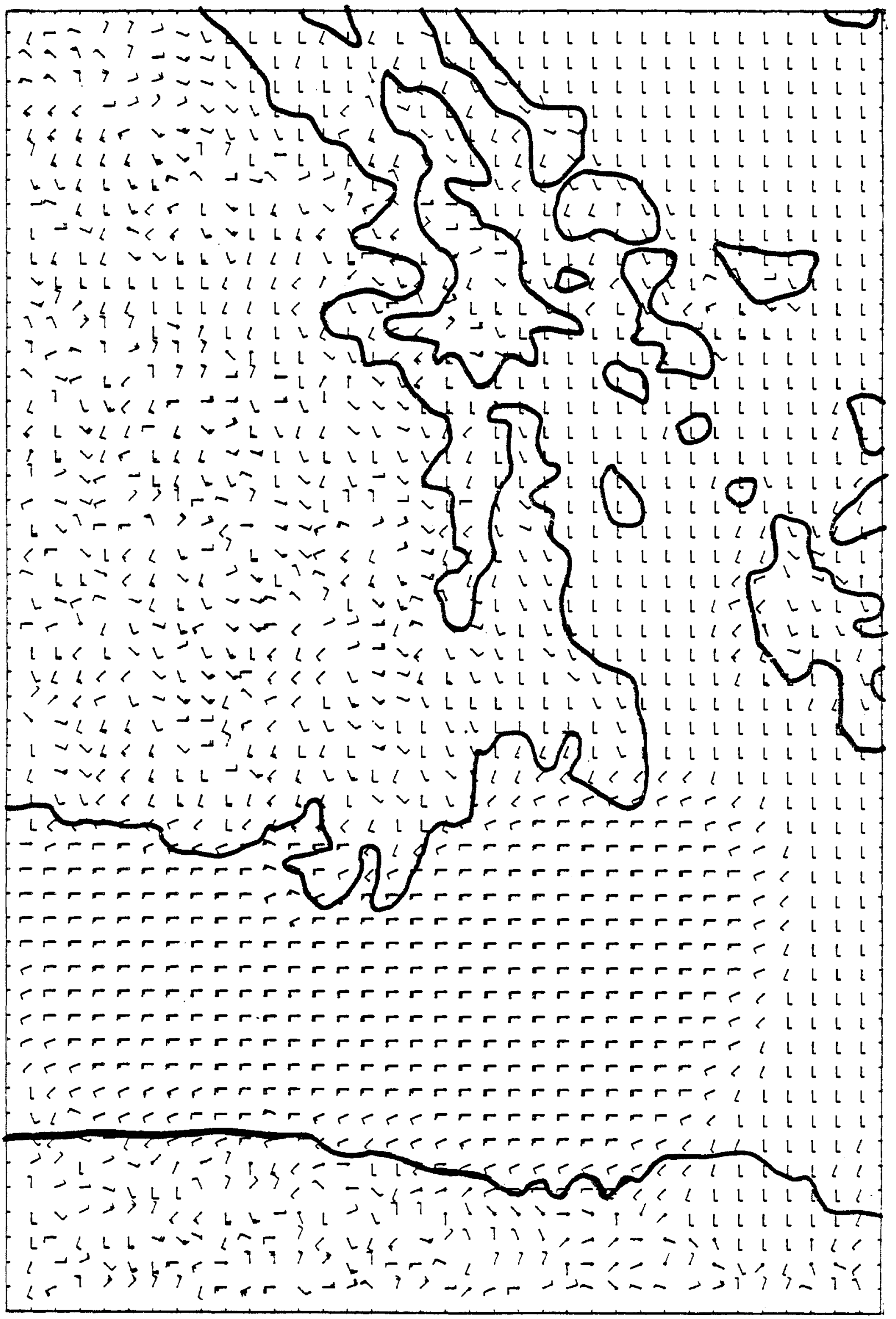




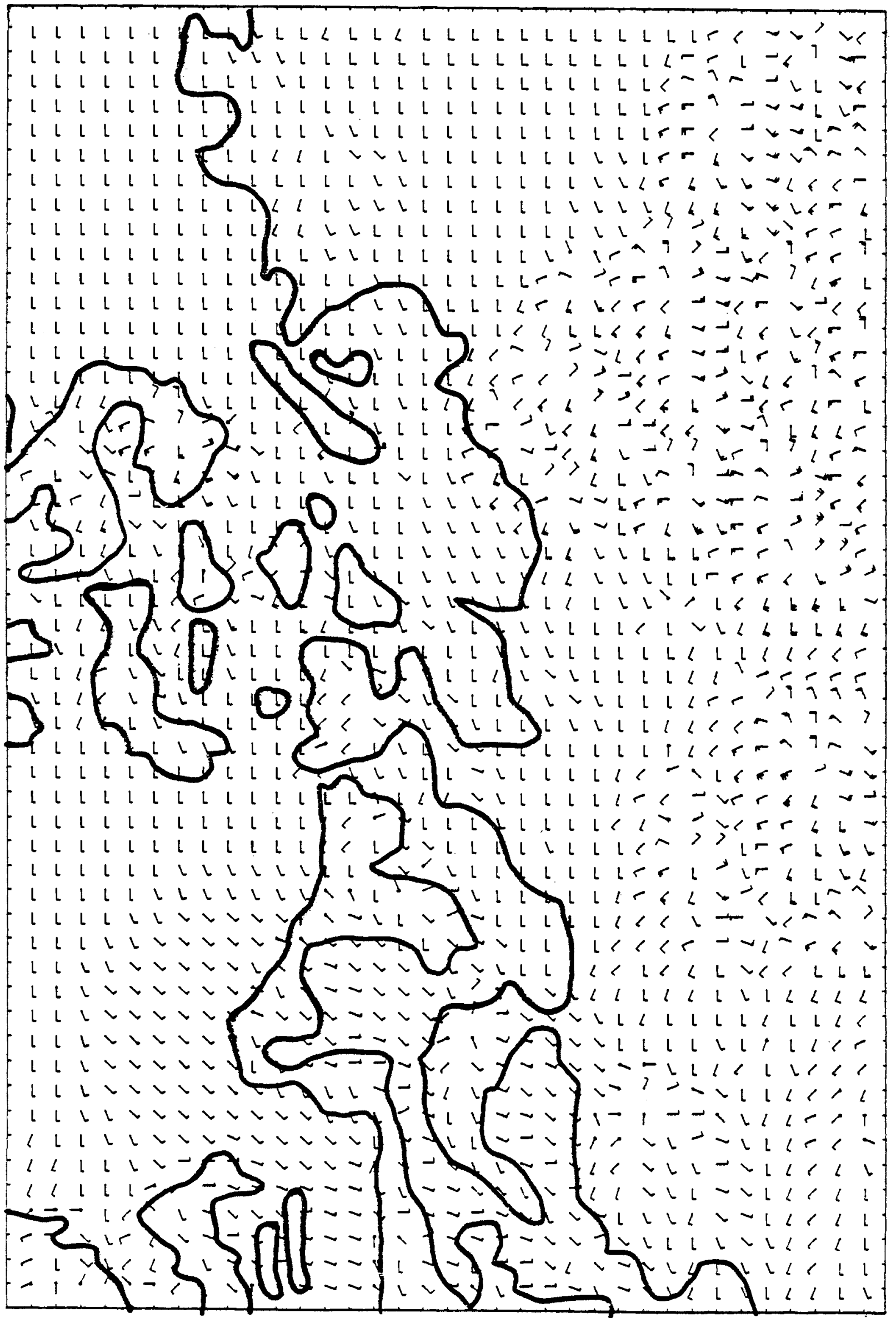


Figure E18. Windfield Analysis of Northern Puget Sound Study Area for January 24, 1975 at 1900 PST. Background flow input: 225.0 degrees at 1.0 meters per second $(<)$.

Wind Flag Codes: $\begin{array}{r}\frac{\mathrm{m} / \mathrm{s}}{5.0} \\ 2.0 \\ 7 \\ ! \quad<1.0\end{array}$




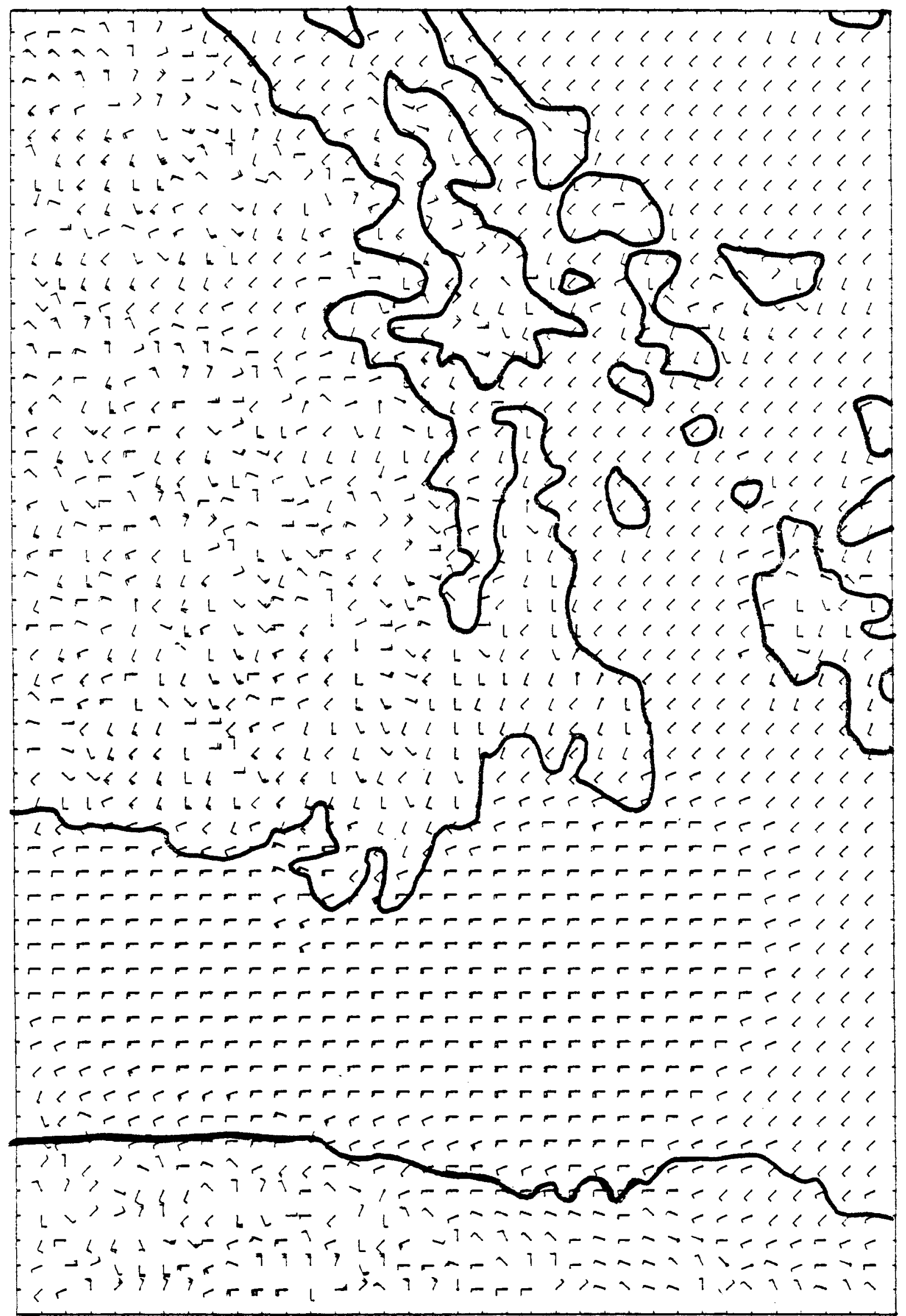




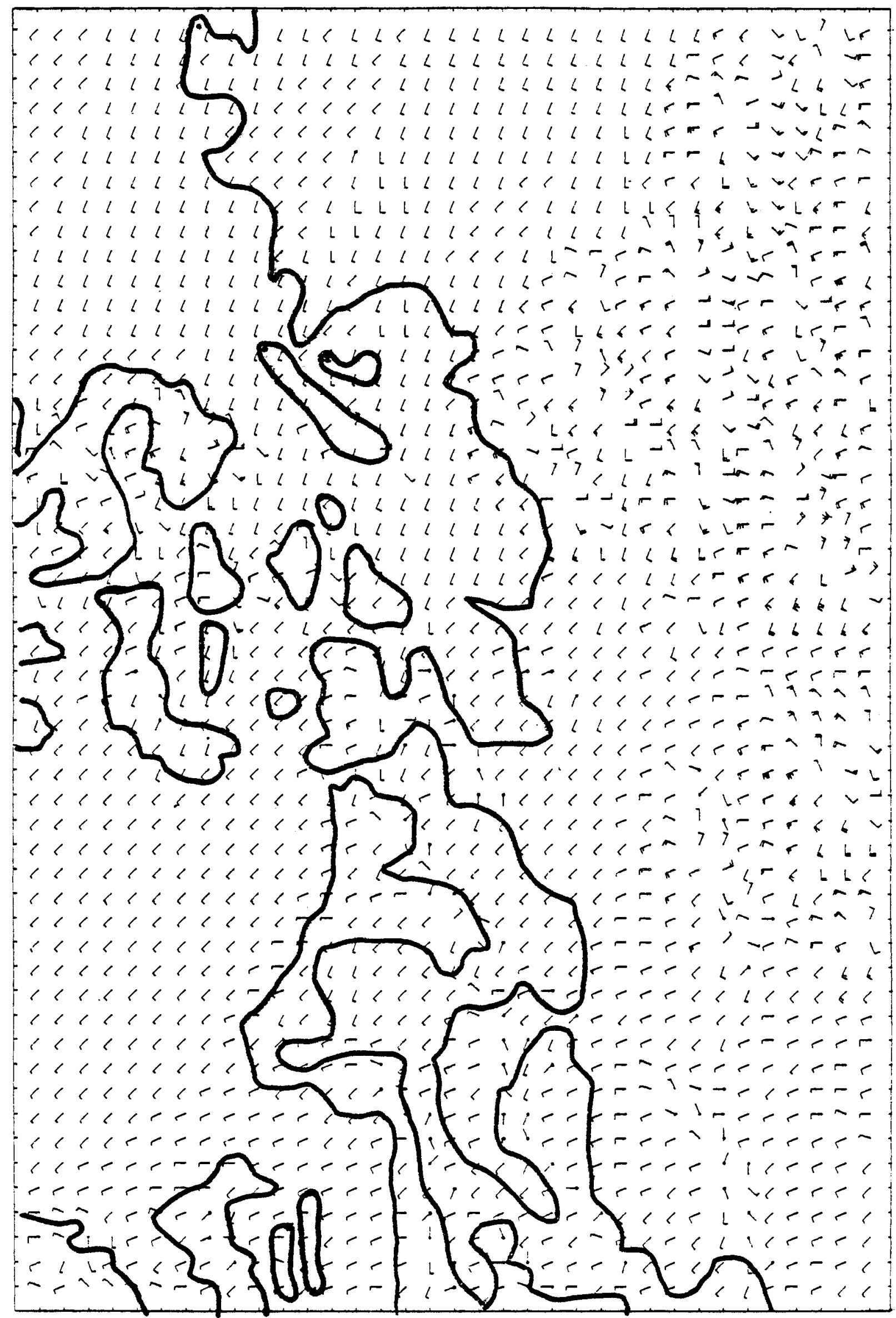


Figure E19. Windfield Analysis of Northern Puget Sound Study Area for January 24, 1975 at 2300 PST. Background flow input: 360.0 degrees at 1.0 meters per second $(\Gamma)$.

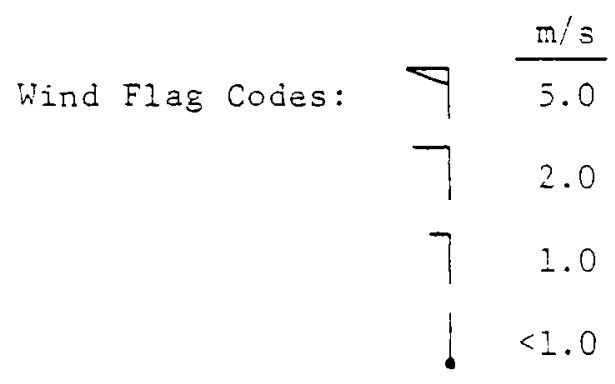




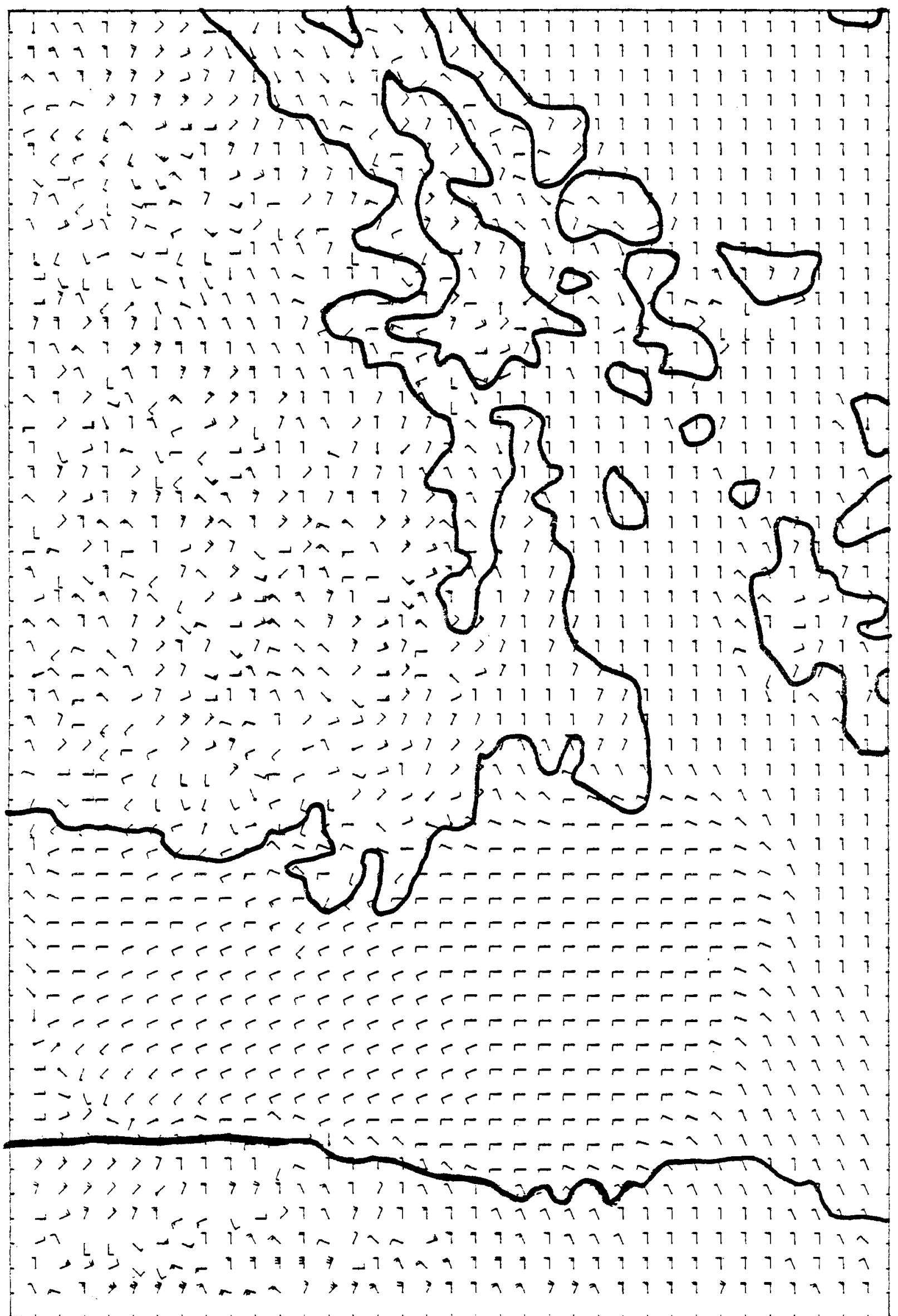




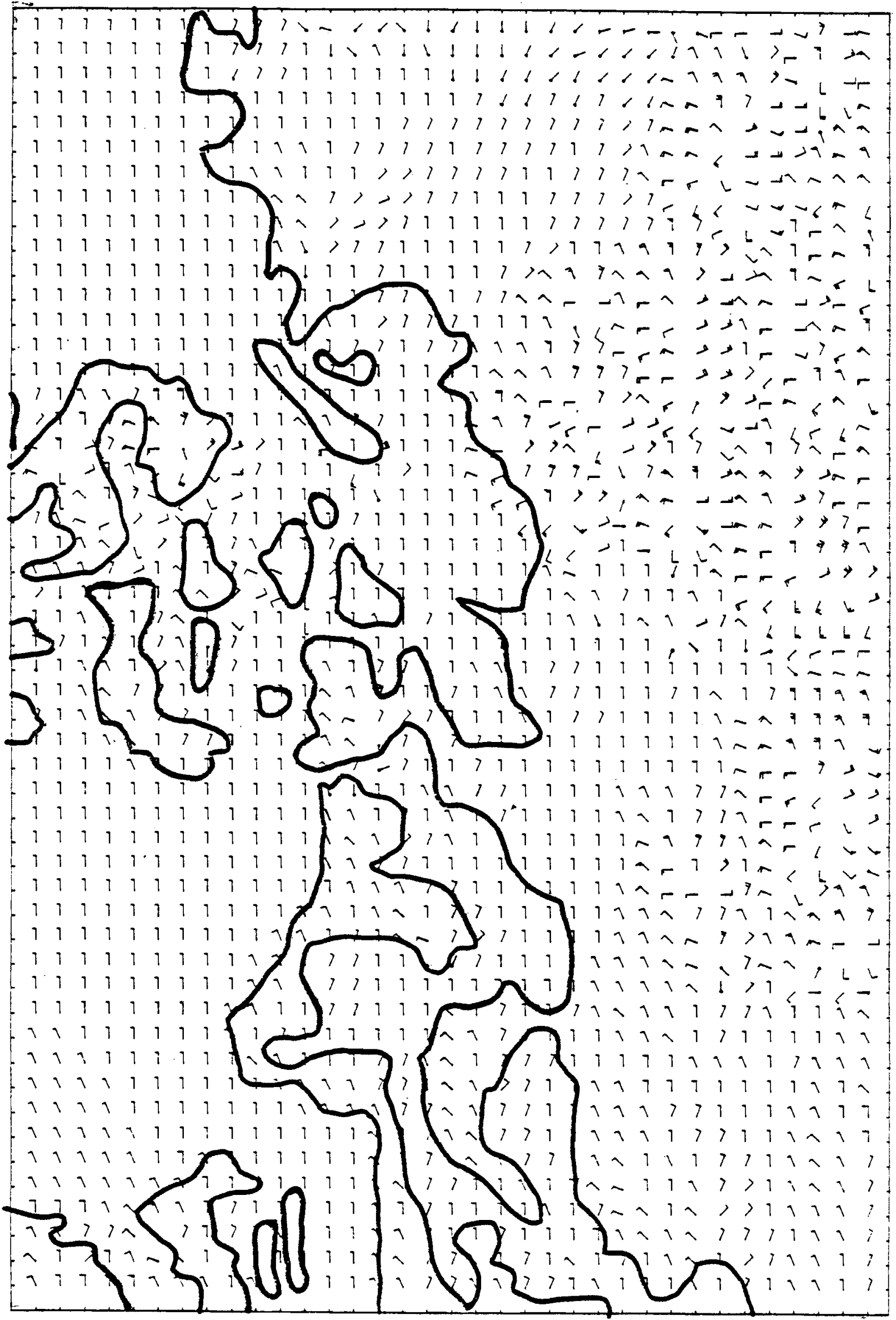


FIGURE 20 . PI ( $\pi$ ) MATRIX FOR JANUARY 24, 1975 AT 03:00

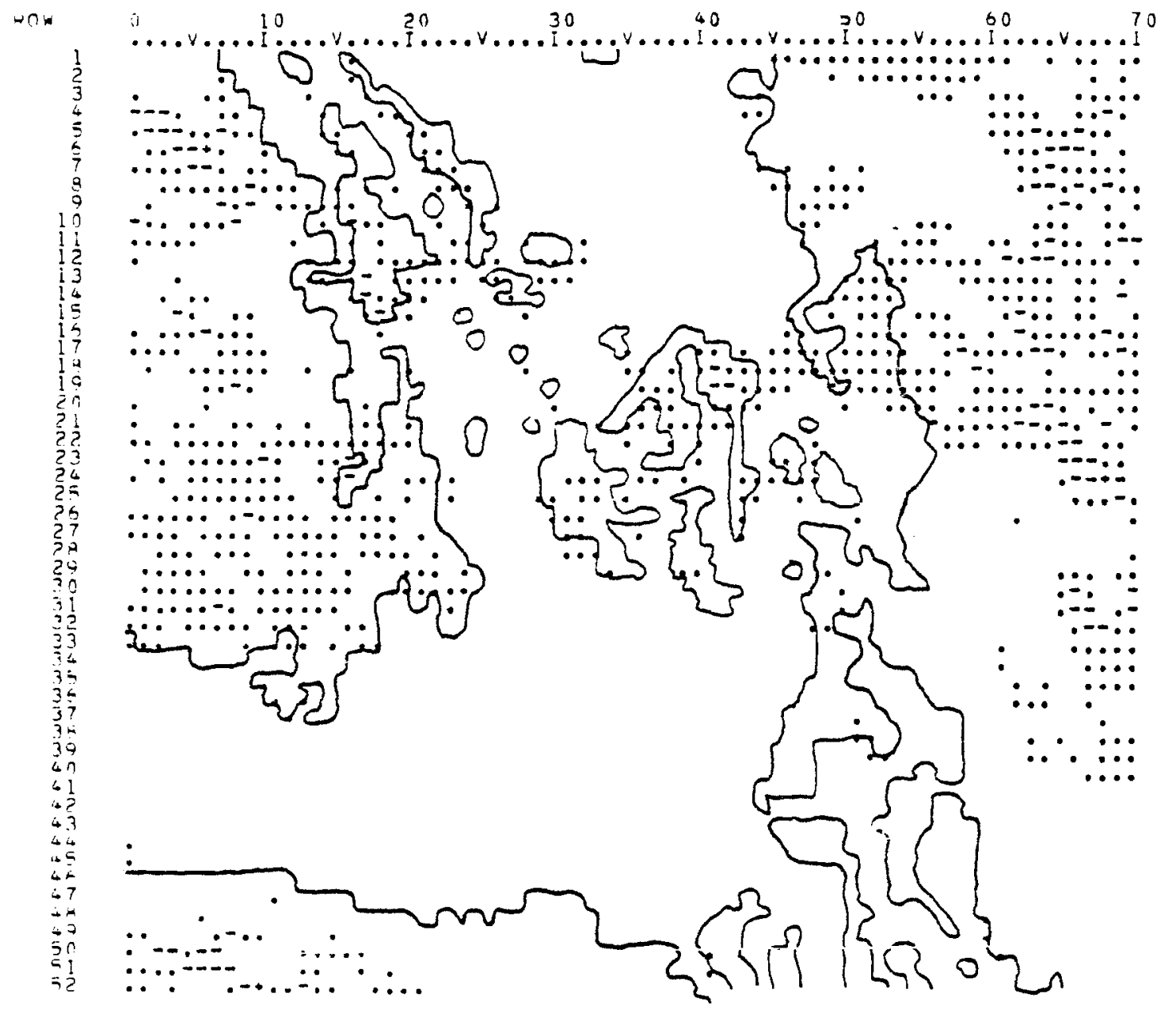

EMISSION RESTRICTION CODES:

\begin{tabular}{|c|c|c|c|c|c|c|c|c|c|c|c|}
\hline CODE & & Blank & . & - & + & $=$ & * & ;t & $\$$ & स & $x$ \\
\hline E!ISSION & $\operatorname{RATE}(3 / 3)$ & $<30$ & $30-59$ & $50-89$ & $90-119$ & $120-149$ & $150-179$ & $180-209$ & $210-239$ & $240-269$ & $\geq 270$ \\
\hline
\end{tabular}


FIGURE F.21. PI ( $\pi$ ) MATRIX FOR JANUARY 24, 1975 AT 07:00

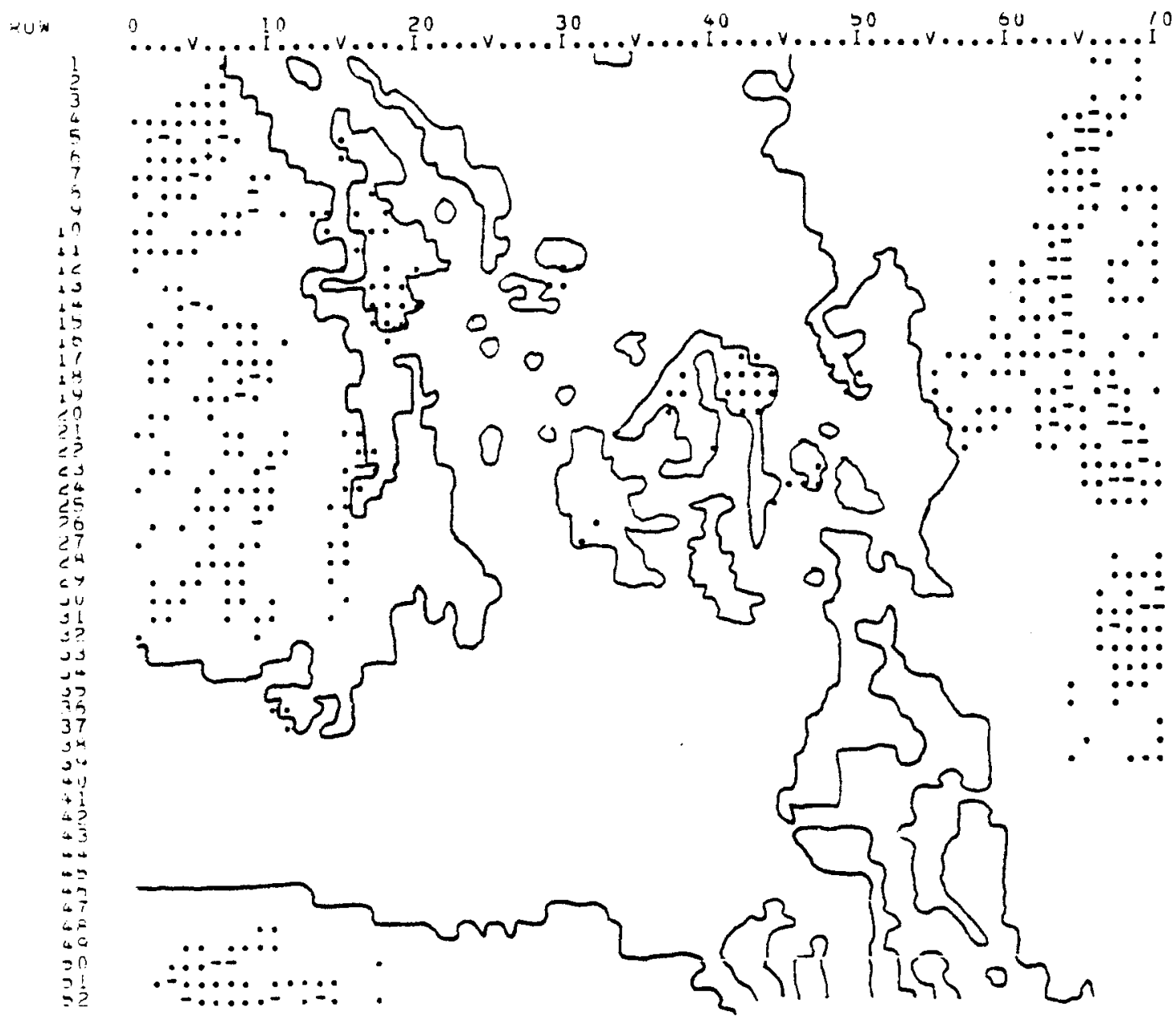

EMISSION RESTRICTION CODES:

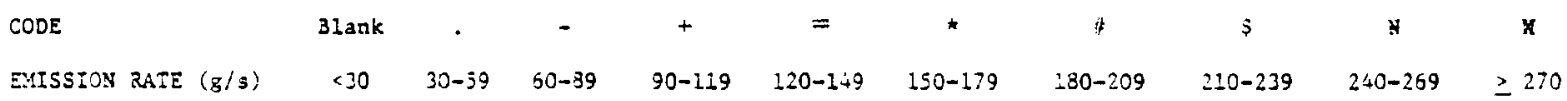

$\mathrm{SO}_{2}$ Standards are $.4 \mathrm{ppm}$ 
FIGURE E22. PI (T) MATRIX FOR JANUARY 24, 1975 AT 11:00

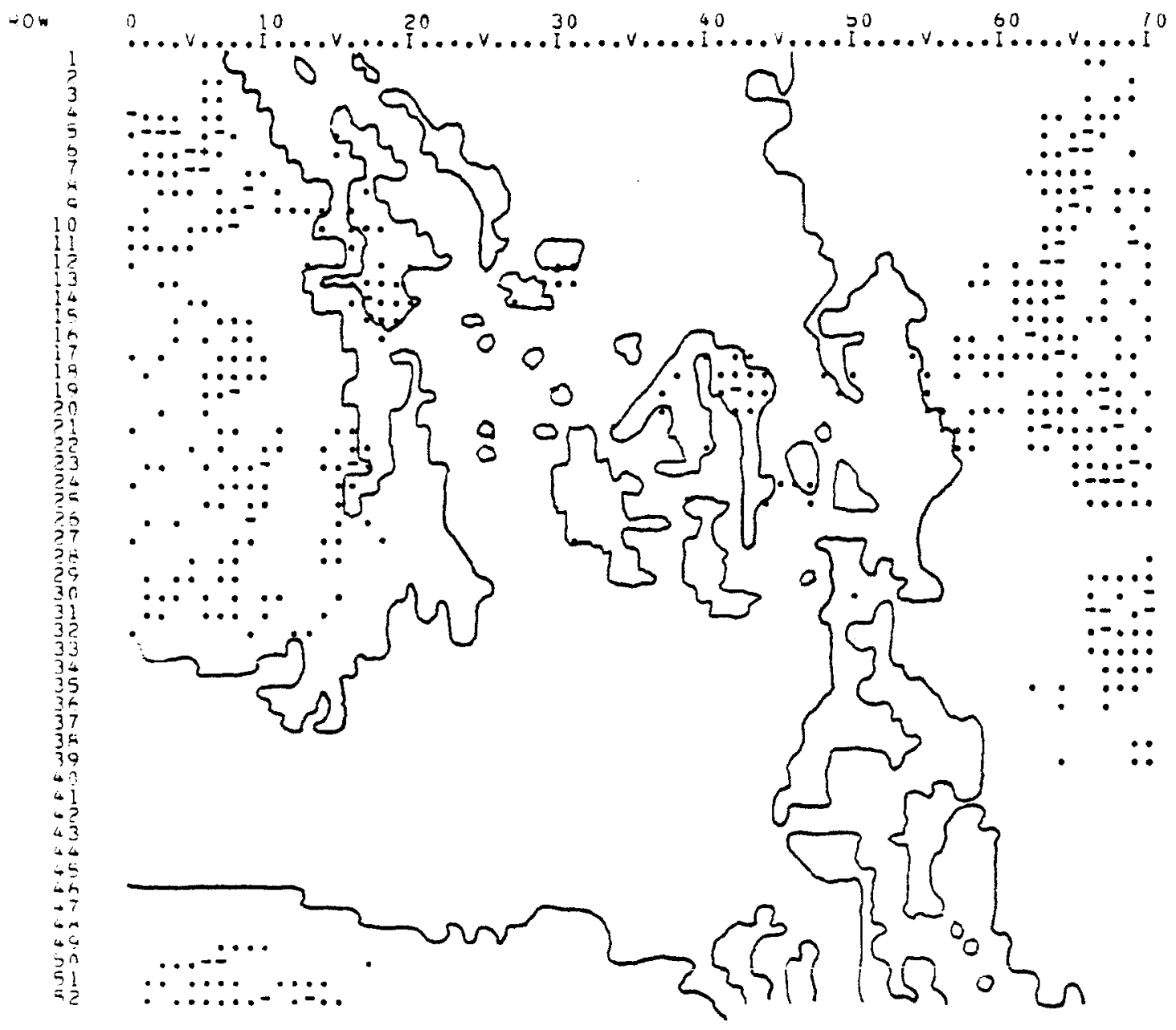

EMISSION RESTRICTION CODES:

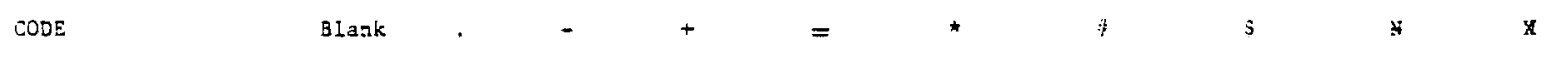

DIISSION RATE $(\mathrm{g} / \mathrm{s}) \quad<30 \quad 30-59 \quad 60-89 \quad 90-119 \quad 120-149 \quad 150-179 \quad 180-209 \quad 210-259 \quad 240-269 \quad \geq 270$

$\mathrm{SO}_{2}$ Standards are $.4 \mathrm{ppm}$ 
FIGURE E23. PI ( $\pi$ ) MATRIX FOR JANUARY 24, 1975 AT 15:00

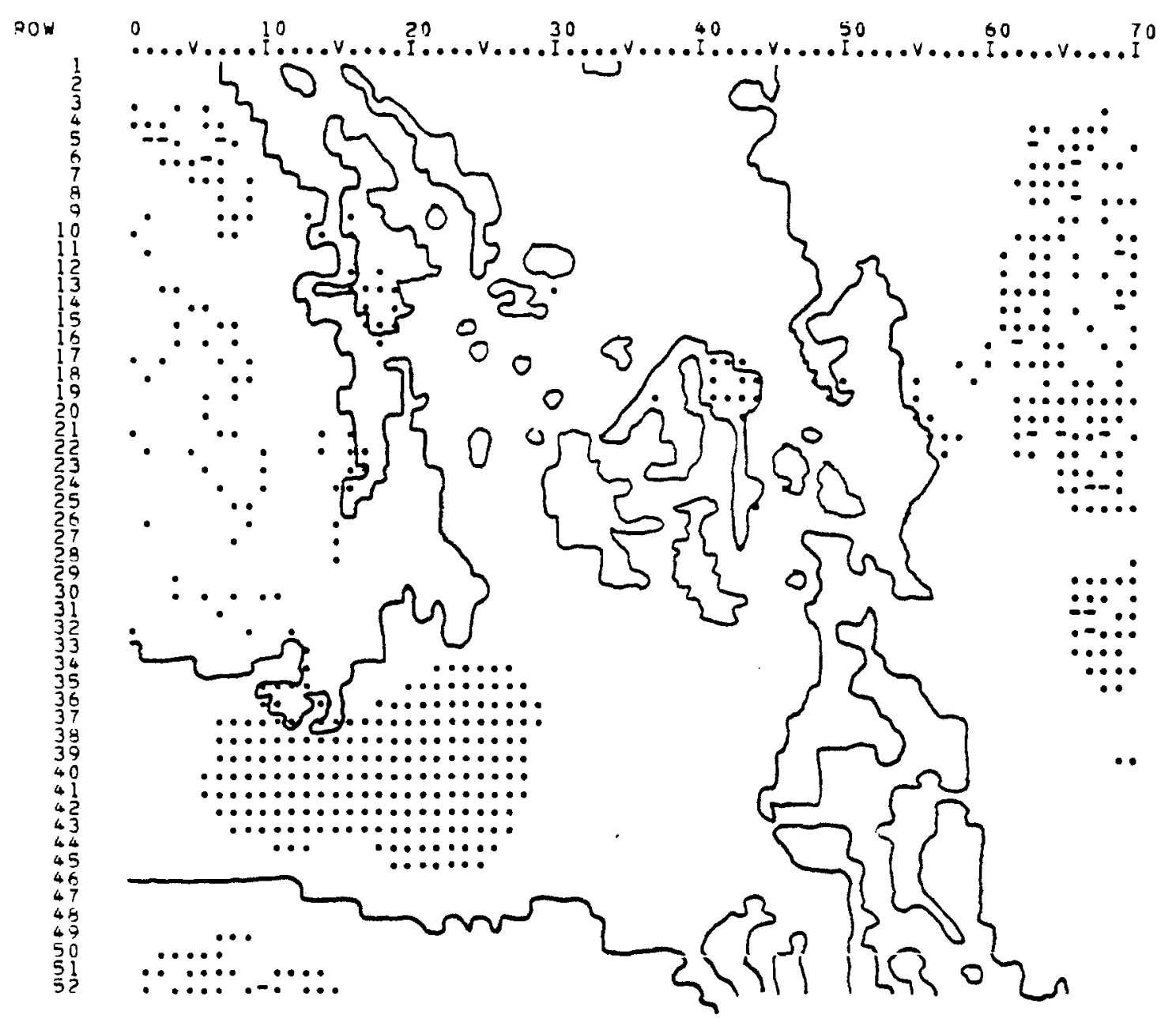

EMISSION RESTRICTION CODES:

\begin{tabular}{|c|c|c|c|c|c|c|c|c|c|c|c|}
\hline CODE & & 3 lank & . & - & + & $=$ & $\star$ & 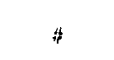 & $\$$ & $H$ & x \\
\hline EUISSION & $\operatorname{RATE}(\mathrm{g} / \mathrm{s})$ & $<30$ & $30-39$ & $60-89$ & $90-119$ & $120-149$ & $150-i 79$ & $180-209$ & $210-239$ & $240-269$ & $\geq 270$ \\
\hline
\end{tabular}


FIGURE E24. PI ( $\pi$ ) MATRIX FOR JANUARY 24, 1975 AT 19:00

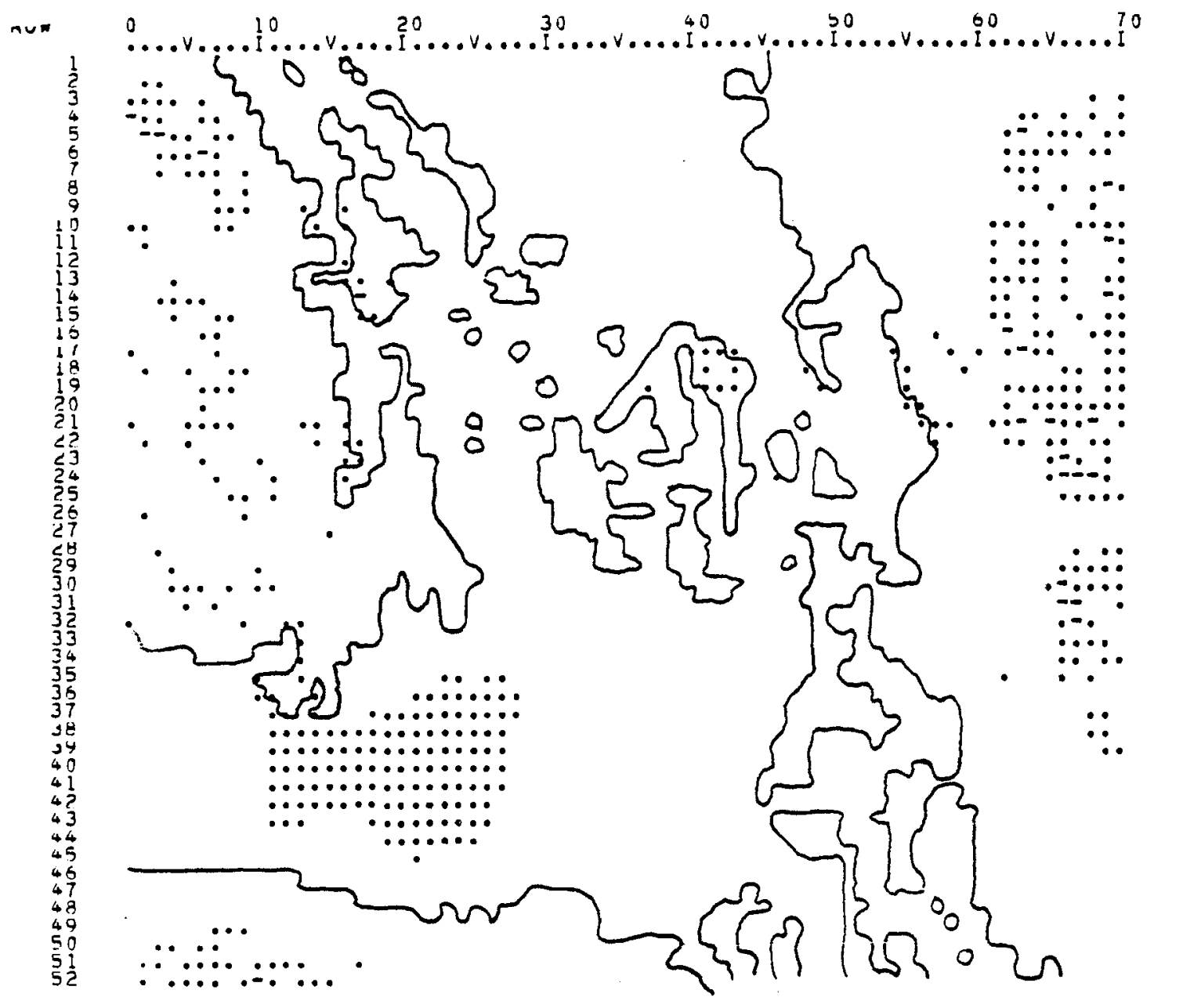

EMISSION RESTRICTION CODES:

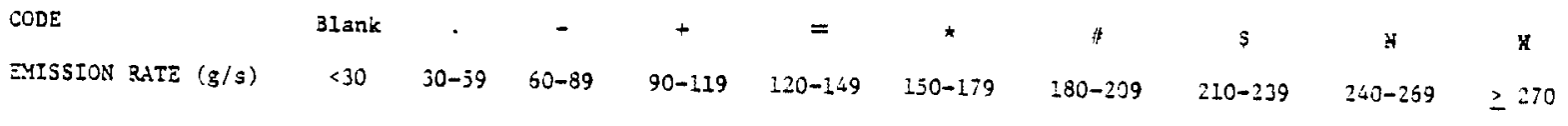

$\mathrm{SO}_{2}$ Standards are $.4 \mathrm{ppm}$ 
FIGURE E25. PI ( $\pi$ ) MATRIX FOR JANUARY 24, 1975 AT 23:00

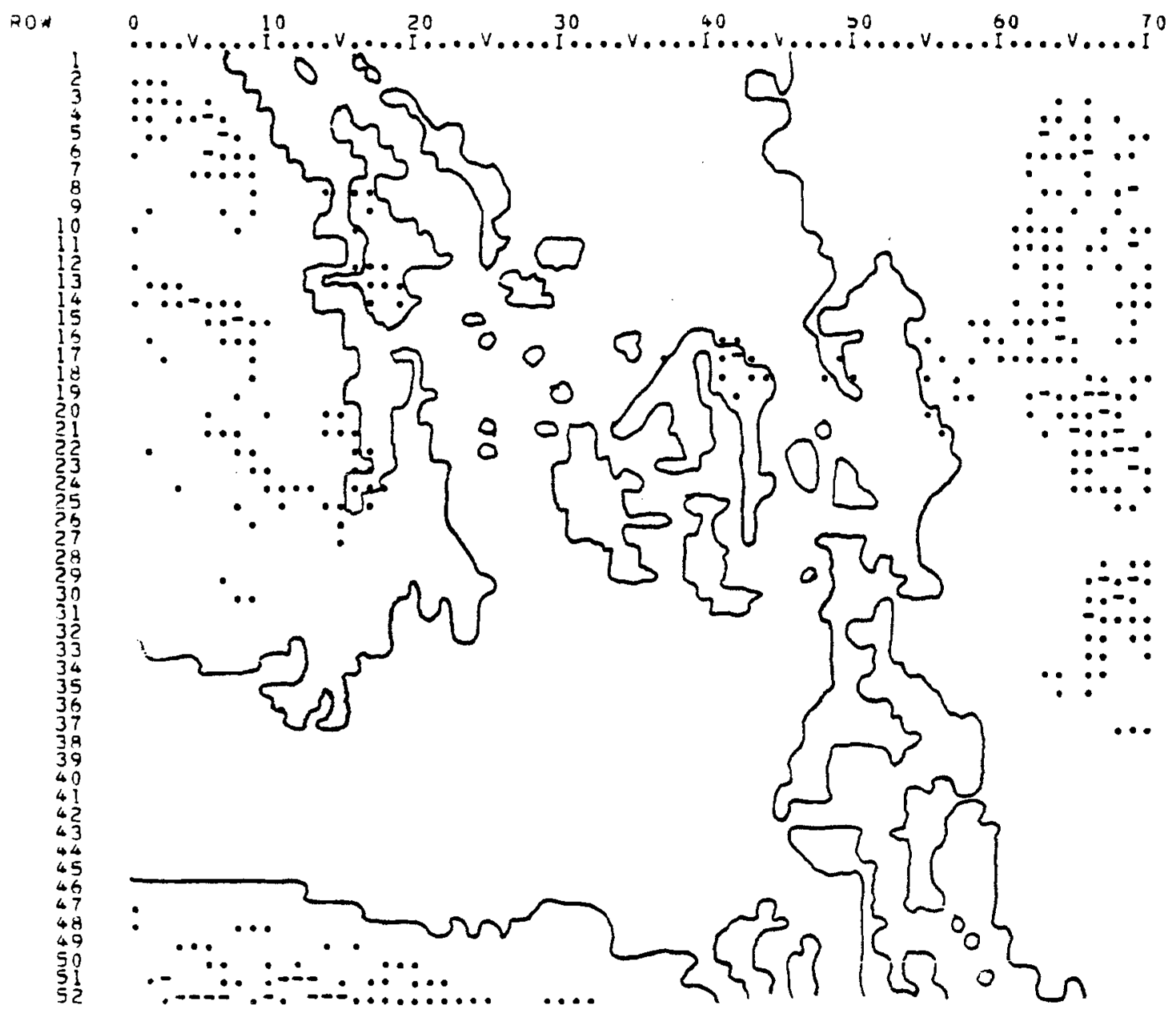

EMISSION RESTRICTION CODES:

$\begin{array}{lcccccccccc}\text { CODE } & \text { Blank } & * & - & + & = & * & * & * & * & x \\ \text { EMISSION RATE }(\mathrm{g} / \mathrm{s}) & <30 & 30-59 & 60-89 & 90-119 & 120-149 & 150-179 & 180-209 & 210-239 & 240-259 & \geq 270\end{array}$

$\mathrm{SO}_{2}$ Standards are $.4 \mathrm{ppm}$ 
FIGURE E26. PI ( $\pi$ ) MATRIX FOR JANUARY 24, 1975 COMBINED

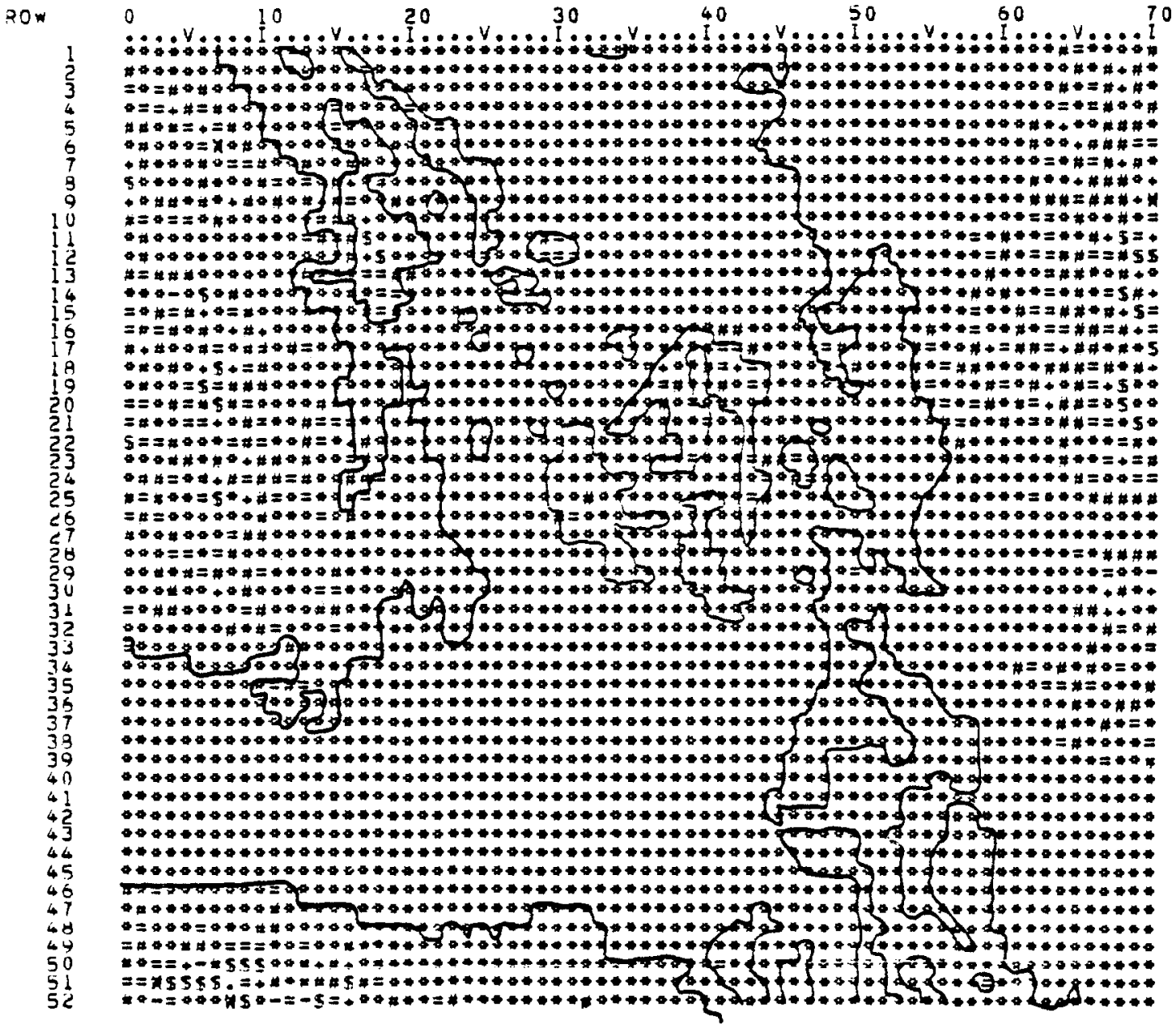

EMISSION RESTRICIION CODES:

CODE

Blank

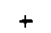

*

\#

s

$x$

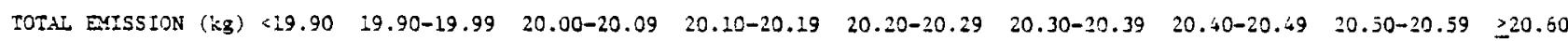

$\mathrm{SO}_{2}$ Standards are $.1 \mathrm{ppm}$ 
Figure E27. Windfield Analysis of Northern Puget Sound Study Area for January 30,1975 at 0300 PST. Background flow input: 112.5 degrees at 4.0 meters per second $(w)$.

$$
\text { Wind Flag Codes: } \begin{array}{r}
\frac{\mathrm{m} / \mathrm{s}}{5.0} \\
2.0 \\
7 \quad 1.0 \\
! \quad<1.0
\end{array}
$$




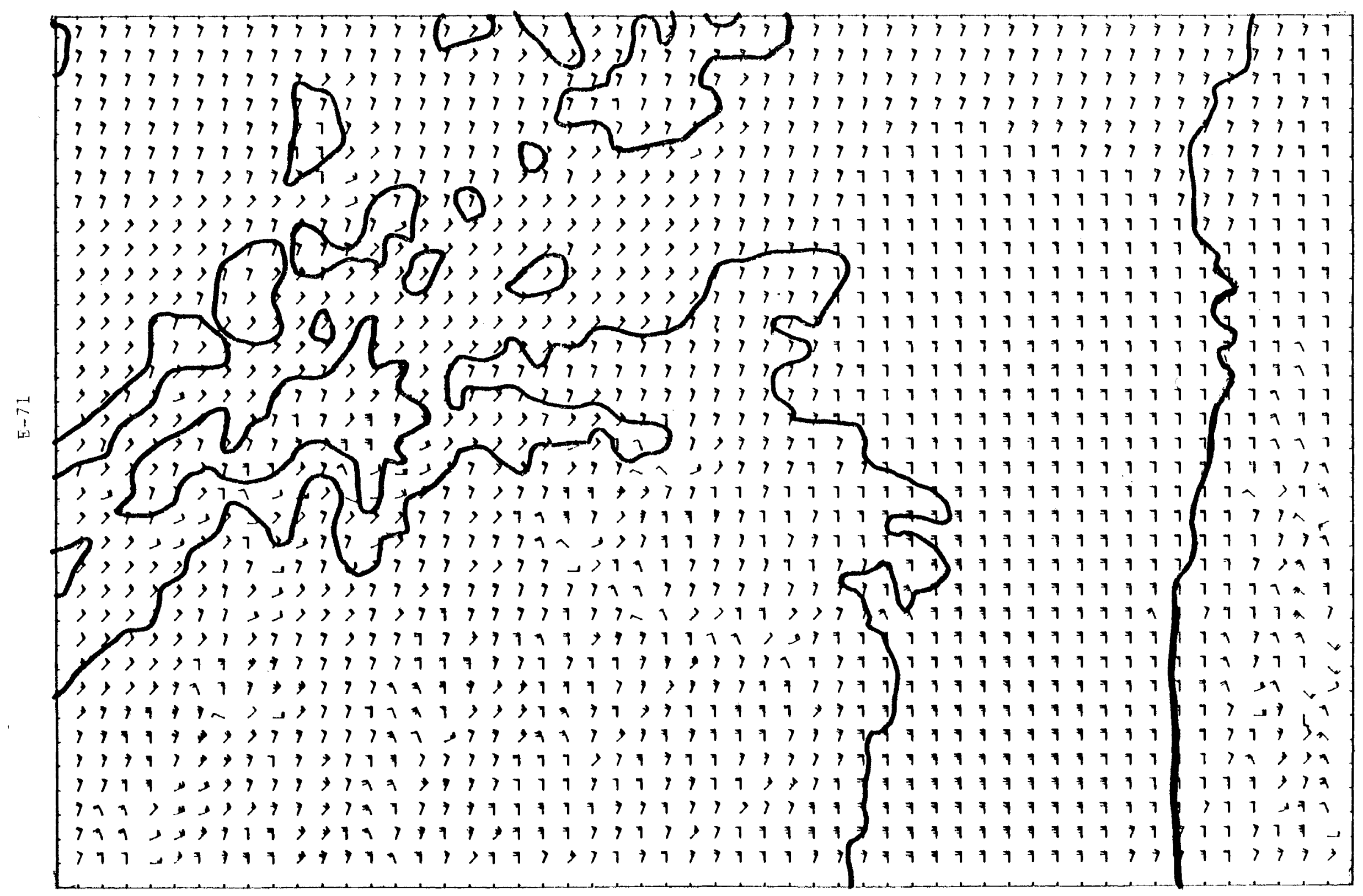




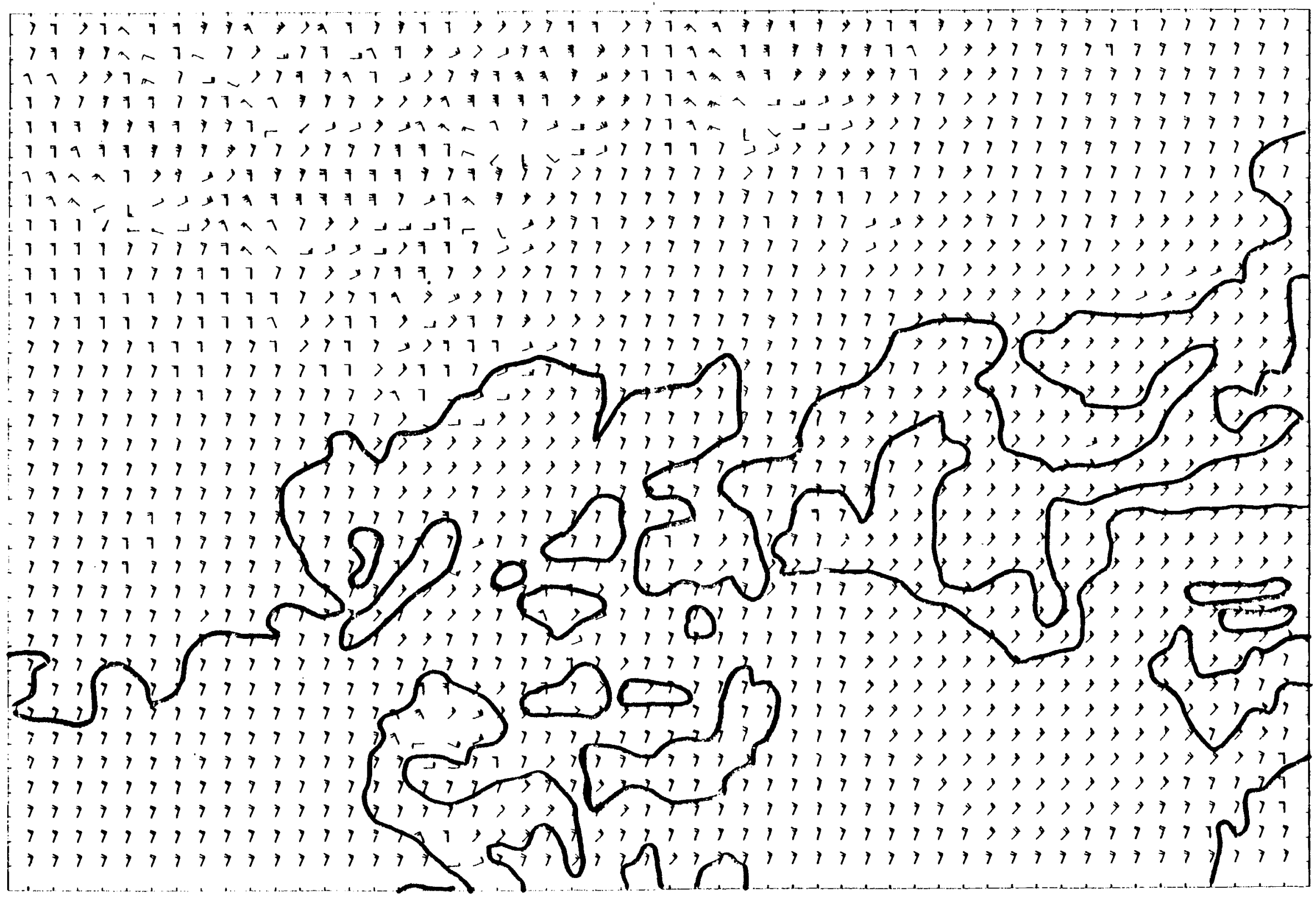


Figure E28. Windfield Analysis of Northern Puget Sound Study Area for January 30,1975 at 0700 PST. Background flow input: 112.5 degrees at 4.0 meters per second $(-ل /)$.

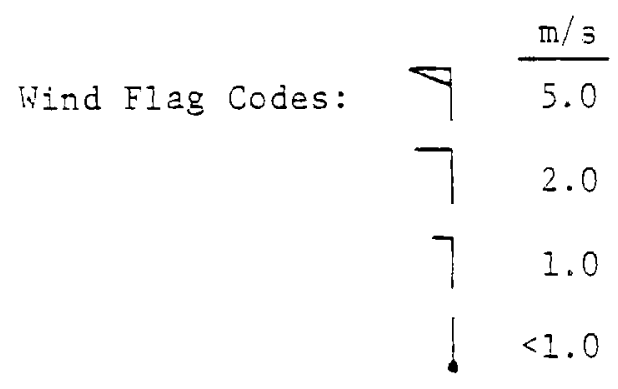




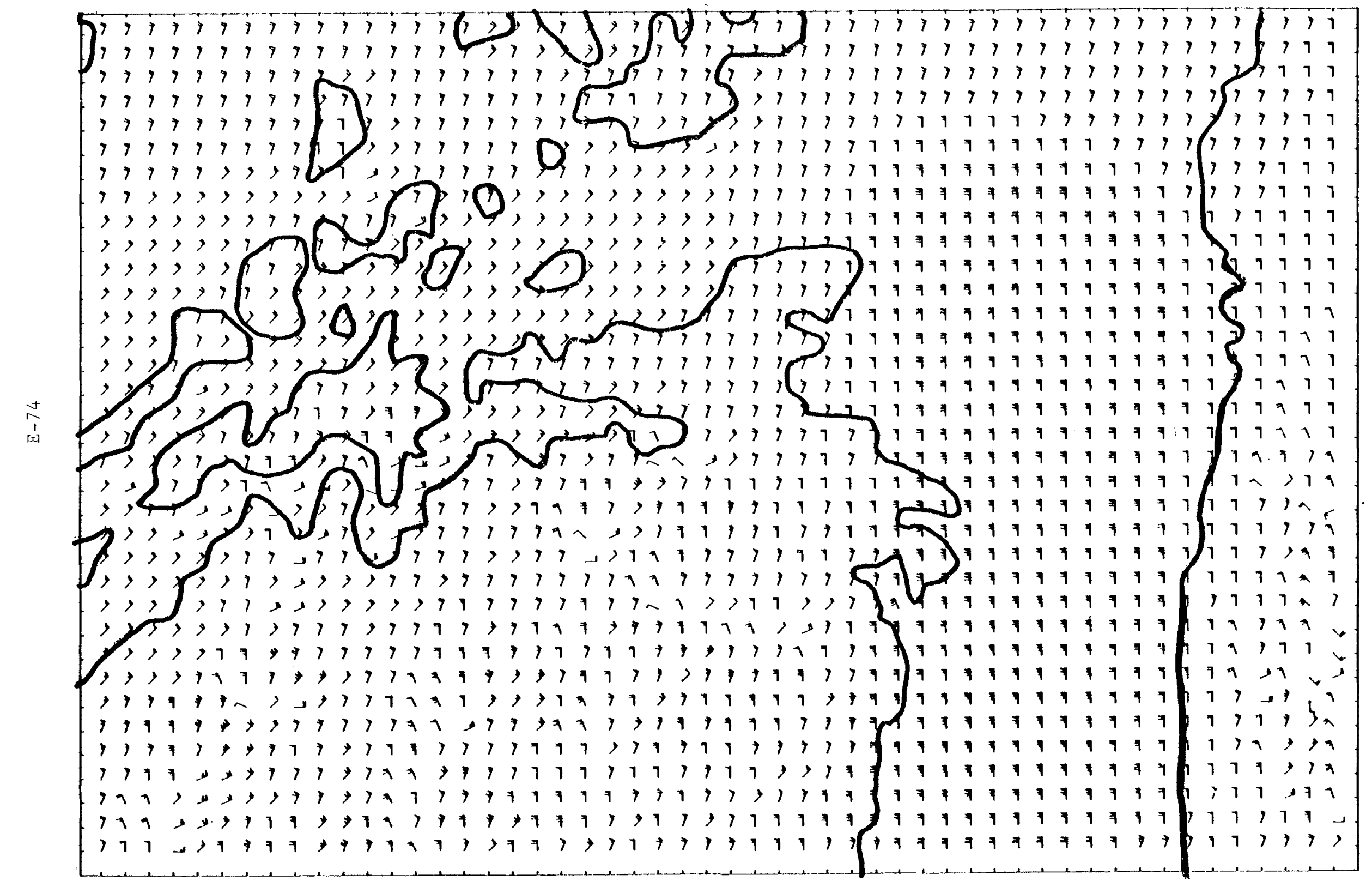




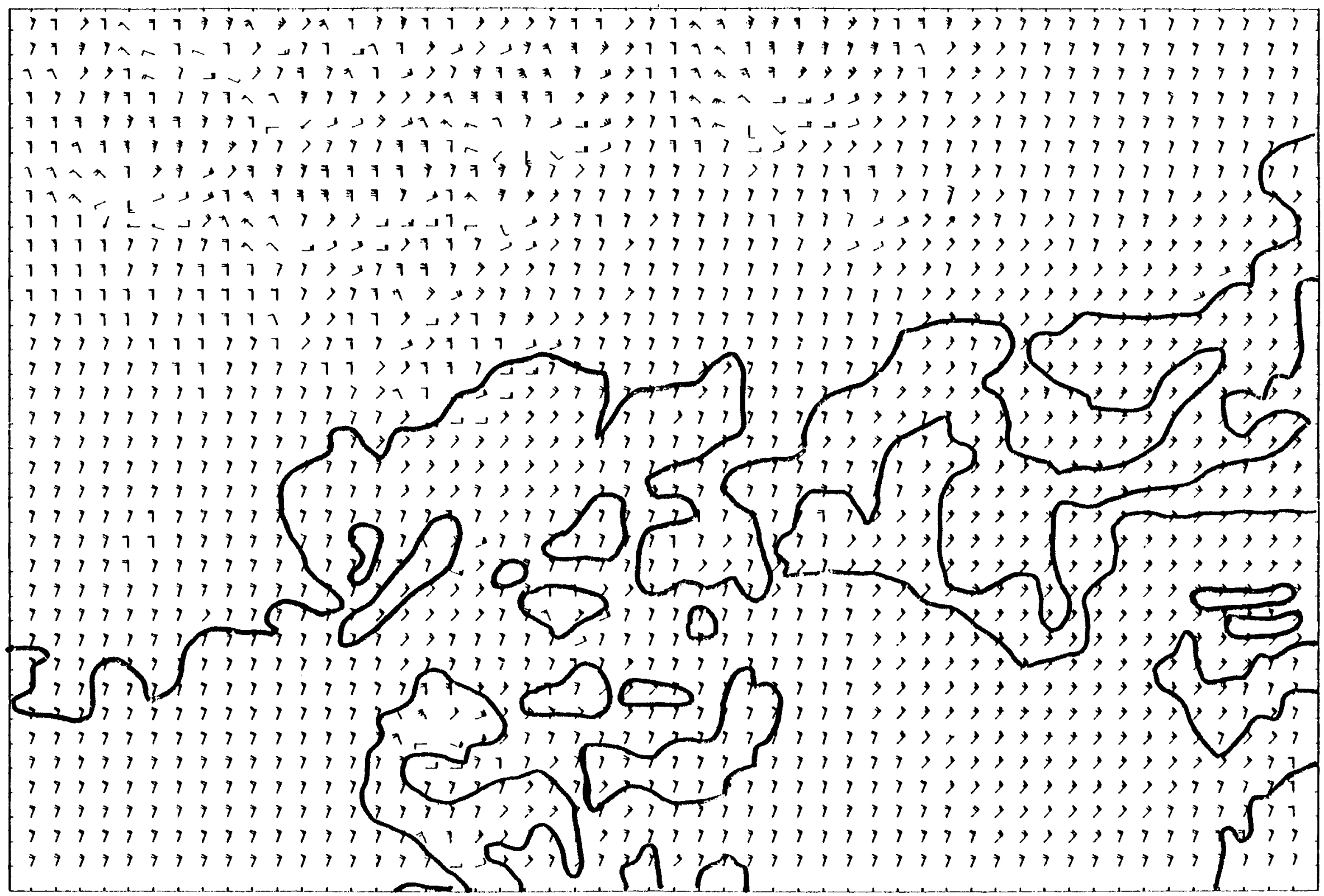


Figure E29. Windfield Analysis of Northern Puget Sound Study Area for January 30,1975 at 1100 PST. Background flow input: 112.5 degrees at 5.5 meters per second (-4).

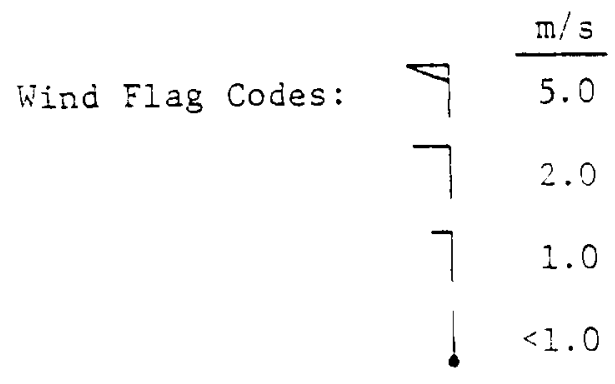




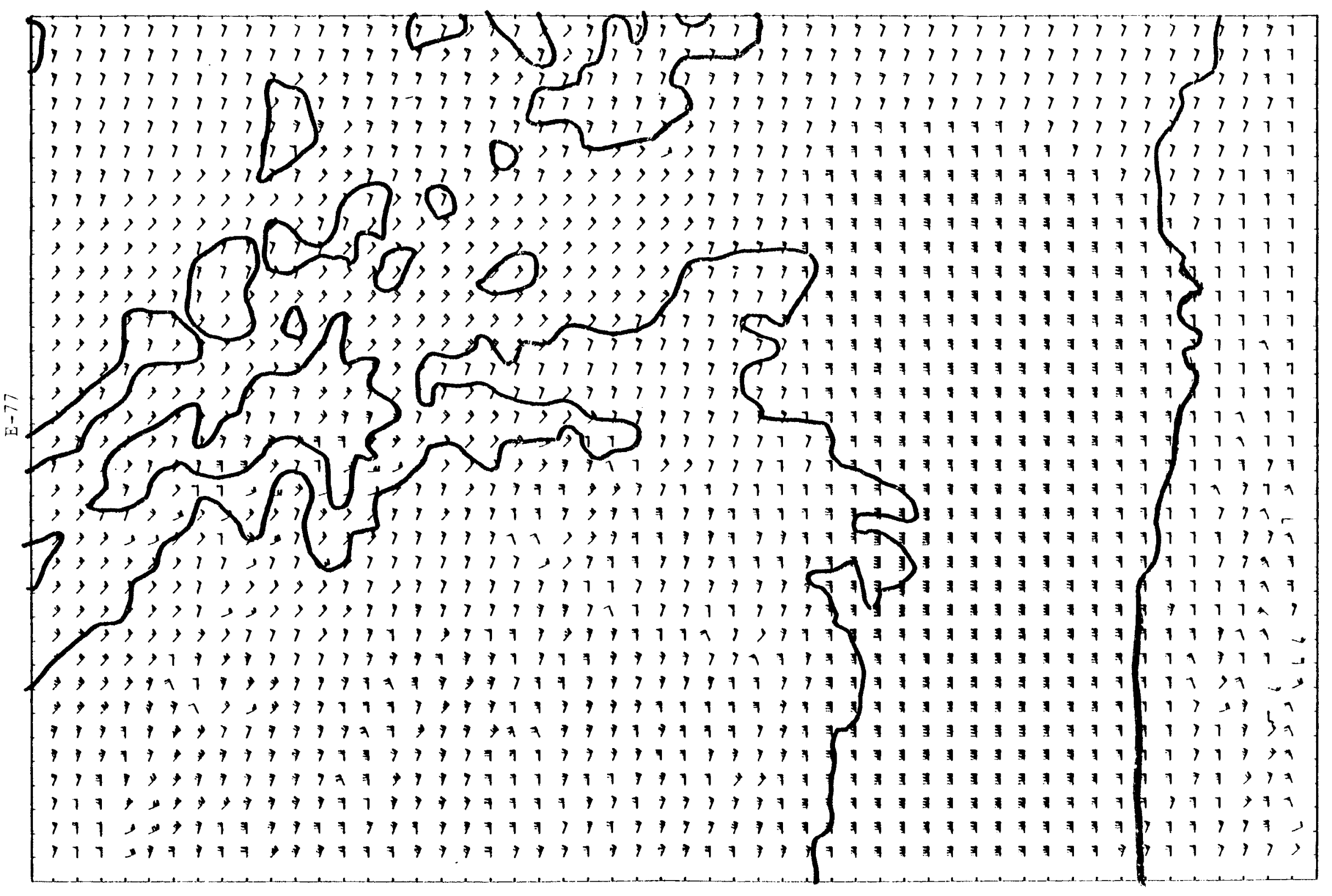




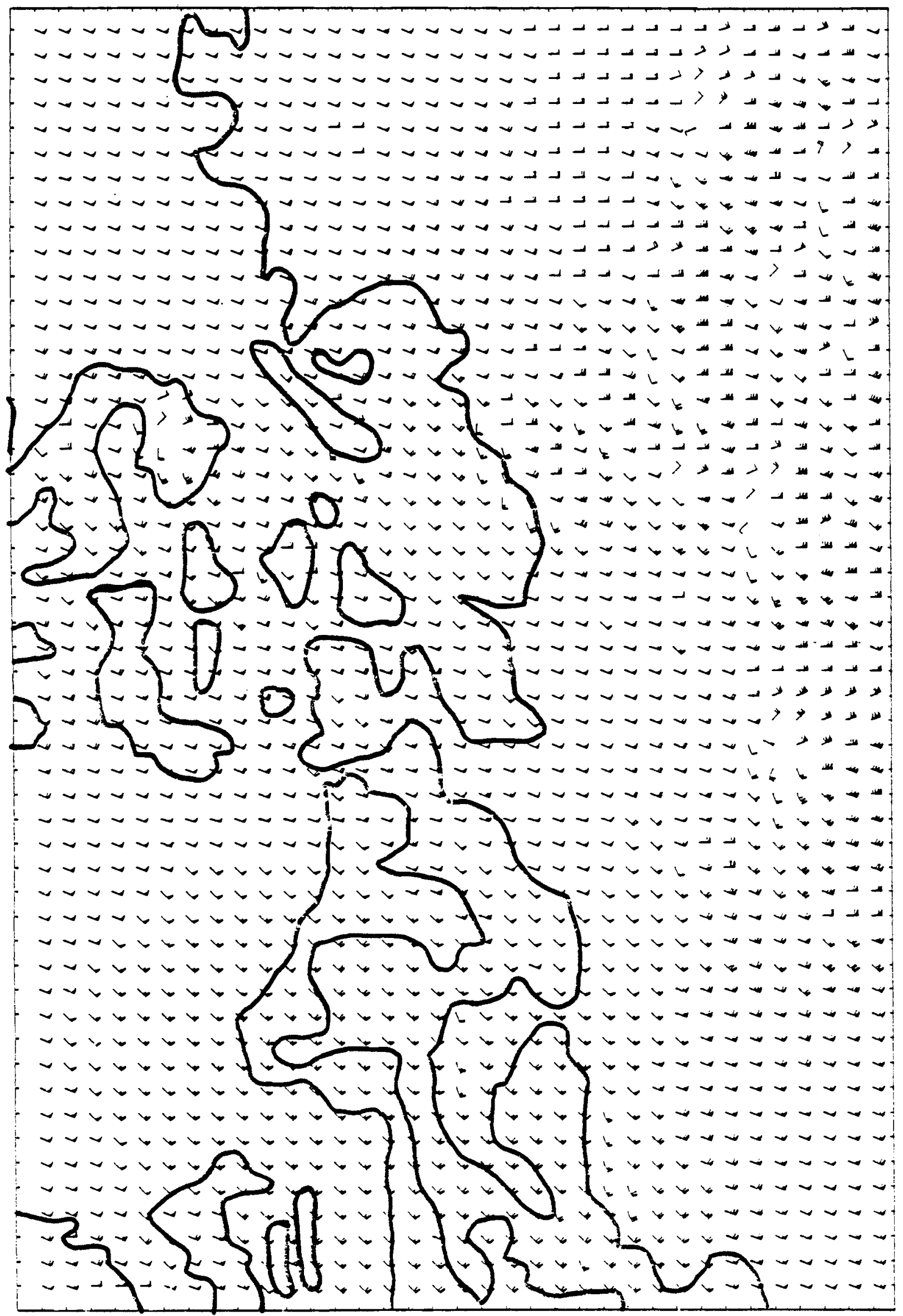


Figure E30. Windfield Analysis of Northern Puget Sound Study Area for January 30,1975 at 1500 PST. Background flow input: 112.5 degrees at 4.0 meters per second $(W)$.

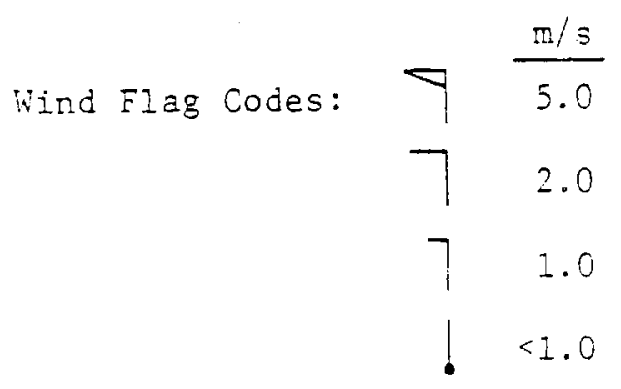




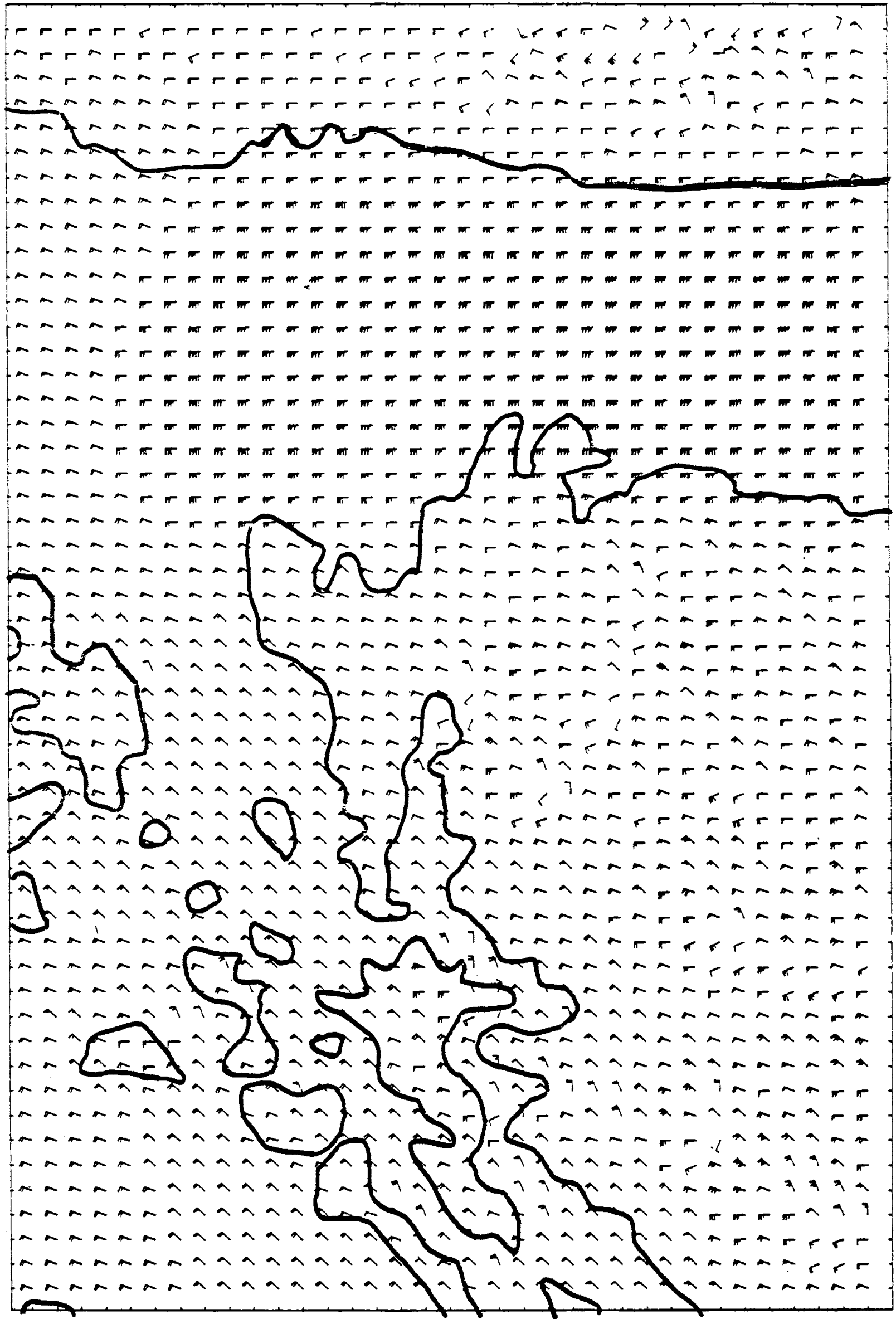

68- 


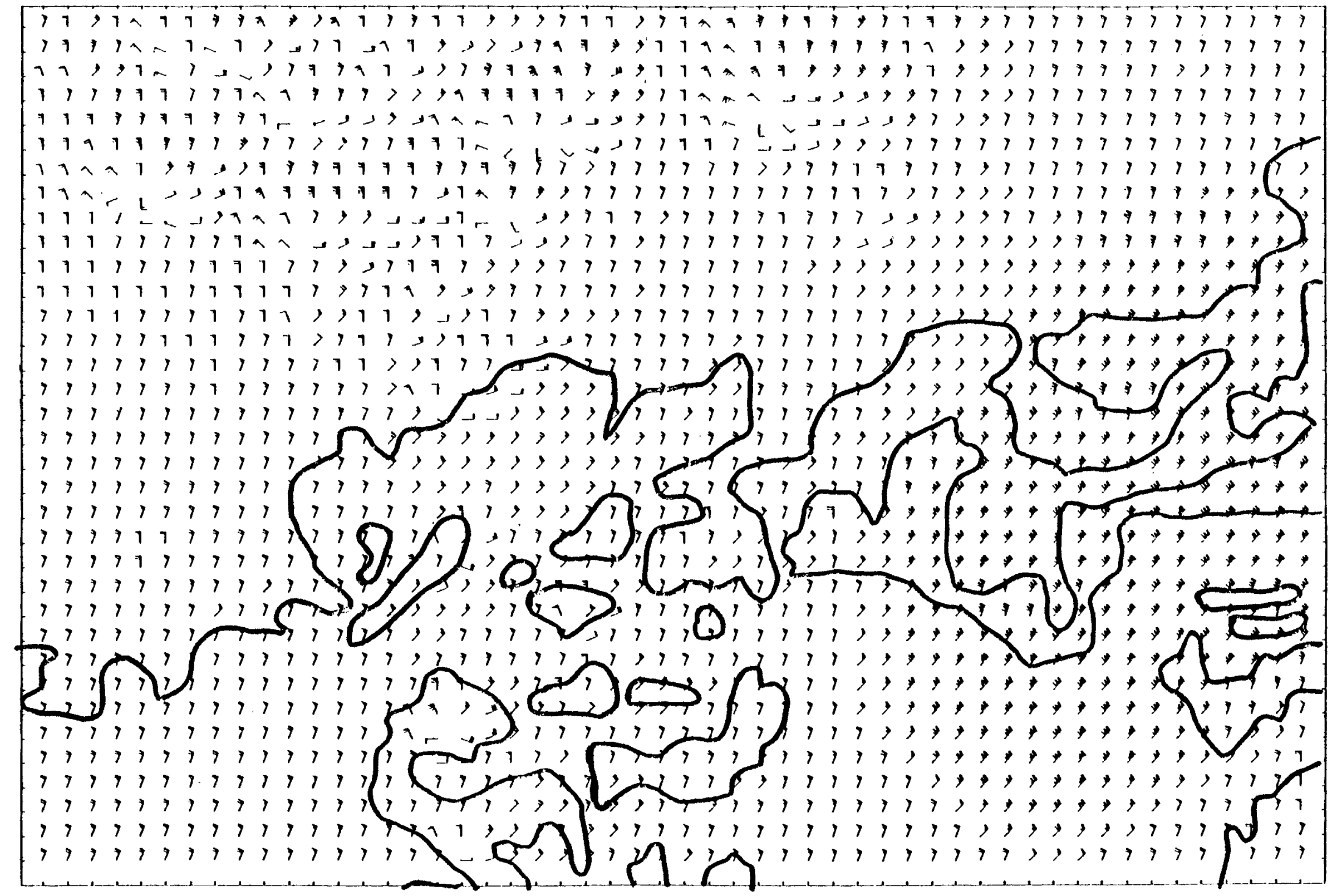


Figure E31. Windfield Analysis of Northern Puget Sound Study Area for January 30,1975 at 1900 PST. Background flow input: 112.5 degrees at 6.5 meters per second ( $-\phi)$.

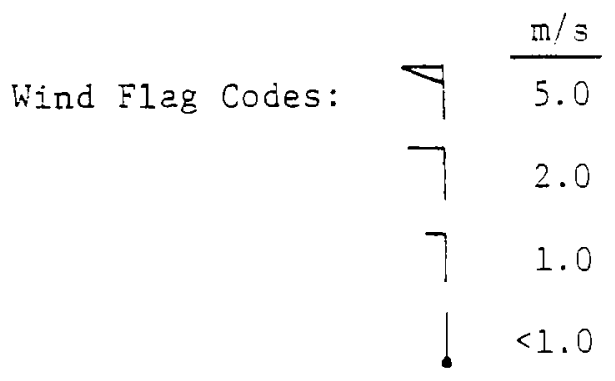




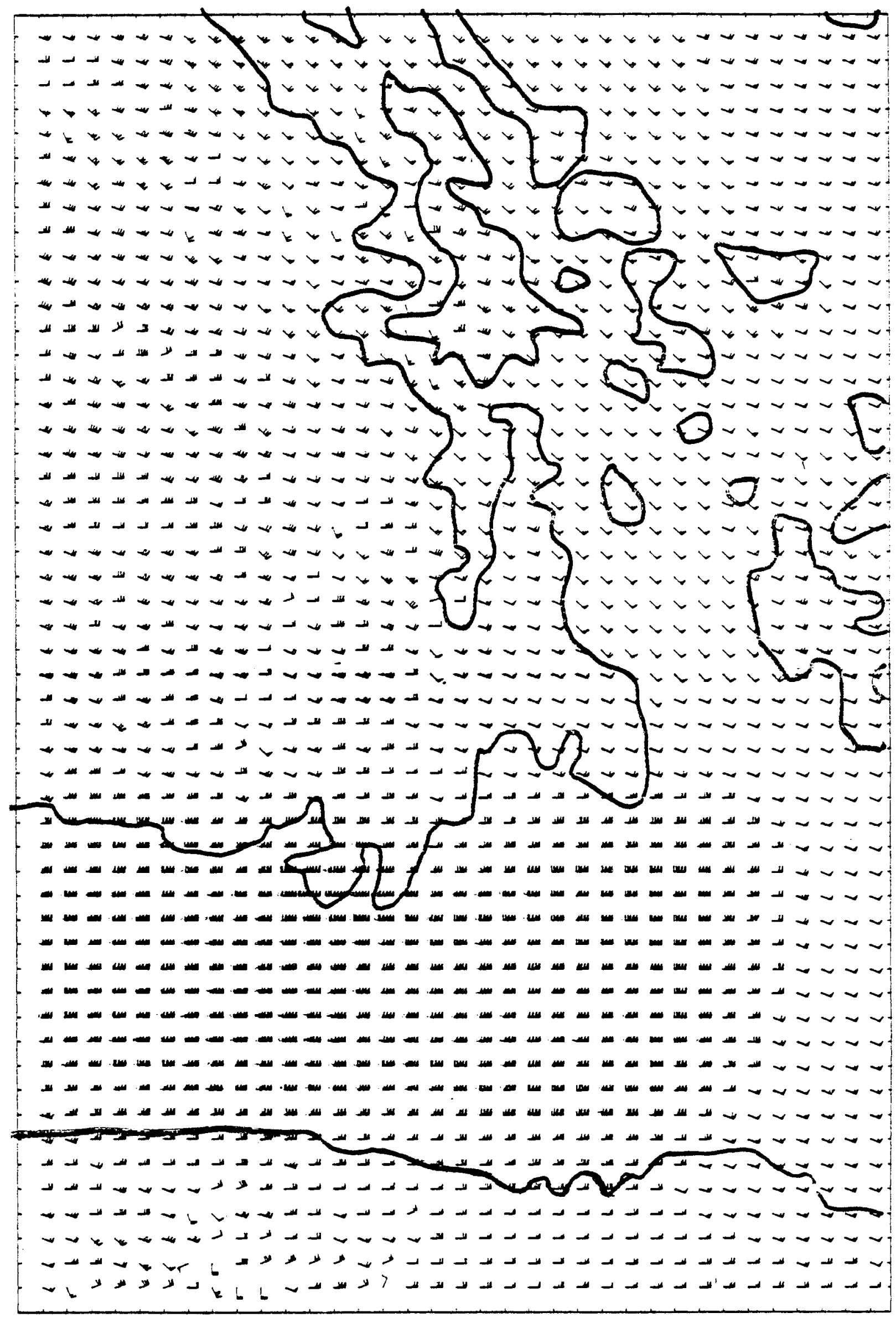




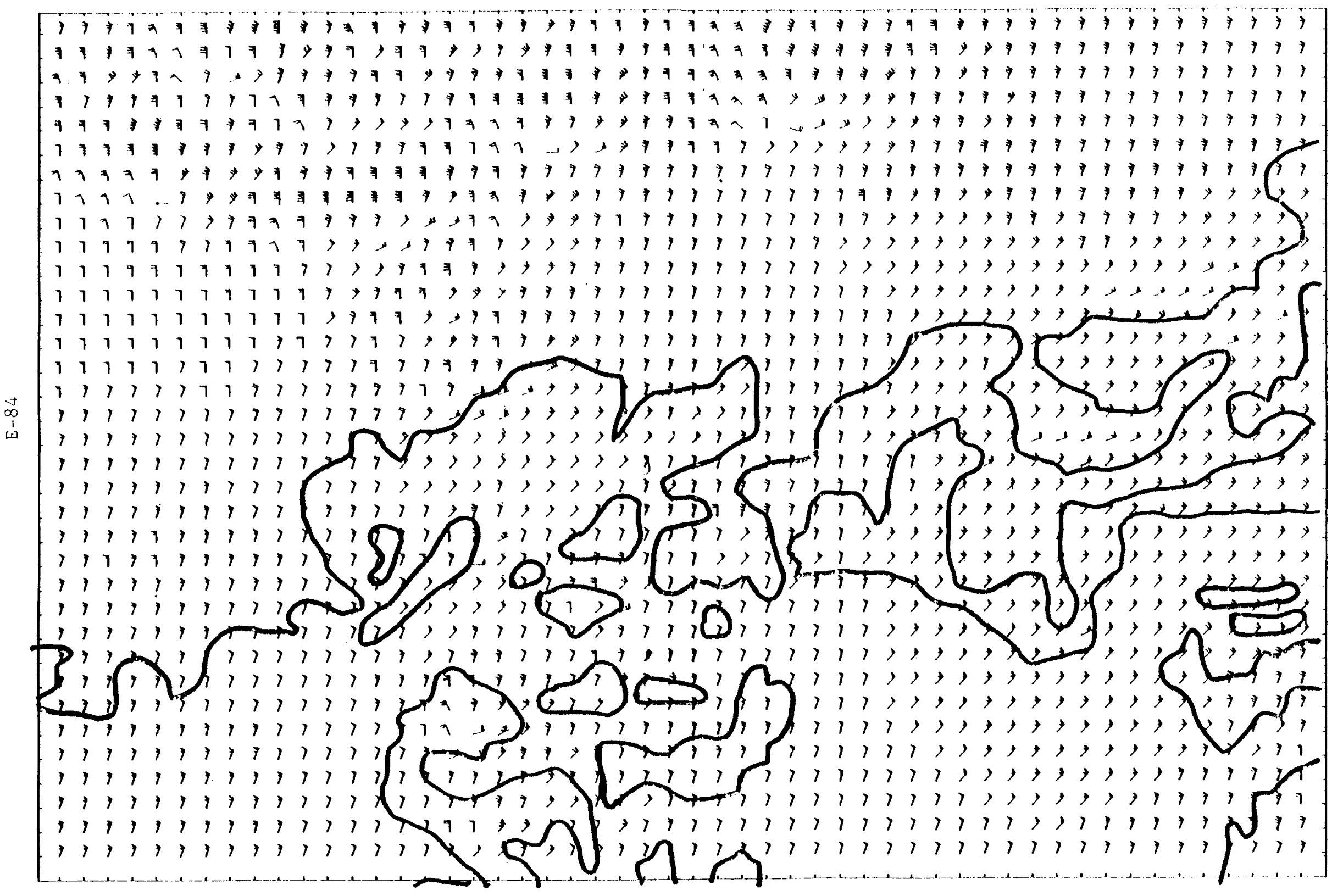


Figure E32. Windfield Analysis of Northern Puget Sound Study Area for January 30,1975 at 2300 PST. Background flow input: 112.5 degrees at 6.0 meters per second $(-)$.

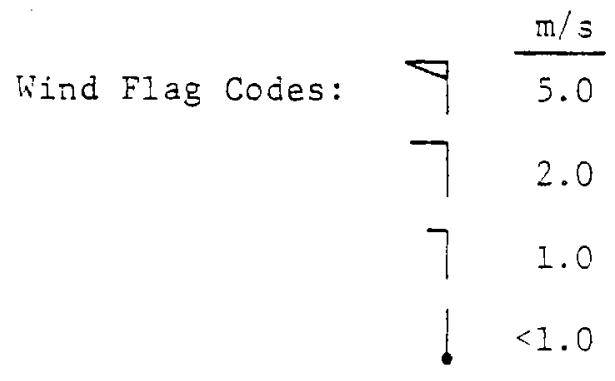




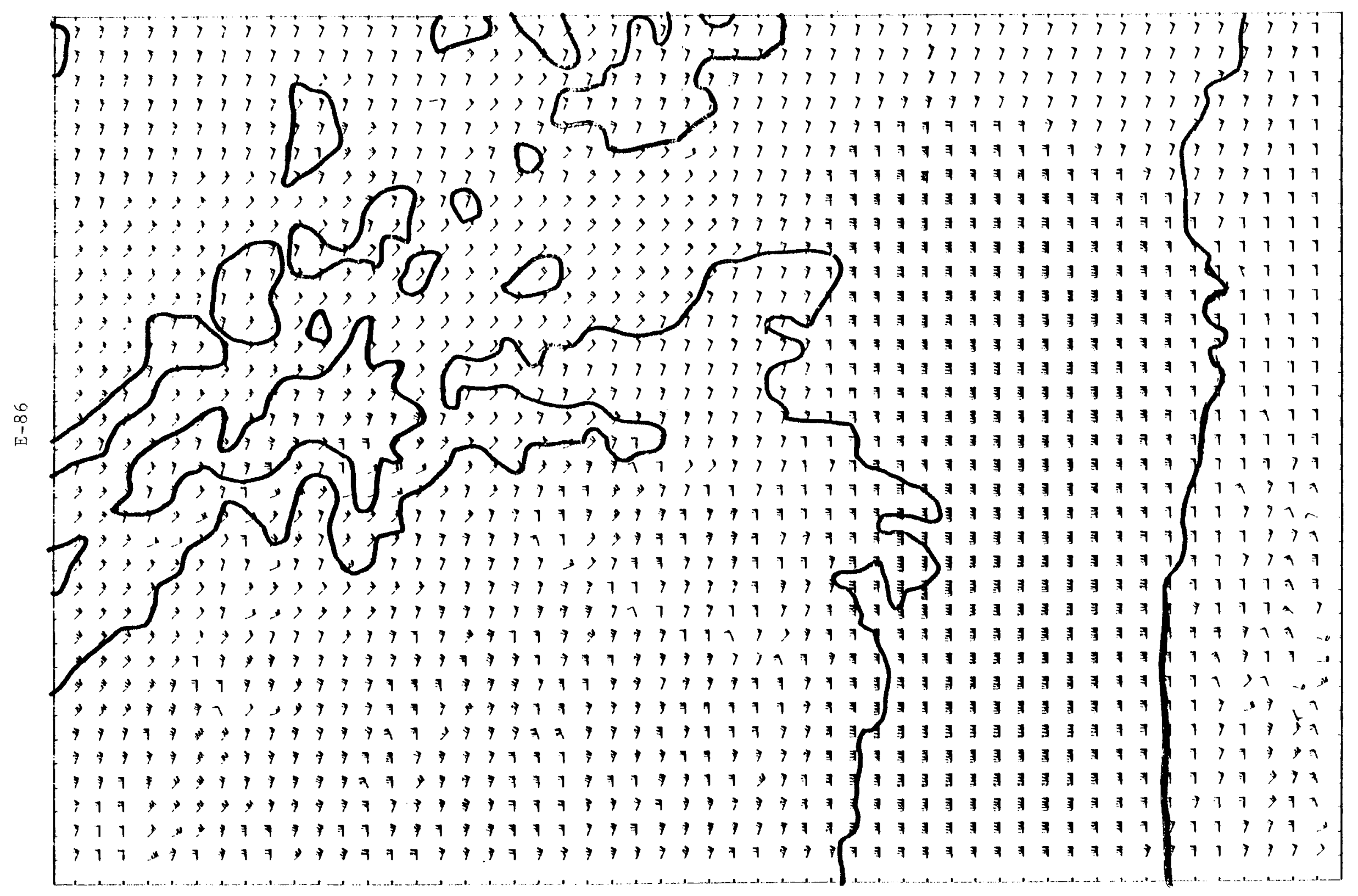




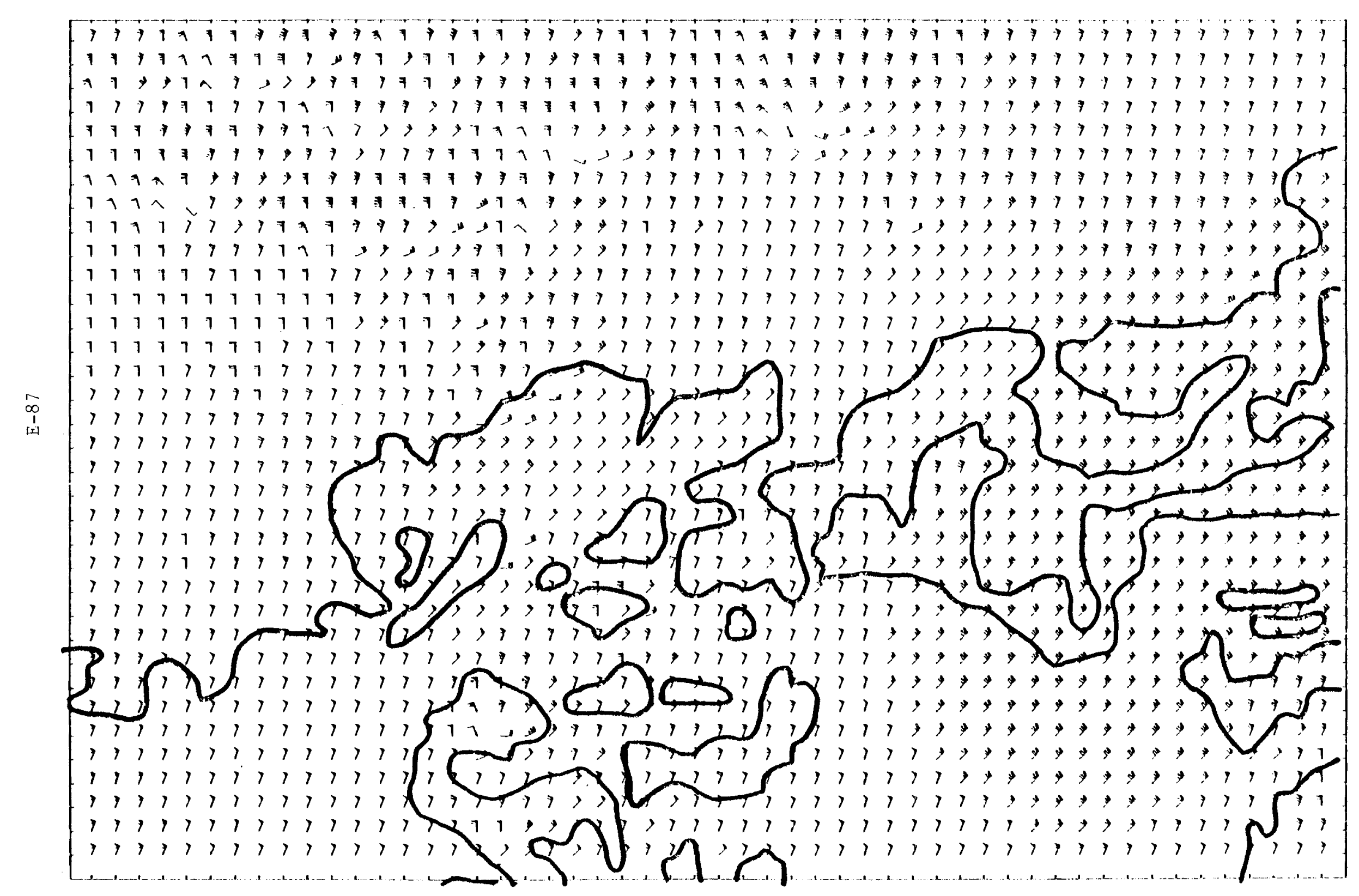


FIGURE E33. PI ( $\pi$ ) IAATRIX FOR JANUARY 30, 1975 AT 03:00

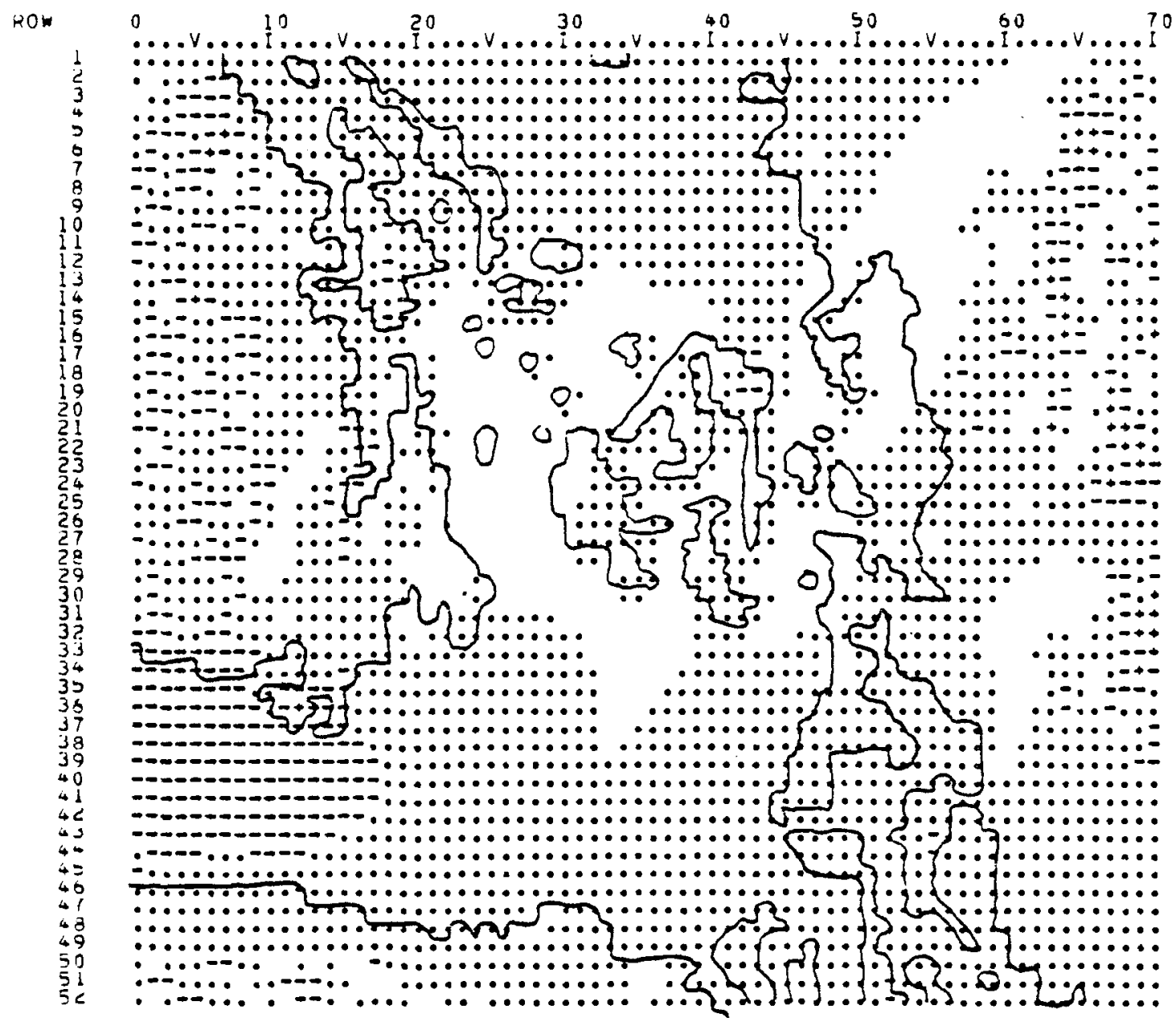

EMISSION RESTRICTION CODES:

CODE

gISSION RITE (g/s)

Blank

$<30 \quad 30-59 \quad 50-89$

$+$

$=$

(150-10

;

$\$$

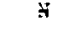

$\mathrm{x}$

(2)

$\mathrm{SO}_{2}$ Standards are $.4 \mathrm{ppm}$ 


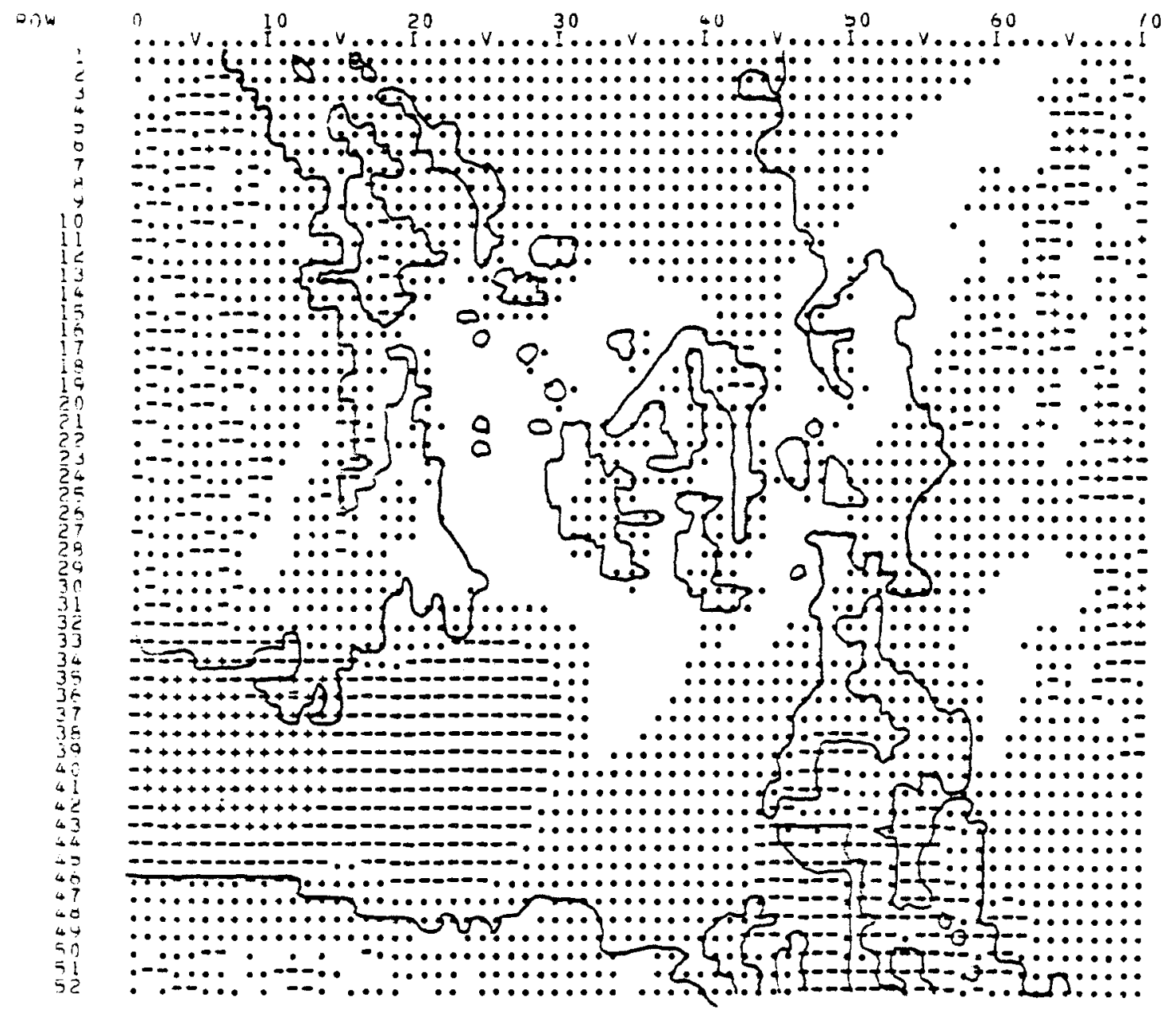

EMISSION RESTRICTION CODES:

\begin{tabular}{|c|c|c|c|c|c|c|c|c|c|}
\hline CODE & Blank & . & - & + & \multicolumn{2}{|l|}{$=$} & ; & $\$$ & \\
\hline EMISSION RATE $(\mathrm{g} / \mathrm{s})$ & $<30$ & $30-59$ & $50-39$ & $90-119$ & $120-149$ & $150-179$ & $180-209$ & $=10-239$ & $240-269$ \\
\hline
\end{tabular}

$\mathrm{SO}_{2}$ Standards are $.4 \mathrm{ppm}$ 


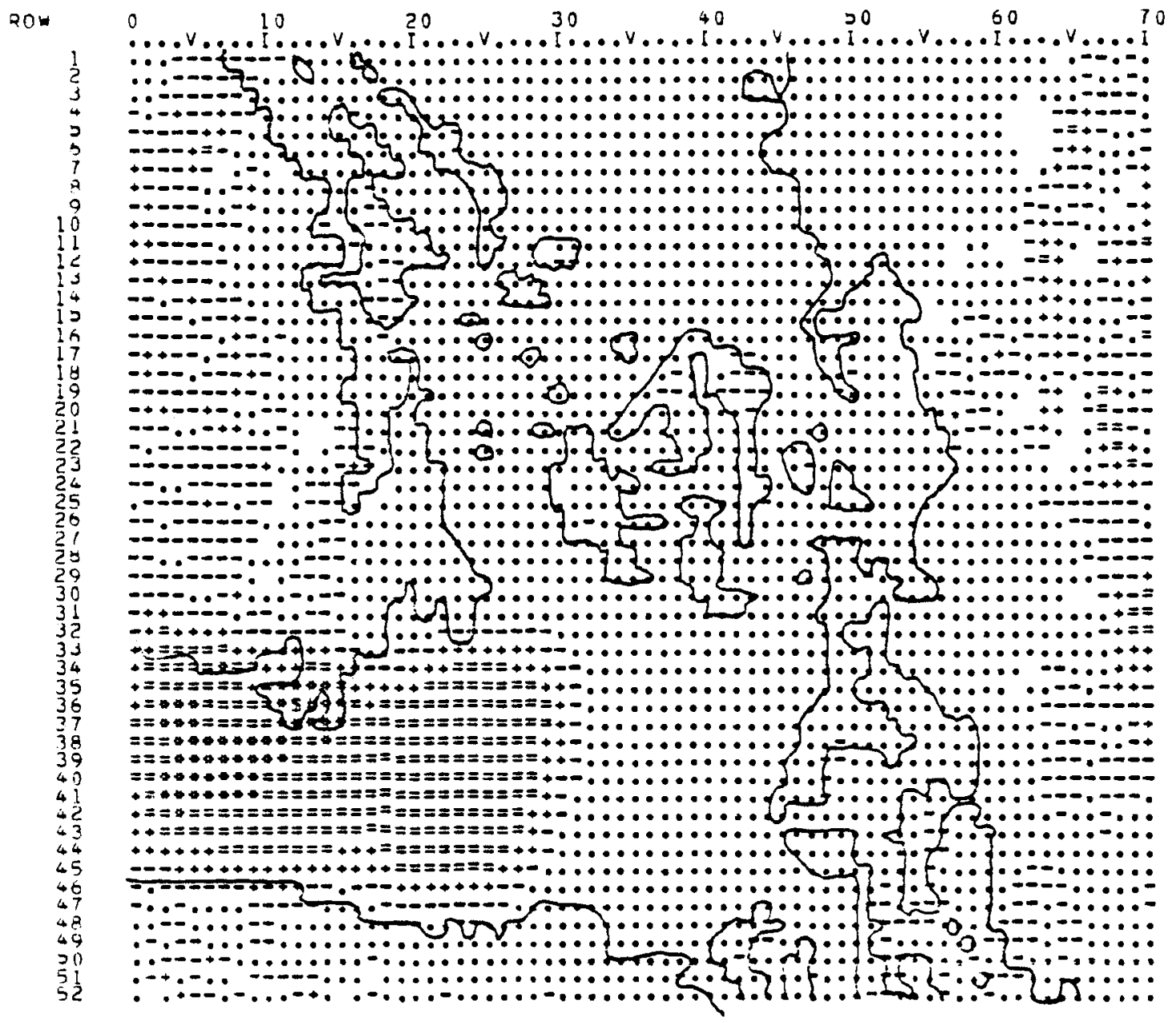

EMISSTON RESTRICTION CODES:

\begin{tabular}{|c|c|c|c|c|c|c|c|c|c|c|}
\hline CODE & Blank & . & - & + & $=$ & * & if & s & ت & $x$ \\
\hline EIISSION RATE ( $\mathrm{g} / \mathrm{s}$ ) & $<30$ & $30-59$ & $60-89$ & $90-119$ & $120-140$ & $150-179$ & $180-209$ & $210-239$ & $240-269$ & $\geq 270$ \\
\hline
\end{tabular}

$\mathrm{SO}_{2}$ Standards are $.4 \mathrm{ppm}$ 
FIGURE E36. PI ( $\pi$ ) MATRIX FOR JANUARY 30, 1975 AT 15:00

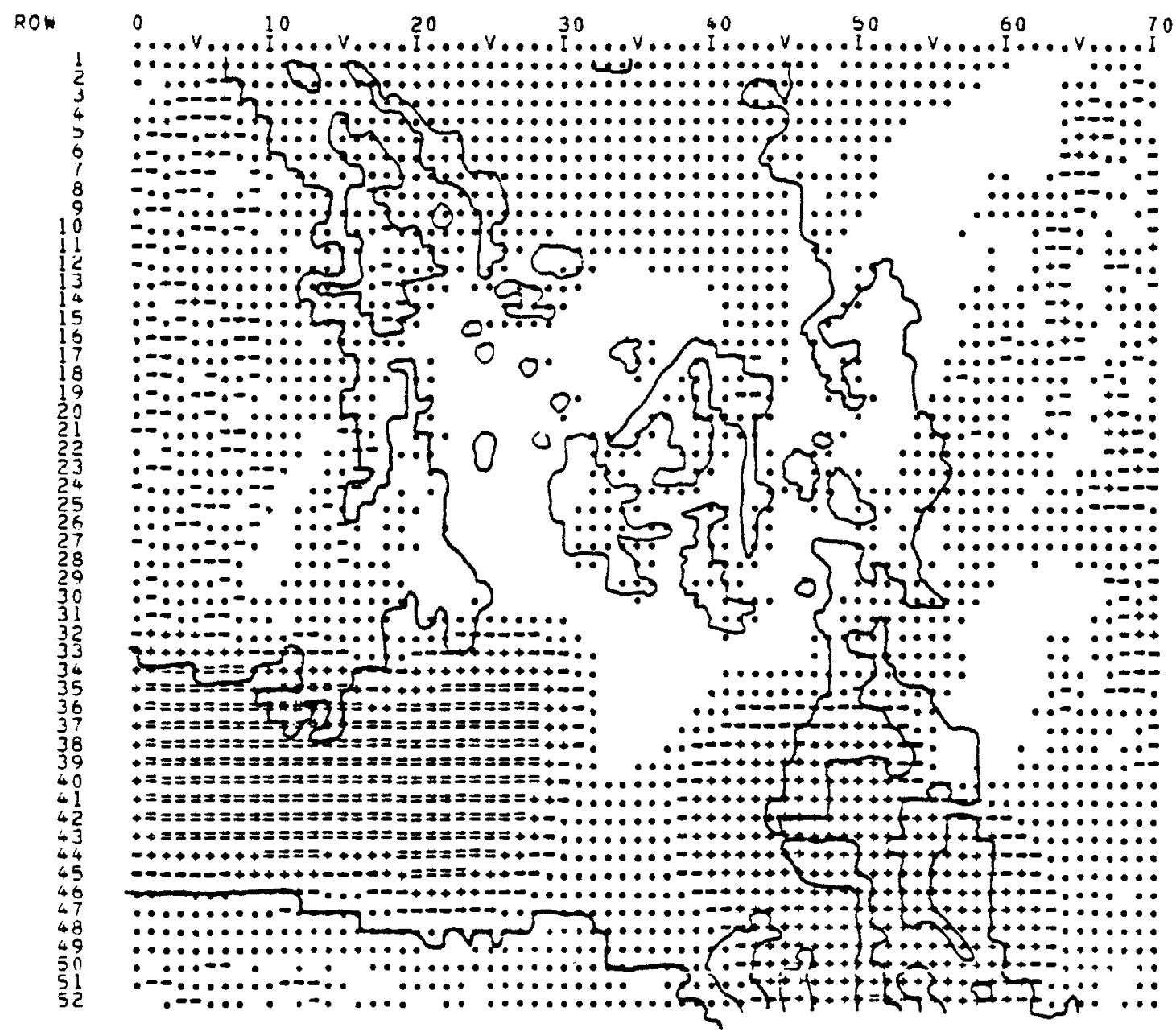

EMISSION RESTRICTION CODES:

CODE

Blanik
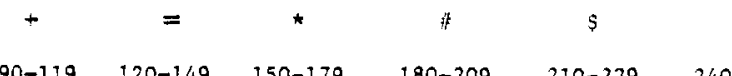

$x$

MISSION RATE ( $/ \mathrm{g} / \mathrm{s}$ )

$<30 \quad 30-59 \quad 60-89$

$90-119 \quad 120-149 \quad 150-179$

$180-209$

$210-239$

$240-269$ 
FIGURE E37. PI ( $\pi$ ) MATRIX FOR JANUARY 30, 1975 AT 19:00

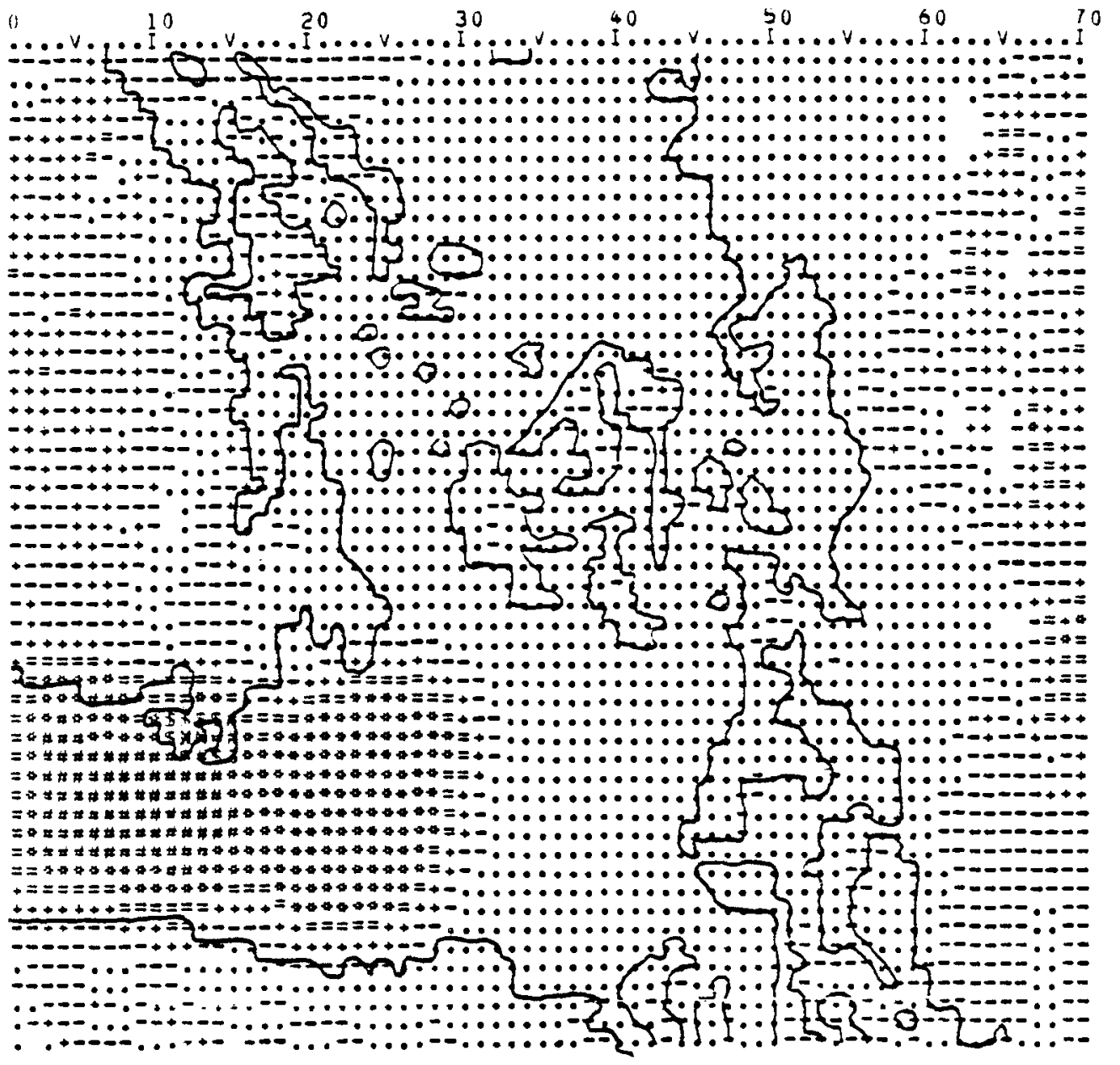

ZIISSION RESTRICTION CODES:

\begin{tabular}{|c|c|c|c|c|c|c|c|c|c|c|}
\hline CODE & Blank & . & - & + & $=$ & * & ; & \$ & 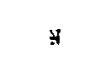 & $\mathrm{x}$ \\
\hline ISSION RATE (g/s) & $<30$ & $30-59$ & $60-89$ & $90-119$ & $120-149$ & $150-179$ & $180-209$ & $210-239$ & $240-269$ & $\geq 2$ \\
\hline
\end{tabular}

$\mathrm{SO}_{2}$ Standards are $.4 \mathrm{ppm}$ 


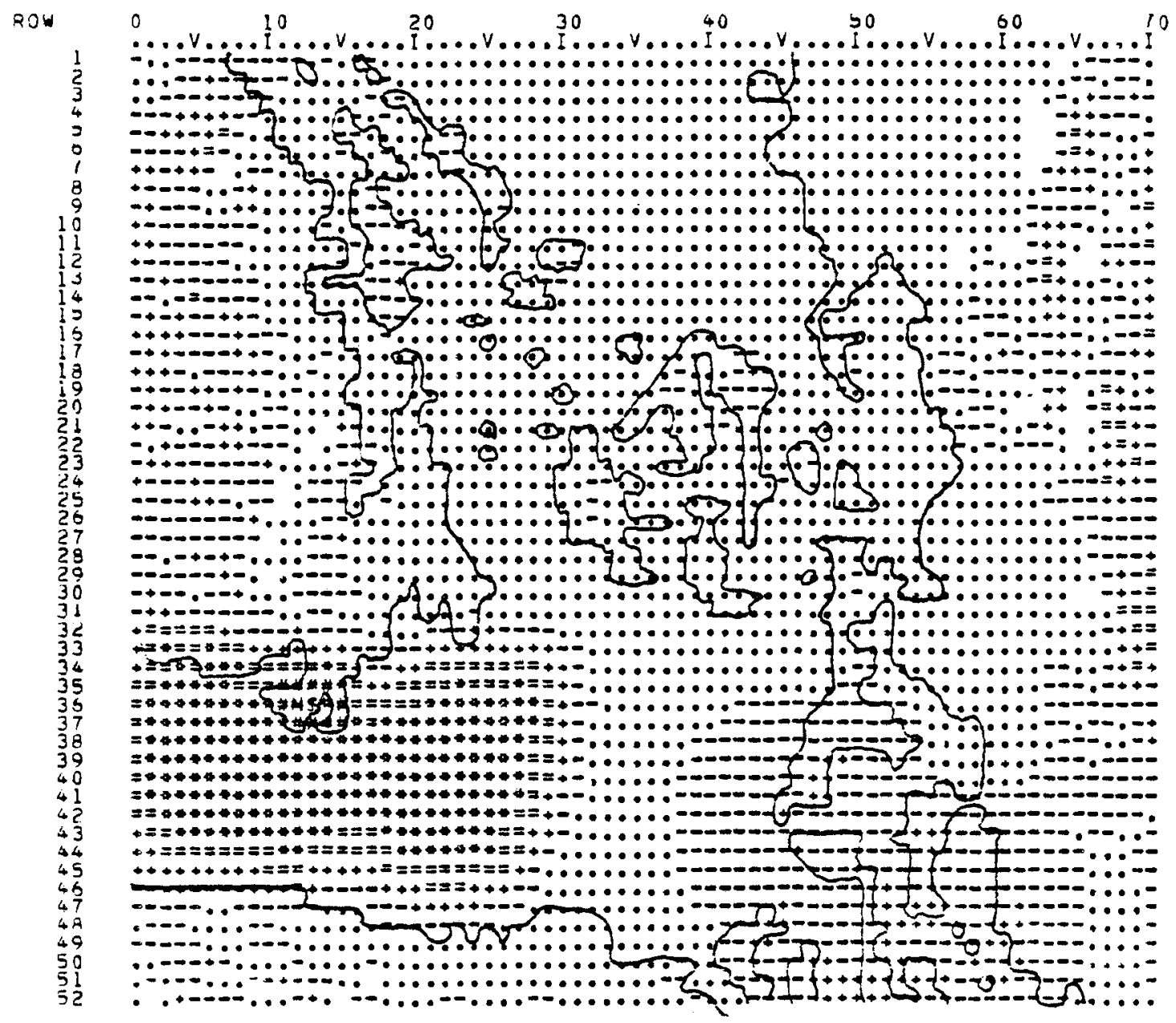

EMISSION RESTRICTION CODES:

\begin{tabular}{|c|c|c|c|c|c|c|c|c|c|c|}
\hline CODE & Siank & - & - & + & $=$ & $\star$ & if & $\$$ & $y$ & $x$ \\
\hline EIISSION RATE $(g / 3)$ & $<30$ & $30-59$ & $60-89$ & $90-119$ & $120-149$ & $150-179$ & $180-209$ & $210-239$ & $240-269$ & $\geq 270$ \\
\hline
\end{tabular}

$\mathrm{SO}_{2}$ Standards are $.4 \mathrm{ppm}$ 
FIGURE E39. PI ( $\pi$ ) ILATRIX FOR JANUARY 30, 1975 COMBINED

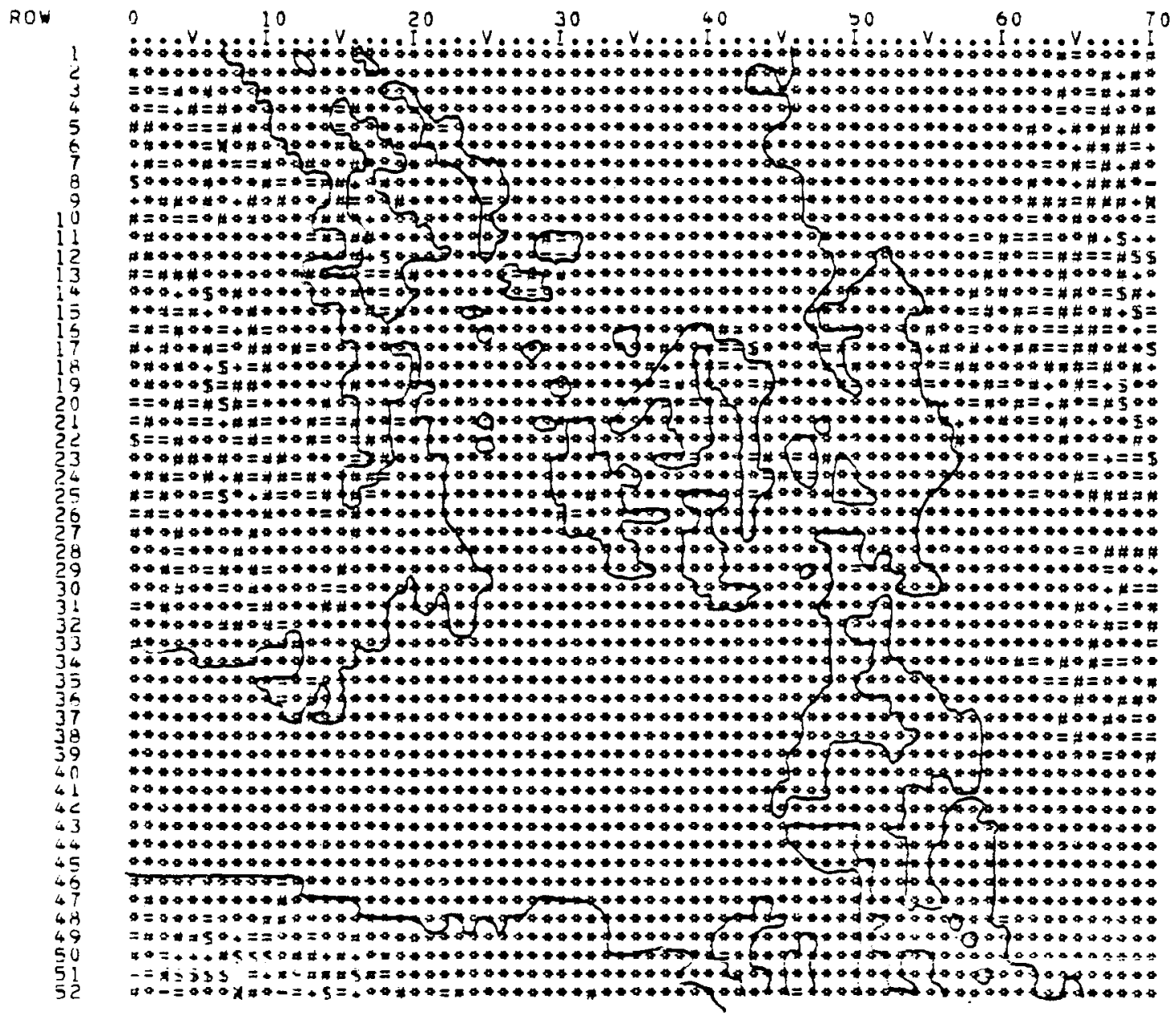

EAISSION RESTRICTION CODES:

CODE Blank $\quad$ - $\quad+\quad$ - $\quad$ * $\mathrm{A}$

TOTAL EMISIION $(\mathrm{kg})<19.90 \quad 19.90-19.99 \quad 20.00-20.09 \quad 20.10-20.19 \quad 20.20-20.29 \quad 20.30-20.39 \quad 20.40-20.49 \quad 20.50-20.59 \quad \geq 20.60$

$\mathrm{SO}_{2}$ Standards are .1 ppm 
Figure E40. Windfield Analysis of Northern Puget Sound Study Area for February 12, 1975 at 0300 PST. Background flow input: 112.5 degrees at 5.0 meters per second ( $)$.

$$
\text { Wind Flag Codes: } \begin{array}{r}
\frac{\mathrm{m} / \mathrm{s}}{5.0} \\
2.0 \\
7<1.0
\end{array}
$$




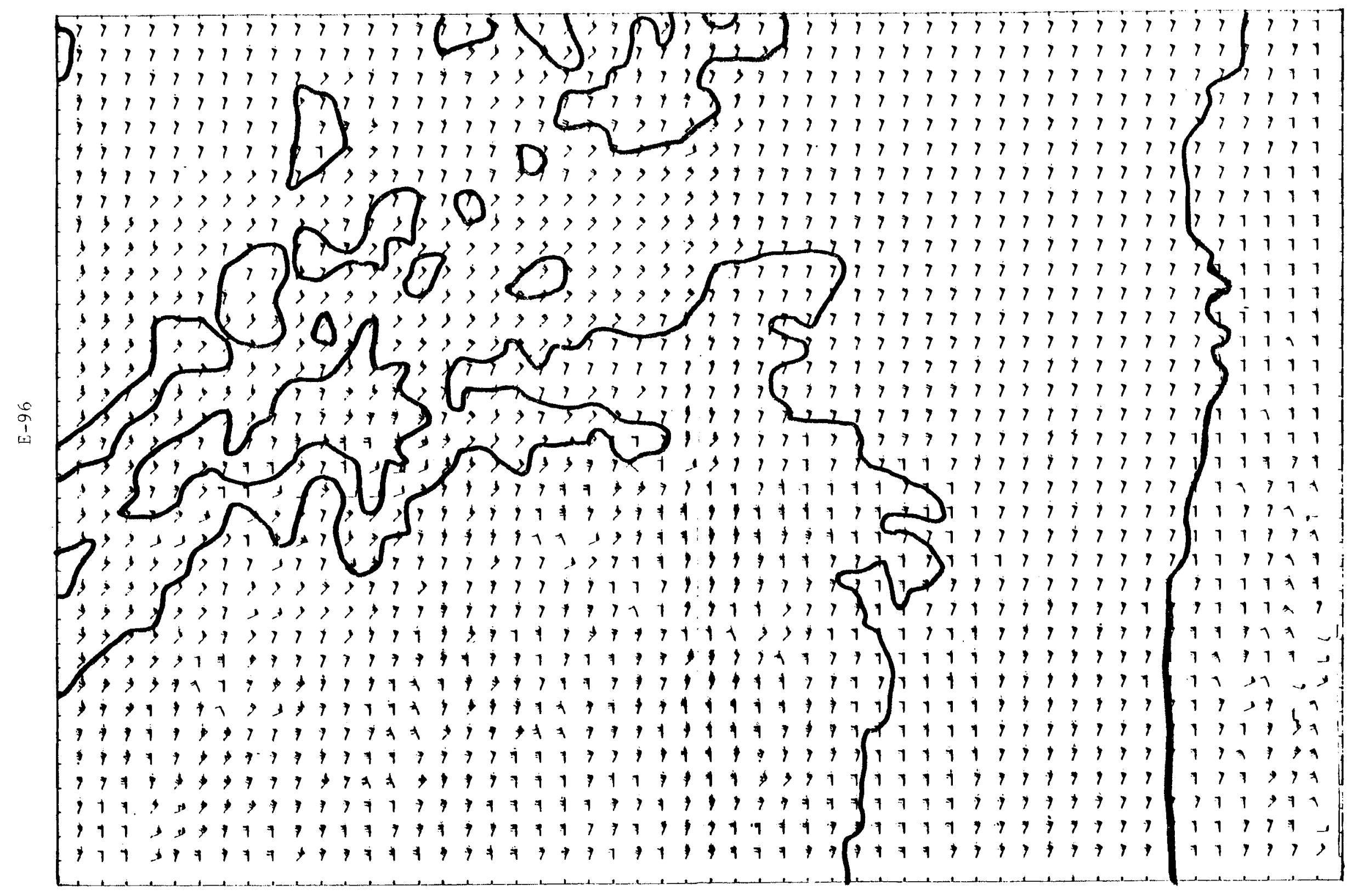




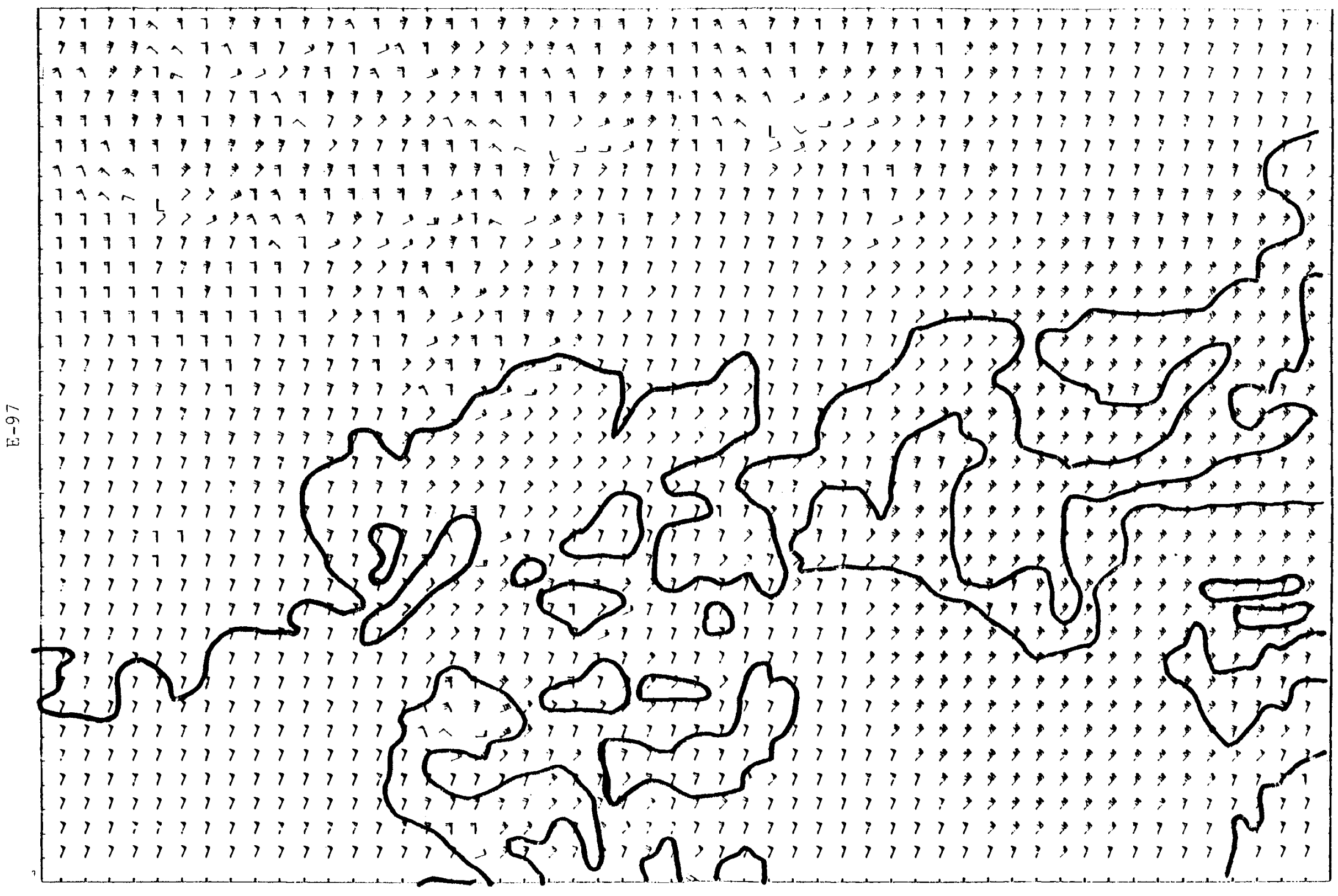


Figure E41. Windfield Analysis of Northern Puget Sound Study Area for February 12, 1975 at 0700 PST. Background flow input: 157.5 degrees at 6.0 meters per second ( $b$ ).

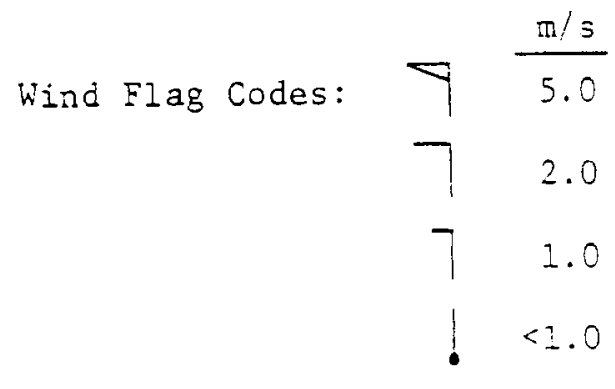




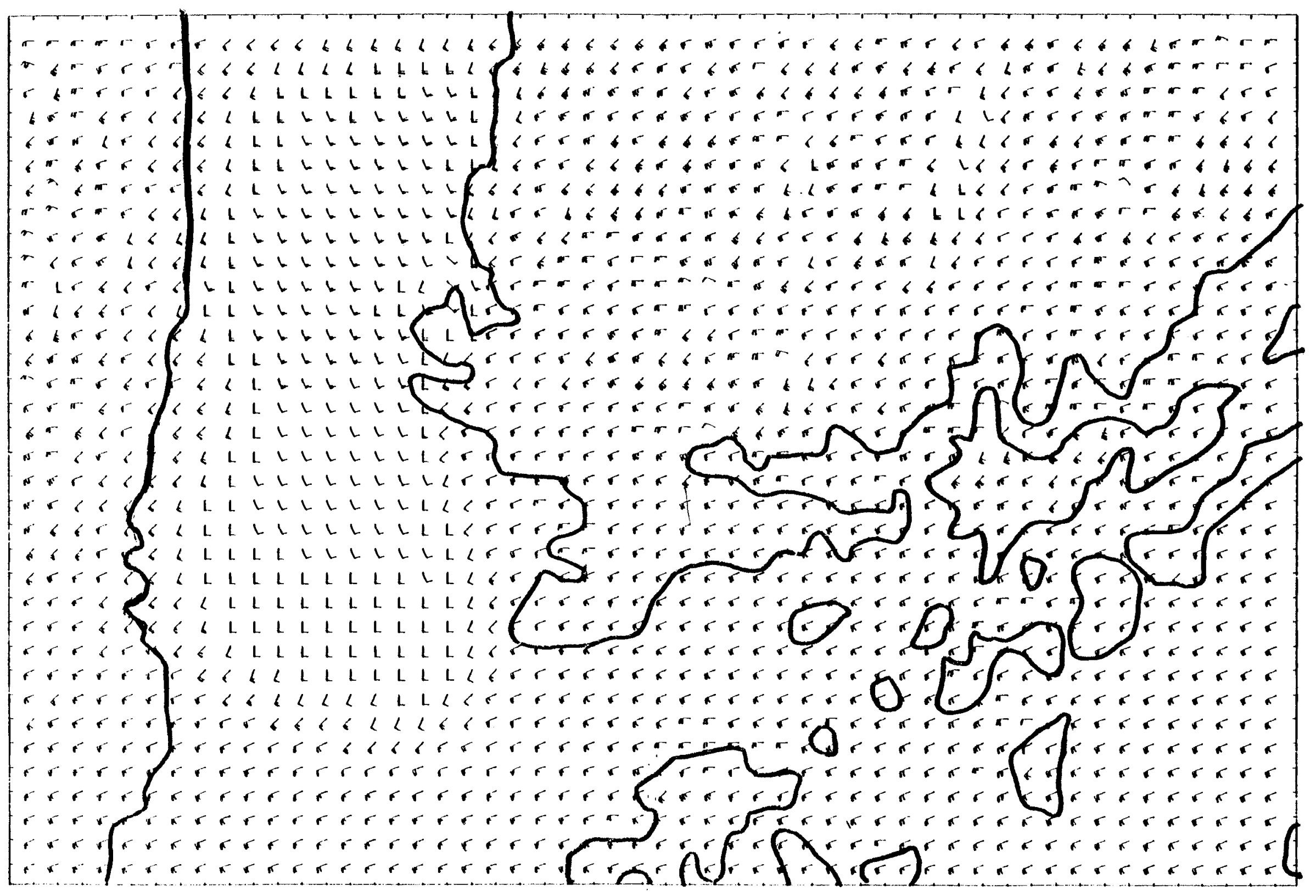




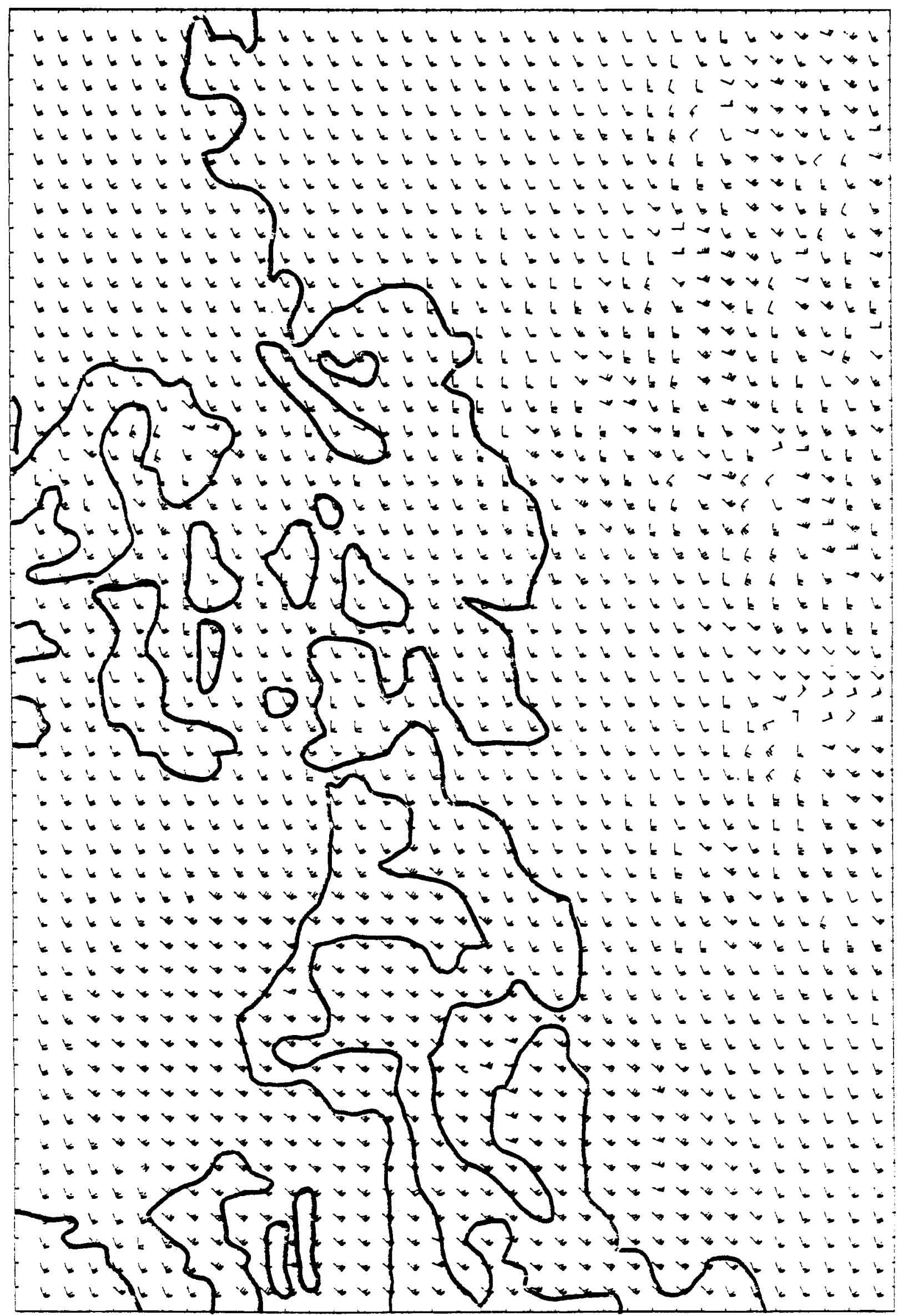


Figure E42. Windfield Analysis of Northern Puget Sound Study Area for February 12, 1975 at 1100 PST. Background flow input: 180.0 degrees at 6.5 meters per second $(E)$.

$$
\text { Wind Flag Codes: } \begin{array}{r}
\frac{\mathrm{m} / \mathrm{s}}{5.0} \\
2.0 \\
7.0 \\
\end{array}
$$




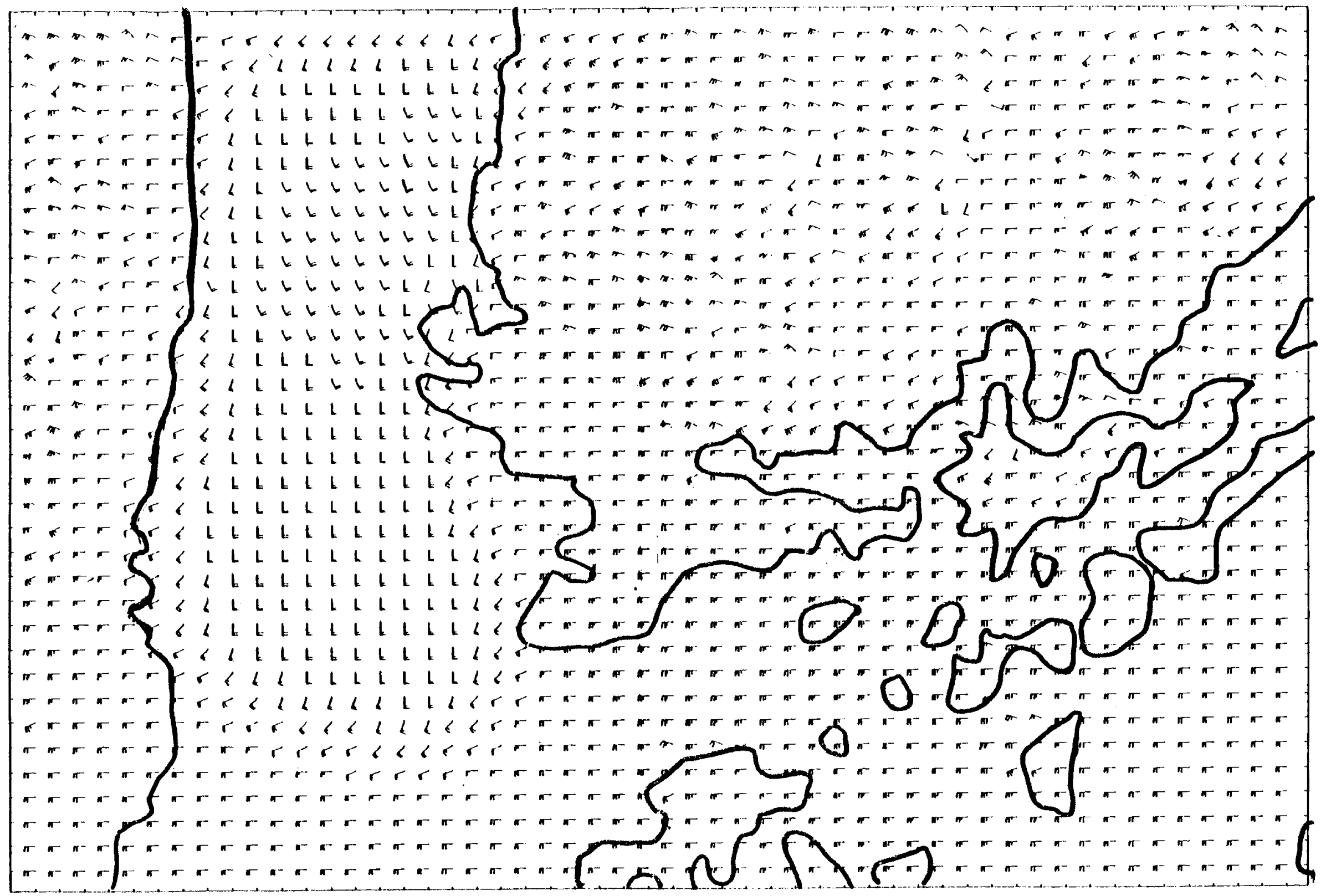




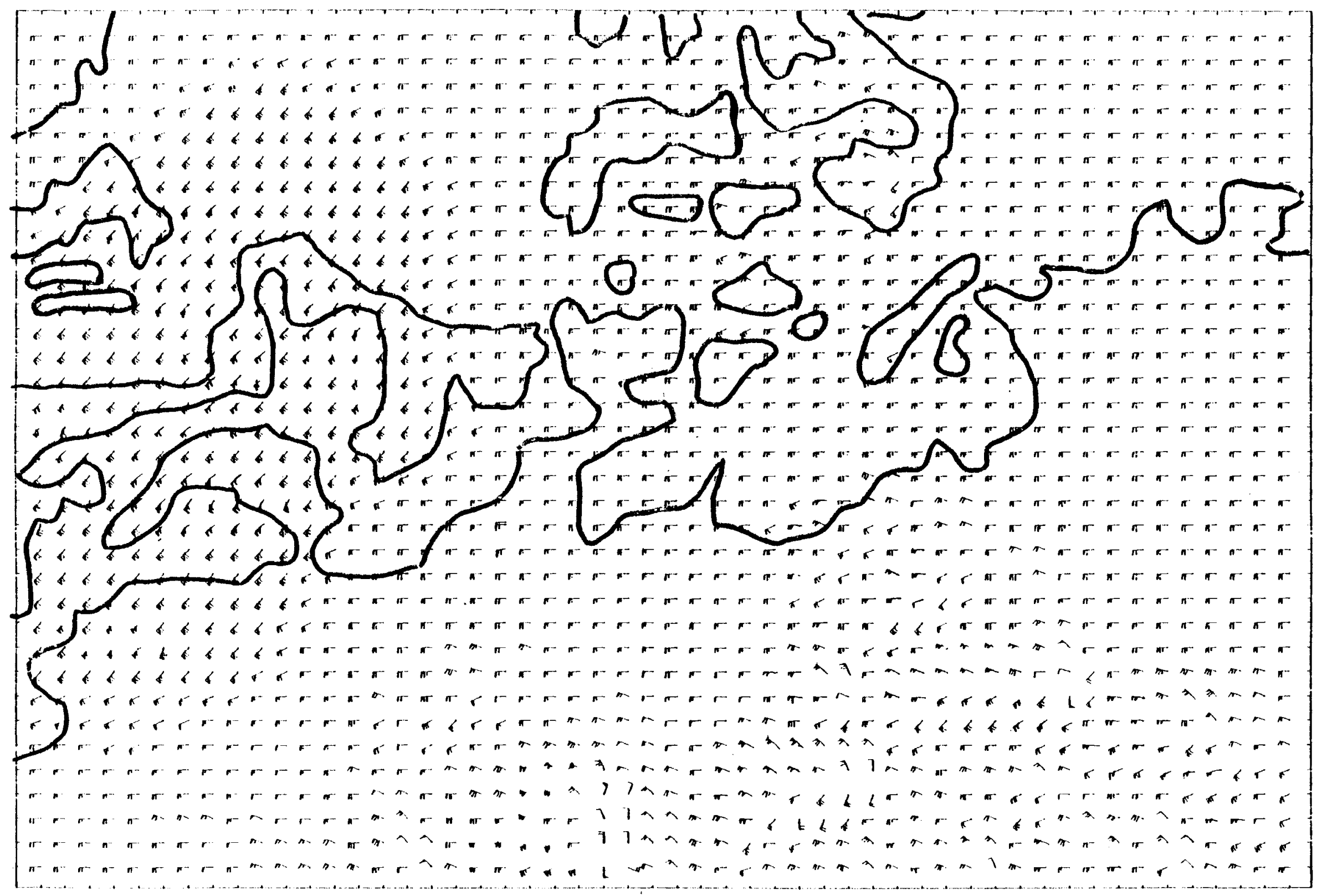


Figure E43. Windfield Analysis of Northern Puget Sound Study Area for February 12, 1975 at 1500 PST. Background flow input: 247.5 degrees at 5.5 meters per second $(\pi)$.

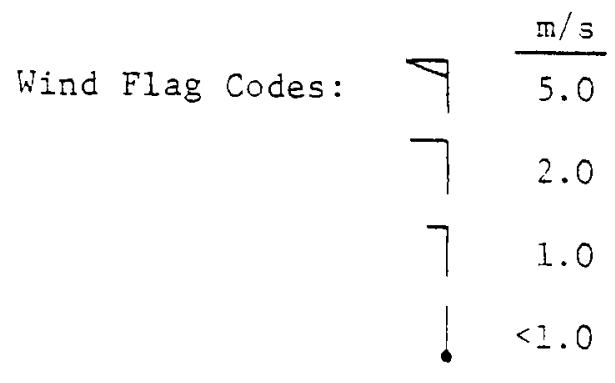




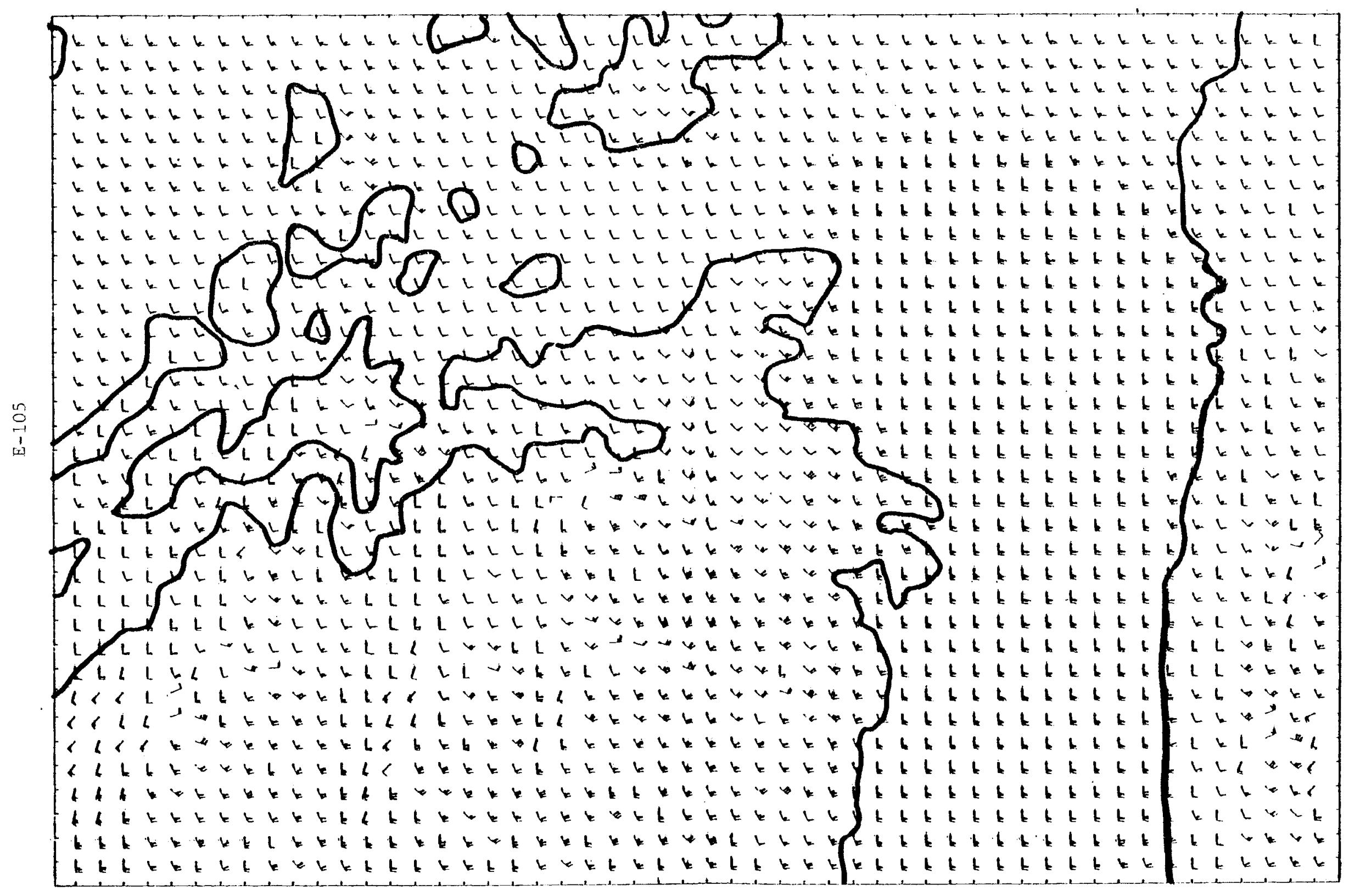




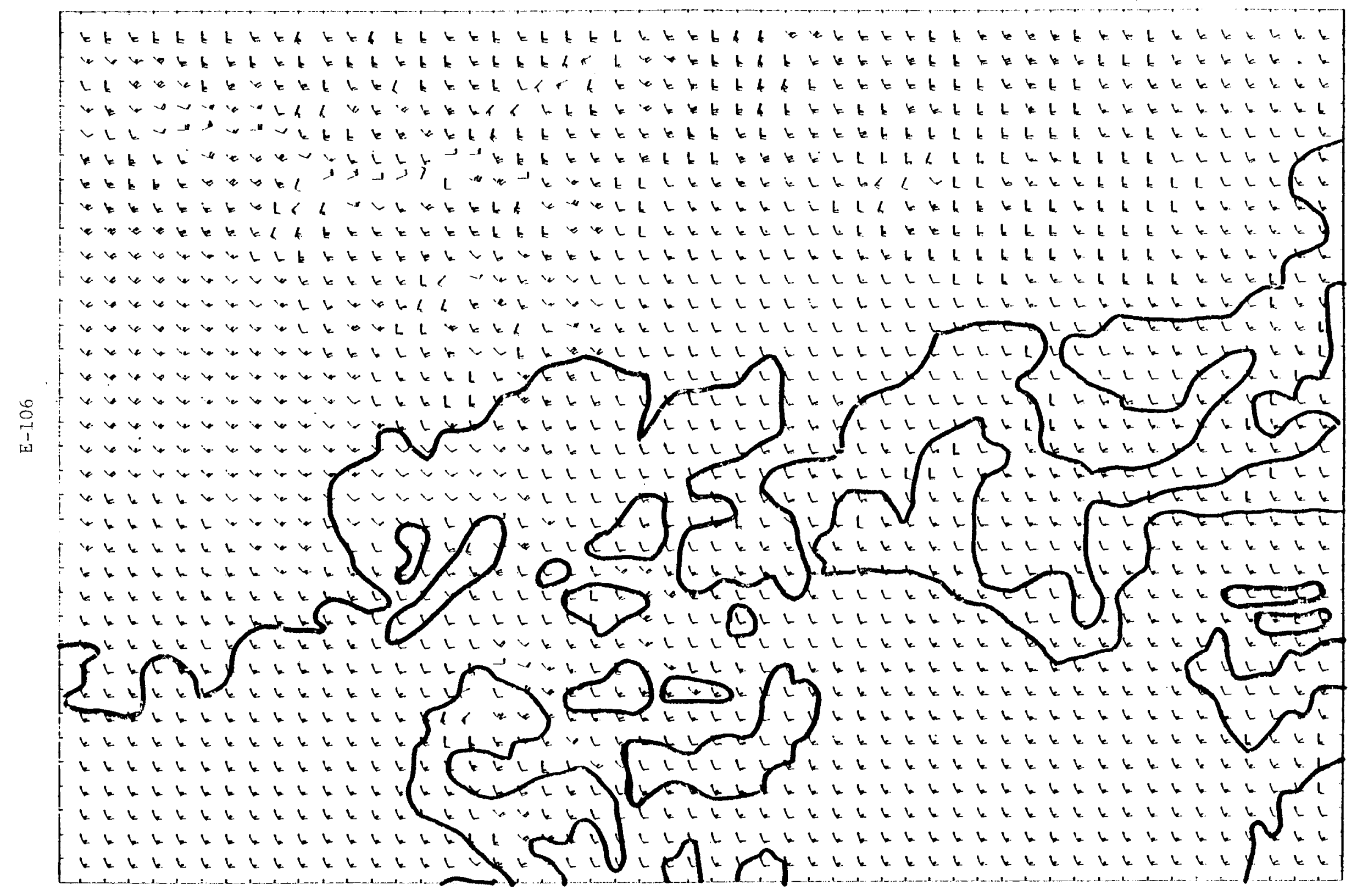


Figure E44. Windfield Analysis of Northern Puget Sound Study Area for February 12, 1975 at 1900 PST. Background flow input: 157.5 degrees at 2.5 meters per second $(t)$.

$$
\text { Wind Flag codes: } \begin{array}{r}
\frac{\mathrm{m} / \mathrm{s}}{5.0} \\
2.0 \\
7 \begin{array}{r}
1.0 \\
\end{array} \quad<1.0
\end{array}
$$




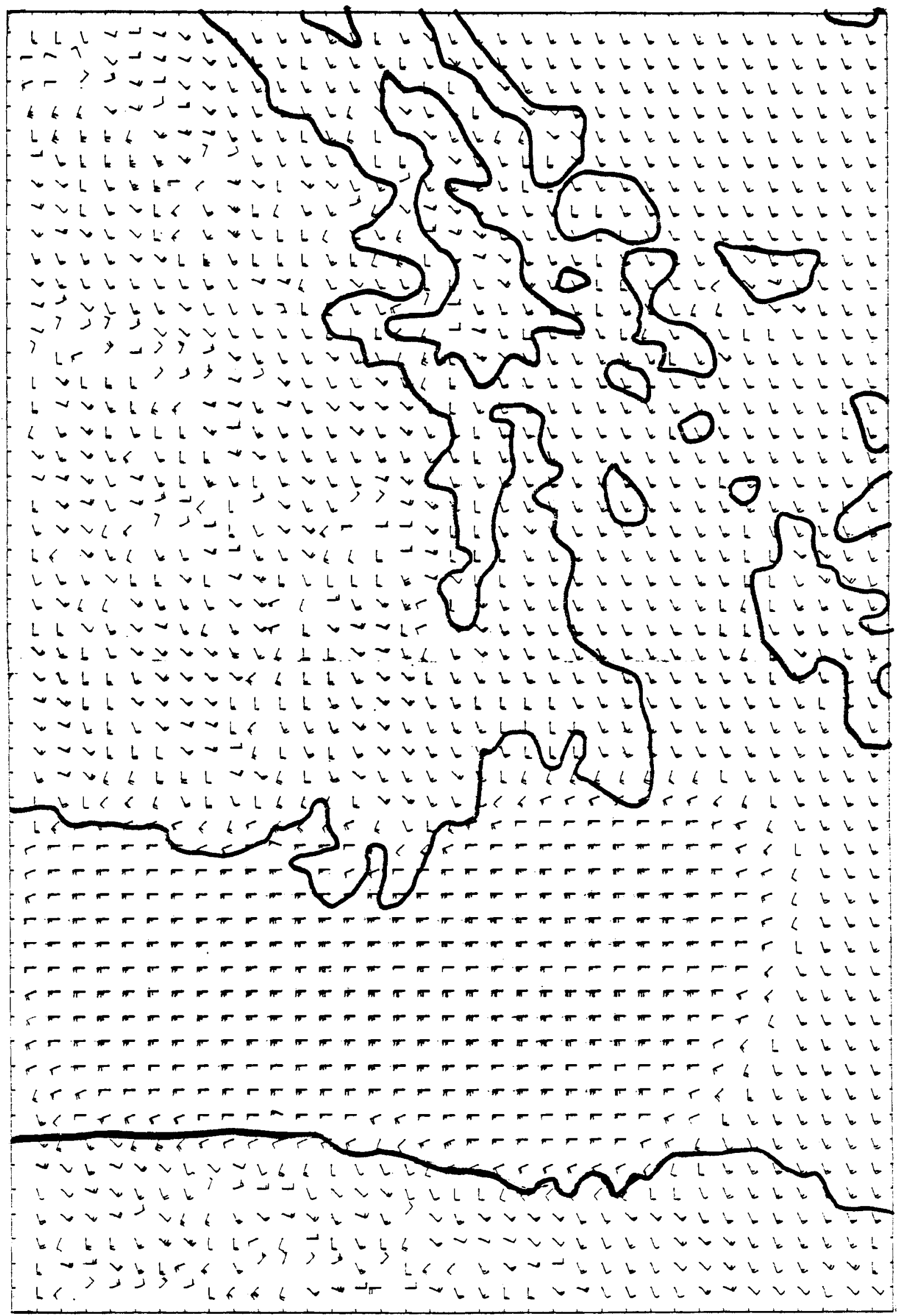




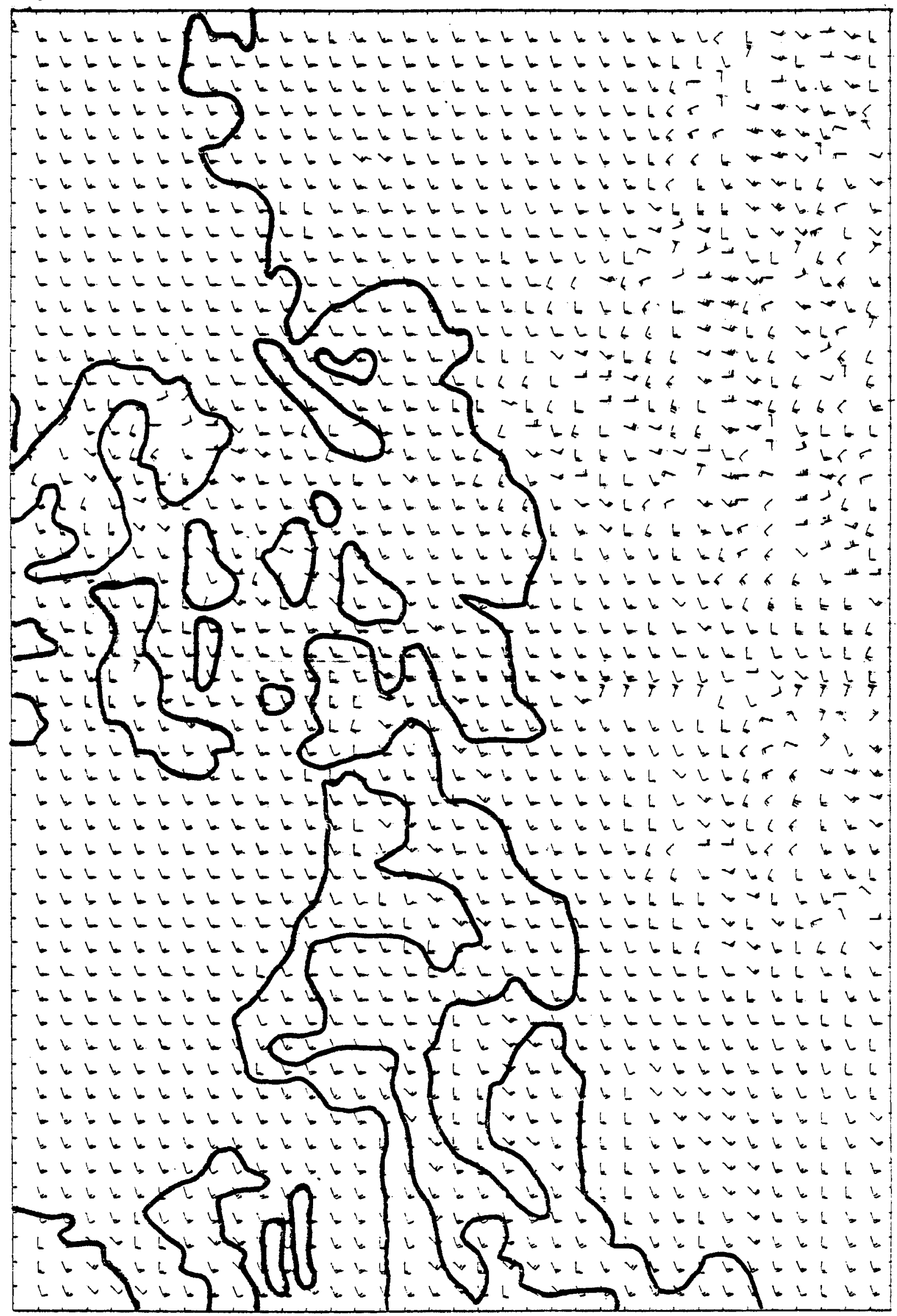


Figure E45. Windfield Analysis of Northern Puget Sound Study Area for February 12, 1975 at 2300 PST. Background flow input: 157.5 degrees at 1.5 meters per second $(L)$.

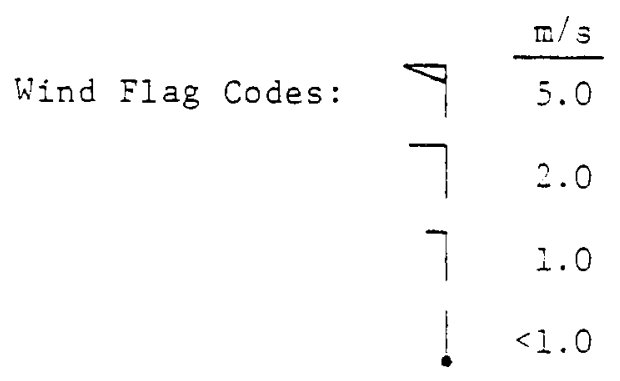




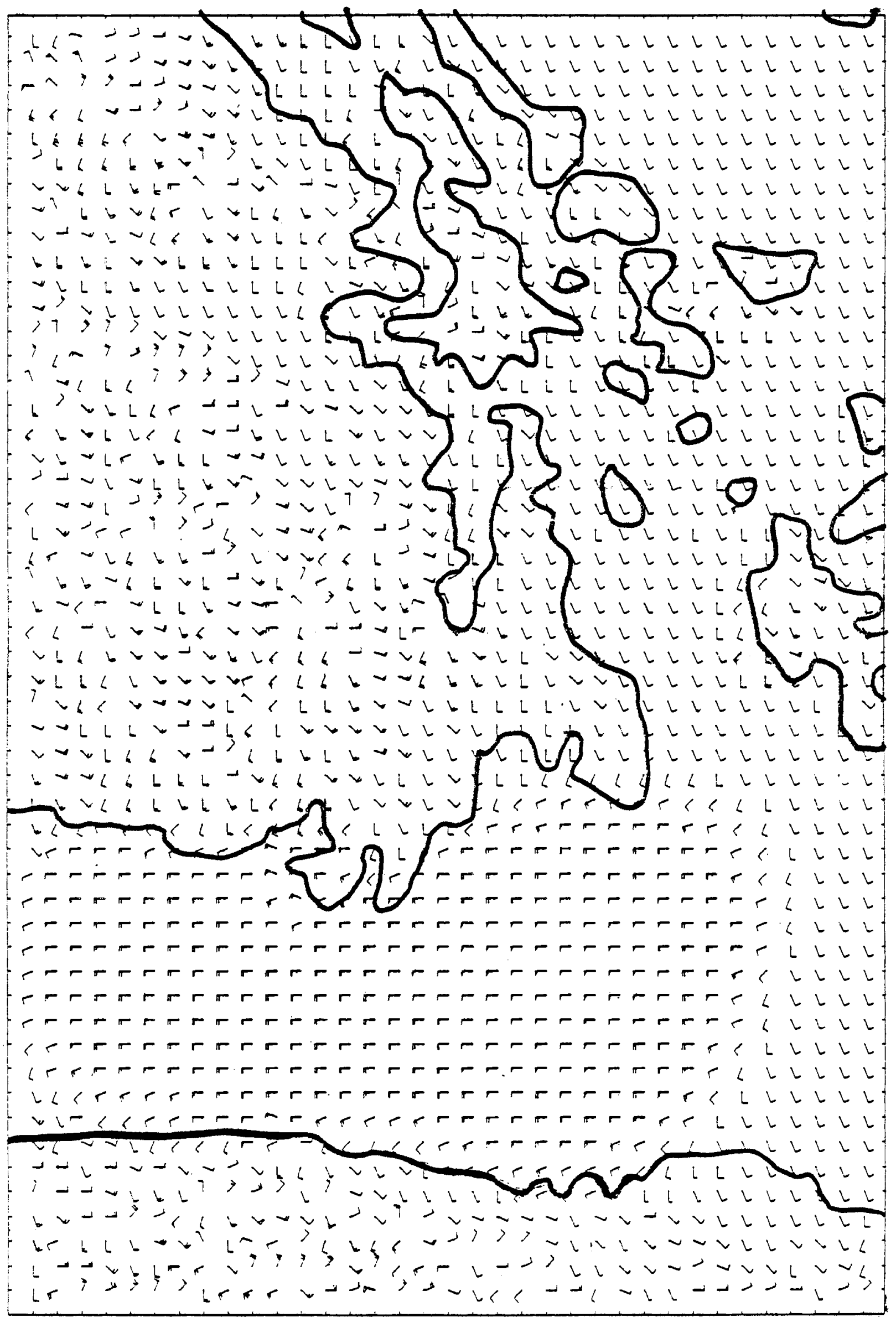




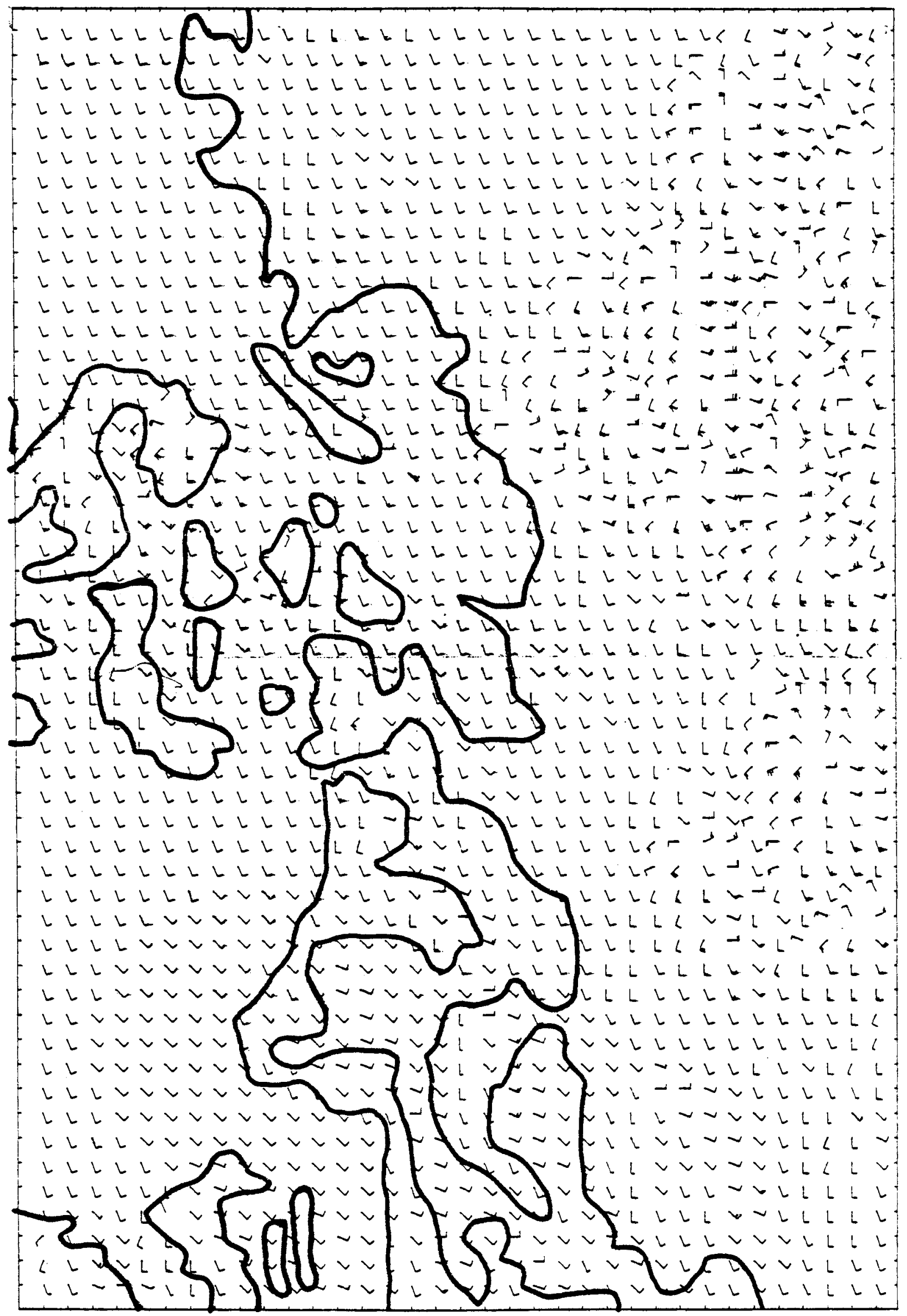

2โ工ーヨ 


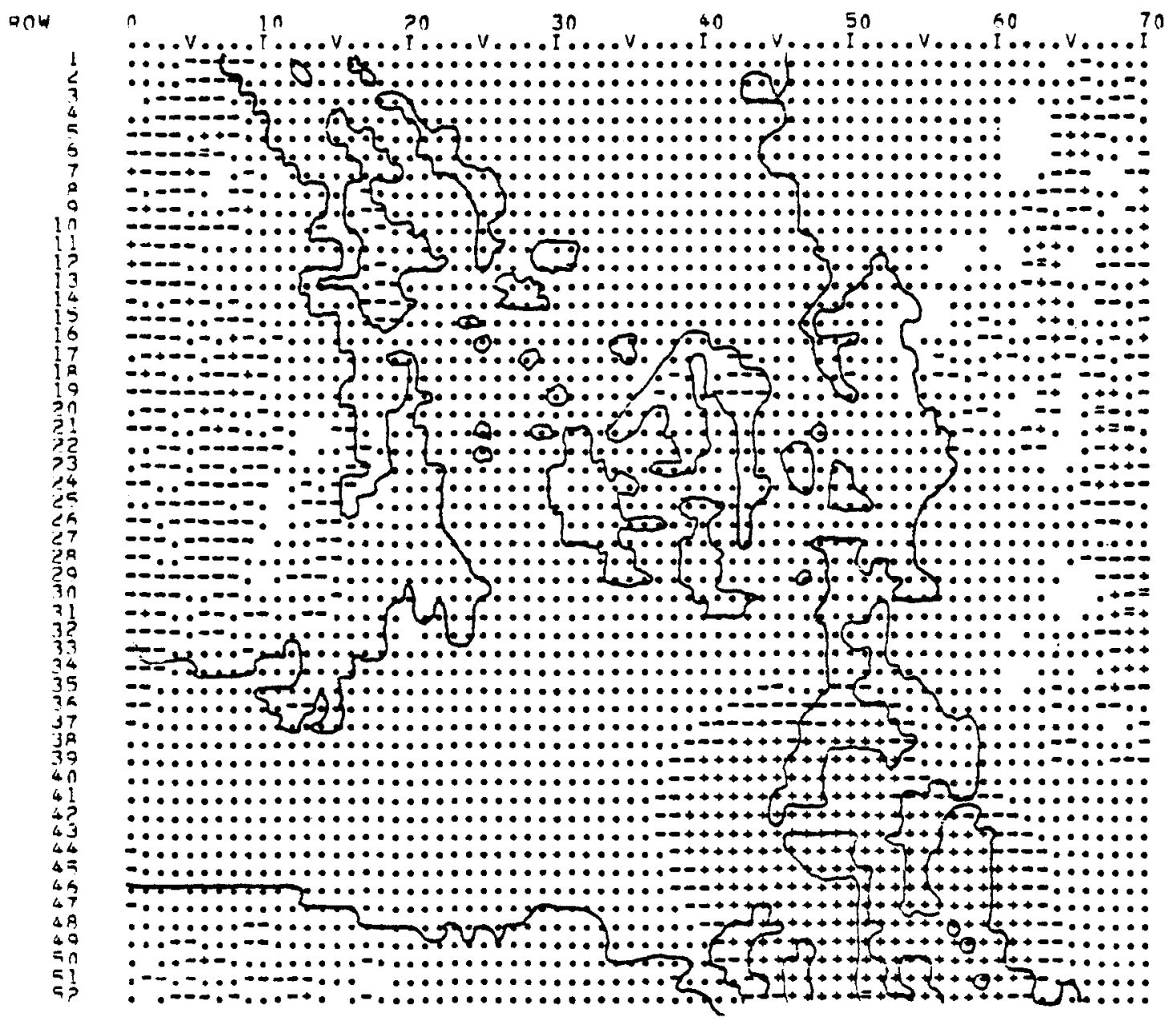

EMISSION RESTRICTION CODES:

CODE

EMISSTON RATE $(g / s)$

Blank

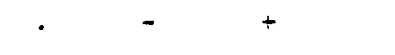

$=$
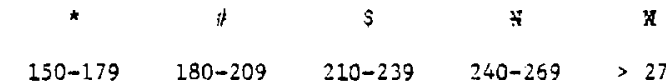

$<30 \quad 30-59 \quad 50-89 \quad 90-119 \quad 120-149$

$150-179$

$180-209$

$210-239 \quad 240-259$

$\geq 270$

$\mathrm{SO}_{2}$ Standards are $.4 \mathrm{ppm}$ 


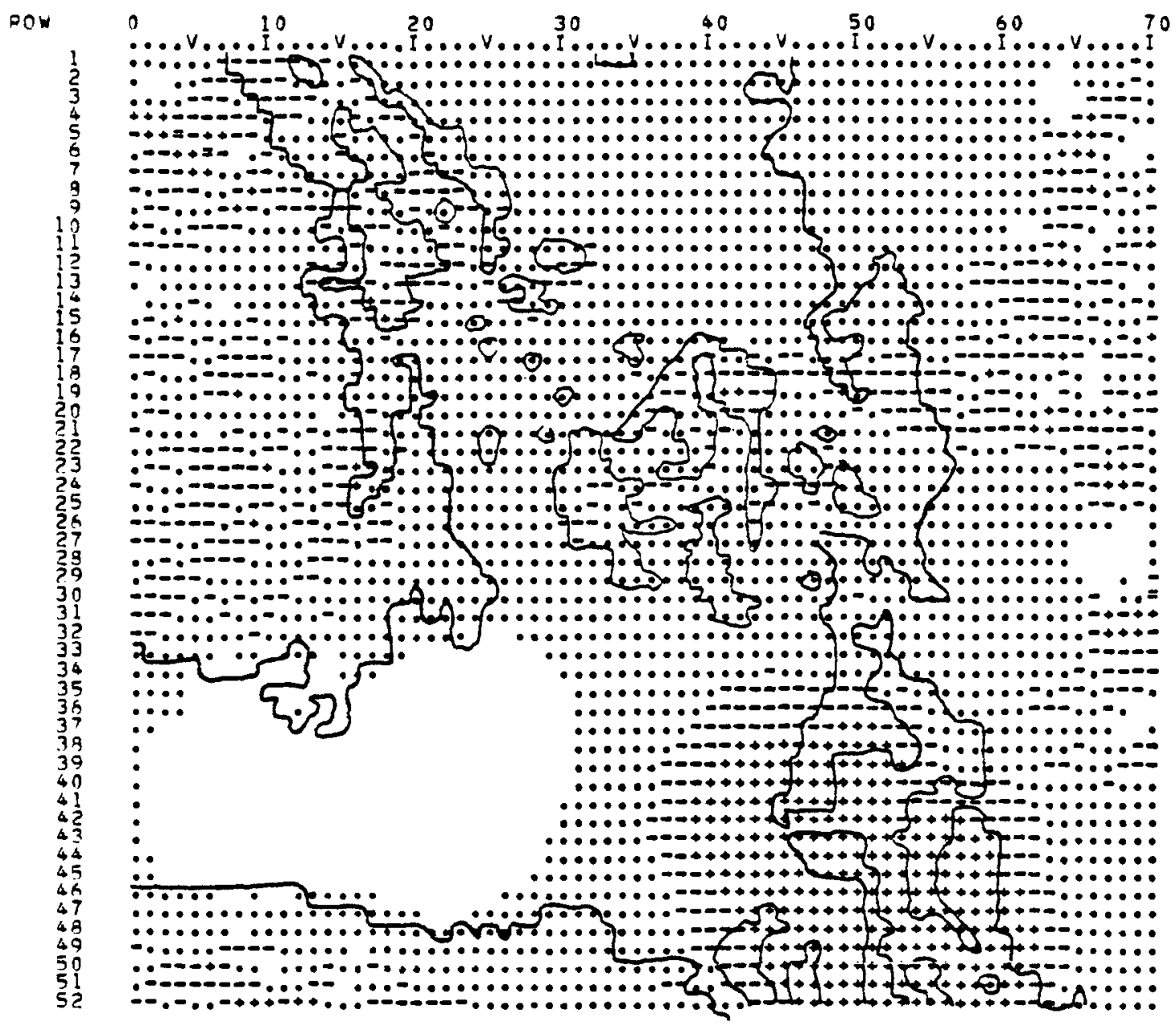

ZMISSION RESTRICTION CODES:

$\begin{array}{lcccccccccc}\text { CODE } & \text { Blank } & * & - & + & = & * & * & \$ & \$ & \mathbb{N} \\ \text { EMTSSION RATE }(\mathrm{g} / \mathrm{s}) & <30 & 30-59 & 60-89 & 90-119 & 120-149 & 150-179 & 180-209 & 210-239 & 240-259 & \geq 270\end{array}$

$\mathrm{SO}_{2}$ Standards are .4 ppm 
FIGURE E48. PI ( $\pi$ ) MATRIX FOR FEBRUARY 12, 1975 AT 11:00

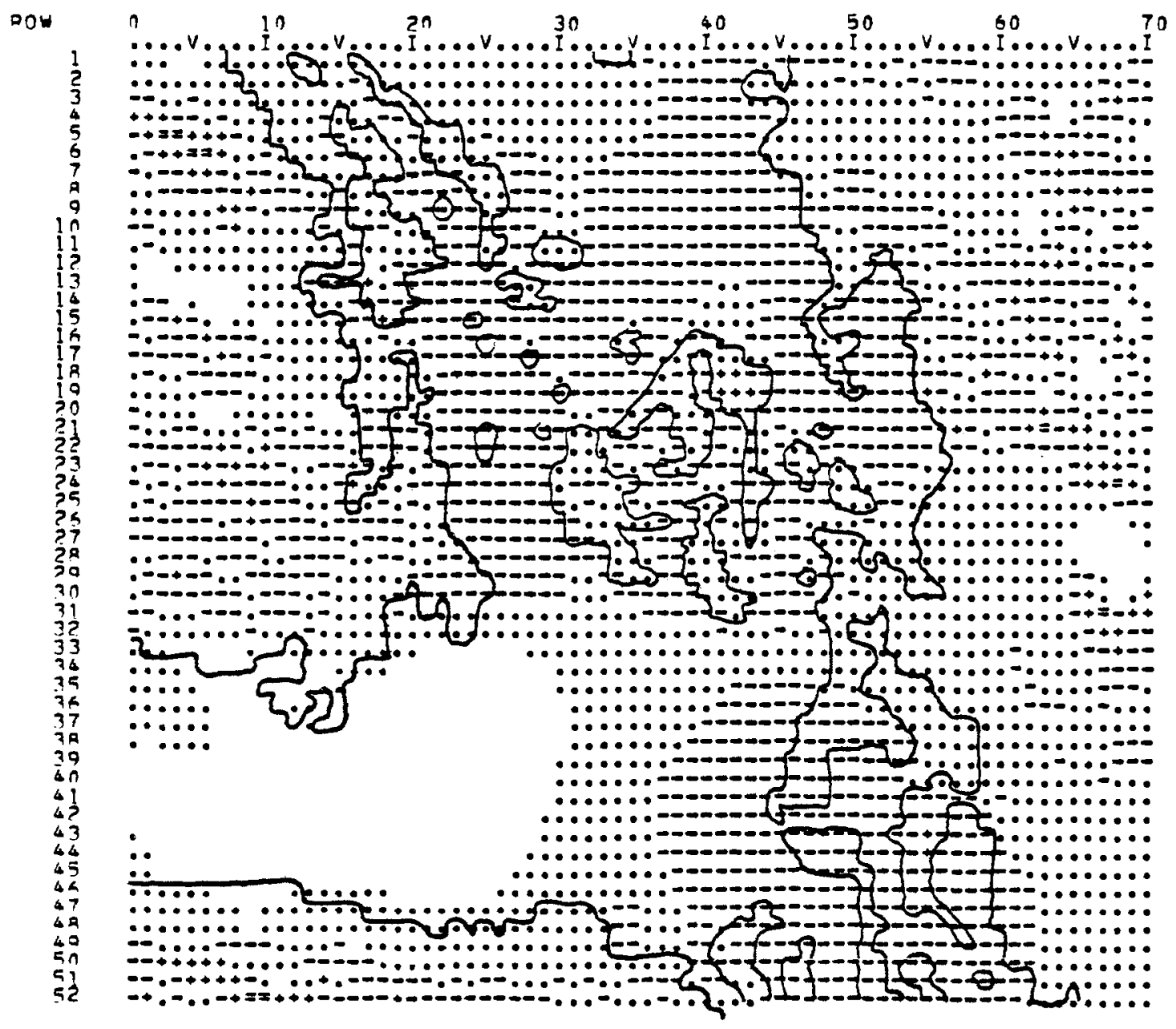

בMISSION RESTRICTION CODES:

\begin{tabular}{|c|c|c|c|c|c|c|c|c|c|c|}
\hline CODE & Blark & . & - & + & $=$ & * & $\#$ & $\$$ & 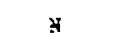 & $x$ \\
\hline EMISSION RATE $(\mathrm{g} / \mathrm{s})$ & $<30$ & $30-59$ & $50-89$ & $90-i 19$ & $120-149$ & $250-179$ & $180-209$ & $210-239$ & $240-269$ & $\geq 270$ \\
\hline
\end{tabular}

$\mathrm{SO}_{2}$ Standards are $.4 \mathrm{ppm}$ 
FIGURE E49. PI ( $\pi$ ) MATRIX FOR FEBRUARY 12, 1975 AT 15:00

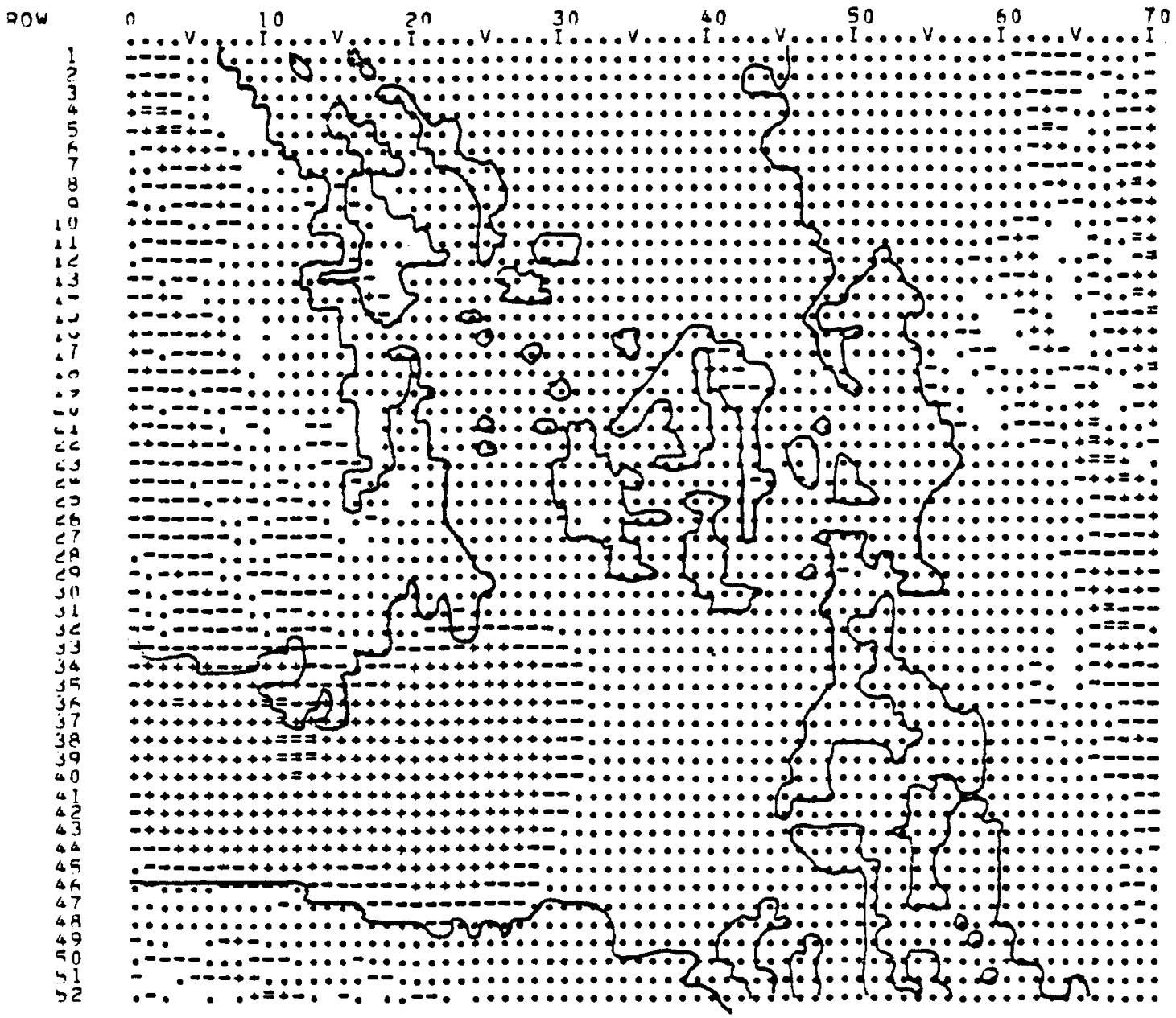

ZMISSION RESTRICTION CODES:

\begin{tabular}{|c|c|c|c|c|c|c|c|c|c|c|}
\hline CODE & B1ank & . & - & + & $=$ & * & $*$ & $\$$ & $\because$ & $x$ \\
\hline EIISSION RATE $(\mathrm{g} / \mathrm{s})$ & $<30$ & $30-59$ & $60-89$ & $90-119$ & $120-149$ & $150-179$ & $280-209$ & $210-239$ & $240-269$ & $\geq 270$ \\
\hline
\end{tabular}

$$
\mathrm{SO}_{2} \text { Standards are } .4 \mathrm{ppm}
$$


FIGURE E50. PI ( $\pi$ ) MATRIX FOR FEBRUARY 12, 1975 AT 19:00

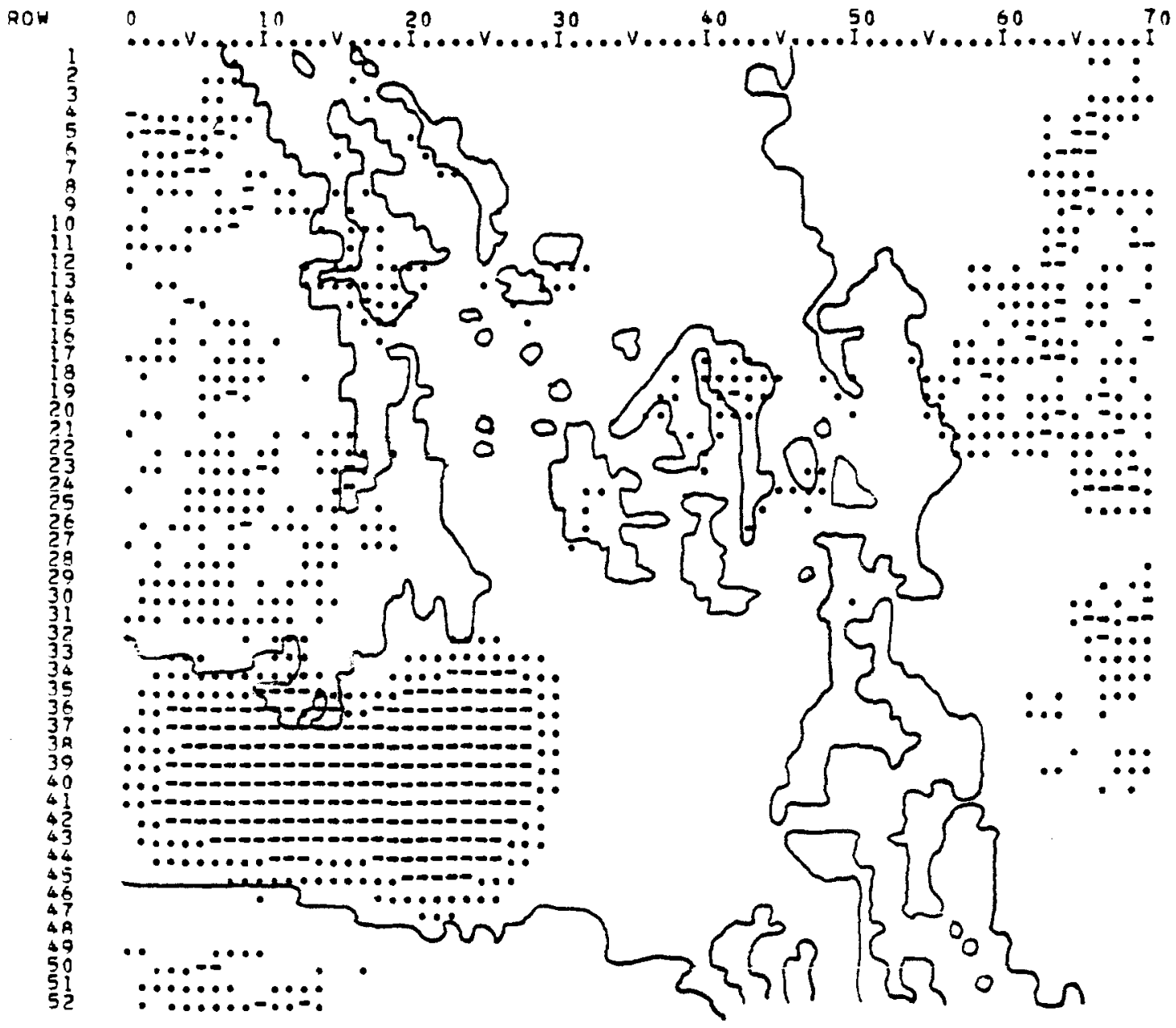

ZMISSION RESTRICTION CODES:

\begin{tabular}{|c|c|c|c|c|c|c|c|c|c|c|}
\hline CODE & Blank & . & - & + & $=$ & * & 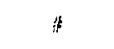 & $\$$ & $\forall$ & $\mathbf{x}$ \\
\hline BISSION RATE $(\mathrm{g} / \mathrm{s})$ & $<30$ & $30-39$ & $60-89$ & $90-119$ & $1=0-149$ & $150-179$ & $180-209$ & $210-239$ & $240-269$ & $\geq 270$ \\
\hline
\end{tabular}


FIGURE E51. PI ( $\pi$ ) MATRIX FOR FEBRUARY 12, 1975 1T 23:00

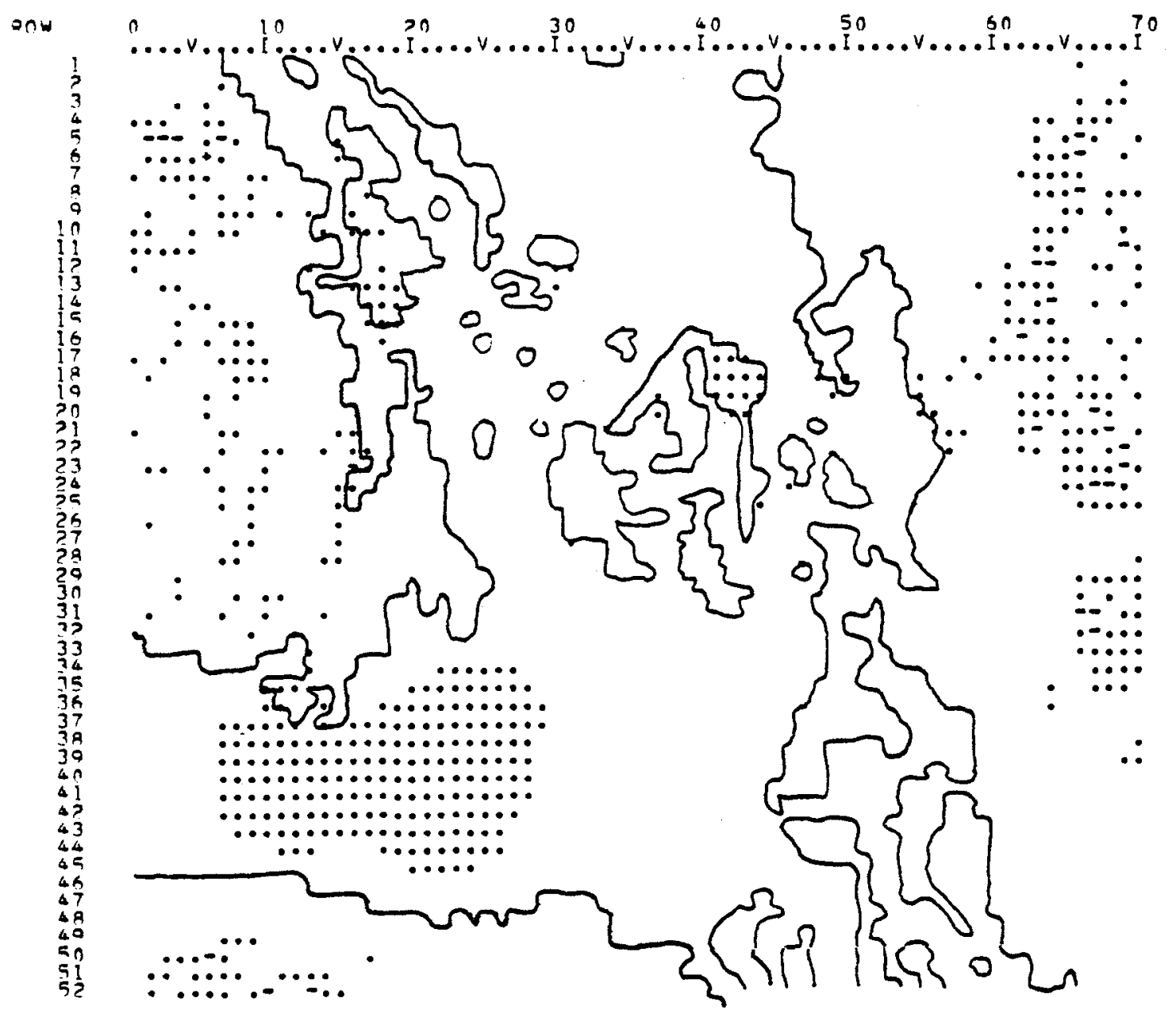

EMISSION RESTRICTION CODES:

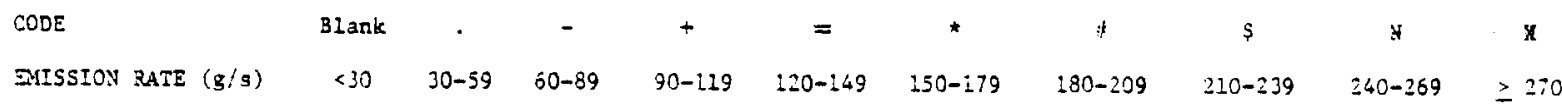

$\mathrm{SO}_{2}$ Standards are $.4 \mathrm{ppm}$ 


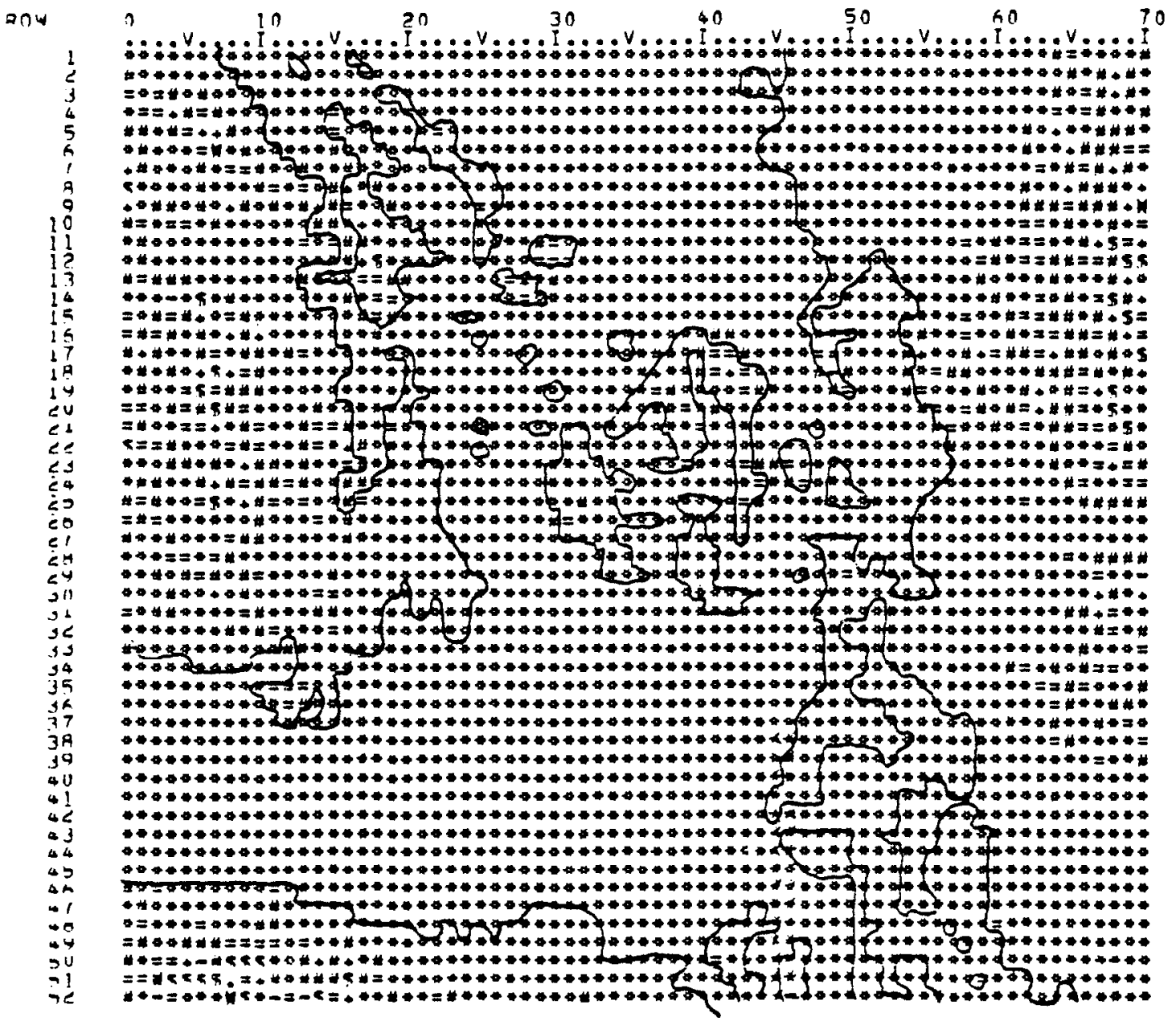

EIISSION BESTRICTION CODES:

CODE

Blank

$+$

*

*

$\$$

$x$

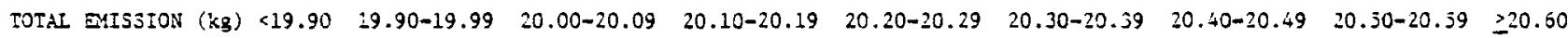

$\mathrm{SO}_{2}$ Standards are .1 ppm 
Figure E53. Windfield Analysis of Northern Puget Sound Study Area Annual Case North. Background flow input: 0.0 degrees at 5.5 meters per second ( 9 ).

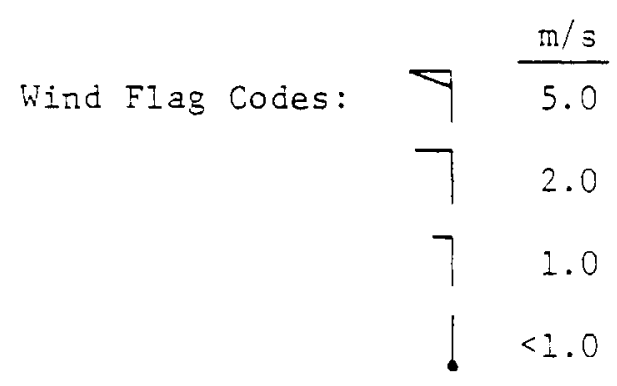


E-121

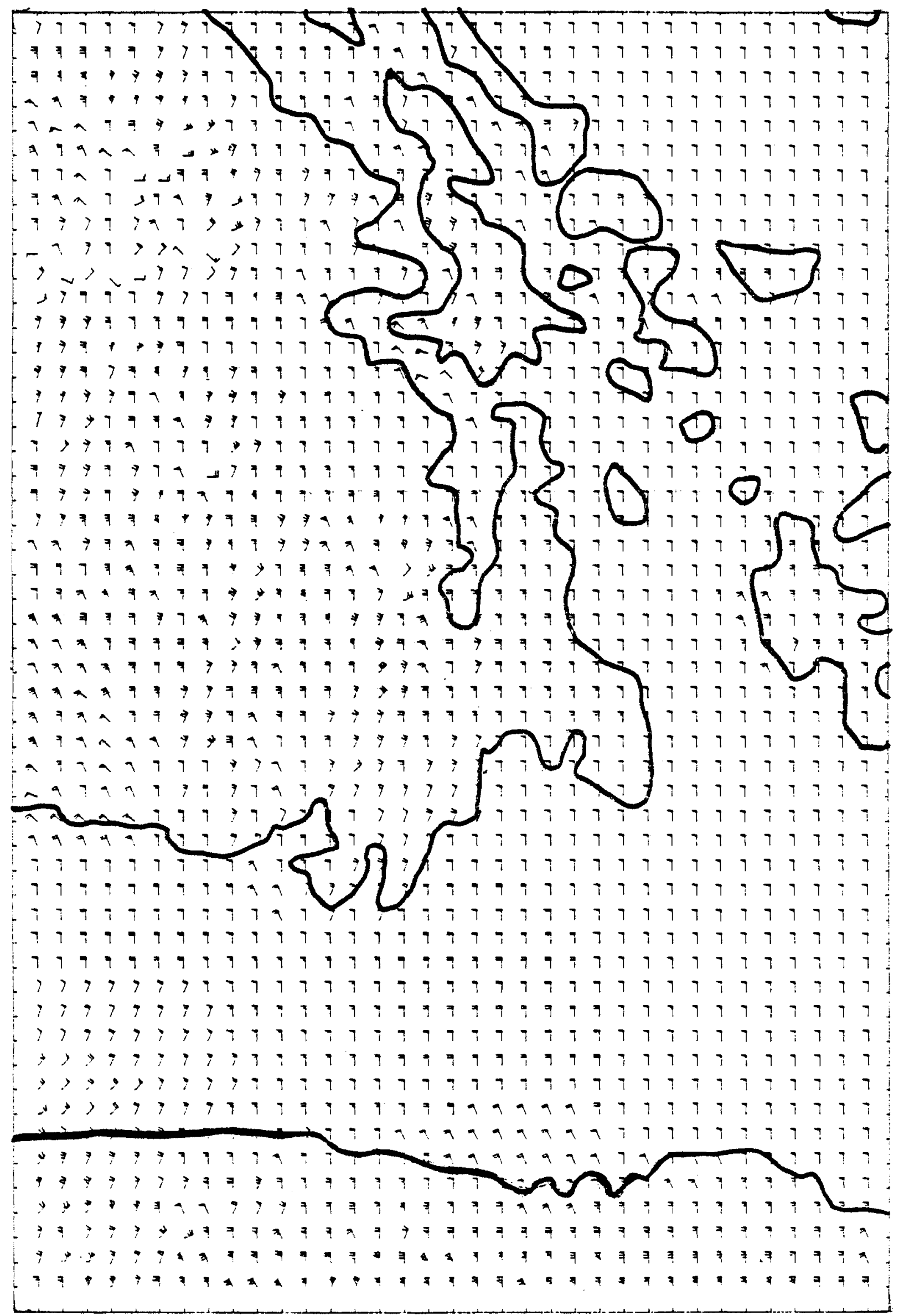




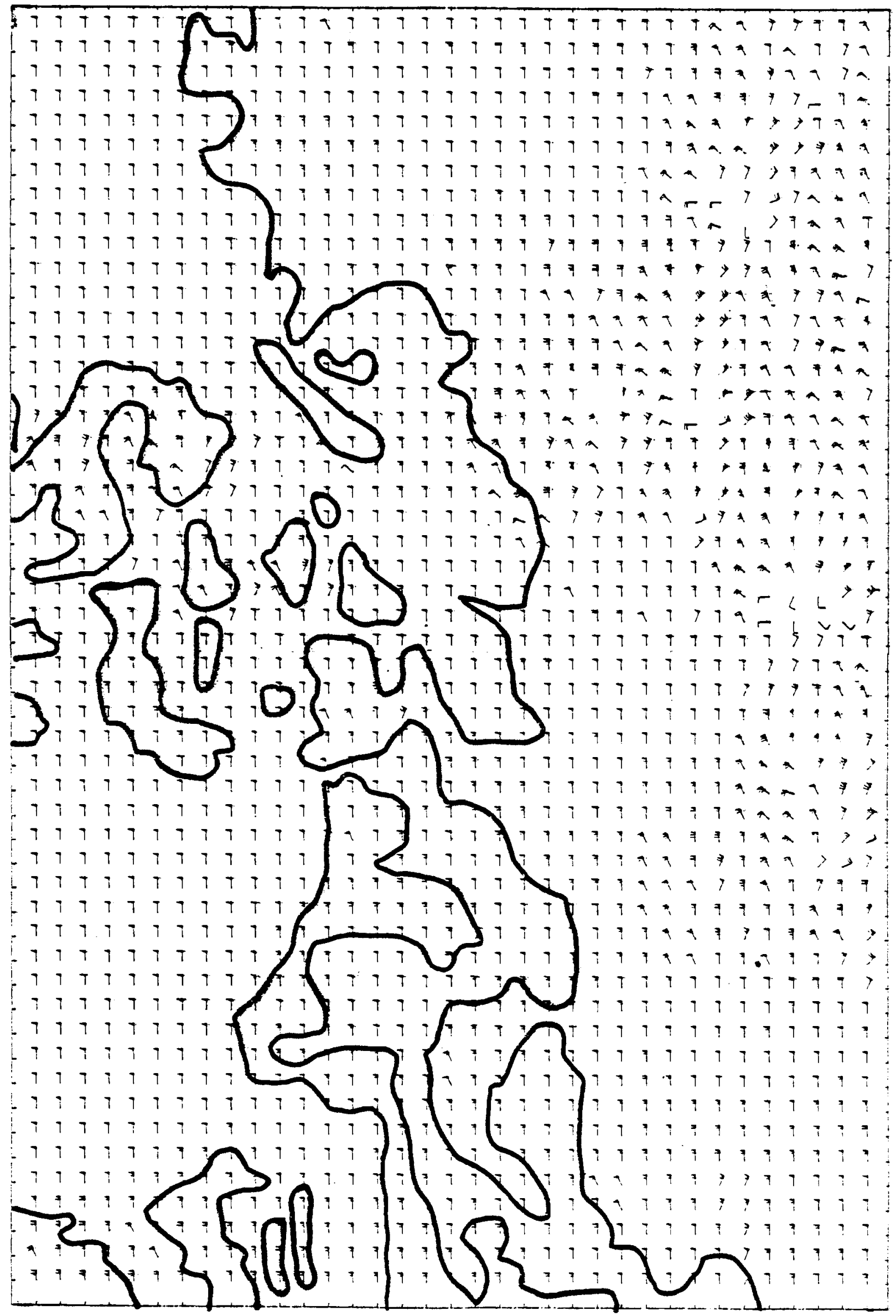


Figure E54. Windfield Analysis of Northern Puget Sound Study Area Annual Case Northeast. Background flow input:

45.0 degrees at 5.5 meters per second $(\xi)$.

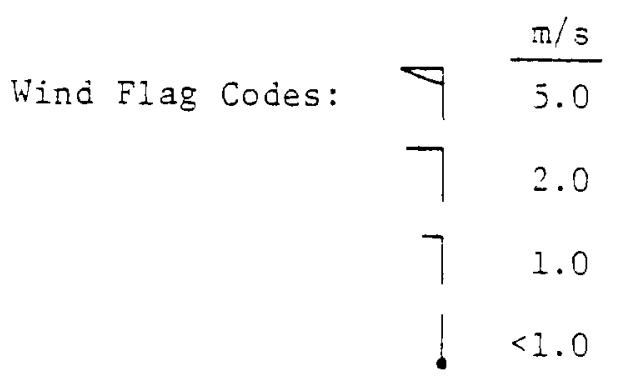




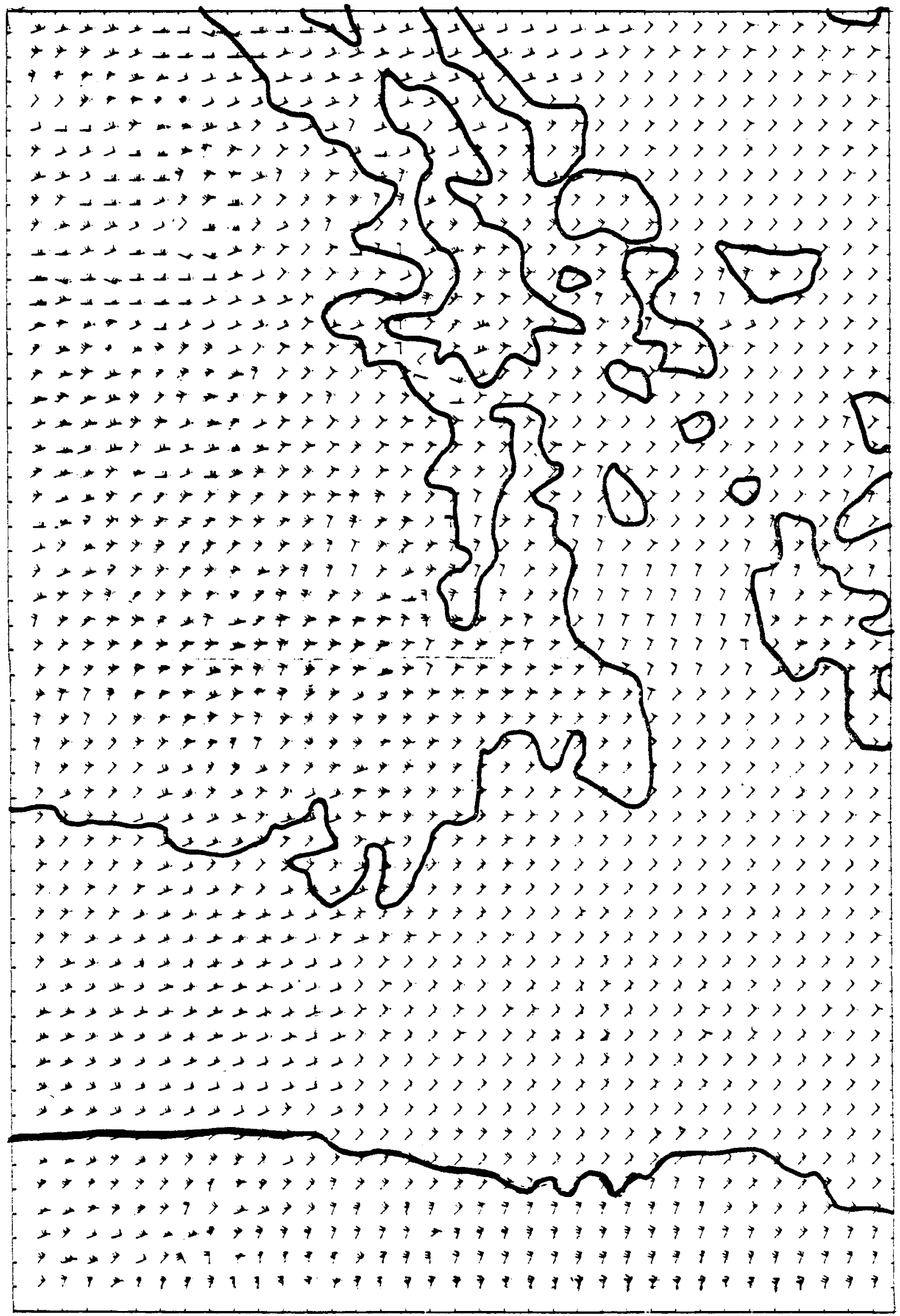




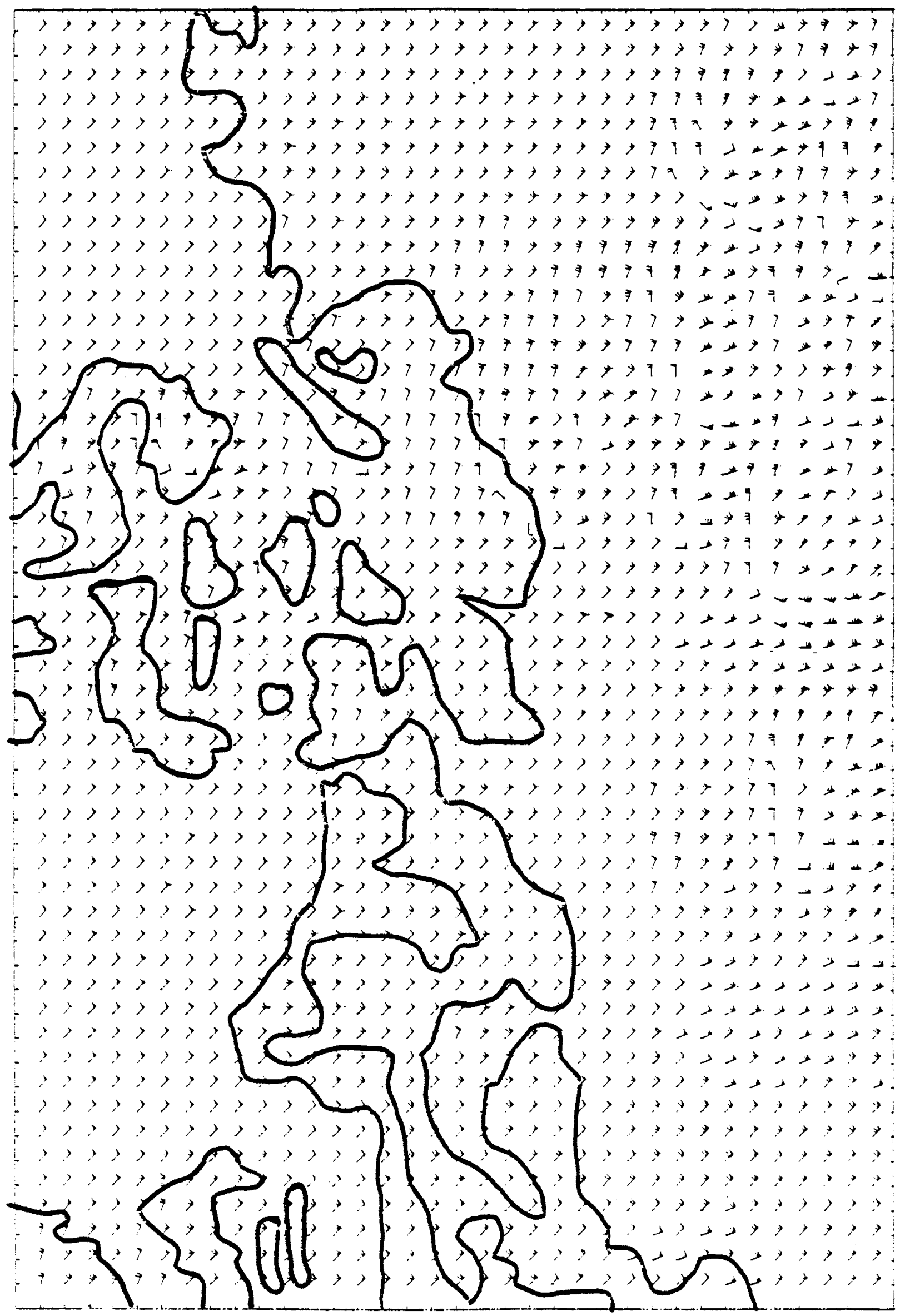


Figure E55. Windfield Analysis of Northern Puget Sound Study Area Annual Case East. Background flow input: 90.0 degrees at 5.5 meters per second $(\stackrel{\Delta}{-})$.

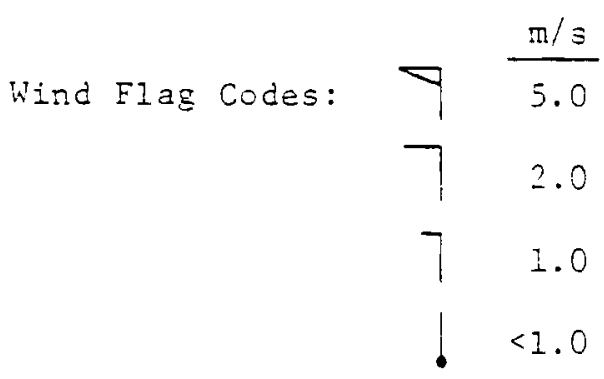




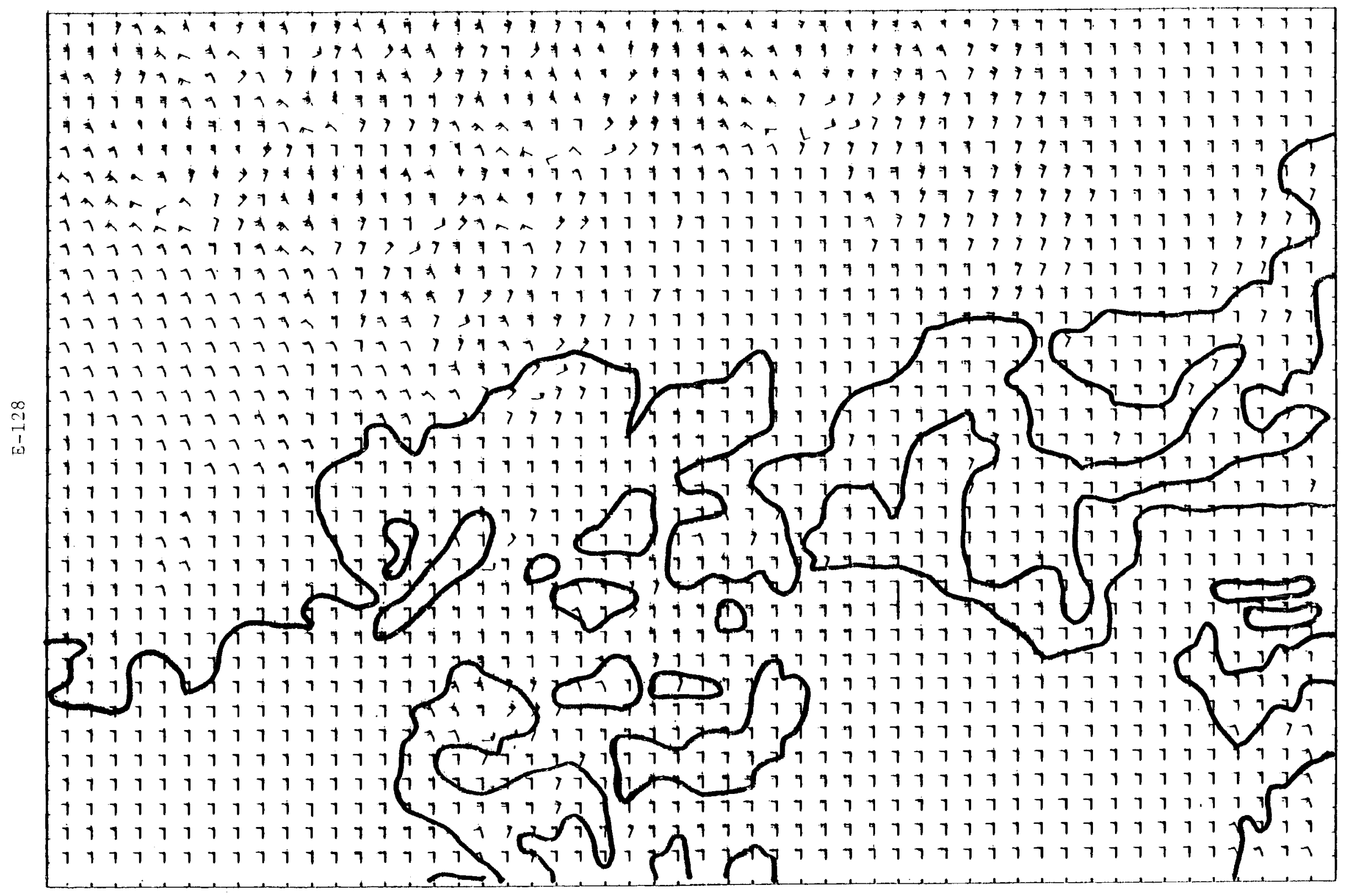




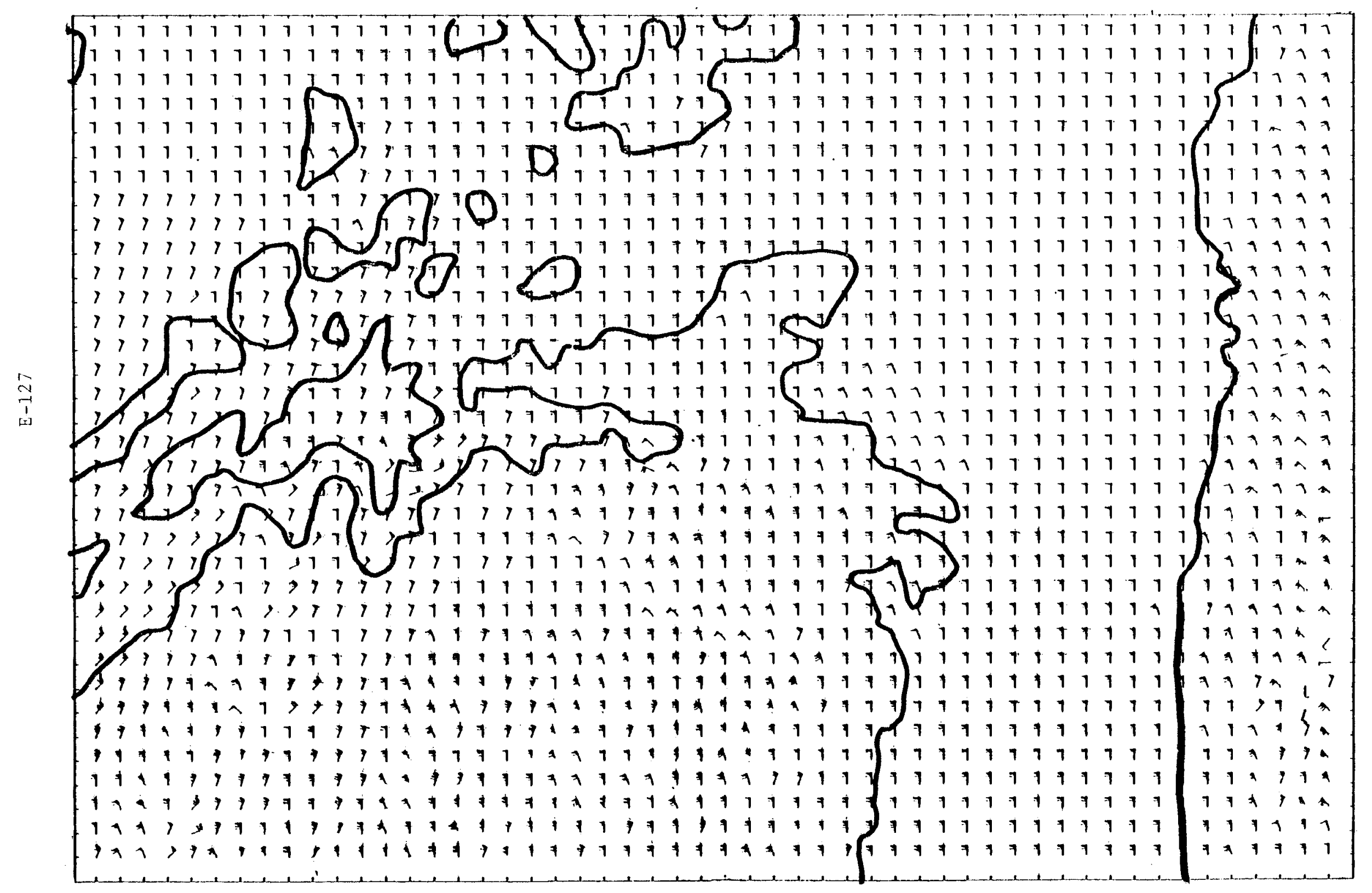


Figure E56. Windfield Analysis of Northern Puget Sound Study Area Annual Case Southeast. Background flow input:

135.0 degrees at 5.5 meters per second (4).

$$
\text { Wind Flag codes: } \begin{array}{r}
\frac{\mathrm{m} / \mathrm{s}}{5.0} \\
2.0 \\
7.0 \\
\end{array}
$$




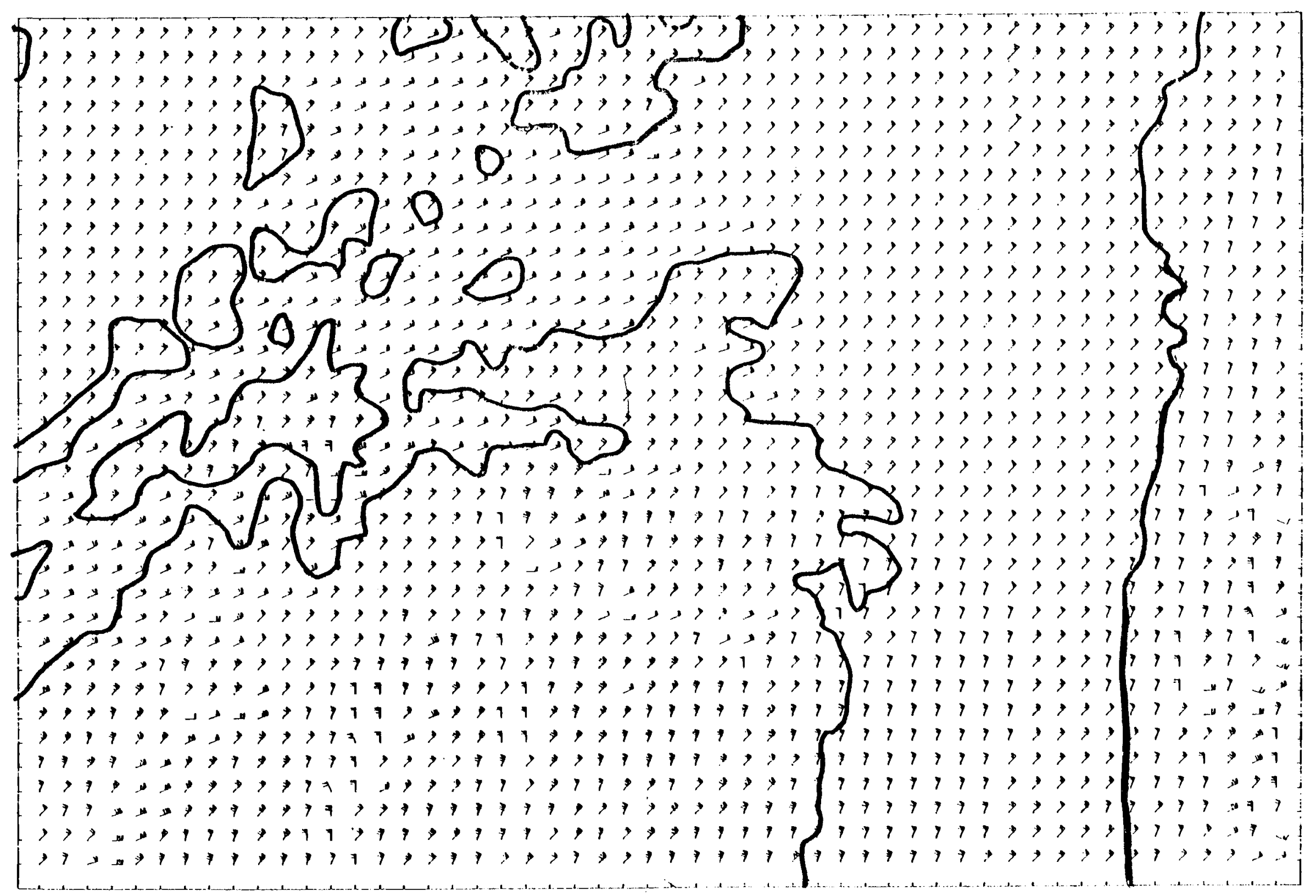




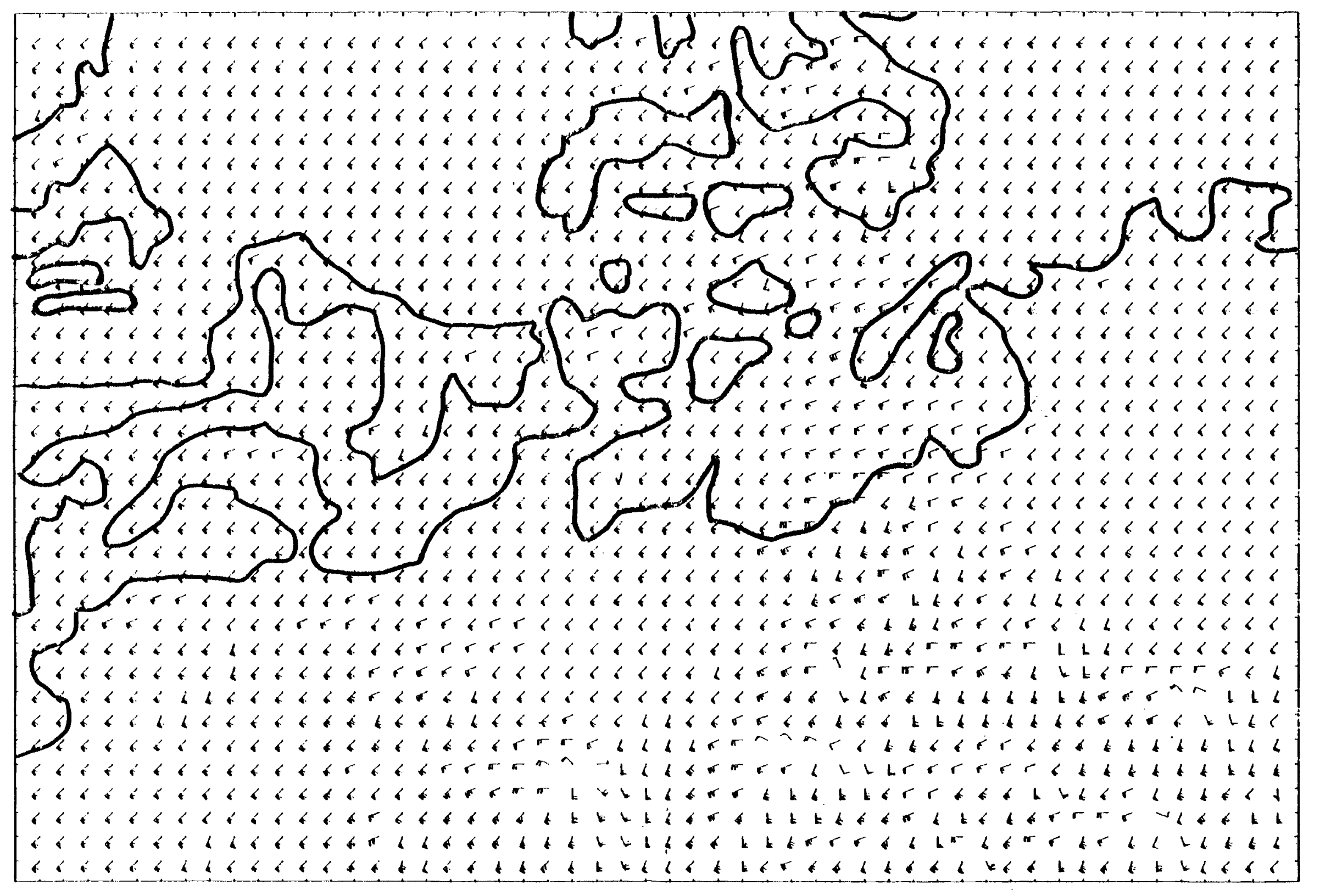


Figure E57. Windfield Analysis of Northern Puget Sound Study Area Annual Case South. Background flow input: 180.0 degrees at 5.5 meters per second $(k)$.

$$
\text { Wind Flag codes: } \begin{array}{r}
\frac{\mathrm{m} / \mathrm{s}}{5.0} \\
2.0 \\
7 \begin{array}{r}
1.0 \\
\end{array}
\end{array}
$$




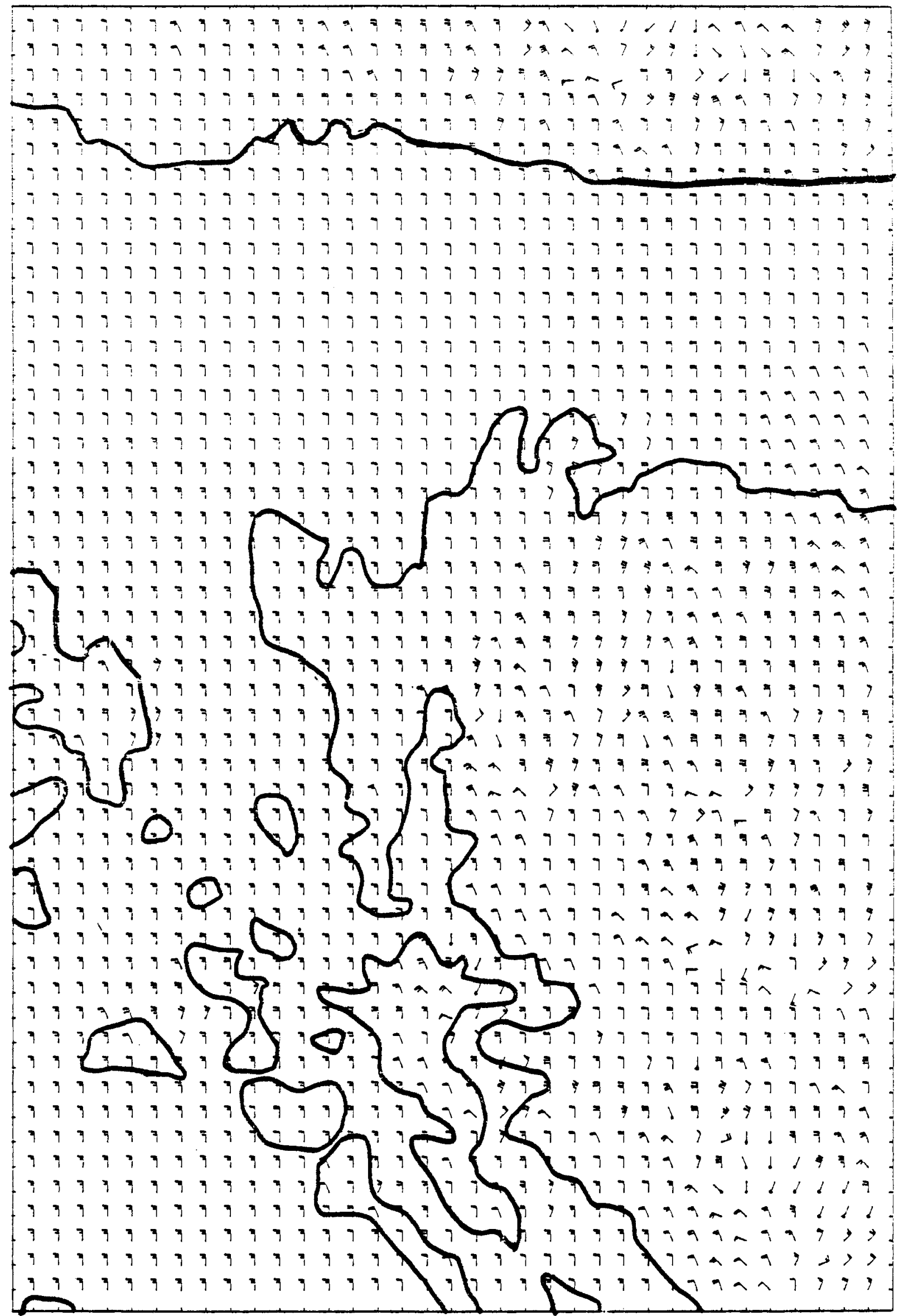




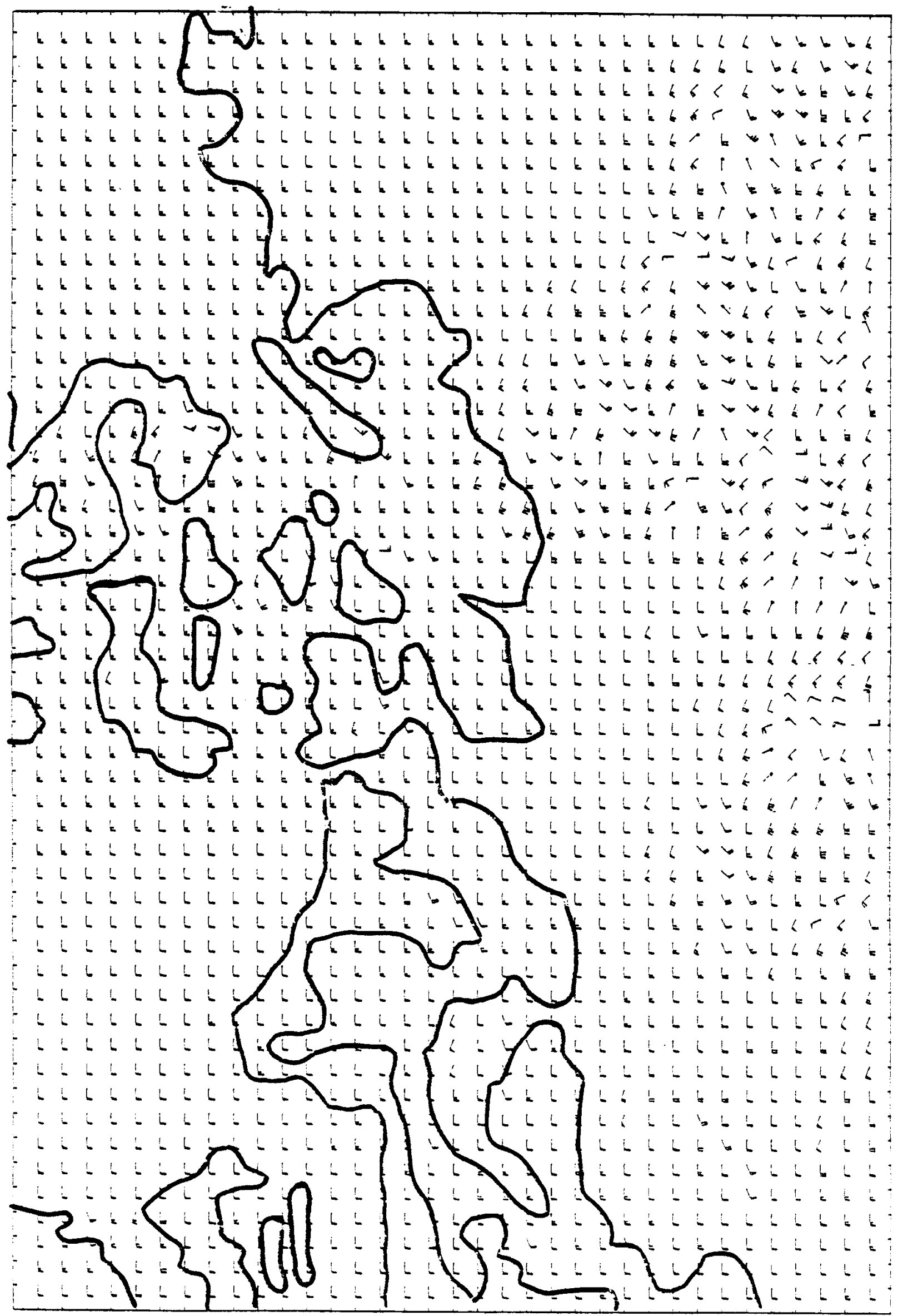


Figure E58. Windfield Analysis of Northern Puget Sound Study Area Annual Case Southwest. Background flow input: 225.0 degrees at 5.5 meters per second ( $($ ).

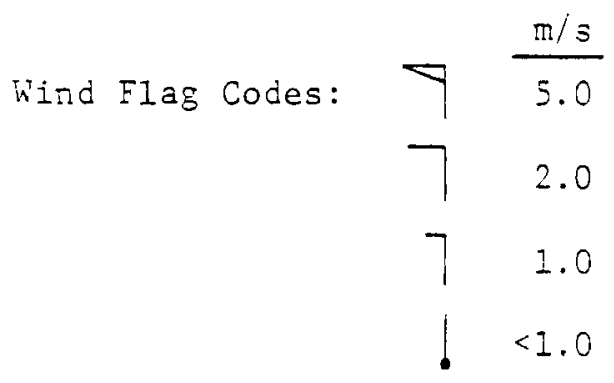




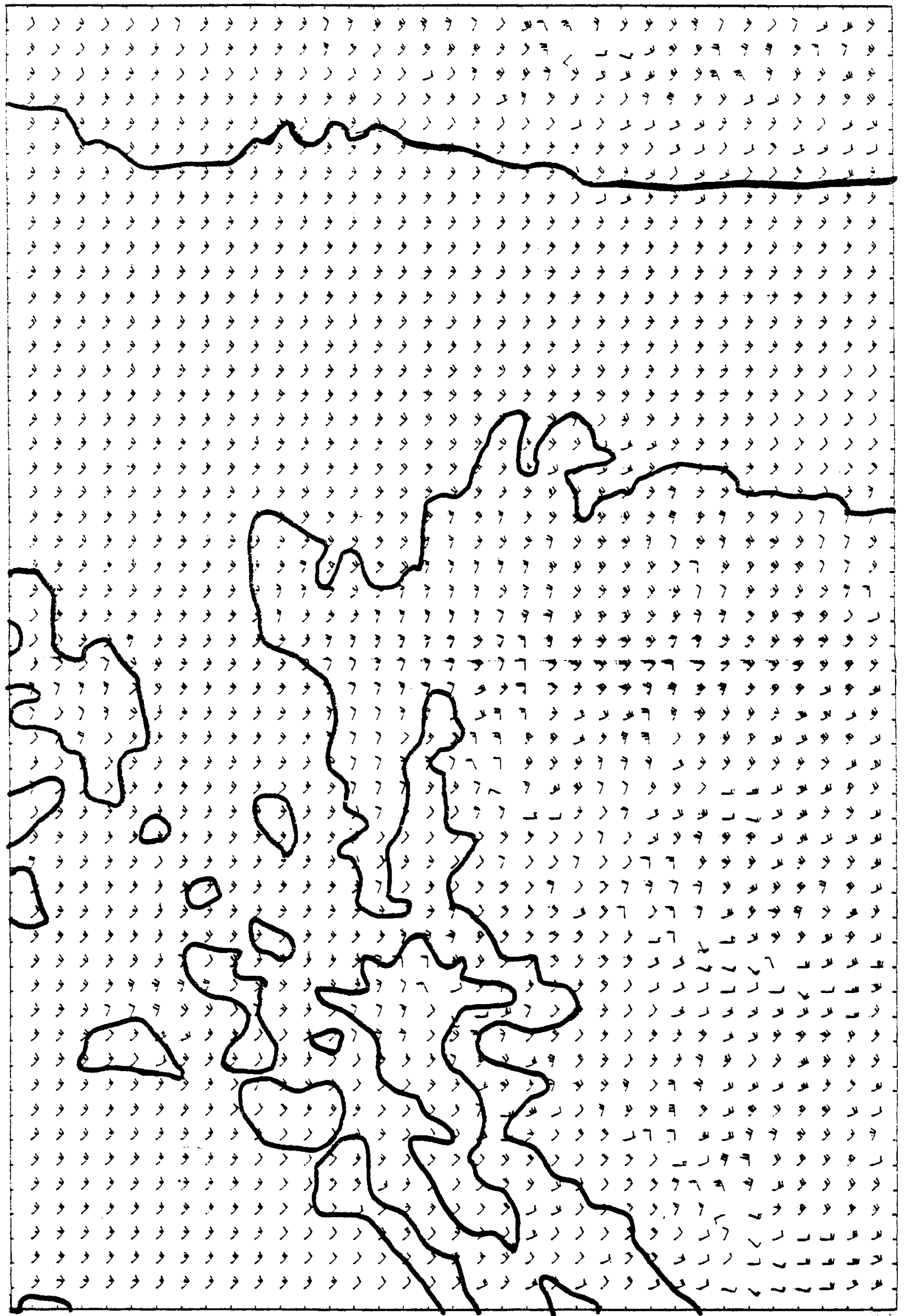

$9 \varepsilon \tau-\exists$ 


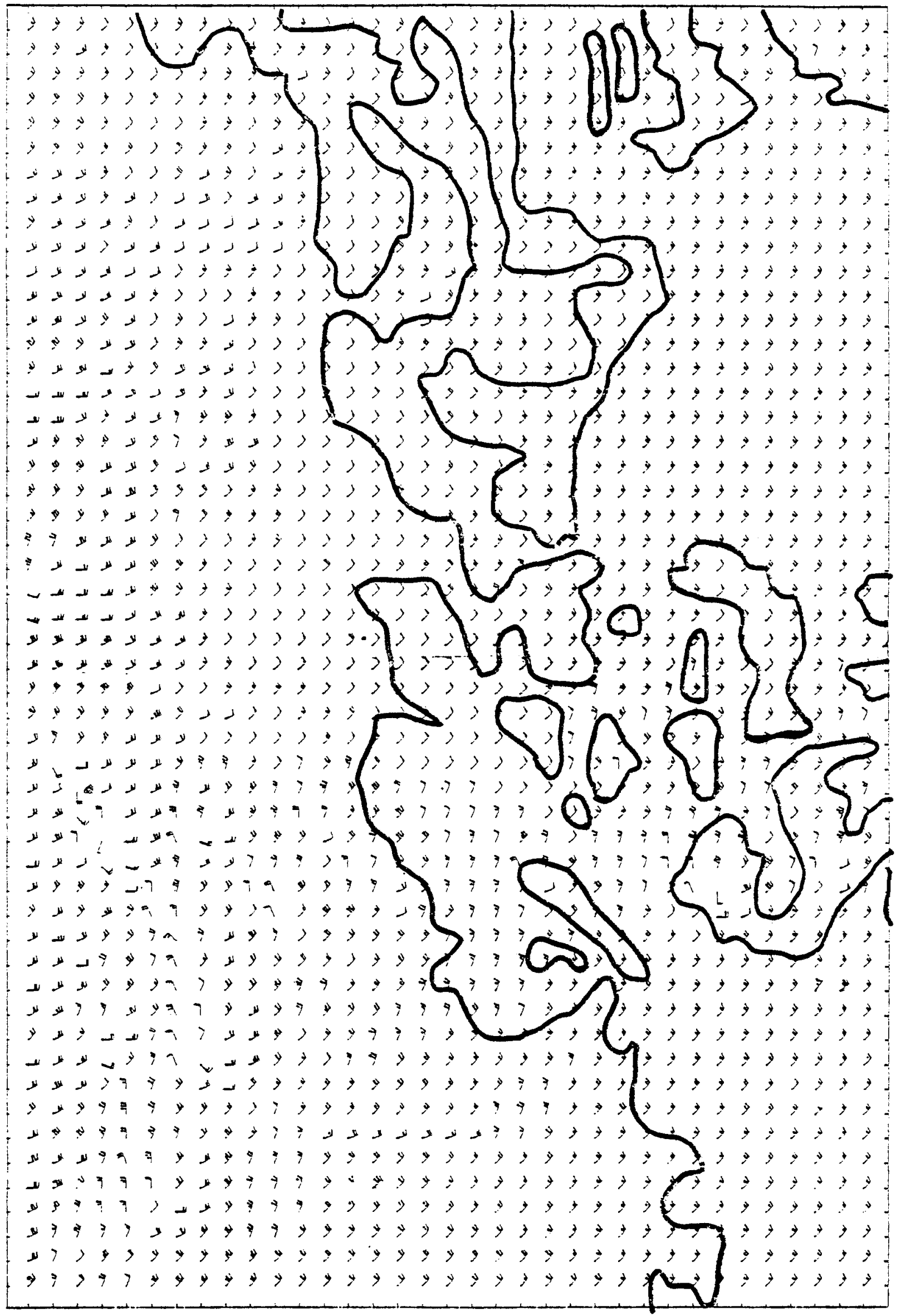


Figure E59. Windfield Analysis of Northern Puget Sound Study Area Annual Case West. Background flow input: 270.0 degrees at 5.5 meters per second $(\pi)$.

$$
\text { Wind Flag codes: } \begin{array}{r}
\frac{\mathrm{m} / \mathrm{s}}{5.0} \\
2.0 \\
7.0 \\
\downarrow
\end{array}
$$




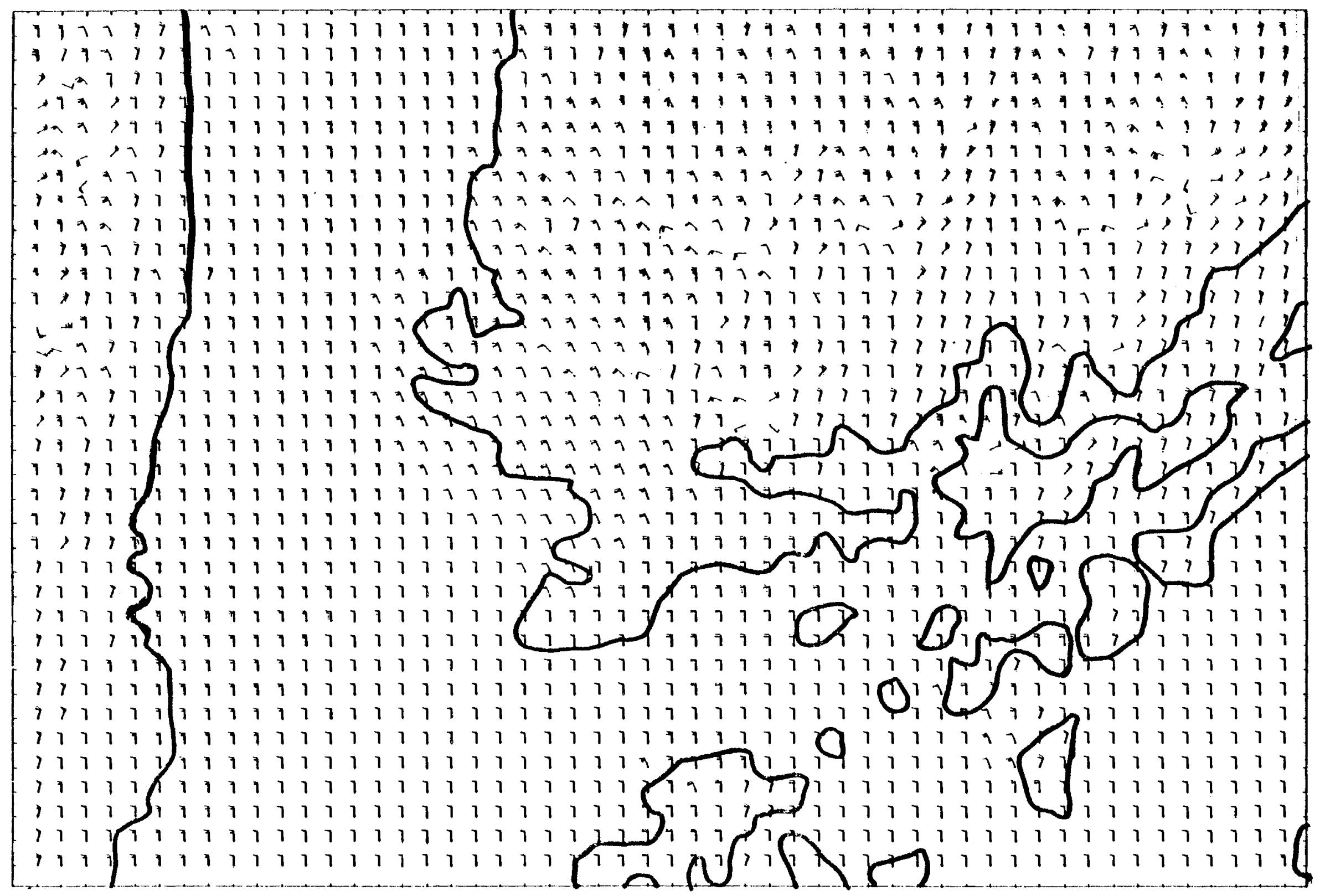




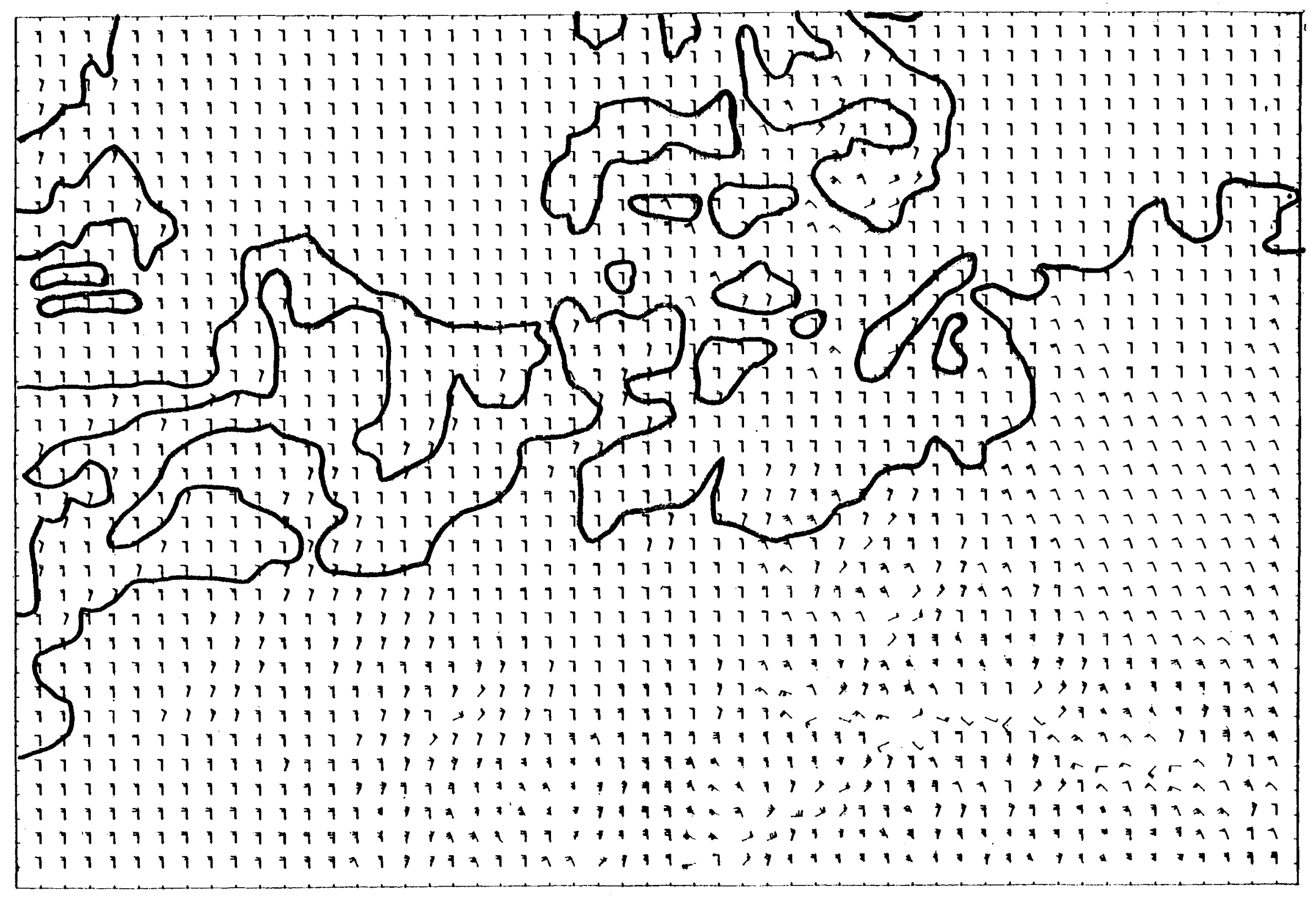


Figure E60. Windfield Analysis of Northern Puget Sound Study Area Annual Case Northwest. Background flow input: 315.0 degrees at 5.5 meters per second ( $\lambda$ ).

$$
\text { Wind Flag codes: } \begin{array}{r}
\frac{\mathrm{m} / \mathrm{s}}{5.0} \\
2.0 \\
7 \quad 1.0 \\
\end{array}
$$




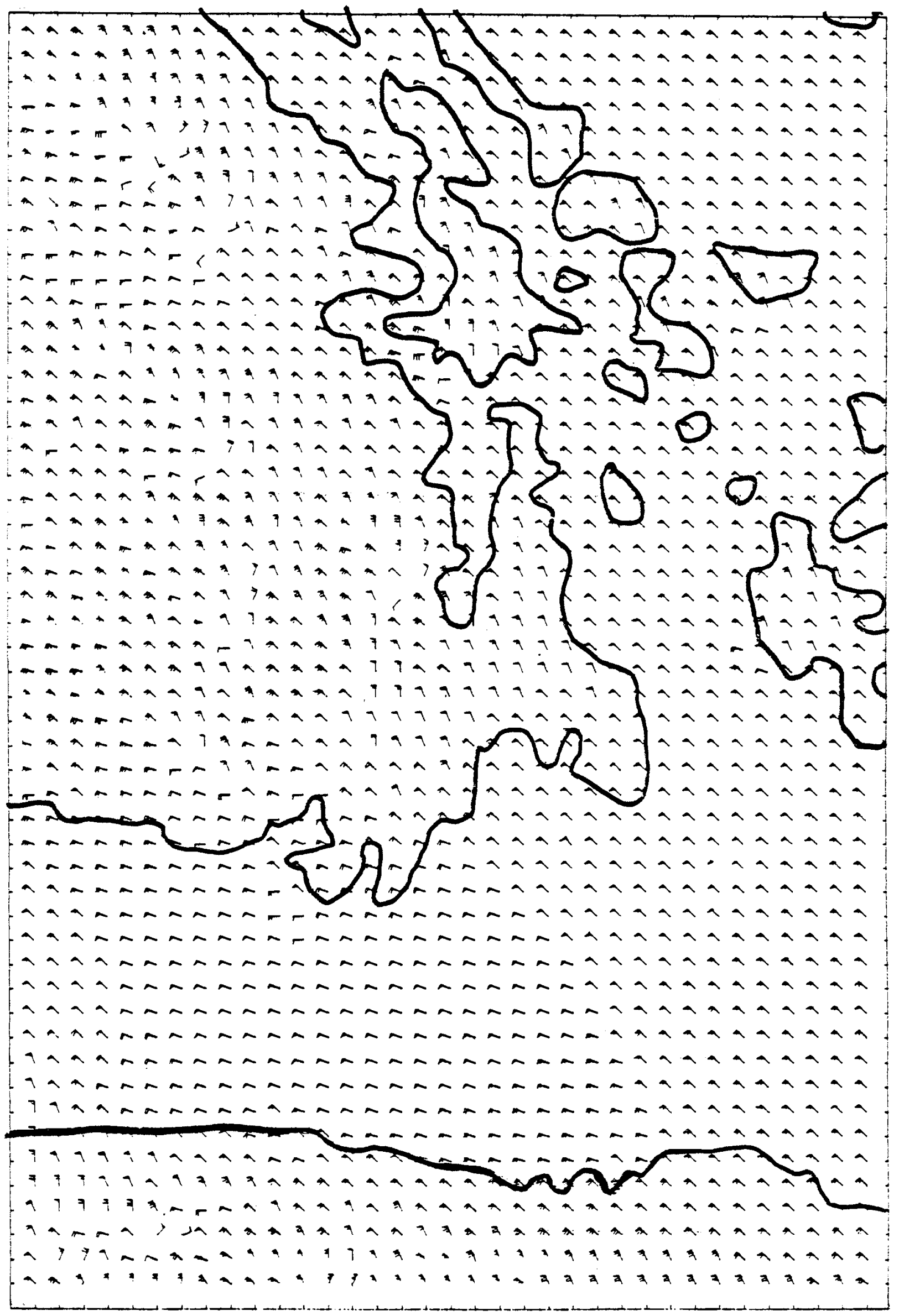




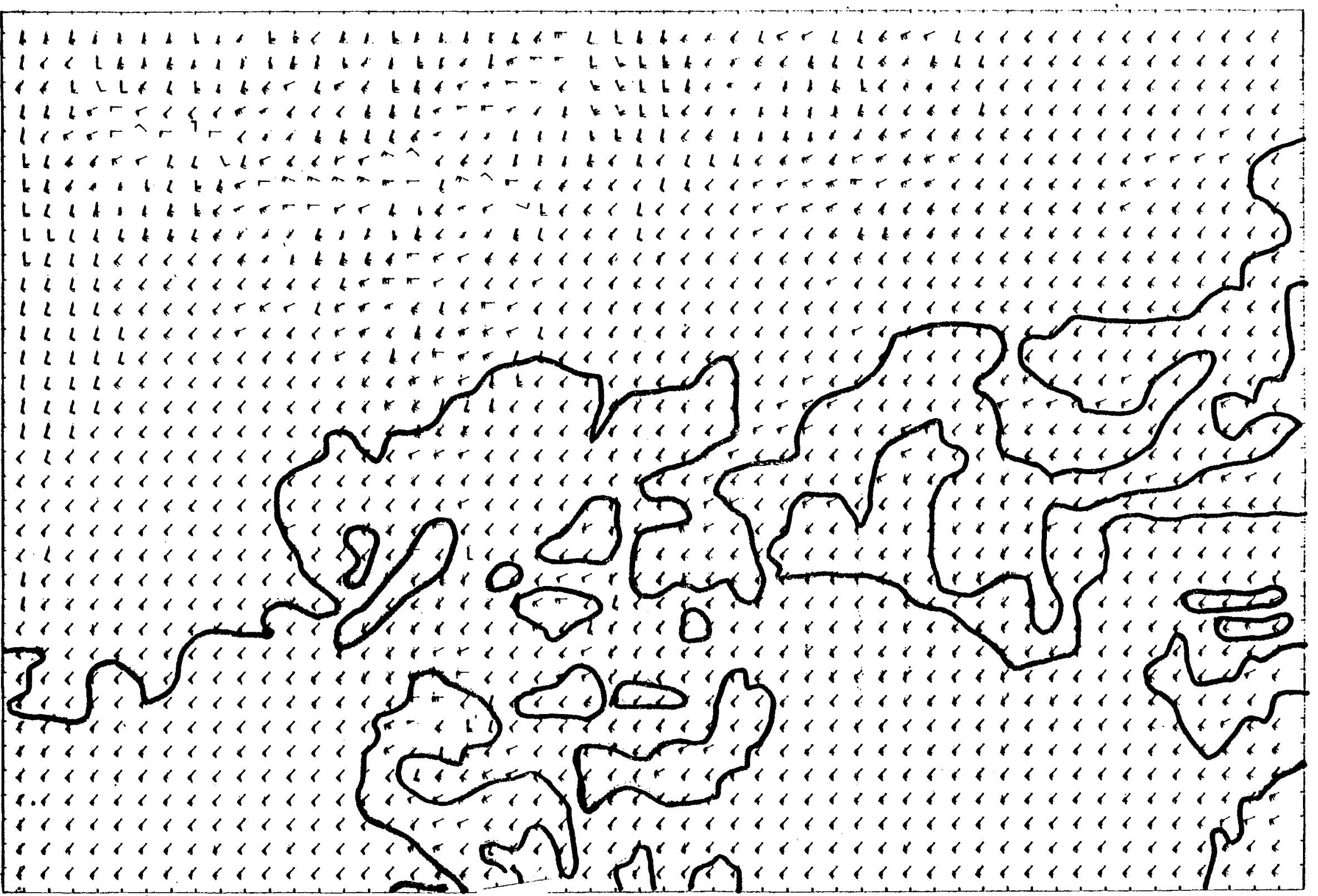


FIGURE I61. MIXING VOLUME INDEX FOR ANNUAL CASE-WIND FROM NORTH.

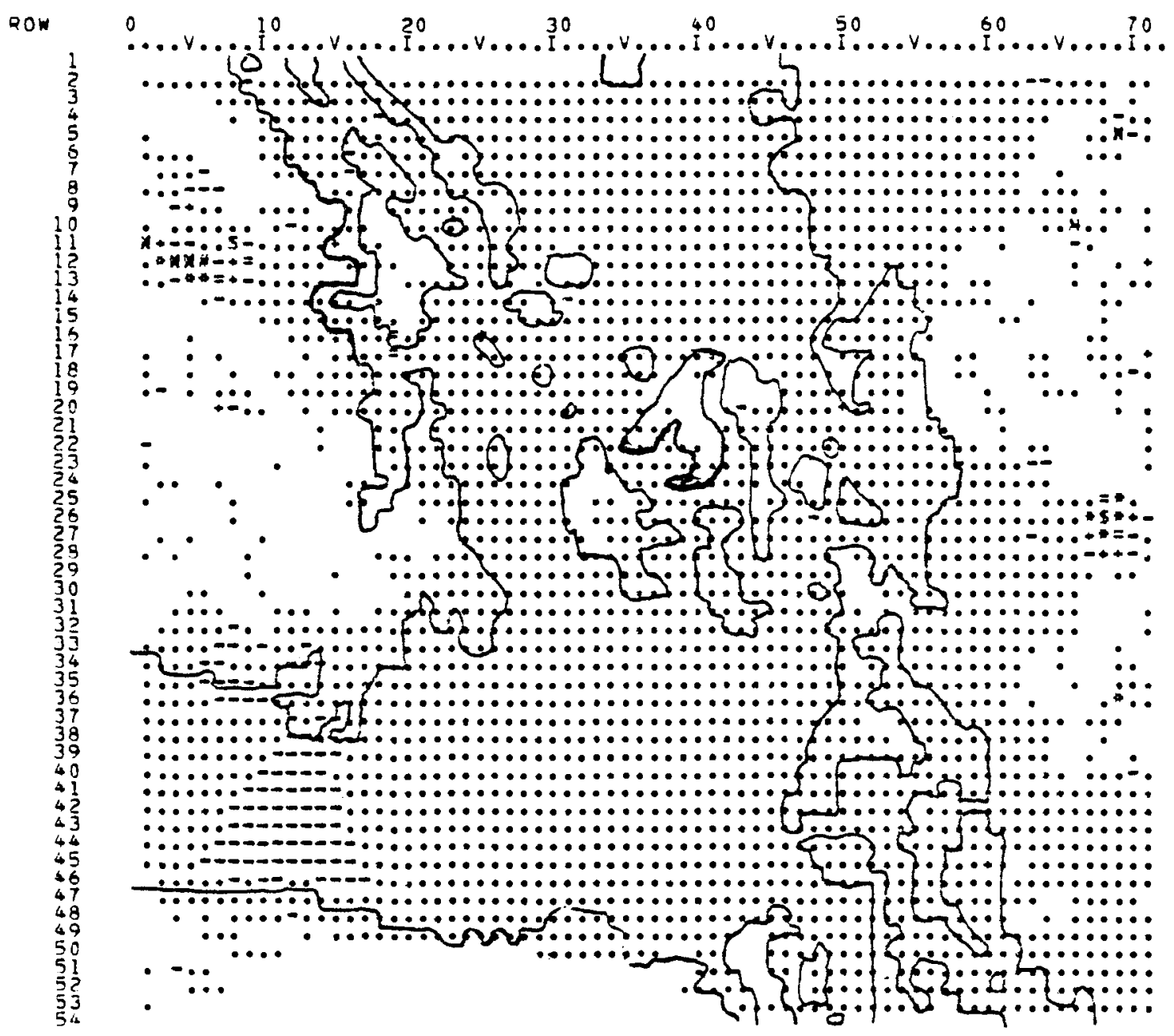

MIXENG VOLUSE IXDER:

\begin{tabular}{|c|c|c|c|c|c|c|c|c|c|c|}
\hline CODE & 3lank & . & - & + & $=$ & * & 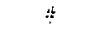 & $\$$ & $\ddot{*}$ & $x$ \\
\hline $\operatorname{CNEx}\left(\mathrm{sec} / \mathrm{m}^{3}\right)$ & $<20$ & $20-60$ & $40-50$ & $50-80$ & $30-100$ & $100-120$ & $120-1 \div 0$ & $140-160$ & $160-180$ & $>180$ \\
\hline
\end{tabular}


FIGURE E62. MIXING VOLIME INDEX FOR ANNUAL CASE-WIND FROM NORTHEAST.

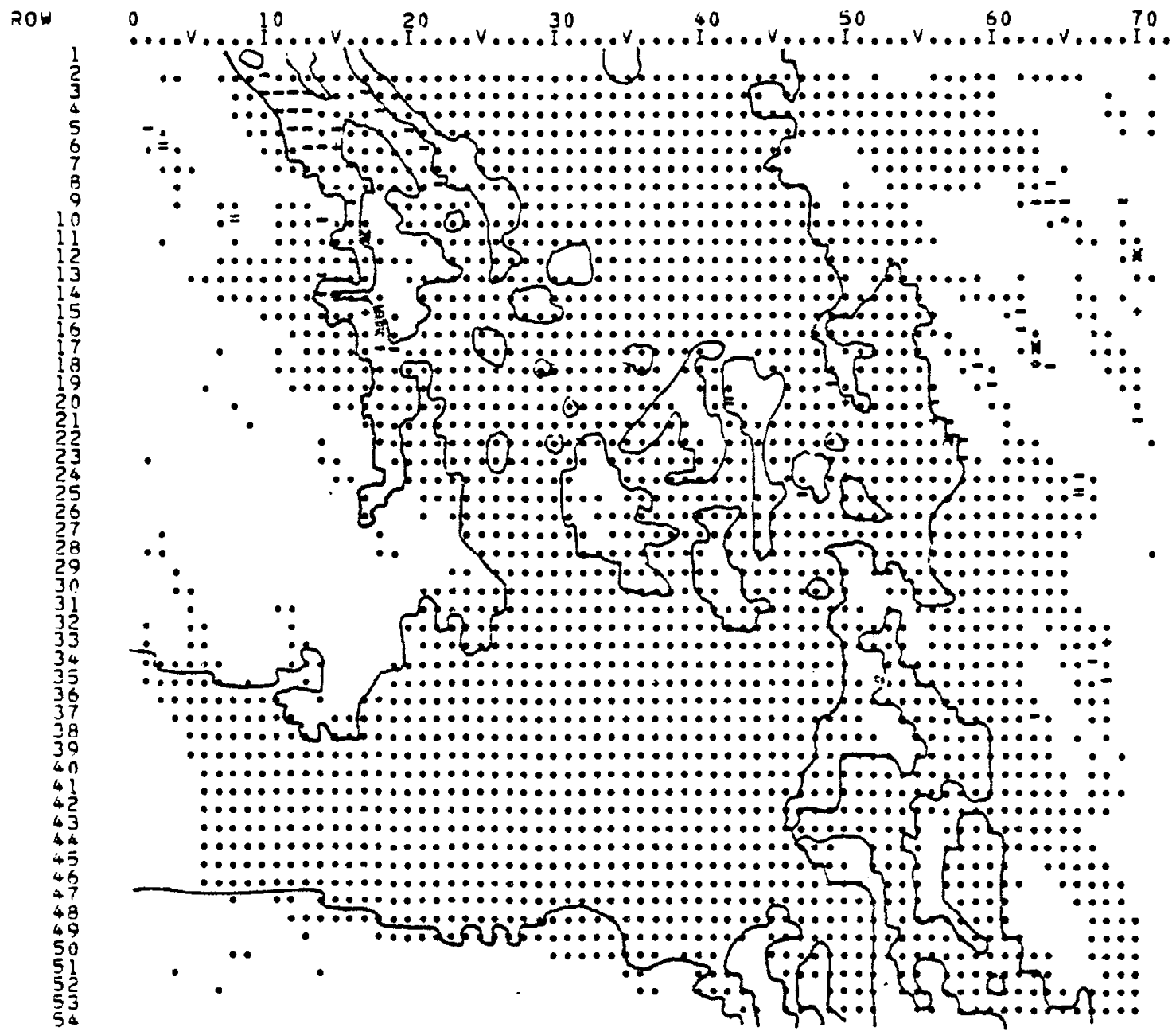

YIXING VOLLIE INDEX:

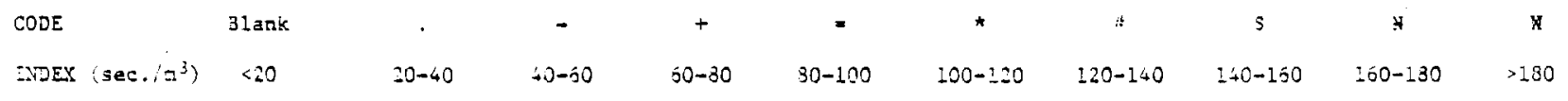


FIGURE E63. MIXING VOLUME INDEX FOR ANNUAL CASE-WIND FROM EAST.

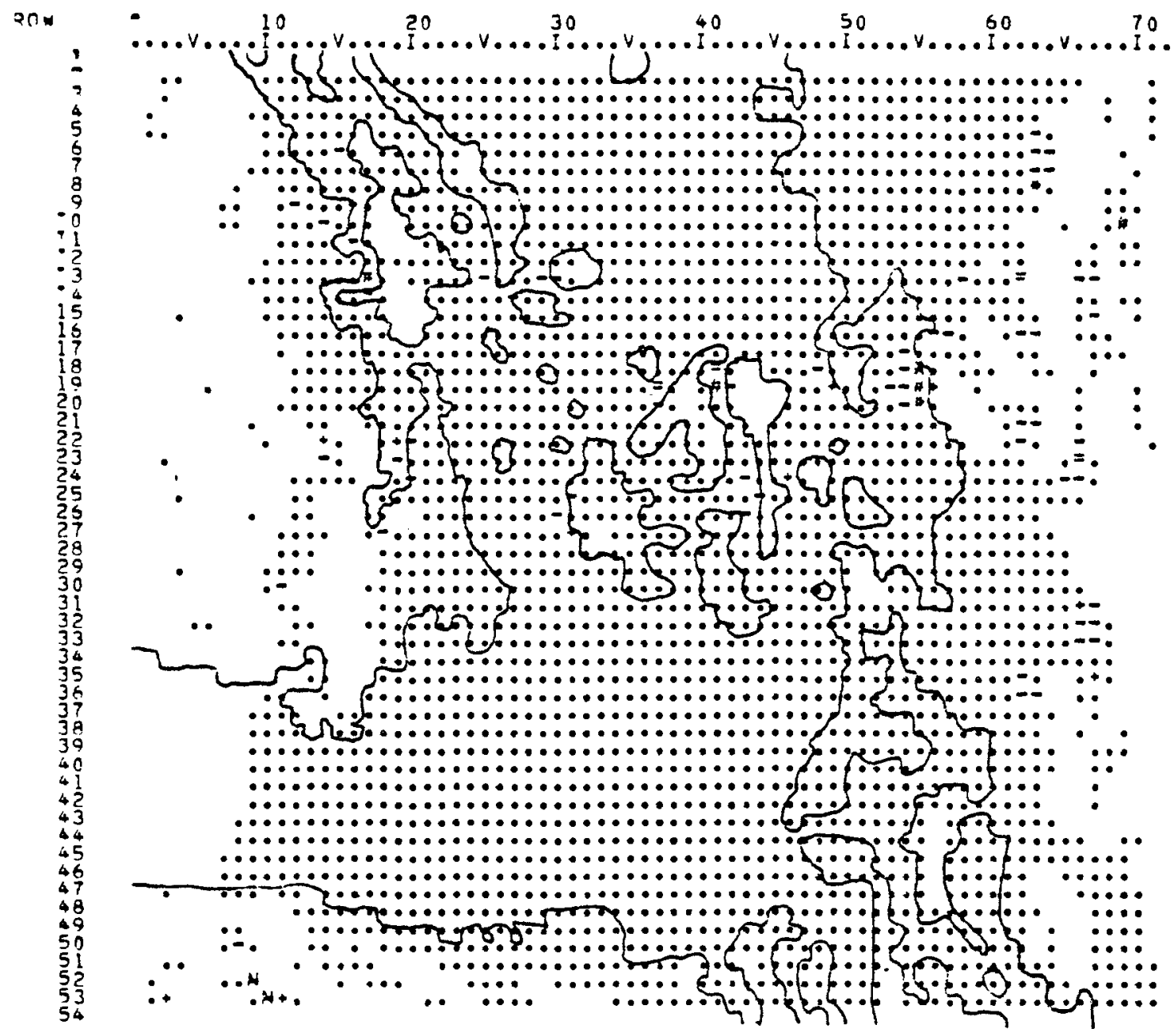

MIXIXC YOLUNE LIDEX:

\begin{tabular}{|c|c|c|c|c|c|c|c|c|c|c|}
\hline CODE & 31 ank & . & - & + & 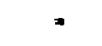 & * & 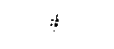 & s & $*$ & $x$ \\
\hline $\operatorname{ZDEX}\left(\right.$ sac. $\left(\mathrm{I}^{3}\right)$ & $<20$ & $20-40$ & $\angle 0=50$ & $60-30$ & $30-100$ & $100-120$ & $120-1 \div 0$ & $140-i 60$ & $160-180$ & $>i \varepsilon 0$ \\
\hline
\end{tabular}


FIGURE E64. MIXING VOLUME INDEX FOR ANNUAL CASE-WIND FROM SOUTHEAST.

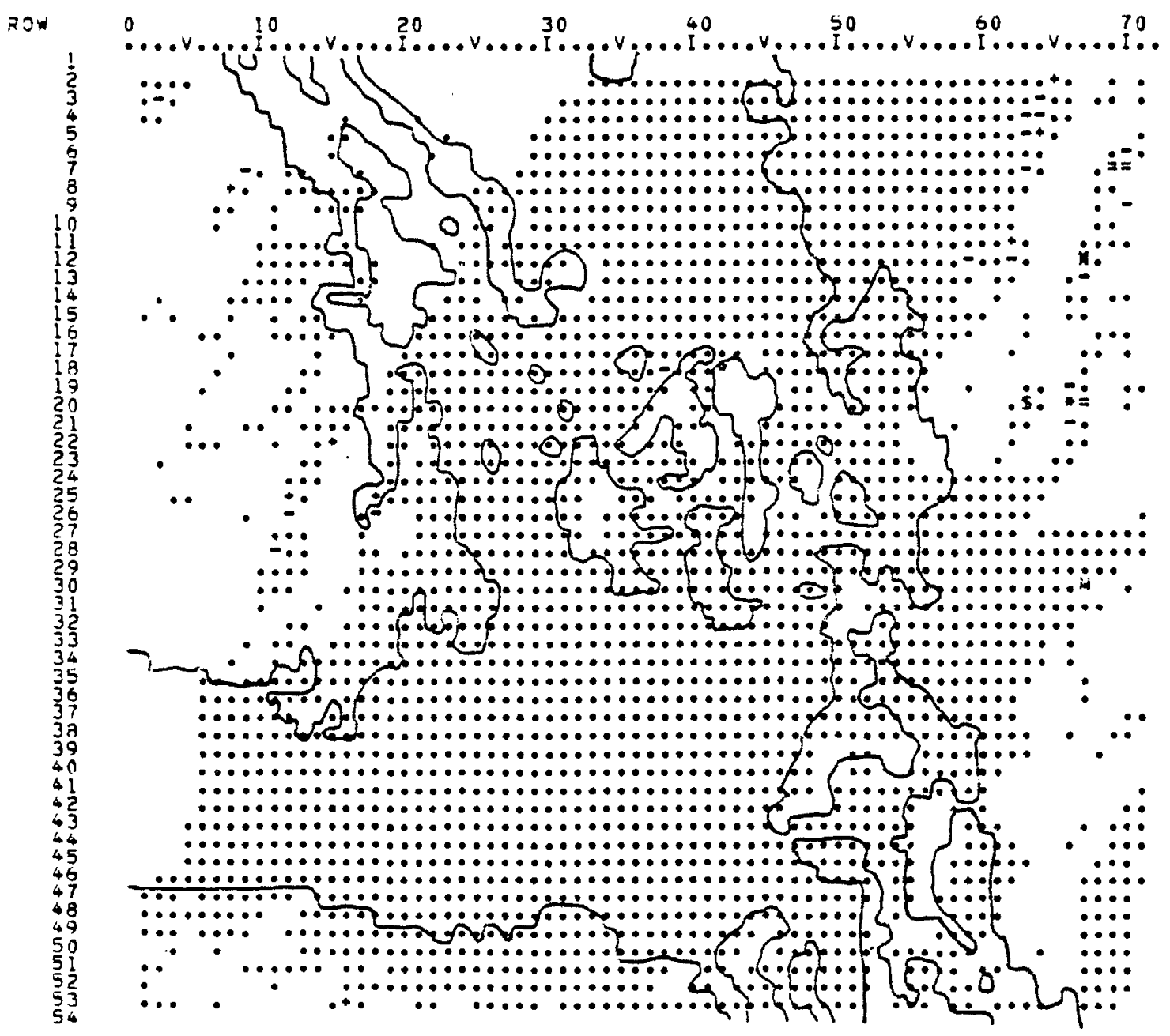

MIXING VOLUNE INDEX:

\begin{tabular}{|c|c|c|c|c|c|c|c|c|c|c|}
\hline CODE & 3ienk & . & - & - & $=$ & * & $\therefore$ & ș & $\because$ & $y$ \\
\hline $\operatorname{trosx}\left(\sec . / \pi^{3}\right)$ & $<20$ & $=0-40$ & $40-50$ & $50-30$ & $30-100$ & $100-120$ & $120-140$ & $1 \div 0-150$ & $150-130$ & $>1.30$ \\
\hline
\end{tabular}


FIGURE E65. MIXING VOLUME INDEX FOR ANNUAL CASE-WIND FROM SOUTH.

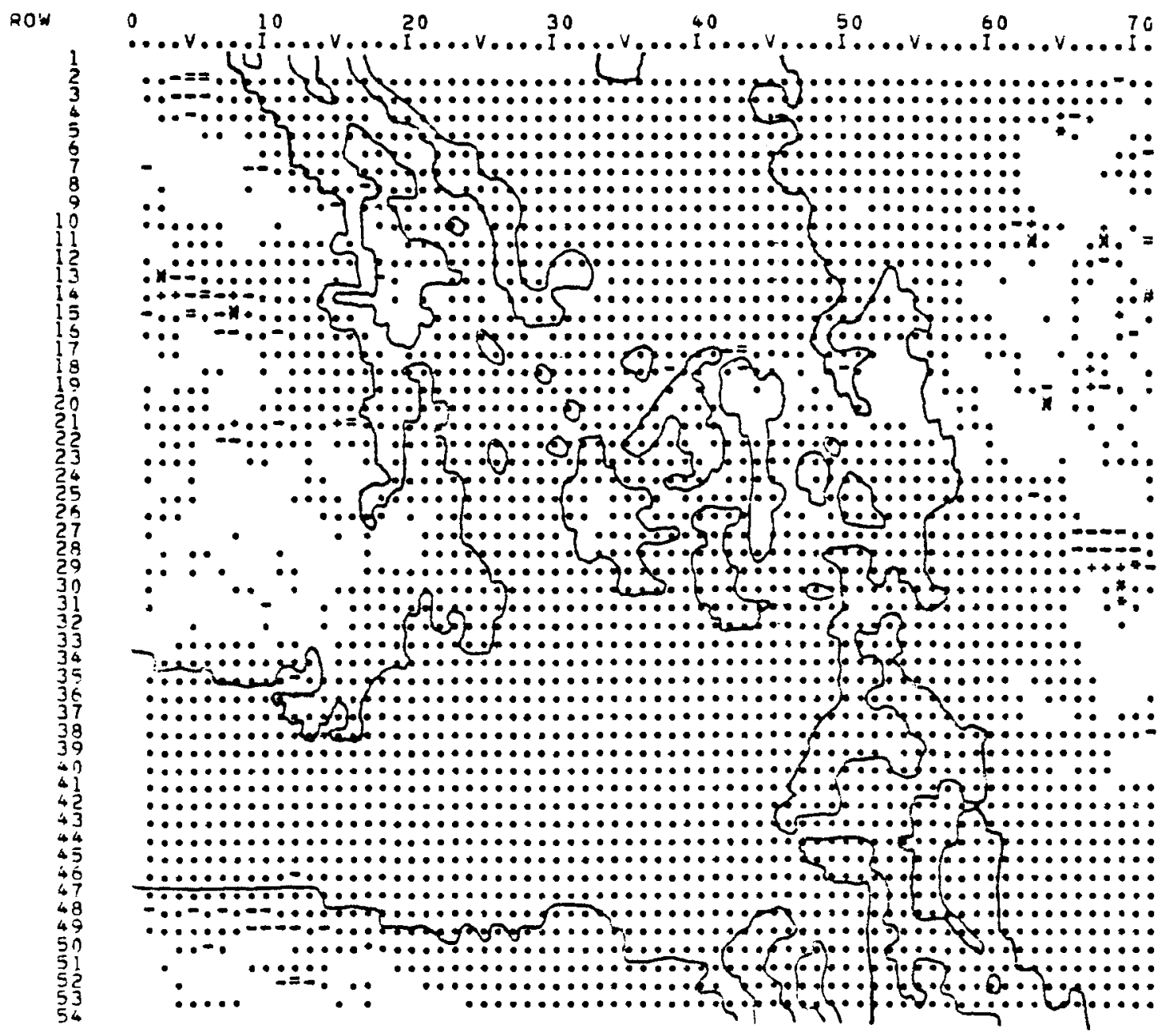

SIXING VOLUME INDER:

\begin{tabular}{|c|c|c|c|c|c|c|c|c|c|c|}
\hline CODE & 3lank & - & - & - & $=$ & * & 4 & s & 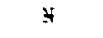 & $x$ \\
\hline INDEX $\left(\sec / / \beth^{3}\right)$ & $\leqslant 20$ & $20-40$ & $60-50$ & $60-30$ & $30-100$ & $100-120$ & $220-140$ & $140-160$ & $260-180$ & $>130$ \\
\hline
\end{tabular}


FIGURE E66. MIXING VOLUME INDEX FOR ANNUAL CASE-WIND FROM SOUTHWEST.

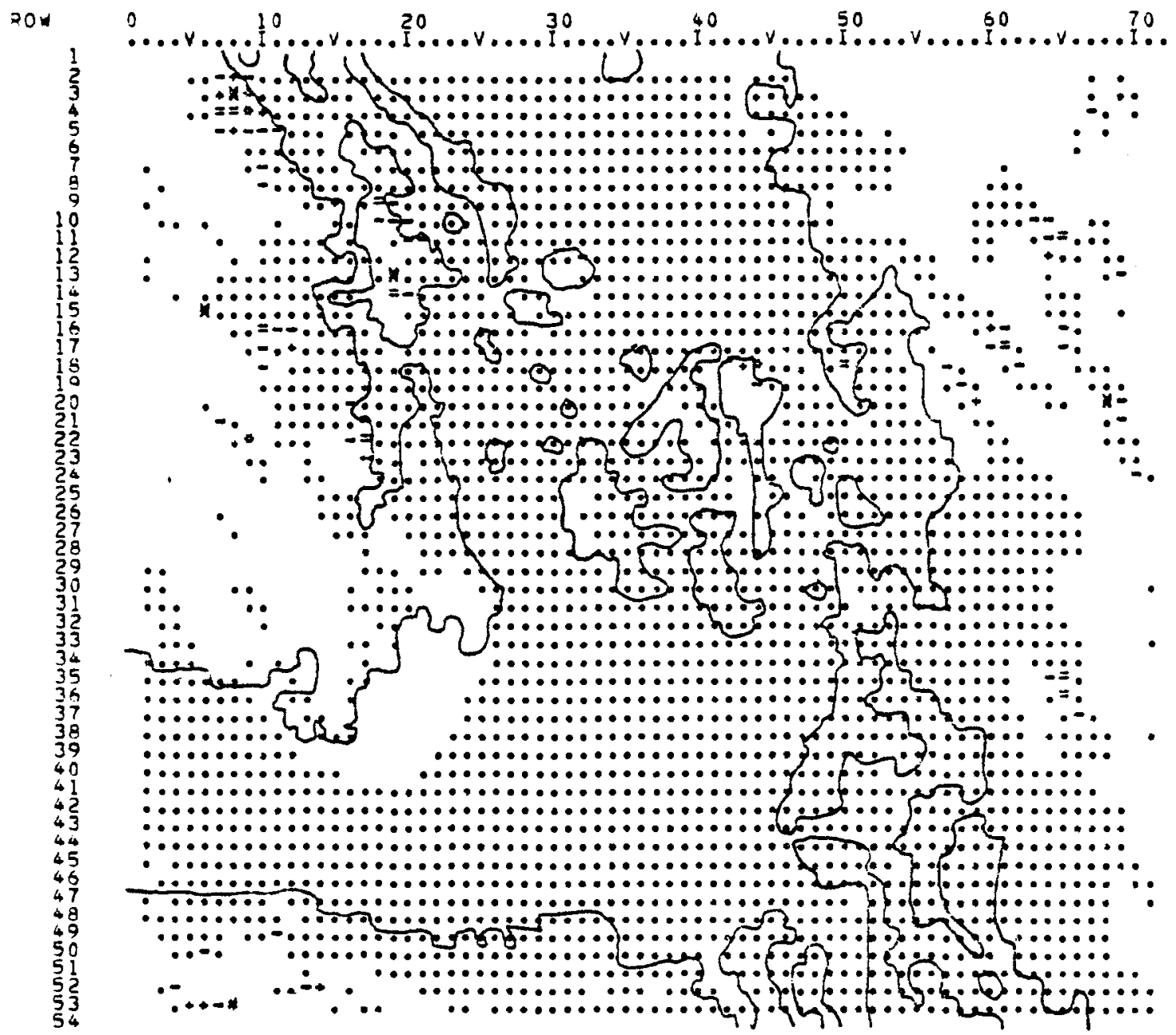

MIXING YOLTME INDEX:

\begin{tabular}{|c|c|c|c|c|c|c|c|c|c|c|}
\hline $\operatorname{cose}$ & 31 ank & . & - & + & $=$ & * & 4 & i & $\because$ & $x$ \\
\hline INDES $\left(\mathrm{sec} . / \mathrm{I}^{3}\right)$ & $=20$ & $20-40$ & $40-50$ & $50-30$ & $30-100$ & $100-120$ & $220-160$ & $140-150$ & $150-180$ & $>180$ \\
\hline
\end{tabular}


FIGURE E 57. MIXING VOLUME INDEX FOR ANNUAL CASE-WIND FROM WEST.

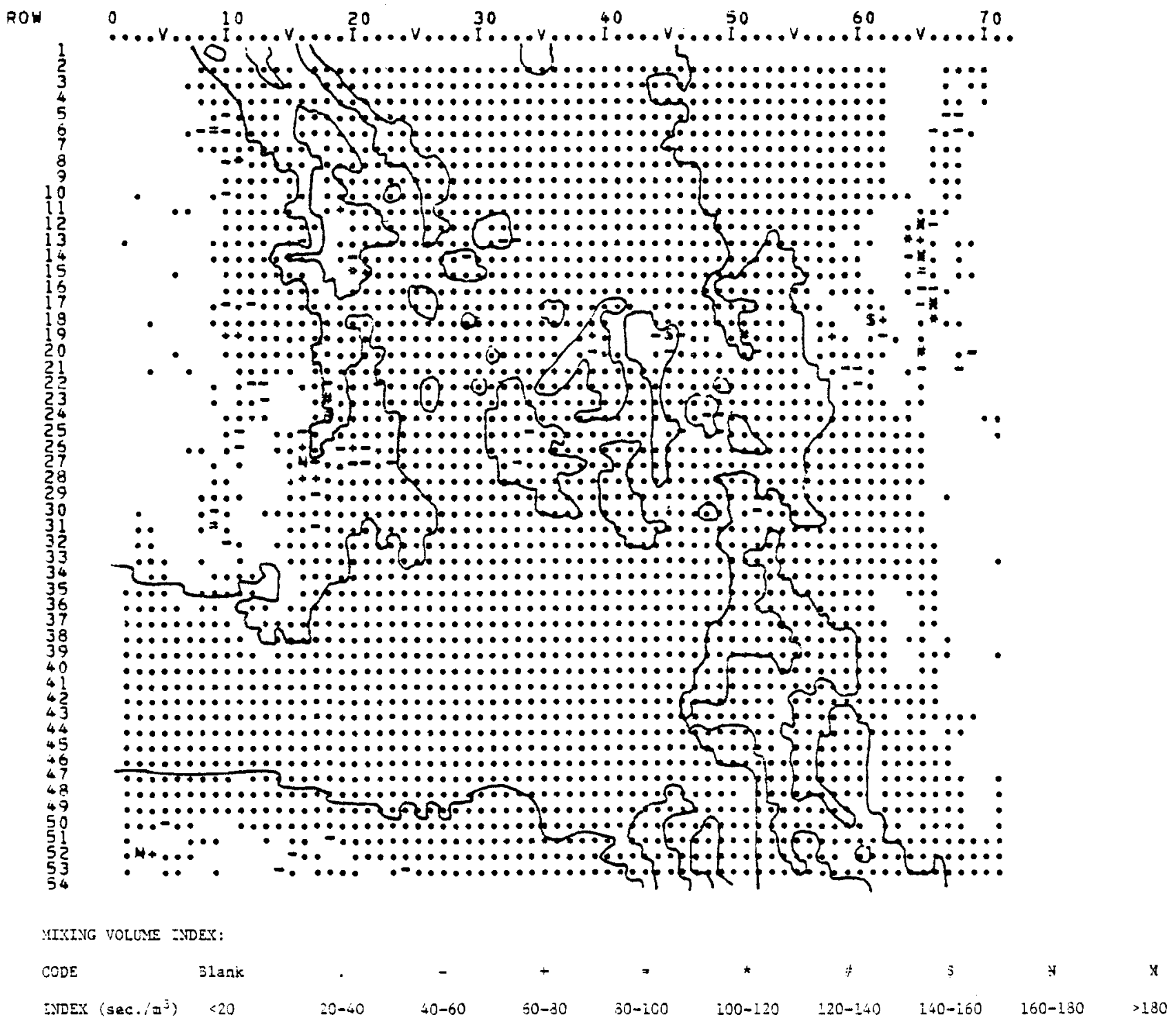


FIGURE E 68. MIXING VOLLAE INDEX FOR ANNUAL CASE-WIND FROM NORTHWEST.

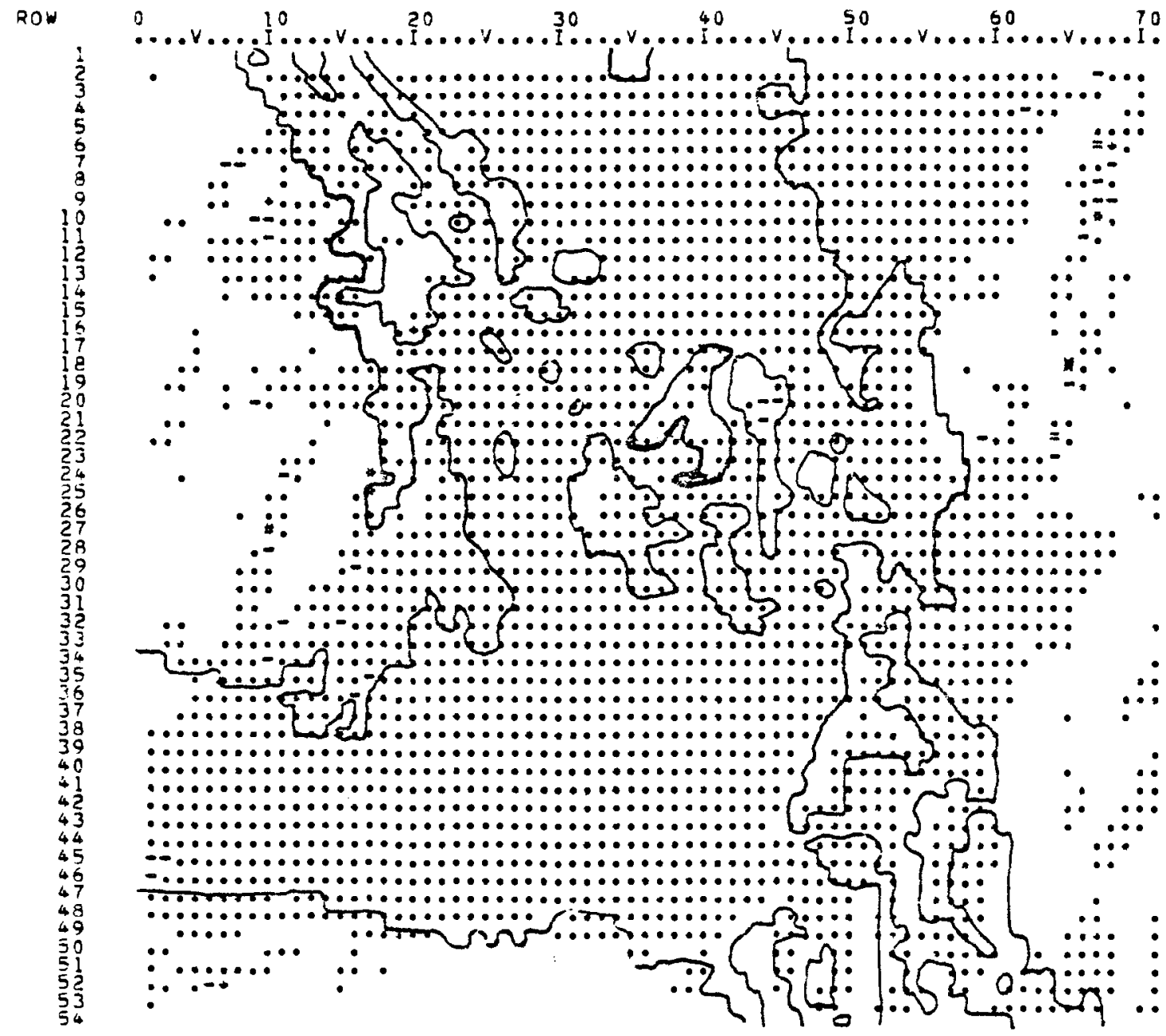

YIXESG YOLUE INDEX:

\begin{tabular}{|c|c|c|c|c|c|c|c|c|c|c|}
\hline $\cos 5$ & $31 \mathrm{ank}$ & . & - & + & $=$ & * & $:$ & 5 & $\sharp$ & x \\
\hline $\operatorname{ADER}\left(\mathrm{sec} \cdot / \pi^{3}\right)$ & $<20$ & $20-40$ & $\div 0-610$ & $50-80$ & $80-100$ & $100-120$ & $220-1<0$ & $140-i 60$ & $160-180$ & $>180$ \\
\hline
\end{tabular}





\section{$\underline{\text { DISTRIBUTION }}$}

No. of

Copies

OFFSITE

A. A. Churm Department of Energy Chicago Patent Group 9800 South Cass Avenue

Argonne, IL 60439

27 Department of Energy

Technical Information Center

Mrs. R. Clusen

Assistant Secretary for Environment Department of Energy (M/S E-201)

washington, DC 20545

P. W. House

Office of Environmental Assessments

Department of Energy (M/S E-201)

Washington, DC 20545

W. E. Marlott

Marlatt \& Associates

3611 Richmond Drive

Fort Collins, CO 80526

R. D. Shull

Office of Environmental Assessments

Department of Energy (M/S E-201)

Washington, DC 20545
No. of

Copies

PACIFIC NORTHWEST LABORATORY

W. J. Bair

D. L. Hessel

D. S. Renne

W. F. Sandusky

Economics Library (3)

Publishing Coordination (1)

Technical Information Files (3)

(3)
tion Files

ONSITE

\section{DOE-RICHLAND}

H. E. Ransom 
